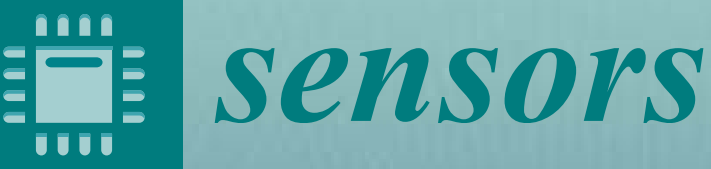

Volume 2

Sensors in

Agriculture

Edited by

Dimitrios Moshou

Printed Edition of the Special Issue Published in Sensors 
Sensors in Agriculture 



\section{Sensors in Agriculture}

\section{Volume 2}

Special Issue Editor

Dimitrios Moshou

\section{MDPI}


Special Issue Editor

Dimitrios Moshou

Aristotle University of Thessaloniki

Greece

\section{Editorial Office}

MDPI

St. Alban-Anlage 66

4052 Basel, Switzerland

This is a reprint of articles from the Special Issue published online in the open access journal Sensors (ISSN 1424-8220) from 2017 to 2018 (available at: https: / /www.mdpi.com/journal/sensors/special_ issues/agriculture)

For citation purposes, cite each article independently as indicated on the article page online and as indicated below:

LastName, A.A.; LastName, B.B.; LastName, C.C. Article Title. Journal Name Year, Article Number, Page Range.

Volume 2

ISBN 978-3-03897-744-5 (Pbk)

ISBN 978-3-03897-745-2 (PDF)
Volume 1-2

ISBN 978-3-03897-858-9 (Pbk)

ISBN 978-3-03897-859-6 (PDF)

(C) 2019 by the authors. Articles in this book are Open Access and distributed under the Creative Commons Attribution (CC BY) license, which allows users to download, copy and build upon published articles, as long as the author and publisher are properly credited, which ensures maximum dissemination and a wider impact of our publications.

The book as a whole is distributed by MDPI under the terms and conditions of the Creative Commons license CC BY-NC-ND. 


\section{Contents}

About the Special Issue Editor $\ldots \ldots \ldots \ldots \ldots \ldots \ldots \ldots \ldots \ldots$

Preface to "Sensors in Agriculture" $\ldots \ldots \ldots \ldots \ldots \ldots \ldots \ldots \ldots \ldots$

Qinghan Dong, Jia Liu, Limin Wang, Zhongxin Chen and Javier Gallego

Estimating Crop Area at County Level on the North China Plain with an Indirect Sampling of

Segments and an Adapted Regression Estimator

Reprinted from: Sensors 2017, 17, 2638, doi:10.3390/s17112638 . . . . . . . . . . . . . . .

Wojciech Wojnowski, Tomasz Majchrzak, Tomasz Dymerski, Jacek Gębicki and

Jacek Namieśnik

Portable Electronic Nose Based on Electrochemical Sensors for Food Quality Assessment

Reprinted from: Sensors 2017, 17, 2715, doi:10.3390/s17122715 . . . . . . . . . . . . 10

Hania AL-Saddik, Jean-Claude Simon and Frederic Cointault

Development of Spectral Disease Indices for 'Flavescence Dorée' Grapevine

Disease Identification

Reprinted from: Sensors $\mathbf{2 0 1 7}, 17,2772$, doi:10.3390/s17122772 . . . . . . . . . . . . . . .

Annamaria Castrignanò, Gabriele Buttafuoco, Ruggiero Quarto, Carolina Vitti,

Giuliano Langella, Fabio Terribile and Accursio Venezia

A Combined Approach of Sensor Data Fusion and Multivariate Geostatistics for Delineation of Homogeneous Zones in an Agricultural Field

Reprinted from: Sensors 2017, 17, 2794, doi:10.3390/s17122794 . . . . . . . . . . . . . . . 49

Yingchi Mao, Hai Qi, Ping Ping and Xiaofang Li

Contamination Event Detection with Multivariate Time-Series Data in Agricultural Water Monitoring ${ }^{\dagger}$

Reprinted from: Sensors 2017, 17, 2806, doi:10.3390/s17122806 . . . . . . . . . . . . . .

Naresh Kumar Ravichandran, Ruchire Eranga Wijesinghe, Seung-Yeol Lee,

Muhammad Faizan Shirazi, Hee-Young Jung, Mansik Jeon and Jeehyun Kim

In Vivo Non-Destructive Monitoring of Capsicum Annuum Seed Growth with Diverse $\mathrm{NaCl}$

Concentrations Using Optical Detection Technique

Reprinted from: Sensors 2017, 17, 2887, doi:10.3390/s17122887

Søren Skovsen, Mads Dyrmann, Anders Krogh Mortensen, Kim Arild Steen, Ole Green, Jørgen Eriksen, René Gislum, Rasmus Nyholm Jørgensen, Henrik Karstoft

Estimation of the Botanical Composition of Clover-Grass Leys from RGB Images Using Data

Simulation and Fully Convolutional Neural Networks

Reprinted from: Sensors 2017, 17, 2930, doi:10.3390/s17122930 . . . . . . . . . . . . . . 10

José M. Bengochea-Guevara, Dionisio Andújar, Francisco L. Sanchez-Sardana,

Karla Cantuña and Angela Ribeiro

A Low-Cost Approach to Automatically Obtain Accurate 3D Models of Woody Crops

Reprinted from: Sensors 2018, 18, 30, doi:10.3390/s18010030 . . . . . . . . . . . . . . . 118

James Brinkhoff, John Hornbuckle and Thomas Dowling

Multisensor Capacitance Probes for Simultaneously Monitoring Rice Field

Soil-Water-Crop-Ambient Conditions

Reprinted from: Sensors 2018, 18, 53, doi:10.3390/s18010053 . . . . . . . . . . . . . . . 135 
Delun Meng, Fanjia Meng, Wei Sun and Shuang Deng

A Compound Sensor for Simultaneous Measurement of Packing Density and Moisture Content of Silage

Reprinted from: Sensors 2018, 18, 73, doi:10.3390/s18010073

Alberto L. Barriuso, Gabriel Villarrubia González, Juan F. De Paz, Álvaro Lozano and Javier Bajo

Combination of Multi-Agent Systems and Wireless Sensor Networks for the Monitoring of Cattle

Reprinted from: Sensors 2018, 18,108, doi:10.3390/s18010108 . . . . . . . . . . . . . . . . . . 159

Jongguk Lim, Giyoung Kim, Changyeun Mo, Kyoungmin Oh, Geonseob Kim, Hyeonheui Ham, Seongmin Kim and Moon S. Kim

Application of Near Infrared Reflectance Spectroscopy for Rapid and Non-Destructive Discrimination of Hulled Barley, Naked Barley, and Wheat Contaminated with Fusarium

Reprinted from: Sensors 2018, 18, 113, doi:10.3390/s18010113 . . . . . . . . . . . . . . . . 186

Marcin Kafarski, Andrzej Wilczek, Agnieszka Szypłowska, Arkadiusz Lewandowski,

Piotr Pieczywek, Grzegorz Janik and Wojciech Skierucha

Evaluation of Apple Maturity with Two Types of Dielectric Probes

Reprinted from: Sensors 2018, 18, 121, doi:10.3390/s18010121 . . . . . . . . . . . . . . . . . 202

Shaoqing Cui, Peter Ling, Heping Zhu and Harold M. Keener

Plant Pest Detection Using an Artificial Nose System: A Review

Reprinted from: Sensors 2018, 18, 378, doi:10.3390/s18020378

Pengcheng Nie, Tao Dong, Yong He and Shupei Xiao

Research on the Effects of Drying Temperature on Nitrogen Detection of Different Soil Types by

Near Infrared Sensors

Reprinted from: Sensors 2018, 18, 391, doi:10.3390/s18020391 _ . . . . . . . . . . . . . . . 233

J. Behmann , K. Acebron, D. Emin, S. Bennertz, S. Matsubara, S. Thomas, D. Bohnenkamp, M.T. Kuska, J. Jussila, H. Salo, A.-K. Mahlein, U. Rascher

Specim IQ: Evaluation of a New, Miniaturized Handheld Hyperspectral Camera and Its Application for Plant Phenotyping and Disease Detection

Reprinted from: Sensors 2018, 18, 441, doi:10.3390/s18020441

Sérgio Francisco Pichorim, Nathan J. Gomes and John C. Batchelor

Two Solutions of Soil Moisture Sensing with RFID for Landslide Monitoring

Reprinted from: Sensors 2018, 18, 452, doi:10.3390/s18020452 . . . . . . . . . . . . . . . . . 275

Jiyu Peng, Fei Liu, Tingting Shen, Lanhan Ye, Wenwen Kong, Wei Wang, Xiaodan Liu and Yong He

Comparative Study of the Detection of Chromium Content in Rice Leaves by $532 \mathrm{~nm}$ and 1064 nm Laser-Induced Breakdown Spectroscopy

Reprinted from: Sensors 2018, 18, 621, doi:10.3390/s18020621 . . . . . . . . . . . . . . . . . 286

Ahmed Laamrani, Renato Pardo Lara, Aaron A. Berg, Dave Branson and Pamela Joosse

Using a Mobile Device "App" and Proximal Remote Sensing Technologies to Assess Soil Cover Fractions on Agricultural Fields

Reprinted from: Sensors 2018, 18, 708, doi:10.3390/s18030708 . . . . . . . . . . . . . . . . . 304 
David Sánchez-Álvarez, Marino Linaje and Francisco-Javier Rodríguez-Pérez

A Framework to Design the Computational Load Distribution of Wireless Sensor Networks in Power Consumption Constrained Environments

Reprinted from: Sensors 2018, 18, 954, doi:10.3390/s18040954 . 



\section{About the Special Issue Editor}

Dimitrios Moshou has been a full Professor at AUTH since 2018 and Lab Head of the Laboratory of Agricultural Engineering since 2012. He has a PhD from the Departments of Electrical Engineering and Biosystems, Faculty of Engineering, K.U. Leuven, Belgium; an MSc in Control Systems from the University of Manchester, UK; and an MSc in Electrical Engineering from Democritus University of Thrace, Greece. His research interests include the theory and applications of bio-inspired information processing, neuroscience, self-organization, and computational intelligence. He is interested in applications of these techniques in intelligent control, pattern recognition, data fusion, food safety, traceability, and cognitive robotics. He is the author of one research monograph on self-organizing networks and learning (Dimitrios Moshou, 'Artificial Neural Maps', ISBN: 978-3639150568, 2009) and more than 200 papers (with a total of $2600+$ citations). He has contributed in research and management tasks of 40 local and EU research projects. 



\section{Preface to "Sensors in Agriculture"}

Agriculture requires technical solutions for increasing production while lessening environmental impact by reducing the application of agro-chemicals and increasing the use of environmentally friendly management practices. A benefit of this is the reduction of production costs. Sensor technologies produce tools to achieve the abovementioned goals. The explosive technological advances and developments in recent years have enormously facilitated the attainment of these objectives, removing many barriers for their implementation, including the reservations expressed by farmers. Precision agriculture and 'smart farming' are emerging areas where sensor-based technologies play an important role. Farmers, researchers, and technical manufacturers are joining their efforts to find efficient solutions, improvements in production, and reductions in costs. This book brings together recent research and developments concerning novel sensors and their applications in agriculture. Sensors in agriculture are based on the requirements of farmers, according to the farming operations that need to be addressed.

Dimitrios Moshou

Special Issue Editor 

Article

\title{
Estimating Crop Area at County Level on the North China Plain with an Indirect Sampling of Segments and an Adapted Regression Estimator
}

\author{
Qinghan Dong ${ }^{1, *}$, Jia Liu ${ }^{2}$, Limin Wang ${ }^{2}$, Zhongxin Chen ${ }^{2}$ and Javier Gallego ${ }^{3}$ \\ 1 Department of Remote Sensing, Flemish Institute of Technological Research, 2400 Mol, Belgium \\ 2 Institute of Agricultural Resources and Regional Planning, Chinese Academy of Agricultural Sciences, \\ Beijing 100081, China; liujia06@caas.cn (J.L.); wanglimin01@caas.cn (L.W.); chenzhongxin@caas.cn (Z.C.) \\ 3 Joint Research Centre, The European Commission, 21027 Ispra, Italy; Javier.GALLEGO@ec.europa.eu \\ * Correspondence: qinghan.dong@vito.be; Tel.: +32-14-33-6856
}

Received: 1 September 2017; Accepted: 4 November 2017; Published: 16 November 2017

\begin{abstract}
Image classifications, including sub-pixel analysis, are often used to estimate crop acreage directly. However, this type of assessment often leads to a biased estimation, because commission and omission errors generally do not compensate for each other. Regression estimators combine remote sensing information with more accurate ground data on a field sample, and can result in more accurate and cost-effective assessments of crop acreage. In this pilot study, which aims to produce crop statistics in Guoyang County, the area frame sampling approach is adapted to a strip-like cropping pattern on the North China Plain. Remote sensing information is also used to perform a stratification in which non-agricultural areas are excluded from the ground survey. In order to compute crop statistics, 202 ground points in the agriculture stratum were surveyed. Image classification was included as an auxiliary variable in the subsequent analysis to obtain a regression estimator. The results of this pilot study showed that the integration of remote sensing information as an auxiliary variable can improve the accuracy of estimation by reducing the variance of the estimates, as well as the cost effectiveness of an operational application at the county level in the region.
\end{abstract}

Keywords: crop area; remote sensing image classification; area frame sampling; stratification; regression estimator

\section{Introduction}

Much research has been conducted in the field of crop mapping and area assessment using satellite image classification. Most often, using high-resolution images such as those generated by the sensors on Landsat satellites, including Landsat 5 Thematic Mapper (TM), Landsat 7 Enhanced Thematic Mapper Plus (ETM+) and Landsat 8 Operational Land Imager (OLI), each pixel is assigned to a specific class of land use or crop [1]. Other methods use an approach of soft or sub-pixel classification, often with coarser spatial resolution images, to increase the geographic coverage and temporal resolution of analysis [2,3]. The classification of spatial objects, which are often called segments, has also become popular, especially for agricultural areas [4].

In the early times of satellite image analysis, crop mapping and crop area estimation were not very well distinguished. Land cover area estimation, and in particular crop area estimation, was considered to be a direct spin-off of classified images that could be obtained by simply counting pixels classified into a particular class [5]. Image analysts became soon conscious that pixel counting generally introduces a bias, and that correcting this bias requires a large amount of field data from a properly sampled set of locations. The most frequent approaches to combine field data with classified images to correct the bias of pixel counting are the calibration and regression estimators [6-9]. The Group 
on Earth Observations (GEO) published a more generic discussion of area estimation issues from the remote sensing perspective [10].

The bias of area estimates by pixel counting is related to the inaccuracy of image classification, and can be estimated from the difference between the commission and omission errors for a given class [11]. An important issue that has been studied less is the margin for subjectivity of an area estimation by pixel counting: if the analyst has an idea of the reasonable area of each land cover class (crop, for example), they can tune the image classification parameters, the training sample composition, or the outlier cleaning process to have a pixel counting estimate close to the a priori belief [12]. This type of bias or discrepancy was already reported in other contexts, such as the Activity B "Rapid crop area change estimates at level of European Union" within the European Monitoring Agricultural Resources (MARS) project [13]. It is also well known in the agricultural statistics community that the remote sensing imagery generally gives sound crop area estimates only when it is used together with ground statistical surveys [14].

In this study, the information derived from remote sensing is used both for the design of the sampling frames, and for the final area estimation. First, the satellite images were used to perform a stratification from which non-agricultural areas were excluded from ground survey. In a later stage, the classification of satellite images was included as an auxiliary variable for a regression estimator. On the other hand, the area frame sampling is adapted to the complex landscape of the North China Plain.

The study was carried out in 2013 in the county of Guoyang, on the southern edge of the plain, in Anhui province (Figure 1). The official statistics data of the year 2012 showed that the county counted 1,545,908 inhabitants within a surface of 210,700 hectares [15]. However, the county border vector generated a figure for the total area of about 214,880 hectares. Similar to elsewhere on the North China Plain, the predominant cropping system consists of two growth seasons for each calendar year, with winter wheat followed by more diversified summer crops, include soybean, maize, cotton, and other vegetables. Although the local government publishes the official crop areas statistics each year, much caution should be paid to these figures, as the planting areas outside of the administrative regulation, such as river beds, are not included in the official statistics. These areas constitute a non-negligible part of the sowing area (up to $20 \%$ ).

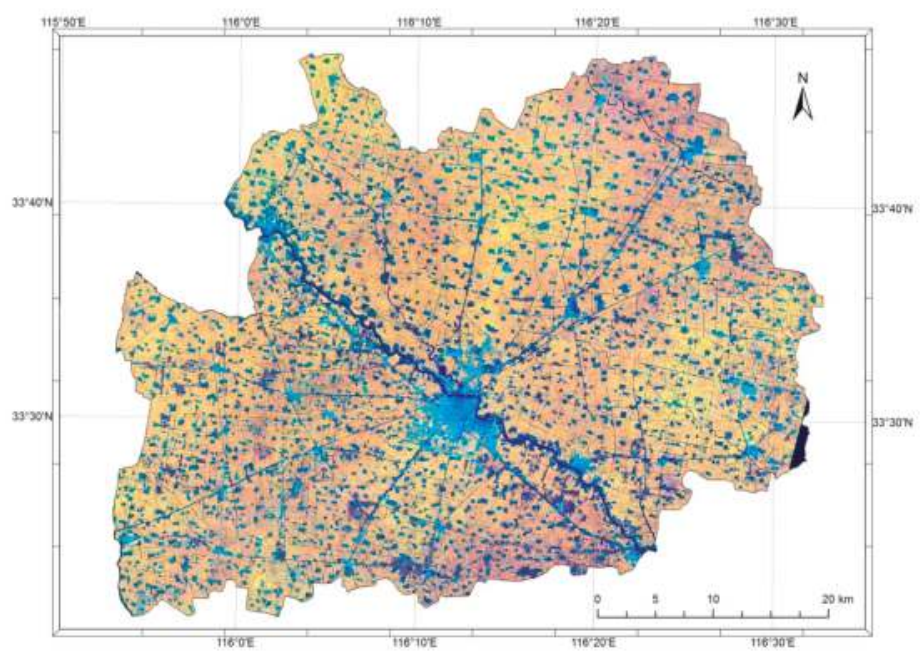

(a)

Figure 1. Cont. 


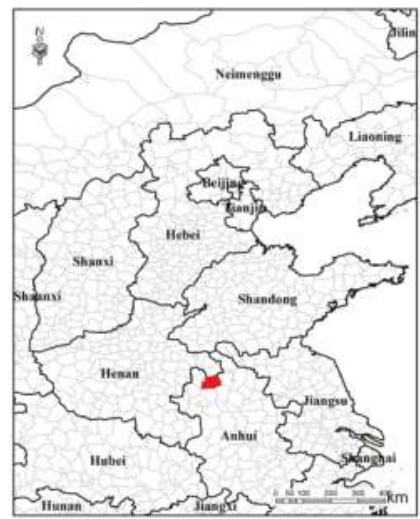

(b)

Figure 1. Guoyang County (a) is located in the north of Anhui province, and on the south edge of the North China Plain (b).

\section{Materials and Methods}

\subsection{Data}

The high resolution images used in this study are two scenes of RapidEye at $5 \mathrm{~m}$ resolution, registered on 5 and 13 August 2013, respectively.

The statistical data were acquired from the local statistical office of the Guoyang County government.

\subsection{Area Frame Sampling-Stage 1: Stratification}

Area frame sampling is defined on the geographic space. The units of an area frame can be points, transects (lines of a certain length), or pieces of territory, which are often named segments. When the units of the frame are points, the survey may be called a point survey.

The area frame sampling in this study was carried out in two stages: stratification and segment survey. Prior to the ground survey, a stratification step is performed to separate the agricultural stratum from other strata.

The stratification starts with a regular grid of $0.01^{\circ} \times 0.01^{\circ}$ overlaid to Google Earth in the study region most often composed by 2012 and 2013 imagery (Figure 2a).

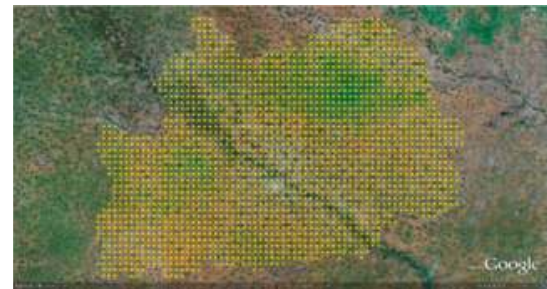

(a)

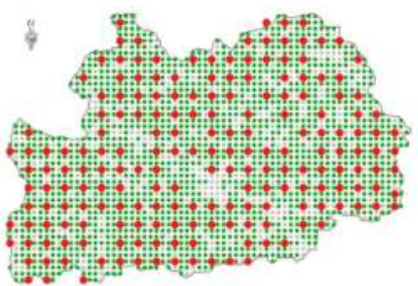

(b)

Figure 2. A grid of $0.01^{\circ}$ was overlaid on the Google Earth imagery (a). There are 2074 grid points within the boarder of the Guoyang County. The sub-sampling of the agricultural stratum was carried out in a systematic way. Two hundred and two grid points plot were thus selected for field survey to assess the crop proportions in this sample/grid point $(\mathbf{b})$. 
The grid points located within the border of Guoyang County were stratified by photo-interpretation into two strata: non-agricultural and agricultural.

The agricultural stratum is subsampled for survey purpose. The number of points to be surveyed is determined by the available resources. In this study, 202 points that were stratified to the agricultural stratum were selected for survey (Figure 2b).

A printout of Google Earth with an approximate scale 1:10,000 was used for guiding the ground survey.

\subsection{Area Frame Sampling-Stage 2: Ground Survey}

The region dispays a very characteristic cropping landscape, where fields of a few hectares are divided into thin stripes and cultivated by different households. Due to this stripe-shaped pattern, the sampling procedure is adapted, starting with 202 points. The points are then expanded to generate "segments" (Figure 3). A segment is thus conceived as a set of parallel stripes that does not straddle any permanent linear element (dirt road, hedge, etc.). The proportion of the targeted crop in segment is assessed by the proportion along the transect. The geographical location was performed using a Trimble GeoXT in stand-alone mode.

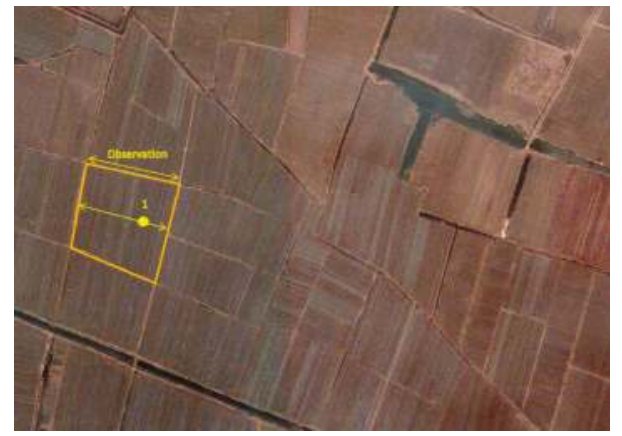

(a)

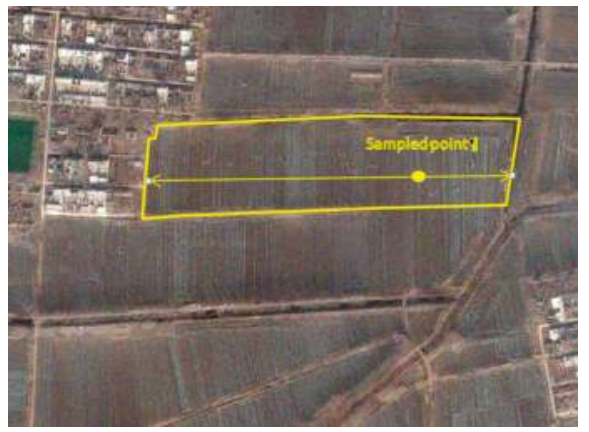

(b)

Figure 3. The crop proportion of a particular grid/sample point was assessed by expanding the point to a segment according to the physical boundaries of the field plot harboring the grid/sample point. The proportion of each crop was assessed by global positioning system (GPS) measuring on one border of the plot, perpendicular to the field strips (a). The expanded segment can be located on the edge of the agricultural stratum $(\mathbf{b})$.

From a statistical point of view, as only the agricultural stratum is sampled in our approach, we identify below this stratum with the population, and we omit for the moment the stratum sub-index. A segment $i$ with area $A_{i}$ is selected with probability $\pi_{i}=\frac{n A_{i}}{D}$, where $D$ is the total area and $n=202$ is the sample size. The usual Horvitz-Thompson estimator for the total area of crop $c$ with unequal sampling probability is:

$$
\hat{Y}_{c}=\sum_{i} \frac{y_{i}}{\pi_{i}}=D \sum_{i} \frac{y_{i}}{n A_{i}}=D \sum_{i} \frac{p_{i}}{n}
$$

where $y_{i}$ is the area of crop $c$ in segment $i$, and $p_{i}$ is the proportion. Thus, the unbiased unequal sampling probability estimator using the area of each crop in each segment coincides with the estimator based on the proportions and assuming equal probability. Therefore, estimators based on proportions are easier to manage, including regression estimators.

This ground survey will generate crop area estimates for each of the surveyed segments (y in the Section 2.5) and its mean $(\bar{y})$. The estimation is obtained by measuring the width of strips cultivated with one crop type. 


\subsection{Image Classification}

The RapidEye scenes were interpreted using the maximum likelihood classifier [16]. The ground truth data derived from the survey segments were split into two sets according to a ratio of 1:7 in a systematic sampling way:

- according to the field numbering in a set of eight plots, the first seven plots are used to train the classifier;

- the eighth plot of the set was assigned to the validation dataset to assess the accuracy of the classifications.

The image classification will generate the values for the variables used in the subsequent regression estimation analysis (Section 2.5): the variable $\mathrm{p}$ represents the crop area proportion in each of the 202 segments, its mean is $(\overline{\mathrm{p}})$, and $\overline{\mathrm{p}}_{\text {pop }}$ is the percentage of the pixels classified as the target crop in the entire agriculture stratum.

\subsection{Regression Estimator}

The regression estimator improves the accuracy of area estimates by adjusting the estimate of mean and reducing the variance $[17,18]$.

In this study:

$$
\bar{y}_{\text {reg }}=\bar{y}+b\left(\bar{p}_{\text {pop }}-\bar{p}\right)
$$

where $\bar{y}_{\text {reg }}$ is the regression estimate for a target crop area; $\bar{y}$ is the area mean derived from the ground survey for the target crop; $\overline{\mathrm{p}}_{\text {pop }}$ is the proportion of pixels classified as the target crop in the agriculture stratum of the county; and $\bar{p}$ is the average proportion of pixels classified as the target crop in the surveyed segments. Also, $\mathrm{b}$ is the slope of the regression between y (the target crop proportion in each segment obtained from the ground survey) and $\mathrm{p}$ (the target crop proportion in each segment generated by image classification).

For large random samples (202 in this study), the variance of the regression estimator var $\left(\bar{y}_{\text {reg }}\right)$ can be approximated by:

$$
\operatorname{var}\left(\bar{y}_{\text {reg }}\right)=\operatorname{var}(\bar{y})\left(1-R_{\text {py }}^{2}\right)=\frac{1}{n} \operatorname{var}(y)\left(1-R_{\text {py }}^{2}\right)
$$

where $R_{\text {py }}^{2}$ is the coefficient of determination for the regression.

The relative efficiency of the regression estimator $\left(\eta_{\text {reg }}\right)$ can be approximated as the ratio of the estimator's variances, and defined as:

$$
\eta_{\text {reg }}=\frac{\operatorname{var}(\bar{y})}{\operatorname{var}\left(\bar{y}_{\text {reg }}\right)}
$$

Relative efficiency can be interpreted as the relative sample size of the original ground survey required to achieve the certainty of the estimates derived from the regression estimation.

\section{Results}

\subsection{Stratification and Segment Survey}

As described above, two strata were defined: (1) agriculture (arable land) and (2) non-agriculture (urban, artificial, water). In this study case, all of the grid points could be exactly photo-interpreted with a priori knowledge. For regions in which ambiguity of interpretation is a relevant problem, a third stratum, "doubts", can be considered.

Each of the 2074 grid points in total was assigned to one of two strata. Out of all of the points, 1502 points, or $72.42 \%$ (155,616 hectares) were identified as belonging to the stratum "arable land", 
and 572 points or $27.58 \%(59,264$ hectares) were interpreted as belonging to the non-agricultural stratum. In the next stage of the sampling, 202 points sampled in the agricultural stratum were expanded to segments, as described in Section 2.3 and surveyed in August 2013. The results of the survey for these expended segments are summarized in Table 1, where the average size of 202 surveyed segments is also indicated.

Table 1. Statistics on the 202 segment survey.

\begin{tabular}{ccccc}
\hline & Maize & Soybean & Other Crops & Non-Agriculture \\
\hline Surface proportion (\%) & $6.21 \%$ & $91.58 \%$ & $2.01 \%$ & $0.20 \%$ \\
Standard deviation (\%) & $1.85 \%$ & $0.34 \%$ & $7.70 \%$ & $8.25 \%$ \\
Average segment size (ha) & & & 4.69 & \\
\hline
\end{tabular}

\subsection{Image Classification}

Figure 4 and Table 2 show the results of image classification using two registrations of the RapidEye imagery. They illustrated that the crop land covers $84.5 \%$ of the classified image, with soybean and maize sharing $85.29 \%$ and $14.64 \%$ of the total crop land area, respectively. It is worth noting that an estimate of the agriculture area generated by photo-interpretation is somehow lower $(72 \%)$. The results also reveal the area percentages of maize and soybean classes in the 202 survey segments (Table 2).

Figure 4 also reveals that maize appears to be under-cultivated in this county in comparison with the neighboring counties, and is more commonly found on the southeastern part of the county, especially near the border with Mengcheng County [19].

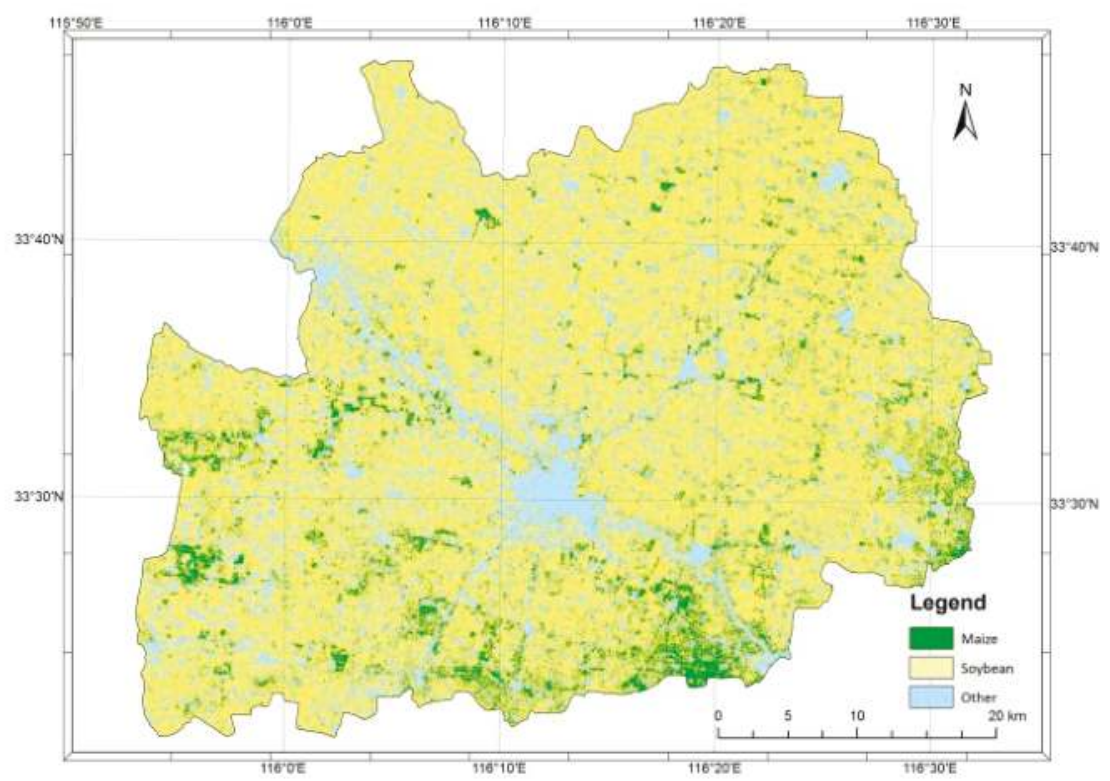

Figure 4. Classification using two scenes of RapidEye imagery. 
Table 2. Percentage of each class derived from the image classification.

\begin{tabular}{cccc}
\hline & \% Area of the County & \% of Crop Land Area & $\begin{array}{c}\text { \% of Classified Pixels in } \\
\text { Surveyed Segments }\end{array}$ \\
\hline Soybean & 72.07 & 85.29 & 88.36 \\
Maize & 12.37 & 14,64 & 10.32 \\
Other crops & 0.06 & 0.07 & $\mathrm{n} / \mathrm{a}$ \\
Other land use & 15.50 & $\mathrm{n} / \mathrm{a}$ & $\mathrm{n} / \mathrm{a}$ \\
\hline
\end{tabular}

The class "other crops", which includes various crops such as cotton and peanuts, is a very small proportion of what is cultivated in this county. However, the image classification provides a still smaller area for this class, as it is marginally represented.

The validation results of the classifications were carried out using a confusion matrix (data not shown). The overall accuracy was found to be high—around 95\%—which was contributed mostly by the highly dominant soybean crop in the county.

\subsection{Regression Estimator}

The regressions between the percentages of crops in the 202 sampled segments derived from the field survey and those computed from image classification for the same segments are illustrated in Figure 5.

The $\mathrm{R}_{\mathrm{py}}^{2}$ of the regressions between the crop proportions (from the 202 segments) derived from the ground survey and from the classification are 0.58 and 0.56 for the classes "soybean" and "maize", respectively. The standard deviations (SD) of the estimates obtained with the regression estimator are subsequently reduced from 1.72 to 1.14 for maize and 0.34 to 0.22 for soybean, when the image classification is used as an auxiliary variable (Table 3).

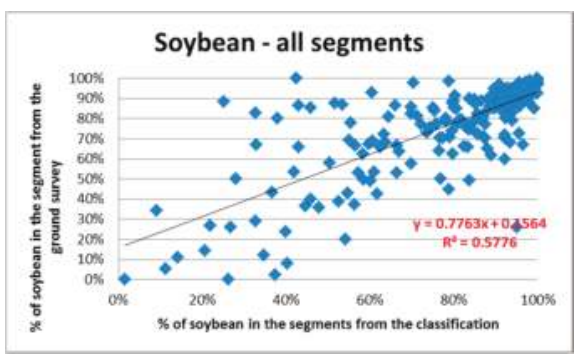

(a)

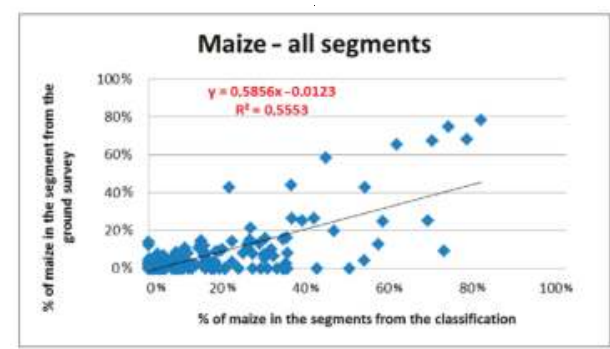

(b)

Figure 5. Regressions of crop proportions for the soybean (a) and maize (b) derived from 202 surveyed segments against those derived from the classification for the same segments. 
Table 3. Crop area proportions derived from a ground survey, image classification, and the regression estimator. $\mathrm{SD}=$ standard deviation.

\begin{tabular}{ccc}
\hline & Maize & Soybean \\
\hline Mean of the area percentage from surveyed segments (and its SD) & $6.21 \%$ & $91.58 \%$ \\
& $(1.72 \%)$ & $(0.34 \%)$ \\
& 0.59 & 0.78 \\
Regression slope (b) and coefficient of determination $\left(\mathrm{R}_{\mathrm{py}}^{2}\right)$ & $(0.56)$ & $(0.58)$ \\
Area percentage from classification in the agriculture stratum & $14.55 \%$ & $85.29 \%$ \\
Mean of the area percentage from classified segments & $10.32 \%$ & $88.36 \%$ \\
Regression estimates in the arable stratum (and its SD) & $8.76 \%$ & $89.20 \%$ \\
Relative efficiency of remote sensing & $(1.14 \%)$ & $(0.22 \%)$ \\
Number of ha in the county (assuming 155,616 ha arable area) & 16,558 ha & 138,809 ha \\
\hline
\end{tabular}

For example, the regression estimated soybean percentage in Guoyang County in 2013 can be deduced:

$$
\overline{\mathbf{y}}_{\text {reg, soybean }}=91.58+0.78 \times(85.29-88.36)=89.02
$$

The relative efficiency of the regression estimation approach for soybean and maize becomes 2.4 and 2.5, respectively, when Equation (4) is applied.

\section{Discussion}

In summary, this study tries to demonstrate the efficiency of the regression estimator approach in estimating the crop areas at a county level. The study region was located in Guoyang County on the North China Plain. The contribution of remote sensing was made on two levels. (1) It helped in area frame stratification, therefore in optimizing the sampling design prior to the ground survey. (2) Remote sensing was incorporated as an auxiliary variable in the analysis of regression estimation by means of image classification, and therefore improved the precision of estimates by reducing the variance of estimation.

More specifically, the area proportions for soybean and maize in the surveyed segments were regressed against those derived from the image classification. The regression estimator allowed adjusting the crop area estimates while reducing the variance of these estimates by a factor between 2.4 and 2.5 in this study. An efficiency value of 2.5 means that the accuracy obtained from sampling 202 segments corrected with remote sensing will be approximately equivalent to the accuracy obtained from sampling 505 segments by ground survey only. From a cost efficiency point of view, the approach will greatly reduce the ground survey costs, whereas relatively low costs for image acquisition and analysis need to be added.

As the use of high resolution imagery (such as Sentinel 2) becomes more generalized and data costs drop sharply, the application by combining ground survey data and satellite information will further show both its scientific soundness and cost-effectiveness in agricultural statistic surveys, especially in remote areas or parceled farming landscapes, such as those on the North China Plain.

Acknowledgments: This study was supported by the 7th EU framework programme (FP7) project E-AGRI.

Author Contributions: Javier Gallego and Jia Liu conceived and designed the experiments; Jia Liu, Limin Wang, Zhongxin Chen and Qinghan Dong performed the experiments; Qinghan Dong and Jia Liu analyzed the data; Qinghan Dong and Javier Gallego wrote the paper.

Conflicts of Interest: The authors declare no conflict of interest. 


\section{References}

1. Richards, J.A. Remote Sensing Digital Image Analysis: An Introduction, 5th ed.; Springer: Berlin, Germany, 2013; pp. 1-494.

2. Dong, Q.; Eerens, H.; Chen, Z. Crop area assessment using remote sensing on the North China Plain. In Proceedings of the 21ST ISPRS Congress, Commission VI, Beijing, China, 3-11 July 2008; Volume 37, pp. 699-709.

3. Sharma, R.; Goyal, A.K.; Dwivedi, R.K. A review of soft classification approaches on satellite image and accuracy assessment. In Proceedings of Fifth International Conference on Soft Computing for Problem Solving; Pant, M., Deep, K., Bansal, J., Nagar, A., Das, K., Eds.; Advances in Intelligent Systems and Computing; Springer: Singapore, 2015; Volume 437, pp. 629-639.

4. Kussul, N.; Lemoine, G.; Gallego, J.; Skakun, S.; Lavreniuk, M. Parcel based classification for agricultural mapping and monitoring using multi-temporal satellite image sequences. In Proceedings of the International Geoscience and Remote Sensing Symposium (IGARSS), Milan, Italy, 26-31 July 2015; pp. 165-168.

5. Mac Donald, R.B.; Hall, F.G. Global crop forecasting. Science 1980, 208, 670-679. [CrossRef] [PubMed]

6. Bauer, M.E.; Hixon, M.M.; Davis, B.J.; Etheridge, J.B. Area estimation of crops by digital analysis of Landsat data. Photogramm. Eng. Remote Sens. 1978, 44, 1033-1043.

7. Card, D.H. Using known map category marginal frequencies to improve estimates of thematic map accuracy. Photogramm. Eng. Remote Sens. 1982, 48, 431-439.

8. Hay, A.M. The derivation of global estimates from a confusion matrix. Int. J. Remote Sens. 1988, 9, 1395-1398. [CrossRef]

9. Czaplewski, R.L.; Catts, G.P. Calibration of remotely sensed proportion or area estimates for misclassification error. Remote Sens. Environ. 1992, 39, 29-43. [CrossRef]

10. Gallego, J.; Craig, M.; Michaelsen, J.; Bossyns, B.; Fritz, S. Workshop on Best Practices for Crop Area Estimation with Remote Sensing Data: Summary of Country Inputs; Group on Earth Observations (GEO), GEOSS Community of Practice Ag Task 0703a, EC JRC: Ispra, Italy, 2008.

11. Gallego, F.J. Remote sensing and land cover area estimation. Int. J. Remote Sens. 2004, 25, 3019-3047. [CrossRef]

12. Gallego, F.J.; Carfagna, E.; Baruth, B. Accuracy, Objectivity and Efficiency of Remote Sensing for Agricultural Statistics. In Agricultural Survey Methods; Benedetti, R., Bee, M., Espa, G., Piersimoni, F., Eds.; John Wiley \& Sons: Hoboken, NJ, USA, 2010; pp. 193-211.

13. Gallego, F.J. Review of the Main Remote Sensing Methods for Crop Area Estimates. In Proceedings of the ISPRS Workshop Proceedings: Remote Sensing Support to Crop Yield Forecast, Stresa, Italy, 30 November1 December 2007; Volume 36, pp. 65-70.

14. Carfagna, E.; Gallego, F.J. Using Remote Sensing for Agricultural Statistics. Int. Stat. Rev. 2005, 73, 389-404. [CrossRef]

15. Guoyang County Government Web Site. Available online: http://www.gy.gov.cn/content/channel/ 564d9172bd68b114c994fdc5 (accessed on 2 May 2017).

16. Duda, R.O.; Hart, P.E.; Stork, D.G. Pattern Classification, 2nd ed.; Wiley-Interscience: New York, NJ, USA, 2000.

17. Gallego, F.J.; Delince, J. Crop area estimation through remote sensing: Stability of the regression correction. Int. J. Remote Sens. 1993, 14, 3433-3445. [CrossRef]

18. Gonzales-Alonso, F.; Cuevas, J.M. Remote sensing and agricultural statistic: Crop area estimation through regression estimators and confusion matrices. Int. J. Remote Sens. 1993, 14, 1215-1219. [CrossRef]

19. Kerdiles, H.; Dong, Q.; Spyratos, S.; Gallego, F.J. Use of high resolution imagery and ground survey data for estimating crop areas in Mengcheng county, China. In Proceedings of the 35th International Symposium on Remote Sensing of Environment (ISRSE35), Beijing, China, 22-26 April 2013. [CrossRef]

(C) 2017 by the authors. Licensee MDPI, Basel, Switzerland. This article is an open access article distributed under the terms and conditions of the Creative Commons Attribution (CC BY) license (http:/ / creativecommons.org/licenses/by/4.0/). 
Article

\title{
Portable Electronic Nose Based on Electrochemical Sensors for Food Quality Assessment
}

\author{
Wojciech Wojnowski ${ }^{1, *}$, Tomasz Majchrzak ${ }^{1}$, Tomasz Dymerski ${ }^{1}$, Jacek Gębicki ${ }^{2}$ \\ and Jacek Namieśnik ${ }^{1}$ \\ 1 Department of Analytical Chemistry, Faculty of Chemistry, Gdańsk University of Technology, \\ 80-233 Gdańsk, Poland; tomasz.majchrzak@pg.edu.pl (T.M.); tomasz.dymerski@pg.gda.pl (T.D.); \\ jacek.namiesnik@pg.edu.pl (J.N.) \\ 2 Department of Chemical and Process Engineering, Faculty of Chemistry, Gdańsk University of Technology, \\ 80-233 Gdańsk, Poland; jacek.gebicki@pg.gda.pl \\ * Correspondence: wojciech.wojnowski@pg.edu.pl; Tel.: +48-583-486-411
}

Received: 18 September 2017; Accepted: 22 November 2017; Published: 24 November 2017

\begin{abstract}
The steady increase in global consumption puts a strain on agriculture and might lead to a decrease in food quality. Currently used techniques of food analysis are often labour-intensive and time-consuming and require extensive sample preparation. For that reason, there is a demand for novel methods that could be used for rapid food quality assessment. A technique based on the use of an array of chemical sensors for holistic analysis of the sample's headspace is called electronic olfaction. In this article, a prototype of a portable, modular electronic nose intended for food analysis is described. Using the SVM method, it was possible to classify samples of poultry meat based on shelf-life with $100 \%$ accuracy, and also samples of rapeseed oil based on the degree of thermal degradation with $100 \%$ accuracy. The prototype was also used to detect adulterations of extra virgin olive oil with rapeseed oil with $82 \%$ overall accuracy. Due to the modular design, the prototype offers the advantages of solutions targeted for analysis of specific food products, at the same time retaining the flexibility of application. Furthermore, its portability allows the device to be used at different stages of the production and distribution process.
\end{abstract}

Keywords: agriculture; meat spoilage; vegetable oil; quality assessment; electronic nose; electrochemical sensors

\section{Introduction}

The development and optimization of control methods at each stage of the production process, from raw materials to final products, is becoming increasingly important in food processing technology. There is a growing interest in food safety and food quality assessment issues, which have become one of the main priorities in food analysis. This is evidenced by the novel monitoring and quality control methods both for raw and processed products' samples.

Apart from classic instrumental techniques (mainly chromatographic), methods based on electronic sensors are increasingly being employed in food analysis [1,2]. In particular, electronic noses are being used for analyses of the samples' aromatic profiles without prior separation of the volatile fraction into individual components. These devices consist of an array of non- or partially selective gas sensors and are coupled with a data processing and pattern recognition system capable of identifying even complex aromatic profiles [3]. The use of this technique allows rapid non-destructive analysis and in certain applications can be an alternative to relatively costly and time-consuming techniques such as gas chromatography coupled with mass spectrometry (GC-MS) and/or olfactometry (GC-O), infrared spectroscopy (IR), and classical sensory analysis. The last one remains the standard in quality analysis partly because its results can be more directly related to the consumers' perception of the 
final product. However, sensory analysis is relatively costly as it requires the participation of trained panellists who can only work for several hours each day due to sensory fatigue [4]. It could be easily supplemented with electronic olfaction, in which also a holistic analysis of the sample's aromatic profile is performed. Food control is one of the main application areas of electronic noses, in particular in quality assessment and process operations in the food industry $[5,6]$. In fact, nearly half of the publications in the area of electronic olfaction are related to the food industry [7].

In the past, analytical devices equipped with arrays of sensors, such as electronic noses electronic tongues were developed for analyses of a large variety of food products, e.g., fish [7], meat [8], honey [9], coffee [10], cheese [11], spirits [12], or wine [13-15], and also more generic devices for general food analysis [16,17]. The latter ones have been developed into commercially available devices, as less specific solutions can be used in a wider area of applications, despite the fact that a more targeted approach might lead to a higher overall sensitivity and specificity of the system. In this paper, a portable electronic nose intended for food analysis is presented. It is equipped with an array of electrochemical (EC) sensors mounted in easily replaceable modules. In order to adapt it to a particular application, individual sensor modules can be easily changed without the need to modify the platform in any way. Due to its portability, it can be used at all stages of the manufacturing process, whether during monitoring of production, quality evaluation during processing, or at the retail level for freshness assessment.

\section{E-Nose System}

The electronic nose system has been developed to analyse the volatile fraction of various food products. Its pneumatic assembly for dynamic sampling consists of a thermostated sample block, a two-way valve, four replaceable sensor modules connected in sequence, two gas filters, and a pump. A schematic representation of the pneumatic assembly is depicted in Figure 1.

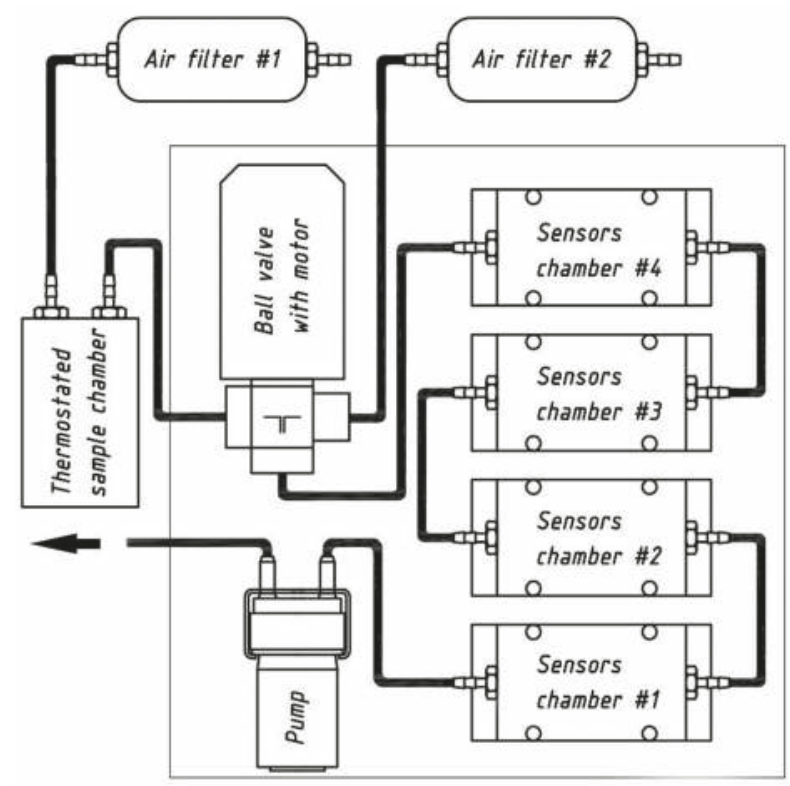

Figure 1. Pneumatic assembly of the electronic nose prototype.

The device is controlled through a custom interface printed circuit board (PCB) equipped with a microprocessor, $12 \mathrm{~V}$ power supply, signal inputs and outputs, and an analogue-digital signal converter. 
The main board processes and analyses output signals from chemical gas sensors and other sensors (temperature, relative humidity, pressure) and controls the sample block's thermostat and pneumatic systems. The processed signals are then transmitted to a personal computer, where the results are displayed in an in-house designed software which can also be used to control the device's operation. The prototype can also be operated manually using controls and LEDs situated on the front panel of the enclosure (Figure 2).

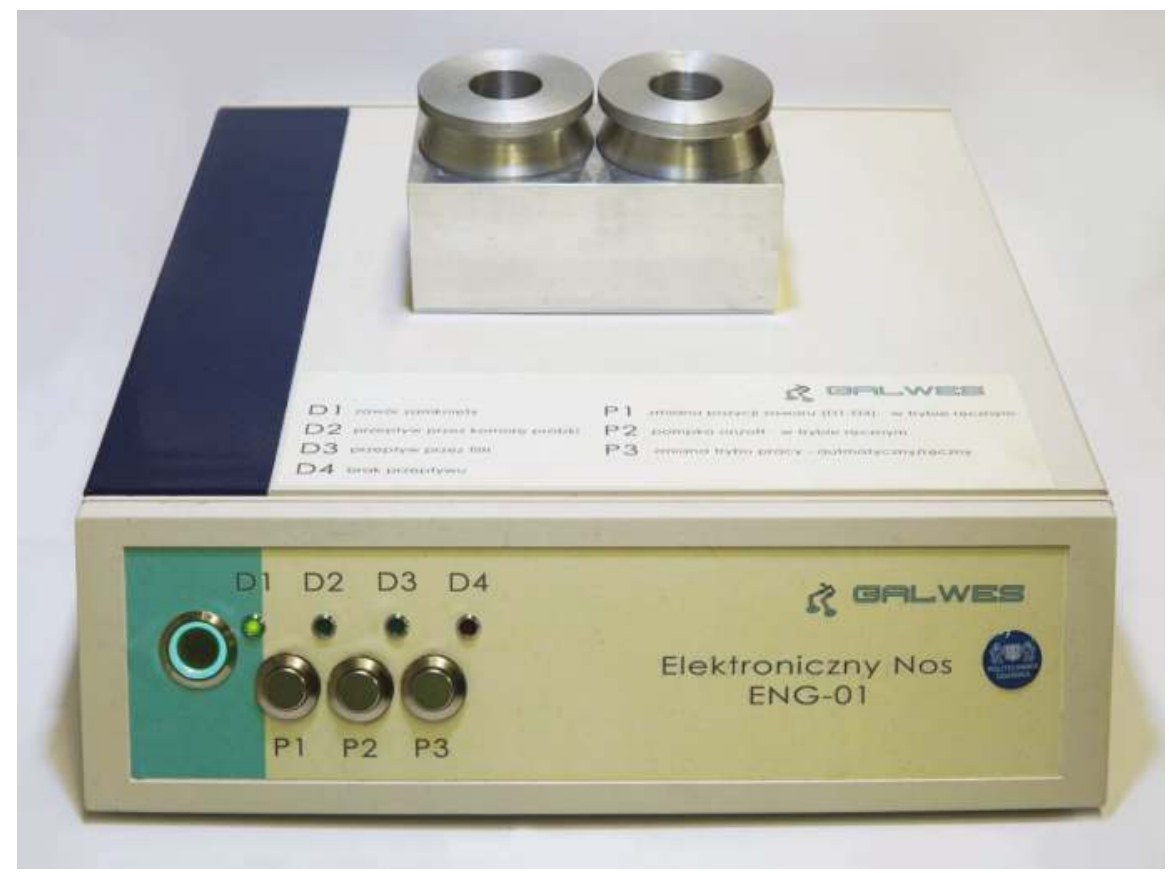

Figure 2. The view of an electronic nose prototype based on the electrochemical sensor array.

\subsection{Sensor Arrays}

In food analysis with the use of electronic noses, there is a need to tune the hardware to the specific application [6], which usually means selecting chemical sensors sensitive to the main components of the sample's volatile fraction. However, regardless of the intended use, there is much overlap in the overall design of the electronic, pneumatic, and data processing systems. For that reason, in order to increase the device's versatility without losing the advantages of using sensor arrays tailored to the specific application, gas sensors were placed in four easily interchangeable and replaceable modules, with two sensors in each. Moreover, since the circuitry of each module is printed on a custom PCB board, each unit is compact and can be plugged into the main board and removed without much effort. Individual modules are connected in-line, which means that only one needs to be connected for the electronic nose to work. The response delay between the same sensor chamber placed at the beginning and at the end of the series is app. $1 \mathrm{~s}$, which does not affect the outcome of the analysis.

Electrochemical sensors manufactured using screen printing on FR4 plates (SPEC Sensors, Newark, CA, USA) were chosen because of their 10-year estimated lifetime, as opposed to the more usual two-year lifetime. This will improve the system's robustness and reduce the frequency of maintenance. Two additional electrochemical sensors with an estimated lifetime of two years (City Technology Ltd., Portsmouth, UK) were also used. All the sensors used in the prototype device are listed in Table 1. It can be seen that these electrochemical sensors present cross sensitivity to some chemical compounds. 
When used as a single sensor for specific compound detection, cross-sensitivity is a real limitation. However, in the case of electronic noses, it increases the range of potential applications of the device. Electrochemical sensors are particularly useful for portable applications due to a low power consumption and the fact that they are relatively unaffected by changes in relative humidity, which is especially important when considering field deployment $[18,19]$.

Table 1. Sensors used in the prototype electronic nose.

\begin{tabular}{|c|c|c|c|c|c|}
\hline Sensor & Designation & Manufacturer & Measurement Range & Resolution & Cross-Sensitivity \\
\hline $\mathrm{CO}$ & $\begin{array}{c}\text { DGS-CO } \\
968-034\end{array}$ & SPEC Sensors & 0 to $1000 \mathrm{ppm}$ & $100 \mathrm{ppb}$ & Hydrogen, Isopropyl Alcohol \\
\hline Ethanol & $\begin{array}{l}\text { DGS-Ethanol } \\
\text { 968-035 }\end{array}$ & SPEC Sensors & 0 to $800 \mathrm{ppm}$ & $300 \mathrm{ppb}$ & $\begin{array}{l}\text { Carbon monoxide, Hydrogen Sulphide, } \\
\text { Nitric Oxide, Sulphur Dioxide, Chlorine }\end{array}$ \\
\hline $\mathrm{H}_{2} \mathrm{~S}$ & $\begin{array}{l}\text { DGS- } \mathrm{H}_{2} \mathrm{~S} \\
968-036\end{array}$ & SPEC Sensors & 0 to $10 \mathrm{ppm}$ & $10 \mathrm{ppb}$ & $\begin{array}{l}\text { Chlorine, Nitrogen Disulphide, Sulphur } \\
\text { Dioxide, Nitric Oxide, Carbon Monoxide }\end{array}$ \\
\hline $\mathrm{NO}_{2}$ & $\begin{array}{c}\text { DGS-NO } \\
968-037\end{array}$ & SPEC Sensors & 0 to $10 \mathrm{ppm}$ & $20 \mathrm{ppb}$ & Hydrogen Sulphide, Ozone \\
\hline $\mathrm{SO}_{2}$ & $\begin{array}{c}\text { DGS-SO } \\
968-038\end{array}$ & SPEC Sensors & 0 to $20 \mathrm{ppm}$ & $50 \mathrm{ppb}$ & $\begin{array}{l}\text { Hydrogen Sulphide, Nitric Oxide, } \\
\text { Carbon Monoxide }\end{array}$ \\
\hline VOC & $\begin{array}{l}\text { DGS-RESPIRR } \\
968-041\end{array}$ & SPEC Sensors & 0 to $20 \mathrm{ppm}$ & $20 \mathrm{ppb}$ & $\begin{array}{l}\text { Hydrogen Sulphide, Ozone, Chlorine, } \\
\text { Ethanol, Nitrogen Dioxide, Sulphur Dioxide }\end{array}$ \\
\hline TBM & $2 \mathrm{E} 50$ & City Technology & 0 to $50 \mathrm{ppm}$ & $500 \mathrm{ppb}$ & Nitrogen Dioxide \\
\hline $\mathrm{NH}_{3}$ & 3E $100 \mathrm{SE}$ & City Technology & 0 to $100 \mathrm{ppm}$ & $1 \mathrm{ppm}$ & Hydrogen Sulphide \\
\hline
\end{tabular}

In order to improve the response time of the system and facilitate sensor recovery, thus achieving high measurement frequencies, it is important to minimize dead volumes, especially in the sensors chamber [20]. The dead volume in a single module's sensor chamber is app. $1 \mathrm{ccm}$. However, reducing the volume above the sensor's active element might increase the flow turbulence, which in turn might have a detrimental effect on the stability of the response signal. In order to reduce this effect, and even out the flow velocity along the sensors surface, a perforated plate designed using Finite element method Computational fluid dynamics software (Autodesk CFD 2018) was introduced into the sensor modules, as shown in Figure 3.
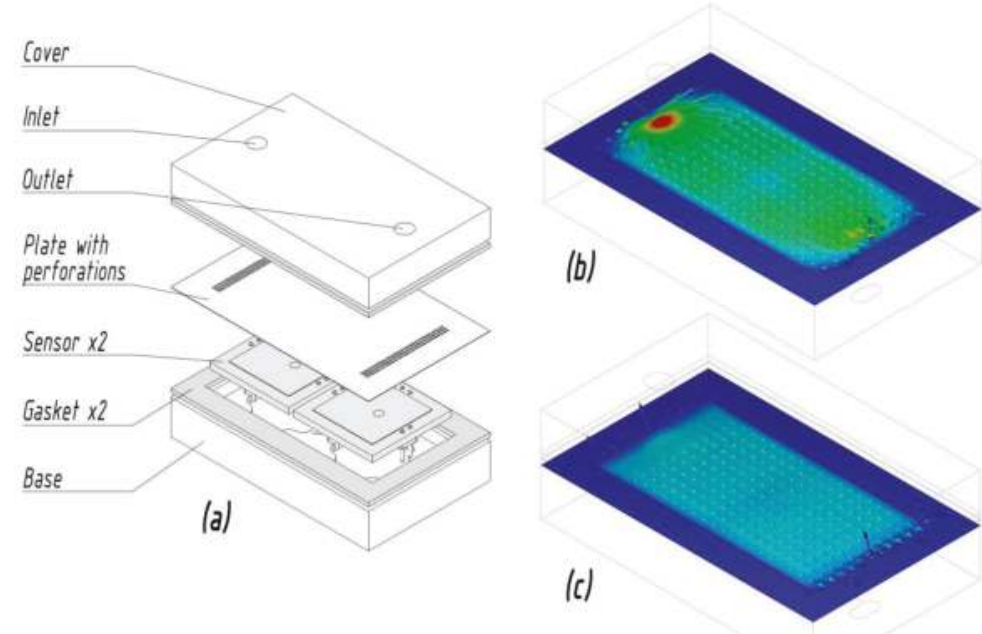

Figure 3. (a) Exploded view drawing of the sensor module; (b) flow velocity magnitude along the sensors' surface without the perforated plate and (c) with the perforated plate. 


\subsection{Sampling System}

In order to facilitate rapid consecutive measurements, samples of food products are placed in standard $60 \mathrm{~mL}$ or $20 \mathrm{~mL}$ glass headspace vials. These can be placed in a thermostated aluminium block operating in the $25^{\circ} \mathrm{C}$ to $45^{\circ} \mathrm{C}$ range to induce the transfer of analytes to the sample's volatile fraction. An aluminium adapter is used to accommodate the smaller $20 \mathrm{~mL}$ headspace vials. Since the heating block has two slots, an empty vial can be used as a reference.

There are two basic operating modes, namely 'sample', in which the carrier gas (by default ambient air passed through a carbon filter) is directed using a two-way ball valve through the sample's headspace and into the sensor chamber, and 'purge', in which it bypasses the sample and goes directly into the sensor chamber to flush any remaining analytes and condition the sensors before the next measurement. As the sensors' response characteristic is dependent on temperature and, to a lesser degree, humidity, an integrated sensor which measures these parameters (SHT25, Sensirion, Staefa, Switzerland) is placed in-line. In order to protect the pump in the event of a clog in the pneumatic system, the electronic nose is equipped with an MPX pressure sensor (NXP Semiconductors, Eindhoven, The Netherlands) and the measurement will stop automatically when a threshold pressure level is exceeded.

\subsection{Data Acquisition System}

Signals from the chemical gas sensors and other sensors are processed by the integrated microprocessor, at which point their raw output is converted to a digital signal using the LTC2433 ADC (Linear Technology, Milpitas, CA, USA) and transformed into concentration values (by default $\mathrm{ppbv}$ ) after temperature corrections are applied. The digital output signal is then transmitted to a PC-class computer via a USB driver (FTDI, Glasgow, UK) and read using custom software written in Python, which enables further processing of the data and also automatic control of the electronic nose's functions. The user can define the parameters of the method which will be used during the analysis. Apart from recording the sensors' responses in discrete 1-s intervals, a separate file is created for an entire experiment, consisting of $i$ analysed samples, which expedites the subsequent statistical data analysis and pattern recognition. In order to limit the impact of baseline noise on the result of the analysis, the average of $\mathrm{n}$ last seconds of the 'purge' mode is subtracted from the average of $\mathrm{n}$ last seconds of the 'sample' mode. All data can be easily exported as comma separated values (csv) to facilitate the system's integration with the existing data analysis workflows.

\section{Materials and Methods}

Two preliminary experiments were conducted to verify whether the prototype e-nose could possibly be used in real-life conditions for animal and vegetable agricultural products analysis, namely meat shelf-life assessment and vegetable oil quality evaluation.

First, a volatile fraction of fish, pork, and poultry meat that was stored at room temperature over a period of two days was analysed in order to test the behaviour of the sensors and of the data acquisition system.

In the below-described experiments, a single analysis cycle consisted of $100 \mathrm{~s}$ purge time, $20 \mathrm{~s}$ sample time and another $100 \mathrm{~s}$ purge time and the flow rate was set to $1 \mathrm{dm}^{3} / \mathrm{min}$. The measured relative humidity of the air ranged from $50 \%$ to $70 \%$.

\subsection{Poultry Meat Analysis}

The prototype was used to determine the shelf-life of refrigerated poultry meat. The fresh chicken breast meat was procured at a local distribution centre in Gdańsk, Poland. The animals were slaughtered in the evening on the day preceding the first day of the analysis, and the carcasses were then transported at $2.4{ }^{\circ} \mathrm{C}$ to the distribution centre where they were dismembered. Breast muscles of two different birds were then ground together in a thoroughly cleaned industrial grinder and 
transported under refrigeration to the laboratory on the morning of the first day of the analysis. Samples of $5 \mathrm{~g}$ were placed in $20 \mathrm{~mL}$ glass headspace vials, covered with polyethylene foil and stored at $4{ }^{\circ} \mathrm{C}$. Prior to analysis, the vials were sealed with disposable caps lined with a silicon-PTFE membrane. During the analysis cycle, each sample was incubated at $37^{\circ} \mathrm{C} .15$ samples were analysed every day with a total of 75 samples.

\subsection{Vegetable Oil Quality Assessment}

Refined rapeseed oil, virgin olive oil and unrefined sunflower oil were obtained at a local distribution centre in Gdańsk, Poland. Samples of $5 \mathrm{~g}$ were poured into $20 \mathrm{~mL}$ glass vials and sealed with a cap lined with a silicon-PTFE membrane. In order to classify samples of rapeseed oil based on the degree of thermal degradation samples were incubated at five different temperatures: $20^{\circ} \mathrm{C}, 60^{\circ} \mathrm{C}, 100{ }^{\circ} \mathrm{C}, 140{ }^{\circ} \mathrm{C}$, and $180^{\circ} \mathrm{C}$, respectively. In total, 50 samples were analysed, 10 for each incubation temperature.

Subsequently, for virgin olive oil adulteration detection model mixtures of virgin olive oil and unrefined sunflower oil were prepared: $95 \%, 90 \%, 80 \%$, and $50 \%(\mathrm{~g} / \mathrm{g})$ of olive oil, respectively. Additionally, samples of $100 \%$ olive oil and $100 \%$ sunflower oil were also prepared. During the analysis cycle, each sample was incubated at $40{ }^{\circ} \mathrm{C}$.

\subsection{Statistical Analysis}

The chemometric analysis was performed using Orange version 3.6 [21] and Minitab version 17.1 (Minitab, Ltd., Coventry, UK) software. Features were normalised trough centring by mean and scaling by the standard deviation. Features (response signals of particular sensors) most relevant in a particular scenario were then chosen based on the analysis of variance or principal component analysis loadings. Support vector machines method with RBF kernel was chosen as a classifier based on the results of stratified 10-fold cross-validation [22]. Subsequently, the method was evaluated, with $66 \%$ of data used for training and $34 \%$ for testing through random sampling.

\section{Results and Discussion}

\subsection{Meat Shelf Life Evaluation}

Presently, commonly used methods of meat freshness assessment are sensory analysis, total volatile base nitrogen (TVB-N) [23] and total bacterial count (TBC) [24]. Each of these methods is time-consuming, requires trained staff, and increases the cost of freshness evaluation. Due to the present regulations and good manufacturing practice, TBC measurement is commonly used in meat production plants. Unfortunately, in this method, a single analysis can take up to $72 \mathrm{~h}$, which means that the batch of meat leaves the production plant before the results of shelf life evaluation are available.

The analysis of volatile compounds, carried out using classical sensory analysis or instrumental methods, can be a source of valuable information regarding meat quality $[25,26]$. The instrumental analysis used to characterize the aroma of food is usually approached in one of two ways: the first is to examine the individual compounds responsible for the odour of a product, typically by gas chromatography, and the second is based on the so-called holistic aroma analysis. Therefore, it is possible to use electronic noses for this purpose. There is plenty of information in the literature on the use of electronic noses to assess freshness of meat, but there are few solutions that use electrochemical sensors [8]. The following is an example of using this type of device.

Shelf life differs depending on the type of meat and it is the shortest for fish meat and the longest for pork. Shown in Figure 4 are radar plots of sensor response signals for different types of meat. During the meat spoilage process, the response signals of sensors such as $\mathrm{SO}_{2}, \mathrm{VOCs}$, and $\mathrm{NH}_{3}$ are increased. Poultry and fish significantly deteriorated after two days of storage, whereas pork exhibited similar freshness each day of measurement. In the case of pork, the main constituents of the volatile fraction are compounds that react with $\mathrm{SO}_{2}, \mathrm{H}_{2} \mathrm{~S}$, and $\mathrm{CO}$ sensors. Also, significant negative cross 
sensitivity signals for the ammonia sensor have been noted for the fresh sample of pork, which also provides valuable analytical information. This preliminary test was intended to check the response signals of the sensors, as simple sensory evaluation is sufficient to assess the freshness of meat stored at room temperature for two days.

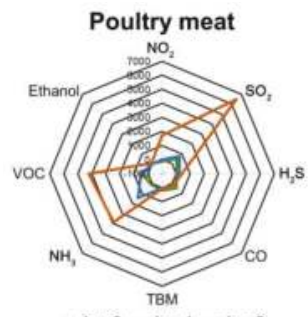

- day 0 -day 1 -day 2

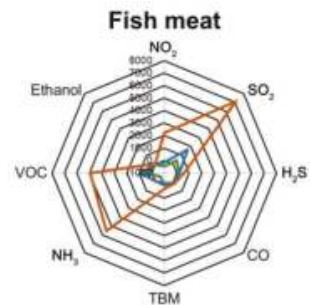

-day 0 -day 1 -day 2

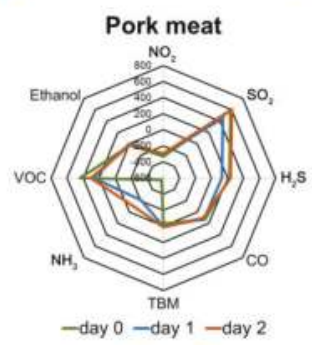

Figure 4. Radar plots for fish, poultry, and pork samples at different time of incubation.

However, under real conditions fresh meat is stored in temperatures from $2{ }^{\circ} \mathrm{C}$ to $7^{\circ} \mathrm{C}$. In these cases, changes in the composition of the volatile fraction with the elapse of storage time is subtle. Therefore, a simple comparison of response signals may not produce satisfactory results and more sophisticated statistical analysis methods are required. In subsequent studies, the possibility to use the prototype electronic nose to classify samples of chicken breast meat based on shelf-life was evaluated. Based on the results of the analysis of variance it was determined, that the signals from four sensors, namely $\mathrm{NO}_{2}, \mathrm{H}_{2} \mathrm{~S}, \mathrm{SO}_{2}$, and $\mathrm{NH}_{3}$ show the highest correlation with class in this particular scenario. Shown in Figure 5 is the result of principal component analysis of these four features. Two first principal components explain $99 \%$ of the total variance.

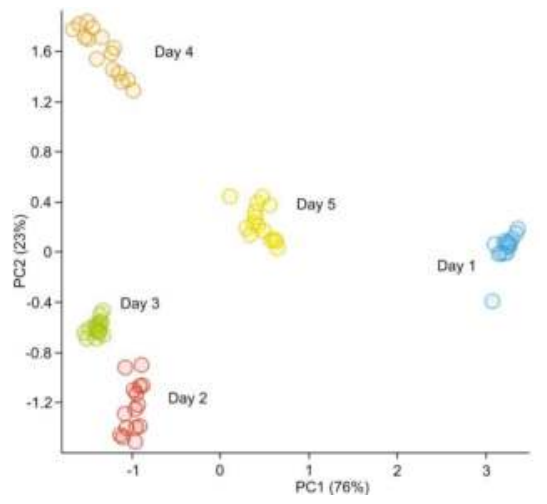

Figure 5. Principal component analysis (PCA) of poultry meat stored at $4{ }^{\circ} \mathrm{C}$ over a period of five days. 
Through a stratified 10-fold cross-validation, it was determined that the overall classification accuracy of the SVM algorithm was $98.7 \%$. The SVM method was then trained using $66 \%$ of the data chosen at random to avoid bias and tested on the remainder of the data. It was possible to repeatedly and accurately obtain a $100 \%$ correct classification based on the shelf-life. A sample confusion matrix for the classification is shown in Figure 6a.

(a)

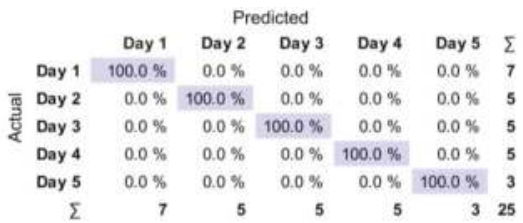

(b)

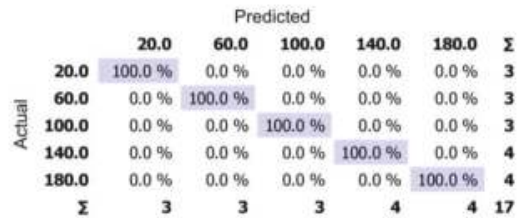

(c)

Predicted

\begin{tabular}{|c|c|c|c|c|c|c|}
\hline & 0 olive & 100_olive & 50_olive & 80 ollve & 90_ollve & 95_olive \\
\hline O_olve & $100.0 \%$ & $0.0 \%$ & $0.0 \%$ & $0.0 \%$ & $0.0 \%$ & $0.0 \%$ \\
\hline 100_olive & $0.0 \%$ & $100.0 \%$ & $0.0 \%$ & $0.0 \%$ & $0.0 \%$ & $0.0 \%$ \\
\hline 50_olive & $0.0 \%$ & $0.0 \%$ & $100.0 \%$ & $0.0 \%$ & $0.0 \%$ & $0.0 \%$ \\
\hline 80_olive & $0.0 \%$ & $0.0 \%$ & $0.0 \%$ & $100.0 \%$ & $0.0 \%$ & $0.0 \%$ \\
\hline 90_olive & $0.0 \%$ & $0.0 \%$ & $0.0 \%$ & $0.0 \%$ & $60.0 \%$ & $0.0 \%$ \\
\hline 95_olve & $0.0 \%$ & $0.0 \%$ & $0.0 \%$ & $0.0 \%$ & $40.0 \%$ & $100.0 \%$ \\
\hline$\Sigma$ & 4 & 3 & 3 & 3 & 5 & 2 \\
\hline
\end{tabular}

Figure 6. Confusion matrices for Support Vector Machine SVM classification of (a) poultry meat based on shelf-life; (b) rapeseed oil based on the degree of thermal degradation; (c) olive oil based on the admixture of adulterations (purple: classified correctly; pink: misclassified).

In the same study, cluster analysis was used to determine the groups of sensors that are used in the meat freshness classification. This approach can be used to find correlations between sensor signal values, for example by highlighting a group of compounds that can affect the sensor signal. In order to find the correlation, a cluster analysis using Ward linkage and absolute correlation coefficient distance (Figure 7) was used. It can be noticed that two main clusters are formed. The first one includes sensors that respond to carbon-based substances, namely CO, VOC, and Ethanol sensors. The second cluster contains sensors that detect nitrogen and sulphur compounds, whose presence in the volatile fraction of the meat may induce an unpleasant odour, and which had the greatest impact on the classification result based on ANOVA.

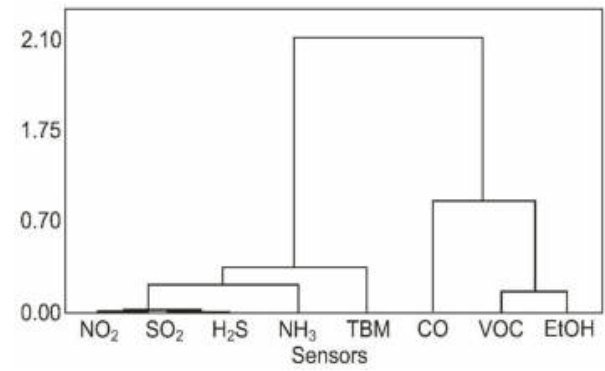

Figure 7. Cluster analysis of variables (sensors) during poultry shelf-life evaluation. 
In subsequent tests, in order to increase the sensor response signal, gaseous sample circulation was used. Unlike the classic DHS, where the carrier gas stream transfers analytes to the detection system, in the proposed approach the flow leaving the sensor chamber is directed back to the sample chamber. As a result, the sensor response signal is increased, as shown in Figure 8. This solution can be found in other agricultural applications, for example in the classification of diluted samples. In the matrices in which volatile chemicals are at high concentrations circulating may cause overloading of the signal and entail measurement errors. It was determined that this procedure does not significantly impact the outcome of classification of meat samples based on shelf-life and the results do not justify increasing the time of a single analysis.

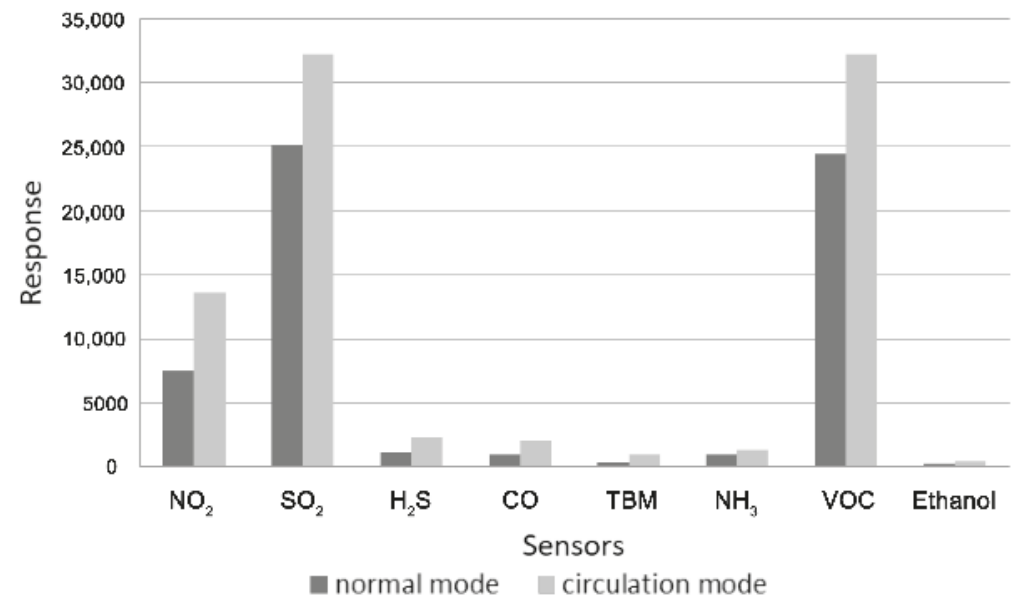

Figure 8. Sensor response signals for poultry meat samples after five days of storage with and without circulation mode.

\subsection{Assessment of the Degree of Vegetable Oil's Thermal Degradation}

The increasing demand for food containing easily digestible micro- and macroelements can lead to a more prevalent use of edible oils for thermal processing of food. Consumption of fried foodstuffs provides assimilable proteins and other nutrients to the human body. Due to the fact that fried foods are characterized by good taste, pleasant aroma, colour, and desirable texture, frying is one of the most popular methods of food preparation $[27,28]$. Frying occurs when the temperature of oil in which the food is immersed ranges between $150{ }^{\circ} \mathrm{C}$ and $190^{\circ} \mathrm{C}$. However, this is a contractual temperature range. In practice, the oil temperature, especially in domestic applications, is not controlled, so the food could be cooked at too low or too high temperatures [29]. This may decrease the quality of the fried product, but it can also be detrimental to consumers. As a result of frying, thermal degradation of oils results in generation of short chain or aromatic volatile organic compounds, e.g., aldehydes such as acrolein, alcohols, ketones, and simple hydrocarbons [30].

Current quality assessment methods, such as sensory analysis, peroxide value measurements, colour determination, and gas chromatography are not in-situ measurement methods and may be labour-intensive and time-consuming. The use of electronic nose devices in which these drawbacks are reduced may be one of the solutions.

Shown in Figure 9 is the result of principal component analysis according to the thermal degradation degree of rapeseed oil samples. The inputs were response signals of all the sensors with which the prototype is equipped. There is a visible separation between samples incubated at different temperatures. Three first principal components explain $99 \%$ of the variance. Samples incubated at $20^{\circ} \mathrm{C}$ and $60{ }^{\circ} \mathrm{C}$ show similarities in the composition of the volatile fraction, as was also demonstrated 
in previous studies [31]. As the degree of thermal degradation increased, variation within the groups also increased.

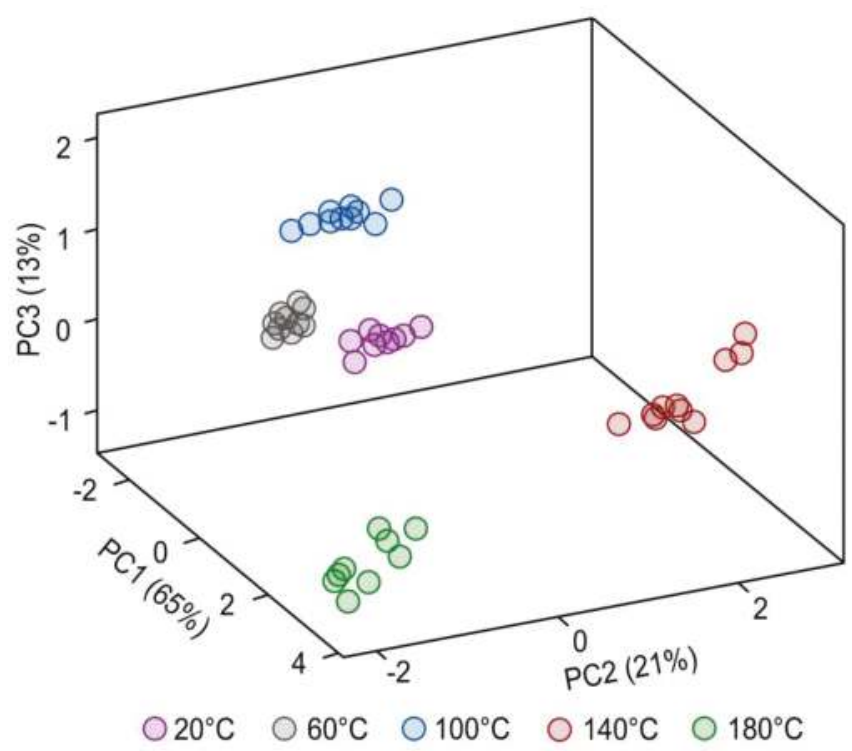

Figure 9. 3D projection of PCA of rapeseed oil samples incubated at different temperatures.

Analysing the loadings for the principal components, it can be noticed that the first component is determined by an overall increase in the response signal values of all sensors (Figure 10). Classification along the second principal component is based primarily on mercaptan and ammonia sensors' responses. Thermal degradation products are primarily aldehydes, ketones, and alcohols, so it is to be assumed that the high loading provided by these sensors results more from its cross sensitivity than from the presence of mercaptans and ammonia in the volatile fraction. Similar conclusions can be drawn for other principal components for which the highest loadings are for the nitrogen dioxide sensor for the PC3 and for the mercaptan, ammonia, and hydrogen sulphide sensors for the PC4. In total, the first four major components explain nearly $99.5 \%$ of the data variance. Analysis of loadings for major components can provide information on how to limit the number of chemical sensors used. Then, due to the modular nature of the device, it is possible to reduce the number of chemical sensor modules and thereby maintain satisfactory classification results. In the case of the assessment of the degree of thermal degradation, the TBM, $\mathrm{NH}_{3}, \mathrm{NO}_{2}$, and $\mathrm{H}_{2} \mathrm{~S}$ sensors had the highest impact on the PCA outcome.

Subsequently, SVM method was used for classification of samples of rapeseed oil according to the degree of thermal degradation. The inputs were features selected based on the interpretation of PCA loadings. As was the case with the classification of meat samples, stratified 10-fold cross-validation was used, and the method was trained using $66 \%$ of the data set chosen at random. The use of SVM resulted in 100\% correct classification, as shown in Figure $6 \mathrm{~b}$. The use of the presented electronic nose to assess the degree of thermal degradation of edible oils allows clear classification. In the future, it is planned to use this device to determine the quality of edible oil during and after the frying process. 

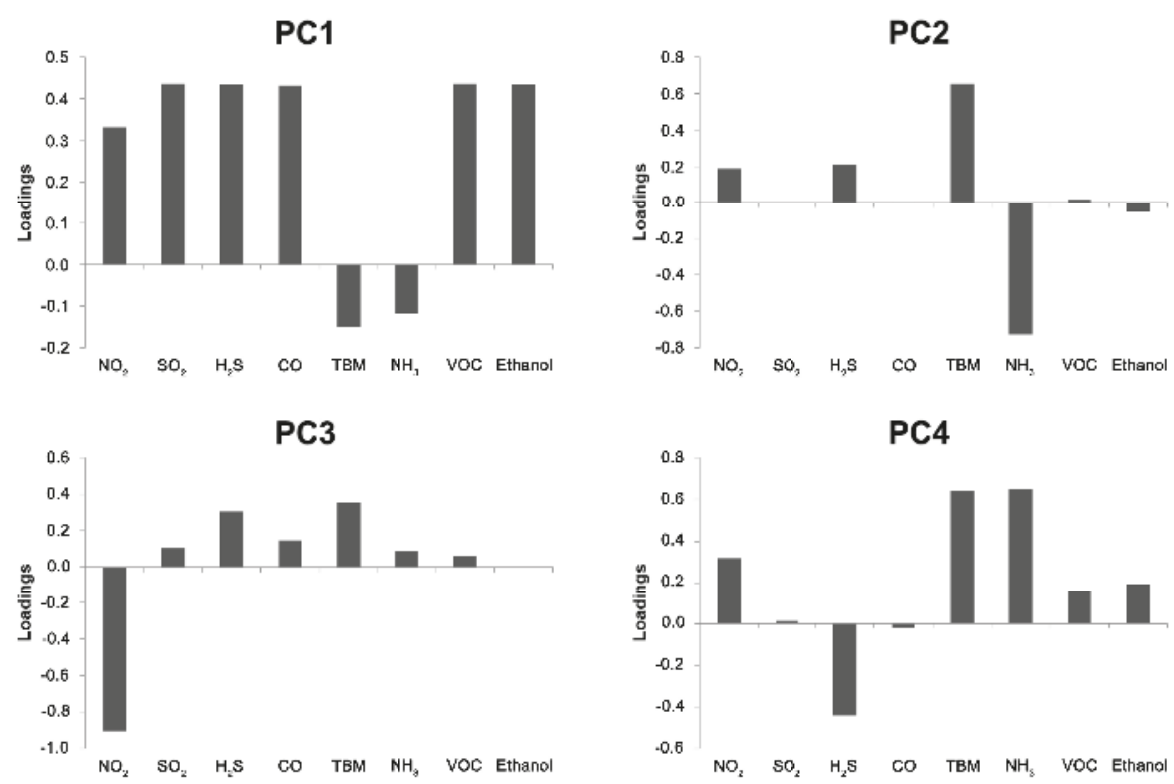

Figure 10. Loadings for the first four principal components in an analysis of rapeseed oil's thermal degradation.

\subsection{Detection of Virgin Olive Oil Adulteration}

The choice of vegetable oil for consumption is dictated mostly by its palatability and nutritional properties. Because of concern for consumers' well-being, the verification of the authenticity of edible oils is one of the key challenges in food analysts [32]. The most commonly adulterated vegetable oil is olive oil. The reason why other oils are added to olive oil is its high price. The inacceptable practice of some oil manufacturers is to admix poor quality oil, which results in an unsatisfactory flavour and nutritional characteristics [33,34]. The most common oil additives are sunflower oil, olive oil of poor quality or hazelnut oil $[32,33,35]$. The use of electronic noses to detect the adulterations of edible oils, mainly extra virgin olive oil, has been a subject of many research projects [36-39]. The use of electrochemical sensors in e-nose systems for detecting oil falsification is presented for the first time in this paper.

The SVM algorithm with RBF kernel was used to classify samples of extra virgin olive oil based on the volume of admixed sunflower oil. Based on the results of ANOVA signals from six sensors, namely $\mathrm{NO}_{2}, \mathrm{SO}_{2}, \mathrm{H}_{2} \mathrm{~S}, \mathrm{VOC}, \mathrm{CO}$, and Ethanol were used as features in the statistical analysis. Cross-validation was performed in the same way as in the previously-described experiments, and the classification accuracy was $82.4 \%$. The method was trained on $66 \%$ of the data set, chosen at random, and the remaining test set was on average classified correctly with $90 \%$ accuracy. A sample confusion matrix for the classification is shown in Figure 6c. It can be noted that misclassifications are contained within the group of samples with $5 \%$ and $10 \%$ admixture of sunflower oil. It can, however, be determined whether a given olive oil sample was adulterated with $100 \%$ accuracy.

\section{Conclusions}

In this article, a portable electronic nose equipped with new-generation electrochemical sensors is described. The use of replaceable sensor modules increases the application potential of this device. Electrochemical sensors are characterized by low electricity consumption, insusceptibility to humidity changes, and long usage life. The use of a perforated plate inside the sensor chamber guarantees 
the elimination of flow turbulence, which contributes to signal stability, and the easy-to-use interface allows the device to be used even by inexperienced personnel.

Also shown are examples of real-time applications in food quality assessment. The prototype electronic nose was used to assess meat shelf-life, thermal degradation of edible oils, and to detect adulterations of extra virgin olive oil with good results, although the application examples were preliminary in character and will in the future be followed by more in-depth studies of the particular matrixes with tailored arrays of electrochemical sensors. Also, work is underway on developing a hand-held solution in which electrochemical sensors will also be used. Electronic nose systems can in the near future be used to complement the existing food quality assessment methods, in particular in screening tests at the production line.

Acknowledgments: The investigations were financially supported by Grant No. UMO-2015/19/B/ST4/02722 from the National Science Centre.

Author Contributions: Wojciech Wojnowski and Tomasz Majchrzak conceived, designed, and performed the experiments; Tomasz Dymerski and Jacek Gẹbicki analysed the data; Jacek Namieśnik contributed reagents/materials/analysis tools; Wojciech Wojnowski and Tomasz Majchrzak wrote the paper.

Conflicts of Interest: The authors declare no conflict of interest.

\section{References}

1. Sanaeifar, A.; ZakiDizaji, H.; Abdolabbas, J.; de la Guardia, M. Early detection of contamination and defect in foodstuffs by electronic nose: A review. TrAC Trends Anal. Chem. 2017, 97, 257-271. [CrossRef]

2. Gliszczyńska-Świgło, A.; Chmielewski, J. Electronic Nose as a Tool for Monitoring the Authenticity of Food. A Review. Food Anal. Methods 2017, 10, 1800-1816. [CrossRef]

3. Gardner, J.W.; Bartlett, P.N. A brief history of electronic noses. Sens. Actuators B Chem. 1994, 18, $210-220$. [CrossRef]

4. Hong, X.; Wang, J. Discrimination and Prediction of Pork Freshness by E-nose. In CCTA 2011: Computer and Computing Technologies in Agriculture V, International Conference on Computer and Computing Technologies in Agriculture, Beijing, China, 29-31 October 2011; Springer: Berlin/Heidelberg, Germany, 2012; pp. 1-14.

5. Peris, M.; Escuder-Gilabert, L. A 21st century technique for food control: Electronic noses. Anal. Chim. Acta 2009, 638, 1-15. [CrossRef] [PubMed]

6. Loutfi, A.; Coradeschi, S.; Mani, G.K.; Shankar, P.; Rayappan, J.B.B. Electronic noses for food quality: A review. J. Food Eng. 2015, 144, 103-111. [CrossRef]

7. O'Connell, M.; Valdora, G.; Peltzer, G.; Martín Negri, R. A practical approach for fish freshness determinations using a portable electronic nose. Sens. Actuators B Chem. 2001, 80, 149-154. [CrossRef]

8. Wojnowski, W.; Majchrzak, T.; Dymerski, T.; Gębicki, J.; Namieśnik, J. Electronic noses: Powerful tools in meat quality assessment. Meat Sci. 2017, 131, 119-131. [CrossRef] [PubMed]

9. Dymerski, T.; Gẹbicki, J.; Wardencki, W.; Namieśnik, J. Application of an electronic nose instrument to fast classification of Polish honey types. Sensors 2014, 14, 10709-10724. [CrossRef] [PubMed]

10. Severini, C.; Derossi, A.; Fiore, A.G.; Ricci, I.; Marone, M. The electronic nose system: Study on the global aromatic profile of espresso coffee prepared with two types of coffee filter holders. Eur. Food Res. Technol. 2016, 1-9. [CrossRef]

11. Gursoy, O.; Somervuo, P.; Alatossava, T. Preliminary study of ion mobility based electronic nose MGD-1 for discrimination of hard cheeses. J. Food Eng. 2009, 92, 202-207. [CrossRef]

12. Dymerski, T.; Gębicki, J.; Wardencki, W.; Namieśnik, J. Quality evaluation of agricultural distillates using an electronic nose. Sensors 2013, 13, 15954-15967. [CrossRef] [PubMed]

13. Macías Macías, M.; Agudo, J.E.; García Manso, A.; García Orellana, C.J.; González Velasco, H.M.; Gallardo Caballero, R. A compact and low cost electronic nose for aroma detection. Sensors 2013, 13, 5528-5541. [CrossRef] [PubMed]

14. Rodríguez-Méndez, M.L.; De Saja, J.A.; González-Antón, R.; García-Hernández, C.; Medina-Plaza, C.; García-Cabezón, C.; Martín-Pedrosa, F. Electronic Noses and Tongues in Wine Industry. Front. Bioeng. Biotechnol. 2016, 4, 1-12. [CrossRef] [PubMed] 
15. Cetó, X.; González-Calabuig, A.; Crespo, N.; Pérez, S.; Capdevila, J.; Puig-Pujol, A.; del Valle, M. Electronic tongues to assess wine sensory descriptors. Talanta 2017, 162, 218-224. [CrossRef] [PubMed]

16. Di Natale, C.; Macagnano, A.; Davide, F.; D’Amico, A.; Paolesse, R.; Boschi, T.; Faccio, M.; Ferri, G. An electronic nose for food analysis. Sens. Actuators B Chem. 1997, 44, 521-526. [CrossRef]

17. Cetó, X.; Voelcker, N.H.; Prieto-Simón, B. Bioelectronic tongues: New trends and applications in water and food analysis. Biosens. Bioelectron. 2017, 79, 608-626. [CrossRef] [PubMed]

18. Wilson, A.D. Review of Electronic-nose Technologies and Algorithms to Detect Hazardous Chemicals in the Environment. Procedia Technol. 2012, 1, 453-463. [CrossRef]

19. Gębicki, J. Application of electrochemical sensors and sensor matrixes for measurement of odorous chemical compounds. TrAC Trends Anal. Chem. 2016, 77, 1-13. [CrossRef]

20. Bourgeois, W.; Romain, A.-C.; Nicolas, J.; Stuetz, R.M. The use of sensor arrays for environmental monitoring: Interests and limitations. J. Environ. Monit. 2003, 5, 852-860. [CrossRef] [PubMed]

21. Demšar, J.; Curk, T.; Erjavec, A.; Hočevar, T.; Milutinovič, M.; Možina, M.; Polajnar, M.; Toplak, M.; Starič, A.; Stajdohar, M.; et al. Orange: Data Mining Toolbox in Python. J. Mach. Learn. Res. 2013, 14, 2349-2353.

22. Boser, B.E.; Guyon, I.M.; Vapnik, V.N. A training algorithm for optimal margin classifiers. In Proceedings of the Fifth Annual Workshop on Computational Learning Theory-COLT'92, Pittsburgh, PA, USA, 27-29 July 1992; ACM Press: New York, NY, USA, 1992; pp. 144-152.

23. Fishler, F. Commission Decision of 8 March 1995 Fixing The Total Volatile Basic Nitrogen (TVB-N) Limit Values for Certain Categories of Fishery Products and Specifying the Analysis Methods to Be Used. Off. J. Eur. Communities 1995, 97, 95-98.

24. Timsorn, K.; Thoopboochagorn, T.; Lertwattanasakul, N.; Wongchoosuk, C. Evaluation of bacterial population on chicken meats using a briefcase electronic nose. Biosyst. Eng. 2016, 151, 116-125. [CrossRef]

25. Lovestead, T.M.; Bruno, T.J. Detection of poultry spoilage markers from headspace analysis with cryoadsorption on a short alumina PLOT column. Food Chem. 2010, 121, 1274-1282. [CrossRef]

26. Alexandrakis, D.; Brunton, N.P.; Downey, G.; Scannell, A.G.M. Identification of Spoilage Marker Metabolites in Irish Chicken Breast Muscle Using HPLC, GC-MS Coupled with SPME and Traditional Chemical Techniques. Food Bioprocess Technol. 2012, 5, 1917-1923. [CrossRef]

27. Senter, S.D.; Arnold, J.W.; Chew, V. APC values and volatile compounds formed in commercially processed, raw chicken parts during storage at 4 and $13{ }^{\circ} \mathrm{C}$ and under simulated temperature abuse conditions. J. Sci. Food Agric. 2000, 80, 1559-1564. [CrossRef]

28. Bordin, K.; Kunitake, M.T.; Aracava, K.K.; Trindade, C.S.F. Changes in food caused by deep fat frying-A review. Arch. Latinoam. Nutr. 2013, 63, 5-13. [PubMed]

29. Boskou, G.; Salta, F.N.; Chiou, A.; Troullidou, E.; Andrikopoulos, N.K. Content of trans,trans-2,4-decadienal in deep-fried and pan-fried potatoes. Eur. J. Lipid Sci. Technol. 2006, 108, 109-115. [CrossRef]

30. Firestone, D. Official Methods and Recommended Practices of the American Oil Chemists' Society, 4th ed.; American Oil Chemists' Society: Champaign, IL, USA, 1992.

31. Choe, E.; Min, D.B. Chemistry of deep-fat frying oils. J. Food Sci. 2007, 72, 1-10. [CrossRef] [PubMed]

32. Majchrzak, T.; Lubinska, M.; Różańska, A.; Dymerski, T.; Gębicki, J.; Namieśnik, J. Thermal degradation assessment of canola and olive oil using ultra-fast gas chromatography coupled with chemometrics. Monatshefte Chem. 2017, 148, 1625-1630. [CrossRef] [PubMed]

33. Aparicio, R.; Morales, M.T.; Alonso, V. Authentication of European Virgin Olive Oils by Their Chemical Compounds, Sensory Attributes, and Consumers' Attitudes. J. Agric. Food Chem. 1997, 45, 1076-1083. [CrossRef]

34. Monfreda, M.; Gobbi, L.; Grippa, A. Blends of olive oil and sunflower oil: Characterisation and olive oil quantification using fatty acid composition and chemometric tools. Food Chem. 2012, 134, 2283-2290. [CrossRef] [PubMed]

35. De La Mata-Espinosa, P.; Bosque-Sendra, J.M.; Bro, R.; Cuadros-Rodríguez, L. Olive oil quantification of edible vegetable oil blends using triacylglycerols chromatographic fingerprints and chemometric tools. Talanta 2011, 85, 177-182. [CrossRef] [PubMed]

36. Mildner-Szkudlarz, S.; Jeleń, H. The potential of different techniques for volatile compounds analysis coupled with PCA for the detection of the adulteration of olive oil with hazelnut oil. Food Chem. 2008, 110, 751-761. [CrossRef] 
37. Concepción, O.C.; Pérez Pavón, L.J.; García Pinto, C.; Fernández Laespada, E.; Moreno Cordero, B.; Forina, M. Electronic nose based on metal oxide semiconductor sensors as a fast alternative for the detection of adulteration of virgin olive oils. Anal. Chim. Acta 2002, 459, 219-228. [CrossRef]

38. Buratti, S.; Benedetti, S.; Cosio, M.S. An electronic nose to evaluate olive oil oxidation during storage. Ital. J. Food Sci. 2005, 17, 203-210.

39. Mildner-Szkudlarz, S.; Jeleń, H.H. Detection of olive oil adulteration with rapeseed and sunflower oils using MOS electronic nose and SPME-MS. J. Food Qual. 2010, 33, 21-41. [CrossRef]

(C) 2017 by the authors. Licensee MDPI, Basel, Switzerland. This article is an open access article distributed under the terms and conditions of the Creative Commons Attribution (CC BY) license (http://creativecommons.org/licenses/by/4.0/). 


\title{
Article \\ Development of Spectral Disease Indices for 'Flavescence Dorée' Grapevine Disease Identification
}

\author{
Hania AL-Saddik *, Jean-Claude Simon and Frederic Cointault \\ INRA, UMR 1347 Agroecology, 21000 Dijon, France; jean-claude.simon@agrosupdijon.fr (J.-C.S.); \\ frederic.cointault@agrosupdijon.fr (F.C.) \\ * Correspondence: hania.al-saddik@agrosupdijon.fr
}

Received: 19 October 2017; Accepted: 26 November 2017; Published: 29 November 2017

\begin{abstract}
Spectral measurements are employed in many precision agriculture applications, due to their ability to monitor the vegetation's health state. Spectral vegetation indices are one of the main techniques currently used in remote sensing activities, since they are related to biophysical and biochemical crop variables. Moreover, they have been evaluated in some studies as potentially beneficial for detecting or differentiating crop diseases. Flavescence Dorée (FD) is an infectious, incurable disease of the grapevine that can produce severe yield losses and, hence, compromise the stability of the vineyards. The aim of this study was to develop specific spectral disease indices (SDIs) for the detection of FD disease in grapevines. Spectral signatures of healthy and diseased grapevine leaves were measured with a non-imaging spectro-radiometer at two infection severity levels. The most discriminating wavelengths were selected by a genetic algorithm (GA) feature selection tool, the Spectral Disease Indices (SDIs) are designed by exhaustively testing all possible combinations of wavelengths chosen. The best weighted combination of a single wavelength and a normalized difference is chosen to create the index. The SDIs are tested for their ability to differentiate healthy from diseased vine leaves and they are compared to some common set of Spectral Vegetation Indices (SVIs). It was demonstrated that using vegetation indices was, in general, better than using complete spectral data and that SDIs specifically designed for FD performed better than traditional SVIs in most of cases. The precision of the classification is higher than $90 \%$. This study demonstrates that SDIs have the potential to improve disease detection, identification and monitoring in precision agriculture applications.
\end{abstract}

Keywords: spectral analysis; feature selection; genetic algorithms; classification; vegetation indices; vineyard; diseases

\section{Introduction}

Plant pathogens pose a major threat to crops and reduce yields worldwide [1]. Marks occurring due to two different infections can be quite similar. Also, patches resulting of the same infection do not appear the same way depending on the crop variety and surrounding conditions. Thus, identifying crop diseases based on symptomatology alone is a complicated and subjective task and is often not sufficient. Additional laboratory tests are usually required to confirm the visual diagnosis.

Since 2013, about half of the French vineyard (400,000 ha) is placed in compulsory control zone against the FD and its insect vector [2]. The FD has also spread to other southern European countries (Italy, Portugal, Serbia and Switzerland) where it induced serious yield losses [2,3] and was declared as a quarantine body by the European Union. FD disease may result in the deterioration of the European grape quality. When FD is identified, massive amounts of pesticides are applied to prevent the propagation of the infection. This implies other problems such as chemical pollution and soil contamination. In order to efficiently apply pesticides, detecting and mapping initial established symptoms of diseases seems crucial. 
Traditional methods for damage identification in crops include, first, visual inspection, a subjective and time-consuming approach particularly when large fields are scouted; second, laboratory-based techniques (Enzyme Linked Immunosorbent Essay (ELISA) or Polymerase Chain Reaction (PCR)), laborious biological techniques that require a strictly defined protocol in order to give a reliable result. Conversely, recent Remote sensing (RS) technology is capable of continuously analyzing information acquired by a device placed at a distance from the phenomenon of interest. RS devices can be grouped into two categories: imaging and non-imaging devices. In the first category, we find Hyperspectral/Multispectral Imaging, Fluorescence Imaging, Thermal Imaging and RGB Imaging. In the second category, we have Visible/Near Infra-Red Spectroscopy, Thermal Spectroscopy and Fluorescence Spectroscopy. RS techniques were exploited in the agricultural field in general $[4,5]$ and have shown great potential in crop monitoring and yield mapping by providing new techniques that can replace or enhance classical approaches used in cultivar management [6,7]. Published scientific literature on the use of RS techniques to detect diseases are numerous. Hyperspectral Imaging was used by [8] to detect fusarium infection and by [9] to identify pathogens and necrosis in sugar beet. The authors in [10], used Multispectral Imaging to detect Huanglongbing in citrus trees. The study [11] consisted on using near-range and aerial hyperspectral sensors to detect fungal diseases. Other researchers combined existing techniques, for example, in [12] hyperspectral reflectance and multi-spectral imaging techniques were used together for fungal diseases detection in arable crops. Spectral reflectance is a valid tool to monitor vegetation's health status. In fact, infections induce changes in pigments [13,14], water content and tissue functionality. These changes often alter the spectral characteristics of leaves. The spectral reflectance from sugarcane infected by thrips (Fulmekiola serrata Kobus) was significantly different between several damage severity levels and the highest difference occurred in the red edge region [15]. Other researchers, such as in [16], also studied reflectance alteration to identify leaf blight in rice.

One way to analyze vegetative spectral modifications due to an infection is the use of spectral vegetation indices (SVIs), calculated as ratios of reflectance at different wavebands. Many researchers investigated the potential of SVIs in detecting diseases. For example, [17] studied some sugar beet fungal diseases, [18] analyzed basal stem rot infection in oil palm trees. Focusing mainly on grapevines, [19], analyzed the spectral reflectance of red-berried leaves infected by Grapevine Leaf Roll disease (GLD) and found a set of variables capable of detecting pathogen presence. Another study was conducted by [20], it identified a feature vector made of 11 indices, able to spectrally differentiate healthy from GLD infected data.

In the above-mentioned studies, the SVIs used are common ones, however, the impact of plant diseases on the physiology and phenology of plants, varies with the host-pathogen interaction and each disease may influence the spectral signature in a different way. Common SVIs are not disease-specific (or disease-dependent); hence, it seems beneficial to design special indices for each infection (SDIs), as these might simplify the disease detection by spectral sensors. SDIs, unlike general SVIs, are designed to identify a specific damage in plants. They couple information from parts of the electromagnetic spectra which are characteristic of an infection and not visible to the human eye. Therefore, they may have the potential to automate the disease detection procedure, not only replacing to some extent the pathologist but also predicting the presence of an infection before its symptoms become noticeable.

In our study, spectral reflectance was acquired under production conditions, directly in the field. Furthermore, unlike other research works, that tested only one variety, we took into consideration 4 different grapevine varieties ( 2 red-berried grapevine and 2 white-berried grapevine). Based on the above background, the main objectives of this paper were (i) to identify disease specific single wavelengths and wavelength differences based on a GA feature selection tool, (ii) to combine these specific wavelengths to spectral disease indices and (iii) to compare the accuracy of the developed indices with respect to common SVIs. 


\section{Materials and Methods}

\subsection{FD Grapevine Disease and Identification Tests}

The distinction between different grapevine diseases is a complicated task [21]. In fact, various diseases can cause almost identical symptoms; also, different symptoms may appear due to the same virus depending on the grapevine variety. Furthermore, marks can be the result of a fusion of many infections affecting the plant at the same time. Some factors such as bad weather conditions, nutrient deficiencies, pollution and pesticides can produce expressions indistinguishable from those of diseases; moreover, the time of infection and the overall environment can affect the appearance of signs on leaves. Present in the national territory since the mid-twentieth century, FD disease is transmitted to the vine by the Scaphoideus Titanus leafhopper and is progressing regularly in France [22].

Three symptoms must be present simultaneously (some are shown in Figure 1) and on the same branch to conclude the presence of FD [2-23]: the change in leaf coloration, the absence of lignification of the new shoots and the mortality of the inflorescences and the berries. For more details, [24] have reviewed in their paper the biology and the ecology of FD. In this study, we are assessing the possibility of FD detection based exclusively on foliar symptoms by employing spectral technology. The FD is difficult to detect because the characteristic symptoms usually appear at least one year after inoculation, not necessarily every year, nor on all the branches. In addition, grapevine varieties have different sensitivities with respect to FD, so the symptoms are not expressed the same fashion. Mainly, the discoloration of leaves varies according to the grape varieties (yellow for white-berried grapevines, red for red-berried grapevines). Other complications that arise when detecting FD is the similarity between its symptoms and those of other yellows of the vine such as 'Bois Noir' (BN); however, new chemical methods based on Polymerase Chain Reaction (PCR) are capable of detecting and differentiating BN from FD.

When FD is diagnosed in the field and in order to control the overall risk, uprooting contaminated vines regularly and applying pesticides to limit the population of leafhoppers, are the currently applied approaches.

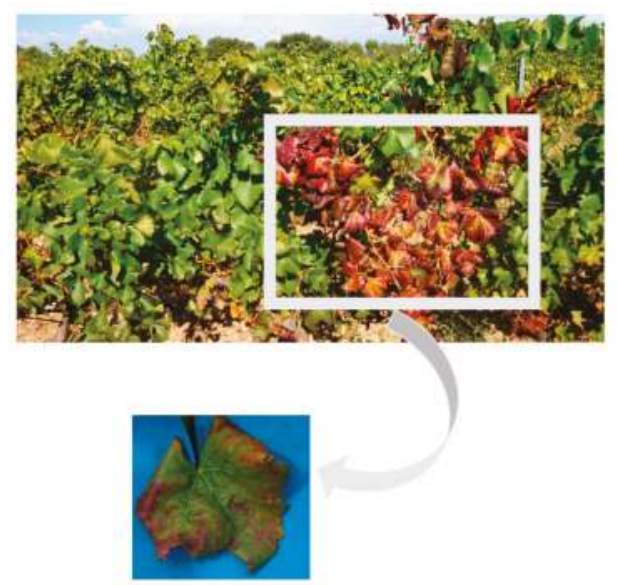

(a)
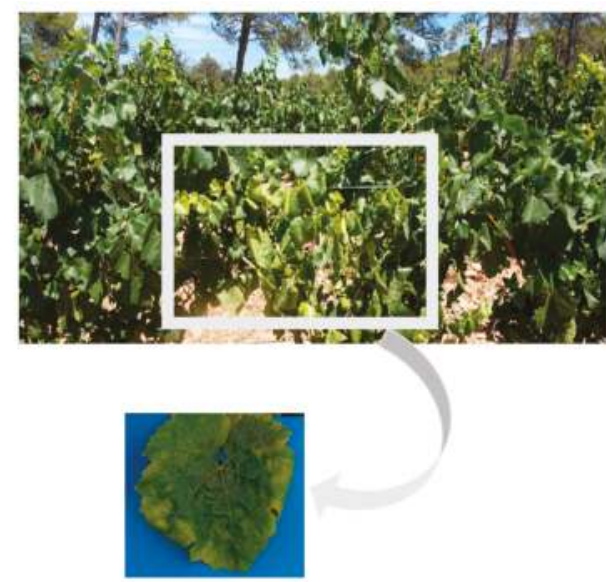

(b)

Figure 1. Some symptoms of FD on leaves: a red discoloration on a red grapevine variety (a) and a yellow discoloration on a white grapevine variety (b); windings of leaves can also be noticed.

\subsection{Sampling Set-Up}

In 2016, spectral signatures were considered from Provence-Alpes Côte d'Azur (PACA) French region (Figure 2). Two acquisition campaigns were conducted at the time in the PACA region. The first 
one took place on the 9th of August, where, symptoms affected only parts of the leaves. The second one took place on the 27 th of September, where symptoms affected complete leaves.

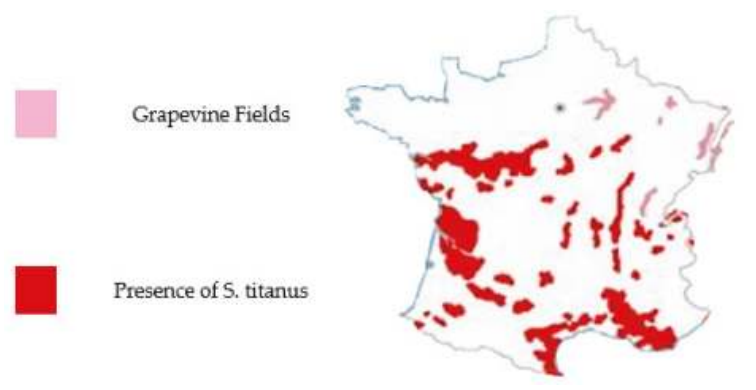

Figure 2. Vineyard distribution and S. titanus presence in France (from [24] modified).

Four grapevine varieties were tested, 2 red-berried ones (Marselan, Grenache) and 2 white-berried ones (Vermentino, Chardonnay). Red-berried fields were measured first in the morning from (10:00 to 12:00) and white-berried fields were measured next in the afternoon (14:00 to 16:00).

Measurements were performed on 2-4 leaves per grapevine and 2-4 measurements were made on each leaf. Four diseased and four healthy grapevines were considered for each grapevine variety. In total, there were 213 diseased and 201 healthy samples (63 Diseased Grenache and 64 Healthy Grenache; 63 Diseased Marselan and 64 Healthy Marselan; 47 Diseased Vermentino and 40 Healthy Vermentino, 42 Diseased Chardonnay and 34 Healthy Chardonnay). A range of healthy leaves of different ages was selected; however, for the infected leaves, a set is chosen in order to get a complete and representative range of FD symptoms. In order to ensure timely follow-up, the grapevines were located using a GPS and leaves were labeled. We tried to consider the same leaves during both acquisition campaigns but after testing them in August, leaves were not necessarily present in September; they either naturally fell or were cut by the winegrower. Thus, when the leaf was not found, we considered another candidate located on the same branch.

An inspector from the Regional Federation of Defense against Pests of PACA was there to confirm the presence of the disease and its severity stage. Furthermore, extra laboratory tests (PCR analysis) were done after the end of the acquisition campaigns to support the inspector's claim.

\subsection{Reflectance Measurements}

Spectral reflectance is the ratio of incident to reflected radiant flux measured from a surface over a defined range of wavelengths. Spectral reflectance measurements from leaf surfaces, in this study, were acquired using a portable Spectro-radiometer (FieldSpec 3, Analytical Spectral Devices, Boulder, CO, USA). Measurements were made on each leaf using a plant probe, specially designed with a low power source, for sensible vegetation surfaces, leaving no observable damage. It has the advantage of reducing the effect of environmental light scattering to insure better measurement accuracy. Each sample data was taken every $1 \mathrm{~nm}$ from $350 \mathrm{~nm}$ to $2500 \mathrm{~nm}$. This was the result of an interpolation performed by the software because the true spectral resolution of the instrument is about $3 \mathrm{~nm}$ at $700 \mathrm{~nm}$ wavelengths and about $10 \mathrm{~nm}$ at 1400 or longer wavelengths. Before starting the acquisitions, the spectro-radiometer was warmed up for a minimum of $20 \mathrm{~min}$, then a calibration was performed to absolute reflectance using a Teflon calibration disk. The number of samples for Spectrum was set to 30, the number of samples for Dark Current and White Reference were set to 100. It took approximately four hours to complete the measurements directly in the field.

Spectral measurements were taken from the same locations on leaves (shown in Figure 3) for both acquisition campaigns and one measurement is taken per location. The locations were chosen 
according to the disease that has tendency to start growing between the veins first. A range of 2-4 measurements is considered depending on the leaf surface with respect to the probe diameter. When the leaf is small, only 2 reflectance spectra from 2 locations are acquired and when the leaf is wide enough, 4 spectral tests are taken from all the 4 locations. The same procedure is applied for healthy leaves and infected leaves.

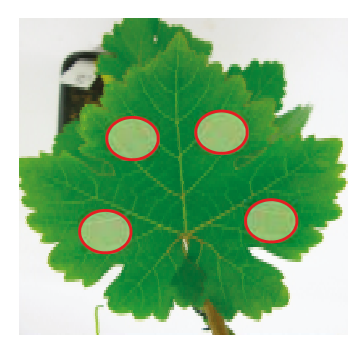

Figure 3. Locations of the measurements on a sample leaf.

\subsection{Spectral Data Analysis for Disease Detection}

Each spectral measurement, acquired in this study, is the reflectance in a large number of contiguous narrow bands (350-2500 nm). Analyzing such high dimensional data is a complex and time-consuming task; therefore, reducing the dimensionality of the data, by selecting optimal wavebands, seems crucial. Techniques, such as SDIs, that uses only few spectral bands, are useful in the hyperspectral data analysis.

\subsubsection{Spectral Disease Vegetation Indices Development Based on GA Feature Selection (SDIs)}

In this section, the procedure is detailed from the acquisition of spectral signatures till the creation of disease-specific (or disease-dependent) indices. Figure 4 shows the approach that was adapted to compute and to evaluate SDIs.

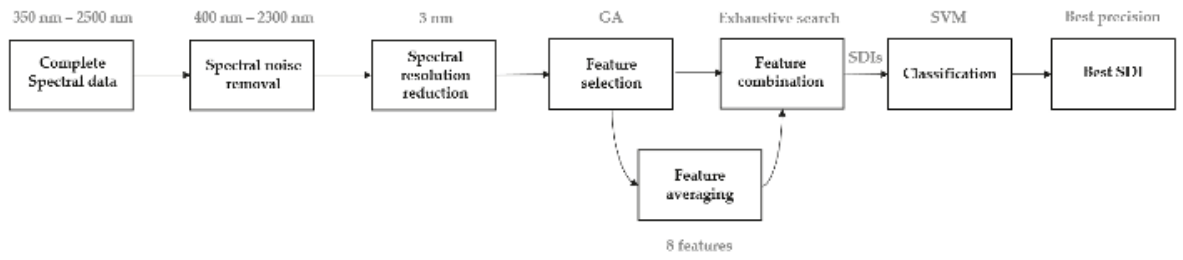

Figure 4. Systematical approach and development of SDIs from hyperspectral reflectance data.

After the acquisition of the spectral signatures of leaves from the field, we obtained a set of healthy and diseased observations ranging from 350 to $2500 \mathrm{~nm}$, with a total of 2151 features or wavelengths. Since the spectral data were noisy at the extremities, values between $400 \mathrm{~nm}$ and $2100 \mathrm{~nm}$ were only considered and adopted, giving in total 1901 wavelengths. Following this, the spectral resolution was reduced by a factor of 3 due to high correlation between adjacent wavelengths. In consequence, only 633 wavelengths were considered for the rest of the analysis (Figure 5).

From the modified set of observations obtained, the best wavelengths were chosen by applying a GA feature selection tool. Genetic Algorithms (GA) provides a valid tool for solving optimization and search problems; it imitates the natural human evolution process [25]. GA manipulates one population to produce a new one based on some genetic operators. The five important steps in GA [26] are: (1) chromosome encoding, (2) fitness evaluation, (3) selection mechanisms, (4) genetic operators and (5) criteria to stop the GA (Figure 6). 


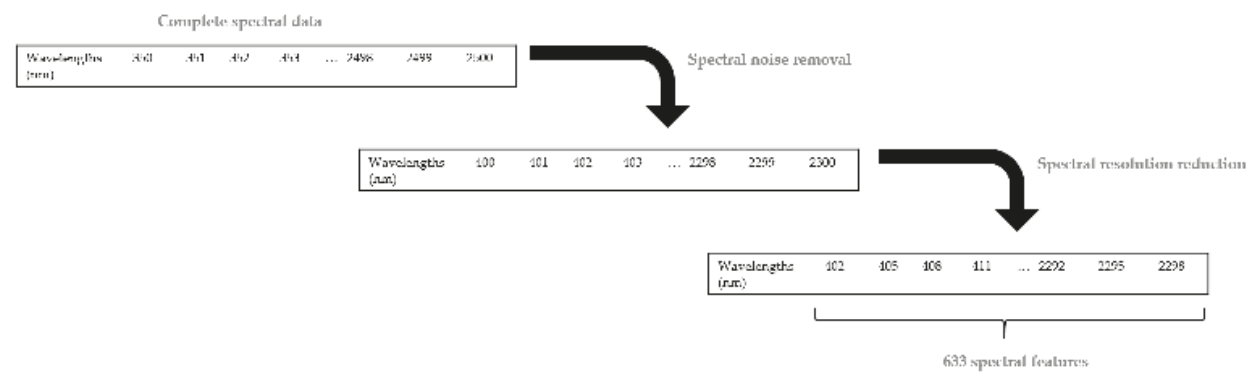

Figure 5. Noise removal and resolution reduction of spectral data.

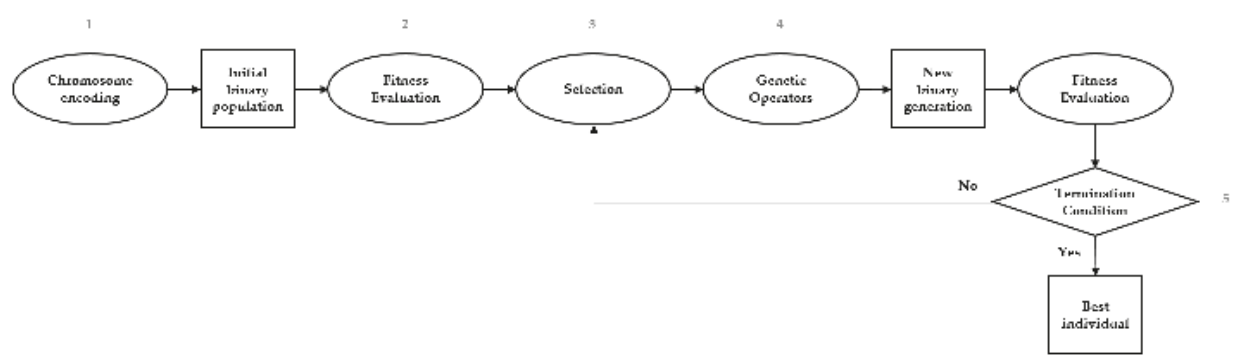

Figure 6. GA-Based Feature Selection.

Human genetics vocabulary is often used in GA, chromosomes are the bit strings (individuals that form the population), gene is the feature [27]. In this study, a binary space is assumed: a gene value " 1 " indicates that the feature indexed by the " 1 " is chosen. Contrarily, (i.e., if it is 0 ), the feature is not chosen for evaluation. At the beginning, a matrix of dimension (Population size (300 samples) $\times$ Number of wavelengths (633 spectral features)) containing random binary digits is created, which forms the initial population. A fitness function evaluates the discriminative capacity of the population, made by chromosomes, each selecting a subset of features. In this work, the loss obtained by cross-validated SVM (Support Vector Machine) classification model is used. Individuals are ranked, based on the values reported by the fitness function; then, the Elite kids with the best fitness values, are selected to survive and are, hence, transferred to the next generation. The selection operation provides individuals for genetic cross-over and mutation; it ensures that the population is being constantly improved. Tournament Selection was used here due to its simplicity, speed and efficiency. Cross-over consists on combining two parent individuals to form children in the new generation. XOR operation is performed in this case since parent chromosomes are binary [28]. The number of new children produced due to the cross-over operator, is defined based on the cross-over fraction. Mutation is another genetic operator and induces a perturbation of chromosomes by applying a bit flipping procedure depending on the mutation probability. Mutation ensures genetic diversity, eliminating premature convergence. A uniform mutation is applied in this study. The number of new children produced due to the mutation operator, is defined by subtracting the population size from the number of elite children and the number of children obtained by cross-over. Each new generation, formed by GA, contains individuals from Elite kids, crossover kids and mutation kids [29]. The new population is evaluated again and the GA continues to evolve until the stopping condition is met. Two stopping conditions are applied in this study: Maximum Number of Generations and Stall Generation Limit. GA terminates if the average changes in the fitness values among the chromosomes over Stall Generation Limit generations is less than or equal to tolerance function. The goal is to insure genetic homogeneity. All the GA parameters used in our study are described in Table 1. 
Table 1. Numerical values of different GA parameters considered in this study.

\begin{tabular}{cc}
\hline GA Parameter & Value \\
\hline Population size (Number of Chromosomes) & 300 \\
Genome length (Number of genes/features) & 300 \\
Population type & Bit strings \\
Fitness Function & 300 \\
Number of generations & 50 \\
Stall generation limit & Arithmetic \\
Crossover & 0.8 \\
Crossover Probability & Uniform Mutation \\
Mutation & 0.2 \\
Mutation Probability & Tournament of size 2 \\
Selection scheme & 2 \\
Elite Count & \\
\hline
\end{tabular}

When GA terminates, one individual is chosen providing the convergence. This individual contains the optimal features, it is a binary set with " 1 " meaning that the feature at this specific index is considered. Since the initial population is randomly created, the number of selected wavelengths by the GA tool cannot be predicted and is function of the data, in fact, the GA keeps evolving until convergence and the number of features might be big. In order to reduce computational cost, we averaged the selected wavelengths chosen by GA to obtain only 8 wavelengths representative of the set (Figure 7). However, this feature averaging step is optional and all wavelengths selected by GA can be used in the feature combination step.
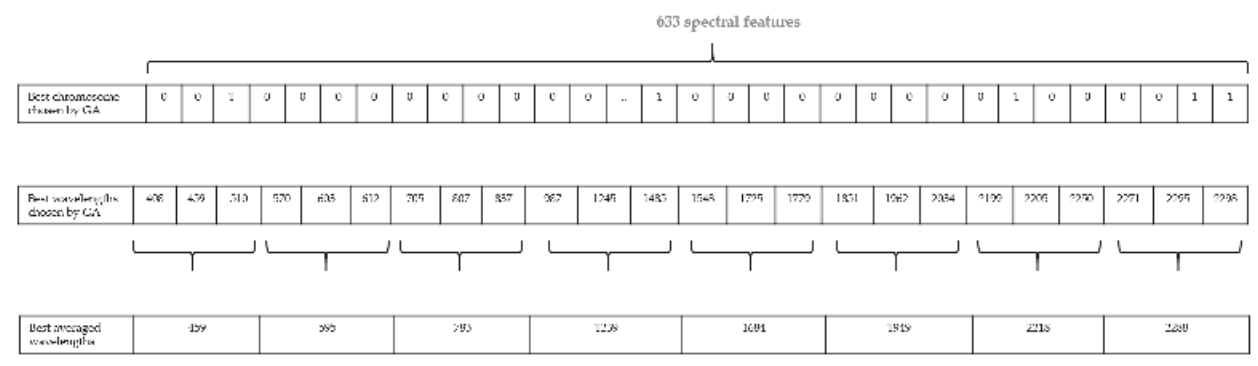

Figure 7. Example of feature averaging.

The indices to be developed aim at identifying a specific plant disease. Thus, a combination of a single wavelength and a normalized wavelength difference seemed suitable. A weighting factor for the single wavelength was determined and the possible weights were: $-1,-0.5,0.5$ and 1. An exhaustive search of the best SDI is undertaken, combinations of an individual wavelength and a normalized wavelength difference are tested. Each combination of 3 wavelengths and a weighting factor forms an index (Equation (1)). When feature averaging is applied: 8 wavelengths $\times 7$ wavelengths $\times 6$ wavelengths $\times 4$ weighting factors $=1344$ possible combinations or SDIs were tested. The ideal case would be, again, to consider directly the wavelengths selected by GA with no averaging and evaluate all possible combinations. The indices were assessed for their classification ability using a 10-fold cross validation SVM model and the configuration providing the best classification precision is retained, this optimal configuration is the best SDI.

$$
\mathrm{SDI}=\mathrm{ab}+(\mathrm{c}+\mathrm{d}) /(\mathrm{c}-\mathrm{d})
$$

where $a, c, d$ are wavelengths chosen from the pool of the 8 best averaged wavelengths $(a \neq c \neq d)$ and $b$ is the weighting factor. 


\subsubsection{Common Spectral Vegetation Indices computation (SVIs)}

In the context of vegetation status monitoring, identifying a specific disease or stress, can be done using spectral reflectance measurements. Discrimination between healthy and infected plants is performed based on some optimal wavelengths or a combination of wavelengths. The principal aim of SVIs is to highlight a certain property of the vegetation; they are combinations of reflectance at 2 or many wavelengths. Several vegetation indices have been proposed in the scientific literature, most of them relate the physiological status of crop to hyperspectral data through their correlation to biochemical constituents (chlorophyll, carotenoids, water, cellulose, lignin, dry matter ... ). Pigment-specific vegetation indices are, currently, an effective data analysis tool for disease discrimination. The ability to identify FD with vegetation indices, found in the literature, was tested in this segment. The classification accuracies of the NDVI, the PRI, the ARI, the SIPI, the mCAI, the PSSRa, PSSRb and PSSRc, the GM1 and GM2, the ZTM and the TCARI/OSAVI were compared to those obtained by SDIs (Table 2).

Table 2. Typical SVIs in literature and applied in this study.

\begin{tabular}{|c|c|c|c|}
\hline Index Name & Formula & Association with Relevant Plant Pigment & $\begin{array}{l}\text { Reference } \\
\text { Example }\end{array}$ \\
\hline $\begin{array}{l}\text { Normalized Difference } \\
\text { Vegetation Index (NDVI) }\end{array}$ & $\begin{array}{l}\text { NDVI_705 }=(\text { R750 - } \\
\text { R705) } /(\text { R750 + R705 })\end{array}$ & $\begin{array}{l}\text { NDVI is a very typical index. Positive values suggest } \\
\text { vegetated areas. }\end{array}$ & {$[30,31]$} \\
\hline $\begin{array}{l}\text { Photochemical Reflectance } \\
\text { index (PRI) }\end{array}$ & $\begin{array}{l}\text { PRI }=(\text { R570 - } \\
\text { R531 }) /(\text { R570 + R531 })\end{array}$ & $\begin{array}{l}\text { PRI index is a function of the reflectance at the } 531 \\
\mathrm{~nm} \text {, this reflectance is related to xanthophyll. When } \\
\text { the xanthophyll activity is high, the light use } \\
\text { efficiency is low, meaning a possible stress occurred. }\end{array}$ & [32-34] \\
\hline $\begin{array}{l}\text { Anthocyanin Reflectance } \\
\text { Index (ARI) }\end{array}$ & $\mathrm{ARI}=(1 / \mathrm{R} 550)-(1 / \mathrm{R} 700)$ & $\begin{array}{l}\text { ARI index is designed to estimate the stack of } \\
\text { anthocyanin in senescing and stressed leaves. }\end{array}$ & [35] \\
\hline $\begin{array}{l}\text { The structure insensitive } \\
\text { pigment index (SIPI) }\end{array}$ & $\begin{array}{l}\mathrm{SIPI}=(\mathrm{R} 800- \\
\mathrm{R} 445) /(\mathrm{R} 800+\mathrm{R} 680)\end{array}$ & $\begin{array}{l}\text { The SIPI index is responsive to the ratio of } \\
\text { carotenoids to chlorophyll. It is very practical to use } \\
\text { when the canopy structure or leaf area index are } \\
\text { inconsistent. }\end{array}$ & {$[36,37]$} \\
\hline $\begin{array}{l}\text { Modified chlorophyll } \\
\text { absorption integral (mCAI) }\end{array}$ & $\begin{array}{l}\mathrm{mCAI}=(\mathrm{R} 545+\mathrm{R} 752) / 2 \times(752- \\
545)-\left(\sum 545-752(1.158 \times \mathrm{R})\right)\end{array}$ & $\begin{array}{l}\text { The mCAI is sensitive to the chlorophyll content. } \\
\text { It calculates the area between a straight line } \\
\text { connecting two points (the green peak at } 545 \mathrm{~nm} \text { and } \\
752 \mathrm{~nm} \text { ) and the curve itself }\end{array}$ & {$[38]$} \\
\hline $\begin{array}{l}\text { Pigment specific simple } \\
\text { ratio chlorophyll a (PSSRa) }\end{array}$ & PSSRa $=$ R800/R680 & \multirow{3}{*}{$\begin{array}{l}\text { The pigment specific ratio indices were suggested to } \\
\text { estimate the pigment's content at the leaf level. } \\
\text { Samples from trees at different senescence stages } \\
\text { were studied aiming to empirically determine the } \\
\text { best individual wavebands for pigment assessment } \\
\text { ( } 680 \mathrm{~nm} \text { for chlorophyll a, } 635 \mathrm{~nm} \text { for chlorophyll b, } \\
470 \mathrm{~nm} \text { for the carotenoids). }\end{array}$} & {$[36,39,40]$} \\
\hline $\begin{array}{l}\text { Pigment specific simple } \\
\text { ratio chlorophyll b (PSSRb) }\end{array}$ & $\mathrm{PSSRb}=\mathrm{R} 800 / \mathrm{R} 635$ & & {$[36,41,42]$} \\
\hline $\begin{array}{l}\text { Pigment specific simple } \\
\text { ratio carotenoids (PSSRc) }\end{array}$ & PSSRc $=$ R800 $/$ R470 & & [36-42] \\
\hline $\begin{array}{l}\text { Gitelson and Merzlyak } \\
1 \text { (GM1) }\end{array}$ & GM1 = R750/R550 & \multirow{2}{*}{$\begin{array}{l}\text { GM1 and GM2 were created to measure the } \\
\text { chlorophyll content in vegetation leaves. }\end{array}$} & {$[43,44]$} \\
\hline $\begin{array}{l}\text { Gitelson and Merzlyak } \\
2 \text { (GM2) }\end{array}$ & GM2 = R750/R700 & & {$[45]$} \\
\hline Zarco-Tejada Miller (ZTM) & $\mathrm{ZTM}=\mathrm{R} 750 / \mathrm{R} 710$ & $\begin{array}{l}\text { ZTM is a Red edge index highly correlated to } \\
\text { chlorophyll content. At the canopy level, it has the } \\
\text { advantage of minimizing shadow effects. }\end{array}$ & {$[46,47]$} \\
\hline $\begin{array}{l}\text { Ratio of the Transformed } \\
\text { Chlorophyll Absorption in } \\
\text { Reflectance Index and } \\
\text { Optimized Soil-Adjusted } \\
\text { Vegetation Index } \\
\text { (TCARI/OSAVI) }\end{array}$ & $\begin{array}{l}\text { TCARI }=3 \times((\mathrm{R} 700-\mathrm{R} 670)-0.2 \\
\times(\mathrm{R} 700-\mathrm{R} 550) \times(\mathrm{R} 700 / \mathrm{R} 670)) \\
\mathrm{OSAVI}=(1+0.16) \times(\mathrm{R} 800- \\
\mathrm{R} 670) /(\mathrm{R} 800+\mathrm{R} 670+0.16)\end{array}$ & $\begin{array}{l}\text { A combination of the Transformed Chlorophyll } \\
\text { Absorption in Reflectance Index (TCARI) and the } \\
\text { Optimized Soil-Adjusted Vegetation Index (OSAVI). } \\
\text { It is sensitive to chlorophyll content variations and } \\
\text { resistant to variations in Leaf Area Index (LAI) and } \\
\text { underlying soil background effect. }\end{array}$ & {$[48,49]$} \\
\hline
\end{tabular}

\subsection{Classification}

There are hundreds of classifiers in the literature and it is often difficult for researchers to choose an appropriate classifier for a certain application. The easiest approach that is used to address this issue is to try several classifiers and select the one having the highest accuracy. In this work, we selected only one classifier, the Support Vector Machines since it is one of the most widely used classifiers in the field and gave good performance in several applications [50,51]. 


\subsubsection{Support Vector Machines (SVM)}

SVM is a supervised machine learning algorithm, mostly used to solve classification problems. It consists on defining a boundary (line/hyperplane) that best separates two classes [52]. The closest points to the boundary are called support vectors, the margin is the perpendicular distance calculated from the boundary to the support vectors. A maximal-margin classifier defines a hyperplane separating two classes and having the largest margin. However, a soft-margin classifier allows points to lie between the margins or on the wrong side of the plane. It is usually used when classes are not fully separable.

In practice, SVM are implemented using kernels. When applying non-linear Kernels (polynomial or radial), non-linear boundaries are created and the accuracy improves. Due to its flexibility, the Radial Basis Function (RBF) kernel is however the most used, so we employed it also in our study. One of the most known methods for fitting SVM is the Sequential Minimal Optimization (SMO) method. The concept and the applications of SVM are discussed in detail in [53].

\subsubsection{Data Configuration}

In our study, we employed a binary classification involving only 2 classes: we considered the healthy group vs. the diseased group in total (medium infested measurements from the August acquisition campaign + high infested measurements from the September acquisition campaign). Since there are four grapevine varieties tested in this study (Marselan, Grenache, Vermentino and Chardonnay), it is possible to analyze the measurements of each variety alone, or measurements can be combined. Based on the grapevine color, we can analyze red types and white types; it is also feasible to combine all leaf measurements together (Figure 8).

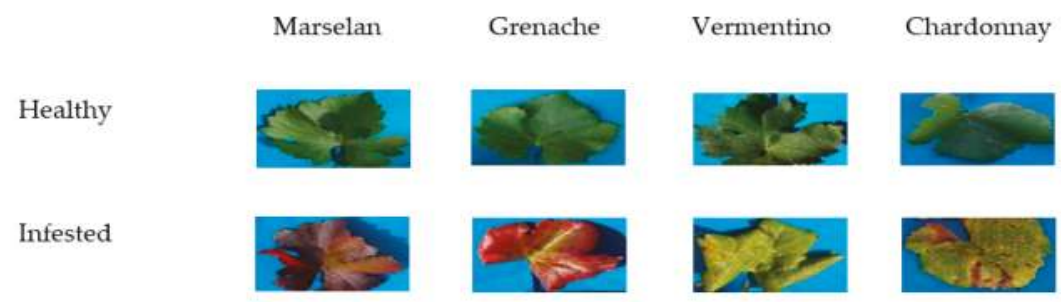

Figure 8. Configuration of analyzed data, infested against healthy groups for a binary classification.

\section{Results}

Spectroscopic and imaging techniques have demonstrated good potential in detecting disease and stress in crops. Currently, researchers tend to apply spectral vegetation indices (SVIs) to identify different plant diseases.

\subsection{Reflectance Spectra of Diseased Grapevine Leaves}

When comparing spectral signatures of healthy and infected red/white berried leaves in Figure 9, obvious differences can be depicted, suggesting that the spectral response was affected by the infestation. For the Marselan variety (a red-berried variety), the healthy spectra were higher than the infested ones in the visible (VIS) region (mainly between 500-700 nm) but the opposite occurred in the region NIR (800-1300 $\mathrm{nm}$ ) and in the IR region (>1300 nm). It seems like when the infestation arises, the spectral signature is lower in the VIS region and higher in the NIR-IR region, the same trend was also observed for the Grenache type (data not shown here). On the other hand, for the Chardonnay variety (a white berried variety), the healthy spectra were lower than the infested ones in the VIS region (mainly between 500-700 $\mathrm{nm}$ ) but the opposite occurred in the region NIR (800-1300 $\mathrm{nm}$ ) and in the IR region (>1300 nm). It seems like when the infestation occurs, the spectral signature is higher in the 
VIS region and lower in the NIR-IR region, the same trend was also observed for the Vermentino type (data not shown here). These changes prove that the spectral signature depends on the pathogen-host interaction. In other words, the grapevine variety does not show the same pattern when the same infestation occurs.

The mean value from a 10-fold cross-validation was reported for the classification; in this manner, all the data were taken into account and variances between different experiments under similar conditions were considered.

The model accuracy defined the percentage of testing set samples correctly classified and the False Negative Rate (FNR) defines the percentage of negative results that are, in fact, positive; in contrast, False Positive Rate (FPR) defines the percentage of positive results that are, in fact, negative. When plotting on a single graph, the FPR values on the abscissa and the TPR values on the ordinate, the resulting curve is called ROC (Receiver Operating Characteristic) curve, AUC (Area Under Curve) refers to the area under the curve. The advantage of using dimension reduction techniques based on GA will be demonstrated next.

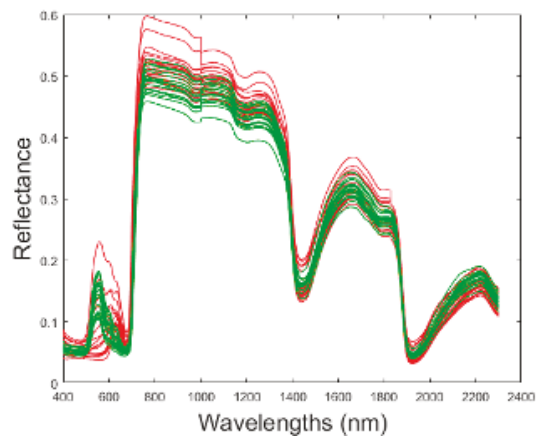

(a)

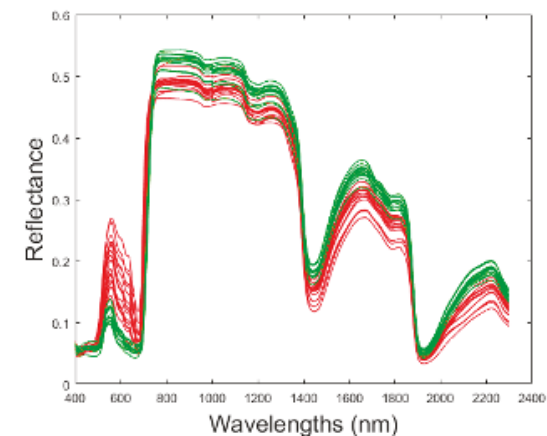

(b)

Figure 9. Reflectance of infected (Red) and healthy (green) leaves for Marselan (a) and Chardonnay grapevines (b).

\subsection{No Dimension Reduction, Use of Complete Spectral Data}

In this section, all spectral data are considered $(400-2100 \mathrm{~nm})$ in the analysis; this means that no dimension reduction method is applied in this case.

Table 3 presents the result of using complete spectra measurements from August (slightly infected leaves). The best classification accuracy is for Vermentino (93.75\%) and the worst is for Marselan variety (70.97\%). The Grenache and Chardonnay measurements gave similar precision (90.63\%). What can be critical in disease diagnosis is probably the FNR, which means that a diseased case was claimed to be healthy. In general, the lower the FNR, the better the classifier is. Here, the best FNR was also for the Vermentino (6.67\%). When considering combined measurements depending on the color of the grapevines, the observations of the White measurements were better than Red ones $(92.19 \%>87.3 \%)$.

Table 4 presents the result of using complete spectra measurements from September (highly infected leaves). The accuracy from September, in general, is better than that of August for all kinds of measurements. This seems logical, since symptoms at the end of the season become well established, diseased spectral reflectance are more influenced by the disease and can be more easily discriminated from healthy ones. White berried grapevines performed better that red-ones when each grapevine type is considered alone or combined (Marselan-Grenache 94.79-95.06\% vs. Vermentino-Chardonnay 98.18-97.73\%; Red 96.61\% < White 98.99\%). Furthermore, for White-berried leaves no FPR was reported. The hardest classification scenario is when all measurements were combined because observations from all grapevine types having different characteristics were put together. However, we obtained a satisfying SVM precision (96.01\%) and a good AUC (0.99). 
Table 3. Results of using complete spectra in classifying different groups of spectral data acquired in the August acquisition campaign (Severity of infestation $=1$ ).

\begin{tabular}{ccccc}
\hline Grapevine Variety & Accuracy (\%) & FNR (\%) & FPR (\%) & AUC \\
\hline Marselan & 70.97 & 27.27 & 30.00 & 0.82 \\
Grenache & 90.63 & 7.14 & 11.11 & 0.73 \\
Vermentino & 93.75 & 6.67 & 5.88 & 0.93 \\
Chardonnay & 90.63 & 12.50 & 6.25 & 0.95 \\
Red & 87.30 & 7.69 & 16.22 & 0.96 \\
White & 92.19 & 9.38 & 6.25 & 0.93 \\
All & 88.19 & 13.85 & 9.68 & 0.95 \\
\hline
\end{tabular}

Table 4. Results of using complete spectra in classifying different groups of spectral data acquired in the September acquisition campaign (Severity of infestation $=2$ ).

\begin{tabular}{ccccc}
\hline Grapevine Variety & Accuracy (\%) & FNR (\%) & FPR (\%) & AUC \\
\hline Marselan & 94.79 & 4.35 & 6.00 & 0.99 \\
Grenache & 95.06 & 2.38 & 7.69 & 0.97 \\
Vermentino & 98.18 & 3.23 & 0.00 & 0.96 \\
Chardonnay & 97.73 & 3.85 & 0.00 & 0.96 \\
Red & 96.61 & 2.22 & 4.60 & 0.99 \\
White & 98.99 & 1.75 & 0.00 & 0.93 \\
All & 96.01 & 2.08 & 6.06 & 0.99 \\
\hline
\end{tabular}

Table 5 presents the result of using complete spectra measurements from August in addition to those from September (slightly + highly infected leaves). Here the accuracy in general was better than considering moderately infected leaves (from August) but was less than using only highly infected leaves for the analysis (from September). The classification's accuracy was above $92 \%$ for all cases: the best was for Chardonnay (97.37\%), no FPR was found for this variety. When combining measurements was applied, similar results were found for Red, White and All configurations (around 95-96\% of accuracy).

Table 5. Results of using complete spectra in classifying different groups of spectral data acquired in the August and September acquisition campaigns (Severity of infestation $=1 \& 2$ ).

\begin{tabular}{ccccc}
\hline Grapevine Variety & Accuracy (\%) & FNR (\%) & FPR (\%) & AUC \\
\hline Marselan & 92.91 & 9.23 & 4.84 & 0.98 \\
Grenache & 96.77 & 1.72 & 4.55 & 0.89 \\
Vermentino & 96.55 & 2.22 & 4.76 & 0.97 \\
Chardonnay & 97.37 & 4.65 & 0.00 & 0.97 \\
Red & 96.41 & 3.28 & 3.88 & 0.99 \\
White & 95.09 & 5.56 & 4.11 & 0.93 \\
All & 95.65 & 1.98 & 6.60 & 0.99 \\
\hline
\end{tabular}

\subsection{Dimension Reduction Using Vegetation Indices (SVIs)}

In this section, the results of the classification using the common SVIs are presented. Only the best SVIs will be presented next, for more details refer to Tables A1-A3.

Table 6 presents the result of calculating the best traditional SVI from August measurements (slightly infected leaves). The classification accuracies were satisfying ( $>90 \%)$ and they were more advantageous than using the complete spectra. No FNR was reported for Grenache leaves. The best SVIs were ARI, ZTM, TCARI/OSAVI. ARI is convenient for Red-grapevine varieties when considered individually or combined. ZTM behaved well for Vermentino and Chardonnay but when combined, TCARI/OSAVI performed better. This index was also robust when all observations are considered together $(92.13 \%)$. 
Table 6. Results of using the best SVIs in classifying different groups of spectral data acquired in the August acquisition campaign (Severity of infestation $=1$ ).

\begin{tabular}{cccccc}
\hline Grapevine Variety & Accuracy $\mathbf{( \% )}$ & FNR $(\%)$ & FPR $(\mathbf{\%})$ & AUC & Best SVIs \\
\hline Marselan & 90.32 & 11.11 & 7.69 & 0.94 & PRI-ARI \\
Grenache & 96.88 & 0.00 & 6.25 & 1.00 & ARI \\
Vermentino & 93.75 & 5.88 & 6.67 & 0.94 & ZTM \\
Chardonnay & 90.63 & 11.11 & 7.14 & 0.91 & NDVI-ZTM \\
Red & 95.24 & 5.88 & 3.45 & 0.99 & ARI \\
White & 92.19 & 11.11 & 3.57 & 0.92 & TCARI/OSAVI \\
All & 92.13 & 13.51 & 1.89 & 0.92 & TCARI/OSAVI \\
\hline
\end{tabular}

Table 7 presents the result of calculating the best traditional SVI from September measurements (highly infected leaves). In this case, all accuracies were enhanced with respect to those of August $(>94 \%)$. When compared to using complete spectra, SVIs used less wavebands and gave better accuracies except for the case of mixing all measurements together $(96.01 \%>94.02 \%)$. The best results were associated with White-berried signatures and no FNR were found (97-98\%). ARI, ZTM, GM1 and mCAI accomplished best precisions. Similar to the first acquisition campaign, the index ARI was interesting for the red-berried signatures. Moreover, ZTM was chosen for Vermentino and Chardonnay. However, when combined, GM1 was selected. mCAI was the most robust index when All measurements are mixed together $(94.02 \%)$.

Table 7. Results of using the best SVIs in classifying different groups of spectral data acquired in the September acquisition campaign (Severity of infestation $=2$ ).

\begin{tabular}{cccccc}
\hline Grapevine Variety & Accuracy (\%) & FNR (\%) & FPR (\%) & AUC & Best SVIs \\
\hline Marselan & 96.88 & 4.00 & 2.17 & 0.9805 & ARI \\
Grenache & 97.53 & 2.70 & 2.27 & 0.9988 & ARI \\
Vermentino & 98.18 & 0.00 & 3.23 & 0.9613 & NDVI-mCAI-PSSRb-ZTM \\
Chardonnay & 97.73 & 0.00 & 3.85 & 0.9579 & NDVI-mCAI-PSSRb-GM1-GM2-ZTM-TCARI/OSAVI \\
Red & 98.31 & 2.33 & 1.10 & 0.9991 & ARI \\
White & 98.99 & 0.00 & 1.75 & 0.9792 & ARI-GM1 \\
All & 94.20 & 7.52 & 2.80 & 0.9540 & mCAI \\
\hline
\end{tabular}

Table 8 presents the result of calculating the best traditional SVI from August measurements in addition to those from September (slightly + highly infected leaves). The performance was less than using only spectra with well-established disease marks from September. When compared to using complete spectra, Marselan, White-berried data were better or very similar to using complete spectra. However, for Grenache and mixed data the use of all wavelengths was more accurate. The best SVIs were ARI, GM1, ZTM and mCAI. ARI was again chosen to be the best SVI for classifying White grape leaves and was also selected when red grape leaves reflectance was tested (93.23\%). GM1 was found to be interesting for Vermentino but ZTM was more convenient for Chardonnay. mCAI, like the above case, was the most robust index when all measurements are mixed together $(88.41 \%)$.

Table 8. Results of using the best SVIs in classifying different groups of spectral data acquired in the August and the September acquisition campaigns (Severity of infestation $=1$ \& 2).

\begin{tabular}{cccccc}
\hline Grapevine Variety & Accuracy (\%) & FNR (\%) & FPR (\%) & AUC & Best SVIs \\
\hline Marselan & 93.70 & 8.70 & 3.45 & 0.9824 & ARI \\
Grenache & 91.13 & 13.70 & 1.96 & 0.9753 & ARI \\
Vermentino & 95.40 & 6.98 & 2.27 & 0.9730 & GM1 \\
Chardonnay & 96.05 & 5.56 & 2.50 & 0.9672 & GM2-ZTM \\
Red & 93.23 & 11.19 & 0.93 & 0.9888 & ARI \\
White & 95.09 & 8.64 & 1.22 & 0.9433 & ARI \\
All & 88.41 & 16.96 & 5.98 & 0.9184 & mCAI \\
\hline
\end{tabular}




\subsection{Dimension Reduction Using Spectral Disease Indices (SDIs)}

The discriminatory capacity of the best single wavelengths and wavelength differences chosen by the GA were tested. This data reduction procedure was the foundation for spectral disease index development. In this section, the results of the classification using the SDIs are presented.

Table 9 presents the result of calculating SDIs from August measurements (slightly infected leaves). A 100\% success with no FNR in classifying individual grapevine measurements was obtained, except for Chardonnay. When combining observations, the results were also satisfying (precision > 94.44\%). In general, better percentage was reached when applying the SDIs than using the complete spectra on one hand and applying conventional SVIs on the other hand.

Table 9. Results of using SDIs in classifying different groups of spectral data acquired in the August acquisition campaign (Severity of infestation $=1$ ).

\begin{tabular}{|c|c|c|c|c|c|c|c|c|}
\hline \multirow{2}{*}{ Grapevine Variety } & \multirow{2}{*}{ Accuracy (\%) } & \multirow{2}{*}{ FNR (\%) } & \multirow{2}{*}{ FPR (\%) } & \multirow{2}{*}{ AUC } & \multicolumn{4}{|c|}{ SDIs } \\
\hline & & & & & $\mathbf{a}$ & c & d & b \\
\hline Marselan & 100.00 & 0.00 & 0.00 & 1.00 & 702 & 957 & 2133 & 1 \\
\hline Grenache & 100.00 & 0.00 & 0.00 & 1.00 & 861 & 2094 & 921 & -1 \\
\hline Vermentino & 100.00 & 0.00 & 0.00 & 1.00 & 735 & 2097 & 1029 & 0.5 \\
\hline Chardonnay & 96.87 & 5.88 & 0.00 & 0.93 & 543 & 876 & 1380 & 1 \\
\hline Red & 95.23 & 6.06 & 3.33 & 0.97 & 1506 & 2214 & 507 & 0.5 \\
\hline White & 96.87 & 3.12 & 3.12 & 0.98 & 792 & 2151 & 654 & -0.5 \\
\hline All & 94.48 & 6.15 & 4.83 & 0.98 & 1401 & 2205 & 501 & -0.5 \\
\hline
\end{tabular}

Table 10 presents the result of calculating SDIs from September measurements (highly infected leaves). A 100\% success with no FNR in classifying leaves measurements each variety at a time and when considering White-reflectance spectra together was obtained. In general, these accuracies are better than those corresponding to the first acquisition campaign. However, when the red varieties are grouped together, it seems that the ARI was better than the SDI $(96.6 \%<98.31 \%)$. When observations were combined, using complete spectra gave a slightly better result than the SDI $(96.01 \%>94.20 \%)$

Table 10. Results of using SDIs in classifying different groups of spectral data acquired in the September acquisition campaign (Severity of infestation $=2$ ).

\begin{tabular}{ccccccccc}
\hline \multirow{2}{*}{ Grapevine Variety } & \multirow{2}{*}{ Accuracy (\%) } & FNR (\%) & FPR (\%) & AUC & \multicolumn{4}{c}{ SDIs } \\
\cline { 6 - 9 } & & & & & $\mathbf{a}$ & $\mathbf{c}$ & $\mathbf{d}$ & $\mathbf{b}$ \\
\hline Marselan & 100.00 & 0.00 & 0.00 & 1.00 & 546 & 708 & 597 & -1 \\
Grenache & 100.00 & 0.00 & 0.00 & 1.00 & 528 & 540 & 1383 & 1 \\
Vermentino & 100.00 & 0.00 & 0.00 & 1.00 & 708 & 1656 & 1755 & 0.5 \\
Chardonnay & 100.00 & 0.00 & 0.00 & 1.00 & 462 & 570 & 888 & -1 \\
Red & 96.61 & 5.68 & 1.12 & 0.97 & 738 & 1650 & 573 & 1 \\
White & 100.00 & 0.00 & 0.00 & 1.00 & 726 & 2166 & 927 & 1 \\
All & 94.20 & 8.95 & 2.81 & 0.98 & 498 & 675 & 1581 & -1 \\
\hline
\end{tabular}

Table 11 presents the result of calculating SDIs from August measurements in addition to those from September (slightly + highly infected leaves). No FPR was present for White-berried data. In general, these accuracies are better than those corresponding to the first acquisition campaign. However, in accordance with the last case, when the red varieties are grouped together, it seems that the ARI was better than the SDI $(92.03 \%<93.23 \%)$. For the White-berried measurements and all mixed ones, it seems that using complete spectra is a bit more advantageous than the SDI but with more computation burden. 
Table 11. Results of using SDIs in classifying different groups of spectral data acquired in the August and the September acquisition campaigns (Severity of infestation $=1 \& 2$ ).

\begin{tabular}{|c|c|c|c|c|c|c|c|c|}
\hline \multirow{2}{*}{ Grapevine Variety } & \multirow{2}{*}{ Accuracy (\%) } & \multirow{2}{*}{ FNR (\%) } & \multirow{2}{*}{ FPR (\%) } & \multirow{2}{*}{ AUC } & \multicolumn{4}{|c|}{ SDIs } \\
\hline & & & & & a & c & d & $\mathbf{b}$ \\
\hline Marselan & 93.70 & 7.57 & 4.91 & 0.95 & 1653 & 2181 & 687 & -1 \\
\hline Grenache & 95.16 & 7.46 & 1.75 & 0.96 & 651 & 1944 & 549 & 1 \\
\hline Vermentino & 96.55 & 6.97 & 0.00 & 0.97 & 687 & 1908 & 762 & -0.5 \\
\hline Chardonnay & 98.68 & 2.85 & 0.00 & 0.98 & 486 & 558 & 966 & -0.5 \\
\hline Red & 92.03 & 7.87 & 8.06 & 0.95 & 1725 & 2226 & 1485 & -0.5 \\
\hline White & 98.15 & 3.89 & 0.00 & 0.98 & 714 & 1404 & 936 & 0.5 \\
\hline All & 89.37 & 12.79 & 8.37 & 0.92 & 1770 & 2208 & 2019 & -0.5 \\
\hline
\end{tabular}

\section{Discussion}

SDI indices were put in place in this article to improve and simplify FD disease detection in grapevines based on hyperspectral data. At the beginning, the most significant wavebands from the VIS, Red-edge, NIR or SWIR (Short-Wave Infrared) needed to be selected.

Feature selection is often used in data pre-processing to identify relevant features having significance in the classification task. The results obtained in this study, confirmed the effectiveness of the GA algorithm in improving the robustness of the feature selection procedure. In fact, GA was able to reach a global optimum despite local peaks that might be caused by noise or interdependencies in the data set. This conclusion was also confirmed in other studies in different fields. In [54] GA selected the best subset of features for breast cancer diagnosis system. Furthermore, in [55] GA feature selection algorithm was applied for hand writing recognition. The complexity of the feature set was reduced using less features and achieved recognition rates similar to those reached when no feature selection is applied.

After the choice of certain wavebands by the GA tool was made, the SDIs were normalized in order to reduce the impact of change in lighting, land, crop variety or sensor specific effects. This helped producing more robust and more generalized indices. SDIs were more advantageous than complete spectra and SVIs in the beginning of the season (August measurements), hence, great promise for early detection of diseases. Using complete spectra was better for the case of combined measurements (August + September) for the Grenache, red-varieties and all data. However, the proposed indices proved high accuracy in general with the advantage of reducing data dimensionality by speeding up the disease detection. SDIs gave, in general, higher accuracy than SVIs but, the ARI index performed a bit better in September measurements for red varieties and all combined data than the corresponding SDIs. The ARI index is documented as a performant feature in many studies. The study [56] concluded that ARI had a persistent response to yellow rust disease at 4 out of 5 growth stages and mentioned that the ARI index was selected for diagnosis of yellow rust in other studies like the one conducted by [57]. Among the indices investigated in the research made by [58], only the ARI index could differentiate healthy from rust infected leaves. However, it was not capable of distinguishing stem rust from leaf rust pustules. In addition to the ARI index, ZTM, GM1, mCAI and TCARI/OSAVI were found to be the best SVIs in this study. The ratio of the TCARI and the OSAVI indices formed a good Chlorophyll estimator, this was done independently of Leaf Area Index (LAI) and illumination state. The ratio demonstrated good results not only in continuous closed crop canopies [49] but also in open tree canopy orchards [59]. Authors in [58] found that the TCARI index, was the only index capable of discriminating stem and leaf rust, among all others. Chlorophyll content is a potential indicator of vegetation stress because of its direct role in the photosynthesis process of light harvesting, initiation of electron transport [46], this was confirmed in our study as the ZTM vegetation index was chosen for white-berried data. Loss of chlorophyll in response to infestation by sap feeding insects like aphids [60] and leafhoppers [61] has been reported earlier. GM1 was also selected in this study; in fact, differences in reflectance between healthy and stressed vegetation due to changes in Chl; $a, b$ levels have been 
detected previously in the green peak and along the red-edge spectral region of 690-750 nm [62]. The CAI index indicates exposed surfaces containing dried plant material [63]. Absorptions in the $2000 \mathrm{~nm}$ to $2200 \mathrm{~nm}$ range are sensitive to cellulose. It was stated in [64] that the CAI index is useful to monitor vegetation coverage for biomass estimation.

Many studies tended to manage pest occurrence in commercially important agricultural crops by designing new and adapted vegetation indices. Research [65] monitored damage by green bugs in wheat by using a hyperspectral spectrometer and a digital camera. They designed 2 indices based on possible band combinations and their correlation with the severity damage. Optimal bands were 509, 537, $572,719,747,873,901 \mathrm{~nm}$. The study detailed in [66], on the other hand, used 2 or 3 narrow bands to design hyperspectral indices in order to assess severity grades of leafhopper in cotton. Two indices gave better results than traditional SVIs from literature and were consistent across tested fields. Interesting bands were: 550, 691, 715, 761, $1124 \mathrm{~nm}$. In the research of [67], two indices were proposed and found to be capable of estimating leaf rust disease. The difficulty was to detect early symptoms due to the resemblance between spectral signatures between lightly infected areas and healthy ones. Based on this research, new indices performed better than other common SVIs. Optimal wavebands found were: 455 , 605, $695 \mathrm{~nm}$. (Mahlein et al., 2013) designed four SDIs and proved high specificity and sensitivity for identifying healthy leaves Cercospora leaf spot, Sugar beet rust and Powdery mildew. Detection of early symptoms of infection was the most difficult part in this research. Chosen bands varied from: 513, 520, $534,570,584,698,704,724,734 \mathrm{~nm}$.

For August data, the majority of the selected bands were found in the NIR region; however, for September data, VIS bands were mostly selected since symptoms became more visually pronounced. In this case, bands from blue (450-520 nm), green $(530-570 \mathrm{~nm})$, red $(580-700 \mathrm{~nm})$ in the VIS were selected. This was in accordance with [19] the 2 maximum differences in the VIS region appeared at the green peak $(550 \mathrm{~nm})$ and in the red peak $(680 \mathrm{~nm})$ indicating less chlorophyll absorption in the infected leaves. Furthermore, changes in Cab levels were translated as modifications occurring over the spectral red edge region, this explains why many optimal bands were selected in the specific range of $690-750 \mathrm{~nm}$. Reflectance near $700 \mathrm{~nm}$ was pointed out by [68] as an essential feature of green vegetation produced by an equilibrium between biochemical and biophysical plant characteristics. Since plant diseases influence the chlorophyll content of crop plants, increased reflectance around $700 \mathrm{~nm}$ can be a first but unspecific indicator to detect diseased crops. Many chosen bands were also mixed with the water absorption bands; the research conducted in [69], demonstrated that the sensitivity to water content was greatest in spectral bands centered at 1450, 1940 where water has its major absorption features.

It can be concluded, as seen from the tables, that the SDIs were dependent on the disease infestation level and the grapevine variety considered; the best wavelengths selected were different from one case to another. As a consequence, although the SDIs tested gave good results, there was no single best index for FD in all situations. In fact, the sensitivity of an index differs depending on the soil, the vegetation and the weather conditions. Therefore, no single index with the same spectral bands was found to be applicable to quantify FD in this research. SDIs were found to be interesting for precision agricultural applications; additional work will be needed in order to apply SDIs in practice. The proposed indices need to be tested on different varieties of grapevines before it can be effectively applied in precision farming. Our study enhances the ability to detect and map FD when foliar symptoms are becoming visible, hence, further tests need to be carried on hosts which do not provide any clear symptoms of infestation, to check if the computed SDIs are capable of predicting early the FD occurrence. Besides, suggested SDIs need to be tested in changing environments, scales and field conditions; other types of diseases must be also taken into consideration to investigate whether SDIs are capable of distinguishing FD from various infestations in general and BN in particular. At the end, it was proven, through this study that the development of indices based on spectral variations due to vegetation diseases, is feasible. 


\section{Conclusions and Perspectives}

Plants display the occurrence of infections in a number of ways. RS is an effective way to detect crop diseases, based on the fact that a pest modifies the photosynthesis phenomenon and the physical structure of the plant, altering the absorption of light by the plant's surface. The difference between spectral signatures of healthy and diseased plants can be the key to identify efficient wavelengths correlated with a specific disease. The transformation of reflectance into vegetation indices is a widely used technique to detect leaf contents (pigments, water, ...); nevertheless, these indices, based on only few wavelengths, showed potential for disease detection. Common vegetation indices are not yet capable of identifying a particular disease. In this article, a data analysis technique was exposed to design specific grapevine disease indices.

Our data set contains a large number of features, in order to reduce the cost and running time, as well as achieving an acceptably high recognition rate, we have selected the most useful ones by applying a GA feature selection tool. GA is one of the most advanced techniques used in the field of predictive analysis, it is computationally expensive but it performs better than common selection techniques and has the advantage of manipulating large data sets with no need for specific knowledge about the problem under study. After being selected, wavelengths are then combined to design the SDI. Depending on the disease severity, on one hand and on the grapevine variety on the other hand, a combination of a single and normalized wavelengths is required each time to correctly identify the FD.

Our study extracted some wavelengths bands sensible to FD occurrence at the leaf-scale. Based on these findings, it might be possible to conceive a multispectral camera, for example and mount the sensor on a movable platform to localize infection foci in a field. However, when going from considering leaves to examining a complete branch, or maybe the whole grapevine, some corrections need to be taken into account. Geometric and radiometric improvements capable to solve shadowing problems, branch structures and interfering reflectance from other surrounding objects are necessary. When applying a multispectral sensor, we will not only have spectral information but also spatial data. Available spatial data will enable adding advanced image processing algorithms to make the detection of FD more robust. Actually, two other FD symptoms cannot be detected spectrally, so, in order to better conclude the presence of the condition, additional pattern recognition algorithms can be integrated directly in the sensor to detect the absence of lignification and the berry mortality.

In accordance with other studies, we found that SDIs performed better than traditional SVIs. The advantages of using SDIs include the dimensionality reduction and the efficiency of computation and processing. The proposed method for SDIs development, in this article, can be transferred to hyperspectral data from different kinds of sensors, it can be used for other crops varieties and for different kinds of diseases or biotic and abiotic stress of crops.

Acknowledgments: We would like to thank Alice Dubois and Sylvain Bernard from the Regional Federation of Defense against Pests of Provence Alpes Côtes Azur, Corinne Trarieux and Jocelyn Dureuil from the Interprofessional Office of Burgundy Wine for their expertise. We also would like to thank the funders of the DAMAV project (Automatic Detection of Grapevine Diseases) and of course the winegrowers for their cooperation.

Author Contributions: Hania AL-Saddik acquired the data with the help of Jean-Claude Simon; Hania AL-Saddik analyzed the data with the help of Frederic Cointault; Hania AL-Saddik wrote the paper, then Jean-Claude Simon and Frederic Cointault corrected it.

Conflicts of Interest: The authors declare no conflict of interest.

\section{Abbreviations}

The following abbreviations are used in this manuscript:

FD

$\mathrm{BN}$

GA

SVI

SDI

\author{
'Flavescence Dorée' \\ 'Bois Noir' \\ Genetic Algorithm \\ Spectral Vegetation Index \\ Spectral Disease Index
}




$\begin{array}{ll}\text { VIS } & \text { Visible } \\ \text { NIR } & \text { Near-Infra red } \\ \text { PACA } & \text { Provence Alpes Côte d'Azur } \\ \text { SVM } & \text { Support Vector Machines } \\ \text { FPR } & \text { False Positive Rate } \\ \text { FNR } & \text { False Negative Rate } \\ \text { ROC } & \text { Receiver Operating Characteristic } \\ \text { AUC } & \text { Area Under the ROC } \\ \text { NDVI } & \text { Normalized Difference Vegetation Index } \\ \text { PRI } & \text { Photochemical Reflectance index } \\ \text { ARI } & \text { Anthocyanin Reflectance Index } \\ \text { SIPI } & \text { Structure insensitive pigment index } \\ \text { mCAI } & \text { Modified chlorophyll absorption integral } \\ \text { PSSRa } & \text { Pigment specific simple ratio chlorophyll a } \\ \text { PSSRb } & \text { Pigment specific simple ratio chlorophyll b } \\ \text { PSSRc } & \text { Pigment specific simple ratio carotenoids } \\ \text { GM1 } & \text { Gitelson and Merzlyak 1 } \\ \text { GM2 } & \text { Gitelson and Merzlyak 2 } \\ \text { ZTM } & \text { Zarco-Tejada Miller } \\ \text { TCARI/OSAVI } & \text { Ratio of the Transformed Chlorophyll Absorption in Reflectance Index and } \\ & \text { Optimized Soil-Adjusted Vegetation Index }\end{array}$

\section{Appendix A}

Table A1. Results of SVIs in classifying different groups of spectral data acquired in the August acquisition campaign (Severity of infestation $=1$ ).

\begin{tabular}{lccccc}
\hline Grapevine Variety & SVIs & Accuracy (\%) & FNR (\%) & FPR (\%) & AUC \\
\hline \multirow{6}{*}{ Marselan } & NDVI & 0.8710 & 0.1176 & 0.1429 & 0.9580 \\
& PRI & 0.9032 & 0.1111 & 0.0769 & 0.9160 \\
& ARI & 0.9032 & 0.1111 & 0.0769 & 0.9412 \\
& SIPI & 0.4516 & 0.5000 & 0.6667 & 0.4244 \\
& mCAI & 0.5806 & 0.3889 & 0.4615 & 0.5924 \\
& PSSRa & 0.5806 & 0.4091 & 0.4444 & 0.5126 \\
& PSSRb & 0.7742 & 0.1875 & 0.2667 & 0.7605 \\
& PSSRc & 0.4839 & 0.4762 & 0.6000 & 0.4244 \\
& GM1 & 0.5484 & 0.4286 & 0.5000 & 0.5462 \\
& GM2 & 0.8387 & 0.1250 & 0.2000 & 0.8992 \\
& ZTM & 0.8387 & 0.1250 & 0.2000 & 0.9244 \\
& TCARI/OSAVI & 0.8387 & 0.2000 & 0.0909 & 0.8193 \\
\hline \multirow{6}{*}{ Grenache } & NDVI & 0.9375 & 0.1053 & 0 & 0.9608 \\
& PRI & 0.9375 & 0.1053 & 0 & 0.9608 \\
& ARI & 0.9688 & 0 & 0.0625 & 1 \\
& SIPI & 0.8750 & 0.1579 & 0.0769 & 0.9529 \\
& mCAI & 0.8750 & 0.1905 & 0 & 0.8902 \\
& PSSRa & 0.5625 & 0.4286 & 0.4545 & 0.5020 \\
& PSSRb & 0.9375 & 0.1053 & 0 & 0.9451 \\
& PSSRc & 0.5938 & 0.4091 & 0.4000 & 0.6549 \\
& GM1 & 0.6563 & 0.2500 & 0.4000 & 0.6471 \\
& GM2 & 0.9375 & 0.1053 & 0 & 0.9529 \\
& ZTM & 0.9375 & 0.1053 & 0 & 0.9490 \\
& TCARI/OSAVI & 0.8750 & 0.1905 & 0 & 0.8314 \\
\hline \multirow{6}{*}{ Vermentino } & NDVI & 0.9063 & 0.1111 & 0.0714 & 0.9412 \\
& PRI & 0.9063 & 0.1111 & 0.0714 & 0.8863 \\
& ARI & 0.6875 & 0.3333 & 0.2727 & 0.6706 \\
SIPI & 0.3438 & 0.5909 & 0.8000 & 0.2627 \\
\hline \multirow{6}{*}{} & & & &
\end{tabular}


Table A1. Cont.

\begin{tabular}{|c|c|c|c|c|c|}
\hline Grapevine Variety & SVIs & Accuracy (\%) & FNR (\%) & FPR (\%) & AUC \\
\hline \multirow{8}{*}{ Vermentino } & mCAI & 0.8438 & 0.2000 & 0.0833 & 0.8667 \\
\hline & PSSRa & 0.5000 & 0.4737 & 0.5385 & 0.5373 \\
\hline & PSSRb & 0.7813 & 0.2222 & 0.2143 & 0.8824 \\
\hline & PSSRc & 0.6250 & 0.3684 & 0.3846 & 0.6000 \\
\hline & GM1 & 0.9063 & 0.1111 & 0.0714 & 0.9608 \\
\hline & GM2 & 0.8750 & 0.1176 & 0.1333 & 0.9255 \\
\hline & ZTM & 0.9375 & 0.0588 & 0.0667 & 0.9490 \\
\hline & TCARI/OSAVI & 0.8438 & 0.2000 & 0.0833 & 0.9490 \\
\hline \multirow{12}{*}{ Chardonnay } & NDVI & 0.9063 & 0.1111 & 0.0714 & 0.9059 \\
\hline & PRI & 0.8438 & 0.2000 & 0.0833 & 0.9647 \\
\hline & ARI & 0.6875 & 0.2941 & 0.3333 & 0.6784 \\
\hline & SIPI & 0.3750 & 0.5652 & 0.7778 & 0.4314 \\
\hline & mCAI & 0.8438 & 0.1667 & 0.1429 & 0.9137 \\
\hline & PSSRa & 0.5938 & 0.4091 & 0.4000 & 0.5725 \\
\hline & PSSRb & 0.8125 & 0.2105 & 0.1538 & 0.9490 \\
\hline & PSSRc & 0.5313 & 0.4286 & 0.5000 & 0.5235 \\
\hline & GM1 & 0.8750 & 0.1176 & 0.1333 & 0.9020 \\
\hline & GM2 & 0.8750 & 0.1176 & 0.1333 & 0.9098 \\
\hline & ZTM & 0.9063 & 0.1111 & 0.0714 & 0.9176 \\
\hline & TCARI/OSAVI & 0.8438 & 0.1667 & 0.1429 & 0.8902 \\
\hline \multirow{12}{*}{ Red } & NDVI & 0.9206 & 0.1316 & 0 & 0.9545 \\
\hline & PRI & 0.9365 & 0.1081 & 0 & 0.9576 \\
\hline & ARI & 0.9524 & 0.0588 & 0.0345 & 0.9980 \\
\hline & SIPI & 0.5556 & 0.4359 & 0.4583 & 0.6768 \\
\hline & mCAI & 0.8254 & 0.2195 & 0.0455 & 0.8566 \\
\hline & PSSRa & 0.5397 & 0.4118 & 0.4483 & 0.6000 \\
\hline & PSSRb & 0.8413 & 0.2250 & 0.0870 & 0.8707 \\
\hline & PSSRc & 0.4444 & 0.5405 & 0.6154 & 0.4313 \\
\hline & GM1 & 0.5079 & 0.3462 & 0.4324 & 0.5081 \\
\hline & GM2 & 0.9206 & 0.1316 & 0 & 0.8758 \\
\hline & ZTM & 0.9206 & 0.1316 & 0 & 0.9414 \\
\hline & TCARI/OSAVI & 0.6825 & 0.270 & 0.2308 & 0.7232 \\
\hline \multirow{12}{*}{ White } & NDVI & 0.6094 & 0.3667 & 0.4118 & 0.6393 \\
\hline & PRI & 0.5313 & 0.4286 & 0.4091 & 0.4633 \\
\hline & ARI & 0.8750 & 0.1795 & 0.0400 & 0.9013 \\
\hline & SIPI & 0.8438 & 0.2051 & 0.0800 & 0.8700 \\
\hline & mCAI & 0.8281 & 0.2439 & 0.0870 & 0.8123 \\
\hline & PSSRa & 0.8125 & 0.2353 & 0.2333 & 0.8485 \\
\hline & PSSRb & 0.7188 & 0.2941 & 0.3000 & 0.7185 \\
\hline & PSSRc & 0.8906 & 0.0938 & 0.1250 & 0.9501 \\
\hline & GM1 & 0.9063 & 0.0882 & 0.0667 & 0.9550 \\
\hline & GM2 & 0.5781 & 0.4194 & 0.4545 & 0.5464 \\
\hline & ZTM & 0.5781 & 0.3704 & 0.4324 & 0.6295 \\
\hline & TCARI/OSAVI & 0.9219 & 0.1111 & 0.0357 & 0.9247 \\
\hline \multirow{12}{*}{ All } & NDVI & 0.6614 & 0.3043 & 0.2931 & 0.7251 \\
\hline & PRI & 0.6063 & 0.4063 & 0.4286 & 0.5593 \\
\hline & ARI & 0.8268 & 0.1884 & 0.1552 & 0.8970 \\
\hline & SIPI & 0.8898 & 0.1549 & 0.0893 & 0.9134 \\
\hline & mCAI & 0.8898 & 0.1471 & 0.1186 & 0.8841 \\
\hline & PSSRa & 0.8661 & 0.1667 & 0.0909 & 0.8605 \\
\hline & PSSRb & 0.5669 & 0.4179 & 0.4333 & 0.6022 \\
\hline & PSSRc & 0.6378 & 0.2833 & 0.3284 & 0.6772 \\
\hline & GM1 & 0.8110 & 0.1940 & 0.1833 & 0.8658 \\
\hline & GM2 & 0.6614 & 0.3158 & 0.2549 & 0.7318 \\
\hline & ZTM & 0.5984 & 0.4154 & 0.4355 & 0.6278 \\
\hline & TCARI/OSAVI & 0.9213 & 0.1351 & 0.0189 & 0.9221 \\
\hline
\end{tabular}


Table A2. Results of SVIs in classifying different groups of spectral data acquired in the September acquisition campaign (Severity of infestation $=2$ ).

\begin{tabular}{|c|c|c|c|c|c|}
\hline Grapevine Variety & SVIs & Accuracy (\%) & FNR $(\%)$ & FPR $(\%)$ & AUC \\
\hline \multirow{12}{*}{ Marselan } & NDVI & 0.7604 & 0.2400 & 0.2391 & 0.7234 \\
\hline & PRI & 0.6250 & 0.3774 & 0.3721 & 0.6018 \\
\hline & ARI & 0.9688 & 0.0400 & 0.0217 & 0.9805 \\
\hline & SIPI & 0.7917 & 0.1778 & 0.2353 & 0.7972 \\
\hline & mCAI & 0.9583 & 0.0213 & 0.0612 & 0.9440 \\
\hline & PSSRa & 0.5104 & 0.4762 & 0.5000 & 0.4774 \\
\hline & PSSRb & 0.6146 & 0.3571 & 0.4074 & 0.6331 \\
\hline & PSSRc & 0.6667 & 0.3509 & 0.3077 & 0.6817 \\
\hline & GM1 & 0.9479 & 0.0769 & 0.0227 & 0.9709 \\
\hline & GM2 & 0.8021 & 0.2222 & 0.1667 & 0.7933 \\
\hline & ZTM & 0.7292 & 0.2653 & 0.2766 & 0.7117 \\
\hline & TCARI/OSAVI & 0.9375 & 0.0784 & 0.0444 & 0.9431 \\
\hline \multirow{12}{*}{ Grenache } & NDVI & 0.6543 & 0.3784 & 0.3182 & 0.6867 \\
\hline & PRI & 0.6914 & 0.3000 & 0.3137 & 0.6873 \\
\hline & ARI & 0.9753 & 0.0270 & 0.0227 & 0.9988 \\
\hline & SIPI & 0.8395 & 0.2273 & 0.0811 & 0.8747 \\
\hline & mCAI & 0.9383 & 0.0789 & 0.0465 & 0.9681 \\
\hline & PSSRa & 0.7407 & 0.2500 & 0.2653 & 0.7377 \\
\hline & PSSRb & 0.5679 & 0.4667 & 0.4118 & 0.5577 \\
\hline & PSSRc & 0.7284 & 0.2222 & 0.2963 & 0.7506 \\
\hline & GM1 & 0.9383 & 0.1190 & 0 & 0.9969 \\
\hline & GM2 & 0.6790 & 0.3514 & 0.2955 & 0.7230 \\
\hline & ZTM & 0.6296 & 0.4054 & 0.3409 & 0.6683 \\
\hline & TCARI/OSAVI & 0.7901 & 0.2500 & 0.1707 & 0.8974 \\
\hline \multirow{12}{*}{ Vermentino } & NDVI & 0.9818 & 0 & 0.0323 & 0.9600 \\
\hline & PRI & 0.9455 & 0.0769 & 0.0345 & 0.9613 \\
\hline & ARI & 0.7455 & 0.2105 & 0.2778 & 0.8080 \\
\hline & SIPI & 0.9273 & 0.0435 & 0.0938 & 0.9560 \\
\hline & mCAI & 0.9818 & 0 & 0.0323 & 0.9613 \\
\hline & PSSRa & 0.8364 & 0.2143 & 0.1111 & 0.8547 \\
\hline & PSSRb & 0.9818 & 0 & 0.0323 & 0.9600 \\
\hline & PSSRc & 0.6727 & 0.3333 & 0.3235 & 0.7320 \\
\hline & GM1 & 0.9455 & 0.0769 & 0.0345 & 0.9573 \\
\hline & GM2 & 0.9636 & 0.0400 & 0.0333 & 0.9600 \\
\hline & $\mathrm{ZTM}$ & 0.9818 & 0 & 0.0323 & 0.9600 \\
\hline & TCARI/OSAVI & 0.9636 & 0.0400 & 0.0333 & 0.9573 \\
\hline \multirow{12}{*}{ Chardonnay } & NDVI & 0.9773 & 0 & 0.0385 & 0.9537 \\
\hline & PRI & 0.9545 & 0 & 0.0741 & 0.9474 \\
\hline & ARI & 0.9318 & 0.0556 & 0.0769 & 0.9053 \\
\hline & SIPI & 0.9318 & 0.1000 & 0.0417 & 0.9516 \\
\hline & mCAI & 0.9773 & 0 & 0.0385 & 0.9474 \\
\hline & PSSRa & 0.9091 & 0.1053 & 0.0800 & 0.9074 \\
\hline & PSSRb & 0.9773 & 0 & 0.0385 & 0.9495 \\
\hline & PSSRc & 0.7955 & 0.1429 & 0.2333 & 0.7853 \\
\hline & GM1 & 0.9773 & 0 & 0.0385 & 0.9495 \\
\hline & GM2 & 0.9773 & 0 & 0.0385 & 0.9516 \\
\hline & ZTM & 0.9773 & 0 & 0.0385 & 0.9474 \\
\hline & TCARI/OSAVI & 0.9773 & 0 & 0.0385 & 0.9579 \\
\hline \multirow{7}{*}{ Red } & NDVI & 0.6836 & 0.3011 & 0.2381 & 0.7092 \\
\hline & PRI & 0.6554 & 0.3684 & 0.3663 & 0.6776 \\
\hline & ARI & 0.9831 & 0.0233 & 0.0110 & 0.9991 \\
\hline & SIPI & 0.8644 & 0.1573 & 0.1136 & 0.8377 \\
\hline & mCAI & 0.9548 & 0.0357 & 0.0430 & 0.9510 \\
\hline & PSSRa & 0.5989 & 0.4143 & 0.4112 & 0.5969 \\
\hline & PSSRb & 0.5763 & 0.4096 & 0.3830 & 0.6084 \\
\hline
\end{tabular}


Table A2. Cont.

\begin{tabular}{cccccc}
\hline Grapevine Variety & SVIs & Accuracy (\%) & FNR (\%) & FPR (\%) & AUC \\
\hline \multirow{4}{*}{ Red } & PSSRc & 0.6893 & 0.3049 & 0.2947 & 0.7210 \\
& GM1 & 0.9492 & 0.0957 & 0 & 0.9792 \\
& GM2 & 0.7175 & 0.2929 & 0.1923 & 0.7063 \\
ZTM & 0.6836 & 0.3889 & 0.3448 & 0.7196 \\
& TCARI/OSAVI & 0.8870 & 0.1538 & 0.0930 & 0.9633 \\
\hline NDVI & 0.6465 & 0.4048 & 0.3158 & 0.6956 \\
White & PRI & 0.5859 & 0.5667 & 0.4348 & 0.5199 \\
ARI & 0.9899 & 0 & 0.0175 & 0.9788 \\
& SIPI & 0.9394 & 0.1064 & 0.0192 & 0.9767 \\
mCAI & 0.9495 & 0.0238 & 0.0351 & 0.9784 \\
PSSRa & 0.9495 & 0.0476 & 0.0526 & 0.9593 \\
PSSRb & 0.8889 & 0.1304 & 0.0566 & 0.9435 \\
PSSRc & 0.9798 & 0.0233 & 0.0179 & 0.9655 \\
GM1 & 0.9899 & 0 & 0.0175 & 0.9792 \\
GM2 & 0.7778 & 0.2955 & 0.2182 & 0.8223 \\
& ZTM & 0.5657 & 0.5000 & 0.4030 & 0.5793 \\
All & TCARI/OSAVI & 0.9697 & 0.0233 & 0.0179 & 0.9950 \\
\hline NDVI & 0.4674 & 0.6184 & 0.4900 & 0.4102 \\
& PRI & 0.8551 & 0.1944 & 0.0833 & 0.9300 \\
ARI & 0.7029 & 0.3411 & 0.2857 & 0.7255 \\
SIPI & 0.7138 & 0.3103 & 0.2938 & 0.7362 \\
mCAI & 0.9420 & 0.0752 & 0.0280 & 0.9540 \\
& PSSRa & 0.6232 & 0.3962 & 0.3706 & 0.6362 \\
PSSRb & 0.7899 & 0.2819 & 0.1575 & 0.8120 \\
PSSRc & 0.8188 & 0.2365 & 0.1094 & 0.8512 \\
GM1 & 0.6775 & 0.3729 & 0.3354 & 0.6991 \\
GM2 & 0.5181 & 0.5049 & 0.4393 & 0.5523 \\
ZTM & 0.5688 & 0.5208 & 0.4500 & 0.5637 \\
& TCARI/OSAVI & 0.8877 & 0.1298 & 0.0897 & 0.9160 \\
\hline & & & &
\end{tabular}

Table A3. Results of SVIs in classifying different groups of spectral data acquired in the August and the September acquisition campaigns (Severity of infestation $=1 \& 2$ ).

\begin{tabular}{cccccc}
\hline Grapevine Variety & SVIs & Accuracy (\%) & FNR (\%) & FPR (\%) & AUC \\
\hline \multirow{5}{*}{ Marselan } & NDVI & 0.6929 & 0.3030 & 0.3115 & 0.7045 \\
& PRI & 0.7165 & 0.2899 & 0.2759 & 0.7318 \\
& ARI & 0.9370 & 0.0870 & 0.0345 & 0.9824 \\
& SIPI & 0.7402 & 0.2143 & 0.2958 & 0.7355 \\
& mCAI & 0.9213 & 0.1014 & 0.0517 & 0.8901 \\
& PSSRa & 0.5433 & 0.4407 & 0.4706 & 0.5330 \\
& PSSRb & 0.5118 & 0.4776 & 0.5000 & 0.5769 \\
& PSSRc & 0.5984 & 0.3833 & 0.4179 & 0.6150 \\
& GM1 & 0.8583 & 0.2099 & 0.0217 & 0.9164 \\
& GM2 & 0.7087 & 0.3056 & 0.2727 & 0.7419 \\
& ZTM & 0.7008 & 0.3151 & 0.2778 & 0.7655 \\
& TCARI/OSAVI & 0.8504 & 0.1714 & 0.1228 & 0.9002 \\
\hline \multirow{5}{*}{ Grenache } & NDVI & 0.7500 & 0.2381 & 0.2623 & 0.7846 \\
& PRI & 0.8226 & 0.1111 & 0.2286 & 0.7971 \\
& ARI & 0.9113 & 0.1370 & 0.0196 & 0.9753 \\
& SIPI & 0.8226 & 0.2083 & 0.1346 & 0.8521 \\
& mCAI & 0.9032 & 0.1286 & 0.0556 & 0.9318 \\
& PSSRa & 0.6048 & 0.3810 & 0.4098 & 0.5828 \\
& PSSRb & 0.6290 & 0.3676 & 0.3750 & 0.6547 \\
& PSSRc & 0.6532 & 0.3279 & 0.3651 & 0.6352 \\
& GM1 & 0.8226 & 0.2558 & 0 & 0.8776
\end{tabular}


Table A3. Cont.

\begin{tabular}{|c|c|c|c|c|c|}
\hline Grapevine Variety & SVIs & Accuracy (\%) & FNR $(\%)$ & FPR $(\%)$ & AUC \\
\hline \multirow{3}{*}{ Grenache } & GM2 & 0.7742 & 0.2429 & 0.2037 & 0.7893 \\
\hline & ZTM & 0.7661 & 0.2388 & 0.2281 & 0.8089 \\
\hline & TCARI/OSAVI & 0.7742 & 0.2857 & 0.1000 & 0.7620 \\
\hline \multirow{12}{*}{ Vermentino } & NDVI & 0.9080 & 0.0976 & 0.0870 & 0.9602 \\
\hline & PRI & 0.8506 & 0.1316 & 0.1633 & 0.9014 \\
\hline & ARI & 0.6207 & 0.3750 & 0.3818 & 0.5536 \\
\hline & SIPI & 0.8161 & 0.2549 & 0.0833 & 0.7937 \\
\hline & mCAI & 0.8391 & 0.2353 & 0.0556 & 0.8489 \\
\hline & PSSRa & 0.6897 & 0.3600 & 0.2432 & 0.7312 \\
\hline & PSSRb & 0.9080 & 0.0976 & 0.0870 & 0.9539 \\
\hline & PSSRc & 0.6437 & 0.3438 & 0.3636 & 0.5938 \\
\hline & GM1 & 0.9540 & 0.0698 & 0.0227 & 0.9730 \\
\hline & GM2 & 0.9080 & 0.0976 & 0.0870 & 0.9491 \\
\hline & ZTM & 0.9310 & 0.0513 & 0.0833 & 0.9655 \\
\hline & TCARI/OSAVI & 0.9425 & 0.0909 & 0.0233 & 0.9459 \\
\hline \multirow{12}{*}{ Chardonnay } & NDVI & 0.9342 & 0.1053 & 0.0263 & 0.9659 \\
\hline & PRI & 0.9342 & 0.0833 & 0.0500 & 0.9484 \\
\hline & ARI & 0.8684 & 0.0968 & 0.1556 & 0.8725 \\
\hline & SIPI & 0.8026 & 0.2917 & 0.0357 & 0.8474 \\
\hline & mCAI & 0.9474 & 0.0811 & 0.0256 & 0.9589 \\
\hline & PSSRa & 0.7237 & 0.3478 & 0.1667 & 0.7582 \\
\hline & PSSRb & 0.9342 & 0.1053 & 0.0263 & 0.9247 \\
\hline & PSSRc & 0.5658 & 0.4688 & 0.4091 & 0.5617 \\
\hline & GM1 & 0.9211 & 0.1081 & 0.0513 & 0.9568 \\
\hline & GM2 & 0.9605 & 0.0556 & 0.0250 & 0.9582 \\
\hline & ZTM & 0.9605 & 0.0556 & 0.0250 & 0.9672 \\
\hline & TCARI/OSAVI & 0.9342 & 0.1053 & 0.0263 & 0.9568 \\
\hline \multirow{12}{*}{ Red } & NDVI & 0.7331 & 0.2908 & 0.2545 & 0.7578 \\
\hline & PRI & 0.6773 & 0.2719 & 0.3285 & 0.7174 \\
\hline & ARI & 0.9323 & 0.1119 & 0.0093 & 0.9888 \\
\hline & SIPI & 0.7968 & 0.2180 & 0.2034 & 0.8010 \\
\hline & mCAI & 0.9203 & 0.1087 & 0.0442 & 0.9126 \\
\hline & PSSRa & 0.5817 & 0.3760 & 0.3968 & 0.5854 \\
\hline & PSSRb & 0.6574 & 0.3630 & 0.3621 & 0.6756 \\
\hline & PSSRc & 0.5936 & 0.3740 & 0.3984 & 0.5963 \\
\hline & GM1 & 0.8486 & 0.2289 & 0 & 0.9032 \\
\hline & GM2 & 0.7809 & 0.2414 & 0.1698 & 0.7743 \\
\hline & ZTM & 0.7331 & 0.2727 & 0.2222 & 0.7531 \\
\hline & TCARI/OSAVI & 0.8088 & 0.2437 & 0.0769 & 0.8270 \\
\hline \multirow{12}{*}{ White } & NDVI & 0.6687 & 0.3333 & 0.3398 & 0.6903 \\
\hline & PRI & 0.5644 & 0.4559 & 0.4000 & 0.5352 \\
\hline & ARI & 0.9509 & 0.0864 & 0.0122 & 0.9433 \\
\hline & SIPI & 0.9264 & 0.1395 & 0.0130 & 0.9517 \\
\hline & mCAI & 0.9202 & 0.1125 & 0.0482 & 0.9385 \\
\hline & PSSRa & 0.9202 & 0.1235 & 0.0488 & 0.9477 \\
\hline & PSSRb & 0.8037 & 0.2527 & 0.0972 & 0.8503 \\
\hline & PSSRc & 0.9202 & 0.1395 & 0.0130 & 0.9456 \\
\hline & GM1 & 0.9387 & 0.0988 & 0.0244 & 0.9658 \\
\hline & GM2 & 0.6503 & 0.3200 & 0.2727 & 0.6598 \\
\hline & ZTM & 0.5951 & 0.4151 & 0.4000 & 0.5662 \\
\hline & TCARI/OSAVI & 0.9387 & 0.1098 & 0.0247 & 0.9659 \\
\hline \multirow{4}{*}{ All } & NDVI & 0.5242 & 0.4805 & 0.4692 & 0.5060 \\
\hline & PRI & 0.7850 & 0.2857 & 0.1818 & 0.7996 \\
\hline & ARI & 0.7271 & 0.2786 & 0.2676 & 0.7574 \\
\hline & SIPI & 0.7536 & 0.2764 & 0.2698 & 0.7741 \\
\hline
\end{tabular}


Table A3. Cont.

\begin{tabular}{cccccc}
\hline Grapevine Variety & SVIs & Accuracy (\%) & FNR (\%) & FPR (\%) & AUC \\
\hline \multirow{6}{*}{ All } & mCAI & 0.8841 & 0.1696 & 0.0598 & 0.9184 \\
& PSSRa & 0.7222 & 0.2990 & 0.3000 & 0.7466 \\
& PSSRb & 0.7101 & 0.3103 & 0.2308 & 0.7353 \\
& PSSRc & 0.7343 & 0.3305 & 0.2472 & 0.7374 \\
& GM1 & 0.6884 & 0.3368 & 0.3348 & 0.7130 \\
& GM2 & 0.5459 & 0.4565 & 0.4435 & 0.5687 \\
& ZTM & 0.5580 & 0.4176 & 0.4221 & 0.5605 \\
& TCARI/OSAVI & 0.8744 & 0.1429 & 0.1078 & 0.8727 \\
\hline
\end{tabular}

\section{References}

1. Strange, R.N.; Scott, P.R. Plant disease: A threat to global food security. Annu. Rev. Phytopathol. 2005, 43, 83-116. [CrossRef] [PubMed]

2. Grapevine Flavescence Dorée. Available online: http://www.inra.fr/en/Scientists-Students/Plant-biology/ All-reports/Grapevine-flavescence-doree (accessed on 20 June 2017).

3. Maladies de la Vigne, la Flavescence Dorée Aujourd'hui et Demain. Available online: https: / /inra-dam-front-resources-cdn.brainsonic.com/ressources/afile/259574-408a4-resource-dossierde-presse-journee-la-flavescence-doree-aujourd-hui-et-demain.html (accessed on 26 May 2017).

4. Doraiswamy, P.C.; Moulin, S.; Cook, P.W.; Stern, A. Crop yield assessment for remote sensing. Photogramm. Eng. Remote Sens. 2003, 69, 665-674. [CrossRef]

5. Galvao, L.S.; Roberts, D.A.; Formaggio, A.R.; Numata, I.; Breunig, F.M. View angle effects on the discrimination of soybean varieties and on the relationships between vegetation indices and yield using off-nadir hyperion data. Remote Sens. Environ. 2009, 113, 846-856. [CrossRef]

6. Milton, E.J.; Schaepman, M.E.; Anderson, K.; Kneubühler, M.; Fox, N. Progress in field spectroscopy. Remote Sens. Environ. 2009, 113, 92-109. [CrossRef]

7. Sindhuja, S.; Mishra, A.; Reza, E.; Davis, C. A review of advanced techniques for detecting plant diseases. Comput. Electron. Agric. 2010, 72, 1-13.

8. Bauriegel, E.; Giebel, A.; Geyer, M.; Schmidt, U.; Herppich, W.B. Early detection of Fusarium infection in wheat using hyper-spectral imaging. Comput. Electron. Agric. 2011, 75, 304-312. [CrossRef]

9. Mahlein, A.K.; Steiner, U.; Hillnhutter, C.; Dehne, H.W.; Oerke, E.C. Hyperspectral imaging for small-scale analysis of symptoms caused by different sugar beet diseases. Plant Methods 2012, 8, 1-16. [CrossRef] [PubMed]

10. Garcia-Ruiz, F.; Sankaran, S.; Maja, J.M.; Lee, W.S.; Rasmussen, J.; Ehsani, R. Comparison of two aerial imaging platforms for identification of Huanglongbing-infected citrus trees. Comput. Electron. Agric. 2013, 91, 106-115. [CrossRef]

11. Hillnhütter, C.; Mahlein, A.K.; Sikora, R.; Oerke, E.C. Remote sensing to detect plant stress induced by heterodera schachtii and rhizoctonia solani in sugar beet fields. Field Crops Res. 2011, 122, 70-77. [CrossRef]

12. Moshou, D.; Bravo, C.; Oberti, R.; West, J.S.; Ramon, H.; Vougioukas, S. Intelligent multisensor system for the detection and treatment of fungal diseases in arable crops. Biosyst. Eng. 2011, 108, 311-321. [CrossRef]

13. Gamon, J.A.; Surfus, J.S. Assessing leaf pigment content and activity with a reflectometer. New Phytol. 1999, 143, 105-117. [CrossRef]

14. Pinter, P.J.; Hatfield, J.L.; Schepers, J.S.; Barnes, E.M.; Moran, M.S.; Daugthry, C.S.T. Remote sensing for crop management. Photogramm. Eng. Remote Sens. 2003, 69, 647-664. [CrossRef]

15. Abdel-Rahman, E.M.; Ahmed, F.B.; Van den Berg, M.; Way, M.J. Potential of spectroscopic data sets for sugarcane thrips (Fulmekiola serrata Kobus) damage detection. Int. J. Remote Sens. 2010, 31, 4199-4216. [CrossRef]

16. Yang, C.M. Assessment of the severity of bacterial leaf blight in rice using canopy hyperspectral reflectance. Precis. Agric. 2010, 11, 61-81. [CrossRef]

17. Mahlein, A.K.; Steiner, U.; Dehne, H.W.; Oerke, E.C. Spectral signatures of sugar beet leaves for the detection and differentiation of diseases. Precis. Agric. 2010, 11, 413-431. [CrossRef] 
18. Santoso, H.; Gunawan, T.; Jatmiko, R.H.; Darmosarkoro, W.; Minasny, B. Mapping and identifying basal stem rot disease in oil palms in North Sumatra with QuickBird imagery. Precis. Agric. 2011, 12, 233-248. [CrossRef]

19. Naidu, R.; Perry, E.; Pierce, F.; Mekuria, T. The potential of spectral reflectance technique for the detection of Grapevine leafroll-associated virus-3 in two red-berried wine grape cultivars. Comput. Electron. Agric. 2009, $66,38-45$. [CrossRef]

20. Hou, J.; Li, L.; He, J. Detection of grapevine leafroll disease based on 11-index imagery and ant colony clustering algorithm. Precis. Agric. 2016, 17, 488-505. [CrossRef]

21. Carisse, A.; Bacon, R.; Lasnier, J.; McFadden-Smith, W. Identification Guide to the Major Diseases of Grapes; Agriculture and Agri-Food Canada: Ottawa, ON, Canada, 2006.

22. La Gestion de la Flavescence Dorée en Viticulture Biologique. Available online: http:/ / www.itab.asso.fr/ downloads/viti/flavescence-doreeab-mai2014.pdf (accessed on 10 May 2016).

23. Guide Flavescence. Aide au Diagnostic de la Flavescence Dorée. Available online: http:/ / ecophytopic.fr/sites/ default/files/livret\%20guide\%20flavescence\%20export\%5B1\%5D_0.pdf (accessed on 12 February 2016).

24. Chuche, J.; Thiéry, D. Biology and ecology of the flavescence dorée vector scaphoideus titanus: A review. Agron. Sustain. Dev. 2014, 34, 381-403. [CrossRef]

25. Babatunde, O.; Armstrong, L. A genetic algorithm-based feature selection. Int. J. Electron. Commun. Comput. Eng. 2014, 5, 2278-4209.

26. Babatunde, O.; Armstrong, L.; Leng, J.; Diepeveen, D. Zernike moments and genetic algorithm: Tutorial and application. Br. J. Math. Comput. Sci. 2014, 4, 2217-2236.

27. Sivanandam, S.N.; Deepa, S.N. Introduction to Genetic Algorithms; Springer: Berlin/Heidelberg, Germany, 2008.

28. Marek, O. Introduction to Genetic Algorithms Czech Technical University. 1998. Available online: http://www.obitko.com/tutorials/genetic-algorithms (accessed on 5 September 2017).

29. Matlab, G.A. Toolbox. Available online: https://fr.mathworks.com/help/gads/genetic-algorithm.html (accessed on 1 September 2016).

30. Tucker, C.J. Red and photographic infrared linear combination for monitoring vegetation. Remote Sens. Environ. 1979, 8, 127-150. [CrossRef]

31. Tucker, C.J.; Holben, B.N.; Elgin, J.; James, H.; McMurtrey, I.; James, E. Remote sensing of total dry-matter accumulation in winter wheat. Remote Sens. Environ. 1981, 11, 171-189. [CrossRef]

32. Penuelas, J.; Filella, I.; Gamon, J.A. Assessment of photosynthetic radiation-use efficiency with spectral reflectance. New Phytol. 1995, 131, 291-296. [CrossRef]

33. Penuelas, J.; Gamon, J.A.; Fredeen, A.L.; Merino, J.; Field, C.B. Reflectance indices associated with physiological changes in nitrogen- and water-limited sunflower leaves. Remote Sens. Environ. 1994, 48, 135-146. [CrossRef]

34. Trotter, G.M.; Whitehead, D.; Pinkney, E.J. The photochemical reflectance index as a measure of photosynthetic light use efficiency for plants of varying foliar nitrogen contents. Int. J. Remote Sens. 2002, 23, 1207-1212. [CrossRef]

35. Gitelson, A.; Merzlyak, N.; Chivkunova, O.B. Optical properties and nondestructive estimation of anthocyanin content in plant leaves. Photochem. Photobiol. 2001, 74, 38-45. [CrossRef]

36. Blackburn, G.A. Spectral indices for estimating photosynthetic pigment concentrations: A test using senescent tree leaves. Int. J. Remote Sens. 1998, 19, 657-675. [CrossRef]

37. Penuelas, J.; Baret, F.; Filella, I. Semi-empirical indices to assess carotenoids/chlorophyll a ratio from leaf spectral reflectance. Photosynthetica 1995, 31, 221-230.

38. Laudien, R. Development of a field and GIS-based management information system for the sugar beet industry. In Proceedings of the 2005 EFITA WCCA Congress, Vila Real, Portugal, 25-28 July 2005.

39. Richardson, A.D.; Aikens, M.; Berlyn, G.P.; Marshall, P. Drought stress and paper birch (Betula papyrifera) seedlings: Effects of an organic biostimulant on plant health and stress tolerance, and detection of stress effects with instrument-based, non-invasive methods. J. Arboric. 2004, 30, 52-61.

40. Sims, A.; Gamon, A. Relationships between leaf pigment content and spectral reflectance across a wide range of species, leaf structures and developmental stages. Remote Sens. Environ. 2002, 81, 337-354. [CrossRef]

41. Apan, A.; Held, A.; Phinn, S.; Markley, J. Formulation and assessment of narrow-band vegetation indices from EO-1 hyperion imagery for discriminating sugarcane disease. In Proceedings of the Spatial Sciences Institute Biennial Conference on Spatial Knowledge without Boundaries, Canberra, Australia, 22-26 September 2003; pp. 1-13. 
42. Kooistra, L.; Leuven, R.S.E.W.; Wehrens, R.; Nienhuis, P.H.; Buydens, L.M.C. A comparison of methods to relate grass reflectance to soil metal contamination. Int. J. Remote Sens. 2003, 24, 4995-5010. [CrossRef]

43. Gitelson, A.; Kaufman, J.; Merzlyak, N. Use of a green channel in remote sensing of global vegetation from EOS-MODIS. Remote Sens. Environ. 1996, 58, 289-298. [CrossRef]

44. Gitelson, A.; Merzlyak, N. Remote estimation of chlorophyll content in higher plant leaves. Int. J. Remote Sens. 1997, 18, 2691-2697. [CrossRef]

45. Main, R.; Cho, A.; Mathieu, R.; O'Kennedy, M.; Ramoelo, A.; Koch, S. An investigation into robust spectral indices for leaf chlorophyll estimation. ISPRS J. Photogramm. Remote Sens. 2011, 66, 751-761. [CrossRef]

46. Zarco-Tejada, P.J.; Miller, J.R.; Noland, T.L.; Mohammed, G.H.; Sampson, P.H. Scaling-up and model inversion methods with narrowband optical indices for chlorophyll content estimation in closed forest canopies with hyperspectral data. IEEE Trans. Geosci. Remote Sens. 2001, 39, 1491-1507. [CrossRef]

47. Underwood, E.; Ustin, S.; DiPietro, D. Mapping nonnative plants using hyperspectral imagery. Remote Sens. Environ. 2003, 86, 150-161. [CrossRef]

48. Rondeaux, G.; Steven, M.; Baret, F. Optimization of soil-adjusted vegetation indices. Remote Sens. Environ. 1996, 55, 95-107. [CrossRef]

49. Haboudane, D.; Miller, J.R.; Tremblay, N.; Zarco-Tejada, P.J.; Dextraze, L. Integrated narrow-band vegetation indices for prediction of crop chlorophyll content for application to precision agriculture. Remote Sens. Environ. 2002, 81, 416-426. [CrossRef]

50. Rumpf, T.; Mahlein, A.; Dörschlag, D.; Plümer, L. Identification of combined vegetation indices for the early detection of plant diseases. In Proceedings of the Remote Sensing for Agriculture, Ecosystems, and Hydrology XI, Berlin, Germany, 18 September 2009. [CrossRef]

51. Rumpf, T.; Mahlein, A.; Steiner, U.; Oerke, E.; Dehne, H.; Plümer, L. Early detection and classification of plant diseases with Support Vector Machines based on hyperspectral reflectance. Comput. Electron. Agric. 2010, 74, 91-99. [CrossRef]

52. Support Vector Machines for Machine Learning. Available online: https://machinelearningmastery.com/ support-vector-machines-for-machine-learning/ (accessed on 20 April 2016).

53. Ben-Hur, A.; Weston, J. A User's Guide to Support Vector Machines. Data Min. Tech. Life Sci. 2010, 609, $223-239$.

54. Shokoufeh, A.; Hadi, S.; Alireza, R.; Saeid, E. Feature selection using genetic algorithm for breast cancer diagnosis: Experiment on three different datasets. Iran. J. Basic Med. Sci. 2016, 19, 476-482.

55. Oliveira, L.S.; Sabourin, R.; Bortolozzi, F.; Suen, C.Y. Feature selection using multi-objective genetic algorithms for handwritten digit recognition. In Proceedings of the16th International Conference on Pattern Recognition, Quebec City, QC, Canada, 11-15 August 2002.

56. Jingcheng, Z.; Ruiliang, P.; Wenjiang, H.; Lin, Y.; Juhu, L.; Jihu, W. Using in-situ hyperspectral data for detecting and discriminating yellow rust disease from nutrient stresses. Field Crops Res. 2012, 134, 165-174.

57. Huang, W.; Lamb, D.W.; Niu, Z.; Zhang, Y.; Liu, L.; Wang, J. Identification of yellow rust in wheat using in situ spectral reflectance measurements and airborne hyperspectral imaging. Precis. Agric. 2007, 8, 187-197. [CrossRef]

58. Devadas, R.; Lamb, D.W.; Simpfendorfer, S.; Backhouse, D. Evaluating ten spectral vegetation indices for identifying rust infection in individual wheat leaves. Precis. Agric. 2009, 10, 459-470. [CrossRef]

59. Zarco-Tejada, P.J.; Miller, J.R.; Morales, A.; Berjon, A.; Aguera, J. Hyperspectral indices and model simulation for chlorophyll estimation in open-canopy tree crops. Remote Sens. Environ. 2004, 90, 463-476. [CrossRef]

60. Khawas, E.S.A.M.; Khawas, E.M.A.M. Interactions between Aphis gossypii (Glov.) and the common predators in eggplant and squash fields, with evaluating the physiological and biochemical aspects of biotic stress induced by two different aphid species, infesting squash and cabbage plants. Aust. J. Basic Appl. Sci. 2008, 2, 183-193.

61. Murugesan, N.; Kavitha, A. Host plant resistance in cotton accessions to the leaf hopper Amrasca devastans (Distant). J. Biopestic. 2010, 3, 526-533.

62. Gitelson, A.; Merzlyak, N. Signature analysis of leaf reflectance spectra: Algorithm development for remote sensing of chlorophyll. J. Plant Physiol. 1996, 148, 494-500. [CrossRef]

63. Harris Geospatial, Dry or Senescent Carbon. Available online: https://www.harrisgeospatial.com/docs/ drysenescentcarbon.html\#cellulose_absorption_index (accessed on 28 July 2017).

64. Ren, H.; Zhou, G.; Zhang, F.; Zhang, X. Evaluating cellulose absorption index (CAI) for non-photosynthetic biomass estimation in the desert steppe of Inner Mongolia. Chin. Sci. Bull. 2012, 57, 1716-1722. [CrossRef] 
65. Mirik, M.; Michels, G.J., Jr.; Kassymzhanova-Mirik, S.; Elliott, N.C.; Catana, V.; Jones, D.B.; Bowling, R. Using digital image analysis and spectral reflectance data to quantify damage by greenbug (Hemitera: Aphididae) in winter wheat. Comput. Electron. Agric. 2006, 51, 86-98. [CrossRef]

66. Prabhakar, M.; Prasad, Y.G.; Thirupathi, M.; Sreedevi, G.; Dharajothi, B.; Venkateswarlu, B. Use of ground based hyperspectral remote sensing for detection of stress in cotton caused by leafhopper (Hemiptera: Cicadellidae). Comput. Electron. Agric. 2011, 79, 189-198. [CrossRef]

67. Ashourloo, D.; Mobasheri, M.R.; Huete, A. Developing two spectral disease indices for detection of wheat leaf rust (Pucciniatriticina). Remote Sens. 2014, 6, 4723-4740. [CrossRef]

68. Mahlein, A.K.; Rumpf, T.; Welke, P.; Dehne, H.W.; Plümer, L.; Steiner, U.; Oerke, E.C. Development of spectral indices for detecting and identifying plant diseases. Remote Sens. Environ. 2013, 128, 21-30. [CrossRef]

69. Zarco-Tejada, P.J.; Ustin, S.L.; Whiting, M.L. Temporal and spatial relationships between within-field yield variability in cotton and high-spatial hyperspectral remote sensing imagery. Agron. J. 2003, 97, 641-653. [CrossRef]

(c) 2017 by the authors. Licensee MDPI, Basel, Switzerland. This article is an open access article distributed under the terms and conditions of the Creative Commons Attribution (CC BY) license (http://creativecommons.org/licenses/by/4.0/). 
Article

\title{
A Combined Approach of Sensor Data Fusion and Multivariate Geostatistics for Delineation of Homogeneous Zones in an Agricultural Field
}

\author{
Annamaria Castrignanò ${ }^{1,2}$, Gabriele Buttafuoco ${ }^{2, *}$, Ruggiero Quarto ${ }^{3}$, Carolina Vitti ${ }^{1}$, \\ Giuliano Langella ${ }^{4}$, Fabio Terribile ${ }^{4}$ and Accursio Venezia ${ }^{5}$ \\ 1 CREA Research Centre for Agriculture and Environment, 70125 Bari, Italy; \\ annamaria.castrignano@crea.gov.it (A.C.); carolina.vitti@crea.gov.it (C.V.) \\ 2 National Research Council of Italy, Institute for Agricultural and Forest Systems in the Mediterranean, \\ 87036 Rende (CS), Italy \\ 3 Earth and Geoenvironmental Sciences Department, University of Bari Aldo Moro, 700125 Bari, Italy; \\ ruggiero.quarto@uniba.it \\ 4 Department of Agriculture, University of Naples Federico II, 8055 Portici (NA), Italy; \\ glangella@unina.it (G.L.); fabio.terribile@unina.it (F.T.) \\ 5 CREA Research Center for Vegetable and Ornamental Crops, 84098 Pontecagnano (SA), Italy; \\ accursio.venezia@crea.gov.it \\ * Correspondence: gabriele.buttafuoco@cnr.it; Tel.: +39-0984-841-484
}

Received: 15 October 2017; Accepted: 1 December 2017; Published: 3 December 2017

\begin{abstract}
To assess spatial variability at the very fine scale required by Precision Agriculture, different proximal and remote sensors have been used. They provide large amounts and different types of data which need to be combined. An integrated approach, using multivariate geostatistical data-fusion techniques and multi-source geophysical sensor data to determine simple summary scale-dependent indices, is described here. These indices can be used to delineate management zones to be submitted to differential management. Such a data fusion approach with geophysical sensors was applied in a soil of an agronomic field cropped with tomato. The synthetic regionalized factors determined, contributed to split the 3D edaphic environment into two main horizontal structures with different hydraulic properties and to disclose two main horizons in the $0-1.0-\mathrm{m}$ depth with a discontinuity probably occurring between $0.40 \mathrm{~m}$ and $0.70 \mathrm{~m}$. Comparing this partition with the soil properties measured with a shallow sampling, it was possible to verify the coherence in the topsoil between the dielectric properties and other properties more directly related to agronomic management. These results confirm the advantages of using proximal sensing as a preliminary step in the application of site-specific management. Combining disparate spatial data (data fusion) is not at all a naive problem and novel and powerful methods need to be developed.
\end{abstract}

Keywords: spatial data; sensor; data fusion; change of support; geostatistics; precision agriculture; management zones

\section{Introduction}

After the post-II World War period, an agriculture model aimed at maximizing yield was elaborated and realized, moving towards intensive agriculture and abandoning extensive agriculture. This change required an increase in both energetic inputs (especially fossil fuel) and chemicals (fertilizers, pesticides) with high toxicity potential. It has been now clearly stated that this way of agricultural management has a negative impact on the environment, reducing soil productivity and causing different types of pollution. This has prompted a search for alternative models with the aim of protecting the environment and its related systems through a reasonable use of natural resources 
according to sustainable agriculture management. A new approach to sustainable agriculture, called 'precision farming' or 'precision agriculture', has been developed, aimed at optimizing agriculture productivity while minimizing environmental impact. It is defined as a management strategy that uses information technology to bring data from multiple sources to bear on decisions associated with crop production [1]. This new agricultural model might have a strong economic and environmental impact, since traditional agriculture considers the field as homogenous for physical and chemical characteristics and then applies one treatment. On the contrary, experimental evidence [2-5] as well as many other references have shown that any agricultural area, even of limited extension, presents spatial variation in soil characteristics; moreover, temporal variability occurs not only throughout the years but also within each crop season.

Precision agriculture is much more than a mere set of even advanced technologies but essentially involves extensive changes in management style and strategy. It aims to match agricultural inputs and practices with the local conditions within a field to help farmers in making better decisions and to achieve their many different goals more efficiently. Therefore, the core of precision agriculture is effectively managing spatial and temporal variability related to all aspects of agricultural production for the purpose of improving crop performance and environmental quality. To obtain such a result, technologies and principles have to be used effectively. Without variability, the concepts of precision agriculture would have little meaning [6] and would never have evolved. Therefore, any precision agriculture system must first address measurement to assess and understand variability. While the technology can facilitate the application of precision agriculture, it is only the knowledge and interpretation of variability that makes it feasible. Therefore, assessing variability is the first critical step and a necessary condition in precision agriculture.

Spatial variability is generally exhibited by a lot of properties and state variables in the natural landscape occurring also over different temporal scales and having different effects on crop growth and yield. Quantifying the variability of these properties adequately can then aid the understanding of the processes causing spatial and temporal variation in crop yield and enlighten the proper management of land resources.

The availability of efficient and accurate techniques for measuring within-field variations in soil properties at a very fine spatial scale is then required in precision farming [7]. Traditional soil sampling is not viable, because it is costly and labour intensive and needs a large number of soil samples in order to achieve a good representation of soil properties. To overcome the limitations of spatially scarce data, advances in proximal sensing technology and data analysis techniques are now able to provide information on soil, crops and associated environmental properties. Recently, the number of proximal soil sensing techniques (PSS) has increased due to the advantages of non-invasive techniques, which are time- and cost-efficient. In particular, geophysical methods provide a low cost and non-invasive way of gathering large amounts of information on various physical soil properties. At present, a number of soil sensors are available which use a variety of numerous measurement techniques, such as electromagnetic induction (EMI), electrical resistivity (ER), ground-penetrating radar (GPR), gamma sensors, radiometry, fluorimetry, etc.

These sensors can be coupled to a Global Positioning System (GPS) receiver to measure different physical and chemical properties of the soil and plants. However, such measurements are generally affected by more than one agronomic soil characteristic [8] and consequently, obtaining accurate information about one property, by using only one sensing technique, is extremely difficult. Recently, to obtain a more comprehensive representation of the surveyed area and separate more easily the different effects, a new approach for soil and vegetation sensing based on combining several sensing techniques (sensor fusion system), has been developed [9].

Therefore, such an approach requires defining methods to combine information from heterogeneous sources into a single composite picture, which provides more accurate and complete information than that derived from any single source [10]. The task is not easy at all because proximal sensing data are often massive, taken on different spatial and temporal scales, with different spatial 
resolutions (support sizes) and subject to measurement error biases. Moreover, a statistical approach to data fusion combines the sensor data sets in a manner that is statistically robust because it takes advantage of the differences between the instruments and their complementary features [11,12]. The problem can be treated from different points of view; however, in this work combining multiple sources of information will be handled within a statistical framework. The main advantage of a statistical approach is that explicit probabilistic models are employed to describe the various relationships between the sensors, taking into account the underlying uncertainties and change of support [13]. At present, there exist different statistical methods to jointly analyze heterogeneous data, most of them based on Bayesian [9] and machine-learning methods [14]. Geostatistics, compared with traditional methods, offers the advantage of treating the observations as variables localized in the geometrical and temporal space and to use their degree of autocorrelation to improve estimation precision. Moreover, it provides a measure of the uncertainty of prediction [15].

The objective of this study was to describe an integrated approach by combining multivariate geostatistical data-fusion techniques with multi-source geophysical sensor data, in order to determine simple summary scale-dependent indices that integrate the multi-scale and multi-variate data in a meaningful way. These indices will be used to delineate homogeneous within-field zones (management zones) that could be submitted to differential agricultural management.

\section{Materials and Methods}

\subsection{Study Site and Soil Sampling}

The study area (Figure 1) is a field located in the Sarno Plain (Striano, Salerno, South-Western Italy) that covers more than $150 \mathrm{~km}^{2}$ in the coastal graben of the Campanian Plain. It is bordered by carbonate ridges of the Lattari mountains on the south, the Sarno mountains on the north, the Salerno mountains on the east and the Somma-Vesuvius volcanic complex on the west.

(a)

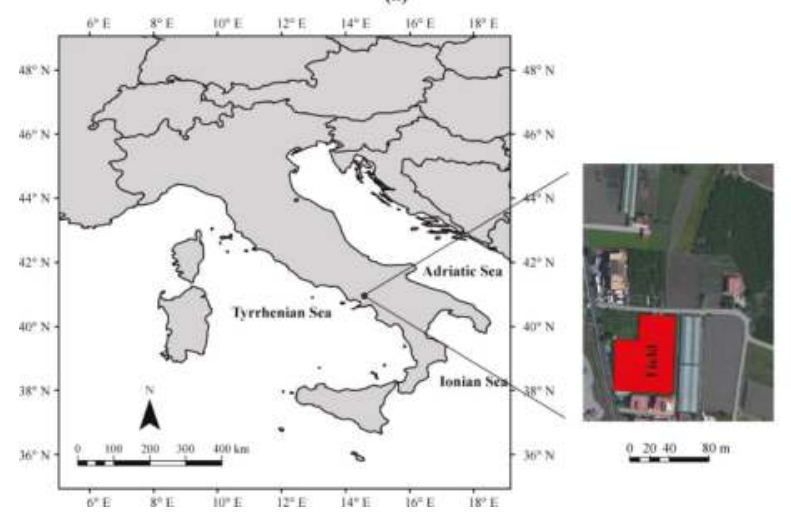

(b)

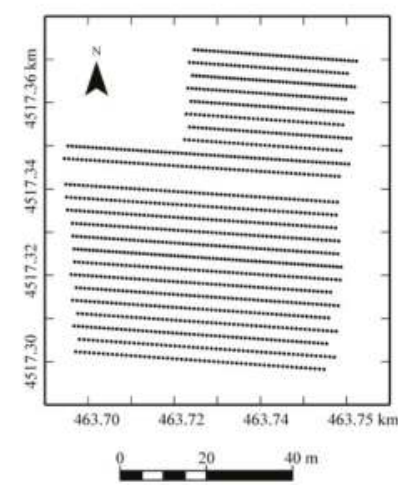

Figure 1. Locations of the experimental field (a) and geophysical surveys (b). The dotted lines are the 24 transects where the geophysical surveys were carried out.

The soils in the alluvial plain landforms at about $14 \mathrm{~m}$ a.s.l. develop over alluvial and volcanoclastic deposits. They are typically deep and generally sub-alkaline; the texture ranges from sandy-loam to silty-loam. Considering their intensive agriculture land management, the studied soils have a good supply of organic carbon (min-max in topsoil: $10.15-18.3 \mathrm{~g} \cdot \mathrm{kg}^{-1}$ ), available phosphorus (min-max in topsoil: $120-160 \mathrm{mg} \cdot \mathrm{kg}^{-1}$ ) and potassium (min-max in topsoil: $574-879 \mathrm{mg} \cdot \mathrm{kg}^{-1}$ ). The carbonate content is always high and it generally increases with depth. Below $90 \mathrm{~cm}$ depth, 
they can have a partially indurated layer with Fe-Mn concretions, segregation, and mottling induced by either ancient or present day water stagnation.

These soils can be classified as Aquic Calciustepts [16] when they show Fe-Mn hydromorphic features (mottles, segregations, etc.) and Typic Calciustepts [16] when they do not show such hydromorphic features.

The climate is Mediterranean with prevailing winds from north and south and abundant rains in autumn, winter, and spring. Drip irrigation was carried out in summer by pumping water from wells.

The tomato cultivation (cv. San Marzano) was cropped with traditional agronomic treatment following the protected designation of origin (PDO) regulation that echoes the age-old tradition but which is submitted to additional constraints.

In order to characterize the soil and then give a physical interpretation to the field-partition provided by proximal sensing, 52 soil samples up to a depth of $0.30 \mathrm{~m}$ were collected according to grid sampling with mesh of about $10 \mathrm{~m}$. The samples were then submitted to a set of laboratory measurements of the main physical properties. These included texture components by using the pipette method: clay (particle size $<0.002 \mathrm{~mm})$, fine silt $(0.002-0.05 \mathrm{~mm})$, coarse silt $(0.05-0.02 \mathrm{~mm})$, fine sand $(0.02-0.2 \mathrm{~mm})$, coarse sand $(0.2-2 \mathrm{~mm})$. In addition, two soil water properties (soil water contents at field capacity and at wilting point) and total carbonate concentration were also determined.

\subsection{Data Acquisition and Protocol of Measurements}

Geophysical surveys (Figure 1) were carried out on 24 geo-referenced transects approximately 5-m apart along the E-W direction using different sensors connected with a differentially corrected GPS system (DGPS, HiPer 27 Pro, Topcon, Tokyo, Japan) on 16 April 2015. The equipment consisted of:

(1) a monostatic GPR sensor with frequency of $250 \mathrm{MHz}$ (Noggin250, Sensors \& Software Inc., Mississauga, ON, Canada);

(2) an electromagnetic induction sensor (EM38DD, Geonics, Ltd., Mississauga, ON, Canada), that provides measurements of either the quad-phase or in-phase component data in both the horizontal and vertical dipole mode;

(3) a multiple frequency electromagnetic profiler GEM $300^{\circledR}$ (Geophysical survey Systems, Inc., Nashua, NH, USA).

Electromagnetic induction (EMI) soil survey is based on the principle that a transmitter coil in contact with the soil surface produces a time-varying primary magnetic field in the subsoil. Any variation in electromagnetic (EM) field induces eddy currents in the soil and generates a secondary magnetic field, which is recorded by a receiver coil in the EM unit. The ratio of the magnitude of the secondary magnetic field to the one of the primary magnetic field allows the calculation of the apparent conductivity near the receiver [17].

The EM38DD EMI sensor, with intercoil spacing of 1 meter and operating frequency of $14.6 \mathrm{kHz}$, was used to measure bulk electrical conductivity $\left(\mathrm{EC}_{\mathrm{a}}\right)$ simultaneously in the horizontal $\left(\mathrm{EC}_{\mathrm{a}} \mathrm{H}\right)$ and vertical $\left(\mathrm{EC}_{\mathrm{a}} \mathrm{V}\right)$ polarization modes with different depth response profile: in a homogeneous soil profile, the vertical maximum sensitivity is at a depth of approximately $0.40 \mathrm{~m}$ and the signal penetrates to a depth of $1.5 \mathrm{~m}$; whereas the horizontal, maximum sensitivity occurs at the surface and the signal penetrates the topsoil up to $0.75 \mathrm{~m}$. The data were recorded at each second and the spatial resolution was $0.70 \mathrm{~m}$ on average along the transects as a function of speed of the tractor which pulled the EMI sensor across the field.

GEM300 Profiler is an EMI sensor, which conceptually works like the previous one. The main difference is the ability to generate at a maximum of 16 pulses in a range of frequencies between 300 and $20,000 \mathrm{~Hz}$. Furthermore, the intercoil distance is $1.67 \mathrm{~m}$ and then the maximum impulse response for the vertical dipole mode is about $0.67 \mathrm{~m}$.

The instrument was moved manually along the same 24 georeferenced transects (Figure 1), with the dipole axis of the coils only in a vertical orientation. This choice was determined by the objective 
of getting deeper information, since the shallow conductivity was already depicted by an EM38DD sensor. The moving speed was about $1 \mathrm{~m} \cdot \mathrm{s}^{-1}$, collecting in this time six measurements at different frequencies decreasing by a factor of $1.54(19,975 ; 12,975 ; 8425 ; 5475 ; 3575$, and $2325 \mathrm{~Hz})$. This set of only six operating frequencies was chosen to avoid too long a delay between the sets of measurements and then a too low moving velocity. Furthermore, frequencies less than the smallest one used $(2325 \mathrm{~Hz})$ were unnecessary for the agricultural purpose of the survey.

Each operating frequency had a different depth of investigation and therefore explored a different soil volume [18]. The maximum depth of investigation was calculated as the square root of the skin depth choosing a soil conductivity of $50 \mathrm{mS} \cdot \mathrm{m}^{-1}[19,20]$. The skin depth is the effective depth of penetration of electromagnetic energy in a conducting medium when displacement currents can be neglected. It is also the depth at which the amplitude of a plane wave is attenuated to $1 / e$ or 37 percent [21].

All the surveys were performed on the same day but separately by sensor type and in soil water conditions close to field capacity after the early spring rainfall events.

Ground Penetrating Radar (GPR) is a noninvasive geophysical tool that is specifically designed to penetrate materials and provide images of subsoil. The radar produces a high-frequency electromagnetic wave, which propagates through the sub-surface materials at the velocity determined by the soil dielectric permittivity. When this propagating wave encounters any change in dielectric properties, it is reflected or scattered back towards the surface where it is recorded [22]. Resistive terrains propagate well the radar waves, while soils with high electrical conductivity (saline or fine textured soils) rapidly attenuate radar energy, which restricts penetration depth and severely limits the effectiveness of GPR.

The GPR data were collected using the reflection, continuous profiling method, recording one radar trace every 5 centimeters after 16 pulses, along the same transect of the EMI survey. A time window of 100 nanoseconds with $2.5 \mathrm{GHz}$ sample frequency was used.

The processing of GPR data was performed with Gradix Software (Interpex Ltd., Golden, CO, USA) and Radar Software (Sensors \& Software Inc., Mississauga, ON, Canada) and included:

(1) a static correction (in this survey only a 'Set Time Zero', since the soil surface was flat and the GPR antennas worked on the ground). The best frequency value of a high pass filter (where the amplitude, in the frequency domain, moving towards the low frequencies in the spectrum, begins to increase) was examined and the best number of points searched;

(2) a 'Dewow filter' by means of a simple 'High Pass Filter' for removing very low frequency components from the data associated with either inductive phenomena or possible instrumentation dynamic range limitations [23];

(3) a 'Band Pass Filter' well known for enhancing useful frequency signals. A trapezoidal filter [24] was used for the 'Band Pass Filter', which is composed of four cut frequencies, like a trapeze, and was more consistent with the amplitude spectra of our data, after making a frequency analysis.

Before 2D data processing to create time-slices, a procedure called 'envelope' was added to improve the attribute analysis for 3D visualizations [20,25,26]. A quadrature filter (Hilbert-Transformation) was used to calculate the instantaneous signal amplitude or envelope of data, which gives an estimation of the reflectivity and is proportional to the square root of the total energy of the signal at a given instant of time [27]. After the application of this procedure, the signal amplitude becomes a relative quantity and gives an overview of the distribution of the underground reflectors (soil layering, in the case of applications for agriculture), their reflectivity or dielectric contrasts, and the intrinsic attenuation of the media or their physical-chemical characteristics (soil structure, mineralogy, water content, salinity).

The mean signal amplitude was calculated within a range of $4 \mathrm{~ns}$ (the period at the frequency of $250 \mathrm{Mhz}$ ) by increasing time intervals, which overlapped (0-4 ns, 2-6 ns, 4-8 ns and so on) up to $40 \mathrm{ns,}$ where the signal shrinks sharply [20]. A depth conversion was performed by means of the analysis of hyperbolae occurring on the radar sections. A mean value of about $10 \mathrm{~cm} \cdot \mathrm{ns}^{-1}$ was calculated 
and then the depth intervals were of $20 \mathrm{~cm}(0-20 \mathrm{~cm}, 10-30 \mathrm{~cm}, 20-40 \mathrm{~cm}$ and so on), since the above mentioned time is actually a two way travel time of the radar waves.

\subsection{Geostatistical Analysis}

To jointly analyze the heterogeneous data set, including data from the different sensors mostly not co-located and with different support and level of uncertainty, a complex but well integrated approach of geostatistical procedures was defined including the following steps:

(1) Sample Data Migration

To perform multivariate analysis on different sensor data, the raw data of GEM and GPR were collocated into the less numerous file containing EM38DD measurements by migrating them to the nearest EMI sample point up to a maximum distance of $5 \mathrm{~m}$ (approximately the distance between the transects);

(2) Gaussian Anamorphosis Modelling

All variables were normalized through a Gaussian anamorphosis [28,29] to zero mean and unity variance to solve the two-type problems occurring in: (a) multivariate approach when the variables differ widely in size and (b) variogram modelling owing to the presence of outliers in highly skewed data distributions. Gaussian anamorphosis is a mathematical function, which transforms a variable $Y$, with a Gaussian standardized distribution, into a new variable $Z$ with any distribution:

$$
Z(\mathbf{x})=\phi[Y(\mathbf{x})]
$$

where $\mathbf{x}$ is the location coordinates vector. As the function $\phi[Y(\mathbf{x})]$ needs to be known for any Gaussian value, it is modelled by fitting a polynomial expansion [28]:

$$
\phi[Y(\mathbf{x})]=\sum_{i=0}^{n} \psi_{i} H_{i}[Y(\mathbf{x})]
$$

where $H_{i}[Y(\mathbf{x})]$ are called Hermite polynomials. In practice, the polynomial expansion is truncated at a generally high order (30-100) and tends to be bijective within the interval defined by the minimum and the maximum of the sample values [29]. Model fitting then consists of calculating the $\Psi i$ coefficients of the expansion; in order to transform the raw variable into a Gaussian one, the anamorphosis function has to be inverted [29].

All the geostatistical techniques were performed on the transformed data and at the end, the estimates were back-transformed to the raw values of the variables through the anamorphosis functions previously calculated;

(3) Fitting of Linear Model of Coregionalization

The linear model of Coregionalization (LMC), developed by Journel and Huijbregts [30], considers all the $n$ studied variables as the result of the same independent physical processes, acting over different spatial scales $u$. The $n(n+1) / 2$ simple and cross semivariograms of the variables are modelled by a linear combination of $N_{S}$ standardized semivariograms of unit sill, $g^{u}(\mathbf{h})$. Using the matrix notation, the LMC can be written as:

$$
\boldsymbol{\Gamma}(\mathbf{h})=\sum_{u=1}^{N_{S}} \mathbf{B}^{u} g^{u}(\mathbf{h})
$$

where $\boldsymbol{\Gamma}(\mathbf{h})=\left[\gamma_{i j}(\mathbf{h})\right]$ is a symmetric matrix of order $n \times n$, whose diagonal and out-of-diagonal elements represent simple and cross variograms, respectively; $\mathbf{B}^{u}=\left[b_{i j}^{u}\right]$ is called the coregionalization matrix and is a symmetrical positive semi-definite matrix of the order $n \times n$ with real elements $b_{i j}^{u}$ 
at a specific spatial scale $u$. The model is properly defined if the functions $g^{u}(\mathbf{h})$ are authorized variograms models [31];

(4) Block (Co)kriging and Change of Support

The solution to most problems of change of supports requires spatial prediction. A common inferential problem consists in predicting a random variable $Z$ defined over a block $B$ from $N$ point samples $Z\left(\mathbf{x}_{i}\right)$. In linear geostatistics, the predictor is called block kriging and is given by:

$$
Z(B)=\sum_{i=1}^{N} \lambda_{i} Z\left(\mathbf{x}_{i}\right)
$$

where optimal weights $\lambda$ are obtained by solving kriging system equations resulting from minimizing prediction mean squared error subject to unbiasedness constraint [28]. The equations are very similar in point- and block kriging; the main difference consists in the calculation of the point-to-block covariance:

$$
\bar{C}\left(B, \mathbf{x}_{i}\right)=\operatorname{cov}\left(Z(B), Z\left(\mathbf{x}_{i}\right)\right)=\int_{B} C\left(v, \mathbf{x}_{i}\right) d u \prime /|B|
$$

where $\bar{C}$ is point-to-block covariance, $|B|$ is the volume of the block which is called spatial support and $C$ is point-to-point covariance.

In practice, $\bar{C}$ is approximated by a summation because $B$ is discretized into points $\left(u^{\prime}\right)$ to compute the integrals. Given the observations at locations with point support, block kriging can be used to predict the average value over a bigger support, accounting for size but also for shape and orientation of the blocks (support). Therefore, block kriging can be considered as a solution to the problem of change of support.

Cokriging is a multivariate spatial predictor, which was developed to improve the prediction of an 'undersampled' variable by determining the spatial correlation with other variables, which are more easily and extensively measured. The optimal ordinary cokriging predictor is a linear combination of all available data and, similarly to kriging, its equations result from minimizing the prediction mean squared error subject to unbiasedness condition. The main difference is that autocovariance and cross-covariance (between two variables) functions need to be estimated. The interested reader can find the full details of cokriging in Wackernagel [29] and Webster and Oliver [32]. The equations are valid regardless of the support of data, however taking into account different supports in the calculation of autocovariance and cross-covariance functions is crucial to obtain valid inference. Once a consistent model for the point covariance functions is estimated, using LMC as described above, block cokriging can be applied by replacing the cross-covariances in point cokriging by their block-averaged counterparts through variogram regularization [33]. The aim of variogram regularization is to transform a variogram model established on points into the corresponding variogram on a given block support. To build a regularized variogram, it needs to have a variogram model fitted on the raw or Gaussian point variable; the model can be also multivariate. The regularized variogram will then be calculated in different directions using a discretization of the blocks into equal cells, where the blocks are replaced by the union of the cell centers. Therefore, regularization consists in calculating a pseudo-experimental variogram on fictious points regularly discretized over the new support. Finally, it will be necessary to fit a new model on the block variogram before using it in the subsequent procedures of kriging or cokriging.

In summary, block cokriging can be used to solve both point-point and point-block problems of change of support although it can be easily extended to solve any type of change of support problems [34].

Finally, factorial block cokriging was applied to analyze the correlation structure between the variables by applying principal component analysis (PCA) at each spatial scale $u$, to obtain independent 
factors which synthesize the multivariate information, to estimate the values of these specific factors at each characteristic scale using block cokriging, and then to map them.

The regionalized principal component analysis breaks down each co-regionalization matrix $\mathbf{B}^{u}$ into eigenvalues and eigenvector matrices [25]:

$$
\mathbf{B}^{u}=\mathrm{Q}^{u} \Lambda^{u} \mathrm{Q}^{u T}=\left(\mathrm{Q}^{u} \sqrt{\Lambda^{u}}\right)\left(\mathrm{Q}^{u} \sqrt{\Lambda^{u}}\right)^{T}=\mathrm{A}^{u} \mathrm{~A}^{u T}
$$

where $Q^{u}$ is the matrix of eigenvectors, i.e., the regionalized factors $Y_{v}^{u}(\mathbf{x})$, and $\Lambda^{u}$ is the diagonal matrix of eigenvalues for each spatial scale $u ; \mathbf{A}^{u}=\mathbf{Q}^{u} \sqrt{\Lambda^{u}}$ is the matrix of order $n \times n$ of the transformation coefficients $a_{i v}^{u}$, which correspond to the covariances between the original variables $Z_{i}(\mathbf{x})$ and the regionalized factors $Y_{v}^{u}(\mathbf{x})$.

The approach breaks down the set of original second-order random variables $\left\{Z_{i}(\mathbf{x}) ; i=1, \ldots, n\right\}$ into $N_{s}$ sets of reciprocally orthogonal factors $\left\{Y_{v}^{u}(\mathbf{x}) ; v=1, \ldots, n ; u=1, \ldots, N_{s}\right\}$ with transformation coefficients $a_{i v}^{u}$ for each spatial scale $u$. Combining the spatial with the multivariate decomposition each variable $Z_{i}$ can be written as:

$$
Z_{i}(\mathbf{x})=\sum_{u=1}^{N_{S}} \sum_{v=1}^{n} \mathrm{a}_{i v}^{u} Y_{v}^{u}(\mathbf{x})
$$

Mapping the regionalized factors $Y_{v}^{u}(\mathbf{x})$ provides an illustration of the joint behavior of the variables and their spatial relationships at the different spatial scales. The estimation of the factors is performed by solving a modified cokriging system, as described by Wackernagel [29] and Castrignanò et al. [31].

All geostatistical analyses were performed using the software Isatis 2017.1 (Geovariances, Avon Cedex, France) [35].

\section{Results and Discussion}

The summary statistics of the selected twenty-eight geophysical variables from the three different sensors, which will be used for field delineation, are reported in Table 1.

From the inspection of Table 1, it is evident that all variables generally show large variability; in more detail GEM variables look quite similar, though with a tendency to decrease on average at lower frequencies, due to the larger volume of soil (support) surveyed by the electromagnetic (EM) signal. However, the EM wave at $3575 \mathrm{~Hz}$ shows a drastic reduction in the mean value of bulk EC, which can be explained in terms of soil properties. The same trend appears in the two variables from the EM38DD sensor: a large decrease of mean bulk EC in depth (vertical polarization) jointly with an increase of variation.

As regards the GPR data at the different depths, a tendency of reduction of the signal amplitude occurs in depth, as is expected owing to the attenuation, especially at depths deeper than 0.60-0.70 m; beneath 1.0-m depth, the signal was critically attenuated.

The values of skewness and kurtosis show that the data distributions are mostly positively skewed, with the exception of the shallower GPR data up to $0.30 \mathrm{~m}$-depth (negatively skewed), and sharply peaked (high kurtosis), which is evidence of great uniformity but with the presence of a few large outliers. Before performing Gaussian anamorphosis, most of the outliers were filtered, especially the ones located along the lower western border, owing to the presence of a metallic gate in the field. The goodness of fitting of Gaussian modelling was tested by cross-validation producing mean errors quite close to 0 and standard deviations of error less than $10 \%$ of the standard deviation of the corresponding raw variable. 
Table 1. Basic statistics of the selected geophysical variables used in the study. $\mathrm{EC}_{\mathrm{a}(-\mathrm{Hz})}$ are the apparent electrical conductivities ( $\mathrm{mS} \cdot \mathrm{m}^{-1}$ ) measured by the GEM Profiler at the different operating frequencies; $\mathrm{EC}_{\mathrm{aH}}$ and $\mathrm{EC}_{\mathrm{aV}}$ are the apparent electrical conductivities $\left(\mathrm{mS} \cdot \mathrm{m}^{-1}\right)$ measured by the EM38DD in horizontal $(\mathrm{H})$ and vertical $(\mathrm{V})$ polarization; $\mathrm{GPR}_{(-\mathrm{m})}$ are the GPR signal amplitude (-) at the different depth slices.

\begin{tabular}{|c|c|c|c|c|c|c|c|}
\hline Variable & Minimum & Maximum & Mean & Median & Stand. Dev. & Skewness & Kurtosis \\
\hline $\mathrm{EC}_{\mathrm{a}(19,975 \mathrm{~Hz})}$ & 34.92 & 122.49 & 43.94 & 43.43 & 4.19 & 9.28 & 162.41 \\
\hline $\mathrm{EC}_{\mathrm{a}(12,975 \mathrm{~Hz})}$ & 29.60 & 106.76 & 35.94 & 35.37 & 4.39 & 7.19 & 97.88 \\
\hline $\mathrm{EC}_{\mathrm{a}(8425 \mathrm{~Hz})}$ & 26.02 & 103.407 & 34.22 & 33.26 & 5.69 & 6.33 & 64.40 \\
\hline $\mathrm{EC}_{\mathrm{a}}(5475 \mathrm{~Hz})$ & 17.49 & 149.06 & 25.27 & 23.80 & 7.84 & 8.75 & 114.65 \\
\hline $\mathrm{EC}_{\mathrm{a}(3575 \mathrm{~Hz})}$ & 0.10 & 200.29 & 5.38 & 3.32 & 11.75 & 12.14 & 185.23 \\
\hline $\mathrm{EC}_{\mathrm{a}(2325 \mathrm{~Hz})}$ & 6.03 & 294.03 & 21.34 & 18.11 & 16.28 & 9.07 & 124.96 \\
\hline $\mathrm{EC}_{\mathrm{a} H}$ & 39.50 & 66.88 & 44.86 & 44.38 & 2.72 & 2.82 & 17.76 \\
\hline $\mathrm{EC}_{\mathrm{a} \mathrm{V}}$ & 2.75 & 73.13 & 8.52 & 8.00 & 3.84 & 8.50 & 124.74 \\
\hline $\mathrm{GPR}_{(0.10 \mathrm{~m})}$ & 1260.00 & $10,387.00$ & 7315.03 & 7481.50 & 1332.98 & -1.08 & 5.42 \\
\hline $\mathrm{GPR}_{(0.20 \mathrm{~m})}$ & 1840.00 & $12,333.00$ & 8191.51 & 8492.00 & 1953.14 & -0.52 & 2.88 \\
\hline $\mathrm{GPR}_{(0.30 \mathrm{~m})}$ & 1115.00 & $10,234.00$ & 6192.77 & 6515.00 & 1957.09 & -0.30 & 2.12 \\
\hline $\mathrm{GPR}_{(0.40 \mathrm{~m})}$ & 634.00 & 7042.00 & 3420.70 & 3521.50 & 1248.71 & 0.01 & 2.29 \\
\hline $\mathrm{GPR}_{(0.50 \mathrm{~m})}$ & 239.00 & 5628.00 & 1658.09 & 1619.50 & 667.15 & 0.58 & 4.25 \\
\hline $\mathrm{GPR}_{(0.60 \mathrm{~m})}$ & 91.00 & 3792.00 & 845.47 & 775.00 & 390.55 & 1.28 & 7.60 \\
\hline $\mathrm{GPR}_{(0.70 \mathrm{~m})}$ & 30.00 & 2085.00 & 439.04 & 427.00 & 191.97 & 1.96 & 14.63 \\
\hline $\mathrm{GPR}_{(0.80 \mathrm{~m})}$ & 24.00 & 1258.00 & 241.15 & 225.00 & 113.79 & 2.13 & 15.30 \\
\hline $\mathrm{GPR}_{(0.90 \mathrm{~m})}$ & 18.00 & 751.00 & 143.91 & 135.00 & 72.70 & 1.76 & 11.11 \\
\hline $\operatorname{GPR}_{(1.00 \mathrm{~m})}$ & 14.00 & 434.00 & 83.99 & 75.00 & 47.31 & 2.03 & 11.15 \\
\hline $\operatorname{GPR}_{(1.10 \mathrm{~m})}$ & 11.00 & 299.00 & 55.55 & 47.00 & 33.42 & 2.39 & 12.46 \\
\hline $\operatorname{GPR}_{(1.20 \mathrm{~m})}$ & 10.00 & 229.00 & 49.96 & 44.00 & 27.35 & 1.85 & 8.77 \\
\hline $\operatorname{GPR}_{(1.30 \mathrm{~m})}$ & 5.00 & 149.00 & 43.07 & 38.00 & 22.93 & 1.30 & 5.30 \\
\hline $\mathrm{GPR}_{(1.40 \mathrm{~m})}$ & 2.00 & 136.00 & 31.65 & 28.00 & 18.84 & 1.65 & 7.26 \\
\hline $\operatorname{GPR}_{(1.50 \mathrm{~m})}$ & 1.00 & 98.00 & 23.37 & 19.00 & 14.08 & 1.53 & 6.24 \\
\hline $\operatorname{GPR}_{(1.60 \mathrm{~m})}$ & 2.00 & 71.00 & 19.08 & 17.00 & 10.34 & 1.14 & 4.44 \\
\hline $\operatorname{GPR}_{(1.70 \mathrm{~m})}$ & 1.00 & 69.00 & 15.79 & 14.00 & 8.24 & 1.14 & 5.47 \\
\hline $\operatorname{GPR}_{(1.80 \mathrm{~m})}$ & 0.00 & 50.00 & 12.12 & 11.00 & 6.17 & 1.10 & 5.35 \\
\hline $\operatorname{GPR}_{(1.90 \mathrm{~m})}$ & 0.00 & 30.00 & 8.90 & 8.00 & 4.44 & 0.90 & 4.36 \\
\hline $\operatorname{GPR}_{(2.00 \mathrm{~m})}$ & 0.00 & 27.00 & 6.48 & 6.00 & 3.33 & 0.99 & 5.38 \\
\hline
\end{tabular}

As no heteroscedasticity effect of variance with direction was manifest in the experimental point variograms, an isotropic LMC model was fitted to the direct and cross-variograms of the twenty-eight Gaussian transformed variables, consisting of the three following basic spatial structures: a nugget effect, a spherical model with a range of $10.74 \mathrm{~m}$ and a spherical model with a range of $55 \mathrm{~m}$. Then, the data were interpolated with block cokriging on a regular grid with a mesh of $1 \mathrm{~m}$, after regularization of the previous point LMC using block size equal to the grid mesh. Regularization did not change the type of the mathematical models but modified the number of the structures by eliminating the nugget, reduced the sills, and increased the ranges slightly. In more details, the regularized LMC included two basic structures: a spherical model with range $=11.66 \mathrm{~m}$ and a spherical model with range $=60.55 \mathrm{~m}$. The associated variances to the two spatial structures were quite comparable though a light prevalence for the structure at longer scale, as it results from the cumulative values of their corresponding eigenvalues. The effect of regularization was showed in Figure 2, where, as an example, some of the point variograms compared with the corresponding regularized variograms are reported.

The goodness of fitting was satisfactory as the mean errors were close to zero and variances of the standardized errors close to 1 (data not shown). 
(a) Point variograms
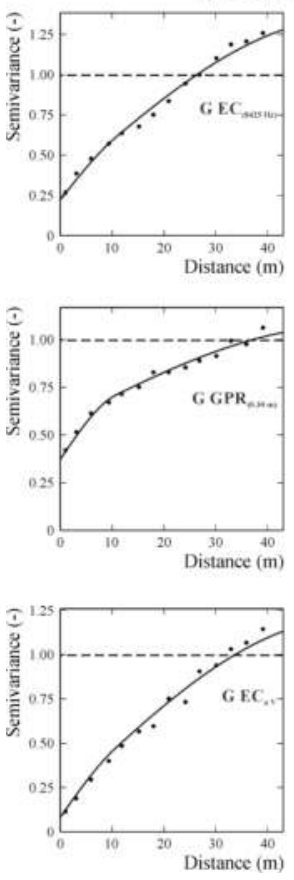
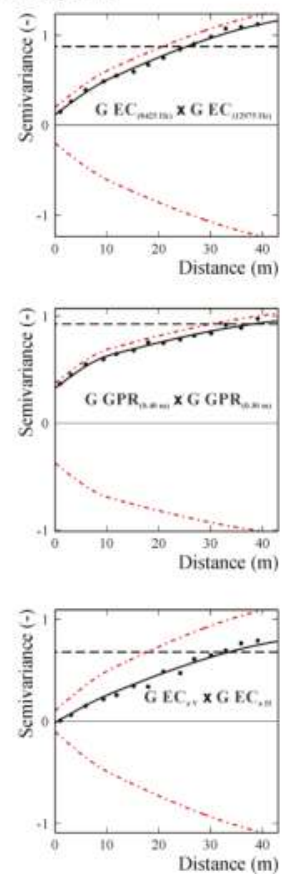

(b) Regularized variograms
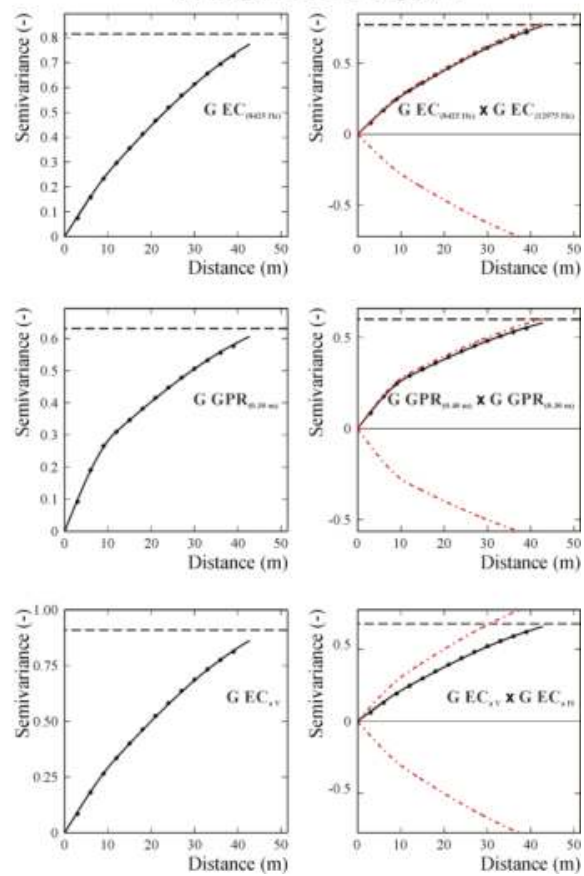

Figure 2. Examples of point (a) and reguralized (b) auto- and cross-variograms of the Gaussian variables of $\mathrm{EC}_{\mathrm{a}(-\mathrm{Hz})}$ (apparent electrical conductivities measured by the GEM Profiler), $\mathrm{EC}_{\mathrm{a}} \mathrm{H}$ and $\mathrm{EC}_{\mathrm{a} V}$ (apparent electrical conductivities measured by the EM38DD in horizontal and vertical polarization) and GPR signal amplitude at different depth slices. The experimental values are the plotted points and the solid lines are of the model of coregionalization. The dash-dotted lines are the hulls of perfect correlation and the dashed lines are the experimental variances.

The maps of the geophysical variables are shown in Figures 3-5: a great similarity among the six bulk $\mathrm{EC}_{\mathrm{a}}$ maps (Figure 3) from the GEM sensor is evident, with a wide central area (blue) characterized by the lowest values (the least conductive zone), and three less extensive but more conductive zones (brown) north, south, and along the western border of the field (Figure 3a). As regards the map corresponding to the frequency of $3575 \mathrm{~Hz}$, it is evident an expansion of the central lower-value area (blue) is at the expense of the other three (brown), with a reduction of the overall minimum and mean values compared with the corresponding values of the other GEM maps but not of the maximum values. What is observed above, is indicative of some sort of discontinuity along the profile up to $2.0 \mathrm{~m}$-depth, though it is not possible from the only cumulative EMI measurements to localize where it occurs. This finding could be associated with the evidence that in the area two soils (Aquic Calciustepts and Typic Calciustepts) coexist, either having or not having in the subsoil Fe-Mn concretion/segregations and mottles. 

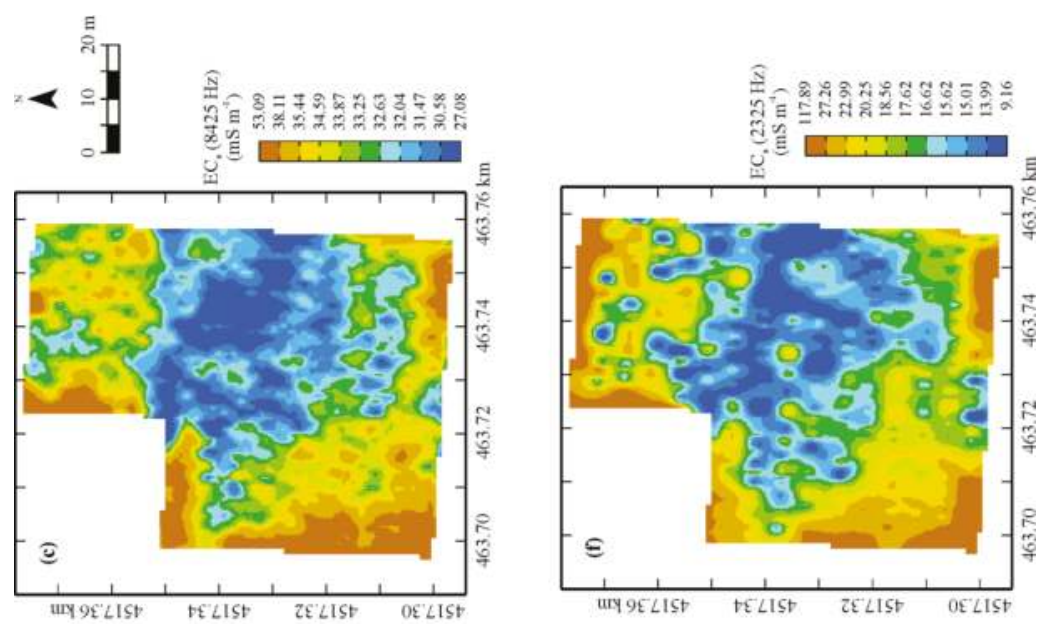

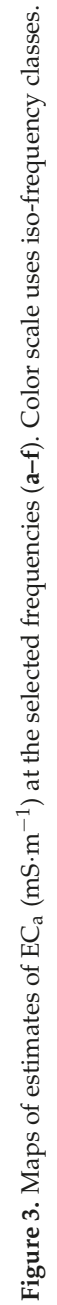
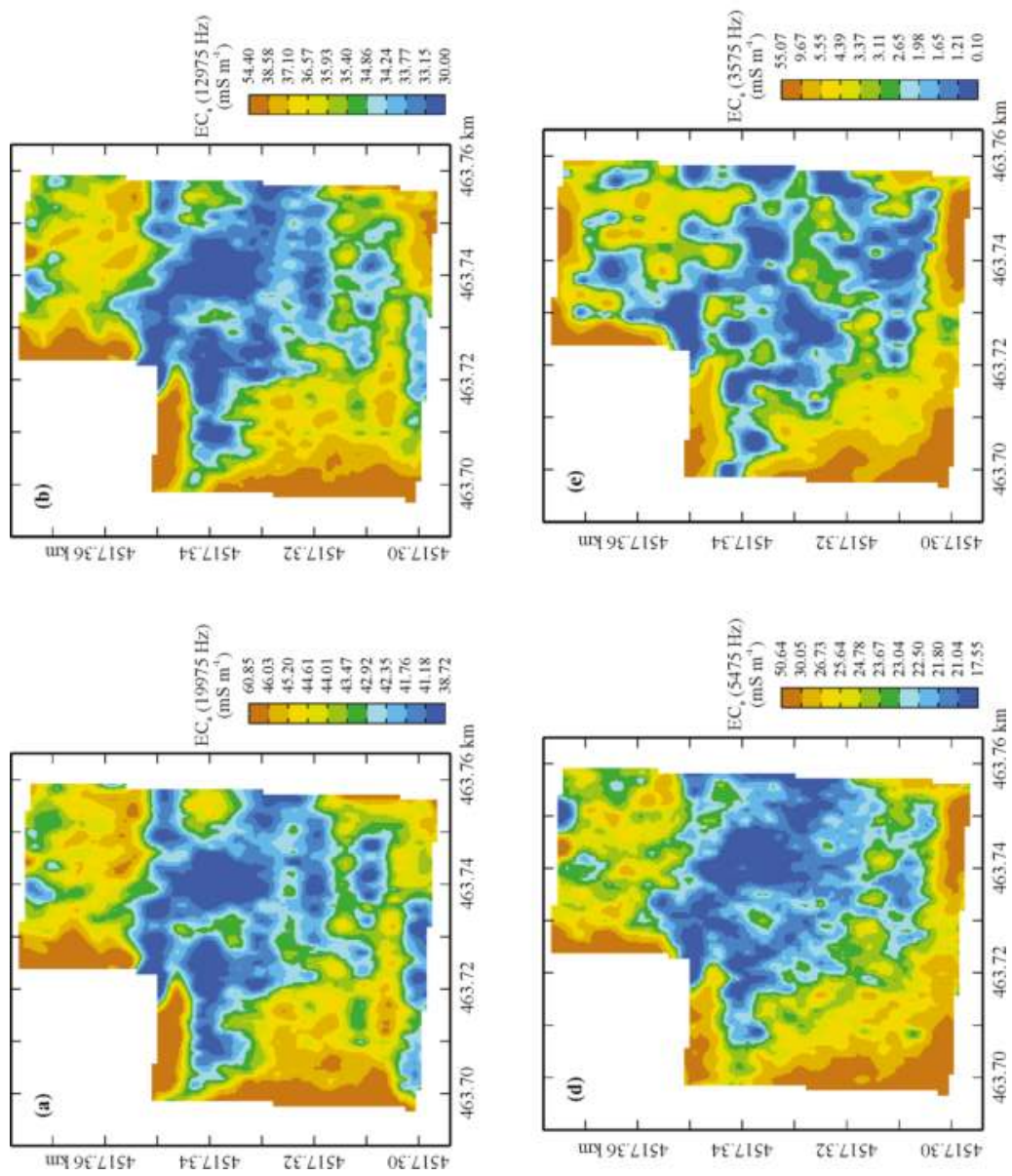

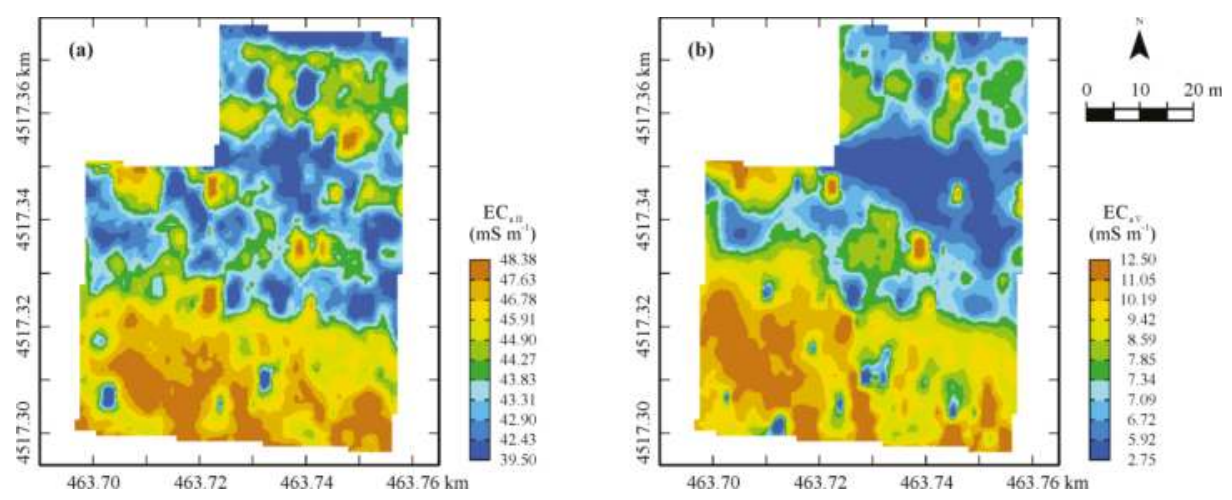

Figure 4. Maps of estimates of $\mathrm{EC}_{\mathrm{a}}\left(\mathrm{mS} \cdot \mathrm{m}^{-1}\right)$ in the (a) horizontal and (b) vertical orientations. Color scale uses iso-frequency classes.

The $\mathrm{EC}_{\mathrm{a}}$ maps in both orientation modes look similar enough to the previous ones, although the area characterized by lower values (blue) is much larger and extends also towards the northern part of the field (Figure 4).

The zone with the highest values (brown) in the map in horizontal mode, which are comparable with the ones of $\mathrm{EC}_{\mathrm{a}}$ maps from the GEM sensor, is restricted only to a strip in the southern part of the field. It is a little surprising to see the large decrease in bulk $\mathrm{EC}_{\mathrm{a}}$ values for the mode in vertical polarization, which is a further indication of the lack of soil continuity in depth in terms of moisture and/or granulometry and/or structure. Again, this type of sensor, which provides an integrated signal along the surveyed soil profile, is not capable of localizing the discontinuity and displaying the vertical stratification. This was the reason why it was decided to integrate the previous EMI sensors with a different type of geophysical sensor, a GPR, whose outcomes can be localized in a 3D space.

In Figure 5, the horizontal sections of block cokriging estimates of GPR signal amplitude at each 0.10-m depth-increment are shown. The maps appear sufficiently similar and in some way reversed compared to $\mathrm{EC}_{\mathrm{a}}$ maps, at least up to about 0.60 -m depth.

This behavior is quite predictable, since the more conductive areas cause greater attenuation of the radar signal. The spatial pattern changes drastically at depths deeper than $0.60 \mathrm{~m}$, becoming quite erratic without clear structures of spatial dependence, which is indicative of some discontinuity along the profile occurring at this depth. At depths deeper than $1 \mathrm{~m}$ the GPR looked strongly attenuated, therefore the corresponding maps were not displayed and any further discussion was restricted to the shallow 1-m depth of soil. The advantage of including GPR in the multi-sensor set then appears evident, compared to an EMI sensor, because it allows one to detect significant structural changes along the soil profile, which can affect the conditions of root development and the nutrient and water uptake of the tomato crops grown in the field. 

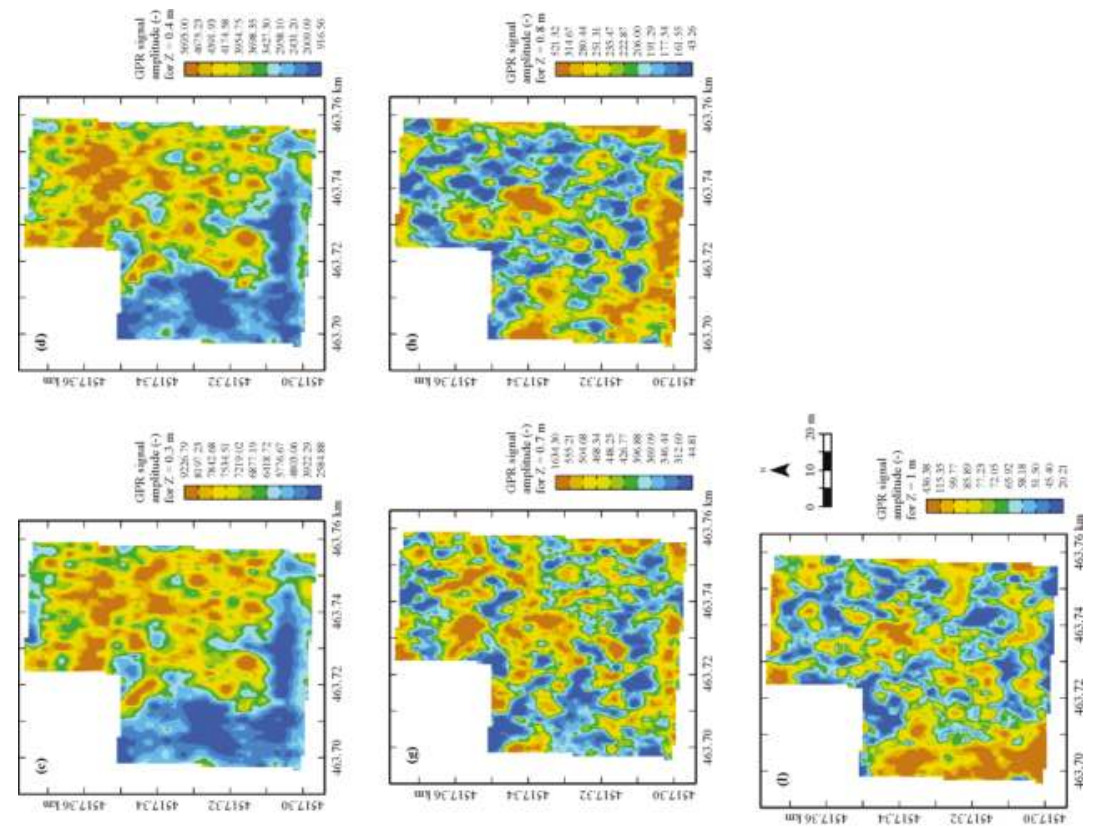

$\frac{\pi}{0}$
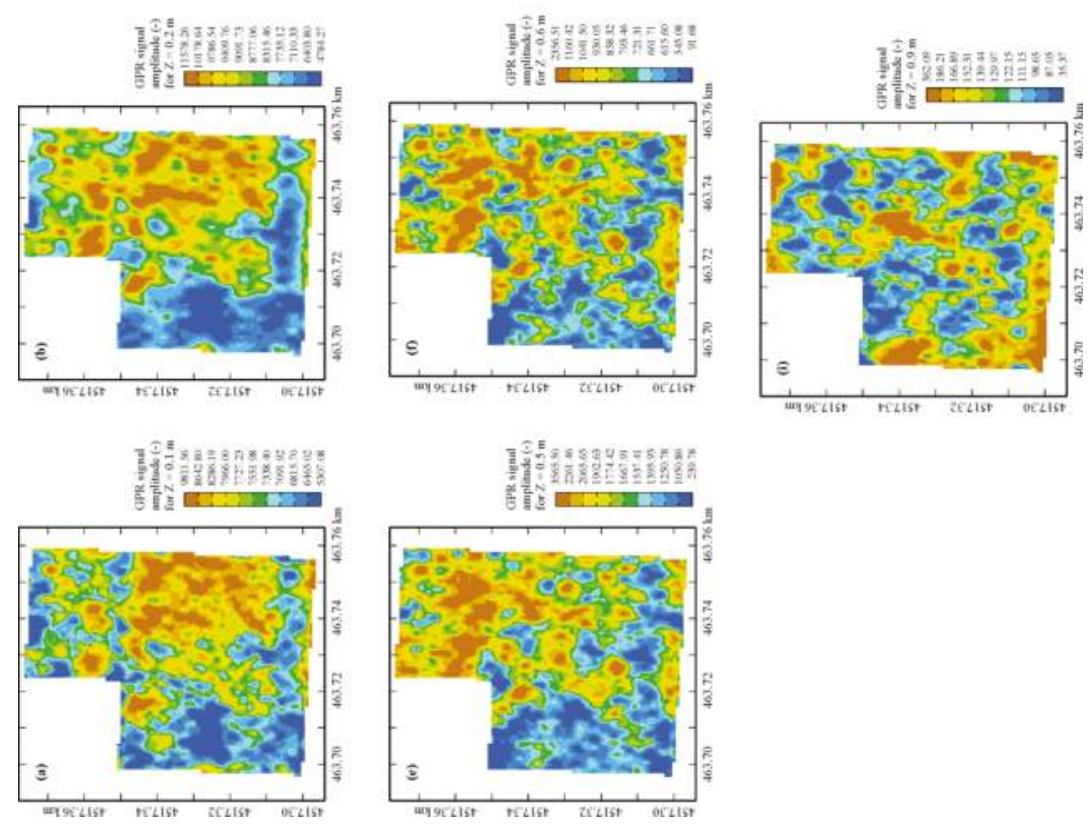

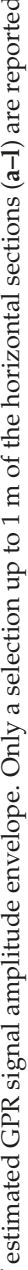

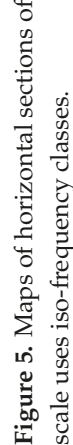


In order to obtain a delineation of the field into homogenous areas, among the block factorial cokriging results, the shorter-range structure was ignored because it is related to variability occurring at a scale (about $11 \mathrm{~m}$ ) deemed to be too small for actual agronomic management. Therefore, only the first two regionalized factors of the spherical model at longer range $(60 \mathrm{~m})$, corresponding to eigenvalues greater than 1 , were retained and mapped.

The first factor is mainly affected positively by the outcomes of the eight EMI sensors (both GEM and EM38 DD sensors) and negatively by the more superficial GPR outcomes up to the depth of $0.30 \mathrm{~m}$, explaining about $64 \%$ of the variance at the $60-\mathrm{m}$ scale (Table 2 ). It could then be interpreted as a synthetic indicator of the conductive properties of the shallow $0.30-\mathrm{m}$ depth. On the second factor, the GPR outcomes between 0.40 and 0.60 weigh mainly and positively and, to a less extent, the other GPR outcomes at deeper depths, explaining only about $18 \%$ of the variance at the same spatial scale $(60 \mathrm{~m})$. These results show clearly that some structural change is occurring along the vertical profile within the transition $0.40-0.60-\mathrm{m}$ horizon of the soil.

Table 2. Structural composition of the first two regionalized factors of the spherical model at longer range $(60 \mathrm{~m})$ with eigenvalue and explained variance $(\%)$.

\begin{tabular}{|c|c|c|c|c|c|}
\hline Variable & Factor 1 & Factor 2 & Variable & Factor 1 & Factor 2 \\
\hline $\mathrm{EC}_{\mathrm{a}(19,975 \mathrm{~Hz})}$ & 0.3012 & 0.1436 & $\mathrm{GPR}_{(0.70 \mathrm{~m})}$ & -0.0457 & 0.2016 \\
\hline $\mathrm{EC}_{\mathrm{a}(12,975 \mathrm{~Hz})}$ & 0.3282 & 0.1280 & $\mathrm{GPR}_{(0.80 \mathrm{~m})}$ & 0.0534 & 0.1673 \\
\hline $\mathrm{EC}_{\mathrm{a}(8425 \mathrm{~Hz})}$ & 0.3432 & 0.1052 & $\operatorname{GPR}_{(0.90 \mathrm{~m})}$ & 0.0264 & 0.2113 \\
\hline $\mathrm{EC}_{\mathrm{a}(5475 \mathrm{~Hz})}$ & 0.3471 & 0.0980 & $\operatorname{GPR}_{(1.00 \mathrm{~m})}$ & 0.0774 & 0.1595 \\
\hline $\mathrm{EC}_{\mathrm{a}(3575 \mathrm{~Hz})}$ & 0.2772 & 0.1348 & $\operatorname{GPR}_{(1.10 \mathrm{~m})}$ & 0.1044 & 0.1699 \\
\hline $\mathrm{EC}_{\mathrm{a}(2325 \mathrm{~Hz})}$ & 0.2987 & 0.1317 & $\operatorname{GPR}_{(1.20 \mathrm{~m})}$ & 0.0908 & 0.1614 \\
\hline $\mathrm{EC}_{\mathrm{a} H}$ & 0.2464 & -0.0880 & $\operatorname{GPR}_{(1.30 \mathrm{~m})}$ & 0.0639 & 0.1445 \\
\hline $\mathrm{EC}_{\mathrm{a} \mathrm{V}}$ & 0.2991 & -0.1266 & $\operatorname{GPR}_{(1.40 \mathrm{~m})}$ & 0.0423 & 0.1699 \\
\hline $\mathrm{GPR}_{(0.10 \mathrm{~m})}$ & -0.2161 & -0.0707 & $\operatorname{GPR}_{(1.50 \mathrm{~m})}$ & 0.0325 & 0.1640 \\
\hline $\operatorname{GPR}_{(0.20 \mathrm{~m})}$ & -0.2396 & 0.1463 & $\operatorname{GPR}_{(1.60 \mathrm{~m})}$ & -0.0071 & 0.1602 \\
\hline $\operatorname{GPR}_{(0.30 \mathrm{~m})}$ & -0.2178 & 0.3279 & $\operatorname{GPR}_{(1.70 \mathrm{~m})}$ & -0.0447 & 0.1621 \\
\hline $\mathrm{GPR}_{(0.40 \mathrm{~m})}$ & -0.1797 & 0.4011 & $\operatorname{GPR}_{(1.80 \mathrm{~m})}$ & -0.0579 & 0.1651 \\
\hline $\mathrm{GPR}_{(0.50 \mathrm{~m})}$ & -0.1120 & 0.3634 & $\operatorname{GPR}_{(1.90 \mathrm{~m})}$ & -0.0137 & 0.1502 \\
\hline $\mathrm{GPR}_{(0.60 \mathrm{~m})}$ & -0.0806 & 0.2802 & $\operatorname{GPR}_{(2.00 \mathrm{~m})}$ & 0.0301 & 0.0747 \\
\hline Eigenvalue & & & & 6.3576 & 1.7734 \\
\hline $\begin{array}{l}\text { Variance } \\
\text { Percent }\end{array}$ & & & & 63.74 & 17.78 \\
\hline
\end{tabular}

Figure 6 shows the maps of the two previous factors that realize the decomposition of the 3D spatial variance of the soil into two orthogonal components: the first related to the shallow soil (0-0.30 m depth) horizon; the second to depths deeper than $0.30 \mathrm{~m}$ at least up to $1.0 \mathrm{~m}$, owing to the strong attenuation of the GPR signal amplitude below this depth.

The map of the first factor displays a wide central area characterized by lower values (blue) of the EMI sensor outcomes but less attenuation of the GPR signal amplitude, probably ascribable to better structural properties of the shallow soil (0-0.30 m-depth). In the map of the second factor, the main structures of the spatial dependence are less clearly defined; however, a tendency can be observed towards lower values of GPR outcomes in the central area (blue) and towards higher values at the northern and south-western parts of the field (brown). Remembering the interpretation of the factors described above (Table 2), the two factors can be considered as synthetic indicators of the dielectric properties of the two different horizons of soil (above and below 0.30-m depth), confirming the occurrence of some sort of discontinuity along the $1.0-\mathrm{m}$ soil profile. 

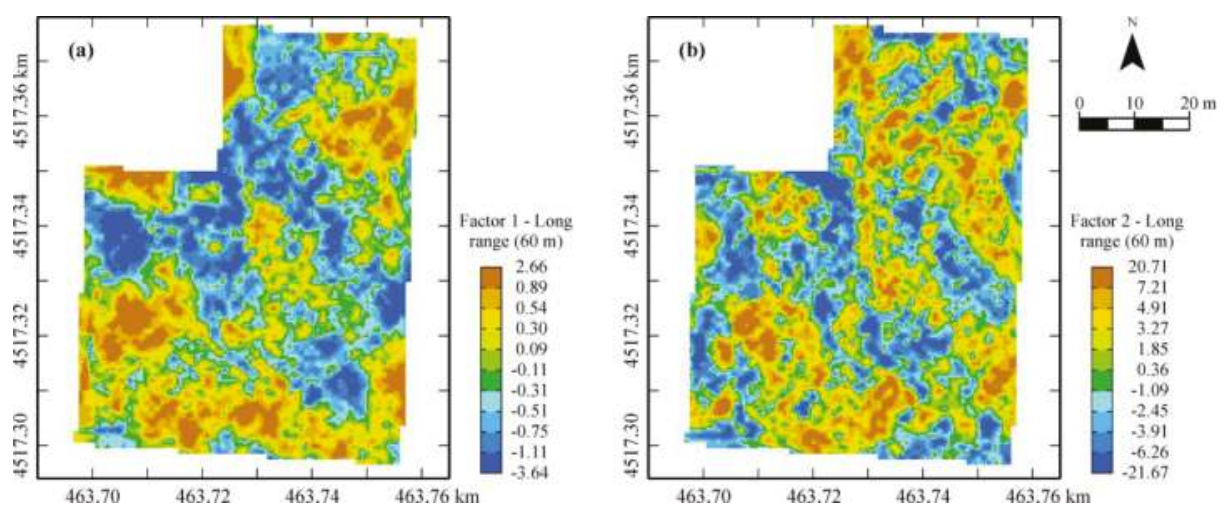

Figure 6. Maps of the first (a) and second (b) regionalized factors from sensor data fusion.

Summing up, the maps of the two factors graphically realize the fusion of the three sensors outputs, providing a partition of the field into zones with different dielectric properties of the soil at different depths. However, in order to interpret the variations shown in the previous maps in terms of actual soil characteristics, which could have an impact on agronomic management, a direct soil sampling of the shallow $0-0.30-\mathrm{m}$ depth was necessary.

The measured topsoil properties of the soil under study are reported in Table 3. Soil is sandy-loam with a coarse sand and silt fractions on average respectively greater than $55 \%$ and $32 \%$; this balanced texture provides the soil with good water draining and retaining properties, as reported by the mean values of the wilting point (WP) and field capacity (FC). Moreover, the soil has a good carbonate content, which also contributes to improving the soil structure [36]. From the correlation matrix (Table 4), it is shown that the total carbonate is significantly and positively correlated with most of the other variables with the exception of coarse sand which, conversely, is negatively correlated with all the other variables, showing a sort of antithetic behavior between the two soil properties (total carbonate and coarse sand). The water retaining properties of soil at low pressure (wilting point) are positively affected by both total carbonates and total silt content (which are positively correlated between them); whereas at high pressure (field capacity) the soil behavior is positively affected only by the fine component of silt. A clear interpretation of the contribution of silt to the hydrologic properties of our soils is difficult to evaluate. This is because fine and medium silt fractions are indeed the main grain sizes of pyroclastic material, but our soils have also a clear alluvial parent material contribution (with other coarser silt fractions) which makes the particle size distribution of the parent material quite varied.

Table 3. Basic statistics of soil properties.

\begin{tabular}{|c|c|c|c|c|c|c|c|}
\hline Variable & Minimum & Maximum & Mean & Median & Stand. Dev. & Skewness & Kurtosis \\
\hline $\begin{array}{l}\text { Total carbonates } \\
\quad\left(\mathrm{g} \cdot \mathrm{kg}^{-1}\right)\end{array}$ & 13.21 & 46.22 & 25.37 & 24.39 & 5.93 & 0.79 & 4.87 \\
\hline Clay $(\%)$ & 9.09 & 17.46 & 12.38 & 12.38 & 1.48 & 0.50 & 4.71 \\
\hline Coarse sand (\%) & 24.96 & 37.95 & 32.48 & 32.48 & 2.34 & -0.58 & 4.67 \\
\hline Coarse silt $(\%)$ & 12.39 & 16.92 & 13.77 & 13.62 & 0.84 & 0.98 & 4.96 \\
\hline Fine silt (\%) & 14.35 & 28.87 & 18.00 & 18.65 & 2.69 & 1.47 & 6.02 \\
\hline Fine sand (\%) & 19.23 & 27.25 & 22.71 & 22.71 & 1.55 & 0.31 & 3.10 \\
\hline Field capacity (\%) & 0.14 & 0.35 & 0.24 & 0.24 & 0.04 & 0.26 & 4.18 \\
\hline Wilting point $(\%)$ & 0.10 & 0.19 & 0.13 & 0.13 & 0.01 & 1.31 & 10.22 \\
\hline
\end{tabular}


Table 4. Correlation matrix of soil variables.

\begin{tabular}{ccccccccc}
\hline Variable & $\begin{array}{c}\text { Total } \\
\text { Carbonates }\end{array}$ & Clay & $\begin{array}{c}\text { Coarse } \\
\text { Sand }\end{array}$ & $\begin{array}{c}\text { Coarse } \\
\text { Silt }\end{array}$ & Fine Silt & $\begin{array}{c}\text { Fine } \\
\text { Sand }\end{array}$ & $\begin{array}{c}\text { Field } \\
\text { Capacity }\end{array}$ & $\begin{array}{c}\text { Wilting } \\
\text { Point }\end{array}$ \\
\hline Total carbonates & 1.00 & & & & & & & \\
$\quad$ Clay & 0.05 & 1.00 & & & & & & \\
Coarse sand & -0.32 & -0.26 & 1.00 & & & & & \\
Coarse silt & 0.22 & 0.21 & -0.21 & 1.00 & & & & \\
Fine silt & 0.23 & -0.40 & -0.46 & -0.47 & 1.00 & & & \\
Fine sand & -0.08 & 0.01 & -0.35 & 0.39 & -0.40 & 1.00 & & \\
Field capacity & 0.08 & -0.02 & -0.13 & -0.20 & 0.36 & -0.31 & 1.00 & 1.00 \\
Wilting point & 0.38 & 0.04 & -0.35 & 0.14 & 0.35 & -0.19 & 0.13 & \\
\hline
\end{tabular}

Moreover, fine sand and coarse silt seem to show a very similar behavior, representing a transition phase for the granulometric and structural properties of soil (from coarse sandy to finer textured soils). Summing up, the observed combination of total carbonate content and texture of the topsoil seems to favor the good properties of water retention and drainage observed for these soils.

The occurrence of the previous significant correlations between the soil properties justifies the multivariate approach that was performed. As all variables showed significant departure from Gaussian data distribution, they were previously Gaussian transformed and standardized to mean 0 and variance 1 .

An isotropic LMC was fitted to all experimental variograms including the following spatial structures: nugget effect, spherical model with range $=16.3 \mathrm{~m}$ and spherical model with range $=33.8 \mathrm{~m}$, which realize a decomposition of the total variance in three components: uncorrelated error (about $35 \%$ ), micro-scale variance (about $18 \%$ ), longer-scale variance (about $46 \%$ ). Therefore, more than $50 \%$ of the total variance is ascribable to variation occurring to a scale $(<16.3 \mathrm{~m})$ that is hardly manageable by tillage or other agricultural procedures. Only about $46 \%$ of the total variance, related to spatial scale of $33.8 \mathrm{~m}$, can be attributed to more structural properties of soil.

Point factorial cokriging (as all soil variables have the same support) was then applied exclusively to the longer-range structure of the previous LMC and the first factor was retained because it was associated with an eigenvalue greater than 1, explaining more than $62 \%$ of the variance at this scale. From the description of the structure of this factor (Table 5), it follows that the variables weighing more on it are: positively, total carbonates and wilting point; negatively, coarse sand.

Table 5. Structural composition of the first regionalized factor of the spherical model at longer range $(33.80 \mathrm{~m})$ with eigenvalue and explained variance $(\%)$.

\begin{tabular}{cc}
\hline Variable & Factor $\mathbf{1}$ \\
\hline G Field capacity & 0.0939 \\
G Wilting point & 0.3758 \\
G Coarse sand & -0.4416 \\
G Coarse silt & 0.2533 \\
G Fine sand & -0.0031 \\
G Silt & 0.2766 \\
G Total carbonate & 0.7171 \\
\hline Eigenvalue & 2.0347 \\
Variance Percent & 62.24 \\
\hline
\end{tabular}

The map of the first factor (Figure 7) shows a wide central area (brown) characterized by the highest carbonate contents and the minimum contents of coarse sand (Figure 8). The high content of carbonates (Figure 8) gives soil good structural properties, improving also water drainage and retention. 


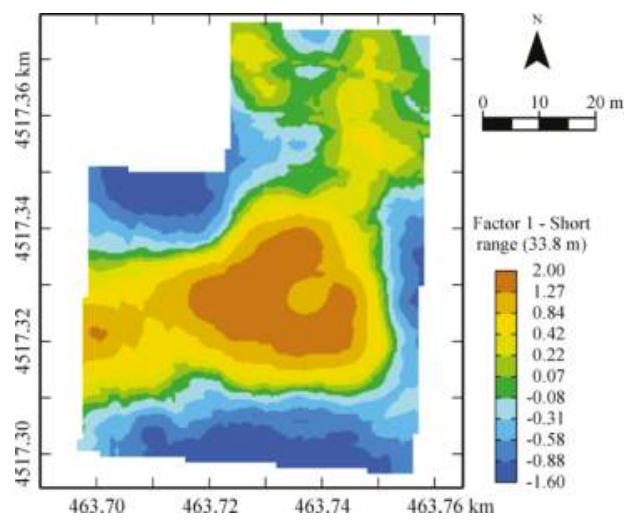

Figure 7. Map of the 33.8-scale factor of soil properties.
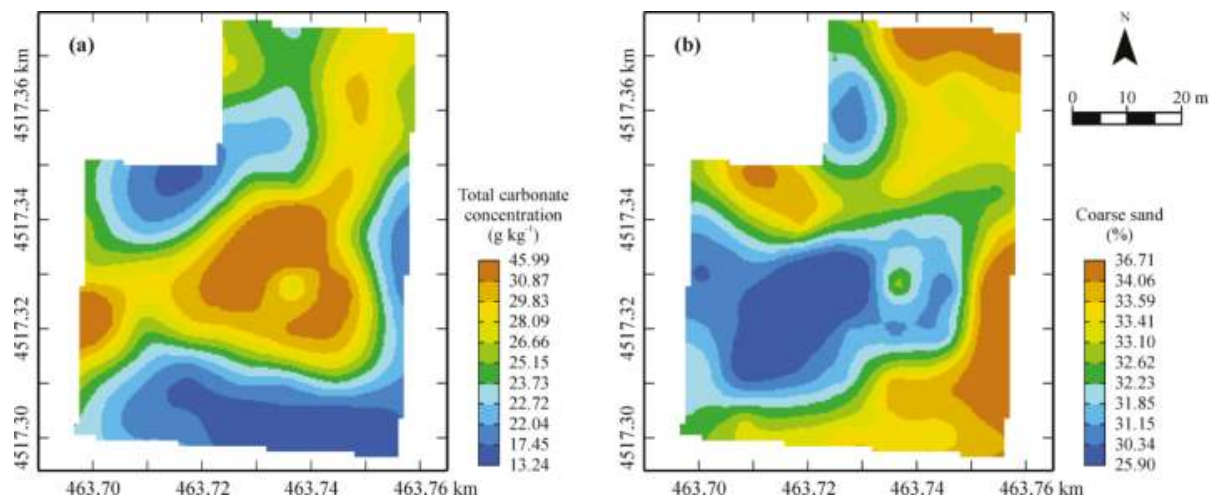

Figure 8. Cokriged maps of total carbonate (a) and coarse sand (b).

Comparing Figure 7 of the topsoil factor with Figure 6a of the first factor of the geophysical variables, which is mainly affected positively by EMI sensor outcomes and negatively by GPR amplitude in the depth $0-0.30 \mathrm{~m}$ (Table 2), it is possible to verify a perfect coherence between them. In fact, the high contents of carbonates increase soil resistivity (low bulk EC) and reduce attenuation of EM signal (high GPR signal amplitude outcomes).

As shown before, the dielectric properties of soil are inverted in depth $(>0.30 \mathrm{~m})$ (Figure 5); the correspondence with actual soil properties could be verified only with soil profiling, because soil sampling was carried out only in the shallow $0-0.30 \mathrm{~m}$ depth more interest for tomato rooting.

The occurrence of this wide area in the central-northern part of shallow soil, creating better water conditions for plants, as verified by higher values (brown) of wilting point and field capacity (Figure 9), can clearly have an impact on crop growth and water requirements, making site-specific management necessary.

The different response of the crop to the varied edaphic conditions could be tested through a remote (satellite, aero or UAV) image of the field, which was not carried out. However, it was proven that what was sensed by a set of different geophysical sensors can be attributed to real physical properties of the soil capable to impact agricultural management. 

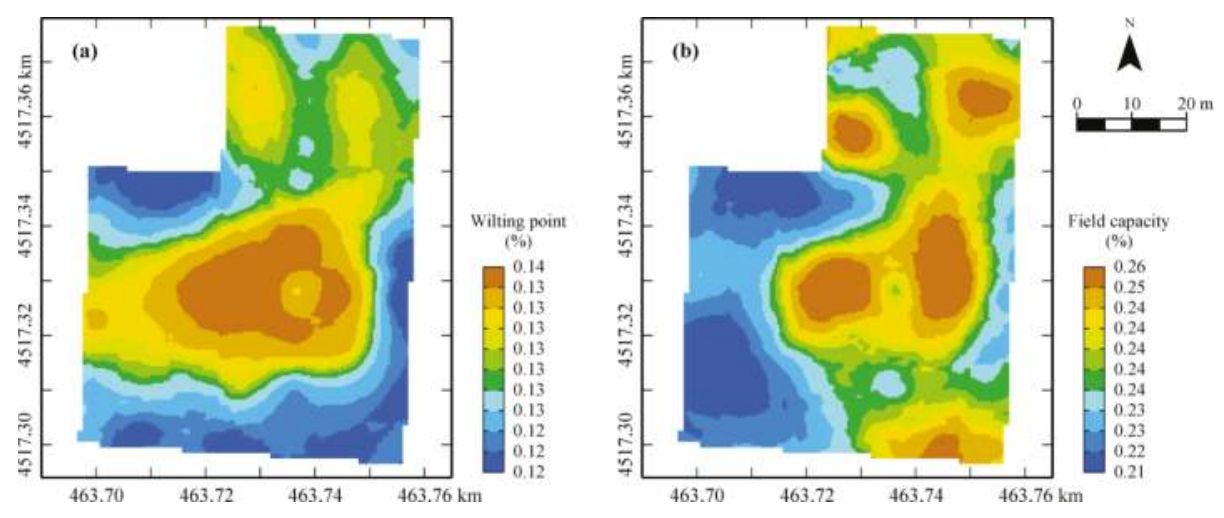

Figure 9. Cokriged maps of wilting point (a) and field capacity (b).

\section{Conclusions}

A data fusion approach based on multivariate geostatistics, for combining spatial data of different types from geophysical sensors, was described and applied in a soil of an agronomic field cropped with tomato. The calculated synthetic regionalized factors contributed in splitting the 3D edaphic environment into two main horizontal structures with different hydraulic properties and to disclose two main horizons in the $0-1.0-\mathrm{m}$ depth with a discontinuity probably occurring between $0.40 \mathrm{~m}$ and $0.70 \mathrm{~m}$. Comparing this partition with the soil properties measured with shallow sampling, it was possible to verify the coherence in the topsoil between the dielectric properties and other properties more directly related to agronomic management. These results confirm the advantages of using proximal sensing as a preliminary step in the application of site-specific management, even if the interpretation of field delineation, obtained by soil sensing in terms of effective management zones, can be done only through direct sampling and field scouting.

Finally, it is worth underlining that combining disparate spatial data (data fusion) is not at all a naive problem and novel and powerful methods should be developed. Although different solutions have already been proposed [37], very few techniques treat the fundamental problem of change of support, associated with the joint analysis of different sensors, explicitly. The geostatistical way of facing the problem is to estimate cross-covariance functions of a multivariate data set over different sample size (block), as was performed in this work with the regularization of LMC used in block cokriging and block factorial cokriging. In some cases, more complex non-linear approaches can still be required, but a measure of prediction uncertainty, as geostatistics effectively does, is needed. What was stated beforehand, points out the necessity of promoting research to solve the problem of change-of-support in sensor data fusion, common to many other disciplines in our time, characterized by an abundance of multi-source information.

Acknowledgments: Financial support for this work comes from the project "M2Q" PON03PE_00180_1 co-funded by the National Operational Program for Research and Competitiveness (PON R\&C) 2007-2013, through the European Regional Development Fund (ERDF) and national resource (Revolving Fund-Cohesion Action Plan MIUR). D.M. MIUR n. 738/05.03.2014. The authors thank the three reviewers of this paper and the academic editor for providing constructive comments, which have contributed to the improvement of the published version.

Author Contributions: A.C. conceived and designed the experiments, analyzed and interpreted the data, and wrote the paper; G.B. analyzed and interpreted the data, designed the figures, and wrote the paper; R.Q. performed the experiments and interpreted the data; C.V analyzed the data and contributed to their interpretation; G.L. and F.T. described the soils and contributed to the interpretation of data; A.V. performed the experiments and contributed to the interpretation of data. All authors revised and approved the final paper version to be published.

Conflicts of Interest: The authors declare no conflict of interest. 


\section{References}

1. National Research Council. Precision Agriculture in the 21st Century: Geospatial and Information Technologies in Crop Management; National Academy Press: Washington, DC, USA, 1997; p. 149.

2. McBratney, A.B.; Santos, M.L.; Minasny, B. On digital soil mapping. Geoderma 2003, 117, 3-52. [CrossRef]

3. Cavallo, G.; De Benedetto, D.; Castrignanò, A.; Quarto, R.; Vonella, V.A.; Buttafuoco, G. Use of geophysical data for assessing 3D soil variation in a durum wheat field and their association with crop yield. Biosyst. Eng. 2016, 152, 28-40. [CrossRef]

4. Shaddad, S.M.; Madrau, S.; Castrignanò, A.; Mouazen, A.M. Data fusion techniques for delineation of site-specific management zones in a field in UK. Precis. Agric. 2016, 17, 200-217. [CrossRef]

5. Buttafuoco, G.; Castrignanò, A.; Cucci, G.; Lacolla, G.; Lucà, F. Geostatistical modelling of within-field soil and yield variability for management zones delineation: a case study in a durum wheat field. Precis. Agric. 2017, 18, 37-58. [CrossRef]

6. Mulla, D.J.; Schepers, J.S. Key processes for site-specific soil and crop management. In The State of Site-Specific Management for Agriculture; Pierce, F.J., Sadler, E.J., Eds.; ASA: Boston, MA, USA; CSSA: Hong Kong, China; SSSA: Madison, WI, USA, 1997; pp. 1-18.

7. Peralta, N.R.; Costa, J.L.; Balzarini, M.; Angelini, H. Delineation of management zones with measurements of soil apparent electrical conductivity in the southeastern pampas. Can. J. Soil Sci. 2013, 93, 205-218. [CrossRef]

8. De Benedetto, D.; Castrignano, A.; Sollitto, D.; Modugno, F. Spatial relationship between clay content and geophysical data. Clay Miner. 2010, 45, 197-207. [CrossRef]

9. Castanedo, F. A review of data fusion techniques. Sci. World J. 2013, 2013, 704504. [CrossRef] [PubMed]

10. David, L.; Hall, D.L.; McMullen, S.A.H. Mathematical Techniques in Multisensor Data Fusion, 2nd ed.; Artech House, Inc.: Norwood, MA, USA, 2004.

11. Moshou, D.; Bravo, C.; Oberti, R.; West, J.; Bodria, L.; McCartney, A.; Ramon, H. Plant disease detection based on data fusion of hyper-spectral and multi-spectral fluorescence imaging using Kohonen maps. Real-Time Imaging 2005, 11, 75-83. [CrossRef]

12. Gotway, C.A.; Young, L.J. A geostatistical approach to linking geographically aggregated data from different sources. J. Comput. Graph. Stat. 2007, 16, 115-135. [CrossRef]

13. Castrignanò, A.; Quarto, R.; Venezia, A.; Buttafuoco, G. A geostatistical approach for modelling and combining spatial data with different support. Adv. Anim. Biosci. 2017, 8, 594-599. [CrossRef]

14. Pinto, A.R.; Montez, C.; Araújo, G.; Vasques, F.; Portugal, P. An approach to implement data fusion techniques in wireless sensor networks using genetic machine learning algorithms. Inf. Fusion 2014, 15, 90-101. [CrossRef]

15. Goovaerts, P. Geostatistics for Natural Resource Evaluation; Oxford University Press: Oxford, UK, 1997.

16. USDA. Keys to Soil Taxonomy, 11th ed.; Soil Survey Staff; United States Department of Agriculture Natural Resources Conservation Service: Washington, DC, USA, 2010.

17. McNeill, J.D. Electromagnetic Terrain Conductivity Measurement at Low Induction Numbers; Geonics Limited: Mississauga, ON, Canada, 1980.

18. Daniels, J.J.; Ehsani, M.R.; Allred, B.J. Ground-Penetrating Radar Methods (GPR). In Handbook of Agricultural Geophysics; Allred, B.J., Daniels, J.J., Ehsani, M.R., Eds.; CRC Press: Boca Raton, FL, USA; Taylor and Francis Group: Didcot, UK, 2008; pp. 129-146.

19. Huang, H. Depth of investigation for small broadband electromagnetic sensors. Geophysics 2005, 70, G135-G142. [CrossRef]

20. Reynolds, J.M. Introduction to Applied and Environmental Geophysics, 2nd ed.; Reynolds International Ltd.: Mold, UK; John Wiley \& Sons, Ltd.: Hoboken, NJ, USA, 2011.

21. Sheriff, R.E. Encyclopedic Dictionary of Applied Geophysics, 4th ed.; Society of Exploration Geophysicists: Tulsa, OK, USA, 2002.

22. Davis, J.L.; Annan, A.P. Ground-penetrating radar for high-resolution mapping of soil and rock stratigraphy. Geophys. Prospect. 1989, 37, 531-551. [CrossRef]

23. Annan, A.P. Ground penetrating radar in near-surface geophysics. In Near-Surface Geophysics, Investigations in Geophysics No. 13; Butler, D.K., Ed.; Society of Exploration Geophysics: Tulsa, OK, USA, 2005; pp. 357-438. 
24. Woodward, J.; Ashworth, P.I.; Best, J.L.; Sambrook Smith, G.H.; Simpson, C.J. The use and application of GPR in sandy fluvial environments: Methodological considerations. In Ground Penetrating Radar in Sediments; Bristow, C.S., Jol, H.M., Eds.; Geological Society: London, UK, 2003; Volume 211, pp. 127-142.

25. Annan, A.P. Ground Penetrating Radar Principles, Procedures \& Applications; Sensors \& Software Incorporated: Mississauga, ON, Canada, 2003.

26. Allred, B.J.; McCoy, E.L.; Redman, J.D. Ground-penetrating radar investigation of a golf course green. Computer processing and field survey setup considerations. In Handbook of Agricultural Geophysics; Allred, B.J., Daniels, J.J., Ehsani, M.R., Eds.; CRC Press: Boca Raton, FL, USA, 2008; pp. 353-362.

27. Claerbout, J.F. Fundamental of Geophysical Data Processing; Blackwell Scientific Publications: Hoboken, NJ, USA, 1985.

28. Chilès, J.P.; Delfiner, P. Geostatistics: Modeling Spatial Uncertainty, 2nd ed.; John Wiley \& Sons, Inc.: New York, NY, USA, 2012.

29. Wackernagel, H. Multivariate Geostatistics: An Introduction with Applications; Springer: Berlin/Heidelberg, Germany, 2003.

30. Journel, A.; Huijbregts, C. Mining Geostatistics; Academic Press: London, UK, 1978; 600p.

31. Castrignanò, A.; Giugliarini, L.; Risaliti, R.; Martinelli, N. Study of spatial relationships among some soil physico-chemical properties of a field in central Italy using multivariate geostatistics. Geoderma 2000, 97, 39-60. [CrossRef]

32. Webster, R.; Oliver, M.A. Geostatistics for Environmental Scientists; John Wiley \& Sons: New York, NY, USA, 2007.

33. Myers, D.E. Cokriging: New Developments in Geostatistics for Natural Resource Characterization; Verly, G., David, M., Journel, A.G., Marechal, A., Eds.; D. Reidel Pub. Co.: Dordrecht, The Netherlands, 1984; pp. 295-305.

34. Gotway, C.C.A.; Young, L.J. Geostatistics for Environmental Applications: Proceedings of the Fifth European Conference on Geostatistics for Environmental Applications; Springer: New York, NY, USA, 2005; pp. 1-14.

35. Geovariances. ISATIS Software: Technical References Release 2017.1; Geovariances and Ecole des Mines de Paris: Paris, French, 2017.

36. Gargiulo, L.; Mele, G.; Terribile, F. Image analysis and soil micromorphology applied to study physical mechanisms of soil pore development: An experiment using iron oxides and calcium carbonate. Geoderma 2013, 197-198, 151-160.

37. Khaleghi, B.; Khamis, A.; Karray, F.O.; Razavi, S.N. Multisensor data fusion: A review of the state-of-the-art. Inf. Fusion 2013, 14, 28-44. [CrossRef]

(C) 2017 by the authors. Licensee MDPI, Basel, Switzerland. This article is an open access article distributed under the terms and conditions of the Creative Commons Attribution (CC BY) license (http:/ / creativecommons.org/licenses/by/4.0/). 
Article

\title{
Contamination Event Detection with Multivariate Time-Series Data in Agricultural Water Monitoring ${ }^{\dagger}$
}

\author{
Yingchi Mao ${ }^{1, *}$, Hai $\mathrm{Qi}^{1}$, Ping Ping ${ }^{1}$ and Xiaofang $\mathrm{Li}^{2}$ \\ 1 College of Computer and Information, Hohai University, Nanjing 211100, China; qihai@hhu.edu.cn (H.Q.); \\ amazingapple@hhu.edu.cn (P.P.) \\ 2 School of Computer Information \& Engineering, Changzhou Institute of Technology, Changzhou 213032, \\ China; lixf@czu.cn \\ * Correspondence: yingchimao@hhu.edu.cn; Tel.: +86-139-5102-9973 \\ + This paper is an extended version of an earlier conference paper: 21st International Conference on Database \\ Systems for Advanced Applications (DASFAA 2016), Dallas, TX, USA, 16-19 April 2016.
}

Received: 17 September 2017; Accepted: 30 November 2017; Published: 4 December 2017

\begin{abstract}
Time series data of multiple water quality parameters are obtained from the water sensor networks deployed in the agricultural water supply network. The accurate and efficient detection and warning of contamination events to prevent pollution from spreading is one of the most important issues when pollution occurs. In order to comprehensively reduce the event detection deviation, a spatial-temporal-based event detection approach with multivariate time-series data for water quality monitoring (M-STED) was proposed. The M-STED approach includes three parts. The first part is that M-STED adopts a Rule $\mathbf{K}$ algorithm to select backbone nodes as the nodes in the CDS, and forward the sensed data of multiple water parameters. The second part is to determine the state of each backbone node with back propagation neural network models and the sequential Bayesian analysis in the current timestamp. The third part is to establish a spatial model with Bayesian networks to estimate the state of the backbones in the next timestamp and trace the "outlier" node to its neighborhoods to detect a contamination event. The experimental results indicate that the average detection rate is more than $80 \%$ with M-STED and the false detection rate is lower than $9 \%$, respectively. The M-STED approach can improve the rate of detection by about $40 \%$ and reduce the false alarm rate by about $45 \%$, compared with the event detection with a single water parameter algorithm, S-STED. Moreover, the proposed M-STED can exhibit better performance in terms of detection delay and scalability.
\end{abstract}

Keywords: event detection; back propagation model; multivariate water quality parameters; time-series data; spatial-temporal model; connected dominating set; water supply network

\section{Introduction}

Water is an important resource of the ecological environment in the agricultural industry. Polluted water can have directly negative impact on the agricultural irrigation and result in the low quality and output of the crops. When a large-scale water contamination event occurs, it is one of the most important issues to detect and warn contamination events and prevent pollution from spreading. One effective way is to deploy a large-scale number of water quality sensors to monitor generic water quality parameters and detect contamination events [1]. At present, online water quality sensors are deployed in the waters networks (WSNs), which can measure many contaminants and provide an early indicator of possible pollution [2,3]. Contamination event detection approaches focus on identifying abnormal system behavior. Accurate detection should consider the response strategies, operation optimization, and overall system efficiency [4]. It is an important to establish how to detect contamination events in real-time and in an accurate way with the multivariate time 
series of water quality parameters in a WSN. The main water quality parameters are free chlorine, electrical conductivity (EC), $\mathrm{pH}$, temperature, total organic carbon (TOC), and turbidity [5]. Almost all existing contamination event detection approaches are based on only a single water quality parameter, which results in high false negative rates and low false positive rates [6]. It is difficult to detect a potential contamination event in the WSNs due to the number of parameters involved [7].

In real application, many redundant sensor nodes are deployed in the water supply networks to improve the robustness of the monitoring systems. On the other hand, due to large-scale sensor node deployment, the amount of data from dissimilar sensors has increased tremendously. To reduce the computational cost of event detection and improve the response time, sensor node selection needs to cut down the amount of data for the spatio-temporal correlation analysis. To avoid forwarding and collecting the sensed data from all of the sensors in the WSNs, Connected Dominating Set (CDS) $[8,9]$ is introduced to select the backbone nodes and construct an effective connected structure in a WSN to reduce the amount of transmitted data and improve the response speed for contamination event detection. In this paper, a distributed and effective algorithm to construct CDS called Rule K [9] is adopted to select backbone nodes and forward the sensed data of water quality.

Meanwhile, real-time event detection (e.g., water pollution incidences) in the WSNs should consider both spatial and temporal correlation and dynamically record the propagation path of the contamination event. This paper focuses on real-time contamination event detection with a spatio-temporal correlation model in a water supply network to achieve high accuracy and low false alarm rates. Therefore, a spatial-temporal-based event detection approach with multivariate time-series data for water quality monitoring (M-STED) was proposed. The M-STED approach includes three parts. The first part is that M-STED adopts Rule $\mathbf{K}$ algorithm to select backbone nodes as the nodes in the CDS, and forward the sensed data of multiple water parameters. The second part is to determine the state of each backbone node with back propagation neural network (BP) models and the sequential Bayesian analysis in the current timestamp. The third part is to establish the spatial model with Bayesian networks to estimate the state of the backbones in the next timestamp and trace the "outlier" node to its neighborhoods to detect a contamination event.

The contributions of this work are as follows.

(1) A connected dominated set (CDS) approach is introduced to select backbones from a large number of sensors, which can cut down the amount of data for the spatio-temporal correlation analysis.

(2) A data-driven event detection algorithm is developed, which relies on multivariate water quality time series with Bayesian sequential analysis and BP model from a WSN. Multiple parameters of water quality are fused to provide a unified decision support about a contamination event.

(3) Bayesian network (BN) is used to model spatial dependency.

The experimental results indicate that the proposed M-STED approach can achieve $90 \%$ accuracy with BP model and improve the rate of detection by about $40 \%$ and reduce the false alarm rate by about $45 \%$, compared to temporal abnormal event detection of univariate spatial-temporal event detection algorithm (S-STED). Furthermore, we found that M-STED can achieve better performance in terms of response time and scalability, compared to the simple threshold algorithm and the BN-only algorithm.

The rest of this paper is organized as follows. Related work is discussed in Section 2. Section 3 presents the overall water supply network model with multivariate time-series of water quality parameters. The CDS-based node selection strategy is proposed in Section 4. In Section 5, the temporal event detection with multivariate time-series data algorithm for a single node is proposed. Section 6 presents the spatial correlation model for event detection. The performance evaluation is given in Section 7. Section 8 is conclusions.

\section{Related Work}

The contamination event detection approaches based on water quality measurements consist of two phases. The first phase is to set up the prediction model with the historical data as the 
training dataset. The second one is to determine the water quality by comparing the predicted values with the measurements. Various approaches have been proposed for addressing the problem of contamination detection, using single or multiple-type measurements which are analyzed separately or in combination, from one or more locations in the network, using model-based or model-free approaches.

In the threshold-based approaches, the threshold values are set through statistical models. Simplicity is the main advantage since raw data can be directly processed. The abnormal event detection with two thresholds were adopted in [10]. However, the threshold-based approaches cannot obtain the spatio-temporal feature of water quality data, which results in low accuracy and high false positives of event detection. In the pattern matching approaches, the pattern is established and verified with water quality sensor readings to infer the contamination event [11]. Byer and Carlson assumed that the water quality parameters obey a Gaussian distribution. One statistical model was established to detect contamination events [12]. The statistical model detection methods cannot be used in the applications.

Learning-based methods can make inference of the possibility of contamination events based on the temporal and spatial correlation of water quality measurements $[4,13,14]$. It was promising to make full use of the spatial and temporal correlation to detect contamination events. Markov random fields (MRFs), Bayesian network (BN), dynamical BN, and SVM are common models in the WSNs with the high density. MRFs were adopted to model spatial context and stochastic interaction among observable quantities [15]. BN is considered as a means for unsupervised learning and anomaly detection in gas monitoring sensor networks for underground coal mines [13]. Using a single water parameter-free chlorine, radial basis function (RBF) neural network and wavelet analysis were applied to determine the contamination event occurrence with on-line data of water quality [16]. Perelman proposed a BN-based contamination event detection method to determine the event occurrence through estimating the possible locations of potential contaminants in WSNs [17]. One improved water-contamination events detection based on D-S theory was proposed to predict water quality parameters with on-line water quality sensors [18]. The contamination event detection algorithm based on principal component analysis (PCA) has been presented [19]. The PCA was applied to the normalized measurement. Then, the alarm index and the threshold of the alarm were obtained. In addition, a spatio-temporal model was adopted to detection the contamination events with water sensor networks [20].

A multiple type measurement approach at a single location was proposed in [12], where each parameter was compared to its three bounds. Control charts and Kalman filters have also been proposed in [21]. When multiple types of sensors are available, these can be used to compute distance metrics to detect contamination [22]. The probability of contamination events could be computed and compared to an adaptive threshold by utilizing a sequential Bayesian rule [23]. The estimation error of event detection was computed with respect to the measurements from a moving window [24]. Moreover, the US Environmental Protection Agency provides the event detection tool-CANARY [25,26] for time series analysis of multiple water quality parameters. Fluctuations in water distribution networks may cause significant variability, a Bayesian belief network was presented to infer the probability of contamination [27].

Based on the above analysis, most approaches for contamination event detection have been discussed via using single type water quality parameters. There are multiple components to indicate water quality in a WSN, such as free chlorine, EC, $\mathrm{pH}$, temperature, TOC, and turbidity. Unfortunately, a single parameter of water quality cannot reflect the real water quality in a WSN. When a contamination event occurred, the observable values of multiple water quality parameters changed in a significant way. The contamination event detection methods based on single parameter may result in low detection accuracy and high false alarms. To improve the detection accuracy and reduce the false alarms, multiple parameters of water quality should be considered to make a decision with data fusion. Liu et al. [28] proposed a method for real-time contamination detection using 
multiple conventional water quality sensors. Eight sensors were used in the case study. Furthermore, they extended their work by determining how the number of sensors influences the detection performance and identifying the optimal combination of sensor deployment [29]. Unfortunately, the development of multi-parameter water quality models and their calibration is challenging due to the large amount of information and number of parameters involved. A large amount of data should be transmitted and processed, which results in the great overhead for event detections. Thus, their application in the context of contamination event detection is complicated. On the other hand, changes in sensor readings caused by one contamination event usually exhibit a strong spatio-temporal correlation. The spatial and temporal correlation is critical to improve the accuracy of event detection. In this paper, the proposed M-STED utilizes a back propagation neural networks model to estimate the relationships between water quality parameters in a WSN. The proposed M-STED can detect potential contamination events for temporary analysis of multivariate water quality time series with Bayesian sequential analysis.

\section{Water Supply Network Model}

To clearly illustrate the event detection with spatio-temporal correlation in water sensor networks, the structure of a water supply network is shown in Figure 1. All of water quality sensors are deployed in the water supply network and each sensor can communicate with others via single-hop communication. The topology of the water supply network can be represented as a graph $G=(V, E)$, where $V$ is the set of water quality sensor nodes. Each directed edge $(u, v) \in E$ in the graph $G$ can be represented as the single-hop transmission link. In the water supply network, a set of sensors is deployed to monitor water quality. Each sensor node has a unique ID, from 1 to $n$. Sensors can sense a variety of attributed, such as free chlorine, electrical conductivity (EC), $\mathrm{pH}$, temperature, total organic carbon (TOC), and turbidity.

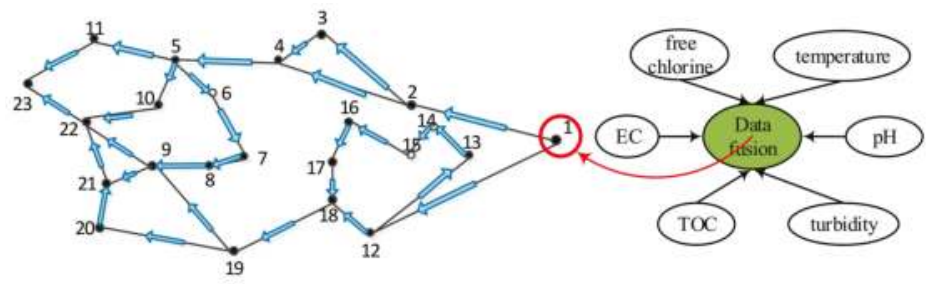

Figure 1. The topology of a water supply network.

Before contamination event detection, the spatio-temporal correlation is modeled based on normal historical data. For a large water supply network, it is difficult to simultaneously model spatial and temporal relationships due to the complexity of the data. In this paper, spatial and temporal relationships are separately modeled. BP models and the sequential Bayesian analysis are used to detect the outlier nodes for each water quality sensor node with multivariate time series of six water quality parameters. The conditional probability of $\mathrm{BN}$ is used to represent the spatial relationship. $S_{i}^{t}$ is denoted as the observed value from sensor $S_{i}$ at the time interval $t$. Once $n-t u p l e\left(S_{1}^{t}, S_{2}^{t}, \ldots, S_{m}^{t}\right)$ can be used as one of the training data cases for learning the parameters of the BN. The maximum likelihood parameter estimation algorithm is adopted to compute the parameters of the BN. If the states of corresponding sensor nodes deviate from the learned spatial relationship, we consider that a contamination event has occurred.

In this paper, the proposed M-STED approach keeps monitoring streams from the backbone nodes at every timestamp and traces from one outlier to its neighborhood to find a contamination event. First, the water supply network keeps monitoring data from the sensor nodes in the CDS at every timestamp, and then the selected nodes analyze whether a temporal outlier appears with BP models 
and sequential Bayesian analysis for each node in the CDS. If it does not, the system waits to monitor the data streams from the backbone nodes in the next timestamp. Otherwise, a candidate CDS set is generated for each "outlier" node and its children nodes of each "outlier". Then, in the next timestamp, the monitoring scope will be expanded, including the backbone nodes and the nodes in the candidate sets. Once a node in a candidate set is detected as a spatio-temporal outlier node, the children nodes of the outlier node will be added to the candidate set. When the number of spatio-temporal outliers is equal to or greater than the threshold $\theta$, we consider that a contamination event has happened and the corresponding warning is broadcast.

\section{Backbone Node selection}

Thousands of water quality sensors are deployed in a water supply network to sense, transmit, and forward the massive sensed data, which brings heavy burdens to further contamination event detection in the context of real-time analysis. To avoid forwarding and collecting all of the sensed data from all sensors in the WSNs, optimal sensor node selection is needed to reduce the amount of transmitted data and improve the response time for event detection. CDS is one of the optimal node selection strategies from Graph Theory [8].

A dominating set (DS) of an undirected graph $G=(V, E)$ is a subset of $V^{\prime}$ of the vertex set $V$ such that every vertex in $V-V^{\prime}$ is adjacent to a vertex in $V^{\prime}$. A dominating set $V^{\prime}$ is connected if for any two vertices $u$ and $v$, a path $\left(u, v_{1}\right),\left(v_{1}, v_{2}\right), \ldots,\left(v_{n}, v\right),\left(v_{i} \in V^{\prime}, 1 \leq i \leq n\right)$ in $E$ exists. Rule $\mathbf{K}$ algorithm was proposed in our previous work [9], which is an energy efficient and distributed algorithm for CDS selection. Through the selection of the appropriate communication range, a set of CDS can be constructed to meet the requirements of network connectivity and coverage. In each round of selection, the sensors adjust the appropriate communication range and select the nodes as nodes in the CDS. The sensors in the CDS as the backbones execute the tasks of monitoring and data forwarding. After the collected data from the CDS are analyzed, the system can detect the contamination event in the context of real-time analysis. The backbone nodes can keep monitoring several streams from backbone nodes instead of all streams, therefore, the CDS-based node selection strategy can reduce the amount of transmitted data and improve the response time for real-time event detection. In this paper, Rule $\mathbf{K}$ algorithm [9] is adopted to select the backbone nodes and construct an effective connected structure in a WSN.

Rule $\mathbf{K}$ algorithm consists of two parts: marking process and pruning rule.

In the marking process, all of the water quality sensor nodes are non-dominated nodes. Each node $u$ exchanges its neighbor's information in its communication radius $R_{u}$ with all its neighbors. According to two neighbors' information, node $u$ judges whether it can directly communicate with its neighbor nodes $v$ and $w$ or not. That is $d(u, v) \leq R_{u}, d(u, w) \leq R_{u}$, while $d(w, v)>\min \left\{R_{w}, R_{v}\right\}$. If two nodes $v$ and $w$ can directly communicate, node $u$ can be as a candidate dominant node and participate in the construction CDS. Otherwise, node $u$ is controlled by other nodes and is not in the CDS.

In the pruning process, it should reduce the size of dominant node set because the number of candidate dominant node selected via marking process is excessive. The basic idea of pruning rules is that if the $k$ neighbor nodes of candidate dominant node $u$ can make communication with other nodes, then node $u$ can be deleted from the CDS. The CDS-based node selection strategy can reduce the amount of transmitted data and improve the response time of the event detection.

\section{Temporal Event Detections with Multivariate Time Series in a Single Node}

\subsection{Main Idea}

The temporal outlier detection approach consists of two phases: an off-line phase and an on-line phase. At first, the system is in the off-line phase. During the off-line phase, one data-driven model-BP neural network is established. The historical data is trained for temporary analysis of multivariate water quality time series. If the obtained model has low residuals, the system enters into the on-line 
phase manually. In the on-line phase, Bayesian sequential analysis and BP model are adopted to compute the new measured parameter's value of each water parameter. Six parameters of water quality are fused to provide a unified decision support about a contamination event. After a period of time, with the increase of the measured values, it needs to retrain the historical data to refine the assessment model. A summary of the overall temporal outlier detection framework is presented in Figures 2 and 3.

Off-line phase: First, six BP neural networks were established and trained to simulate the six water quality parameters (free chlorine, EC, $\mathrm{pH}$, temperature, TOC, turbidity), respectively. Then, the residuals are calculated for each new measured value of each quality parameter as the total error. The possible outliers can be identified based on the residuals. The next step is to evaluate the sensitivity of the BP model and assess its accuracy.

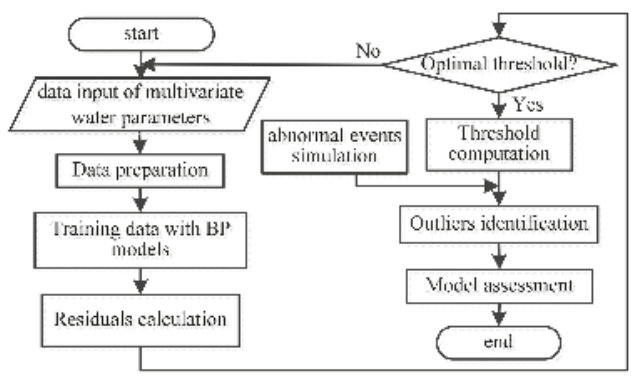

Figure 2. Procedure in the off-line phase.

The on-line phase includes four main steps for each incoming measured value: (1) data-driven model training - the relationships between water quality parameters are examined using BP models; (2) error threshold setting-calculated residuals are classified as normal or abnormal values using thresholds for each water quality parameter; (3) identification of events—the probability of an event is updated using sequential Bayesian analysis based on the residual classification; and (4) information fusion-univariate event probabilities are fused to provide a unified multivariate event probability reflecting the likelihood of an event based on all water quality parameters. Each water quality parameter is assigned a weight reflecting its influence on the fusion decision. Steps (1) and (2) are initially trained using available data collected from the WSN. Steps (1) to (4) are then repeatedly executed in real-time for each new incoming measured value.

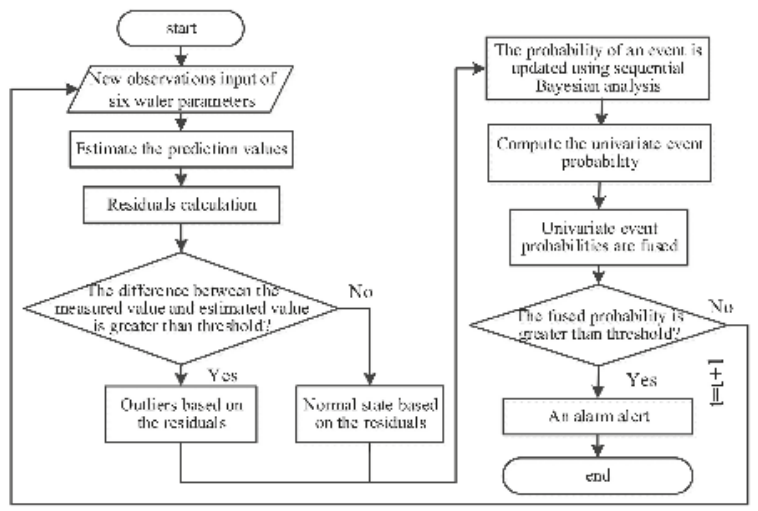

Figure 3. Procedure in the on-line phase. 


\subsection{Water Parameters Simulation Based on BP Model}

BP model is adopted to estimate the relationships between six water quality parameters through nonlinear, weighted functions during normal operation. The development of BP model does not require any knowledge of the physical and chemical laws affecting water quality parameters. Thus, BP model can be available to model the multivariate reactions in the WSN.

In the water supply networks, contamination events heavily rely on multiple water quality factors, which results in the difficulties to pre-specify the events. In the case of free chlorine, BP model is just established with the historical data from single water parameter-free chlorine to estimate the predicted value. When there is a small difference between the measured and estimated free chlorine values, it has obvious great deviations between the measured values of other water parameters and their normal states. The reason is that the measured values of free chlorine exhibit great deviations from the normal operation conditions due to the interplay among some of water quality parameters. The prediction model based on a single water quality parameter would obtain a lower accuracy of estimated values. To improve the accuracy of the prediction model, BP neural networks are adopted and trained for modeling the relationships between multiple variate water quality parameters in WSNs. One of the most important components in the BP model are multi-layer perceptrons (MLPs). MLPs are represented by an input layer, a hidden layer, and an output layer and correlate input variables to output variables depending on the nonlinear, weighted, parameterized functions. The BP model is shown in Equation (1):

$$
f_{k}(x ; w)=\varphi_{0}\left(w_{0}+\sum_{j} w_{j k} \varphi\left(w_{0 j}+\sum_{i} w_{i j} x_{i}\right)\right)
$$

where $w_{j k}, w_{i j}$ are weights, $w_{0}, w_{0 j}$ are biases, $\varphi$ and $\varphi_{0}$ are activation function and output function, respectively, $x_{i}$ is one of water quality parameters, and $f_{k}(x ; w)$ is used to estimate the target value $y$. Data is passed from the input layer with $p$ inputs $x=\left(x_{1}, \ldots x_{p}\right)$ to hidden layer having $m$ neurons. Each neuron in the hidden layer receives the summed weighted outputs of the preceding layer. The output layer with $k$ targets $y=\left(y_{1}, \ldots, y_{k}\right)$ receives the summed weighted outputs of the preceding layer again. The final output is the function $f_{k}(x ; w)$.

To avoid the interplay among multiple water quality parameters, for each target water quality parameter, the corresponding BP model is established with the input measured time series of all predictive parameters and lagged target parameter, as formulated in Equation (2).

$$
\hat{x}_{i}(t)=f\left(x_{1}(t), \ldots, x_{i-1}(t), x_{i}(t-1), x_{i+1}(t), \ldots, x_{n}(t)\right)
$$

where $x_{i}(t)$ and $\hat{x}_{i}(t)$ are the measured and estimated water parameters at time $t$ respectively, $n$ is the number of water quality parameters, and $f(\cdot)$ is a function defined by the BP model, as in Equation (1).

Water quality parameters include free chlorine, electrical conductivity (EC), $\mathrm{pH}$, temperature, total organic carbon (TOC), and turbidity. Six BP models can be created and trained. One of each water quality parameter $x_{i}(t), i=1,2, \ldots, 6$ with six water parameters, respectively, to predict the target water quality parameters and the relationships between them. A BP model consists of the size of the BP neural network, its inputs, and a training algorithm and testing the model with measured values. According to Equation (2), one model was constructed for each target water quality parameter with the input vector containing measured time series of all predictive parameters at the same time as lagged target parameters. For example, as shown in Figure 4, the BP model for estimating free chlorine takes the following inputs:

$$
\hat{x}_{\text {free_chlorine }}(t)=f\left(x_{E C}(t), x_{p H}(t), x_{\text {temperature }}(t), x_{\text {TOC }}(t), x_{\text {turbidity }}(t), x_{\text {free_chlorine }}(t-1)\right)
$$

The accuracy of BP model can be evaluated through mean error, standard deviation (STD), and correlation $\left(R^{2}\right)$ between the measured values and predicted values of water quality parameters. 


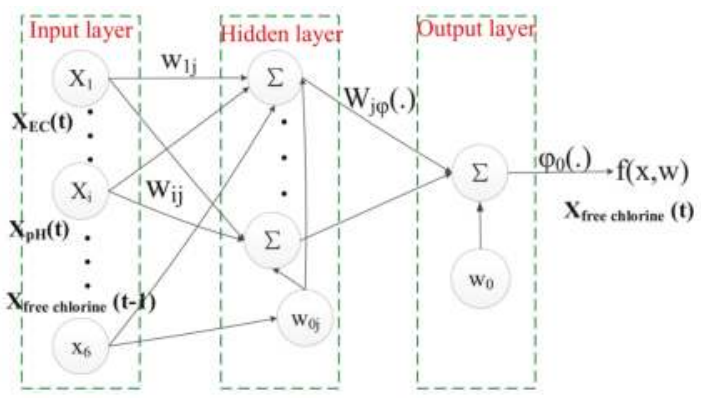

Figure 4. BP (Back Propagation) neural network structure of free chlorine.

\subsection{Residual Estimation and Classification}

The established BP models through training the historical data can be utilized to predict the values of water quality parameters. The next step is to calculate the residuals as the difference between the predicted and measured values of parameters and then to classify the residuals. Residuals can be calculated as shown in Equation (4), represented as time series.

$$
E R_{i}(t)=x_{i}(t)-f(\cdot)=x_{i}(t)-\hat{x}_{i}(t)
$$

where $x_{i}(t), \hat{x}_{i}(t)$, and $E R_{i}(t)$ represent the measured value, the estimated values, and the estimated residual for parameter $i$ at the time $t$, respectively, $f(\cdot)$ is defined by Equation (1).

To distinguish the normal operation of a water supply network from the contamination events, the estimated residuals $E R_{i}(t)$ can be classified into two types: normal and outlier. For each BP model, the estimated residuals are bounded within the upper and lower limits. If the measured values exceeding the threshold are considered to represent outliers. During the training phase, the experimental results indicate that the majority of residual within the bound $[96 \%, 99 \%]$ can be acceptable. If the residuals cannot reside within the upper and lower limits, the residual should be considered as outlier.

\subsection{Sequential Bayesian Updating}

In this stage, the probability of a contamination event is updated using sequential Bayesian analysis for each incoming value. In the sequential Bayesian analysis, the number of incoming values is unknown in advance. In fact, the measured values come in sequence and a decision is required to be made about the current state. The performance of the BP model is measured through a confusion matrix during the training [30]. The confusion matrix represents the model's classification of all measured values to one of four classes: true positive $(T P)$, the residual is classified as an outlier during an actual event; false oositive ( $F P$ ), the residual is classified as an outlier during normal operation condition; true negative (TN), the residual is classified as reasonable model error during normal operation; false negative $(F N)$, the residual is classified as a reasonable model error during a contamination event, as shown in Table 1.

Table 1. Notations of four conditions.

\begin{tabular}{ccc}
\hline Estimated Conditions & Actual Normal Operation & Actual Outlier Operation \\
\hline Normal operation & True Negative (TN) & False Negative (FN) \\
Outlier operation & False Positive (FP) & True Positive (TP) \\
\hline
\end{tabular}

Additional common metrics, two others can be derived from the confusion matrix as described in Equation (5).

$$
R D=\frac{T P}{T P+F N} \times 100 \%, F A R=\frac{F P}{T N+F P} \times 100 \%
$$


where $R D$ represents the ratio of the number of the detected contamination events to the number of the actual contamination events, and FAR is the ratio of the number of the detected false alarms to the total number of the determined contamination events.

To determine the possibility of a contamination event occurrence from previously classified states (normal or outlier), the probability of a contamination event is updated based on the classification of the residuals. The state of an event has two states: normal and outlier, as shown in Equation (6). In the sequential Bayesian analysis for each new incoming data, the number of measured values is not known in advance. The measured values come in sequence, and a decision is made based on the current state.

$$
P\left(E_{t}\right)=\left\{\begin{array}{l}
P\left(E_{t} \mid O_{t}\right) \text { if } \text { Residual }_{t} \text { is Outlier } \\
P\left(E_{t} \mid \overline{O_{t}}\right) \text { if Residual } \text { is Normal }^{\text {is Nes }}
\end{array}\right.
$$

At first, the probability of a contamination event is assumed to be rare. With each new incoming measured value, the probability of an event is updated with sequential Bayesian rule, as shown in Equation (7).

$$
\begin{gathered}
P\left(E_{t} \mid O_{t}\right)=\frac{P(O \mid E) \times P\left(E_{t-1}\right)}{P(O \mid E) \times P\left(E_{t-1}\right)+P(O \mid \bar{E}) \times P\left(\bar{E}_{t-1}\right)}=\frac{R D \times P\left(E_{t-1}\right)}{R D \times P\left(E_{t-1}\right)+F A R \times\left(1-P\left(E_{t-1}\right)\right)} \\
P\left(E_{t} \mid \bar{O}_{t}\right)=\frac{P(\bar{O} \mid E) \times P\left(E_{t-1}\right)}{P(\bar{O} \mid E) \times P\left(E_{t-1}\right)+P(\bar{O} \mid \bar{E}) \times P\left(\bar{E}_{t-1}\right)}=\frac{(1-R D) \times P\left(E_{t-1}\right)}{(1-R D) \times P\left(E_{t-1}\right)+(1-F A R) \times\left(1-P\left(E_{t-1}\right)\right)}
\end{gathered}
$$

where $P\left(E_{t}\right)$ is the probability of an event at time $t, O_{t}$ and $\bar{O}_{t}$ are "Outlier" and "Normal" states at time $t$, respectively. $P\left(E_{t} \mid O_{t}\right)$ is the conditional probability of a contamination event when the residual is classified as an outlier. $P\left(E_{t} \mid \bar{O}_{t}\right)$ is the conditional probability of a contamination event when the residual is classified as a normal state.

From Equation (7), each event probability is updated through the sequential Bayesian rule depending on the new measured value and on the RD and FAR. The posterior probability is updated for each water quality parameter independently resulting in univariate event probabilities. The probability of events designates the likelihood of a contamination event based solely on the target parameter. If the probability exceeds the pre-defined threshold value, it is considered that a contamination event has occurred.

In the application of contamination event detection, the initial probability of an event is set to $10^{-5}$, and the default threshold probability for a contaminating event is set to $P_{\text {threshold }}=0.7$. Real data are available from CANARY database. The data covers four months with five-minute intervals of operations. Table 2 shows the average results of multiple runs for ten contamination events. In the contamination events simulation, the update probability of each water quality parameter can be found in Table 2 . In the simulation, if the updated probability of single water parameter above $9.4 \times 10^{-1}$ indicates an event. Alarm (1) represents at least one water quality parameter raised an alarm. Alarm (2) shows that an alarm is raised if at least two water quality parameters or more are fused. In this case, only one event is undetected with one false alarm. As shown in Table 2, one of the six water quality parameters identified a contamination event at 8:20, and three of the six parameters raised an alarm at 9:00. Moreover, during the contamination event spreading phase, the updated probabilities of a single water parameter gradually increased and more alarms are declared from 8:20 to 16:20. When the event ends, the pollution disappears slowly and the corresponding probability decreases.

Table 2. Updated probability of contamination events in a single node.

\begin{tabular}{ccccccccc}
\hline Time & Mon & Mon & Mon & Mon & Mon & Mon & Mon & Mon \\
& $\mathbf{0 8 : 0 0}$ & $\mathbf{0 8 : 2 0}$ & $\mathbf{0 8 : 4 0}$ & $\mathbf{0 9 : 0 0}$ & $\mathbf{1 6 : 0 0}$ & $\mathbf{1 6 : 2 0}$ & $\mathbf{1 6 : 4 0}$ & $\mathbf{1 7 : 0 0}$ \\
\hline true state & FALSE & TRUE & TRUE & TRUE & TRUE & TRUE & TRUE & TRUE \\
free chlorine $[\mathrm{mg} / \mathrm{L}]$ & $4.19 \times 10^{-5}$ & $4.05 \times 10^{-1}$ & $\underline{9.50 \times 10^{-1}}$ & $\underline{9.50 \times 10^{-1}}$ & $\underline{8.69 \times 10^{-2}}$ & $4.18 \times 10^{-2}$ & $3.50 \times 10^{-1}$ & $1.52 \times 10^{-1}$ \\
EC $[\mathrm{mS} / \mathrm{cm}]$ & $1.00 \times 10^{-5}$ & $1.00 \times 10^{-5}$ & $\underline{1.00 \times 10^{-5}}$ & $\underline{1.00 \times 10^{-5}}$ & $\underline{2.59 \times 10^{-1}}$ & $\underline{8.01 \times 10^{-1}}$ & $3.02 \times 10^{-1}$ & $2.16 \times 10^{-2}$ \\
pH & $1.00 \times 10^{-5}$ & $1.00 \times 10^{-5}$ & $1.00 \times 10^{-5}$ & $1.00 \times 10^{-5}$ & $1.00 \times 10^{-5}$ & $1.00 \times 10^{-5}$ & $1.00 \times 10^{-5}$ & $1.00 \times 10^{-5}$ \\
Temperature & $1.00 \times 10^{-5}$ & $1.00 \times 10^{-5}$ & $1.00 \times 10^{-5}$ & $1.00 \times 10^{-5}$ & $1.00 \times 10^{-5}$ & $1.00 \times 10^{-5}$ & $1.00 \times 10^{-5}$ & $1.00 \times 10^{-5}$ \\
TOC [ppb] & $1.87 \times 10^{-5}$ & $\underline{9.46 \times 10^{-1}}$ & $\underline{9.50 \times 10^{-1}}$ & $\underline{9.50 \times 10^{-1}}$ & $\underline{9.50 \times 10^{-1}}$ & $\underline{9.50 \times 10^{-1}}$ & $2.01 \times 10^{-1}$ & $4.02 \times 10^{-1}$ \\
Turbidity [NTU] & $1.00 \times 10^{-5}$ & $\underline{1.00 \times 10^{-5}}$ & $\underline{5.34 \times 10^{-2}}$ & $\underline{9.50 \times 10^{-1}}$ & $\underline{\underline{9.50 \times 10^{-1}}}$ & $\underline{\underline{9.50 \times 10^{-1}}}$ & $\underline{9.50 \times 10^{-1}}$ & $4.02 \times 10^{-1}$ \\
\hline
\end{tabular}


Table 2. Cont.

\begin{tabular}{|c|c|c|c|c|c|c|c|c|}
\hline Time & $\begin{array}{l}\text { Mon } \\
\text { 08:00 }\end{array}$ & $\begin{array}{l}\text { Mon } \\
\text { 08:20 }\end{array}$ & $\begin{array}{l}\text { Mon } \\
08: 40\end{array}$ & $\begin{array}{l}\text { Mon } \\
\text { 09:00 }\end{array}$ & $\begin{array}{l}\text { Mon } \\
16: 00\end{array}$ & $\begin{array}{l}\text { Mon } \\
16: 20\end{array}$ & $\begin{array}{l}\text { Mon } \\
16: 40\end{array}$ & $\begin{array}{l}\text { Mon } \\
\text { 17:00 }\end{array}$ \\
\hline Alarm (1) & FALSE & FALSE & TRUE & TRUE & TRUE & FALSE & FALSE & FALSE \\
\hline Alarm (2) & FALSE & FALSE & FALSE & FALSE & FALSE & TRUE & FALSE & FALSE \\
\hline Alarm (4) & FALSE & FALSE & FALSE & FALSE & FALSE & FALSE & FALSE & FALSE \\
\hline Alarm (5) & FALSE & TRUE & TRUE & TRUE & TRUE & TRUE & FALSE & FALSE \\
\hline Alarm (6) & FALSE & FALSE & FALSE & TRUE & TRUE & TRUE & TRUE & FALSE \\
\hline
\end{tabular}

Notes: the underline italic numbers denote that the values are greater than the threshold. The bold texts mean that the results of alarms are true.

\subsection{Multivariate Fuse Decision}

To improve the model's estimation of a temporal event and to lower the number of false alarms, a process of multivariate fusion decision is invoked. This process ensures that only when a predetermined number of parameters indicates an event, an alarm is raised. This process reduces the number of false alarms and improves the alarms' reliability. The univariate event probabilities are fused to compute the multivariate event probability reflecting the likelihood of a contamination event based on all measured water parameters.

Figure 5 illustrates the univariate event probabilities for each water quality parameter during the simulated ten contamination events. In the simulations, the threshold probability for a contamination event is set to $P_{\text {threshold }}=0.7$. If the updated probability of one water parameter is above 0.7, the corresponding event for single parameter occurs. As shown in Figure 5, free chlorine can detect eight out of ten events with three false alarms. EC can detect six out of ten events with one false alarm. $\mathrm{pH}$ can detect five out of ten events with one false alarm. Temperature detects five out of ten events without false alarm. TOC detects nine out of two events with three false alarms. Turbidity can detect eight out of ten events with two false alarms.

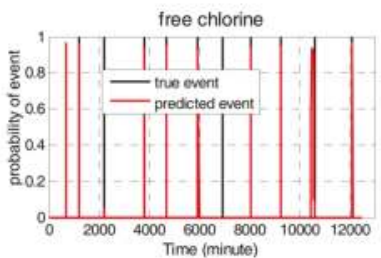

(a)

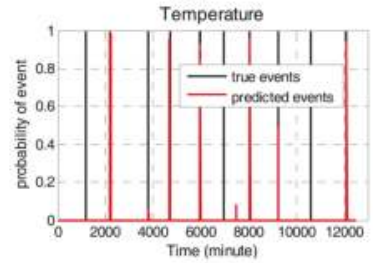

(d)

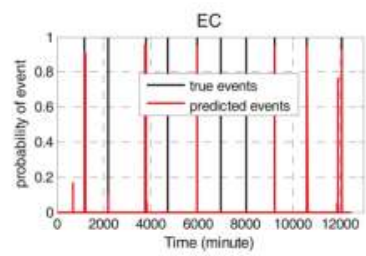

(b)

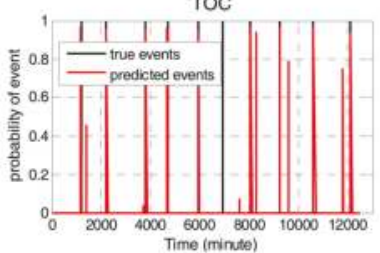

(e)

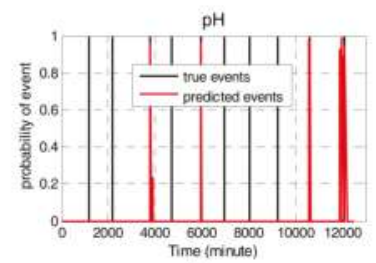

(c)

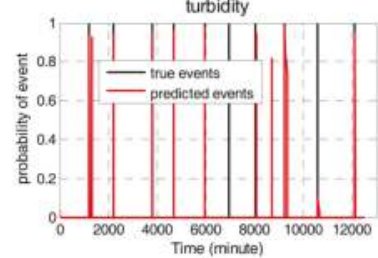

(f)

Figure 5. Event probability of single water quality parameter. (a) free Chlorine; (b) EC; (c) $\mathrm{pH}$; (d) Temperature; (e) TOC; (f) turbidity.

Since water quality parameters differ in their predictive ability of contamination events, weights reflecting their influence on the synchronized decision should be allocated. In this paper, uniform weights were given to reflect no prior information. Figure 6 depicts the multivariate fused alarms with six water parameters. In Figure 6, warning (1) represents that a contamination event is detected when the probability from one water parameter is above the threshold. In this case, almost contamination 
events can be detected, however, it also results in high false alarms. As shown in Figure 6, nine out of ten events can be detected with four false alarms. Warning (2) represents that a contamination event is detected when the probabilities from two or more parameters are greater than the threshold. In this case, only one true event cannot be detected with two false alarms. When the probabilities from three parameters are fused to indicate an event, there are no false alarms. This reflects the better tradeoff between the detection accuracy and the false alarm rate. Therefore, when the updated probabilities of three or more water parameters through the sequential Bayesian analysis are greater than the threshold 0.7, the M-STED event detection method can determine a temporal outlier occurrence after multiple fusion decisions in the water supply network.

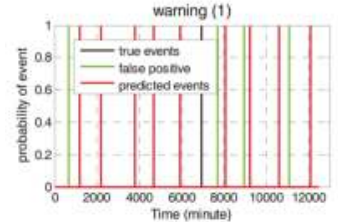

(a)

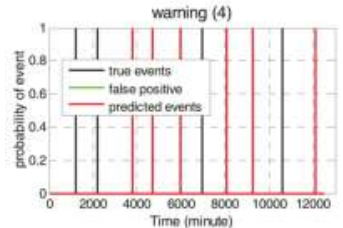

(d)

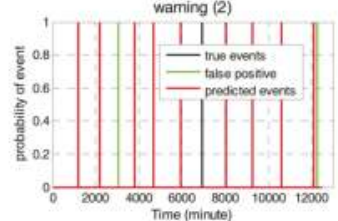

(b)

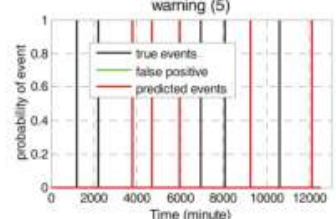

(e)

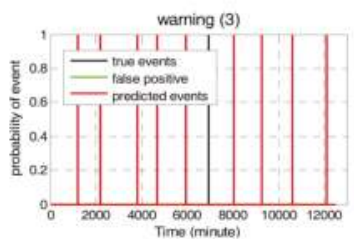

(c)

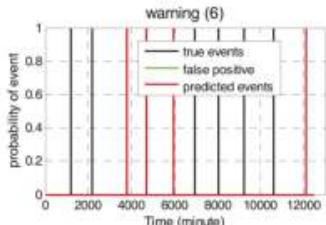

(f)

Figure 6. Event probability of multiple water quality parameters.

\section{Spatial Event Detection in the CDS}

In Section 5, single water quality sensor node in the water supply network can determine whether it is "outlier" or "normal" with multivariate time series of six water quality parameters. Unfortunately, it cannot obtain an accurate judgement for contamination event detection without considering the spatial correlations among the sensor nodes. After the determination the state of a single node, it should establish a spatial correlation model to further detect the contamination events based on the spatial relationship among the "outlier" sensor nodes. A BN is used to model causal and spatial relationship between the sensor stream and its neighbor sensor streams. As illustrated in Figure 7, a causal relationship exists between the upstream nodes and the downstream nodes, so the structure of $\mathrm{BN}$ is the same as the topological structure of water supply network.

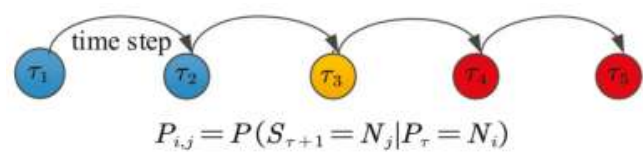

Figure 7. State transitions of the fused values of six water quality parameters at the moment $\tau_{1}$

Assume that there are three sensor nodes $S_{1}, S_{2}$ and $S_{3}$ to measure the multivariate water quality, with values varying from 0.0 to 1.0. There is a total of two states. Based on the experts' experiences, if the values are in $[0.0,0.5)$, the state of sensor node is denoted as state 1 . While the values are in $[0.5,1.0]$, the state is denoted as state 2 . During the training phase, it can compute the parameters of $\mathrm{BN}$ by adopting the maximum likelihood parameter estimation algorithm. 
After the training phase, the conditional probability table of each sensor can be learned. The temporal event probabilities of sensor $S_{1}$ are shown in Table 3. The conditional probabilities of sensor $S_{2}$ and $S_{3}$ are shown in Table 4. From Table 3, the probability of two states of sensor $S_{1}$ are 0.2 and 0.8 , respectively. In Table 4 , if the states of sensor $S_{1}$ is state 1 , the state of sensor $S_{2}$ is state ${ }_{1}$, then the conditional probability is 0.8. That is to say, $P\left(S_{2}=\right.$ state $_{1} \mid S_{1}=$ state $\left._{1}\right)=0.8$. During the reasoning phase, if the probability of sensor $S_{1}$ at a certain interval time is 0.24 , the state of sensor $S_{2}$ will more likely at state $e_{2}$ and the state of sensor $S_{3}$ will at state 1 , according to the given conditional probability table. If the real value of sensor nodes $S_{2}$ and $S_{3}$ is not within that state, it can be considered that the sensed value deviates from the spatial relationship. It can be considered that a contamination event occurred.

Table 3. The temporal event probability table of sensor $S_{1}$

\begin{tabular}{cc}
\hline State & $\boldsymbol{P}\left(\boldsymbol{S}_{\mathbf{1}}\right)$ \\
\hline state $_{1}$ & 0.2 \\
state $_{2}$ & 0.8 \\
\hline
\end{tabular}

Table 4. The conditional probability table of sensor $S_{2}$ and $S_{3}$.

\begin{tabular}{ccc|ccc}
\hline $\boldsymbol{S}_{1}$ & $\boldsymbol{S}_{\mathbf{2}}$ & $\boldsymbol{P}\left(\boldsymbol{S}_{\mathbf{2}}\right)$ & $\boldsymbol{S}_{\mathbf{1}}$ & $\boldsymbol{S}_{\mathbf{3}}$ & $\boldsymbol{P}\left(\boldsymbol{S}_{\mathbf{3}}\right)$ \\
\hline state $_{1}$ & state $_{1}$ & 0.8 & state $_{1}$ & state $_{1}$ & 0.8 \\
state $_{1}$ & state $_{2}$ & 0.4 & state $_{1}$ & state $_{2}$ & 0.3 \\
state $_{2}$ & state $_{1}$ & 0.3 & state $_{2}$ & state $_{1}$ & 0.2 \\
state $_{2}$ & state $_{2}$ & 0.5 & state $_{2}$ & state $_{2}$ & 0.6 \\
\hline
\end{tabular}

The contamination event detection approach, M-STED, based on the spatio-temporal correlation model is as follows:

(1) The nodes in the CDS collect the sensed data. If temporal abnormal events occur, the nodes individually go through the BP model and sequential Bayesian updating with multivaries time series of six water quality parameters for a period of time $\tau$. If the temporal event probability of one backbone node is greater than the threshold, it can consider that backbone node as in the "outlier" state.

(2) If the temporal event occurs in one of the backbone nodes, a candidate set is constructed with each backbone node in the "outlier" state, and its corresponding children nodes. If there is no temporal event in these backbone nodes, these nodes just wait for sensing and will be checked in the next time period.

(3) The reasoning ability of BN can be used to estimate the expected state of the backbone nodes $S_{i}$ in the "Outlier" state. Each backbone node compares the expected state with the observed value $S_{i}^{t}$ to determine whether the value of observed data deviates from the spatial relationship.

(4) If the observed value $S_{i}^{t}$ is greatly different from the expected value, it can be considered as a spatial deviation. The number of nodes in the "outlier" state increases by one.

(5) In the following timestamp, the scope of monitoring is expanded, including the backbone nodes and their candidate nodes. They continue to detect whether there is a temporal and spatial deviation.

(6) If the number of nodes in the "outlier" state in the CDS is equal to or greater than the threshold $\theta$, we consider that a contamination event occurred and the warning should be broadcast.

\section{Performance Evaluation}

\subsection{Experimental Setup}

The methodology is tested on real data from CANARY [27]. The data spans over four months with five-minute intervals. The data consists of six online multivariate water quality parameters: 
free chlorine $(\mathrm{mg} / \mathrm{L}), \mathrm{EC}(\mathrm{mS} / \mathrm{cm}), \mathrm{pH}$, temperature $(\mathrm{C}), \mathrm{TOC}(\mathrm{ppb})$, and turbidity $(\mathrm{NTU})$. In the experiments, the data set is divided into two subsets, $67 \%$ for training and $33 \%$ for testing. The training subset is used to establish the BP neural network model with data-driven model, and the test subset is used to evaluate the accuracy of BP neural model.

Event Simulation: Contamination events in the water supply networks heavily depend on environmental factors, which makes it harder to detect contamination events. To cope with the above difficulty, contamination events are superimposed on normal patterns, characterized by the magnitude, direction, and duration [31], which can reflect water parameter variation caused by contamination events. Contamination events were assumed to be Gaussian in distribution [32]. Figure 8 shows a partial time series plot of six water quality parameters during normal operation and randomly simulated events with real data from CANARY [27].

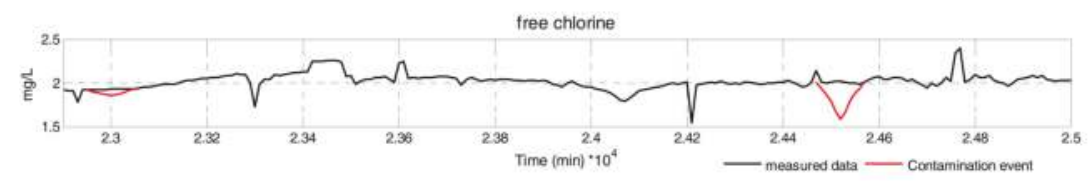

(a)

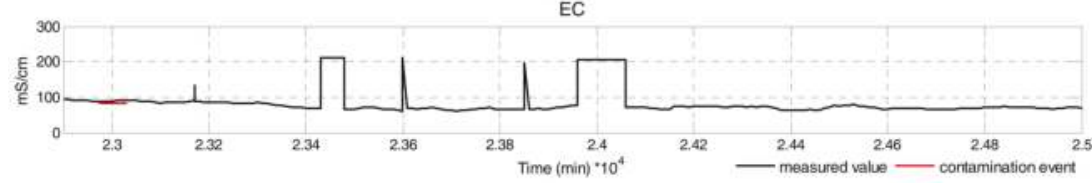

(b)

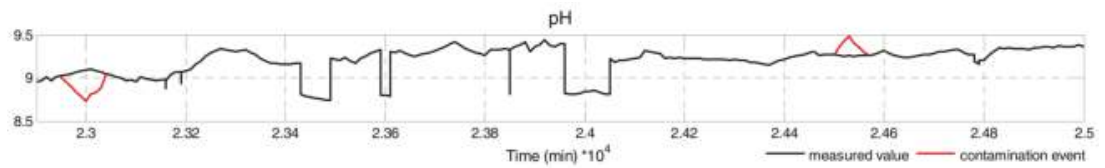

(c)

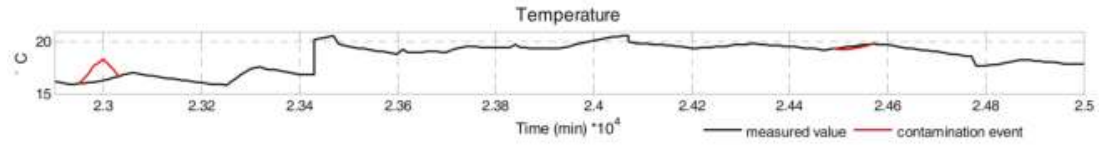

(d)

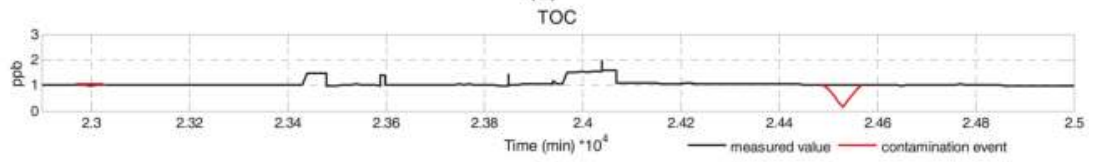

(e)

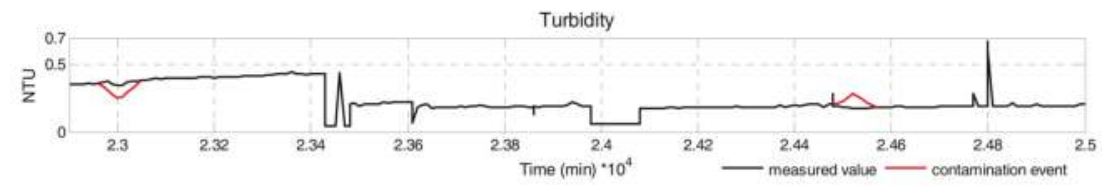

(f)

Figure 8. Time series of multivariate water quality parameters. (a) Free Chlorine; (b) EC; (c) pH; (d) Temperature; (e) TOC; (f) turbidity. 
The experiments include three parts: (1) BP model assessment. The historical training subset of each water parameter was exploited to establish, train, and evaluate the BP model through mean square error $(M S E)$, Correlation coefficient $\left(R^{2}\right)$. The testing subset is used to simulate real-time contamination events and to test performance of the proposed M-STED approach; (2) Due to considering multivariate water quality parameters, several metrics, such as receiver operating characteristic $(R O C)$ curve, rate of detection $(R D)$, and false alarm rate $(F A R)$, are used to evaluate the performance of the M-STED algorithm in terms of the accuracy, compared with the S-STED algorithm depending on a single water parameter; (3) Efficiency. The efficiency performance can be evaluated in terms of delay and scalability, compared with the simple threshold algorithm [10], Bayesian network-based algorithm [16]. The threshold-based approach is the basic one for the event detection in water supply networks. It has the lowest communication complexity. The BN-only approach only considers the spatial relationship among the sensor nodes without temporal relationship. The average delay is the average time from event occurrence to the event being detected. The shorter the average delay, the higher the efficiency.

\subsection{Experimental Results}

\subsubsection{BP Model Assessment}

In the experiments, the data set is divided into two subsets, $67 \%$ for training and $33 \%$ for testing. MSE and $R^{2}$ are adopted to calculate the residuals for each water parameter. Table 5 lists the training results and test results for six water quality parameters with BP models. As shown in Table 5, the computed MSEs of each water parameter are all below 0.035 , which indicates that the training subset does not have much noise data and can reflect the actual water quality conditions. Moreover, the difference between the measured and estimated value for each parameter is quite small in the test phase. The corresponding $R^{2}$ values of each parameter are acceptable. Therefore, it can be seen that the BP neural network model can be used to identify water quality state "normal" or "outlier".

Table 5. Training results and test results with BP model.

\begin{tabular}{|c|c|c|c|c|c|c|c|c|}
\hline Phase & & Parameters & $\begin{array}{c}\text { Free Chlorine } \\
{[\mathrm{mg} / \mathrm{L}]}\end{array}$ & $\begin{array}{c}\mathrm{EC} \\
\mathrm{mS} / \mathrm{cm}\end{array}$ & $\mathrm{pH}$ & $\begin{array}{c}\text { Temperature } \\
{\left[{ }^{\circ} \mathrm{C}\right]}\end{array}$ & $\begin{array}{l}\text { TOC } \\
{[p p b]}\end{array}$ & $\begin{array}{c}\text { Turbidity } \\
\text { [NTU] }\end{array}$ \\
\hline \multirow{6}{*}{ Training phase } & \multirow{2}{*}{ mean $_{1}$} & measured value & 1.945 & 77.670 & 9.039 & 17.253 & 0.966 & 0.220 \\
\hline & & estimated value & 1.947 & 78.320 & 9.040 & 17.253 & 0.960 & 0.223 \\
\hline & \multirow{2}{*}{ mean $_{2}$} & measured value & 1.945 & 77.670 & 9.039 & 17.253 & 0.966 & 0.220 \\
\hline & & estimated value & 1.947 & 78.32 & 9.04 & 17.253 & 0.960 & 0.223 \\
\hline & & $R^{2}$ & 0.923 & 0.986 & 0.999 & 0.999 & 0.685 & 0.639 \\
\hline & & $M S E$ & 0.007 & 0.0347 & 0.000 & 0.005 & 0.076 & 0.008 \\
\hline \multirow{6}{*}{ Test phase } & \multirow[b]{2}{*}{ mean $_{1}$} & measured value & 2.002 & 87.207 & 9.159 & 18.078 & 1.031 & 0.198 \\
\hline & & estimated value & 2.010 & 88.359 & 9.151 & 18.086 & 1.021 & 0.225 \\
\hline & \multirow{2}{*}{ mean $_{2}$} & measured value & 0.145 & 55.804 & 0.240 & 1.302 & 1.214 & 1.351 \\
\hline & & estimated value & 0.075 & 52.244 & 0.215 & 1.189 & 1.452 & 1.439 \\
\hline & & $R^{2}$ & 0.625 & 0.914 & 0.690 & 0.779 & 0.654 & 0.654 \\
\hline & & MSE & 0.016 & 0.502 & 0.018 & 0.377 & 0.732 & 1.379 \\
\hline
\end{tabular}

\subsubsection{Accuracy Analysis}

To improve the accuracy of event detection, M-STED adopts BP models to establish the prediction model for each water quality parameter. Different water quality parameters have different predictive abilities for contamination events; it should allocate the appropriate weights to make a unified decision. Contamination event detection based on the multivariate parameters can greatly reduce the false alarms and improve the detection rate, compared with the detection based on the univariate parameter. As shown in Figures 9 and 10, when the number of simulated contamination events vary from 20 to 100 , the contamination event detection rate falls to a range between 45-65\% with the S-STED method, while the detection rate is greater than $75 \%$ with the M-STED method. The average detection rate is more than $80 \%$ with M-STED. The detection rate of M-STED is $40 \%$ higher than that of S-STED. 
Meanwhile, the false alarm rates of M-STED and S-STED are lower than $9 \%$ and greater than $14 \%$, respectively. Obviously, M-STED can effectively reduce the false alarm rates.

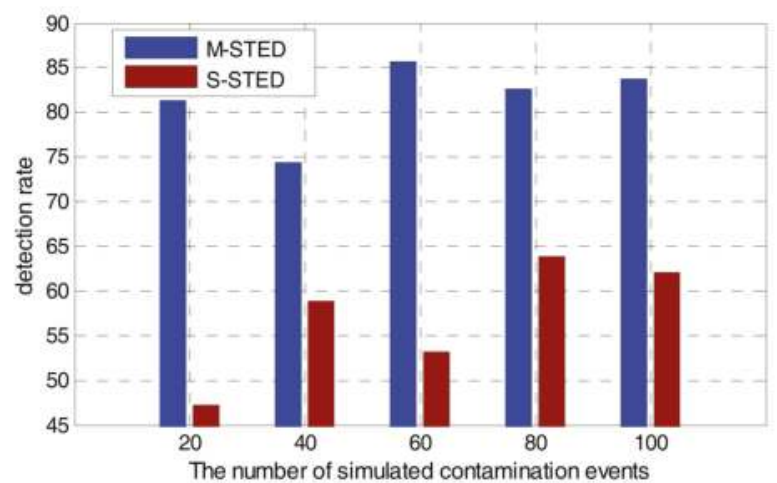

Figure 9. Comparison of detection rates between M-STED and S-STED.

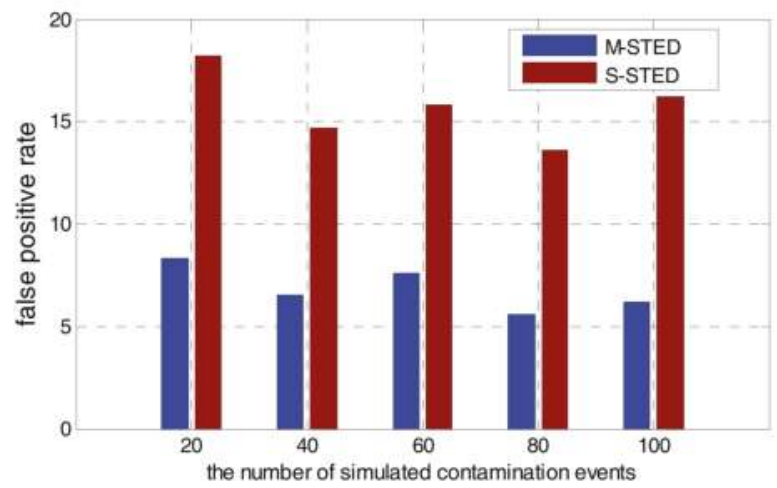

Figure 10. Comparison of false alarm rates between M-STED and S-STED.

In statistics, a $R O C$ (receiver operating curve) is created by plotting the true positive rate against the false positive rate at various threshold settings. In our experiments, the true positive rate is denoted as $R D$, which represents the ratio of the number of the detected contamination events to the number of the actual contamination events. The false positive rate is denoted as FAR, which is the ratio of the number of the detected false alarms to the total number of the determined contamination events. In general, if the probability distributions for both detection and false alarm are known, the ROC curve can be generated by plotting the cumulative distribution function (area under the probability distribution) of detection rate in the $y$-axis versus the cumulative distribution function of the false alarm rate in the $x$-axis. Therefore, $R D$ and FAR can be used to construct $R O C$, which visually depict the same information as the confusion matrix demonstrating the fundamental performance trade-off between $R D$ and FAR in a much more intuitive way. Figure 11 illustrates the ROC of S-STED and M-STED, respectively. As shown in Figure 11, area under ROC of M-STED is significantly greater than that of S-STED. 


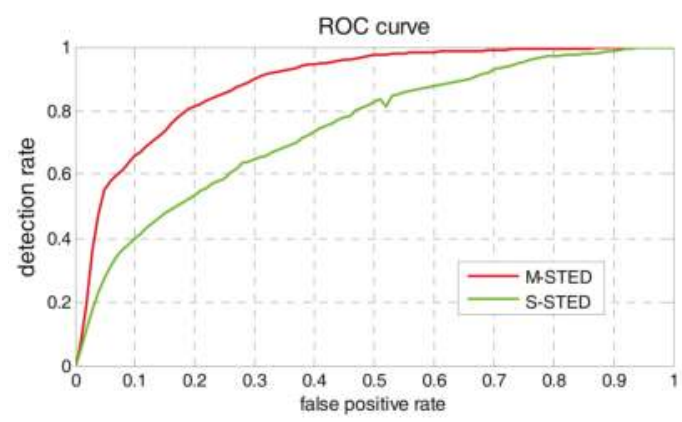

Figure 11. Comparison of ROC (receiver operating curve) between M-STED and S-STED.

Based on the experimental results analysis, M-STED has better performance in terms of the detection rate and false alarm rate than S-STED, which relies on a single water parameter.

\subsubsection{Detection Delay}

To evaluate the detection delay of different methods, different numbers of sensors are selected in the same area to execute the proposed M-STED, S-STED, BN-only, and simple threshold approaches. As shown in Figure 12, with the increase of the number of sensor nodes, the average delay of the four approaches significantly increase. The average delay time of the proposed M-STED is smaller than that of S-STED, BN-only, and the simple threshold one. The results show that the communication overhead increases with the increase of the number of nodes. However, the proposed M-STED approach exhibits a slower increase than the other three approaches. The reason is that Rule $\mathbf{K}$ algorithm is adopted to select part of the nodes as backbone nodes for forwarding the sensed data, which can greatly reduce the communication overhead. Therefore, the proposed M-STED can achieve shorter average delay for contamination event detection. While BN-only and simple threshold approaches need to collect all of the sensed data, resulting in a large communication overhead. The response time of event detection is longer than that of the proposed M-STED approach.

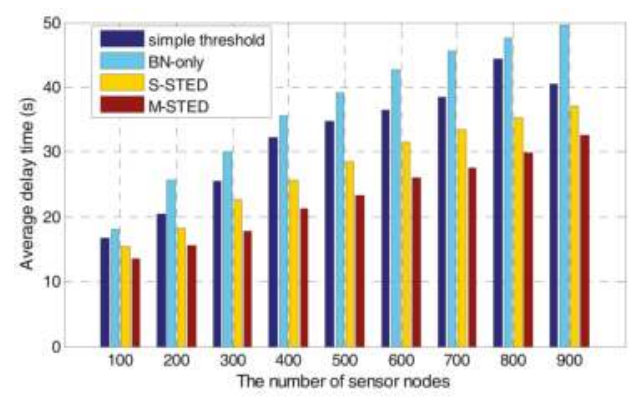

Figure 12. The average delay time in the different node densities.

\subsubsection{Scalability}

The communication complexity of the proposed M-STED approach depends on the local transition of the sensed values. If the nodes are not in the CDS, detecting temporal events requires no communication overhead, because the nodes run the algorithm individually. Whenever a temporal event is detected by the backbone nodes, they broadcast the corresponding messages and collect the sensed data. If the nodes in the CDS are in the "outlier" state, the communication overhead and the 
message transmission increase when the number of sensor nodes increases. From Figure 13, it can be seen that with the increase of the number of sensor nodes, the number of message transmission also increases for all of the event detection methods. It is obvious that the increment speeds of the S-STED and M-STED with Rule K algorithm are slower than those of BN-only and simple threshold approaches. The S-STED and M-STED approaches can achieve better performance on data transmission. The reason is that Rule $\mathbf{K}$ algorithm can optimally reduce the number of sensor nodes, resulting in a low communication overhead. On the contrary, without an optimal node selection procedure, BN-only and simple threshold approaches exhibit a linear increase of communication overhead when the number of sensor nodes increases.

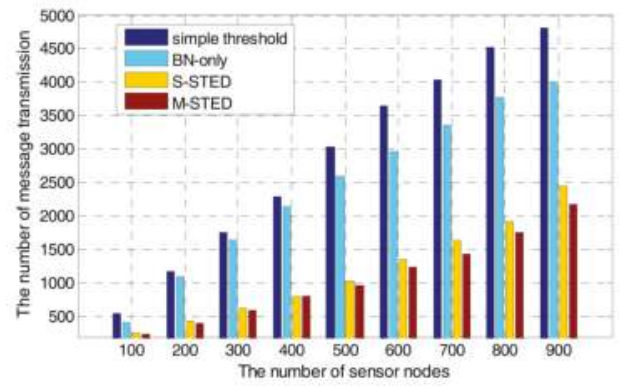

Figure 13. Scalability with an increased number of nodes.

Furthermore, the scale of water supply networks also causes side effect on the efficiency of performance. As the number of sensor nodes increases, the average delay time of the proposed M-STED approach also grows. However, the increase speed is slower than that of BN-only and simple threshold approaches. The weak linear correlation of the number of sensor nodes and communication overhead shows good performance on scalability for the proposed M-STED approach.

\section{Conclusions}

To improve the accuracy of contamination event detection in the agricultural water supply networks, a spatial-temporal-based event detection approach with multivariate time-series data for water quality monitoring (M-STED) was proposed. M-STED adopts Rule K algorithm to select backbone nodes, which collect water quality parameters to reduce the transmitted data. Then, the BP neural networks models and sequential Bayesian analysis are adopted to detect the "outlier" nodes. Finally, a spatial model is established with BN to estimate and trace the state of "outlier" nodes to determine a contamination event. The experimental results indicate that the average detection rate is more than $80 \%$ with M-STED and the false detection rate is lower than $9 \%$, respectively. The M-STED algorithm can improve the rate of detection by about $40 \%$ and reduce the false alarm rate by about $45 \%$, compared with the S-STED algorithm. Moreover, the proposed M-STED can exhibit better performance in terms of detection delay and scalability.

Acknowledgments: This study was supported by the National Key Technology Research and Development Program of the Ministry of Science and Technology of China under Grant No. 2016YFC0400910; Technology Research Program of Huneng Group under Grant No. HNKJ17-H21; the Fundamental Research Funds for the Central Universities under Grant No. 2017B20914, 2017B16814, 2015B22214. The authors are grateful to the reviewers for their comments which greatly improved the quality of the paper.

Author Contributions: All four authors have contributed to the work presented in this paper. Yingchi Mao and Hai Qi formed the initial idea. Yingchi Mao, Hai Qi, and Ping Ping developed the overall theoretical structure of the research. Yingchi Mao and Hai Qi conceived and designed the experiments. Hai Qi and Xiaofang Li performed the experiments. Yingchi Mao, Hai Qi, and Ping Ping analyzed the experimental results. Yingchi Mao and Xiaofang Li provided the experiment dataset. All authors worked collaboratively on writing the main text.

Conflicts of Interest: The authors declare no conflict of interest. 


\section{References}

1. Heidemann, J.; Stojanovic, M.; Zorzi, M. Underwater sensor networks: Applications, advances and challenges. Philos. Trans. Roy. Soc. A Math. Phys. Eng. Sci. 2012, 370, 158-175. [CrossRef] [PubMed]

2. Jayaraman, P.P.; Yavari, A.; Georagakopoulos, D.; Morshed, A.; Zaslavsky, A. Internet of things platform for smart farming: Experiences and lessons learnt. Sensors 2016, 16, 1884. [CrossRef] [PubMed]

3. Zhang, J.; Hu, J.; Huang, L.; Zhang, Z.; Ma, Y. A portable farmland information collection system with multiple sensors. Sensors 2016, 16, 1762. [CrossRef] [PubMed]

4. Eliades, D.G.; Lambrou, T.P.; Panayiotou, C.G.; Polycarpou, M.M. Contamination event detection in water distribution systems using a model-based approach. Procedia Eng. 2014, 89, 1089-1096. [CrossRef]

5. Yang, J.Y.; Haught, C.R.; Goodrich, A.J. Real-time contaminant detection and classification in a drinking water pipe using conventional water quality sensors: Techniques and experimental results. J. Environ. Manag. 2009, 90, 2494-2506. [CrossRef] [PubMed]

6. Huang, T.; Ma, X.; Ji, X. Online detecting spreading events with the spatio-temporal relationship in water distribution networks. In Advanced Data Mining and Applications, Proceedings of the International Conference on Advanced Data Mining and Applications, Hangzhou, China, 4-16 December 2013; Springer: New York, NY, USA, 2013; pp. 145-156.

7. Storey, M.V.; Gaag, B.V.D.; Burns, B.P. Advances in on-line drinking water quality monitoring and early warning systems. Water Res. 2011, 45, 741-747. [CrossRef] [PubMed]

8. Chandra, A.; Tarasia, N.; Kumari, A.; Swain, A.R. A distributed connected dominating set using adjustable sensing range. In Proceedings of the 2014 International Conference on Advanced Communication Control and Computing Technologies, Ramanathapuram, India, 8-10 May 2014; IEEE Press: New York, NY, USA, 2014; pp. 868-871.

9. Mao, Y.C.; Xu, Z.; Liang, Y. An energy efficient connected coverage protocol in wireless sensor networks. In Proceedings of the Joint Asia-Pacific Web and International Conference on Web-Age Information Management Conference on Advances in Data and Web Management, Huang Shan, China, 16-18 June 2007.

10. Yim, S.J.; Choi, Y.H. Fault-tolerant event detection using two thresholds in wireless sensor networks. In Proceedings of the 15th IEEE Pacific Rim International Symposium on Dependable Computing, Shanghai, China, 16-18 November 2009; IEEE: Picataway, NJ, USA, 2009; pp. 331-335.

11. Xue, W.; Luo, Q.; Wu, H. Pattern-based event detection in sensor networks. Distrib. Parallel Datab. 2012, 30, 27-62. [CrossRef]

12. Byrt, D.; Carlson, K.H. Expanded summary: Real-time detection of intentional chemical contamination in the distribution system. J. Am. Water Works Assoc. 2005, 97, 130-133.

13. Wang, X.R.; Lizier, J.T.; Obst, O. Spatiotemporal anomaly detection in gas monitoring sensor networks. In Wireless Sensor Networks, Proceedings of the 5th European Conference, Bologna, Italy, 30 January-1 February 2008; pp. 90-105.

14. Uusital, L. Advantages and challenges of Bayesian networks in environmental modelling. Ecol. Model. 2014, 203, 312-318. [CrossRef]

15. Piao, D.; Menon, P.G.; Mengshoel, O.J. Computing probabilistic optical flow using Markova random fields. In Computational Modeling of Objects Presented in Images; Zhang, Y.J., Tavares, J.M.R.S., Eds.; Springer: New York, NY, USA, 2014; Volume 8641, pp. 241-247.

16. Hou, D.; Chen, Y.; Zhao, H. Based on RBF neural network and wavelet analysis the water quality of anomaly detection method. Transducer Microsyst. Technol. 2013, 32, 138-141.

17. Peremlan, L.; Ostfeld, A. Bayesian networks for source intrusion detection. J. Water Resour. Plan. Manag. 2012, 139, 426-432.

18. Hou, D.; He, H.; Huang, P.; Zhang, G.; Loaiciga, H. Detection of water-quality contamination events based on multi-sensor fusion using an extended Dempster-Shafer method. Meas. Sci. Technol. 2013, $24,055801$. [CrossRef]

19. Kuhnert, C.; Bernard, T.; Arango, I.; Nitsche, R. Water quality supervision of distribution networks based on machine learning algorithm and operator feedback. Procedia Eng. 2014, 89, 189-196. [CrossRef]

20. Mao, Y.; Chen, X.; Xu, Z. Real-Time Event Detection with Water Sensor Networks Using a Spatio-Temporal Model. In Proceedings of the 21st International Conference on Database Systems for Advanced Applications, Dallas, TX, USA, 16-19 April 2016. 
21. Hou, D.; Liu, S.; Zhang, J.; Chen, F.; Huang, P.; Zhang, G. Online monitoring of water-quality anomaly in water distribution systems based on probabilistic principal component analysis by UV-Vis absorption spectroscop. J. Spectrosc. 2014, 150636, 1-9. [CrossRef]

22. Kroll, D.; King, K. Laboratory and flow loop validation and testing of the operational effectiveness of an on-line security platform for the water distribution system. In Proceedings of the Water Distribution Systems Analysis Symposium, Cincinnati, OH, USA, 27-30 August 2006; pp. 1-16.

23. Perelman, L.; Arad, J.; Housh, M.; Ostfeld, A. Event detection in water distribution systems from multivariate water quality time series. Environ. Sci. Technol. 2012, 46, 8212-8219. [CrossRef] [PubMed]

24. Arad, J.; Housh, M.; Perelman, L.; Ostfeld, A. A dynamic thresholds scheme for contaminant event detection in water distribution systems. Water Res. 2013, 47, 1899-1908. [CrossRef] [PubMed]

25. Hart, D.; McKenna, S. CANARY User's Manual, 4.1 ed.; National Security Applications Dept., Sandia National Laboratories: Albuquerque, NM, USA, 2009.

26. Murray, R.; Haxton, T.; Janke, R.; Hart, W.E.; Berry, J.; Phillips, C. Water Quality Event Detection Systems for Drinking Water Contamination Warning Systems-Development, Testing, and Application of CANARY; Technical Report EPA/600/R-10/036; U.S. EPA: Washington, DC, USA, 2010.

27. Murray, S.; Ghazali, M.; McBean, E.A. Real-time water quality monitoring: Assessment of multisensor data using Bayesian belief networks. J. Water Resour. Plan. Manag. 2011, 138, 63-70. [CrossRef]

28. Liu, S.; Che, H.; Smith, K.; Chen, L. Contamination event detection using multiple types of conventional water quality sensors in source water. Environ. Sci. Process. Impacts 2014, 16, 2028-2038. [CrossRef] [PubMed]

29. Che, H.; Liu, S.; Smith, K. Performance evaluation for a contamination detection method using multiple water quality sensors in an early warning system. Water 2015, 7, 1422-1436. [CrossRef]

30. Kong, Y.H.; Jing, M.L. Classification method based on confusion matrix and the integrated learning research. Comput. Eng. Sci. 2012, 34, 111-117.

31. Mckenna, S.A.; Klise, K.A. Multivariate Applications for Detecting Anomalous Water Quality; American Society of Civil Engineers: Reston, VA, USA, 2010; p. 247.

32. Mckenna, S.A.; Wilson, M.; Klise, K.A. Detecting Changes in Water Quality Data. J. Am. Water Works Assoc. 2008, 77, 74-85.

(C) 2017 by the authors. Licensee MDPI, Basel, Switzerland. This article is an open access article distributed under the terms and conditions of the Creative Commons Attribution (CC BY) license (http:/ / creativecommons.org/licenses/by/4.0/). 
Article

\title{
In Vivo Non-Destructive Monitoring of Capsicum Annuum Seed Growth with Diverse $\mathrm{NaCl}$ Concentrations Using Optical Detection Technique
}

\author{
Naresh Kumar Ravichandran ${ }^{1}$, Ruchire Eranga Wijesinghe ${ }^{1}$, Seung-Yeol Lee ${ }^{2}$, \\ Muhammad Faizan Shirazi ${ }^{1}$, Hee-Young Jung ${ }^{2, *}$, Mansik Jeon ${ }^{1, *}$ and Jeehyun Kim ${ }^{1}$ \\ 1 School of Electronics Engineering, College of IT Engineering, Kyungpook National University, 80 Daehak-ro, \\ Buk-gu, Daegu 41566, Korea; nareshr.9169@gmail.com (N.K.R.); eranga@knu.ac.kr (R.E.W.); \\ faizan_shirazi@knu.ac.kr (M.F.S.); jeehk@knu.ac.kr (J.K.) \\ 2 School of Applied Biosciences, Kyungpook National University, 80 Daehak-ro, Buk-gu, Daegu 41566, Korea; \\ leesy1985@gmail.com \\ * Correspondence: heeyoung@knu.ac.kr (H.-Y.J.); msjeon@knu.ac.kr (M.J.); Tel.: +82-53-950-7221 (M.J.)
}

Received: 31 October 2017; Accepted: 11 December 2017; Published: 12 December 2017

\begin{abstract}
We demonstrate that optical coherence tomography (OCT) is a plausible optical tool for in vivo detection of plant seeds and its morphological changes during growth. To investigate the direct impact of salt stress on seed germination, the experiment was conducted using Capsicum annuиm seeds that were treated with different molar concentrations of $\mathrm{NaCl}$. To determine the optimal concentration for the seed growth, the seeds were monitored for nine consecutive days. In vivo two-dimensional OCT images of the treated seeds were obtained and compared with the images of seeds that were grown using sterile distilled water. The obtained results confirm the feasibility of using OCT for the proposed application. Normalized depth profile analysis was utilized to support the conclusions.
\end{abstract}

Keywords: SS-OCT; Capsicum annuum; germination; salt concentration

\section{Introduction}

Germination of seeds is a growth process during which a plant is contained within its seed. Studies on seed germination and their respective morphological changes plays a vital role in attaining quicker harvesting [1-5]. Germination can be affected by a variety of internal factors as well as by external factors, such as the soil temperature [6], water oxygen content availability, light and dark conditions, soil salinity [7], soil pH [8], and soil chemical composition [9]. Most of these external factors vary with the seed type. Soil salinity plays a major role in germination $[7,10,11]$. Only certain (appropriate) soil salinity will promote germination. An excess amount of salinity will result in the soil degradation or in the inhibition of the germination process in seeds [12]. Salt content in water varies greatly from region to region, and from rain water to sea water. For instance, rain water usually has $\mathrm{NaCl}$ concentrations of $0.003 \mathrm{~g} \mathrm{NaCl} / \mathrm{L}$ and sea water concentrations of up to $0.030 \mathrm{~g} \mathrm{NaCl} / \mathrm{L}$. Thus, choosing the right soil salinity is a major concern for farmers to maximize crop yield $[13,14]$. Many previous research studies have determined appropriate soil salinity conditions that promote germination, for a variety of plant species [7]. Earlier studies have shown the effect of seed priming by different salt concentrations, their advantages and disadvantages depending on the concentrations $[4,11]$. However, there have been no studies on in vivo monitoring of seed growth or inhibition of germination for a range of soil salt concentrations. Analysis and/or estimation of the germination growth stage, aiming to determine the effect of the level of inhibition on seed germination, has been accomplished using various methods, such as sectioning of seeds and microscope-assisted inspection $[15,16]$, 
histological methods [17], scanning electron microscopy (SEM) imaging [18], magnetic resonance imaging (MRI) [19,20], and X-radiography [21]. All of these methods are either destructive methods that preclude continuous monitoring of the same seed or are inferior resolution-wise compared with optical coherence tomography (OCT).

OCT is an in vivo, non-contact, non-destructive real-time imaging method that was first introduced in 1991 [22]. The working principle of the OCT imaging method is based on the interferometric technique that uses a low-coherence light source. Because OCT offers non-contact real-time imaging, this method has been profoundly used for diagnostics in medical imaging and research studies that require high-resolution imaging of samples with thicknesses on the order of a few millimeters. Because it provides high-resolution images that are on par with histological images, OCT has been widely used in many fields, such as ophthalmology [23], otorhinolaryngology [24-26], dermatology [27], blood flow measurements $[23,28]$, defect inspection and thickness measurements in electronics devices such as light-emitting diodes (LEDs) [29], liquid crystal displays (LCDs) [30], composite structural analysis [31], and agriculture. In past research studies by various groups, the usefulness of OCT in agricultural studies has been shown to be prominent, for example, for monitoring of leaf diseases [32-34], and for the identification of defects and diseases in seeds and fruits [35,36].

The experimental conditions and parameters of this study were based on previously reported studies on effects of salt stress on seed germination [4,11]. In the published literature articles, they have investigated the effect of salt concentrations of up to $200 \mathrm{mM} \mathrm{NaCl}$ on Capsicum annuum seed priming. This study was carried out as a continuous monitoring process for 9 days. All the seed specimens were inspected throughout the experimental duration, and only the representative OCT images (acquired on Days 1, 3, 5, 7, and 9) are shown in the manuscript, which emphasize representative major morphological changes. By demonstrating this, we show a broader spectrum of OCT applications as a tool for in vivo growth analysis of primed seeds. Two-dimensional (2D) OCT images of seeds exhibiting morphological changes during the growth within the seeds are presented, and the obtained results are supported by averaged and normalized depth profile analysis.

\section{Materials and Methods}

\subsection{The Preperation of Seed Specimens and Experimental Conditions}

To assure homogeneity and viability of the samples used in the experiment, the Capsicum annuum plant seeds that were utilized for experiments were obtained from the Department of Microorganisms, Kyungpook National University, Daegu, South Korea. The experimental procedure was approved by the research ethics committee of Kyungpook National University. All the seed specimens were selected with an average weight of $\sim 1.44 \mathrm{~g}$. The seed samples were washed using distilled water and dried at room temperature $\left(27^{\circ} \mathrm{C}\right)$. The experiment lasted for 9 consecutive days (the seeds exhibited prominent germination by the end of the experiment). Overall, 60 Capsicum annuum seeds were used in our experiments. These 60 seeds were partitioned into 4 groups, with each group containing 15 seeds. Seed specimens were placed on Petri dishes with each base-surfaced with tissue paper, were used as container bases for germinating the seeds, and was filled with individual solutions of sterile distilled water (SDW), $0.1 \mathrm{M} \mathrm{NaCl}$ (molar concentration of sodium chloride), $0.2 \mathrm{M} \mathrm{NaCl}$, $0.3 \mathrm{M} \mathrm{NaCl}$, and $0.4 \mathrm{M} \mathrm{NaCl}$. The solutions were filled to a level slightly above the halfway point of the Petri dishes to ensure complete immersion of the seed samples into their individual solutions. To maintain similar saline environment conditions and to avoid dehydration during the OCT imaging process, each seed specimen was taken out and kept in a Petri dish containing a saline solution similar to its origin. All Petri dishes were handled with extreme care so that the seeds were not subject to any damages during the experiment. An external mark was placed on the top surface of the exact center of seed specimens using a normal marker to indicate the region of interest. This helped in identifying and maintaining the region of interest throughout the experimental duration. 


\subsection{Specimen Preperation Forhistological Analysis}

For histological analysis, the Capsicum annuum seeds that were primed using sterile distilled water (SDW) were utilized. The seeds used for histological analysis were primed with SDW for 1 day. The samples that were used for histology were fixed in $2 \%$ paraformaldehyde and $2.5 \%$ glutaraldehyde in a $0.05 \mathrm{M}$ sodium cacodylate buffer solution, for $24 \mathrm{~h}$. Then, the samples were washed and dehydrated using graded ethanol series of $30 \%, 50 \%, 70 \%, 80 \%$, and $90 \%$ of absolute ethanol for $\sim 30 \mathrm{~min}$. Then, the dehydrated samples were embedded in a Spurr's resin after being infiltrated with propylene oxide. The specimen was then polymerized in $100 \%$ Spurr's resin for $24 \mathrm{~h}$ at $70{ }^{\circ} \mathrm{C}$. Finally, the samples were sectioned using an ultra-microtome (MT-7000, RMC, Tucson, AZ, USA) with a 2-3 $\mu$ m thickness. The sectioned samples were stained with a $2 \%$ methylene blue solution to be inspected using a light microscope (BX50, Olympus, Tokyo, Japan).

\subsection{OCT System Setup}

A custom assembled swept source optical coherence tomography (SS-OCT) was used for OCT imaging of the samples during the experiment. A schematic of the SS-OCT system that was used is shown in Figure 1A. The SS-OCT build was driven by a high-speed, broad bandwidth swept laser source (AXP50125-6, Axsun Technology, Billerica, MA, USA) with a center wavelength of $1310 \mathrm{~nm}$ and a full width at half maximum (FWHM) of $110 \mathrm{~nm}$. The sweeping rate of the source was $50 \mathrm{kHz}$, with an average output power of $20 \mathrm{~mW}$. The laser output from the source was connected to a fiber coupler (OCT-310C32C13, Gooch \& Housego PLC, Ilminster, UK) at an 80:20 ratio. Twenty percent of the output arm of the coupler was then connected to the input arm of a circulator (CIR-1310-50-APC, Thorlabs Inc., Newton, NJ, USA), and the output arm of the circulator was connected to a reference arm setup. The reference arm setup consisted of a collimator followed by a focusing lens and a mirror. Similarly, the remaining $80 \%$ output from the coupler arm was also connected to the input arm of another circulator. The output arm of that circulator was connected to a sample arm setup. The sample arm consisted of a collimator, a galvanometer scanner, and a focusing lens. The final laser power at the sample arm end was $14 \mathrm{~mW}$. The backscattered light beams from both the sample and reference mirrors were collected from their respective redirected circulator arms and connected to the input arms of a fiber coupler at a 50:50 ratio (OCT-310K32C13, Gooch \& Housego PLC, Ilminster, UK). At this stage, the interference of the backscattered light beams from the reference arm and the sample arm occurred. The interference signal was then collected from the output arms of the 50:50 ratio coupler and connected to the positive and negative ports of a balanced photodetector (PDB430C, Thorlabs Inc., Newton, NJ, USA). All of the collimators, lenses, mirrors, and a galvanometer scanner (GVS102, Thorlabs Inc., Newton, NJ, USA) that were utilized in the system configuration were chosen so as to be optimal for working in the broad bandwidth range of wavelengths that was centered at $1310 \mathrm{~nm}$. The signal obtained from the photodetector was digitized using a digitizer (ATS9462, Alazar Technologies Inc., Montreal, QC, Canada).

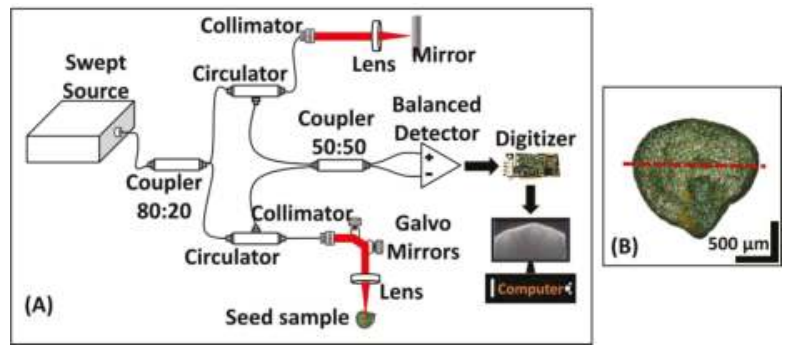

Figure 1. Schematic of the SS-OCT setup. (A) Schematic of the SS-OCT system. (B) Photograph of a sample Capsicum annuum seed. The dashed line represents the place of OCT scanning beam. 
The axial and lateral resolutions in air for the built system were $6.8 \mu \mathrm{m}$ and $14.6 \mu \mathrm{m}$, respectively. The depth roll-off of the built system was less than $5 \mathrm{~dB} / \mathrm{mm}$, and the SNR of the system was $110 \mathrm{~dB}$. To match the optical depth with the true depth of the sample, the obtained signals were multiplied with a factor of 1.42 (refractive index of seed structures). There are other methods to further improve the sensitivity fall-off at increasing depth $[37,38]$. A software-based data-processing technique was designed to construct the 2D OCT images. During the entire experimental duration, OCT imaging was performed in a controlled environment, in which the temperature was maintained at $27 \pm 3{ }^{\circ} \mathrm{C}$, and the humidity was in the $75-80 \%$ range, in a dark room. OCT imaging of the seed was maintained at the center of the seed. The region of interest (ROI) was determined by using a previously published article [6] as a base reference for identifying the important internal structures, and accordingly the ROI was selected. An external mark was placed on the ROI of seed specimens to indicate the position of OCT scanning, and multiple 2D-OCT scans were acquired approximately close to the aforementioned marked position, which made it possible to match the 2D-OCT image at the exact same imaging position as that of the images obtained on earlier days. Within the ROI, the cross-sectional image providing the maximum depth was selected for further analysis. The acquisition of multiple 2D-OCT images minimized the requirement of volumetric analysis, since the acquired cross sections sufficiently confirmed the desired results. Furthermore, the focusing point during scanning was set to be the same. To maintain similar saline environment conditions and to avoid dehydration during the OCT imaging process, each seed specimen was taken out and kept in a Petri dish containing the saline solution similar to its origin. All Petri dishes were handled with extreme care so that the seeds were not subject to any damages during the experiment.

The seeds were placed (expected radical emerging point) facing the OCT scanning sample arm. Figure 1B shows the direction and position in which the seeds were placed during OCT imaging. Figure $1 \mathrm{~B}$ shows the photograph of a representative Capsicum annuum seed and its imaging orientation, for which OCT imaging was maintained at the center of the seed, and the expected radicle emergence point was forward-facing during imaging. The red dashed line in Figure $1 \mathrm{~B}$ indicates the direction and position of scanning by the sample arm. The total depth of the seed structures was not enough for the presented study to be carried out without any error occurrence due to intensity variations.

\subsection{Depth Profile Analysis Algorithm}

To analyze the obtained 2D images, a MATLAB (Mathworks, Middlesex County, NJ, USA) based software program was used to detect the intensity peaks in the depth direction. During the depth profile analysis process, 2D OCT image was loaded and a peak search algorithm based image window with 60 intensity signals (depth profile signals) was applied to select the region of interest. The peak search algorithm detects the maximum intensity in an individual depth scan sequentially. Due to the physical structure of the leaf, the 2D OCT cross-sectional image is an unflattened image containing maximum intensity index positions at different positions. All peaks positions in all 60 depth scans were rearranged to match the peak intensity index in the depth scans to flatten the image. Averaging these consecutive depths scans within a certain selected window aided in reducing the noise in the depth profile plots. The obtained depth profile intensities were then divided by the maximal value to obtain normalized depth profile intensity plots for the 2D OCT images. Thus, the absence of intensity owing to the presence of the air region can be considered negligible and did not negatively affect the plotted depth profiles.

\section{Results and Discussion}

A 2D SS-OCT image and a histologically sectioned image of Capsicum annuum seeds, both of which were acquired during the initial SDW priming stage (Day 1), are shown in Figure 2. Figure 2A shows a 2D SS-OCT image of the primed seed, and Figure 2B shows an enlarged histology sectioned image of the same seed. It usually becomes difficult to distinguish between the cotyledon and the non-micropylar endosperm layers. However, the OCT image is based on change in the refractive 
index of the scattering layers in the sample, and different biological tissues have their respective refractive index coefficients. In seeds, the biological compositions of cotyledon and non-micropylar endosperm are different, which results in a difference in their intensity in OCT images. In Figure 2A, the red color dashed circles depict the corresponding borders of the cotyledon layers and the layer with comparatively lesser intensity; i.e., the non-micropylar endosperm layer around the cotyledon can be differentiated and identified with the help of a histology image. From comparative analysis, it can be concluded that the 2D OCT image reveals a similar growth pattern of seed structures in the testa, inner seed coat, and cotyledon. As per seed growth, the cotyledon and the embryo growth can be observed in 2D OCT images, which are shown later on.

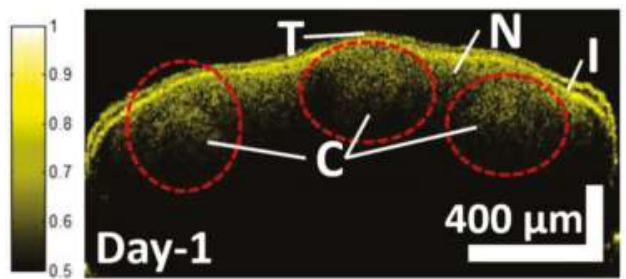

(A)

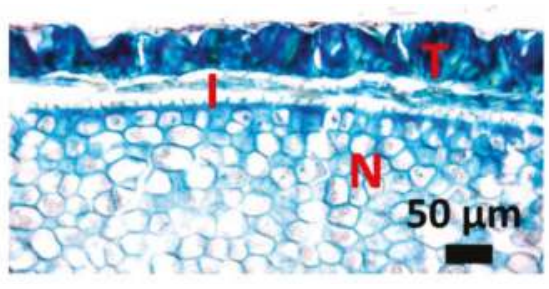

(B)

Figure 2. A 2D SS-OCT image and a histological image. (A) A 2D-SSOCT image of a seed primed with sterile distilled water for one day. (B) An enlarged histology image of the same seed that was used in (A). C: Cotyledon; I: Inner seed coat; N: Non-micropylar endosperm; T: Testa. Dashed red circle shows the enclosed cotyledon regions.

Figure 3 shows the SS-OCT images that were taken on consecutive days, for seeds that were soaked in SDW, $0.1 \mathrm{M} \mathrm{NaCl}, 0.2 \mathrm{M} \mathrm{NaCl}, 0.3 \mathrm{M} \mathrm{NaCl}$, and $0.4 \mathrm{M} \mathrm{NaCl}$ solutions. Figure 3 shows the representative images of each corresponding seed category and provides visualizations of the inner morphological structures. The best seed image, with a visible structural difference in each group, was selected and monitored, and the images of that particular seed are arranged in Figure 3. The OCT in vivo imaging was carried out for all the seeds in each group. The statistical values giving the seed growth has been shown in Figure 4 plots. Table S1 provides the measured seed weight (average, standard deviation, minimum and maximum values) and embryo thickness measurement (average, standard deviation, minimum and maximum values) using depth profile analysis for the entire group throughout the monitoring process. Panels (A1) to (A5) in Figure 3 show the images of seeds soaked in SDW that were taken on consecutive days. It can be clearly seen that on Day 1 the three major distinct layers of seeds are visible: the testa (seed coating), the cotyledon, and the inner seed coat. Starting from Day 3, notable traces of collective vacuum-like deposition begin to appear beneath the inner seed coat layer, owing to the development of embryos. As the seed grows, the embryo becomes clearer and the cotyledon layer forms into multiple lobe-like structures, which is observable in images taken on Day 5 and Day 7. The bottom part of the seed starts to exhibit more structural details as the seed germination increases. This is visible on Day 7 onwards. On Day 9, the embryo is seen in most parts of the seed; in addition, the cotyledon appears to have a smaller area than the area observed during the preceding seed growth days. On Day 9, the seeds are apparently well-germinated. 


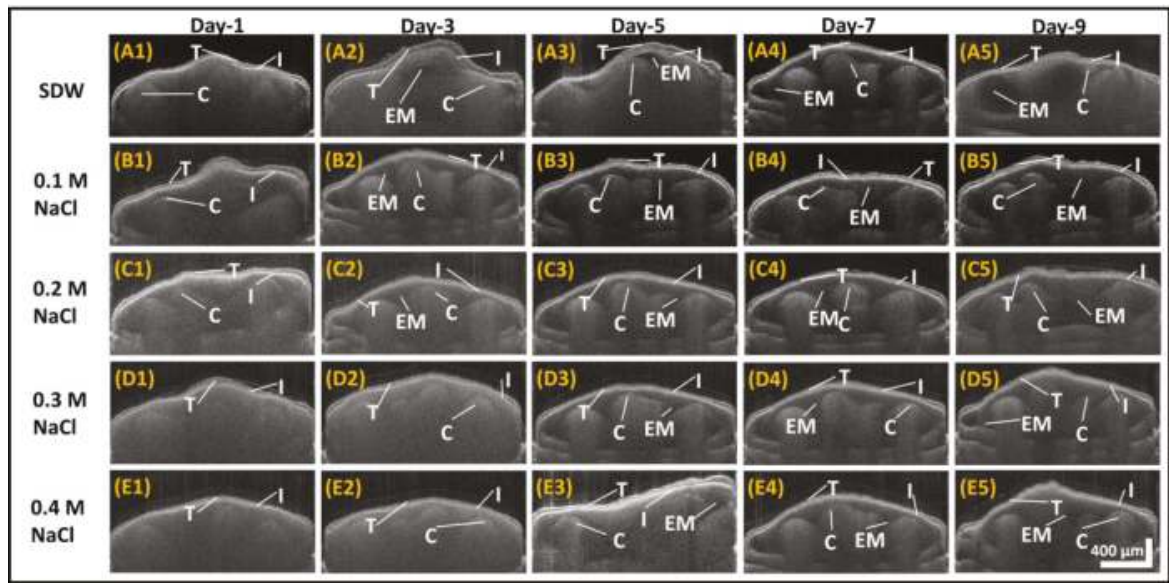

Figure 3. Comparative growth analysis by 2D SS-OCT of Capsicum annuum seeds primed with different salt solutions. The images were taken on consecutive days for seeds that were soaked in sterile distilled water (SDW) (A1-A5), $0.1 \mathrm{M} \mathrm{NaCl}$ solution (B1-B5), $0.2 \mathrm{M} \mathrm{NaCl}$ solution (C1-C5), $0.3 \mathrm{M} \mathrm{NaCl}$ solution (D1-D5), and 0.4 M NaCl solution (E1-E5). C: Cotyledon; EM: embryo; I: Inner seed coat; T: Testa. The scale bar of $400 \mu \mathrm{m}$ applies to all 2D images.

In Figure 3, Panels (B1) to (B5) show the images of the seeds primed with the $0.1 \mathrm{M} \mathrm{NaCl}$ salt solution. On Day 1, the effect of the $0.1 \mathrm{M} \mathrm{NaCl}$ solution on the structural development of the seeds appears to be similar to that of the SDW solution. The development of the embryos and bottom structures of the seeds becomes barely visible from Day 3 onwards. The notable growth of the embryos and cotyledon structures becomes clearer as the seeds grow further. However, the difference between the states of the seeds on Day 7 and Day 9 appears to be insignificant. In addition, on Day 9 the embryos are not as prominent as those of the seeds grown in SDW.

Panels (C1) to (C5) in Figure 3 show morphological changes that occur internally in seeds soaked in the $0.2 \mathrm{M} \mathrm{NaCl}$ solution. Unlike the Day 1 images of SDW-primed seeds and $0.1 \mathrm{M} \mathrm{NaCl}$ primed seeds, the Day 1 images of $0.2 \mathrm{M} \mathrm{NaCl}$ seeds reveal a barely visible bottom structure in the imaged seeds. These structures, along with the embryo growth, appear at Day 3. The images of the seeds soaked in the $0.2 \mathrm{M} \mathrm{NaCl}$ solution, acquired on Day 3 and Day 5, reveal that the seeds exhibit fairly similar changes in their internal morphological structures compared to the Day 3 and Day 5 images of the seeds soaked in the $0.1 \mathrm{M} \mathrm{NaCl}$ solution. The intensities of structures in the Day 7 and Day 9 images of the seeds soaked in the $0.2 \mathrm{M} \mathrm{NaCl}$ solution are slightly higher than those in the Day 7 and Day 9 images of the seeds soaked in the $0.1 \mathrm{M} \mathrm{NaCl}$ and SDW solutions. This can seemingly be the effect of a slower germination rate induced by the $0.2 \mathrm{M} \mathrm{NaCl}$ solution.

Panels (D1) to (D5) in Figure 3 show the images of the Capsicum annuum seeds primed with the $0.3 \mathrm{M} \mathrm{NaCl}$ solution. It can be noted that, unlike on Day 1 and Day 3 for SDW-soaked seeds and those soaked in the $0.1 \mathrm{M} \mathrm{NaCl}$ and $0.2 \mathrm{M} \mathrm{NaCl}$ solutions, the embryos are not prominently visible on Day 1 and Day 3 for the $0.3 \mathrm{M} \mathrm{NaCl}$ primed seeds. The development of the embryos in these seeds appears to start on Day 5. In addition, the Day 7 image is quite similar to the Day 5 image. In the Day 7 and Day 9 images, the bottom part of the seed structures become visible, while in the case of SDW, $0.1 \mathrm{M} \mathrm{NaCl}$, and $0.2 \mathrm{M} \mathrm{NaCl}$ primed seeds, these structures become visible on earlier days. In addition, the intensity of these structures is seemingly higher than that in other solutions.

Panels (E1) to (E5) in Figure 3 show the effect of the $0.4 \mathrm{M} \mathrm{NaCl}$ concentration on the growth of Capsicum annum seeds. It can be observed that the development of embryos starts on Day 5 in the seeds primed with the $0.4 \mathrm{M} \mathrm{NaCl}$ solution, which correlates with the Day 5 onset of development for 
the seeds primed with the $0.3 \mathrm{M} \mathrm{NaCl}$ solution. However, the visibility of the bottom structures of the seeds in Day 7 and Day 9 images for the $0.4 \mathrm{M} \mathrm{NaCl}$ solution is even weaker, compared with those of the seeds primed with the $0.3 \mathrm{M} \mathrm{NaCl}$ solution. In addition, for the seeds primed with the $0.4 \mathrm{M} \mathrm{NaCl}$ solution, the imaged internal morphological changes on Day 9 were much weaker than those on Day 7. Even naked eye examinations of these seeds, compared with the seeds primed with the $0.3 \mathrm{M} \mathrm{NaCl}$ solution, revealed weaker germination.

In Figure 3, the difference in size of the horizontal scale and the vertical scale bar is due to the fact that the scan range and the imaging depth of the OCT system were not same. The horizontal scale bar is dependent on the scan range, whereas the vertical scale bar is dependent on the depth range that can be achieved using the OCT system. The variation of image background intensities in Figure 3 is due to the time-dependent continuous power fluctuations of the swept source laser. Since the imaging procedure was started with SDW primed seeds, the contrast of the entire first row appears to be different from that of the others. The effect of the unstable laser power was compensated by normalizing the overall intensity of the depth profiles. Since time-consuming histological analysis for each seed specimen was not possible for monitoring experiments, the cotyledon, embryo, and other internal layers of the seeds were pre-identified by correlating them with histological images. The unique structural differences as well as the similarities in the seed specimens can be observed in the images shown in Figure 3. Even though at first glance, B5 and C5 might look similar, upon closer inspection, the structure of the testa and the bottom right internal layers of B5 and C5 are uniquely different from each other. These were taken at the center point of both seeds, which may seem to be a reason for the similarity in their appearances. Furthermore, the difference in the appearance of E3 and E4 is due to the change in orientation and the angle of seed specimen, since it had to be slightly shifted during imaging to overcome the negative effects of excessive back-reflection. We selected these images because only these images optimally showed the internal structural differences.

Figure 4 shows the statistical analysis for all groups. Figure 4A shows the average seed weight (providing average and standard deviation for 5 groups 15 seeds) of all five groups for each day during monitoring. Each seed group had an average initial fresh weight of approximately $1.44 \mathrm{~g}$ on Day 0 . Seeds primed in SDW had the highest weight gain at the end of the monitoring period. It is to be noted that seeds primed in $0.1 \mathrm{M} \mathrm{NaCl}$ and $0.2 \mathrm{M} \mathrm{NaCl}$ were almost similar in weight, where $0.1 \mathrm{M} \mathrm{NaCl}$ primed seeds were only a few grams higher than those primed in $0.2 \mathrm{M} \mathrm{NaCl}$. The weight values of the seeds primed in $0.3 \mathrm{M} \mathrm{NaCl}$ and $0.4 \mathrm{M} \mathrm{NaCl}$ were well below the other groups. Furthermore, both of these seed groups had a negligible gain in weight towards the end of the monitoring period. The average weight gain observed in each seed groups, along with its standard deviation value and the maximum and minimum weight of the seeds in each group that was observed during the entire monitoring process, is given in the Supplementary Materials.

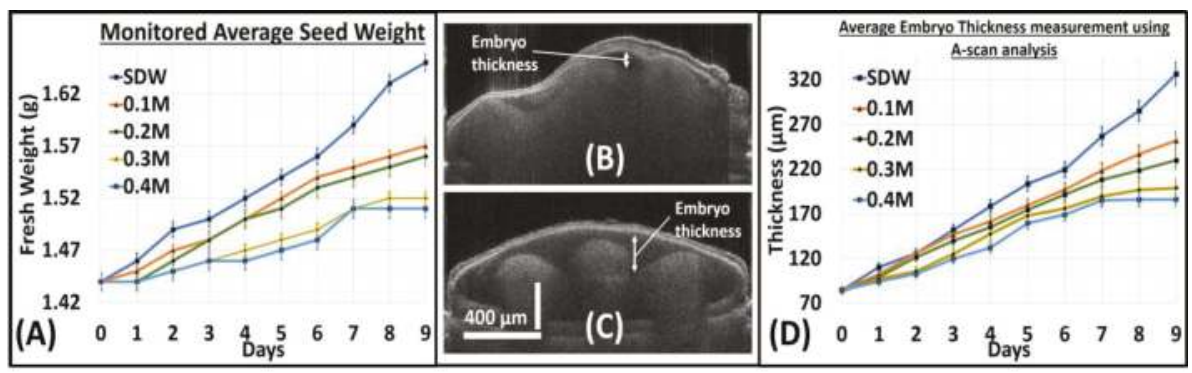

Figure 4. Statistical analysis of 2D OCT images using depth profile analysis. (A) The recorded average seed weight fluctuations during monitoring period. (B,C) Representative 2D-OCT images marked with embryo region in a seed. (D) The measured average embryo thickness using depth profile analysis of all seed groups during the monitoring period. 
For further structural analysis of seed morphology changes, we utilized a single point depth profile analysis procedure. We optimized the MATLAB-based program (stated earlier in Section 2.2 in Materials and Methods) such that the program takes individual point depth profiles of the entire 2D image, from which a single depth profile is chosen. The selection of the depth profile was made, and the maximum embryo thickness was observed. In Figure 4B,C, the embryo region in a 2D OCT image is marked with a double headed arrow for representation. The embryo region growth is a direct indication of the germination progress in a seed. Thus, by measuring the maximum embryo thickness in a seed, the growth progress can be determined. Figure $4 \mathrm{D}$ is a representative graph indicating the average embryo thickness measurement obtained using this depth profile analysis procedure for all the groups throughout the monitoring period. The averaged embryo thickness values for each group with its respective standard deviation, along with the maximum and minimum thickness observed in a seed in every group that was measured throughout the monitoring process is shown in the Supplementary Material. As previously mentioned in the OCT system setup, the axial resolution of the OCT system is $6.8 \mu \mathrm{m}$ in air, while in seed it is $4.8 \mu \mathrm{m}$. Therefore, there can be a least measurement error of $4.8 \mu \mathrm{m}$ in the thickness measurement of the embryo. The variation in the embryo is also proportional to the weight of seed, so a standard deviation of $\sim 16 \mu \mathrm{m}$ was observed, as shown in the Supplementary Material.

The top panel in Figure 5 shows the images acquired on Day 3, for seeds that were primed with different solutions. The bottom panel shows the normalized depth profile graphs for seeds in different priming scenarios. As the embryo development in a seed can be taken as a direct correlation for the germination progress, the ROI in the depth profile analysis was chosen in a way as to get the maximum development of the embryo region in the seed. Sixty consecutive depth profiles were taken within the window size (red rectangular box regions shown in the images in the top panel of Figure 5). The red curve corresponds to the normalized depth profile for the seeds that were primed with SDW, the blue curve is the normalized depth profile for the seeds primed with the $0.1 \mathrm{M} \mathrm{NaCl}$ solution, the green curve corresponds to the results for the seeds primed with the $0.2 \mathrm{M} \mathrm{NaCl}$ solution, the normalized depth profile plot for the seeds primed with the $0.3 \mathrm{M} \mathrm{NaCl}$ solution is given by the black curve, and the pink curve shows the results for the seeds primed with the $0.4 \mathrm{M} \mathrm{NaCl}$ solution.

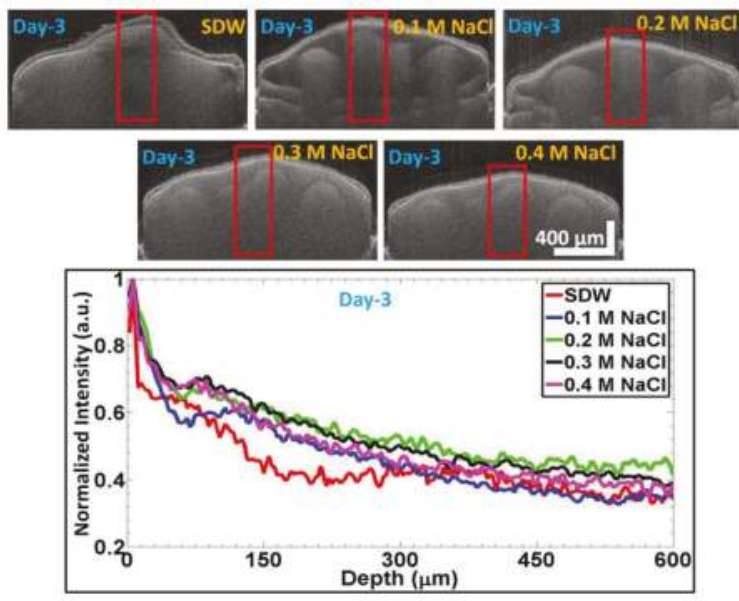

Figure 5. SS-OCT images acquired on Day 3 after priming the Capsicum annuum seeds with different solutions. The bottom figure is the respective averaged and normalized depth profile analysis plot (as discussed in Section 2.4) for SDW, $0.1 \mathrm{M} \mathrm{NaCl}, 0.2 \mathrm{M} \mathrm{NaCl}, 0.3 \mathrm{M} \mathrm{NaCl}$, and $0.4 \mathrm{M} \mathrm{NaCl}$, which are correspondingly shown with colors of red, blue, green, black, and magenta plots. 
The major illustration of the acquired depth profiles was the sudden decrease of the signal intensity due to the change in refractive index of the scattering medium, i.e., the internal structures. The sudden intensity-drop or weaker signal intensity was observed due to the changes of the refractive index of the germinated seed structures. By using this, we can determine the thickness of layers. Moreover, through a continuous monitoring process, it is possible to measure the growth progress internal structures. The intensity falloff in the plots illustrates the extent of growth in the analyzed seeds. Weaker intensity corresponds to higher growth, and vice versa. By comparison, it is observed that the seeds primed with SDW exhibit the highest growth on Day 3, followed by the seeds primed with the $0.1 \mathrm{M} \mathrm{NaCl}$ solution. The maximal intensity (or, in other words, the slowest growth) was found for the seeds primed with the $0.3 \mathrm{M} \mathrm{NaCl}$ and $0.4 \mathrm{M} \mathrm{NaCl}$ solutions.

Figure 6 shows the OCT images of seeds primed with the different solutions; the seeds were imaged on Day 9. The bottom panel shows the respective normalized depth profile plot for the seeds, acquired within the red rectangular boxes in the OCT images in the top panel. As mentioned above, these normalized depth profiles were plotted by averaging and normalizing 60 consecutive depth profile signals in each image. Owing to the maximal growth of embryos in SDW-primed seeds, the red curve shows the least signal intensity. The blue curve shows the second least intensity, showing changes in the seeds' internal morphology. The results for the $0.2 \mathrm{M} \mathrm{NaCl}, 0.3 \mathrm{M} \mathrm{NaCl}$, and $0.4 \mathrm{M} \mathrm{NaCl}$ primed seeds are shown with the green, black, and pink curves, respectively, and reveal much weaker growth than that in the other two solutions. The seed germination rates in SDW and $\mathrm{NaCl}$ solutions were evaluated by the internal seed regions, such as the embryos, the micropylar endosperm growth, and the cotyledons, which were formed by clearing the solid structures within the seed. A low germination rate was noted in seeds with higher $\mathrm{NaCl}$ concentrations. SDW-primed sample seeds yielded full germination as the seedling emerged, but negligible or no seedling emergence was noted for the seeds primed with the highest concentration of $\mathrm{NaCl}$. This was confirmed with OCT images.
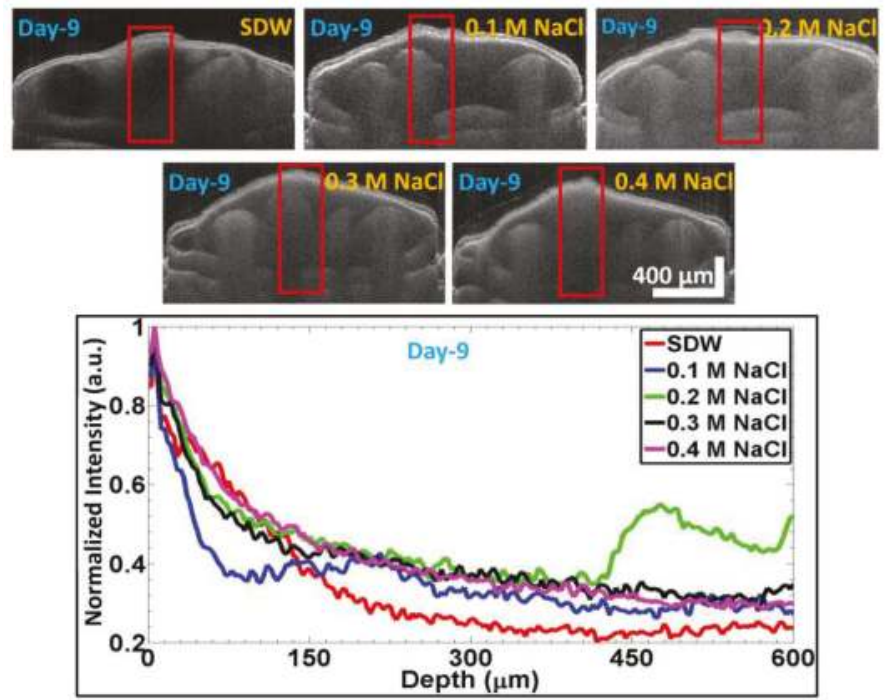

Figure 6. SS-OCT images on Day 9 after priming the Capsicum annuum seeds with different solutions. Bottom figure is the respective averaged and normalized depth profile analysis plot (as discussed in Section 2.4) for SDW, $0.1 \mathrm{M} \mathrm{NaCl}, 0.2 \mathrm{M} \mathrm{NaCl}, 0.3 \mathrm{M} \mathrm{NaCl}$, and $0.4 \mathrm{M} \mathrm{NaCl}$, which are correspondingly shown with colors of red, blue, green, black, and magenta plots. 
The biological nature, such as internal nutrients, illumination, and the refractive index, of the categorized 60 seed specimens ( 15 seeds $\times 4$ groups), can be different from one to another. To minimize the aforementioned drawbacks, seed specimens with the exactly same weight were chosen, and such seeds were stored and imaged in a completely dark environment to maintain homogenous illumination and nutrient access. Therefore, the assumptions for the aforementioned seed biological nature were made on the basis of the weight. Moreover, the proposed study was carried out to examine how a feasible OCT imaging technique for in vivo germination progress assessment and for growth inhibition due to salt concentration. Future experiments aimed with other possible germination affecting factors can be studied for further evaluation of the system applicability.

\section{Conclusions}

We have demonstrated the usefulness of OCT as a tool for continuous in vivo monitoring of morphological changes in seeds. In addition, this study provided useful insights in the way OCT imaging can be used for seed growth analysis and for detecting the state of germination inhibition in seeds by continuous monitoring. Because maintenance of appropriate soil salinity plays a major role for seed growth, we conducted this study by priming Capsicum annuum seeds with different molar concentrations of salt and with sterile distilled water. We assessed the differences in internal morphological changes in seeds as the seeds grew. We observed that the seeds primed with sterile distilled water exhibited prominent growth, while the seeds primed with the $0.1 \mathrm{M} \mathrm{NaCl}$ and $0.2 \mathrm{M} \mathrm{NaCl}$ solutions exhibited slower but constant growth compared with the seeds soaked in sterile distilled water. Seeds primed with a higher concentration of $\mathrm{NaCl}$ showed negligible or no seedling emergence, indicating an impediment in the germination of the seed, which was verified with OCT monitoring images. The results of this study show the potential applications of OCT for agronomical studies related to seed growth. Further OCT studies of seed germination can help to determine appropriate conditions for faster seed germination, thereby reducing the time to harvest.

Supplementary Materials: The following are available online at http:/ / www.mdpi.com/1424-8220/17/12/2887/ s1. Table S1, The average weight gain observed and the averaged embryo thickness values for each group, along with its standard deviation value and the maximum and minimum values of seeds in each group that was observed during the entire monitoring process.

Acknowledgments: This research was supported by the Bio \& Medical Technology Development Program of the NRF funded by the Korean government, MSIP (2017M3A9E2065282). This work was also supported by Korea Institute of Planning and Evaluation for Technology in Food, Agriculture, Forestry and Fisheries (IPET) through Advanced Production Technology Development Program, funded by Ministry of Agriculture, Food and Rural Affairs (MAFRA) (No. 314031-3). Additionally, this study was also supported by the BK21 Plus project funded by the Ministry of Education, Korea (21A20131600011).

Author Contributions: The experimental idea was proposed by Mansik Jeon, Hee-Young Jung, and Jeehyun Kim. Histological experiments and analysis was performed by Seung-Yeol Lee and Hee-Young Jung. OCT system development and experiments were carried out by Naresh Kumar Ravichandran, Ruchire Eranga Wijesinghe, and Muhammad Faizan Shirazi. All authors equally contributed to manuscript preparation.

Conflicts of Interest: The authors declare no conflict of interest. The funding sponsors had no role in the design of the study; in the collection, analyses, or interpretation of data; in the writing of the manuscript; or in the decision to publish the results.

\section{References}

1. Martínez-Maldonado, F.E.; Miranda-Lasprilla, D.; Magnitskiy, S. Sugar apple (Annona squamosa L., annonaceae) seed germination: Morphological and anatomical changes. Agron. Colomb. 2013, 31, 176-183.

2. Smith, P.T.; Cobb, B.G. Accelerated germination of pepper seed by priming with salt solutions and water. HortScience 1991, 26, 417-419.

3. Stoffella, P.J.; Di Paola, M.L.; Pardossi, A.; Tognoni, F. Seedling root morphology and shoot growth after seed priming or pregermination of bell pepper. HortScience 1992, 27, 214-215.

4. Yadav, P.V.; Kumari, M.; Ahmed, Z. Seed priming mediated germination improvement and tolerance to subsequent exposure to cold and salt stress in capsicum. Res. J. Seed Sci. 2011, 4, 125-136. [CrossRef] 
5. Nimmi, V.; Madhu, G. Effect of pre-sowing treatment with permanent magnetic field on germination and growth of chilli (Capsicum annum L.). Int. Agrophy. 2009, 23, 195-198.

6. Watkins, J.T.; Cantliffe, D.J. Mechanical resistance of the seed coat and endosperm during germination of capsicum annuum at low temperature. Plant Physiol. 1983, 72, 146-150. [CrossRef] [PubMed]

7. Bojović, B.; Đelić, G.; Topuzović, M.; Stanković, M. Effects of nacl on seed germination in some species from families brassicaceae and solanaceae. Kragujev. J. Sci. 2010, 32, 83-87.

8. Ramana, S.; Biswas, A.; Kundu, S.; Saha, J.; Yadava, R. Effect of distillery effluent on seed germination in some vegetable crops. Bioresour. Technol. 2002, 82, 273-275. [CrossRef]

9. Fargašová, $\mathrm{A}$. Effect of $\mathrm{Pb}, \mathrm{Cd}, \mathrm{Hg}$, $\mathrm{As}$, and $\mathrm{Cr}$ on germination and root growth of Sinapis alba seeds. Bull. Environ. Contam. Toxicol. 1994, 52, 452-456. [CrossRef] [PubMed]

10. Chartzoulakis, K.; Klapaki, G. Response of two greenhouse pepper hybrids to $\mathrm{NaCl}$ salinity during different growth stages. Sci. Hortic. 2000, 86, 247-260. [CrossRef]

11. Khan, H.; Ayub, C.; Pervez, M.; Bilal, R.; Shahid, M.; Ziaf, K. Effect of seed priming with $\mathrm{NaCl}$ on salinity tolerance of hot pepper (Capsicum annuum L.) at seedling stage. Soil Environ. 2009, 28, 81-87.

12. Silva, C.; Martínez, V.; Carvajal, M. Osmotic versus toxic effects of $\mathrm{NaCl}$ on pepper plants. Biol. Plant. 2008, 52, 72-79. [CrossRef]

13. Zeng, L.; Shannon, M.C. Salinity effects on seedling growth and yield components of rice. Crop Sci. 2000, 40, 996-1003.

14. Francois, L.; Donovan, T.; Maas, E. Salinity effects on seed yield, growth, and germination of grain sorghum. Agron. J. 1984, 76, 741-744. [CrossRef]

15. Débarre, D.; Supatto, W.; Pena, A.-M.; Fabre, A.; Tordjmann, T.; Combettes, L.; Schanne-Klein, M.-C.; Beaurepaire, E. Imaging lipid bodies in cells and tissues using third-harmonic generation microscopy. Nat. Methods 2006, 3, 47-53. [CrossRef] [PubMed]

16. Genda, Y.; Sato, K.; Nunomura, O.; Hirabayashi, T.; Ohnishi, J.; Tsuda, S. Immunolocalization of pepper mild mottle virus in capsicum annuum seeds. J. Gen. Plant Pathol. 2005, 71, 238-242. [CrossRef]

17. Beeckman, T.; De Rycke, R.; Viane, R.; Inzé, D. Histological study of seed coat development in arabidopsis thaliana. J. Plant Res. 2000, 113, 139-148. [CrossRef]

18. Weitbrecht, K.; Müller, K.; Leubner-Metzger, G. First off the mark: Early seed germination. J. Exp. Bot. 2011, 62, 3289-3309. [CrossRef] [PubMed]

19. Neuberger, T.; Sreenivasulu, N.; Rokitta, M.; Rolletschek, H.; Göbel, C.; Rutten, T.; Radchuk, V.; Feussner, I.; Wobus, U.; Jakob, P. Quantitative imaging of oil storage in developing crop seeds. Plant Biotechnol. J. 2008, 6, 31-45. [CrossRef] [PubMed]

20. Melkus, G.; Rolletschek, H.; Radchuk, R.; Fuchs, J.; Rutten, T.; Wobus, U.; Altmann, T.; Jakob, P.; Borisjuk, L. The metabolic role of the legume endosperm: A noninvasive imaging study. Plant Physiol. 2009, 151, 1139-1154. [CrossRef] [PubMed]

21. Foucat, L.; Chavagnat, A.; Renou, J.-P. Nuclear magnetic resonance micro-imaging and x-radiography as possible techniques to study seed germination. Sci. Hortic. 1993, 55, 323-331. [CrossRef]

22. Huang, D.; Swanson, E.A.; Lin, C.P.; Schuman, J.S.; Stinson, W.G.; Chang, W.; Hee, M.R.; Flotte, T.; Gregory, K.; Puliafito, C.A. Optical coherence tomography. Science 1991, 254, 1178-1181. [CrossRef] [PubMed]

23. Wang, Y.; Bower, B.A.; Izatt, J.A.; Tan, O.; Huang, D. In vivo total retinal blood flow measurement by fourier domain doppler optical coherence tomography. J. Biomed. Opt. 2007, 12, 041215. [CrossRef] [PubMed]

24. Cho, N.H.; Lee, S.H.; Jung, W.; Jang, J.H.; Kim, J. Optical coherence tomography for the diagnosis and evaluation of human otitis media. J. Korean Med. Sci. 2015, 30, 328-335. [CrossRef] [PubMed]

25. Lee, J.; Kim, K.; Wijesinghe, R.E.; Jeon, D.; Lee, S.H.; Jeon, M.; Jang, J.H. Decalcification using ethylenediaminetetraacetic acid for clear microstructure imaging of cochlea through optical coherence tomography. J. Biomed. Opt. 2016, 21, 081204. [CrossRef] [PubMed]

26. Cho, N.H.; Jang, J.H.; Jung, W.; Kim, J. In vivo imaging of middle-ear and inner-ear microstructures of a mouse guided by SD-OCT combined with a surgical microscope. Opt. Express 2014, 22, 8985-8995. [CrossRef] [PubMed]

27. Steiner, R.; Kunzi-Rapp, K.; Scharffetter-Kochanek, K. Optical coherence tomography: Clinical applications in dermatology. Med. Laser Appl. 2003, 18, 249-259. [CrossRef] 
28. Zhao, Y.; Chen, Z.; Saxer, C.; Xiang, S.; de Boer, J.F.; Nelson, J.S. Phase-resolved optical coherence tomography and optical doppler tomography for imaging blood flow in human skin with fast scanning speed and high velocity sensitivity. Opt. Lett. 2000, 25, 114-116. [CrossRef] [PubMed]

29. Cho, N.H.; Jung, U.; Kim, S.; Kim, J. Non-destructive inspection methods for LEDs using real-time displaying optical coherence tomography. Sensors 2012, 12, 10395-10406. [CrossRef] [PubMed]

30. Kim, S.-H.; Kim, J.-H.; Kang, S.-W. Nondestructive defect inspection for LCDs using optical coherence tomography. Displays 2011, 32, 325-329. [CrossRef]

31. Shirazi, M.F.; Jeon, M.; Kim, J. Structural analysis of polymer composites using spectral domain optical coherence tomography. Sensors 2017, 17, 1155. [CrossRef] [PubMed]

32. Lee, C.-H.; Lee, S.-Y.; Jung, H.-Y.; Kim, J.-H. The application of optical coherence tomography in the diagnosis of marssonina blotch in apple leaves. J. Opt. Soc. Korea 2012, 16, 133-140. [CrossRef]

33. Ravichandran, N.K.; Wijesinghe, R.E.; Shirazi, M.F.; Park, K.; Lee, S.-Y.; Jung, H.-Y.; Jeon, M.; Kim, J. In vivo monitoring on growth and spread of gray leaf spot disease in capsicum annuum leaf using spectral domain optical coherence tomography. J. Spectrosc. 2016, 2016, 1093734. [CrossRef]

34. Wijesinghe, R.E.; Lee, S.-Y.; Kim, P.; Jung, H.-Y.; Jeon, M.; Kim, J. Optical inspection and morphological analysis of diospyros kaki plant leaves for the detection of circular leaf spot disease. Sensors 2016, 16, 1282. [CrossRef] [PubMed]

35. Lee, C.; Lee, S.-Y.; Kim, J.-Y.; Jung, H.-Y.; Kim, J. Optical sensing method for screening disease in melon seeds by using optical coherence tomography. Sensors 2011, 11, 9467-9477. [CrossRef] [PubMed]

36. Wijesinghe, R.E.; Lee, S.-Y.; Ravichandran, N.K.; Shirazi, M.F.; Moon, B.; Jung, H.-Y.; Jeon, M.; Kim, J. Bio-photonic detection method for morphological analysis of anthracnose disease and physiological disorders of diospyros kaki. Opt. Rev. 2017, 24, 199-205. [CrossRef]

37. Kholodnykh, A.I.; Petrova, I.Y.; Motamedi, M.; Esenaliev, R.O. Accurate measurement of total attenuation coefficient of thin tissue with optical coherence tomography. IEEE J. Sel. Top. Quantum Electron. 2003, 9, 210-221. [CrossRef]

38. Scolaro, L.; McLaughlin, R.A.; Klyen, B.R.; Wood, B.A.; Robbins, P.D.; Saunders, C.M.; Jacques, S.L.; Sampson, D.D. Parametric imaging of the local attenuation coefficient in human axillary lymph nodes assessed using optical coherence tomography. Biomed. Opt. Express 2012, 3, 366-379. [CrossRef] [PubMed]

(C) 2017 by the authors. Licensee MDPI, Basel, Switzerland. This article is an open access article distributed under the terms and conditions of the Creative Commons Attribution (CC BY) license (http:/ / creativecommons.org/licenses/by/4.0/). 
Article

\title{
Estimation of the Botanical Composition of Clover-Grass Leys from RGB Images Using Data Simulation and Fully Convolutional Neural Networks
}

\author{
Søren Skovsen ${ }^{1, *}$, Mads Dyrmann ${ }^{1}$, Anders Krogh Mortensen ${ }^{2}$, Kim Arild Steen ${ }^{3}$, Ole Green ${ }^{3}$, \\ Jørgen Eriksen ${ }^{4}$, René Gislum ${ }^{2}$, Rasmus Nyholm Jørgensen ${ }^{1}$ and Henrik Karstoft ${ }^{1}$ \\ 1 Department of Engineering, Aarhus University, Finlandsgade 22, 8200 Aarhus N, Denmark; \\ madsdyrmann@eng.au.dk (M.D.); rnj@eng.au.dk (R.N.J.); hka@eng.au.dk (H.K.) \\ 2 Department of Agroecology, Aarhus University, Forsøgsvej 1, 4200 Slagelse, Denmark; \\ anmo@agro.au.dk (A.K.M.); rg@agro.au.dk (R.G.) \\ 3 Agro Intelligence ApS, Agro Food Park 13, 8200 Aarhus N, Denmark; kas@agrointelli.com (K.A.S.); \\ olg@agrointelli.com (O.G.) \\ 4 Department of Agroecology, Aarhus University, Blichers Allé 20, 8830 Tjele, Denmark; \\ jorgen.eriksen@agro.au.dk \\ * Correspondence: ssk@eng.au.dk
}

Received: 31 October 2017; Accepted: 12 December 2017; Published: 17 December 2017

\begin{abstract}
Optimal fertilization of clover-grass fields relies on knowledge of the clover and grass fractions. This study shows how knowledge can be obtained by analyzing images collected in fields automatically. A fully convolutional neural network was trained to create a pixel-wise classification of clover, grass, and weeds in red, green, and blue (RGB) images of clover-grass mixtures. The estimated clover fractions of the dry matter from the images were found to be highly correlated with the real clover fractions of the dry matter, making this a cheap and non-destructive way of monitoring clover-grass fields. The network was trained solely on simulated top-down images of clover-grass fields. This enables the network to distinguish clover, grass, and weed pixels in real images. The use of simulated images for training reduces the manual labor to a few hours, as compared to more than $3000 \mathrm{~h}$ when all the real images are annotated for training. The network was tested on images with varied clover/grass ratios and achieved an overall pixel classification accuracy of $83.4 \%$, while estimating the dry matter clover fraction with a standard deviation of $7.8 \%$.
\end{abstract}

Keywords: deep learning; clover-grass; precision agriculture; dry matter composition; proximity sensing

\section{Introduction}

Estimation of the clover/grass ratio is essential for optimal fertilization, as it has significant economic potential for the dairy industry. Clover-grass combines high productivity and low environmental impact if the nitrogen $(\mathrm{N})$ supply is adjusted according to the actual clover content. Nonetheless, manure and fertilizers are used largely irrespective of clover content due to poor ability to assess clover content and lack of knowledge on the correlation between fertilizer $(\mathrm{N})$ and clover content during the season.

A high clover fraction gives a higher feed uptake and thus higher performance for cows when the harvested material is used for fodder. The goal is, therefore, to get a larger clover percentage into the clover pastures. Clover is an $\mathrm{N}$-fixing crop able to capture and utilize free $\mathrm{N}$ from the air, while grass relies on soil available $\mathrm{N}$ for its growth and development. Clover is, however, also able to take up and utilize $\mathrm{N}$ from the soil, which creates competition between grass and clover if there is a sufficient amount of soil $\mathrm{N}$ available. In cases of low soil-available $\mathrm{N}$ the clover will outcompete the grass crop. However, due to its faster growth, the grass will outcompete the clover crop if high amounts of soil $\mathrm{N}$ 
are available. Therefore, it is possible to control the competition and thereby the clover-grass ratio by applying more or less N. Determining the clover-grass ratio thus automatically has great potential for optimizing $\mathrm{N}$ applications.

Clover and grass are often grown in mixtures, as clover-grass leys with different species increase the yield stability [1,2] and herbage quality [3] compared to fertilized grass-only leys. This is due to niche complementarity [4] and the greater protein content of the clover $[5,6]$. Thus, properly managed clover-grass mixtures produce a greater yield compared to pure stands of grass and clover $[7,8]$. In order to implement targeted fertilization in practice, it is important to firstly estimate actual clover content in a sward and, secondly optimize fertilization based on clover content.

In agriculture today, the amount of $\mathrm{N}$ added is based on coarse visual inspections, which are subject to errors and do not cover the variability within a single field or between fields. Automated and precise methods for estimating clover/grass ratio could potentially improve the fertilization strategy, thus improving yield and quality of biomass, resulting in a great financial impact. Positive environmental impacts include reduced $\mathrm{N}$ leaching (as more $\mathrm{N}$ will be absorbed and utilized by the clover-grass), and potentially reduced $\mathrm{CO}_{2}$ emissions, as some fertilization operations can be skipped if the correct amount of clover is present early in the life-span of the clover-grass field $[9,10]$.

Researchers have previously sought to estimate the content of clover-grass in images. Bonesmo et al. [11] developed an image processing software program for pixel-wise classification of clover-grass images. The program used color indexes, edge detection, and morphological operations on the color images to distinguish and quantify the amount of soil, grass, clover, and large weeds. The software estimations and the manually-labeled clover coverage showed a high correlation $\left(r^{2}=0.81\right)$.

Himstedt et al. [12] used digital image analysis on images of grass-legume mixtures from a pot experiment to determine the relative legume dry matter contribution. The image analysis was used to determine the legume coverage (red clover, white clover, or alfalfa) by applying a sequence of morphological erosions and dilations. In each image, the legumes were manually encircled to determine the actual legume coverage. The estimated legume coverage showed a high correlation to both the actual legume coverage $\left(\mathrm{r}^{2}=0.79-0.87\right)$ and the relative legume dry matter contribution $\left(r^{2}=0.82\right)$. Himstedt et al. [13] proposed an extension to estimate the absolute legume contribution using legume coverage, total biomass, and logit transformation. Himstedt et al. [14] improved on the image analysis method by filtering the images in hue, saturation, and lightness (HSL) space and applying color segmentation to separate plants and soil after a sequence of morphological operations.

Rayburn [15] correlated randomly sampled points in clover-grass images to relative legume and grass fractions. A regularly spaced grid was placed on top of each image at a random location, and the pixel at each grid location was manually classified. The distribution of classes was used to estimate the relative fractions. The estimated grass and legume fractions were not significantly different from the measured fractions and they showed a high correlation $\left(r^{2}=0.96-0.98\right)$.

McRoberts et al. [16] used local binary patterns to estimate the grass fraction from color images converted to grayscale. The images were tiled into $64 \times 64$ pixel blocks, which were manually labeled as either legumes, grass, or unknown. The local binary patterns were made to be rotational invariant by grouping rotational similar patterns together. A histogram of the local binary pattern groups along with the height were used to estimate the grass fraction with a strong correlation $\left(r^{2}=0.81-0.85\right)$.

Automatic segmentation of images from clover-grass fields is a research area with great potential in precision agriculture. Nevertheless, there has been limited success in automated estimation of clover and grass content from red, green, and blue (RGB) images so far. The primary limitation of current methods involve the uncertainty in the image recognition process. Although the use of morphological operations has been shown to correlate with the clover content in the images, it lacks robustness with regards to scale invariance, field conditions, and estimation uncertainty. With varying clover sizes, camera or vegetation heights, or camera resolutions, the parameters of the existing methods need 
adjustments. This is illustrated by the drop in performance in the work of Himstedt et al. [13] when moving from green house pots to field conditions.

The aim of this project is to automatically determine the clover fraction in clover-grass fields through the use of machine learning to analyze RGB images for grass, clover, and weeds. We follow the procedure of splitting the challenge of estimating the clover content in dry matter from images into a two-step problem, similarly to Himstedt et al. [12]. However, instead of relying on simple patch detections using morphological operations, we propose training a deep convolutional neural network, based on the fully convolutional network (FCN) architecture [17], to directly classify relevant plant species visible in the images $[18,19]$. This provides the latter stage of clover content estimation with information on the field patch composition and the detected coverage of grasses, clovers, and weeds in the image. Specifically, the selected convolutional neural network is designed to output a semantically segmented image, specifying the plant species of every pixel in the image. An example of such automatic semantic segmentation is shown in Figure 1, where pixels classified as grasses, clovers, and weeds are visualized by blue, red, and yellow overlay, respectively.

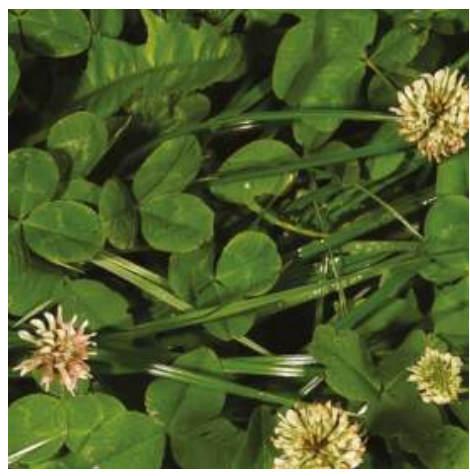

(a)

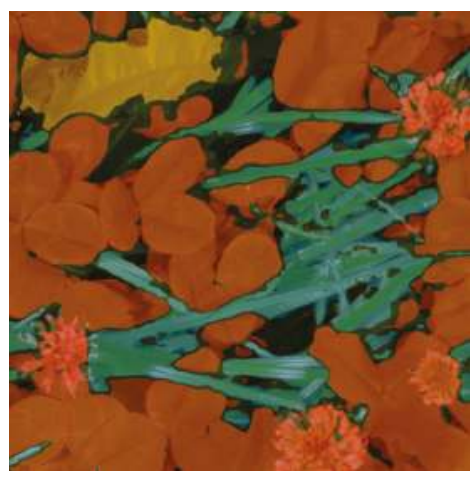

(b)

Figure 1. Example of the image analysis on a real image. Each pixel in the image is automatically analyzed and classified as either grass (blue), clover (red), weeds (yellow), or unidentified (black overlay). (a) Example input image; (b) Automatically analyzed image.

The main challenge of moving from manually designed features of the previous methods to learned features using neural networks is the significant increase in demand for ground truth data. To obviate this demand, a simulation environment was designed to generate labeled images of specific field compositions, from which the discriminating features are learned. The trained neural networks were then evaluated against human-annotated images for pixel-wise classification. Finally, a trained network was combined with the latter processing stage to estimate the clover content of the dry matter directly from images.

\section{Materials and Methods}

\subsection{Data Material}

In order to evaluate the image segmentation, clover-grass field trial samples were photographed, harvested, and separated into distinct fractions of ryegrass, white clover, red clover, and weeds. The images and corresponding biomass samples represent the physical data of this paper. There are two uses for these samples: 
1. Evaluation of the use of convolutional neural networks (CNNs) for semantic segmentation on a hand-annotated subset of real images.

2. Application of the $\mathrm{CNN}$ on the gathered images and validation of the coupling between semantic segmentation of the clover grass canopy and the dry matter species composition in the yield.

\subsubsection{Field and Agricultural Setup}

The primary samples were collected in 2017 within a primary experimental site measuring 2 hectares at Aarhus University, Foulum (world geodetic system 1984 (WGS84): Latitude 56.495365, Longitude 9.569537). The field trial consisted of 60 clover-grass plots, ranging from 1 to 4 years in age (established in 2013-2016). A mixture containing 82\% perennial ryegrass (Lolium perenne L.), $14 \%$ white clover (Trifolium repens L.), and $4 \%$ red clover (Trifolium pratense L.) with respect to the seed weight was seeded. To increase the variations of the clover-grass ratios for this research, five different fertilization strategies $(0,50,100,200$, and $300 \mathrm{~kg} \mathrm{~N} /$ hectare) were applied in a randomized design. All fertilization was applied as cattle slurry injected into the soil and no pesticides were used. The site follows a four cut per year strategy, typical for Danish conditions.

\subsubsection{Clover-Grass Sample Pairs}

Each clover-grass sample gathered in the plots consisted of an image of a defined patch of $0.5 \times 0.5 \mathrm{~m}$ and a botanical dry matter composition of the harvested plants in the same patch. These cross-domain samples of both images and dry matter composition are from this point on referred to as sample pairs.

At the time of this research, three of the four seasonal cuts of the 2017 season were performed and analyzed. Acquisition of one sample pair per plot per cut led to 179 acquired samples (one sample was lost due to human error). The sample pairs were gathered on 29 to 31 May, on 6 July and on 10 August 2017. Subsequent to the sample pair acquisition, the whole trial field was cut evenly.

The procedure for sample acquisition was as follows:

1. Capture of an image of the clover-grass without interfering with the vegetation.

2. Placement of a $0.5 \times 0.5 \mathrm{~m}$ frame onto the vegetation.

3. Capture of an image to record the placement of the frame and cropping of the initial image accordingly.

4. Harvesting of all vegetation with stems inside the frame at a height of $5 \mathrm{~cm}$.

5. Separation of all plants as either ryegrass, white clover, red clover, or weeds.

6. Drying of the fractions at $60^{\circ} \mathrm{C}$ for $14 \mathrm{~h}$.

7. Weighing of the dry matter content of each fraction.

Due to the procedure of harvesting the plants based on stem placement, a potential mismatch was introduced between the photographed canopy and the analyzed dry matter. In homogeneous swards this error will approximately even out, due to the similarity of plants being removed and added to the cut. In sparse or inhomogeneous clover-grass swards, however, this uncertainty adds unwanted noise to the gathered sample pairs, especially in the presence of plants with high coverage such as red clover, dandelions, and thistles.

Figure 2 shows the variations in the collected sample pairs in terms of clover content in the dry matter composition and the total yield. The very low clover fraction in multiple samples of the 2016 establishment is due to poor plot establishment. 


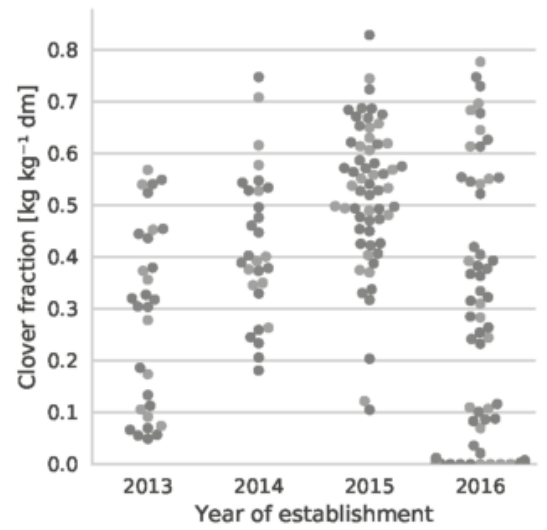

(a)

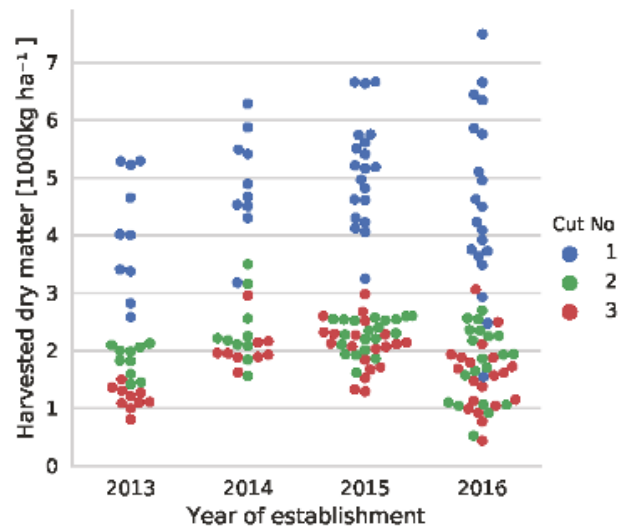

(b)

Figure 2. Overview of the variation in the 179 sample pairs sorted by the age of the swards. (a) Clover content in harvested material; (b) The total dry matter yield of harvested samples. The year axis is discrete, but points are spread out to avoid occlusion of samples.

\subsubsection{Camera Setup}

The core of the camera setup consisted of a Nikon D810A camera with two external light-emitting diode (LED) flashes. This setup was mounted on a push cart to fix the capture height, minimize movement during shooting, and ease the transportation in the field. The complete camera platform is shown in Figure 3 along with a sample image. The full 14-bit image resolution was exploited to reduce shadow regions in the vegetation using gamma correction and to correct for uneven lighting of the flashes as demonstrated in Figure 3c,d. Depending on the vegetation height and camera position, the gathered images had a ground sample distance (GSD) of 4-6 pixels per mm.

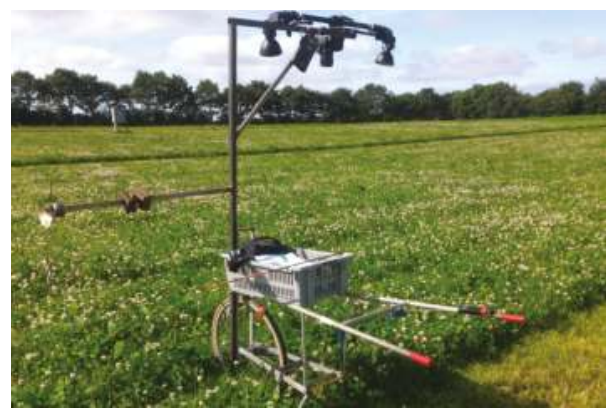

(a)

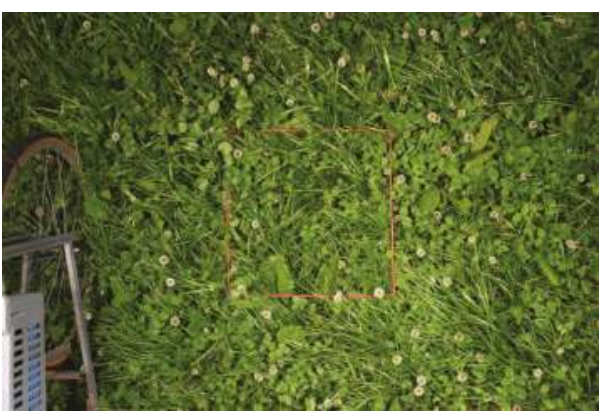

(b)

Figure 3. Cont. 


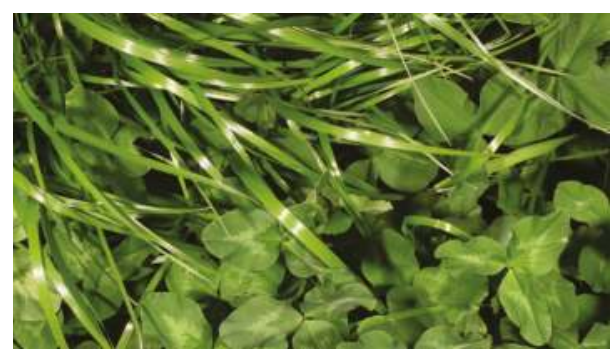

(c)

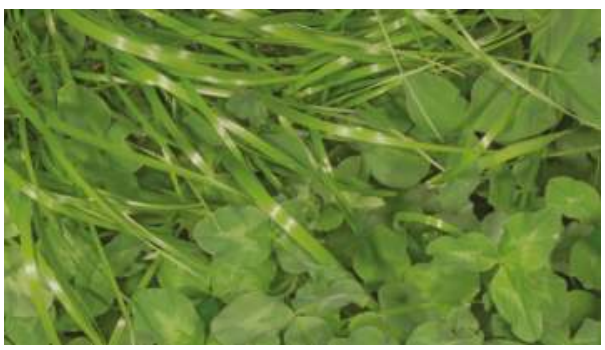

(d)

Figure 3. Depiction of the camera setup used for the sample acquisition and visualization of the gamma correction used to reduce the impact of shadow regions. (a) The pushcart with the camera system mounted on top; (b) Sample of acquired image; (c) Crop of an original captured image to illustrate the level of details; (d) Gamma-corrected version of (c) with a more uniform lighting and increased visibility.

\subsubsection{Image Labeling}

For evaluation of the semantic segmentation performance, a subset consisting of 10 of the sample pairs was selected for hand labeling. The subset was designed to cover the variation in the clover content by selecting the image sample closest to each of the the following clover dry matter contents: $5,10,20,30,40,50,60,70,80$, and $100 \%$. However, a sample with $100 \%$ clover content did not occur naturally in the field plots. Therefore, a single image sample from another dataset was included to maximize the range for semantic segmentation evaluation.

To reduce the time requirement for the hand-annotation task, a center crop of $1000 \times 1000$ pixels marks the area of the final annotation for each image in the subset. Four samples of the subset are shown in Figure 4 in the form of the original framed clover-grass image samples and the corresponding crops.

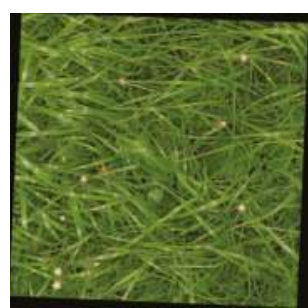

(a1)

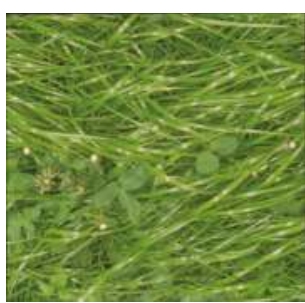

(b1)

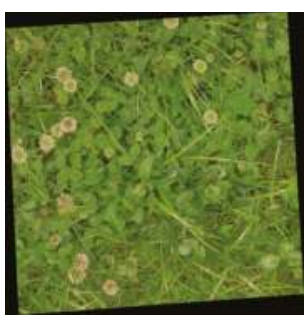

(c1)

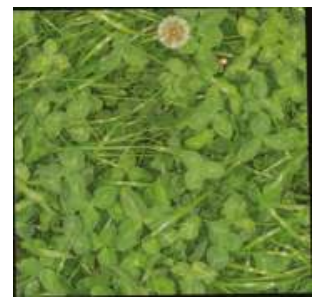

(d1)

Figure 4. Cont. 


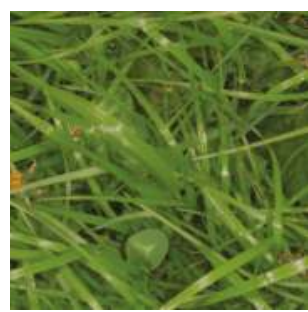

(a2)

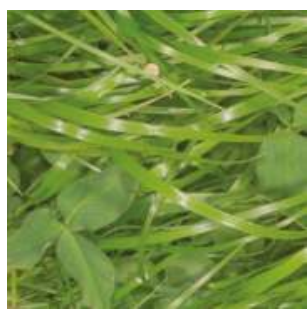

(b2)

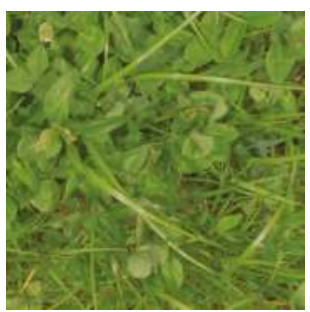

(c2)

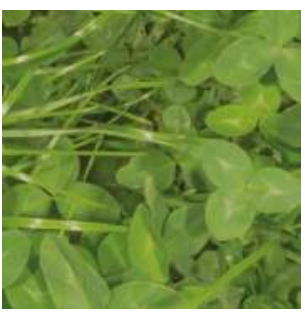

(d2)

Figure 4. Visualization of annotated image samples. Top row shows four samples with different clover contents. Bottom row shows crops of the images from the top row to illustrate the image resolution. The black pixels surrounding the image samples mark the border of the harvested samples. When the orientation of the camera does not align perfectly with the harvested patch, the image sample appears to be rotated. (a1) 5\% clover; (b1) 20\% clover; (c1) 50\% clover; (d1) 70\% clover; (a2) Annotated image patch of (a1); (b2) Annotated image patch of (b1); (c2) Annotated image patch of (c1); (d2) Annotated image patch of $(\mathrm{d} 1)$.

\subsubsection{Validation Field}

To validate the proposed method on a broader basis, additional sample pairs were gathered from field trials at the DLF Seed \& Science clover-grass breeding facility in Stevns, Denmark (WGS84: Latitude 55.33916, Longitude 12.38562). The sample acquisition procedure and equipment follow what was described in Sections 2.1.2 and 2.1.3. The field trials were established in 2015 and 2016 and consisted of ryegrass and white clover mixtures of numerous cultivars. In total, 50 plots were selected by visual inspection to maximize the spread in clover fraction, total yield, time since establishment, stress levels, and phenotypes. The resulting 50 validation sample pairs were acquired on 20 June 2017, as part of the second cut of the season.

\subsection{Image Simulation}

Convolutional neural networks require hundreds, or even thousands of labeled images for training [20]. In the case of semantic segmentation, where each pixel needs to be labeled, achieving this amount of data manually is unfeasible within a reasonable amount of time. This is particularly so in images of species mixtures, where leaves from different species occlude and intertwine with each other and thereby increase the complexity of the labeling task. As an example, it took an average of $3.5 \mathrm{~h}$ to annotate each of the 10 images (described in Section 2.1.4), all of which were of 1 megapixel. If all 179 primary samples were to be annotated for training, this would correspond to more than $3000 \mathrm{~h}$ of labor.

Dyrmann et al. [18] showed how a convolutional neural network can be trained to segment weeds and maize from each other when using only simulated images for training. Inspired by this work, a program which simulates images from clover-grass fields was created. The images are simulated using few hand-segmented samples of clovers, grasses, and weeds, which are placed on top of an image of soil. All the plant samples were acquired from clover-grass field images and selected samples are illustrated in Figure 5. The weeds include dandelion, thistle, and shepherd's purse. While generating simulated images, corresponding label-images are automatically generated. A label can take one of the following four classes: clover, grass, soil, and weed. A sample of a simulated images and its corresponding label is shown in Figure 6. 

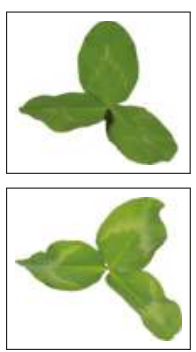

(a)

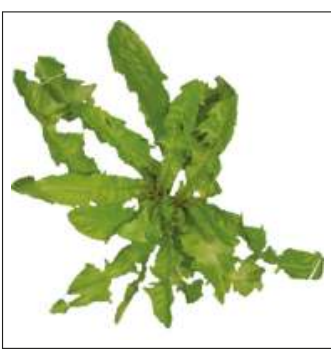

(b)

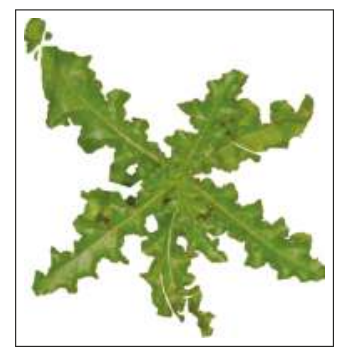

(c)

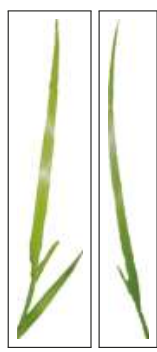

(d)

Figure 5. Samples of single plants that are used for simulating images. (a) clover; (b) dandelion; (c) thistle; and (d) grasses.

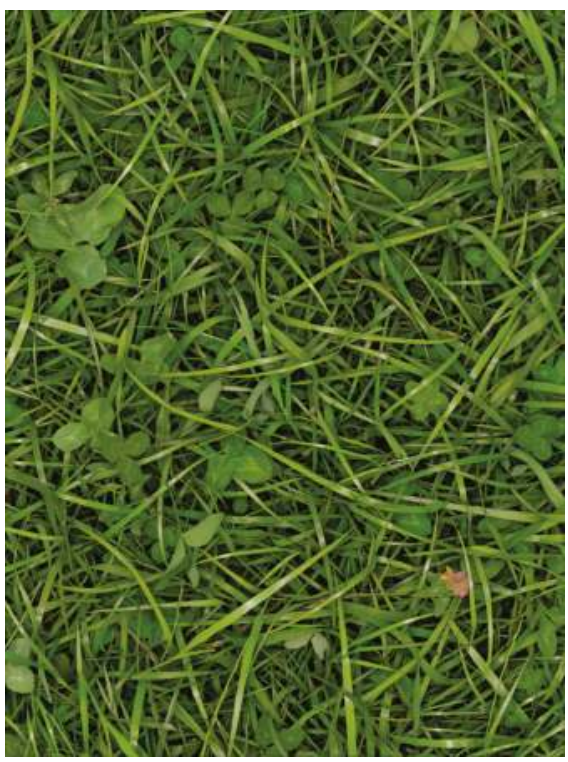

(a)

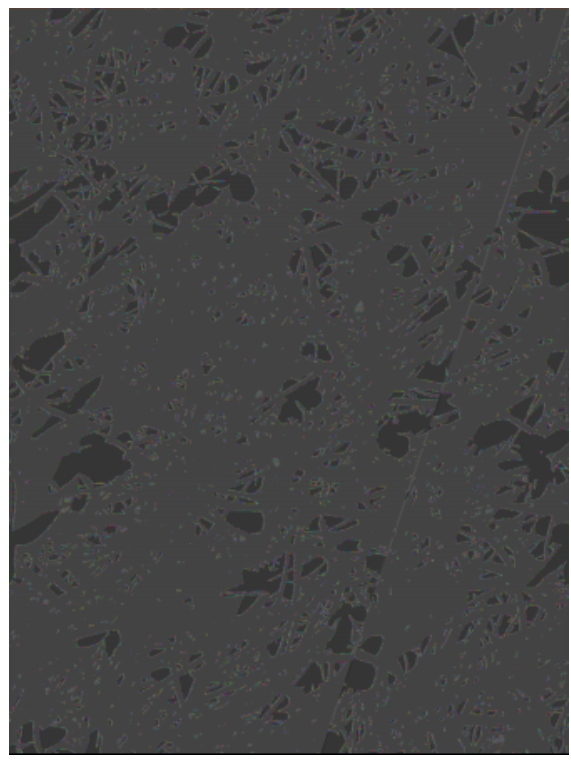

(b)

Figure 6. Simulated training data pair. (a) Simulated red, green, and blue (RGB) image; (b) Corresponding label image. Grass, clover and soil pixels in the RGB image are denoted by blue, red and black pixels in the label image, respectively.

In total, 55 samples of grass, 117 samples of clover, 14 samples of dandelion, 5 samples of thistle, and 4 samples of shepherd's purse were used for simulating the images. The total number of plant samples was 195. The effect of varying the number of plant samples is discussed in Section 3.1.

To reduce biases in the species distributions and introduce high levels of variability in the simulated images, several field compositions were simulated. Throughout the field compositions, the clover-grass plant ratio was uniformly distributed. Three different ratios of weeds were used, ranging from none to one-eighth of all the plants in the image. The simulated field density was varied from approximately 10 plants per square meter to complete coverage of the soil. Finally, the clovers were simulated with and without flowers to imitate differences between seasonal cuts. In total these variations amounted to 46 distinct field compositions with individual minor perturbations per 
simulated image. To train the neural network, 2810 simulated clover-grass images and corresponding label images were generated.

To increase the use of the plant samples and extend the variability of the simulated images, each plant sample was randomly augmented with respect to rotation, scale, and saturation before being placed in the simulated image. The angle of rotation was sampled from a discrete uniform distribution $U\left(0^{\circ}, 359^{\circ}\right)$ with $1^{\circ}$ increments, the scale was sampled from a uniform distribution in the interval $\pm 25 \%$ of the original size, and the saturation was sampled from a uniform distribution in the interval $\pm 25 \%$ of the original saturation. Furthermore, the depth was simulated by applying Gaussian shadows to plants when placing them in the image. Applying the large Gaussian kernel to a binary mask of the plant caused thin grasses to cast less shadow than larger clover leaves, leading to a satisfying visual effect. The shadows were added iteratively together with each plant instance, causing underlying soil and plants to become slowly darker. This effect is clearly seen in Figure 6a where areas with lower-placed vegetation appear darker.

\subsection{Convolutional Neural Network}

Since the introduction of AlexNet by Krizhevsky et al. [21] in 2012, convolutional neural networks (CNNs) have excelled in image classification tasks. These tasks are accomplished by learning abstract features using stacked layers of convolutions and non-linearities by backpropagating thousands of labeled training images through the network and updating the filter kernels accordingly. Contrary to image classification CNNs, the semantic segmentation task of this paper requires a classification output at each spatial position of the input image. This is traditionally accomplished by replacing fully connected layers with convolutions, to maintain spatial feature maps throughout the network, leading to a fully convolutional neural network. In this study, the fully convolutional network with a output stride of 8 (FCN-8s) architecture by Long et al. [17] was used. This model is a modification of the VGG16 architecture [22], which was made fully convolutional by transforming the fully connected layers to convolutional layers, while preserving the learned parameters. The network contains 15 convolutional layers, inbetween which there are five $2 \times 2$ max-pooling layers with a stride of 2 . Each of these pooling layers downscales the input by a factor of 2 horizontally and vertically. After the fifth pooling layer, the image is therefore downscaled by a factor of 32 . The pooling layers enable the network to learn semantics, at the expense of fine details, which are scarified. The last convolutional layer and two intermediate layers are followed by deconvolutional layers that up-sample the network output to the size of the input image. In order to compensate for the loss of details and spatial information, shortcuts are made from pooling layers three and four to the deconvolutional layers, whereby finer details can be partly restored. The network architecture is sketched in Figure 7.

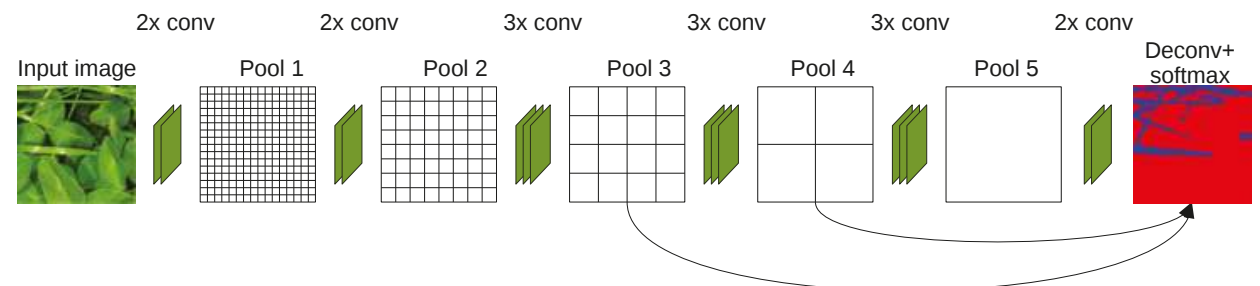

Figure 7. The fully convolutional network with a output stride of 8 (FCN-8s) architecture. The network consists of 15 convolutional layers and 5 max pooling layers. The outputs from pooling layers three and four were routed through deconvolution layers to help restore smaller details in the segmented images. (This figure is redrawn from Long et al. [17]). 
Training

The CNNs trained for this research followed the same procedure with regards to data augmentation and hyperparameters as the original architecture and were exclusively trained on simulated clover-grass images and labels. The networks' weights were initiated based on a pretrained VGG16 for image classification [22], and later trained for 12 epochs with a learning rate of $10^{-3}$, weight decay of $10^{-4}$ and mini-batches of four image crops of $1000 \times 1000$ pixels for semantic segmentation. The resolution of the original simulated images is comparable to the gathered clover-grass images of 4-6 pixels per $\mathrm{mm}$. To increase the robustness of the network towards images captured with various resolutions, at different heights and different plant sizes, the simulated images and corresponding label-images were augmented by scaling the images prior to training. The images were scaled to $100 \%$, $75 \%, 50 \%$, and $25 \%$ of their original size, which effectively increased the training data fourfold.

\section{Results}

As a result of splitting the task of determining the clover fraction into two problems, the presented results were separated similarly. First, the ability to semantically segment 10 test images was evaluated, followed by evaluation of the dry matter contents on all the gathered sample pairs.

\subsection{Image Segmentation}

To investigate the requirements of using real plant samples for clover-grass simulations, as shown in Section 2.2, the same network architecture was trained twice with different numbers of training samples. Therefore, the two trained models only differed in terms of the plant variation in the training images.

One model was trained on simulated images from all 195 plant samples. The other model was trained on simulated images from a subset of the 195 plant samples. The subset of plant samples was selected randomly and made up $25 \%$ of the 195 plant samples.

The FCN-8s models, trained on simulated data, were evaluated with respect to the quality of the semantic segmentation of real images into clover, grass, and weed pixels. This was tested on the 10 hand-annotated image crops. Following the accuracy metrics of Long et al. [17], the pixel-wise accuracy, mean intersection over union, and frequency-weighted intersection over union were calculated for each model and are shown in Table 1.

Table 1. Comparison of the approach by Bonesmo et al. [11] and the obtained results of FCN-8s on simulated images. Our results are presented when using all 195 plant samples $(100 \%)$ to simulate clover grass images and when using only $25 \%$ of the samples. IoU: intersection over union. F.w: frequency weighted.

\begin{tabular}{lcccc}
\hline Method & Trained Plant Samples & Pixel Accuracy & Mean IoU & F.w. IoU \\
\hline Bonesmo et al. [11] & - & 65.5 & 34.3 & 47.9 \\
FCN-8s & $25 \%$ & 82.2 & 62.4 & 70.7 \\
FCN-8s & $100 \%$ & 83.4 & 65.5 & 71.7 \\
\hline
\end{tabular}

For a fair comparison to the state of the art, the morphological approach of Bonesmo et al. [11] was implemented and evaluated. The test images for this approach were downscaled to match the original GSD stated in the work by Bonesmo et al. [11]. Additionally, the parameters were fine-tuned to the images, since the provided parameters led to poor classification results.

The FCN-8s architecture has a receptive field of $404 \times 404$ pixels, defining the area of the input image used for each pixel classification. Along the borders of the image this context information is reduced, leading to a reduced accuracy in the border region. This effect is particularly pronounced when the image size is reduced, as the border region takes up a larger portion of the total image area. 
To avoid this effect, the test images were semantically segmented in their original sizes, and later cropped, leading to a useful context in the evaluation.

From the segmentation results shown in Table 1, the improvement from the previous method is clear. The trained convolutional neural network increased the pixelwise accuracy by 17.9 percentage points due to the much higher level of abstraction.

Qualitative results of the semantic segmentation with the varying clover, grass, and weed densities of Figure 4 are shown in Figure 8. Several observations regarding the FCN-8s models are clearly visible in the four samples:

1. The networks learned to identify both grass and clover despite heavy occlusion.

2. Use of $100 \%$ of the samples resulted in a great improvement in weed detection. The majority of the weeds were correctly identified in the first column, while the number of false positives in the third column was reduced.

3. Both FCN-8s models were slightly biased towards detecting clover. While the classified grass and weed pixels appeared to follow the contours of the plants in the image, the clover classification appeared to be the default choice of the network. This was beneficial in the fourth row, but led to general misclassifications in the second and third rows.

These observations are supported by the quantitative results in Table 1. The networks reached a high pixel-wise accuracy of $82.2 \%$ and $83.4 \%$ for $25 \%$ and $100 \%$ samples, respectively. The increase in mean intersection over union (IoU) was of 3.2 percentage points when moving from $25 \%$ to $100 \%$ samples, while the frequency-weighted increase was of $1 \%$, supporting the argument that the less-present class of weeds is classified much better.
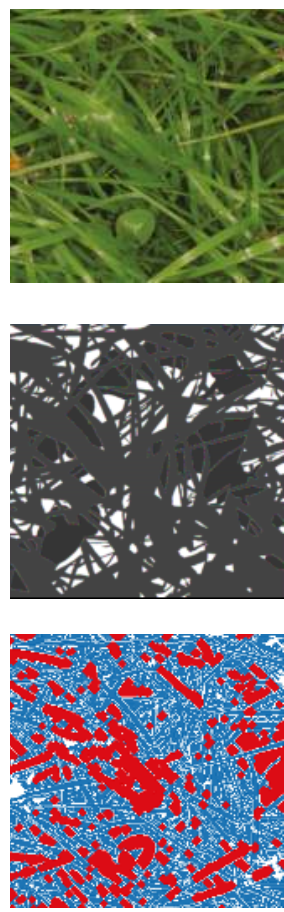
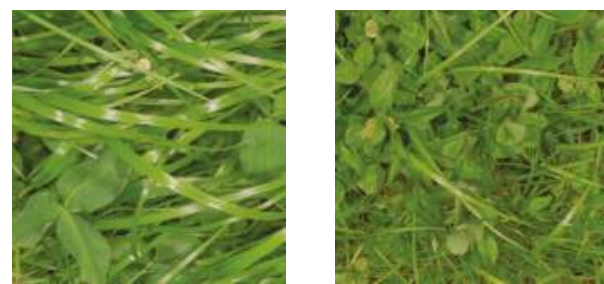

Image patch
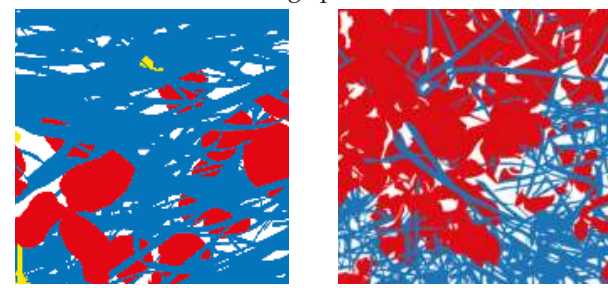

Ground truth
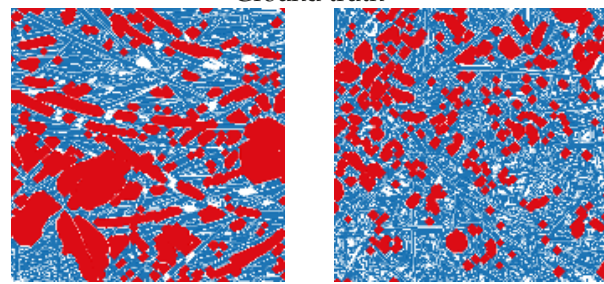

Bonesmo et al. [11]
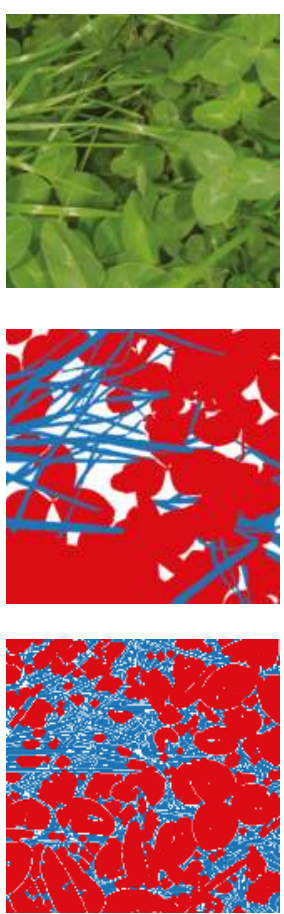

Figure 8. Cont. 

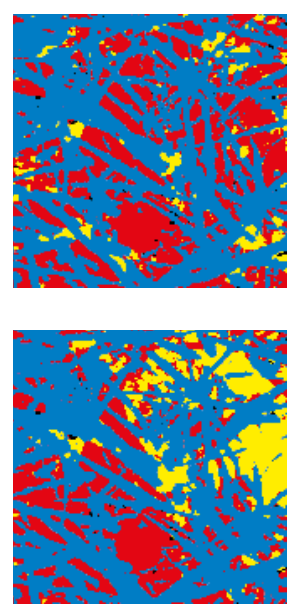
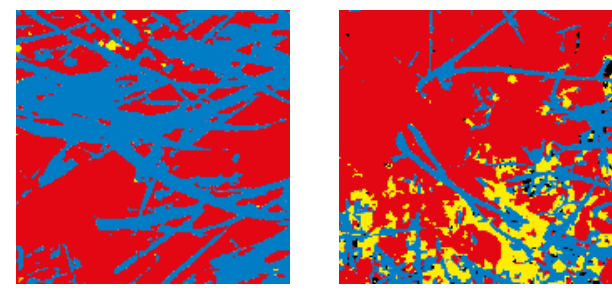

FCN-8s (25\% plant samples)
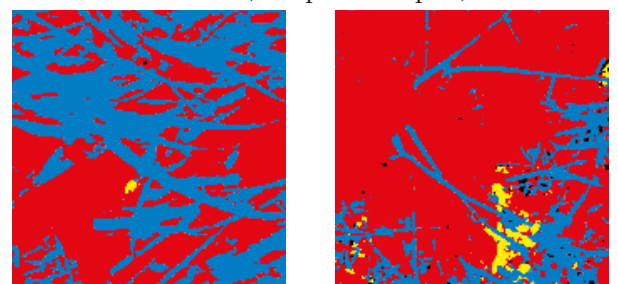

FCN-8s (100\% plant samples)
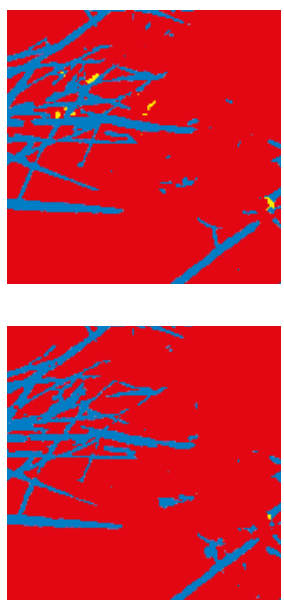

Figure 8. Comparison between four ground truth labels of the hand-annotated images, our implementation of the approach by Bonesmo et al. [11], and the two instances of convolutional neural network (CNN)-driven segmentation. The areas in blue represent grass, those in red represent clover, those in yellow represent weeds, those in black represent soil, and those in white represent areas that could not be distinguished in the manual annotation.

The approach of Bonesmo et al. [11] can in some cases detect clovers and grasses with high accuracy. This was clearly demonstrated in the fourth column. The morphological operations were, however, challenged by the varying leaf sizes across the test images. This was exemplified in the second and third column, where wide grass was classified as clover, and small clovers were classified as grass, respectively.

The possible use of leaf texture and the surrounding context by the FCN-8s network is believed to be of high importance when segmenting clover grass mixtures. Due to the high level of occlusions in the images, only a fraction of every plant is typically visible, while the leaf texture of the visible part remains.

\subsection{Clover Fraction Estimation}

To verify the usefulness of the trained CNN and validate the coupling between visual clover content in the canopy and clover dry matter fraction, the best-performing FCN-8s CNN was utilized to semantically segment the images from the 179 sample pairs.

In contrast to the isolated semantic segmentation case of Section 3.1, it was beneficial to increase the stability of the clover-grass analysis at the cost of sparsity in the semantic segmentation. It was better for shadow regions and foreign objects to be ignored than falsely classified, as they influenced the dry matter composition estimation. To apply this, the traditional non-max suppression used for semantic segmentation was substituted by a custom threshold on the individual softmax score maps of the CNN. By qualitative visual inspection of five images, the softmax thresholds were defined as $0.95,0.8$, and 0.3 for clover, weeds, and grass, respectively. This corresponds well to the overestimation of clover and underestimation of grass in Figure 8 in Section 3.1. Three samples of the thresholded segmentation of clover-grass images are shown in Figure 9.

Qualitatively, the three samples of semantic segmentation demonstrated an excellent understanding of the images by the convolutional neural network across vegetation densities and clover fractions. Visible weeds, clover, and grasses were detected with high accuracy while withered vegetation and ground were correctly ignored. 


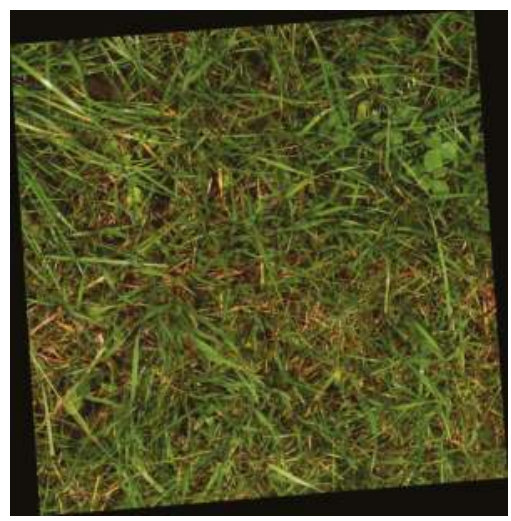

(a1)

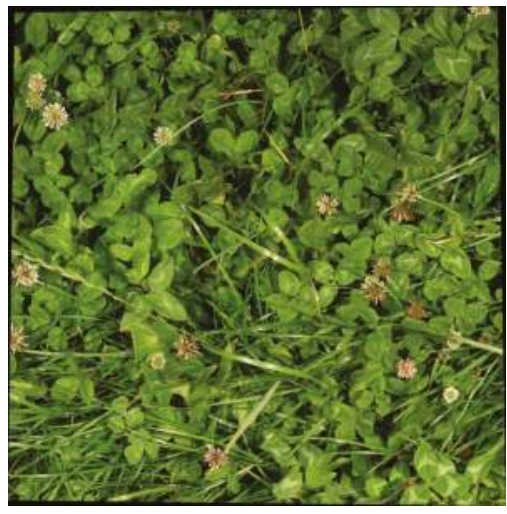

(b1)

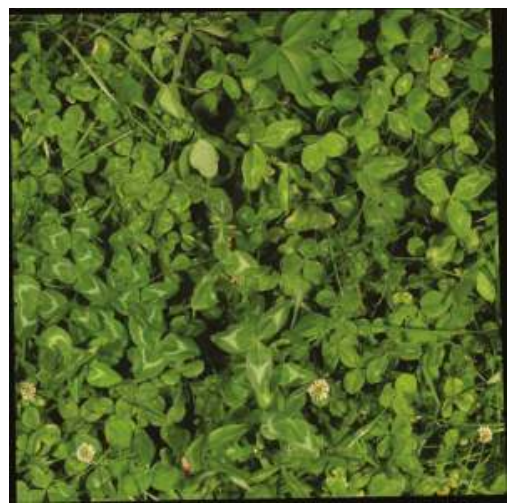

(c1)

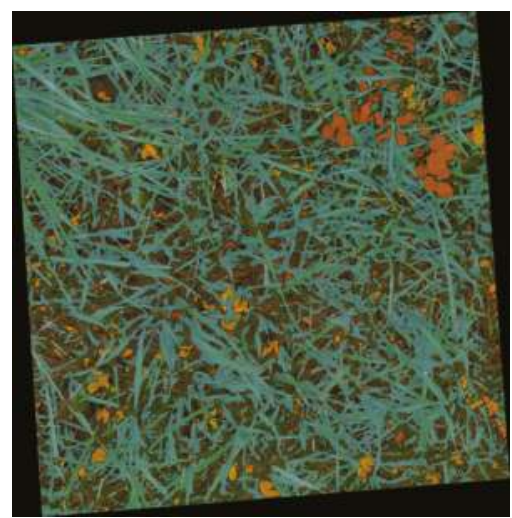

(a2)

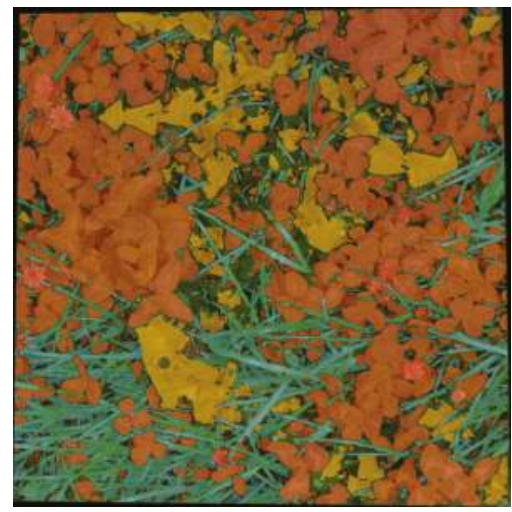

(b2)

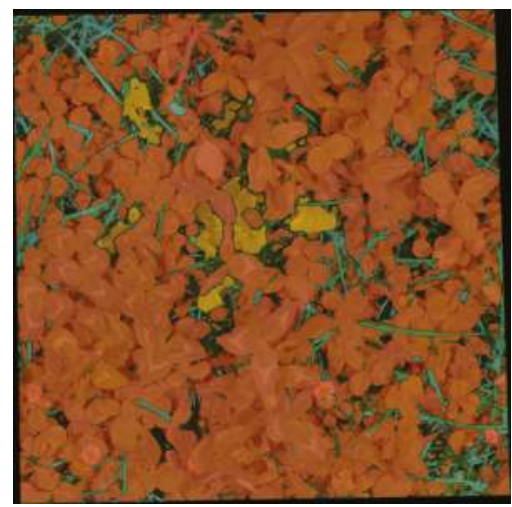

(c2)

Figure 9. Visualization of $\mathrm{CNN}$-driven semantic segmentation of highlighted sample pairs from Figure 10. The first column shows the evaluated image. The second column shows the thresholded identification of grass (blue), clover (red), and weeds (yellow) in the image. (a1) Low clover fraction; (a2) Semantic segmentation of (a1); (b1) Medium clover fraction; (b2) Semantic segmentation of (b1); (c1) High clover fraction; (c2) Semantic segmentation of (c1). 


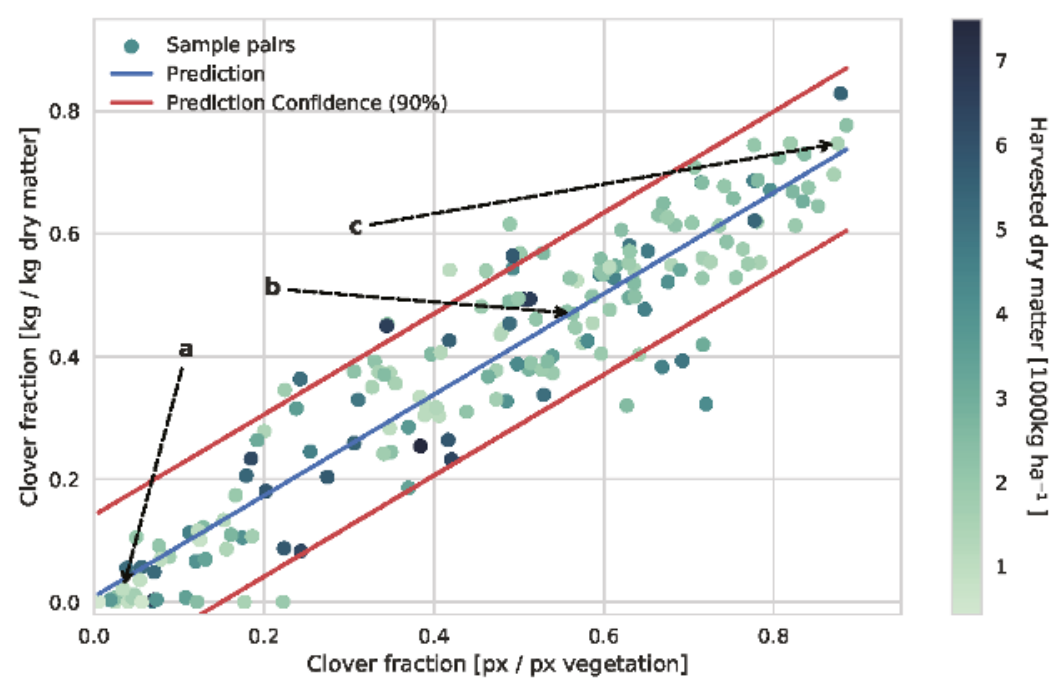

Figure 10. Comparison and linear regression of the visual clover ratio determined by the CNN and the actual clover ratio in the harvested dry matter at the primary field site. The linear relationship between the visual and dry matter fraction of clover in the sample pairs is clear. The annotations $a, b$, and $c$ refer to the three image samples shown in Figure 9.

The coupling between the clover-grass images and dry matter composition was evaluated using the clover fraction metrics defined in Equations (1) and (2) for dry matter samples and image samples, respectively:

$$
\begin{aligned}
\text { fraction }_{\text {clover, } D M} & =\frac{\text { clover }_{\text {dry matter }}}{\text { vegetation }_{\text {dry matter }}} \\
\text { fraction }_{\text {clover, } p x} & =\frac{N_{\text {clover, } \text { pixel }}}{N_{\text {vegetation, pixel }}}
\end{aligned}
$$

The result of the automated CNN-driven image analysis of visual clover content is shown in Figure 10. The relationship between the CNN-driven analysis and the dry matter clover ratio in the photographed clover-grass patches was eminent and similar to the manually obtained relationship shown by Himstedt et al. [14]. The linear regression based on the 179 primary samples pairs led to a clover dry matter fraction prediction with a standard deviation of $7.8 \%$. This was achieved while covering the spread of total yield, clover fraction, weed levels, fraction of red and white clover, and time in season of the sample pairs.

Besides the general fitness of the linear regression, multiple outliers appeared in Figure 10. Looking into the underlying image samples and image analysis, two distinct causes were observed.

1. The image was out of focus in sparse or low-yield vegetation.

The low-yield outliers along the x-axis all suffered from overestimated clover content. Due to the loss of focus in these samples, blurred vegetation on the ground was often misclassified as clover.

2. The clover-covered area of the canopy did not always represent the dry matter composition. In the range of 30-60\% dry matter clover fraction, the canopy could be less representative of the harvested samples. This was the result of the heavy occlusions and was directly linked to the challenges of using the top-down view. The furthest outlier $(0.75,0.32)$ was for example a dense clover grass mixture, covered by a shallow layer of clover leaves, giving the impression of a much higher clover fraction. 
The trained $\mathrm{CNN}$ was also evaluated on the validation field located $210 \mathrm{~km}$ from the primary field site, while preserving the threshold parameters. The sample pair relationship of the validation field is shown in Figure 11. When comparing the results of the two field sites, the validation site led to a similar relationship, with an introduction of an offset. This offset shifts the validation sample pairs towards the upper region of the prediction confidence of the estimator in Figure 10, leading to a general underestimation of the clover fraction in the validation field.

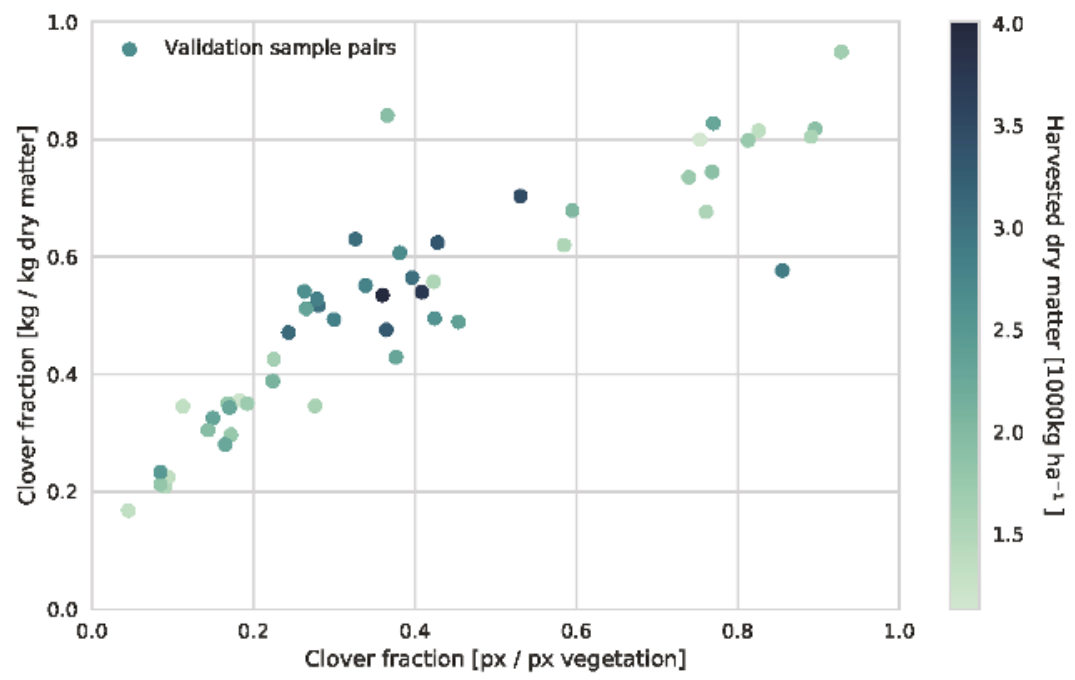

Figure 11. Comparison of the visual clover ratio and the dry matter clover ratio of the validation field site. The linear relationship is more poorly defined, with an introduction of a noticeable offset, possibly due to a less complete segmentation of clovers in the image samples.

\section{Discussion}

The ability to train a CNN for image segmentation using solely simulated images has been shown to have good prospects, as it allows one to train a network for tasks for which it would otherwise be unfeasible to achieve the needed amount of data. The acquired 195 plant samples for training have been additionally been reduced by $75 \%$, leading to a pixelwise accuracy drop of 1.2 percentage points. This drop is mainly a result of worsened distinction between clover and weeds, caused by the use of only six weeds. This low number of samples does not cover the variation of weeds in the test data in terms of number of neither species nor appearances. This demonstrates that high classification accuracies can be achieved on real images with only few plant samples used for training, and this translates to reaching a working prototype for semantic segmentation within hours with manual labor, as opposed to weeks or months if following a traditional work flow of manual image labeling.

Care should be taken when training the convolutional neural network to span the variations in the test data by training using the corresponding variations in the training data. This was the case for vegetation density and varying ratios of clover, grass, and weeds in the simulated images. More care should be taken when simulating training data to imitate natural and common errors, introduced when collecting images. These include lighting conditions, color temperature, image noise, and blurring. Several of the images with a high ratio of misclassified pixels were blurred. As the network was trained on sharp images, it is believed that the number of misclassifications can be reduced by introducing blurred simulated images in the training data. 
From the estimate of the clover content of the dry matter we see that the uncertainty is larger in cases where the clover and grass are mixed compared to cases dominated by one species. This is because the camera can only see the canopy of the plant cover, and plants of one species that is hidden by the others would thereby make the estimated ratio less accurate. In cases where the sward is dominated by one species, the estimated dry matter-ratio would be less affected by this phenomenon. Nonetheless, the estimated ratio is close to the real ratio even in cases where the real ratio is close to $50 \%$.

When evaluating the system on the validation field at a separate location, the automated image-based estimation of the dry matter clover fraction does not translate accurately between the two field sites. The design of the two field trials differs largely, mainly in terms of absence of red clover in the validation field seed mixture, fertilization strategies, organic or conventional farming, and variation of cultivars in the plots. Through experiments outside of this paper, it has been shown that the system accurately translates to the validation field when lowering the threshold value for detecting clover pixels from 0.95 to 0.85 . This suggests a visual difference between the clovers in the two field sites, leading to partly unclassified clovers. By introducing larger variations of clovers from multiple locations in the image simulation, the $\mathrm{CNN}$ should be generalized to better handle natural visual variances between the fields.

It is essential to be aware of the accuracy of alternative methods for estimation the botanical dry matter composition. While separating a forage sample into species by hand does provide the ground truth composition for this paper, this is not feasible for real applications. Fair comparisons include vision-based evaluation by expert consultants in the field and near-infrared reflectance spectroscopy (NIRS)-based estimation, often integrated in grass sward harvesters used for research.

While the visual estimation accuracy of consultants remains undocumented, this method is time consuming and requires the consultant to inspect the clover-grass throughout the field and map it accordingly. NIRS-based methods, such as in [23], show comparable estimation accuracy of grass swards of either white clover or red clover, by use of distinct models for each case. The accuracy on grassland swards with mixed red and white clover species, as in this experiment, has not been investigated.

To utilize the botanical composition of clover-grass leys for targeted fertilization, the information must be available at the time of fertilization. The largest amount of fertilizer is typically applied in the spring prior to the start of the growth period. At this stage NIRS for identifying the botanical composition is ruled out, since the crop is too small for harvesting. This leaves a great potential for the non-destructive approach of monitoring clover-grass mixtures in this paper. Future work includes extending the presented method for clover content estimation to preseason image samples. This extension allows the camera-based system to provide the necessary botanical information for optimizing the fertilizing strategy, for every fertilization. Following the approach presented in this paper, the image analysis of the extension can be operational with a couple of days of labor for gathering relevant image samples and cropping out representative plant samples for data simulation. Other future work relates to extending the parametric information level delivered by the convolutional neural network to further improve the dry matter composition estimates, or directly predicting the dry matter botanical composition in the neural network.

\section{Conclusions}

It has been shown that a fully convolutional neural network can be trained to semantically segment images of clover-grass swards using only simulated images. This fully convolutional neural network has been proven capable of discriminating between clover, grass, and weeds in real images at a pixel level with high accuracies.

The clover fraction that is estimated from the segmentation has been shown to be directly coupled to the clover fraction of the dry matter, making the method a cheap, nondestructive, and applicable method for determining the clover fraction, which is needed to fertilize optimally. Furthermore, 
it presents a research tool for investigating clover and grass dynamics in mixed swards more efficiently and with better spatial and temporal resolution than is possible with laborious separation by hand.

Supplementary Materials: The following are available online at www.mdpi.com/1424-8220/17/12/2930/s1, Figure 1: Example of the image analysis on a real image. Each element in the image is analyzed and classified as either grass (blue), clover (red), weeds (yellow) or unidentified (black overlay). (a) Example input image. (b) Automatically analyzed image.

Acknowledgments: The work was founded by Green Development and Demonstration Programme (GUDP) under the Danish Ministry for Food, Agriculture and Fisheries, and Innovation Fund Denmark. The camera setup was kindly provided by AgroTech, Teknologisk Institut. The validation field was kindly provided by DLF Seed \& Science.

Author Contributions: Søren Skovsen collected the image data. Jørgen Eriksen provided the Foulum field site and the corresponding dry matter analysis. René Gislum provided the DLF field site and the corresponding dry matter analysis. Søren Skovsen and Mads Dyrmann implemented the simulation algorithm and evaluated the algorithm's performance. Søren Skovsen and Anders Krogh Morten developed the Tensorflow environment for training FCN-8s. Anders Krogh Mortensen implemented, fine-tuned, and evaluated the compared method for semantic segmentation. Rasmus N. Jørgensen and Henrik Karstoft advised on the research. Søren Skovsen, Mads Dyrmann, Anders Krogh Mortensen, Rasmus N. Jørgensen, René Gislum, Henrik Karstoft, Kim Arild Steen, and Ole Green wrote the paper.

Conflicts of Interest: The authors declare no conflict of interest.

\author{
Abbreviations \\ The following abbreviations are used in this manuscript: \\ CNN Convolutional neural network \\ FCN-8 Fully convolutional network with a output stride of 8. [17] \\ HSL Hue, saturation, and lightness \\ IoU Intersection over union. Also known as the Jaccard index. \\ LED Light-emitting diode \\ N Nitrogen \\ NIRS Near-infrared reflectance spectroscopy \\ RGB Red, green, and blue \\ WGS84 World geodetic system 1984
}

\title{
References
}

1. Eriksen, J.; Askegaard, M.; Søegaard, K. Complementary effects of red clover inclusion in ryegrass-white clover swards for grazing and cutting. Grass Forage Sci. 2014, 69, 241-250.

2. Frankow-Lindberg, B.E.; Halling, M.; Höglind, M.; Forkman, J. Yield and stability of yield of singleand multi-clover grass-clover swards in two contrasting temperate environments. Grass Forage Sci. 2009, 64, 236-245.

3. Phelan, P.; Moloney, A.P.; McGeough, E.J.; Humphreys, J.; Bertilsson, J.; O’Riordan, E.G.; O'Kiely, P. Forage Legumes for Grazing and Conserving in Ruminant Production Systems. Crit. Rev. Plant Sci. 2015, 34, 281-326.

4. Nyfeler, D.; Huguenin-Elie, O.; Suter, M.; Frossard, E.; Connolly, J.; Lüscher, A. Strong mixture effects among four species in fertilized agricultural grassland led to persistent and consistent transgressive overyielding. J. Appl. Ecol. 2009, 46, 683-691.

5. Egaard, K.S. Nitrogen fertilization of grass/clover swards under cutting or grazing by dairy cows. Acta Agric. Scand. Sect. B-Soil Plant Sci. 2009, 59, 139-150.

6. Suter, M.; Connolly, J.; Finn, J.A.; Loges, R.; Kirwan, L.; Sebastià, M.T.; Lüscher, A. Nitrogen yield advantage from grass-legume mixtures is robust over a wide range of legume proportions and environmental conditions. Glob. Chang. Biol. 2015, 21, 2424-2438.

7. Kirwan, L.; Lüscher, A.; Sebastia, M.T.; Finn, F.; Collins, R.; Porqueddu, C.; Helgadottir, A.; Baadshaug, O.H.; Brophy, C.; Coran, C.; et al. Evenness drives consistent diversity effects in intensive grassland systems across 28 European sites. J. Ecol. 2007, 95, 530-539. 
8. Nyfeler, D.; Huguenin-Elie, O.; Suter, M.; Frossard, E.; Lüscher, A. Grass-legume mixtures can yield more nitrogen than legume pure stands due to mutual stimulation of nitrogen uptake from symbiotic and non-symbiotic sources. Agric. Ecosyst. Environ. 2011, 140, 155-163.

9. Landbrug \& Fødevarer, Planteproduktion. Oversigt Over Landsforsøgene 2014; Videncentret for Landbrug: Midtjylland, Danmark, 2014.

10. Williams, A.; Audsley, E.; Sandars, D. Determining the Environmental Burdens and Resource Use in the Production of Agricultural and Horticultural Commodities; Defra Project Report IS0205; Cranfield University and DEFRA: Bedford, UK, 2006. Available online: http://randd.defra.gov.uk/Default.aspx (accessed on 15 September 2017).

11. Bonesmo, H.; Kaspersen, K.; Bakken, A.K. Evaluating an image analysis system for mapping white clover pastures. Acta Agric. Scand. Sect. B-Soil Plant Sci. 2004, 54, 76-82.

12. Himstedt, M.; Fricke, T.; Wachendorf, M. Determining the contribution of legumes in legume-grass mixtures using digital image analysis. Crop Sci. 2009, 49, 1910-1916.

13. Himstedt, M.; Fricke, T.; Wachendorf, M. The relationship between coverage and dry matter contribution of forage legumes in binary legume-grass mixtures. Crop Sci. 2010, 50, 2186-2193.

14. Himstedt, M.; Fricke, T.; Wachendorf, M. The benefit of color information in digital image analysis for the estimation of legume contribution in legume-grass mixtures. Crop Sci. 2012, 52, 943-950.

15. Rayburn, E.B. Measuring legume content in pastures using digital photographs. Forage Grazinglands 2014, 12, doi:10.2134/FG-2011-0143-MG.

16. McRoberts, K.C.; Benson, B.M.; Mudrak, E.L.; Parsons, D.; Cherney, D.J. Application of local binary patterns in digital images to estimate botanical composition in mixed alfalfa-grass fields. Comput. Electron. Agric. 2016, 123, 95-103.

17. Long, J.; Shelhamer, E.; Darrell, T. Fully Convolutional Networks for Semantic Segmentation. In Proceedings of the IEEE Conference on Computer Vision and Pattern Recognition, Boston, MA, USA, 7-12 June 2015; pp. 3431-3440.

18. Dyrmann, M.; Mortensen, A.K.; Midtiby, H.S.; Jørgensen, R.N. Pixel-wise classification of weeds and crops in images by using a fully convolutional neural network. In Proceedings of the International Conference on Agricultural Engineering, Aarhus, Denmark, 26-29 June 2016.

19. Mortensen, A.K.; Dyrmann, M.; Karstoft, H.; Jørgensen, R.N.; Gislum, R. Semantic segmentation of mixed crops using deep convolutional neural network. In Proceedings of the International Conference on Agricultural Engineering, Aarhus, Denmark, 26-29 June 2016.

20. Oquab, M.; Bottou, L.; Laptev, I.; Sivic, J. Learning and Transferring Mid-level Image Representations Using Convolutional Neural Networks. In Proceedings of the 2014 IEEE Conference on Computer Vision and Pattern Recognition, Columbus, OH, USA, 23-28 June 2014; pp. 1717-1724.

21. Krizhevsky, A.; Sutskever, I.; Hinton, G.E. ImageNet Classification with Deep Convolutional Neural Networks. In Advances in Neural Information Processing Systems 25; Pereira, F., Burges, C.J.C., Bottou, L., Weinberger, K.Q., Eds.; Curran Associates, Inc.: Red Hook, NY, USA, 2012; pp. 1097-1105.

22. Simonyan, K.; Zisserman, A. Very Deep Convolutional Networks for Large-Scale Image Recognition. arXiv 2014, arXiv:1409.1556v6.

23. Wachendorf, M.; Ingwersen, B.; Taube, F. Prediction of the clover content of red clover- and white clover-grass mixtures by near-infrared reflectance spectroscopy. Grass Forage Sci. 1999, 54, 87-90.

(C) 2017 by the authors. Licensee MDPI, Basel, Switzerland. This article is an open access article distributed under the terms and conditions of the Creative Commons Attribution (CC BY) license (http:/ / creativecommons.org/licenses/by/4.0/). 
Article

\title{
A Low-Cost Approach to Automatically Obtain Accurate 3D Models of Woody Crops
}

\author{
José M. Bengochea-Guevara ${ }^{1}$, Dionisio Andújar ${ }^{1,2}$, Francisco L. Sanchez-Sardana ${ }^{1}$, \\ Karla Cantuña ${ }^{1,3}$ and Angela Ribeiro ${ }^{1, *}$ \\ 1 Centre for Automation and Robotics, CSIC-UPM, Arganda del Rey, Madrid 28500, Spain; \\ jose.bengochea@csic.es (J.M.B.-G.); dionisioandujar@hotmail.com (D.A.); fl.sanchez@csic.es (F.L.S.-S.); \\ karlacantunaflores@yahoo.es (K.C.) \\ 2 Institute of Agricultural Sciences, CSIC, Madrid 28006, Spain \\ 3 Departamento de Ingeniería Informática y Sistemas Computacionales, Cotopaxi Technical University, \\ Latacunga 050101, Ecuador \\ * Correspondence: angela.ribeiro@csic.es; Tel.: +34-918-711-900
}

Received: 14 October 2017; Accepted: 20 December 2017; Published: 24 December 2017

\begin{abstract}
Crop monitoring is an essential practice within the field of precision agriculture since it is based on observing, measuring and properly responding to inter- and intra-field variability. In particular, "on ground crop inspection" potentially allows early detection of certain crop problems or precision treatment to be carried out simultaneously with pest detection. "On ground monitoring" is also of great interest for woody crops. This paper explores the development of a low-cost crop monitoring system that can automatically create accurate 3D models (clouds of coloured points) of woody crop rows. The system consists of a mobile platform that allows the easy acquisition of information in the field at an average speed of $3 \mathrm{~km} / \mathrm{h}$. The platform, among others, integrates an RGB-D sensor that provides RGB information as well as an array with the distances to the objects closest to the sensor. The RGB-D information plus the geographical positions of relevant points, such as the starting and the ending points of the row, allow the generation of a $3 \mathrm{D}$ reconstruction of a woody crop row in which all the points of the cloud have a geographical location as well as the RGB colour values. The proposed approach for the automatic 3D reconstruction is not limited by the size of the sampled space and includes a method for the removal of the drift that appears in the reconstruction of large crop rows.
\end{abstract}

Keywords: 3D reconstruction; RGB-D sensor; crop inspection platform

\section{Introduction}

Precise crop monitoring helps farmers improve crop quality and reduce operational costs by making better crop management decisions. Thus, yield estimation is typically based on crop knowledge, historical data, meteorological conditions and crop monitoring that is generally conducted via manual sampling. However, manual sampling is a time-consuming, labour-intensive, and frequently inaccurate process, mainly because the number of samples is often too small to capture the magnitude of variations in a crop, particularly for regions of more than several hectares. Thus, it is extremely important to identify an automated and efficient alternative to manual sampling that can accurately capture the spatial and temporal variations of a crop. Towards this end, vehicles equipped with on-board sensing equipment are a promising choice among the various means of collecting well-structured information. Furthermore, the use of medium-sized platforms for full crop scouting is a suitable choice for minimising soil compaction that, among other applications, enables the performance of more than one sampling throughout the year due to having minimal impacts on the crop. 
Alternatively, 3D reconstruction of woody crop models using non-destructive methods is a valuable technique to improve the decision-making processes. The use of sensors for the characterisation of crops leads to a better understanding of the processes involved in tree development throughout their life cycle. With information obtained from a 3D reconstruction of the crop, important parameters, such as the growth status, height, shape, biomass, need for nutrients, and health status, can be estimated. These parameters are currently mostly estimated by applying equations that assume the trees to be geometric solids (regular polygons) or by applying empiric models [1], which produce inconsistent results. The use of the information extracted from 3D reconstructions can improve the decisions made related to crop management and contribute to creating new protocols to improve the profitability and health of plants.

RGB-D sensors are one of the most promising solutions on the market to obtain data for reconstructing 3D models of a scene. In particular, the Microsoft Kinect v2 sensor has assumed a leading role in recent years because of its low cost and favourable performance. This sensor, based on time-of-flight (ToF) technology, provides additional information to the RGB data, i.e., depth, infrared and skeleton frames that, among other applications, have been used to characterise plants in agriculture. Thus, in [2], the authors compared two low-cost 3D systems, including the Kinect v2 sensor, with an expensive high-precision laser scanner and concluded that the low-cost systems can replace the more expensive scanner in several plant phenotyping scenarios. The use of the Kinect v2 sensor was proposed in [3] to determine the volume of weeds in maize crops and to define their treatment period. The results suggest that this sensor can serve as a high-precision device in estimating the volume of weeds and determining the state of the crop. In [4], a Kinect v2 sensor was used to estimate the volume of onions, showing that the calculated volume was directly related to the estimation based on the measurements from the sensor. As a result, although 3D reconstruction is a research topic associated with numerous and important results involving computer vision, the emergence of sensors, such as the above-mentioned sensors, which provide information on the distance to the objects closest to the scene (depth data), has enabled new possibilities in 3D reconstruction. Several recent research studies have focused on acquiring 3D scene reconstruction using the depth images supplied by these new sensors. Furthermore, various techniques have been proposed that employ distinct types of accelerated data structures using graphics hardware to combine consecutive depth images with a certain degree of overlap. Each technique has its own advantages and disadvantages in terms of speed, scale and quality of reconstruction.

Certain methods use the voxel structure, which represents a value on a regular grid in 3D space to store 3D sensor information [5-7]. A well-known example is the method described in [6], which generates high-quality 3D reconstructions [8] and was adopted by Kinect Fusion $[9,10]$, i.e., it is the $3 \mathrm{D}$ reconstruction method included by Microsoft in its software development kit-Kinect for Windows Software Development Kit 2.0 [11]. However, the method has the important constraint of not allowing the reconstruction of large scenarios with volumes larger than 8 cubic metres. This constraint has motivated the emergence of variants on the method that allow reconstructions of larger volumes using voxel structures [12-14], while other strategies are based on the use of hierarchical data structures, which divide the space effectively but are not easily parallelized, thus resulting in added computational complexity $[15,16]$.

One of the limitations of 3D reconstruction methods is that they estimate the position and orientation of the sensor with the information obtained from the set of images, i.e., slight variations can exist between the calculated position and orientation and the actual values obtained by the sensor. Such differences arise primarily with similar scenes, such as crops, in which the same structure is repeated, appearing as analogous information (e.g., canopies full of similar leaves existing at similar distances from the sensor). These slight variations in calculated position and orientation can give rise to drift that causes deformations in the $3 \mathrm{D}$ reconstruction, with the deformations being more pronounced with the greater size of the reconstruction. 
The overall objective of the present work is to provide a method to automatically generate 3D reconstructions of large zones, such as a complete crop row, from the information directly supplied by the Microsoft Kinect v2 sensor on-board a vehicle. The presented method includes a technique to manage the drift that appears in the reconstruction, which is accentuated with the line length.

\section{Materials and Methods}

\subsection{The Acquisition Equipment at the Field}

The RGB-D sensor Kinect v2 (Microsoft, Redmond, WA, USA), which operates at 30 fps, supplies RGB images with a resolution of $1920 \times 1080$ pixels together with depth information with a resolution of $512 \times 424$ pixels. The depth data is obtained via a ToF system inside the sensor, which modulates a light source using a square wave. The sensor uses phase detection to measure the time for light to travel from the light source to the object and back to the sensor and then estimates the distance from the results. The system calculates the distance from the speed of light in air by estimating the received light phase at each pixel with knowledge of the modulation frequency. The depth measurement range of the sensor is 0.5-4.5 $\mathrm{m}$ [17]; outdoors, however, the maximum range decreases. Specifically, studies conducted outdoors under different daytime illumination conditions [18] show that the sensor provides valid depth measurements up to $1.9 \mathrm{~m}$ during sunny days, while the distance increases up to $2.8 \mathrm{~m}$ under the diffuse illumination of an overcast day.

The Kinect v2 sensor is placed in a field platform (Figure 1) using an aluminium support structure. The field platform is based on a Twizy Urban 80 model (Renault, Valladolid, Spain), which has a $13 \mathrm{~kW}$ electric motor and can travel up to $80 \mathrm{~km} / \mathrm{h}$. The vehicle is ultra-compact, with a length of $2.32 \mathrm{~m}$, width of $1.19 \mathrm{~m}$, height of $1.46 \mathrm{~m}$ and unladen weight of $450 \mathrm{~kg}$; moreover, the completed battery charge allows a total travel distance of over $80 \mathrm{~km}$. The electric motor of the vehicle allows negligible vibration at speeds below $3 \mathrm{~km} / \mathrm{h}$ [19], which is convenient for high-quality information acquisition.

In addition to the Kinect v2, the platform is equipped with another sensor, a digital single-lens reflex camera (EOS 7D, Canon, Tokyo, Japan), which supplies high-quality RGB images at $2 \mathrm{fps}$ with a resolution of $2592 \times 1728$ pixels. Both sensors are connected to the on-board computer, which contains an Intel Core i7-4771@3.5GHz processor, 16 GB of RAM, and an NVIDIA GeForce GTX 660 graphic card. The platform is also equipped with a RTK-GNSS receiver, an R220 receiver (Hemisphere, Scottsdale, AZ, USA), which provides location data at a $20 \mathrm{~Hz}$ sample rate with an accuracy of $20 \mathrm{~mm}+2 \mathrm{ppm}$ (2DRMS, 95\%) according to the manufacturer's specifications. The total cost of the described system was approximately 8000 euros, not including the reflex camera.

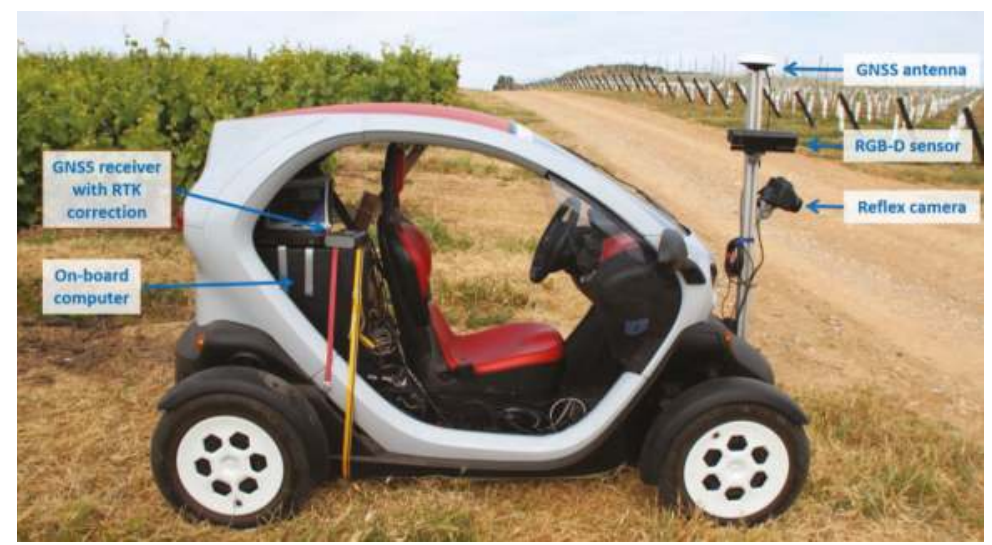

Figure 1. Field platform and on-board equipment. 
To illustrate the implications of a journey of $80 \mathrm{~km}$ (as determined by the autonomy of vehicle) in crop inspection, we can estimate the hectares that would be covered in the case of a woody crop inspected using RGB-D sensors. If the woody crop is a vineyard, then the space between crop rows (lane) is typically $2 \mathrm{~m}$. If two RGB-D sensors are placed on both sides of the vehicle to record images of each row while the vehicle advances along the lane, the vehicle can cover a total of 16 ha. The covered area can be somewhat decreased, considering the consumption of energy of the equipment connected to the battery of the vehicle and the movements made in the crop headers to change lanes.

The inspection plan to be followed by the platform is generated by a path planner [20], which can be formulized as the well-known capacitated vehicle routing problem, as stated in [21]. The fundamental problem consists of determining the best inspection route that provides complete coverage of the field considering features (such as the field shape, crop row direction, and type of crop) and certain characteristics of the platform (such as the turning radius or the number of on-board sensors). Therefore, the planner determines the order for performing the lane analysis in such a manner that an established optimisation criterion is minimised.

While this mobile platform is prepared to inspect both annual (e.g., maize or cereal) and multi-annual (e.g., orchards or vineyards) crops, the present work is focused on the inspection and $3 \mathrm{D}$ reconstruction of woody crops. Note that in the case of arable crops, the platform can only scout the crop during the early season, which is acceptable since this coincides with the time when treatments for weeds are carried out.

\subsection{D Reconstruction Approach}

After studying different 3D reconstruction methods [12-14,16], the algorithm described in [14] was selected for the 3D reconstruction of woody crops, typically formed by several rows of a large length. This method provides satisfactory results in large-zone reconstruction from the information directly supplied by the Kinect sensor.

The method extends the algorithm proposed by [6] to reconstruct large regions using the fusion of different overlapped depth images, storing information only on the voxels closest to the detected object and accessing to the stored information using a hash table. In this way, a complete regular voxel grid stored in the memory is unnecessary, thereby providing computational advantages. Given a new input depth image and known camera position, the ray-casting technique [22] is used to project a ray from the camera focus for each pixel of the input depth image to determine the voxels in the 3D world that cross each ray. In this way, the voxels related to the depth information are determined.

Once the surface of the scene has been extracted using the ray-casting technique, this information is used to estimate the position and orientation of the camera with 6 degrees of freedom when a new input image arrives. The last estimation is conducted with a variant of the iterative closest point (ICP) algorithm [23] and provides a point cloud as the output, which in this case is a woody row that does not appear to be as straight as the original row, showing detectable drift, as shown in Figure 2.

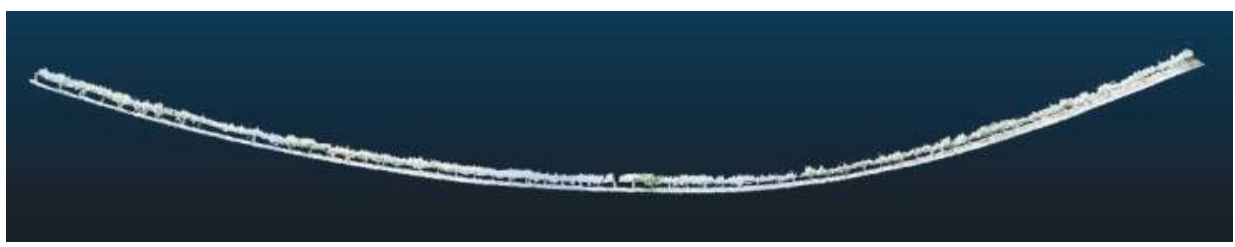

Figure 2. 3D reconstruction of a woody crop row in which the drift problem is observed.

Finally, a method was designed and developed to accommodate this drift using the minimal scene information that can be acquired from the field platform during its travel, such as the geographical 
location of the starting and ending points of each sampling row and the fact that trees are typically planted in straight lines. In the following subsections, the developed method will be explained in detail.

\subsubsection{Filtering}

The first step is to filter the point cloud to eliminate those points that appear isolated. A point is considered an outlier if the average distance to its 64 nearest neighbours is greater than the standard deviation of the distance to the neighbours of all the points. Figure 3 shows a fragment of a reconstruction of a vineyard row before and after the filtering operation.

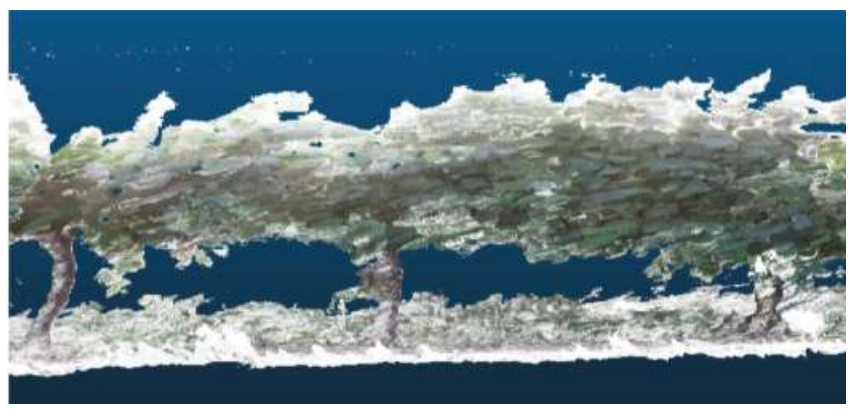

(a)

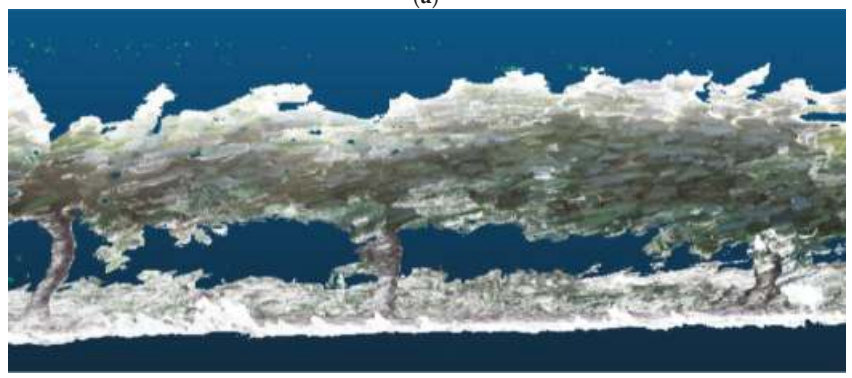

(b)

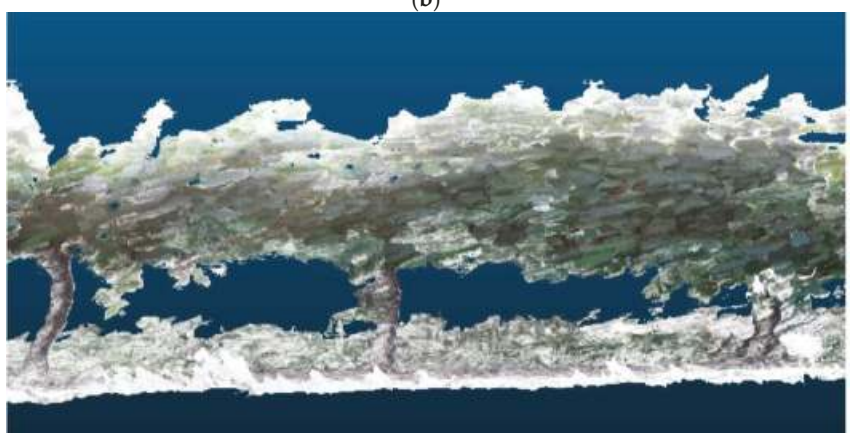

(c)

Figure 3. Section of the 3D reconstruction of a vineyard row: before filtering (a); and after filtering (c).

The removed points are marked in fluorescent green in image $(\mathbf{b})$.

\subsubsection{Estimation of a Model Line}

The next step is to find a line that longitudinally models the 3D reconstruction of the previously generated tree row. This model line should be adjusted as much as possible to the path following the tree 
row and will be used in the next steps to segment the row in sections of the same length. The positions of the point cloud representing the 3D reconstruction of the row are used to model the line.

The point cloud is divided into smaller groups of equal numbers of points and the centroids of the points in each group. Since the points in the cloud are stored consecutively according to their order of appearance, the centroids are also consecutive and determine the path followed by the model line. The number of points of the model line is proportional to the number of groups into which the original point cloud is divided, i.e., more groups correspond to more points and a higher accuracy of the fit to the original tree row. The number of groups is estimated from the total length of the tree row and the known geographical position of the starting and ending points of the row. In general, it is sufficient to divide the row into groups of approximately $5 \mathrm{~m}$, as we have experimentally verified that the effect of the drift over $5 \mathrm{~m}$ can be considered to have a negligible effect on the performance of the proposed method. Note that the groups of points, although they include the same number of points, do not represent the same row length because the density of points is not necessarily uniform (i.e., the density changes depending on the degree of tree coverage); thus, the points that define the model line are not at the same distance, as shown in Figure 4.

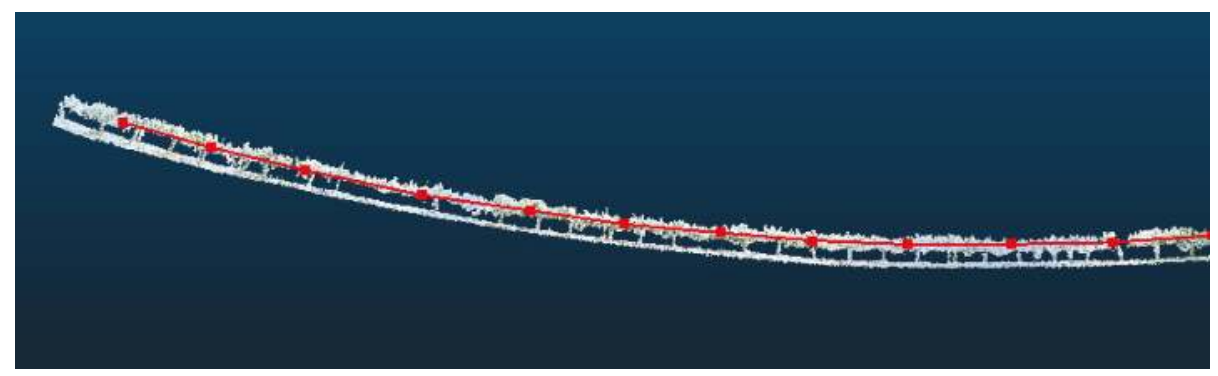

Figure 4. Part of the model line of the 3D reconstruction of Figure 2.

To obtain the model line that covers the tree row end to end, it is necessary to determine the endpoints of the row to incorporate these endpoints to the points that form the model line. To identify the starting point, the points of the first group are projected onto the extension of the line connecting the first and second centroids. The projected point most distant to the first centroid is chosen as the starting point of the model line since, in a frontal view, all other points of the tree row are to the right of the centroid. The procedure to be followed is similar in the case of the ending point estimation. All points of the last group are projected on the extension of the model line that connects the penultimate and last centroids, choosing as the ending point of the model line the point projected as most distant to the last centroid since all the points of the tree row are to the left of this centroid (Figure 5).

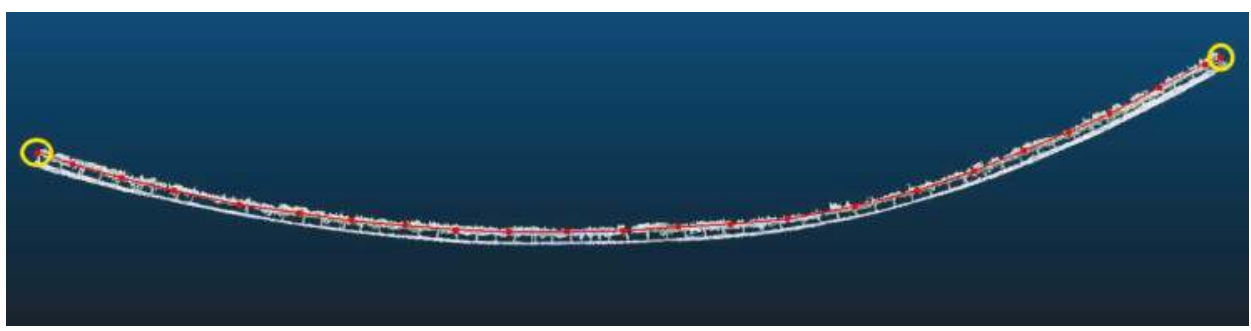

Figure 5. Yellow circles represent the endpoints of the tree row added to the model line of Figure 4. 


\subsubsection{Splitting the Model Line into Sections of the Same Length}

Once an accurate model line of the crop is obtained, the line is divided into segments of equal length. The model line length is estimated by adding the Euclidean distances between the points that define the line. This value is compared with the actual tree row length obtained from the geographical position of the starting and ending points of the row. A slight difference is expected due to the drift of the row, which makes the length of the model line greater than the actual length. To distribute the error along the entire tree row, the desired length of the segment is adjusted proportionally to the ratio between the model line length and the actual tree row length. For example, if the actual row length is $50 \mathrm{~m}$, its corresponding model line is $53 \mathrm{~m}$ in length, and a scale of $0.5 \mathrm{~m}$ is desired; thus, both the actual row and the model line will be divided into 100 sections. Consequently, each actual row segment of $0.5 \mathrm{~m}$ will have a corresponding model line segment of $0.53 \mathrm{~m}$ to maintain the number of sections, thus distributing the error. Subsequently, when the drift is corrected, the change will be undone, and the segment will again measure the actual $0.5 \mathrm{~m}$.

Therefore, once the length of the section is defined, the model line is divided into segments of that length and the 3D reconstruction or point cloud is divided into sections separated by planes perpendicular to the model line at each point of segment separation (Figure 6). In this manner, the point cloud is divided into sections, each containing a fragment of the $3 \mathrm{D}$ reconstruction.

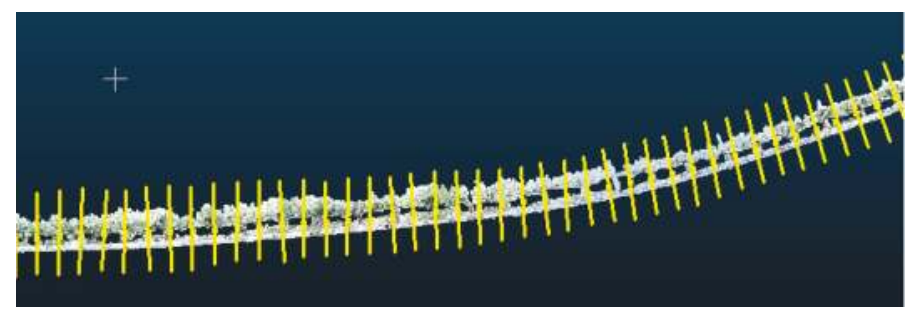

Figure 6. Part of the 3D reconstruction shown in Figure 2 split into sections.

\subsubsection{Section Correction}

First, to facilitate the completion of the developed procedures, the coordinate system must be changed since the origin of the coordinate system for the generated point cloud is placed in the initial position of the Kinect sensor. The applied transformation matrix places the origin in the initial position of the crop, centred in the soil, with the X-axis in the direction of the crop row (sense from the starting point to the ending point of the crop row), the Z-axis in the direction of tree growth, and the Y-axis in the direction of the crop depth (Figure 7).

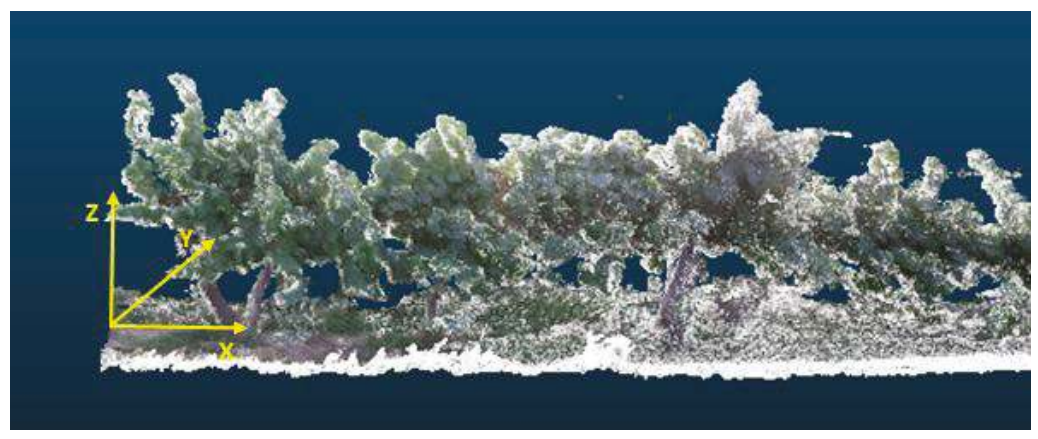

Figure 7. The coordinate system defined in yellow. 
The vector that indicates the direction of the crop row in each section can be calculated using the starting and ending points of the model line segment contained in the section, drawn as a red line in Figure 8. Ideally, the direction of this vector coincides with the direction of the actual tree row (i.e., the reference $X$-axis represented in Figure 7); however, this condition does not occur due to the drift that appears in the 3D reconstruction. To eliminate the drift, each section must be corrected in such a manner that the section must be rotated to align the vector that indicates the direction of the model line in the reconstructed section (Xsection) with the direction of the X-axis of the actual section (Xreference). To accomplish this correction, first, in each section, the desired reference system is placed at the starting point of the segment in the section by performing a translation from the coordinate origin of the reconstructed row to that point. Next, after normalizing the vector Xsection, the rotation matrix R (see Equation (1)), which rotates the unit vector Xsection to align it with the unit vector Xreference, must be determined, as shown in Figure 8.

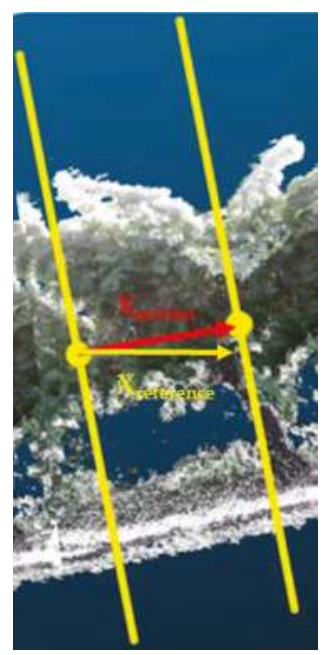

Figure 8. The $\mathrm{X}$-axis of the section (Xsection) is represented in red, and the $\mathrm{X}$-axis of the reference (Xreference) is represented in yellow.

Euler's rotation theorem [24] states that any rotation or set of successive rotations can be expressed as a rotation about a principal axis of rotation or vector $(\mathrm{v})$ that, in this case, can be calculated by the cross product of vectors Xsection and Xreference.

Furthermore, Rodrigues' rotation formula [25] mathematically states the rotation theorem of Euler, enabling the rotation matrix $\mathrm{R}$ calculation, given an axis of rotation $\mathrm{v}$ and an angle of rotation $\theta$, as shown in Equation (1):

$$
\mathrm{R}=\mathrm{I}+[\mathrm{v}]_{\times} \cdot \sin \theta+[\mathrm{v}]_{\times}^{2} \cdot(1-\cos \theta)
$$

where $[\mathrm{v}]_{\times}$represents the $\mathrm{v}$-axis obtained from the cross product and expressed in terms of a skew-symmetric matrix (Equation (2)):

$$
[\mathrm{v}]_{\times}=\left(\begin{array}{ccc}
0 & -\mathrm{v}_{3} & \mathrm{v}_{2} \\
\mathrm{v}_{3} & 0 & -\mathrm{v}_{1} \\
-\mathrm{v}_{2} & \mathrm{v}_{1} & 0
\end{array}\right)
$$


Since Xsection and Xreference are unit vectors, the cosine of the angle between them can be obtained as the dot product of both vectors (Equation (3)):

$$
\cos \theta=\text { Xsection } \cdot \text { Xreference }
$$

In a similar manner, the sine of the angle between vectors Xsection and Xreference can be calculated using Equation (4), i.e., as the norm of the axis obtained from the cross product of both vectors:

$$
\sin \theta=\|\mathrm{v}\|
$$

At this point, all terms necessary to calculate the rotation matrix, $\mathrm{R}$, from the Rodrigues formula (Equation (1)) are available. Applying such a matrix to all the points forming the section, the section is rectified, aligning the direction of the crop in that section with the actual X-axis and thus correcting the drift. With the section correctly placed, the coordinate transformation previously performed is undone so that all section points are referenced with respect to the coordinate origin of the reconstructed row. As explained in Section 2.2.3, once the drift is corrected, the change in the length of the segment is reversed by rescaling the segment such that its initial length is restored.

Once the drift of each section has been corrected, the sections are aligned; thus, the drift produced during 3D reconstruction is corrected. Figure 9 shows a reconstruction of a vineyard row with drift and the result obtained after applying the developed procedure.

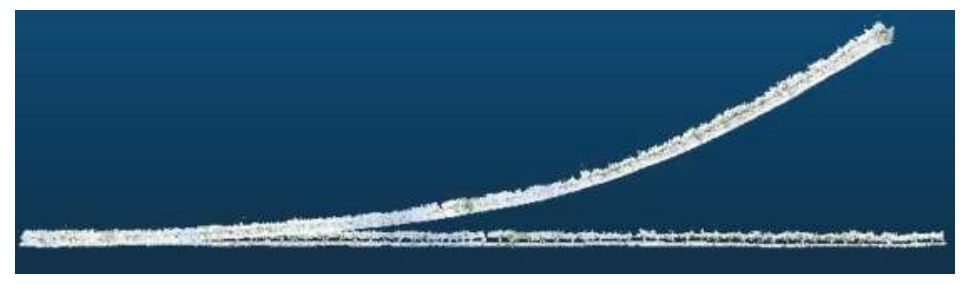

Figure 9. 3D reconstruction of a tree row with drift (up) and the result after the drift correction procedure is applied (down).

\section{Results and Discussion}

Several tests were conducted in 2016 in vineyards owned by Codorniu S.A. (Raimat, Lleida, Spain). The abovementioned inspection platform moving at $3 \mathrm{~km} / \mathrm{h}$ was used to collect the information. The sensor Kinect v2 was mounted at approximately $1.4 \mathrm{~m}$ high with a $10^{\circ}$ pitch angle oriented to the crop rows at a distance of approximately $1 \mathrm{~m}$ from the crop row (Figure 10).

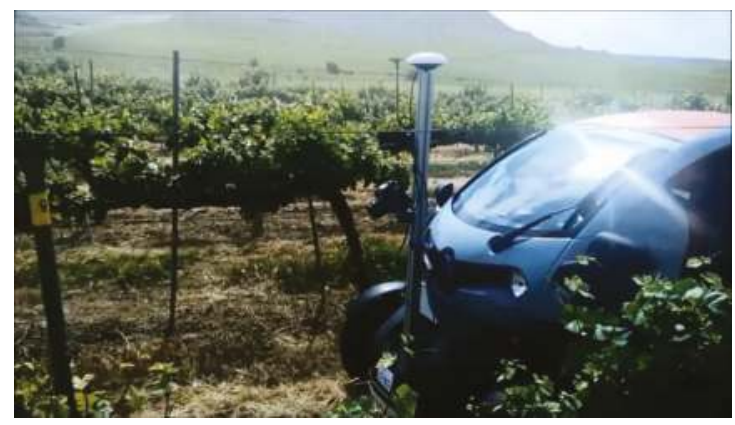

Figure 10. Detail of the sampling in a vineyard using the field platform. 
From each inspected row, the starting and ending geographical positions of the row supplied by the RTK-GPS receiver from the vehicle were stored.

Figure 11 shows examples of the information provided by the Kinect v2 sensor in the vineyard in the tests conducted on May 2016. Figure 11a,c shows the RGB information of two scenes. Figure 11b,d shows a false-colour representation of the depth information, i.e., the distance from the camera to the objects in the scene, where the closest objects appear in red and the furthest objects appear in blue; the intermediate objects are shown in various shades of orange, yellow and green depending on their distance from the sensor. The operating range of the sensor meets the inspection requirements of the vineyard rows since the non-interesting objects are ignored, such as those extremely close and those within distant areas that typically contain other vineyard rows.

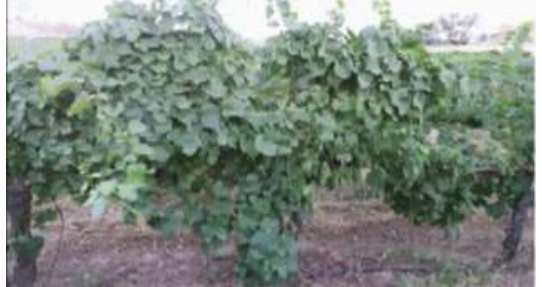

(a)

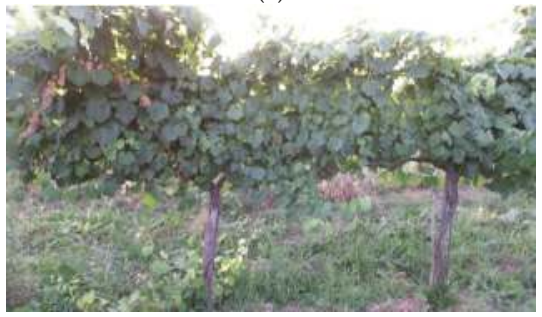

(c)

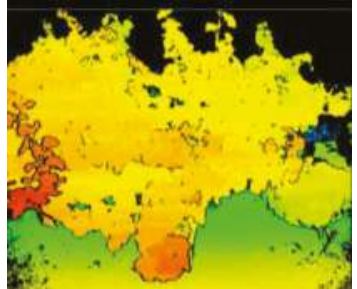

(b)

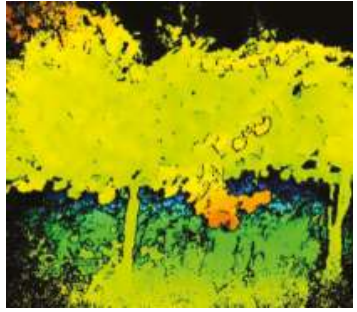

(d)

Figure 11. (a,c) RGB images supplied by the Kinect v2 sensor; and (b,d) examples of the depth images supplied by the Kinect v2 sensor simultaneous to when images $(\mathbf{a}, \mathbf{c})$ were obtained.

With the information supplied by the Kinect v2 sensor and stored in the on-board computer of the vehicle, the $3 \mathrm{D}$ reconstruction of the sampled rows of vines was performed. For that reconstruction, a desktop computer with an Intel Core i7-6900K@3.2GHz processor, 64 GB of RAM, and an NVIDIA GeForce GTX Titan X graphics card was used. Figure 12 shows an example of one of the 3D reconstructions that includes a 3D mesh structure, i.e., a triangular mesh (Figure 13) obtained from the point cloud using the marching cubes algorithm [26].

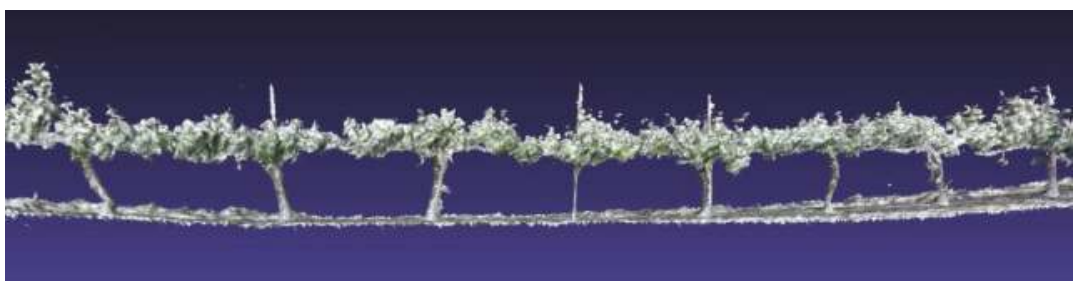

Figure 12. 3D reconstruction of a row of vines. 


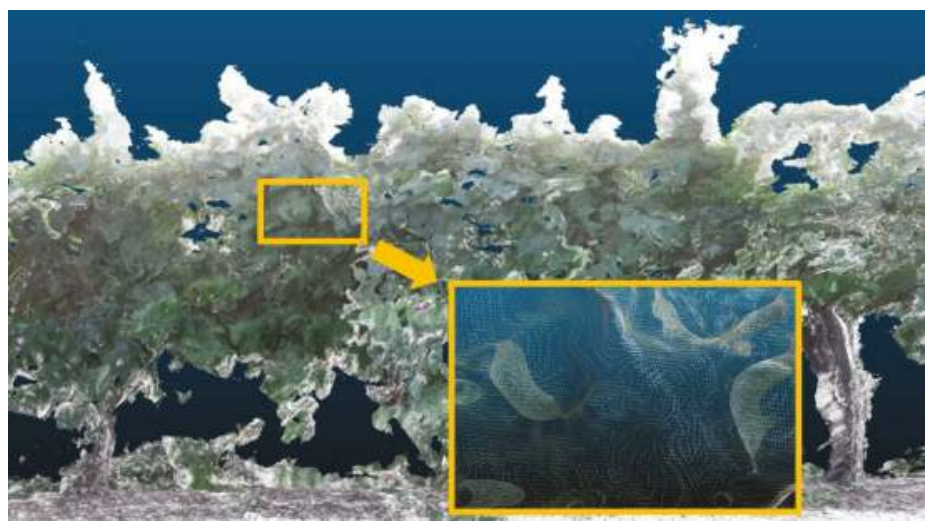

Figure 13. Details of a $3 \mathrm{D}$ reconstruction of a vine that shows the triangular mesh obtained from a point cloud.

As discussed earlier, a drift that causes distortion in the row reconstruction typically appears in the 3D reconstructions of long crop rows. To evaluate the performance of the proposed approach to correct the drift, 3D reconstructions of long crop rows sampled at different times of the year were conducted. Figure 14 shows the condition of the vineyard in the tests conducted in February, May and July 2016.

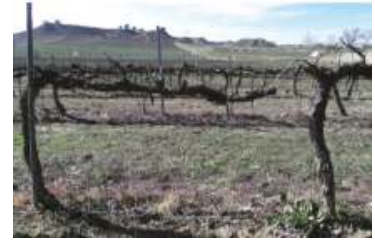

(a)

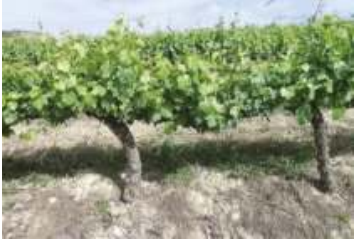

(b)

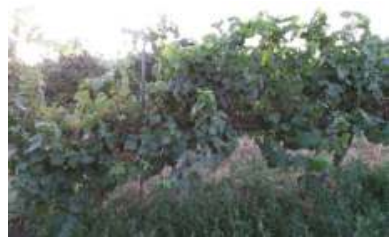

(c)

Figure 14. Condition of the vineyards used in the experiments conducted in: (a) February 2016; (b) May 2016; and (c) July 2016.

Thus, the method was tested with trees at distinct stages of development, in which the structure to be reconstructed was different and the number of points within clouds was smaller or greater depending on the time of year, i.e., without leaves, with leaves, and with both highly developed leaves and vegetation cover. Figure 15 shows the 3D reconstruction of the same part of a vineyard in February, May and July 2016.

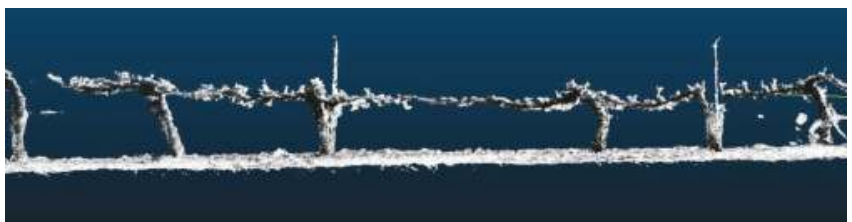

(a)

Figure 15. Cont. 


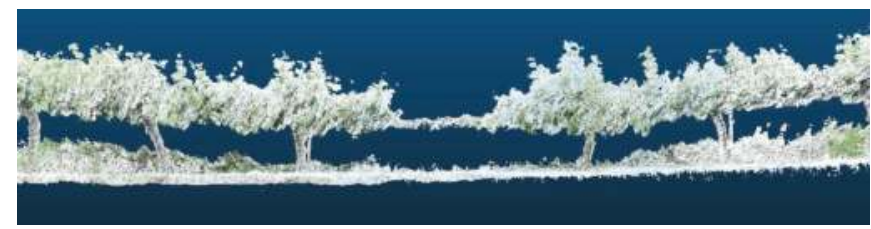

(b)

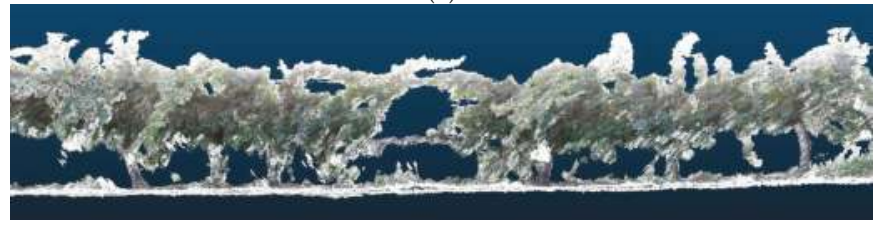

(c)

Figure 15. The same view of the $3 \mathrm{D}$ reconstruction of a vineyard with the information acquired in: (a) February 2016; (b) May 2016; and (c) July 2016.

During the several experiments conducted, although 3D reconstructions of long crop rows were properly performed, drift appears in all the tests conducted throughout the year (Figure 16).

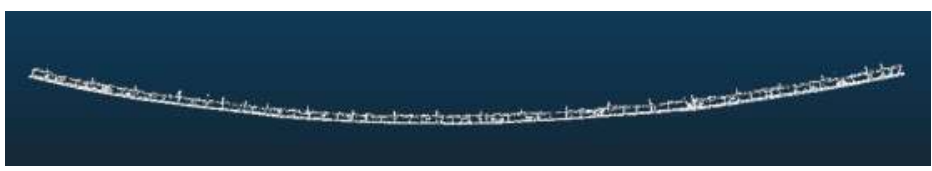

(a)

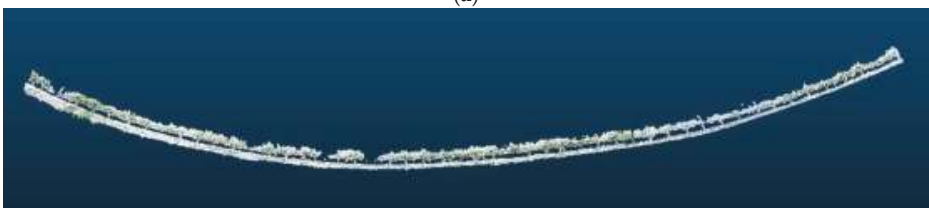

(b)

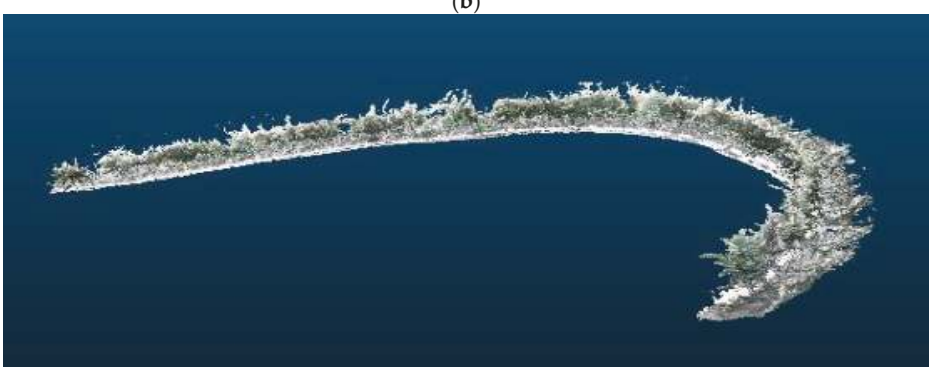

(c)

Figure 16. Examples of 3D reconstructions that exhibit drift. Sampling performed in: (a) February 2016; (b) May 2016; and (c) July 2016.

Using the method described before, it is possible to correct the drift. The vineyard row in Figure 16 is divided in sections of $5 \mathrm{~m}$, and the rotation angle of each section is estimated using Rodrigues' rotation formula to correct the drift. Table 1 shows the calculated rotation angles per section. Thus, using the proposed approach, the drift, shown in Figure 16, was corrected to obtain the images displayed in Figure 17. 


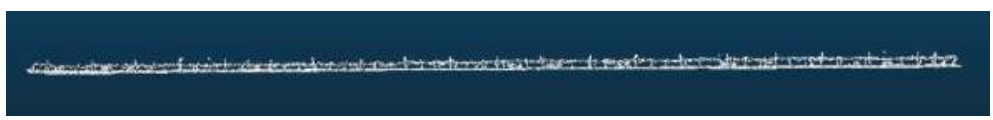

(a)

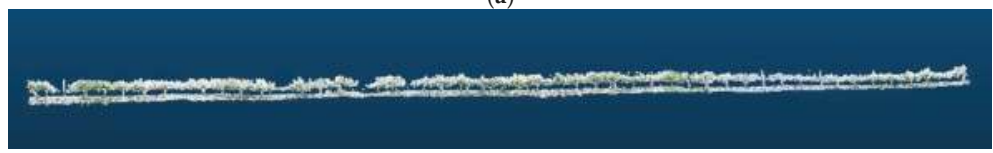

(b)

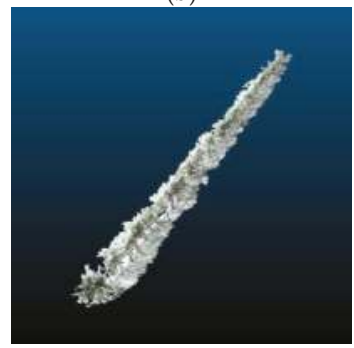

(c)

Figure 17. (a-c) 3D reconstruction of vineyards in Figure 16 after the drift has been removed.

Next, the performance of the proposed approach was analysed through a 3D reconstruction of two rows of the vineyard with lengths of $85 \mathrm{~m}$ and $105 \mathrm{~m}$. Figure 18 shows the rotation angles estimated to correct each section on the rows. Tables 1 and 2 show the mean, standard deviation and maximum of the calculated rotation angles of all the sections for the two considered rows and the number of points of the cloud and the length of the model line that defines each row. From the results, it can be concluded that a smaller number of leaves in the vineyards is correlated with a smaller deformation in the $3 \mathrm{D}$ reconstructions. This behaviour arises because the ICP algorithm, which estimates the position and orientation of the Kinect sensor, is not as effective when the same structure is repeated, i.e., the appearance of similar leaves results in analogous distances. It seems that, when the length of the row to be reconstructed is greater, the deformation that appears in the 3D reconstruction is greater because of the error accumulated along the row. This fact must be confirmed through a specific and deeper analysis.

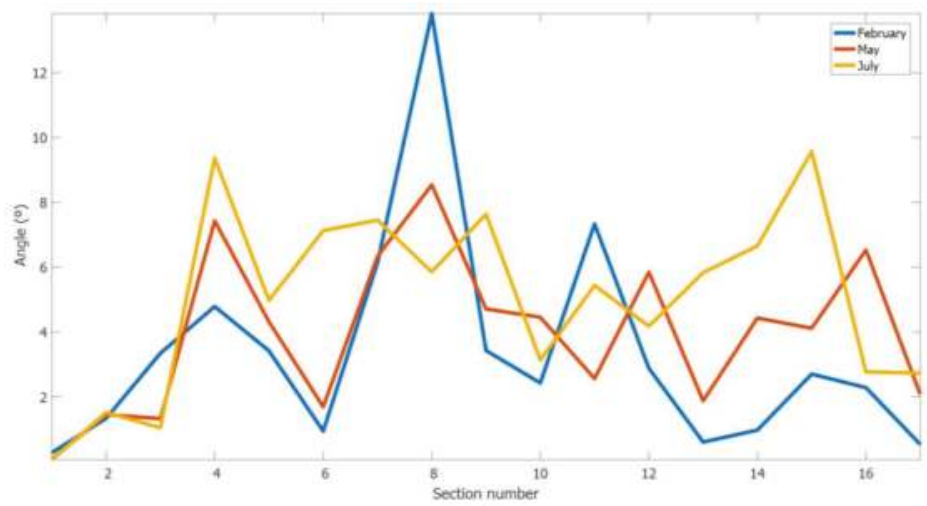

(a)

Figure 18. Cont. 


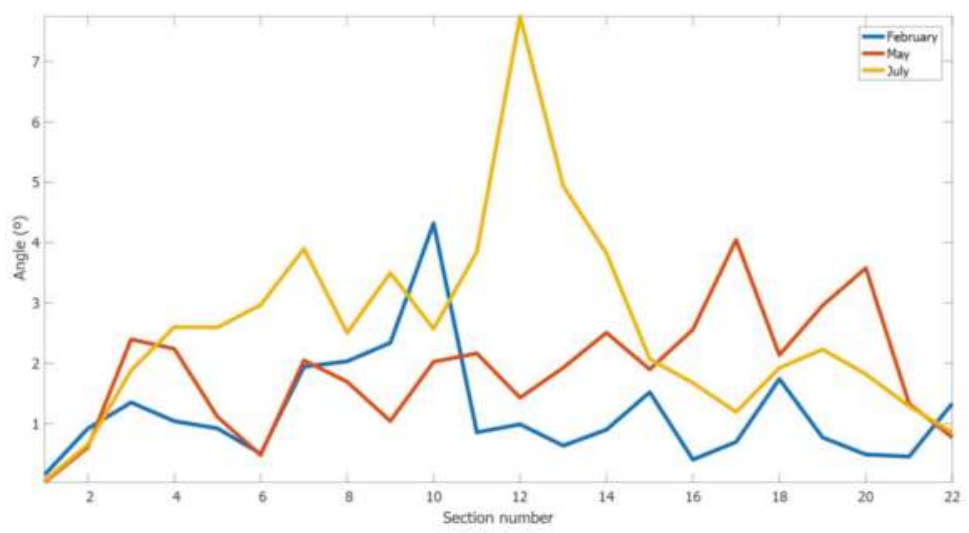

(b)

Figure 18. Rotation angles calculated by the proposed approach to correct each section on the vineyard rows of: (a) $85 \mathrm{~m}$ length; and (b) $105 \mathrm{~m}$ length. Sampling performed during February, May and July 2016.

Table 1. Statistics of the performance of drift correction method for the $85 \mathrm{~m}$ length row.

\begin{tabular}{ccccccc}
\hline Sampling & $\begin{array}{c}\text { Number } \\
\text { of Points }\end{array}$ & $\begin{array}{c}\text { Mean } \\
\text { Angle }\left(^{\circ}\right)\end{array}$ & $\begin{array}{c}\text { Std. Dev. } \\
\text { Angle }\left(^{\circ}\right)\end{array}$ & $\begin{array}{c}\text { Maximum } \\
\text { Angle }\left({ }^{\circ}\right)\end{array}$ & $\begin{array}{c}\text { 3D Reconstruction } \\
\text { Time (s) }\end{array}$ & $\begin{array}{c}\text { Model Line } \\
\text { Length (m) }\end{array}$ \\
\hline February & $4,289,633$ & 3.56 & 3.33 & 13.82 & 42.46 & 86.30 \\
May & $6,441,484$ & 3.99 & 2.42 & 8.54 & 64.03 & 89.69 \\
July & $13,624,626$ & 5.02 & 2.83 & 9.57 & 83.76 & 93.81 \\
\hline
\end{tabular}

Table 2. Statistics of the performance of drift correction method for the $105 \mathrm{~m}$ length row.

\begin{tabular}{ccccccc}
\hline Sampling & $\begin{array}{c}\text { Number } \\
\text { of Points }\end{array}$ & $\begin{array}{c}\text { Mean } \\
\text { Angle }\left(^{\circ}\right)\end{array}$ & $\begin{array}{c}\text { Std. Dev. } \\
\text { Angle }\left(^{\circ}\right)\end{array}$ & $\begin{array}{c}\text { Maximum } \\
\text { Angle }\left(^{\circ}\right)\end{array}$ & $\begin{array}{c}\text { 3D Reconstruction } \\
\text { Time (s) }\end{array}$ & $\begin{array}{c}\text { Length of } \\
\text { Model Line (m) }\end{array}$ \\
\hline February & $5,575,934$ & 2.39 & 1.80 & 8.63 & 60.52 & 107.21 \\
May & $7,941,965$ & 3.72 & 1.96 & 8.09 & 72.85 & 112.00 \\
July & $16,000,906$ & 5.39 & 3.31 & 15.49 & 111.09 & 115.93 \\
\hline
\end{tabular}

Additionally, the processing time to correct the drift depends on the number of the points of the cloud. To reduce this time, one option is to perform a uniform downsampling of the point cloud. For example, when the downsampling percentage equals $75 \%$, the original point cloud is sampled uniformly, preserving $25 \%$ of the points. It may appear that, if the resolution to generate the $3 \mathrm{D}$ reconstruction were reduced, the effect would be the same. However, in this case, the ICP algorithm performs worse, and the drift of the 3D reconstruction increases.

To study the influence of downsampling, differences among the calculated rotation angles applied to each section in the clouds with a distinct downsampling percentage were calculated. Taking the correct values of the angles as those calculated with $100 \%$ of the points, the errors among this value and those obtained values with the downsampled point clouds were obtained. Table 3 shows the mean error of all sections, the maximum error in these sections, and the processing time to correct the drift in the computer described above for different downsampling percentages of the point cloud analyses. 
Table 3. Different measures for different downsampling percentages of the point clouds studied.

\begin{tabular}{|c|c|c|c|c|c|c|c|c|}
\hline \multirow{2}{*}{ Crop Row } & \multirow{2}{*}{ Sampling } & \multirow{2}{*}{ Measures } & \multicolumn{6}{|c|}{ Downsampling Percentage } \\
\hline & & & $0 \%$ & $50 \%$ & $75 \%$ & $90 \%$ & $99 \%$ & $99.9 \%$ \\
\hline \multirow{3}{*}{$85 \mathrm{~m}$} & \multirow{3}{*}{ February } & Mean error $\left(^{\circ}\right)$ & - & 0.06 & 0.18 & 0.27 & 0.97 & 1.30 \\
\hline & & Max. error $\left({ }^{\circ}\right)$ & - & 0.19 & 0.61 & 1.30 & 3.69 & 3.85 \\
\hline & & Time (s) & 119.25 & 58.30 & 50.59 & 12.65 & 2.09 & 0.99 \\
\hline \multirow{3}{*}{$85 \mathrm{~m}$} & \multirow{3}{*}{ May } & Mean error $\left({ }^{\circ}\right)$ & - & 0.05 & 0.09 & 0.11 & 0.23 & 0.75 \\
\hline & & Max. error $\left({ }^{\circ}\right)$ & - & 0.17 & 0.29 & 0.30 & 0.68 & 2.54 \\
\hline & & Time (s) & 213.60 & 89.78 & 65.06 & 18.44 & 2.59 & 1.11 \\
\hline \multirow{3}{*}{$85 \mathrm{~m}$} & \multirow{3}{*}{ July } & Mean error $\left(^{\circ}\right)$ & - & 0.01 & 0.03 & 0.10 & 0.39 & 0.63 \\
\hline & & Max. error $\left({ }^{\circ}\right)$ & - & 0.04 & 0.14 & 0.41 & 1.72 & 2.07 \\
\hline & & Time (s) & 389.49 & 183.65 & 93.38 & 36.04 & 4.62 & 1.67 \\
\hline \multirow{3}{*}{$105 \mathrm{~m}$} & \multirow{3}{*}{ February } & Mean error $\left(^{\circ}\right)$ & - & 0.03 & 0.05 & 0.09 & 0.34 & 0.42 \\
\hline & & Max. error $\left({ }^{\circ}\right)$ & - & 0.10 & 0.22 & 0.26 & 1.61 & 1.61 \\
\hline & & Time (s) & 155.01 & 75.77 & 65.76 & 16.43 & 2.73 & 1.21 \\
\hline \multirow{3}{*}{$105 \mathrm{~m}$} & \multirow{3}{*}{ May } & Mean error $\left(^{\circ}\right)$ & - & 0.05 & 0.05 & 0.16 & 0.40 & 0.85 \\
\hline & & Max. error $\left({ }^{\circ}\right)$ & - & 0.18 & 0.10 & 0.60 & 1.49 & 3.37 \\
\hline & & Time (s) & 263.35 & 110.71 & 85.92 & 22.64 & 3.18 & 1.45 \\
\hline \multirow{3}{*}{$105 \mathrm{~m}$} & \multirow{3}{*}{ July } & Mean error $\left({ }^{\circ}\right)$ & - & 0.04 & 0.11 & 0.26 & 0.33 & 2.34 \\
\hline & & Max. error $\left({ }^{\circ}\right)$ & - & 0.15 & 0.38 & 0.90 & 0.96 & 13.55 \\
\hline & & Time (s) & 457.52 & 214.68 & 109.66 & 42.23 & 5.32 & 1.97 \\
\hline
\end{tabular}

Using $50 \%$ of the points, the maximum error is less than $0.2^{\circ}$. When downsampling is performed at $75 \%$, the maximum error is tripled (to $0.61^{\circ}$ ), although this error is still within an acceptable range of error. Performing a downsampling at $90 \%$, the error further increases until it becomes inappropriate for a proper drift correction, since it is greater than $1^{\circ}$.

When the number of points of the cloud is greater, the effect of downsampling on the processing time is greater. The relationship among the number of points and the processing time is not linear; for example, comparing the processing time downsampling at $75 \%$ with respect to the original point cloud, in the cloud with fewer points (February, $85 \mathrm{~m}$ ), the processing time is reduced by a factor of 2.34, and, in the cloud with the highest number of points (July, $105 \mathrm{~m}$ ), the processing time is reduced by a factor of 4.17 .

For certain applications, it may not be necessary to use such a high number of points (such as measuring the height of the tree), and fewer points would suffice, thus enabling the drift correction process to be accelerated without producing high errors. If the use of all points is required but the process must be accelerated, one solution is to perform the drift correction algorithm on the downsampled point cloud at $75 \%$. In other words, the planes, which delimit each section, and the angle applied to the section to correct it are established via a calculation in the reduced point cloud for later use in the complete point cloud. This strategy could reduce the processing time by a significant amount without considerably increasing the error.

\section{Conclusions}

This paper describes a low-cost crop monitoring system that can automatically create accurate $3 \mathrm{D}$ models of woody crop rows. The system integrates a medium-size platform equipped with various on-board sensors to scan annual crops (maize, cereal, etc.) and multi-annual crops (orchards, vineyards, etc.). It also incorporates software specifically developed to accurately generate 2D and 3D maps of the sampled crops. Particularly, the paper is focused on the automatic 3D reconstruction of woody crops based on the information obtained from an on-board RGB-D sensor.

Based on the results of the samplings conducted, it can be concluded that the implemented algorithm provides good results for the automatic $3 \mathrm{D}$ reconstruction of large areas under uncontrolled 
lighting conditions at different times of the year within commercial vineyard fields. Furthermore, the operating range of the sensor meets the inspection requirements of the vineyard rows since the non-interesting objects are ignored, including objects extremely close to the sensor and those at distant locations that usually correspond to other vineyard rows. The drift that usually appears in the $3 \mathrm{D}$ reconstruction of long rows, those greater than $25 \mathrm{~m}$, can be properly handled with a method that uses information about the scene, such as the geographical position of the starting and ending points of the row and the fact that the woody crops are typically planted in straight rows. Additionally, a smaller number of leaves in the vineyards is correlated with a smaller deformation in the $3 \mathrm{D}$ reconstructions. The processing time to remove the drift depends on the number of points in the $3 \mathrm{D}$ reconstruction and can be reduced by performing a uniform downsampling of the point cloud. Moreover, it has been verified that the greater the number of points in the cloud, the greater the effect of downsampling on the processing time. Finally, it can be concluded that in some cases, it might not be necessary to use such a large number of points in the removal drift process, accelerating the process without producing high errors.

Considering future work, the developed drift removal method should be able to properly handle field crops that are planted in curved rows. A potential strategy might be to suitably incorporate the information of all the GNSS locations taken during the sampling. Additionally, the integration of an inertial measurement unit (IMU) fixed to the Kinect sensor for registering variations in the yaw, pitch and roll angles of the sensor could be a good strategy to obtain complementary and useful information for improving the $3 \mathrm{D}$ reconstruction accuracy.

Acknowledgments: The Spanish Government has provided full and continuing support for this research work through project AGL2014-52465-C4-3-R. The authors wish to thank the Codorniu S.A. company for the use of the facilities on the estate of Raimat and extend their gratitude to Jordi Recasens and his team (Weed Science and Plant Ecology Research Group of the UdL) for their invaluable help in the field trials. Karla Cantuña thanks the service commission for the remuneration given by the Cotopaxi Technical University. The authors also wish to acknowledge the ongoing technical support of Damián Rodríguez.

Author Contributions: The work was developed as a collaboration among all authors. J.M. Bengochea-Guevara and A. Ribeiro designed the study. J.M. Bengochea-Guevara and D. Andújar carried out the system integration. J.M. Bengochea-Guevara performed the programming. F.L. Sanchez-Sardana and K. Cantuña mainly provided support in the field tests with D. Andújar. A. Ribeiro directed the research, collaborating in the testing and the discussion of the results with D. Andújar and J.M. Bengochea-Guevara. The manuscript was mainly drafted by J.M. Bengochea-Guevara and A. Ribeiro and was revised and corrected by all co-authors. All authors have read and approved the final manuscript.

Conflicts of Interest: The authors declare no conflict of interest.

\section{References}

1. West, P.W. Tree and Forest Measurement; Springer: Heidelberg, Germany, 2009; Volume 20.

2. Paulus, S.; Behmann, J.; Mahlein, A.-K.; Plümer, L.; Kuhlmann, H. Low-cost 3D systems: Suitable tools for plant phenotyping. Sensors 2014, 14, 3001-3018. [CrossRef] [PubMed]

3. Andújar, D.; Dorado, J.; Fernández-Quintanilla, C.; Ribeiro, A. An Approach to the Use of Depth Cameras for Weed Volume Estimation. Sensors 2016, 16, 972. [CrossRef] [PubMed]

4. Wang, W.; Li, C. Size estimation of sweet onions using consumer-grade RGB-depth sensor. J. Food Eng. 2014, 142, 153-162. [CrossRef]

5. Hilton, A.; Stoddart, A.; Illingworth, J.; Windeatt, T. Reliable surface reconstruction from multiple range images. In Proceedings of the 4th European Conference on Computer Vision, Cambridge, UK, 15-18 April 1996; pp. 117-126.

6. Curless, B.; Levoy, M. A volumetric method for building complex models from range images. In Proceedings of the 23rd Annual Conference on Computer Graphics and Interactive Techniques, New Orleans, LA, USA, 4-9 August 1996; pp. 303-312.

7. Wheeler, M.D.; Sato, Y.; Ikeuchi, K. Consensus surfaces for modeling 3D objects from multiple range images. In Proceedings of the Sixth International Conference on Computer Vision, Bombay, India, 7 January 1998; pp. 917-924. 
8. Zhou, Q.-Y.; Koltun, V. Dense scene reconstruction with points of interest. ACM Trans. Graph. 2013, 32, 112. [CrossRef]

9. Newcombe, R.A.; Izadi, S.; Hilliges, O.; Molyneaux, D.; Kim, D.; Davison, A.J.; Kohi, P.; Shotton, J.; Hodges, S.; Fitzgibbon, A. KinectFusion: Real-time dense surface mapping and tracking. In Proceedings of the 2011 10th IEEE International Symposium on Mixed and Augmented Reality (ISMAR), Basel, Switzerland, 26-29 October 2011; pp. 127-136.

10. Izadi, S.; Newcombe, R.A.; Kim, D.; Hilliges, O.; Molyneaux, D.; Hodges, S.; Kohli, P.; Shotton, J.; Davison, A.J.; Fitzgibbon, A. Kinectfusion: Real-time dynamic 3d surface reconstruction and interaction. In Proceedings of the ACM SIGGRAPH 2011 Talks, Vancouver, BC, Canada, 7-11 August 2011; p. 23.

11. Microsoft Kinect for Windows Software Development Kit 2.0. Available online: https://developer.microsoft. com/es-es/windows/kinect/develop (accessed on 9 August 2017).

12. Steinbrucker, F.; Kerl, C.; Cremers, D. Large-scale multi-resolution surface reconstruction from RGB-D sequences. In Proceedings of the IEEE International Conference on Computer Vision, Sydney, Australia, 1-8 December 2013; pp. 3264-3271.

13. Whelan, T.; Kaess, M.; Fallon, M.; Johannsson, H.; Leonard, J.; McDonald, J. Kintinuous: Spatially Extended Kinectfusion. Available online: https:/ / dspace.mit.edu/handle/1721.1/71756 (accessed on 9 August 2017).

14. Nießner, M.; Zollhöfer, M.; Izadi, S.; Stamminger, M. Real-time 3D reconstruction at scale using voxel hashing. ACM Trans. Graph. 2013, 32, 169. [CrossRef]

15. Zeng, M.; Zhao, F.; Zheng, J.; Liu, X. Octree-based fusion for realtime 3D reconstruction. Graph. Models 2013, 75, 126-136. [CrossRef]

16. Chen, J.; Bautembach, D.; Izadi, S. Scalable real-time volumetric surface reconstruction. ACM Trans. Graph. 2013, 32, 113. [CrossRef]

17. Pagliari, D.; Pinto, L. Calibration of Kinect for Xbox One and comparison between the Two Generations of Microsoft Sensors. Sensors 2015, 11, 27569-27589. [CrossRef] [PubMed]

18. Fankhauser, P.; Bloesch, M.; Rodriguez, D.; Kaestner, R.; Hutter, M.; Siegwart, R. Kinect v2 for mobile robot navigation: Evaluation and modeling. In Proceedings of the 2015 International Conference on Advanced Robotics (ICAR), Istanbul, Turkey, 27-31 July 2015; pp. 388-394.

19. Anderson, C.D.; Anderson, J. Electric and Hybrid Cars: A history; McFarland: Jefferson, NC, USA, 2010.

20. Conesa-Munoz, J.; Bengochea-Guevara, J.M.; Andujar, D.; Ribeiro, A. Efficient Distribution of a Fleet of Heterogeneous Vehicles in Agriculture: A Practical Approach to Multi-path Planning. In Proceedings of the 2015 IEEE International Conference on Autonomous Robot Systems and Competitions (ICARSC), Vila Real, Portugal, 8-10 April 2015; pp. 56-61.

21. Bochtis, D.D.; Sørensen, C.G. The vehicle routing problem in field logistics part I. Biosyst. Eng. 2009, 104, 447-457. [CrossRef]

22. Roth, S.D. Ray casting for modeling solids. Comput. Graph. Image Process. 1982, 18, 109-144. [CrossRef]

23. Chen, Y.; Medioni, G. Object modelling by registration of multiple range images. Image Vis. Comput. 1992, 10, 145-155. [CrossRef]

24. Euler, L. Formulae generales pro translatione quacunque corporum rigidorum. Novi Acad. Sci. Petrop 1775, 20, 189-207.

25. Rodrigues, O. Des lois géométriques qui régissent les déplacements d'un système solide dans l'espace: Et de la variation des cordonnées provenant de ces déplacements considérés indépendamment des causes qui peuvent les produire. In Journal de Mathématiques Pures et Appliquées; Gauthier-Villars: Paris, France, 1840; pp. 380-440.

26. Lorensen, W.E.; Cline, H.E. Marching cubes: A high resolution 3D surface construction algorithm. In Proceedings of the 14th Annual Conference on Computer Graphics and Interactive Techniques, Anaheim, CA, USA, 27-31 July 1987; Volume 21, pp. 163-169. 
Article

\title{
Multisensor Capacitance Probes for Simultaneously Monitoring Rice Field Soil-Water-Crop-Ambient Conditions
}

\author{
James Brinkhoff ${ }^{1, *}$, John Hornbuckle ${ }^{1}$ and Thomas Dowling ${ }^{2}$ \\ 1 Centre for Regional and Rural Futures Deakin University, Hanwood 3217, New South Wales, Australia; \\ j.hornbuckle@deakin.edu.au \\ 2 Goanna Telemetry Systems, Goondiwindi 4390, Queensland, Australia; tdowling@goannatelemetry.com.au \\ * Correspondence: james.brinkhoff@deakin.edu.au
}

Received: 23 October 2017; Accepted: 22 December 2017; Published: 26 December 2017

\begin{abstract}
Multisensor capacitance probes (MCPs) have traditionally been used for soil moisture monitoring and irrigation scheduling. This paper presents a new application of these probes, namely the simultaneous monitoring of ponded water level, soil moisture, and temperature profile, conditions which are particularly important for rice crops in temperate growing regions and for rice grown with prolonged periods of drying. WiFi-based loggers are used to concurrently collect the data from the MCPs and ultrasonic distance sensors (giving an independent reading of water depth). Models are fit to MCP water depth vs volumetric water content (VWC) characteristics from laboratory measurements, variability from probe-to-probe is assessed, and the methodology is verified using measurements from a rice field throughout a growing season. The root-mean-squared error of the water depth calculated from MCP VWC over the rice growing season was $6.6 \mathrm{~mm}$. MCPs are used to simultaneously monitor ponded water depth, soil moisture content when ponded water is drained, and temperatures in root, water, crop and ambient zones. The insulation effect of ponded water against cold-temperature effects is demonstrated with low and high water levels. The developed approach offers advantages in gaining the full soil-plant-atmosphere continuum in a single robust sensor.
\end{abstract}

Keywords: water depth sensors; soil moisture sensors; temperature sensors; rice field monitoring; irrigation

\section{Introduction}

Multisensor capacitance probes (MCPs) feature multiple sensors along a vertical tube [1]. Some have sensors that can be distributed along the plastic tube as needed, such as Sentek EnviroScan [2] probes. Others are sealed and have sensors fixed at regular intervals, for example $100 \mathrm{~mm}$, as is the case for EnviroPro [3] probes. Each sensor in a probe is typically able to sense volumetric water content (VWC), temperature and conductivity. This makes them useful for characterizing those variables at multiple depths through the soil profile [4].

MCPs are typically used in irrigated crops, to monitor soil moisture status and schedule irrigations [5]. They are widely used in horticulture, cotton and cereal crops. By calculating the total water content, and checking for leeching beyond the root zone, optimal irrigation quantities can be determined [1]. Their characteristics with respect to temperature, salinity and soil type have been well studied, and calibrations developed to accurately determine volumetric water content from raw probe readings for numerous soil types [6,7]. Some probes, such as those used in this study, have calibrations for moisture variation with temperature and conductivity built in [3]. 
The demands of growing rice in temperate regions are quite different from those of other crops. Low temperatures at the microspore stage cause spikelet sterility [8], particularly for low temperatures around the rice plant panicle and to a lesser extent around the root zone. Deep ponded water has been used in temperate rice-growing regions to insulate against cold-temperature events [9]. However, in semi-arid regions, water is scarce, and ponded water leads to an increase in rice crop water use [10]. So careful management of water depth in rice paddies is critical to achieve environmental and productivity goals. This necessitates monitoring and management of water level, as well as temperatures at the root zone, in the water, at pannicle height and ambient. Many of these considerations, specifically for the Australian rice growing environment, are detailed in [11], where a water depth of $250-300 \mathrm{~mm}$ is recommended during the microspore growth phase to guard against cold-induced sterility.

Recently, techniques to minimise water use have been investigated, such as alternate wetting and drying (AWD) and delayed permanent water (DPW) [12,13]. Also, growing rice aerobically in temperate regions is gathering interest $[12,14]$. These developments require the monitoring of soil moisture to ensure sufficient water is available for rice plant growth during periods where the water is drained. Within Australia, water scarcity and increased competition for water is seeing many farmers actively look to dynamically manage water height for controlling the temperature the rice crop is exposed to during critical growth periods. This will aid in maximising yield and improving water use efficiency by maintaining high water levels only when required.

Various sensors can be used to automatically monitor these parameters (water depth, temperatures, soil moisture status). Capacitance-based fluid level sensors have been used in many applications, for example to measure fuel tank levels in [15]. An integrated soil moisture and water depth sensor for rice paddys based on a single capacitance sensor was presented in [16]. An array of sensors was used to monitor air and soil temperature, soil moisture and water height of rice fields in [17].

In this paper, we demonstrate the usefulness of MCPs as a robust option to simultaneously monitor all these parameters in a single probe. This provides advantages over the traditional method of using multiple sets of discrete sensors. Temperatures at multiple heights and volumetric water content (VWC) data is gathered by a single MCP. To provide an accurate estimation of water depth, the sensitivity to water depth within each of an MCP's individual sensor's $100 \mathrm{~mm}$ zone is characterized, and models are fit to the characteristics. Models are first developed for these $100 \mathrm{~mm}$ sensor zones, then these model parameters are used as initial conditions to fit global models covering the whole length of the MCPs.

WiFi-based loggers developed by the authors, named WiFields, were used to gather data from the sensors [18]. They were deployed in a rice field, simultaneously gathering data from EnviroPro MCPs, and MaxBotix MB7389 ultrasonic distance sensors. Comparisons between water depth measured using an ultrasonic distance sensor and a $\mathrm{MCP}$ are provided, as well as demonstration of the utility of gathering the temperature and soil moisture profile in fine $(100 \mathrm{~mm})$ increments.

\section{Materials and Methods}

\subsection{WiFi-Based Data Loggers}

WiFi-based agricultural data loggers (WiFields) were used to gather and upload sensor data. These loggers were developed by the authors, and make use of an Electric Imp (http:/ / www.electricimp.com/) imp002 module which integrates a low-power microcontroller, WiFi radio and antenna. The loggers include common agriculture sensor interfaces such as SDI-12, UART and One-Wire. The loggers are programmed to periodically poll the sensors (typically once per hour), store sensor data, and if a WiFi connection to the Internet is available, upload all stored data to a cloud-based service, such as Google Sheets. The loggers then go into an ultra-low power sleep state until the next reading is due, saving 
power and ensuring battery operation over a whole cropping season. More detail on the WiField logger electronic design and software is available in [18].

\subsection{Sensors}

\subsubsection{Ultrasonic Distance Sensors}

MaxBotix MB7389 ultrasonic distance sensors (https:/ / www.maxbotix.com/Ultrasonic_Sensors / MB7389.htm) were used to provide an independent measure of water depth. They were positioned above the water surface, facing directly downwards. The water depth could then be determined by:

$$
d=d_{\text {ground }}-d_{\text {reading }}
$$

In the equation, $d$ is the water depth, $d_{\text {ground }}$ is the distance between the sensor and the ground under the water, and $d_{\text {reading }}$ is the distance reported by the sensor to the surface of the water, all in $\mathrm{mm}$.

The MB7389 sensors were chosen for a number of reasons. They are robust and suitable for outdoor environments. They operate at $42 \mathrm{kHz}$, and have a narrow field-of-view, so there is less impact from interference from nearby plants or other objects. They include DSP to filter out noise from small reflections, so that the distance to the target with the largest acoustic return (in this case the water surface) is the one reported. They are low power, and operate from $3.3 \mathrm{~V}$, which is the regulated supply voltage for many parts of the WiField logger circuitry. They were connected to the UART interface of the WiField loggers, providing readings with a resolution of $1 \mathrm{~mm}$. The raw strings transmitted over UART were parsed by the logger code to determine $d_{\text {reading }}$ as an integer, with units of $\mathrm{mm}$.

The ultrasonic sensors were used in laboratory characterization of the MCPs, and in field verification. For the field verification, the sensors were suspended above the ponded water of the rice crop as shown in Figure 2. Rice plants under the ultrasonic sensor were removed in order not to obscure the principal reflection from the water surface.

\subsubsection{Multisensor Capacitance Probes (MCPs)}

The multisensor capacitance probes used were manufactured by EnviroPro [3]. The probes were connected to the SDI-12 interface of the WiFields. These MCPs are calibrated and sealed at the factory. They are designed to be used as soil moisture probes with a moisture, conductivity and temperature sensor at $100 \mathrm{~mm}$ intervals along the length of the probe. In this study, we used 8 -sensor $(800 \mathrm{~mm}$ long) and 12-sensor (1200 mm long) probes. The moisture readings are returned as volumetric water content (VWC) in $\mathrm{m}^{3} / \mathrm{m}^{3}$ as a percentage. This VWC is determined inside the probes from dielectric constant readings and is calibrated for a sandy soil. The user does not have access to the raw dielectric constant data, but calibration equations to scale the VWC to other soil types are provided by the manufacturer. The VWC is also calibrated against temperature and conductivity (salinity) variation inside the probes [3]. The moisture, temperature and conductivity readings returned over the SDI-12 interface were parsed by the logger and uploaded to the cloud-based Google Sheet as floating-point values. The spreadsheet is then further processed in Python to generate data directly useful to growers.

A diagram of a probe is shown in Figure 1. The bottom $60 \mathrm{~mm}$ of the probes were found to be insensitive to moisture. For a probe with $\mathrm{N}$ sensors, the total probe length is $N \times 100+60 \mathrm{~mm}$. The bottom $n_{s}$ sensors are buried in the soil. The remaining sensors above the soil can be used to determine the level of the water. The water depth relative to the $n^{\text {th }}$ sensor is $d_{n}$ (with a range of $0-100 \mathrm{~mm}$ ), and the total water depth in $\mathrm{mm}$ is

$$
d=\sum_{n_{s}}^{N-1} d_{n}
$$




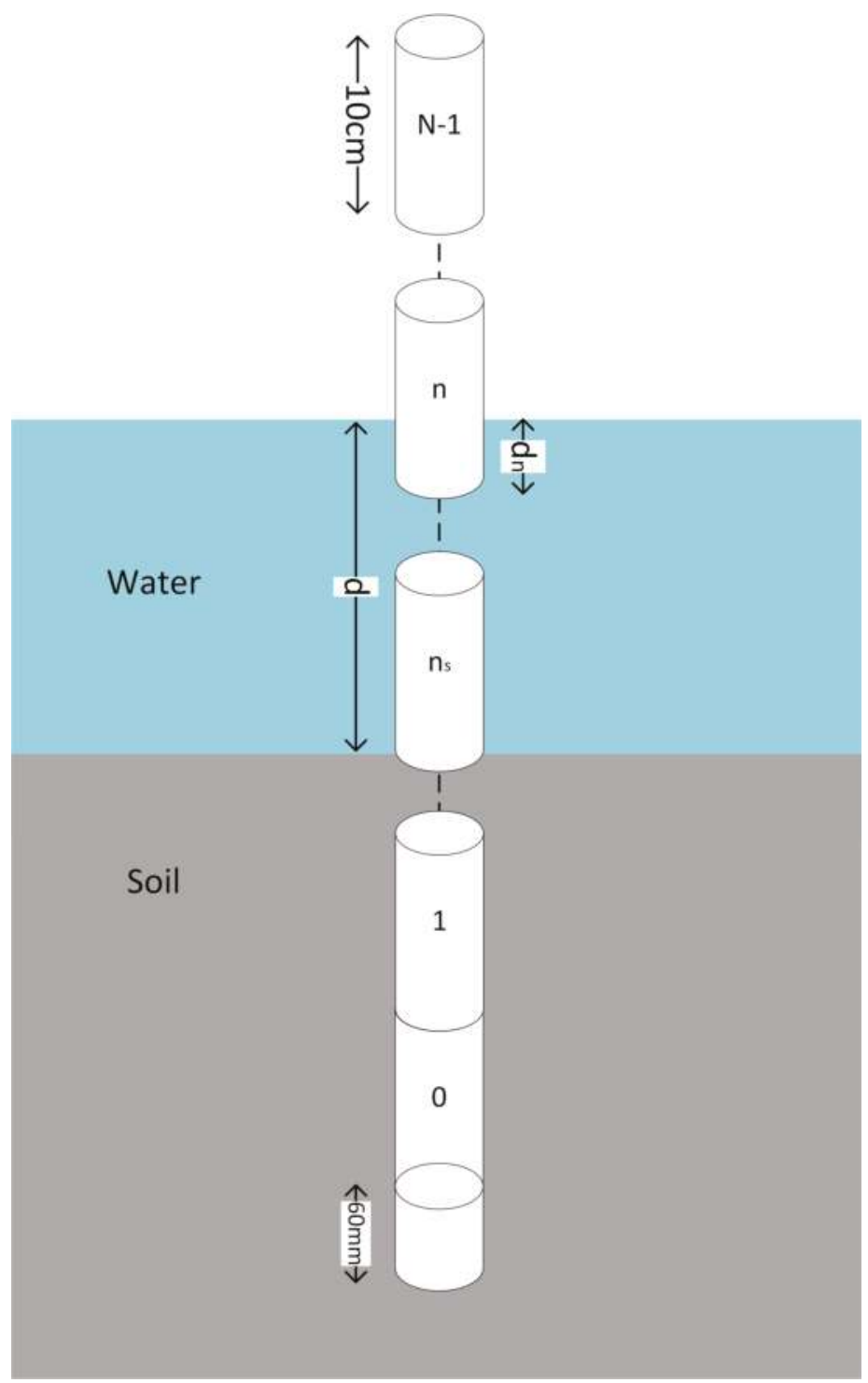

Figure 1. Diagram of a multiple-sensor capacitive soil moisture probe. The probe contains $N-1$ sensors, $d$ is the total water depth in $\mathrm{mm}, d_{n}$ is the water depth relative to the bottom of the $n$-th sensor and $n_{S}$ is the sensor sitting above soil level. Each of the sensors returns volumetric water content (VWC) $(\%)$, temperature $\left({ }^{\circ} \mathrm{C}\right)$ and conductivity $(\mathrm{dS} / \mathrm{m})$ data.

Figure 2 shows a photo of a WiField logger installed in a rice field, with connected ultrasonic and MCP sensors. 


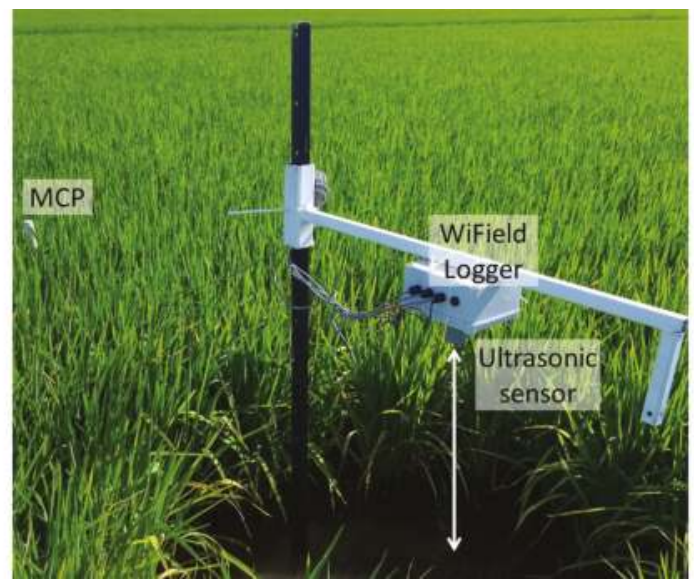

Figure 2. Photograph of a WiField data logger installed in a rice field with ultrasonic water level sensor, temperature sensors and MCP attached.

\subsection{Models for Water Depth as a Function of the MCP VWC}

The characteristics of the MCP VWC as a function of water depth were measured in a laboratory using a WiField logger. The probe was placed inside a $150 \mathrm{~mm}$ diameter PVC pipe. The ultrasonic sensor was used to measure the actual water depth. As the pipe was slowly filled with water, the water depth from the ultrasonic sensor, and the VWC from the MCP were logged frequently. The MCP measures the dielectric content of the surrounding medium, and calculates the VWC (assuming a sandy soil) for each of the $100 \mathrm{~mm}$ spaced sensors as a percentage $\left(\theta_{n_{\text {raw }}}\right)$. Only these VWC readings are available to the user (not the dielectric constant or frequency readings), so we developed the following methodology to calculate water depth from the available VWC data. The raw VWC of each sensor was normalized so that the VWC is between $0 \%-100 \%$ as follows:

$$
\theta_{n_{\text {norm }}}=\frac{\theta_{n_{\text {raw }}}-\theta_{n_{\text {raw min }}}}{\theta_{n_{\text {raw max }}}-\theta_{n_{\text {raw min }}}} \times 100 \quad[\%]
$$

If the $n$-th sensor is in air, the VWC $\theta_{n_{\text {norm }}}$ will be zero, a fully submerged sensor will give of $\theta_{n_{\text {norm }}}=100 \%$, and a partially-submerged sensor will be between $0 \%-100 \%$. A simple linear model for water depth relative to each sensor is then:

$$
d_{n}=\frac{\theta_{n_{\text {norm }}}}{100} \times d_{\text {sensor }} \quad[\mathrm{mm}]
$$

where $d_{\text {sensor }}$ is the spacing of the sensors along the probe, which is $100 \mathrm{~mm}$ in this case. These $d_{n}$ can then be summed to find the total water depth using (2). However, because of the in-built VWC calibrations and because the probe sensor characteristics vary over their $100 \mathrm{~mm}$ region of sensitivity, the curve relating the water depth to VWC is nonlinear. Each of the sensors is less sensitive at the 0 and $100 \mathrm{~mm}$ ends of their range than they are in the middle. Therefore, we evaluated a number of models that can better fit the depth vs VWC characteristic, which has an inverse-sigmoid shape.

The first was a general polynomial model of order $k$ :

$$
d_{n}=\sum_{1}^{k} \alpha_{k} \theta_{n_{\text {norm }}}^{k}
$$


Next was a model using a tangent function, which has the desired inverse-sigmoid shape:

$$
d_{n}=\alpha_{1} \tan \left(\alpha_{2} \theta_{n_{\text {norm }}}+\alpha_{3}\right)+\alpha_{4}
$$

Knowing that $d_{n}=0$ when $\theta_{n_{\text {norm }}}=0$, we can reduce the degrees of freedom of this tangent model to three:

$$
\alpha_{4}=-\alpha_{1} \tan \left(\alpha_{3}\right)
$$

Various other probit functions were also evaluated, but they had similar characteristics and performance to the tangent model, so are not shown here.

The parameters of the above models were fit to depth vs VWC data using the optimizer functions of the SciPy Python library [19]. Reasonable initial guesses were provided for the tangent function parameters to improve convergence.

\section{Results}

\subsection{MCP Laboratory Characterization}

\subsubsection{Per-Sensor Model Fitting}

The laboratory setup used the $150 \mathrm{~mm}$ diameter PVC pipe, with the MCP in the center and the ultrasonic sensor pointing down the pipe. Water was slowly added to the pipe, and the ultrasonic depth and MCP VWC logged continuously. If $d$ is the water depth in the pipe from the ultrasonic depth sensor, and having determined the bottom $60 \mathrm{~mm}$ of the MCP is insensitive, the water depth relative to the $n^{\text {th }}$ sensor (being the currently partially submerged sensor, see Figure 1) of the MCP is

$$
d_{n}=(d-60) \bmod 100
$$

We can then examine the characteristic of each of the $100 \mathrm{~mm}$ sensors individually. The PVC pipe was slowly filled to $660 \mathrm{~mm}$, so the characteristics of 6 sensors could be determined. The modulo'd data is shown as points in Figure 3. The per sensor data was then interpolated and the mean over all sensors at each water depth was computed, and is shown as the solid red line in the figure. The resulting curve fits are shown in Figure 3, and the parameters of the models are shown in Table 1 in the "local" columns. The linear model (4) has up to $10 \mathrm{~mm}$ error over some $\theta_{n_{\text {norm }}}$ values (i.e., at $20 \%$ and $90 \%$ ). For the polynomial model (5), a number of polynomial orders were tried. Finally, a fifth-order polynomial was used, which gave reasonable accuracy while not oscillating between fit points, as higher-order polynomials tended to do.

Table 1. Curve fit parameters from MCP per-sensor VWC to water depth for each of the models. The local columns are the parameters extracted from the per-sensor data, and the global columns are the parameters optimized over the whole MCP.

\begin{tabular}{ccccc}
\hline \multirow{2}{*}{ Parameter } & \multicolumn{2}{c}{ Tangent } & \multicolumn{2}{c}{ Polynomial } \\
\cline { 2 - 5 } & Local & Global & Local & Global \\
\hline$\alpha_{1}$ & 36.0 & 23.9 & 2.36 & 2.61 \\
$\alpha_{2}$ & $16.8 \times 10^{-3}$ & $22.7 \times 10^{-3}$ & $63.5 \times 10^{-3}$ & $-96.0 \times 10^{-3}$ \\
$\alpha_{3}$ & -1.05 & -1.12 & $1.44 \times 10^{-3}$ & $2.46 \times 10^{-3}$ \\
$\alpha_{4}$ & & & $-17.1 \times 10^{-6}$ & $-29.7 \times 10^{-6}$ \\
$\alpha_{5}$ & & & $76.0 \times 10^{-9}$ & $131 \times 10^{-9}$ \\
\hline
\end{tabular}




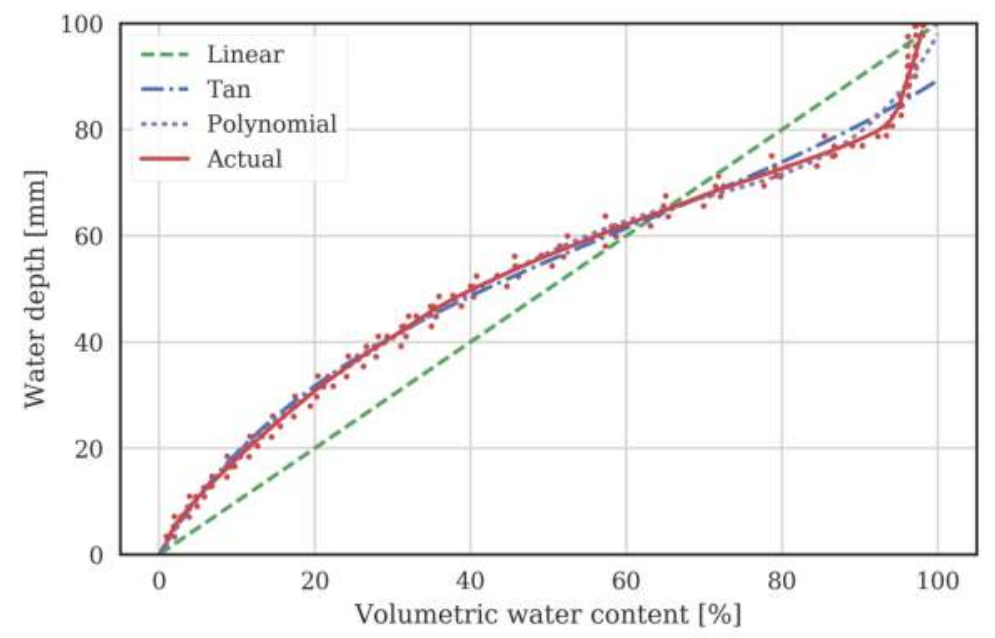

Figure 3. Water depth vs probe normalized VWC $\left(\theta_{n_{\text {norm }}}\right)$. Measured points from each sensor of the $\mathrm{MCP}$ are shown as red points, and the mean over all these sensors as a solid red line. Other lines indicate the water depth calculated from $\theta_{n_{\text {norm }}}$ using various models.

\subsubsection{Whole Probe Model Fitting}

Having fit local models to the mean of the MCP sensors characteristics, the obtained parameters were then used as initial conditions to optimize global models across the whole MCP length, which take account of overlap between the $100 \mathrm{~mm}$ sensitivity regions of the individual sensors. The global model depth is the sum of the individual sensor depths, from (2). The obtained global model parameters are also shown in Table 1. The modelled depth from VWC as a function of the actual water depth is shown in Figure 4. The ideal characteristic would be a straight line, with calculated depth from the MCP equalling measured depth from the ultrasonic sensor. The deviation between each of these models and the ideal straight line is shown in the bottom graph of Figure 4.

The root mean squared error (RMSE) is computed to give a quantitative assessment of the goodness-of-fit for each of the models. RMSE is defined as

$$
\text { RMSE }=\sqrt{\sum_{i=1}^{k} \frac{\left(d_{i}-\hat{d}_{i}\right)^{2}}{k}}
$$

where $d_{i}$ is the actual water depth, $\hat{d}_{i}$ is the modeled depth (both in $\mathrm{mm}$ ) and $k$ is the number of readings. The RMSE is 9.9, 4.4 and $4.7 \mathrm{~mm}$ for the linear, polynomial and tangent models respectively. In the following results, only the tangent model results will be shown as it has similar accuracy to the polynomial model while requiring fewer parameters. 

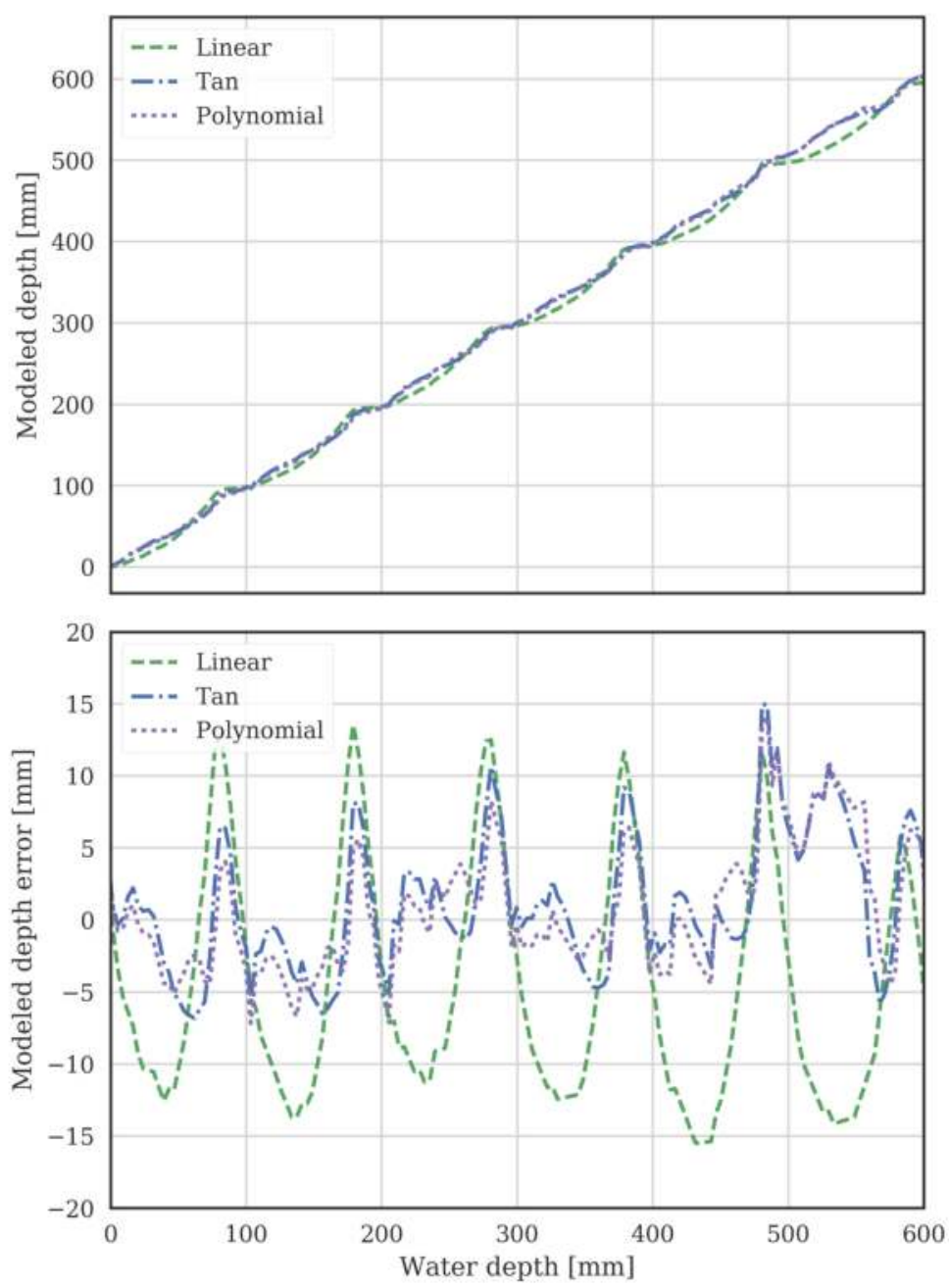

Figure 4. Water depth using the three models processing the characterized MCP's VWC data, with actual water depth measured by the ultrasonic sensor on the x-axis. The bottom graph shows the error between the actual (ultrasonic) and modeled (MCP) depth.

\subsubsection{Probe-to-Probe Variability}

The models above were extracted from a single 12-sensor probe in the laboratory. To assess variability from probe-to-probe, and to test the robustness of the characterization procedure, three additional probes were measured in the same laboratory setup. Two of these were 12-sensor probes, and one was an 8-sensor probe. The errors between measured water depth (from the ultrasonic sensor) and the MCP probe VWC data processed using the tangent model is shown in Figure 5. Note, the global model parameters in Table 1, which were extracted from the initial characterized probe, were used.

The RMSE (9) of the tangent model for the original probe the model was extracted from was $4.7 \mathrm{~mm}$, as described in Section 3.1.2. The RMSE of the tangent model for these additional independent 
3 probes was 4.9, 5.4 and $7 \mathrm{~mm}$ for the two 12 -sensor probes and 8-sensor probe respectively. This gives confidence that the model is well able to predict water level from the probe VWC readings, and the variation between probes is well controlled.

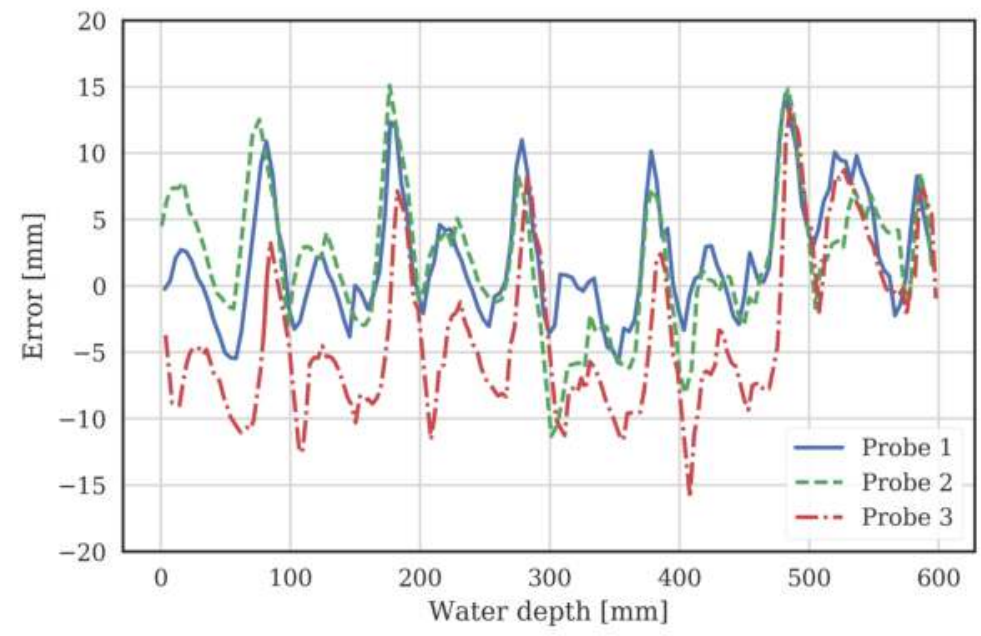

Figure 5. The error between actual (using the ultrasonic sensor) and calculated (using the VWC data from the MCP processed using the tangent model) water depth from 3 additional independent MCPs. Probes 1 and 2 are 12-sensor probes, and probe 3 is an 8 -sensor probe.

\subsection{Rice Field Verification}

In order to assess the usefulness of MCPs in a real rice growing situation, a WiField logger was installed in a field in Whitton, NSW, Australia (Figure 2). The rice variety was Topaz, the emergence date was 18 Novermber 2016, and harvest was on 12 May 2017. The soil type according the World Reference Base for Soil Resources [20] is Luvisol, and according to the local classification system is transitional red brown earth [21]. The WiField logger measured and uploaded data once per hour over the 2016-2017 rice growing season. The logger had the same sensors connected as those in the laboratory setup, a MaxBotix MB7389 ultrasonic sensor to measure ponded water depth and a 12-sensor EnviroPro soil moisture probe. In the example given, the top of the MCP was $700 \mathrm{~mm}$ above the soil, so water depths and above-ground temperatures to $700 \mathrm{~mm}$ could be measured. The remaining 5 sensors were below the soil and could be used to monitor soil moisture and temperature to a depth of $500 \mathrm{~mm}$. Generally, the rice crop root zone is within $200 \mathrm{~mm}$ of the soil surface, so an 8-sensor probe would be sufficient to measure both the rice root zone soil moisture and the ponded water depth. Since the usefulness of the technique was demonstrated in the 2016-2017 season, additional units are being used in additional rice fields in the current season.

Figure 6 shows the water depth measured with the ultrasonic sensor, together with the water depth calculated from the MCP using the linear and tangent models. The RMSE over the season was 9.6 and $6.6 \mathrm{~mm}$ for the linear and tangent models respectively. The units functioned continuously over the season in the commercial farming situation and provided data in real time to the farmer that allowed him to actively adjust water depth on the fields based on the data. Note the farmer ensured the water was deep during the microspore growth period from late January to early February, to provide insulation against possible cold temperature events as recommended in [11].

The field data is shown in Figure 7 as a regression plot, with the actual depth on the $x$-axis, and the modeled depth using the linear and tangent models on the $y$-axis. The equation of the linear model 
regression line is $d_{\text {lin }}=1.036 d-6.8$, with a coefficient of determination $R^{2}=0.985$, and $d$ is the actual depth in $\mathrm{mm}$. The equation of the tangent model regression line is $d_{\tan }=1.009 d-0.56$, with $R^{2}=0.991$. These results show the tangent model from the MCP data gives a very good approximation to the actual water depth, with expected slope near 1 and intercept near 0 .
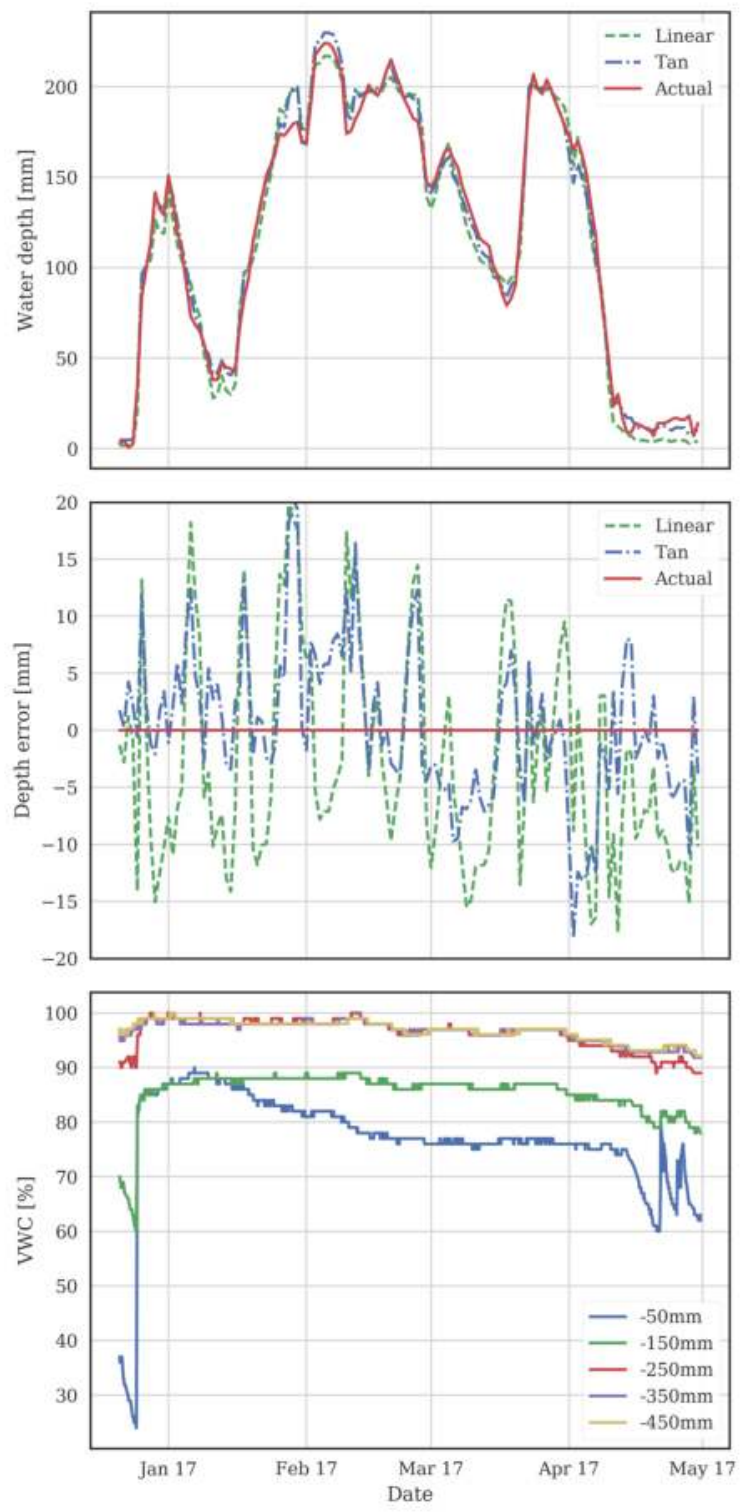

Figure 6. Measurements from a rice field over a growing season. The top graph shows the water depth from the ultrasonic sensor and from the MCP probe using the linear and tangent models. The middle graph shows the difference between the ultrasonic depth and depths calculated from the MCP readings. The bottom graph shows the soil moisture. 

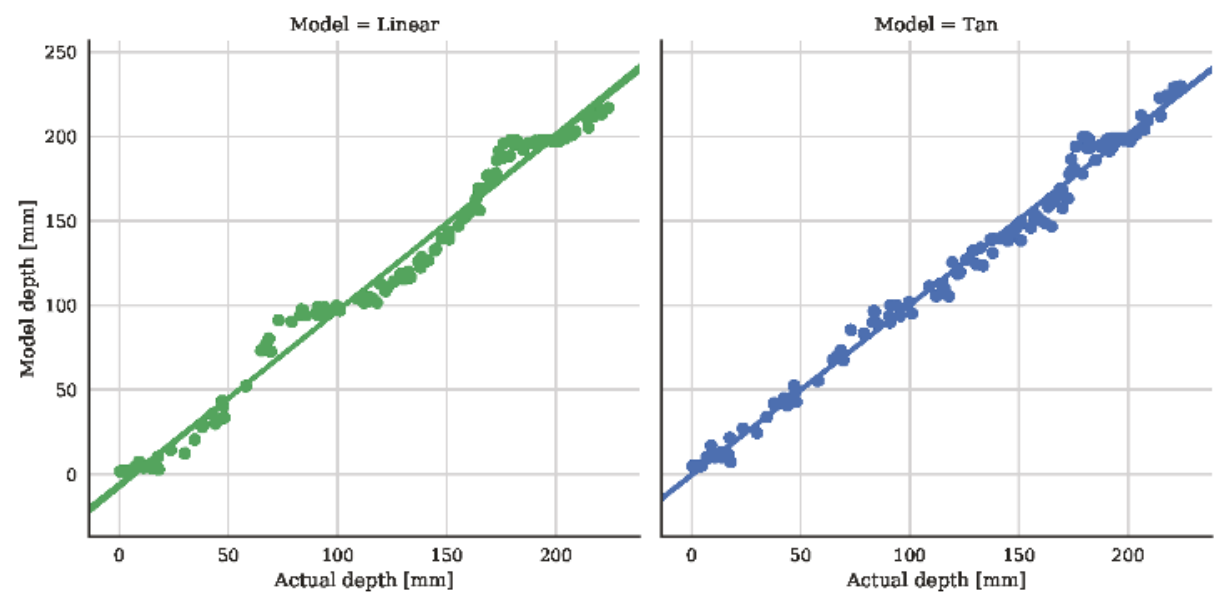

Figure 7. Regression plot of the modeled vs actual water depths from the rice field. The $R^{2}$ of the linear model is 0.985 , and that of the tangent model is 0.991 .

\section{Additional MCP Data}

One advantage of using MCPs in this application is the range of useful data available. As well as water depth, which has been the focus of this paper, data on soil moisture and temperature at fine $(100 \mathrm{~mm})$ intervals is also obtained. The moisture data is shown in the lower graph of Figure 6. The soil never becomes unsaturated at depths below $200 \mathrm{~mm}$. Early in the season before permanent ponded water is applied, the soil at $50 \mathrm{~mm}$ and $150 \mathrm{~mm}$ starts to dry out. It starts to dry again after the ponded water is drained in early-April. Some re-wetting of the soil at $50 \mathrm{~mm}$ due to rain events is evident in mid-April. Using the soil moisture information is particularly important in these early and late parts of the rice growing season to ensure the soil does not reach moisture stress levels, which would be detrimental to yield. The absolute VWC numbers depend on soil type and structure and varies with depth, as described in [22]. Calibration of these effects is beyond the scope of this paper but will be important areas of future study in using MCPs to schedule irrigations of rice fields with prolonged periods without ponded water. This is of particular relevance when managing newer water techniques such as alternate-wetting-drying (AWD) and delayed permanent water (DPW) as well as scheduling irrigation for newer aerobic rice varieties [12]. The variation of the VWC at 50 and $150 \mathrm{~mm}$ while permanent water is applied may be due to effects such as the growing roots affecting the air filled porosity of the soil.

The temperature data from the rice field at two dates is shown in Figure 8. On both of these dates, there were signigicant cold events where the ambient temperature got as low as $6{ }^{\circ} \mathrm{C}$. The first date is 20 February 2017, which for a late-sown crop in NSW, Australia would fall close to the critical microspore phase. If the rice panicle was subject to the cold ambient temperature of $6{ }^{\circ} \mathrm{C}$, there would likely be a significant negative impact on yield. The second date is 12 April 2017, when there would not be such sensitivity to cold because the rice has passed the microspore phase. On the first date, the water was above $200 \mathrm{~mm}$. It can be seen that this provided effective insulation against the cold temperatures at panicle height (around $200 \mathrm{~mm}$ ), keeping the temperature there above $12{ }^{\circ} \mathrm{C}$. The water effectively maintains warmer temperatures above the surface of the water through its stored energy. In contrast, at the second date, no such insulation was provided as the water had been nearly drained. This provides a useful illustration of how temperature at multiple heights can be used to manage rice paddy water depth to effectively insulate the crop from cold temperatures. For temperate growing conditions with such cold events, recommended water heights to guard against cold-induced yield loss are given in [11]. It is anticipated simultaneously gathering water depth and 
the comprehensive temperature profiles that the MCP makes possible, will enable optimization and automation of water depth.
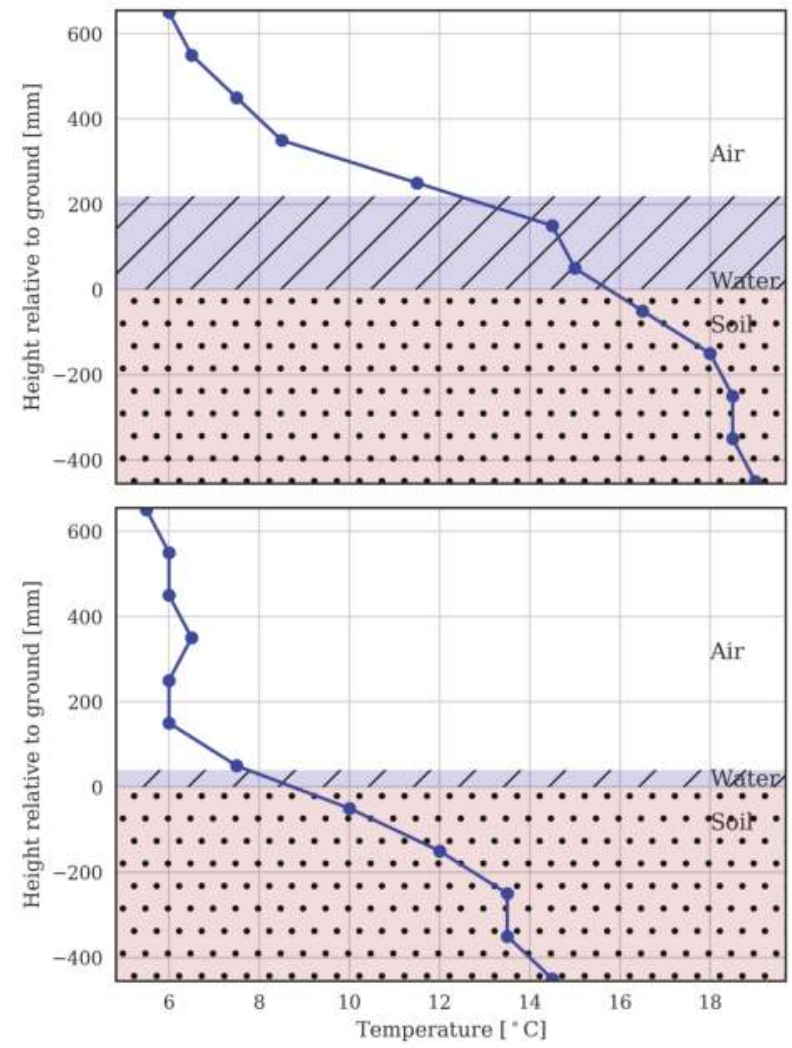

Figure 8. The temperature profile measured by the MCP during cold temperature events (20 February 2017 and 12 April 2017 6AM). The measured water depth is indicated with blue shading.

\section{Discussion}

In rice field monitoring applications, there is a need to monitor temperatures and soil moisture as well as water depth. Table 2 shows typical sensor types used to measure these parameters, and their relative cost, accuracy, robustness in field conditions, and typical interface used to connect the sensor to a data logger. Of course, there are a wide variety of manufacturers producing each of these sensors, so the table is only intended to be indicative of common examples.

Sensors that are typically used to monitor water depth include those using ultrasonic, pressure and capacitance measurements. Pressure sensors are accurate, but expensive. Ultrasonic sensors are subject to interference in open environments such as agricultural fields, particularly from insects, spiders and plants that grow into the field-of-view, so tend to not be robust in field environments and require regular maintenance. Many single capacitance sensors are not very accurate, being subject to variation with temperature and salinity, for example [23]. 
Table 2. Comparison of using an MCP versus other sensor combinations to measure water depth, soil moisture and temperature.

\begin{tabular}{cccccc}
\hline Parameter & Sensor Type & Cost & Accuracy & Robustness & Typical Interface \\
\hline \multirow{3}{*}{ Water depth } & Pressure & $\$ \$ \$$ & +++ & +++ & $40-20 \mathrm{~mA}$ \\
& Ultrasonic & $\$ \$$ & + & + & UART \\
& Capacitance & $\$ \$$ & + & ++ & Voltage \\
Soil moisture & Gypsum block & $\$$ & ++ & ++ & AC resistance \\
& Capacitive & $\$ \$$ & + & ++ & Voltage \\
Temperature & DS18B20 & $\$$ & + & ++ & OneWire \\
All parameters & MCP & $\$ \$$ & ++ & +++ & SDI-12 \\
\hline
\end{tabular}

Soil moisture may be monitored with capacitance probes or gypsum blocks. The latter requires an AC resistance interface in order not to polarize the sensor. This interface is not available on many data loggers. It is usually desired to monitor moisture at multiple depths to gain an understanding of the moisture available throughout the root zone, so multiple sensors are needed.

Temperature sensors come in many types, with the digital DS18B20 sensors being quite common. For rice monitoring, multiple sensors are needed to determine soil, water, crop and ambient temperatures. It can be seen that simultaneously monitoring all the parameters of interest requires loggers with multiple interface types.

In view of the above, a $\mathrm{MCP}$ offers a unique solution. It measures many of the required environmental parameters in one robust unit with a single data interface. So multiple temperature sensors, a separate soil moisture sensor and water depth sensor are not required. This simplicity is attractive in agriculture, where reliability, robustness and lack of clutter are important considerations. For monitoring water level, MCPs offer a good trade-off between cost, accuracy and reliability. Though they are not inexpensive compared to some individual sensors, if the cost and interface requirements for a complete monitoring solution is considered (including soil moisture, water depth and multiple temperatures), their cost is not prohibitive relative to the benefits provided. It is also worth noting that many growers already own MCPs for other purposes. The MCPs can therefore be re-deployed for other uses during alternate seasons/years, so for example they could be used to monitor the water level in a rice field over the summer, and then the soil moisture in a wheat crop over the winter.

This study has developed techniques using the EnviroPro MCP. The same procedure of fitting models to the VWC vs water depth characteristic would be applicable to other MCPs too, provided their individual sensor sensitivity regions overlap, and that temperature and salinity variation is compensated, either by the probe (as is the case for the probes used in this study) or the user.

Acknowledgments: The authors acknowledge the support of AgriFutures Australia project funding, and thank Garry Knagge for allowing loggers to be installed on his rice farm.

Author Contributions: J.B. designed the WiField loggers and software, completed the laboratory and field measurements and analyzed the data. J.H. assisted with field installation and provided information on the rice field monitoring application. T.D. provided the original idea of measuring water level using an MCP.

Conflicts of Interest: The authors declare no conflict of interest.

\section{References}

1. Fares, A.; Polyakov, V. Advances in crop water management using capacitive water sensors. Adv. Agron. 2006, 90, 43-77.

2. Sentek EnviroSCAN Probe. Available online: http://www.sentek.com.au/products/enviro-scan-probe.asp (accessed on 11 October 2017).

3. EnviroPro Soil Probes. Available online: http://www.enviroprosoilprobes.com/ (accessed on 11 October 2017). 
4. Charlesworth, P. Soil Water Monitoring; Land \& Water Australia: Canberra, Australia, 2005.

5. Fares, A.; Alva, A.K. Evaluation of capacitance probes for optimal irrigation of citrus through soil moisture monitoring in an entisol profile. Irrig. Sci. 2000, 19, 57-64.

6. Paltineanu, I.; Starr, J. Real-time soil water dynamics using multisensor capacitance probes: Laboratory calibration. Soil Sci. Soc. Am. J. 1997, 61, 1576-1585.

7. Schwank, M.; Green, T.R.; Mätzler, C.; Benedickter, H.; Flühler, H. Laboratory characterization of a commercial capacitance sensor for estimating permittivity and inferring soil water content. Vadose Zone J. 2006, 5, 1048-1064.

8. Gunawardena, T.; Fukai, S.; Blamey, F. Low temperature induced spikelet sterility in rice. II. Effects of panicle and root temperatures. Crop Pasture Sci. 2003, 54, 947-956.

9. Williams, R.; Angus, J. Deep floodwater protects high-nitrogen rice crops from low-temperature damage. Aust. J. Exp. Agric. 1994, 34, 927-932.

10. Heenan, D.; Thompson, J. Growth, grain yield and water use of rice grown under restricted water supply in New South Wales. Aust. J. Exp. Agric. 1984, 24, 104-109.

11. Rice Growing Guide 2016-17. Available online: https://www.dpi.nsw.gov.au/_data/assets/pdf_file/ 0007/178171/rice-growing-guide-2016-17.pdf (accessed on 9 December 2017).

12. Humphreys, E.; Meisner, C.; Gupta, R.; Timsina, J.; Beecher, H.; Lu, T.Y.; Gill, M.; Masih, I.; Guo, Z.J.; Thompson, J. Water saving in rice-wheat systems. Plant Prod. Sci. 2005, 8, 242-258.

13. Dunn, B.; Dunn, T.; Beecher, H. Nitrogen timing and rate effects on growth and grain yield of delayed permanent-water rice in South-Eastern Australia. Crop Pasture Sci. 2014, 65, 878-887.

14. Kato, Y.; Okami, M.; Katsura, K. Yield potential and water use efficiency of aerobic rice (Oryza sativa L.) in Japan. Field Crops Res. 2009, 113, 328-334.

15. Tward, E.; Junkins, P.D. Multi-Capacitor Fluid Level Sensor. U.S. Patent No. 4,417,473, 3 February 1982.

16. Xiao, D.; Feng, J.; Wang, N.; Luo, X.; Hu, Y. Integrated soil moisture and water depth sensor for paddy fields. Comput. Electron. Agric. 2013, 98, 214-221.

17. Kawakami, Y.; Furuta, T.; Nakagawa, H.; Kitamura, T.; Kurosawa, K.; Kogami, K.; Tajino, N.; Tanaka, M.S. Rice Cultivation Support System Equipped with Water-level Sensor System. IFAC-PapersOnLine 2016, 49, 143-148.

18. Brinkhoff, J.; Hornbuckle, J. WiField, an IEEE 802.11-based Agricultural Sensor Data Gathering and Logging Platform. In Proceedings of the 11th International Conference on Sensing Technology, Sydney, Australia, 4-6 December 2017.

19. Jones, E.; Oliphant, T.; Peterson, P. SciPy: Open Source Scientific Tools for Python. Available online: https://www.scipy.org/ (accessed on 9 December 2017).

20. Food and Agriculture Organisation of the United Nations (FAO). World Reference Base for Soil Resources 2014, International Soil Classification System for Naming Soils and Creating Legends for Soil Maps (Update 2015); World Soil Resources Reports 106; FAO: Quebec, QC, Canada, 2015.

21. Hornbuckle, J.W.; Christen, E. Physical Properties of Soils in the Murrumbidgee and Coleambally Irrigation Areas; CSIRO Land and Water: Canberra, Australia, 1999.

22. Gasch, C.K.; Brown, D.J.; Brooks, E.S.; Yourek, M.; Poggio, M.; Cobos, D.R.; Campbell, C.S. A pragmatic, automated approach for retroactive calibration of soil moisture sensors using a two-step, soil-specific correction. Comput. Electron. Agric. 2017, 137, 29-40.

23. Larson, P.; Runyan, C. Evaluation of a Capacitance Water Level Recorder and Calibration Methods in an Urban Environment. 2009. Available online: http://www.umbc.edu/cuere/BaltimoreWTB/pdf/TM_2009_ 003.pdf (accessed on 16 December 2017).

(C) 2017 by the authors. Licensee MDPI, Basel, Switzerland. This article is an open access article distributed under the terms and conditions of the Creative Commons Attribution (CC BY) license (http:/ / creativecommons.org/licenses/by/4.0/). 


\title{
Article \\ A Compound Sensor for Simultaneous Measurement of Packing Density and Moisture Content of Silage
}

\author{
Delun Meng ${ }^{1}$, Fanjia Meng ${ }^{1, *}$, Wei Sun ${ }^{2}$ and Shuang Deng ${ }^{1}$ \\ 1 Key Laboratory on Modern Precision Agriculture System Integration Research, Ministry of Education, \\ China Agricultural University, Beijing 100083, China; mdlun@126.com (D.M.); Dengs415@163.com (S.D.) \\ 2 Agricultural Information Institute, Chinese Academy of Agricultural Sciences, Beijing 100081, China; \\ sunwei01@caas.cn \\ * Correspondence: meng@cau.edu.cn; Tel.: +86-137-0133-5698
}

Received: 27 October 2017; Accepted: 23 December 2017; Published: 28 December 2017

\begin{abstract}
Packing density and moisture content are important factors in investigating the ensiling quality. Low packing density is a major cause of loss of sugar content. The moisture content also plays a determinant role in biomass degradation. To comprehensively evaluate the ensiling quality, this study focused on developing a compound sensor. In it, moisture electrodes and strain gauges were embedded into an ASABE Standard small cone for the simultaneous measurements of the penetration resistance $(\mathrm{PR})$ and moisture content $(\mathrm{MC})$ of silage. In order to evaluate the performance of the designed sensor and the theoretical analysis being used, relevant calibration and validation tests were conducted. The determination coefficients are 0.996 and 0.992 for PR calibration and 0.934 for MC calibration. The validation indicated that this measurement technique could determine the packing density and moisture content of the silage simultaneously and eliminate the influence of the friction between the penetration shaft and silage. In this study, we not only design a compound sensor but also provide an alternative way to investigate the ensiling quality which would be useful for further silage research.
\end{abstract}

Keywords: silage; packing density; moisture content; compound sensor; simultaneous measurement

\section{Introduction}

Packing density and moisture content of silage are of great significance in guiding the fine processing of silage and improving its quality. A lower packing density means a higher porosity, which could result in more oxygen remaining in the silage. Excessive oxygen will cause carbohydrates reduction, protein denaturation and quality degradation during the aerobic phase [1-9]. In addition, the biomass moisture affects the activity of antioxidant bacteria and the degree of degradation of the biomass $[1,10]$. In general, to prevent the loss of dry matter and sugar content of silage, both factors should be optimally controlled.

The traditional method for determining the packing density of silage is to calculate its gross density from mass and volume. The disadvantage of this method is that it is unable to show density differences at specific sites. The $\gamma$-ray scanner is an effective tool to analyze the packing density in two dimensions and the relative error of measurement could be $\pm 1 \%[11,12]$. However, the $\gamma$-ray scanner is not widely applied due to its high cost and the potential danger to health [2-4]. In recent years, the penetrometer technique has been used to measure the packing density of silage because it is straightforward to calibrate and can provide reliable data [13]. In many penetrometer designs [1,10], the force sensor was placed on the upper end of the penetration shaft. Given that silage was a complex porous elastoplastic medium, the penetration shaft would be extruded laterally by silage in the penetration process. Therefore, penetration resistance $(\mathrm{PR})$ obtained by these penetrometers was generally referred to as a superposition of cone resistance and the friction force between the penetration shaft and the 
tested material [14]. To improve the measuring accuracy of the penetrometer, Y. Sun et al. developed a mathematical filter to correct the friction-induced error of PR measurements $[14,15]$. For the MC measurement of silage, the stoving method and the infrared method have been employed [16,17]. The stoving method has not met the production process's requirements because of its time-consumption and sample destruction. For the infrared method, the configuration of the sensor was complicated and not robust. Silage, like soil, is a complex porous elastoplastic medium and PR is not only strongly dependent on packing density but also on water content [18]. Most instruments applying the above-mentioned measurement methods are designed to obtain a specified parameter and are unable to accurately evaluate the ensiling quality. In this study, a novel compound sensor was designed to measure the PR and MC of silage simultaneously and calibrated in the laboratory. Validation tests were conducted to verify its feasibility and ascertain the correlation between the PR and MC of silage.

\section{Compound Sensor Design}

The compound sensor, shown in a schematic diagram (Figure 1), consists of a probe, a penetration shaft and wires. The probe and the penetration shaft are made of nickel chromium alloy material and designed strictly based on the ASABE Standard [19]. The angle of the cone is 30 degrees, the diameter of the cone is $12.83 \mathrm{~mm}$ and the diameter of the shaft is $9.53 \mathrm{~mm}$. In addition, the wire connects the probe and the measurement circuits through the penetration shaft.

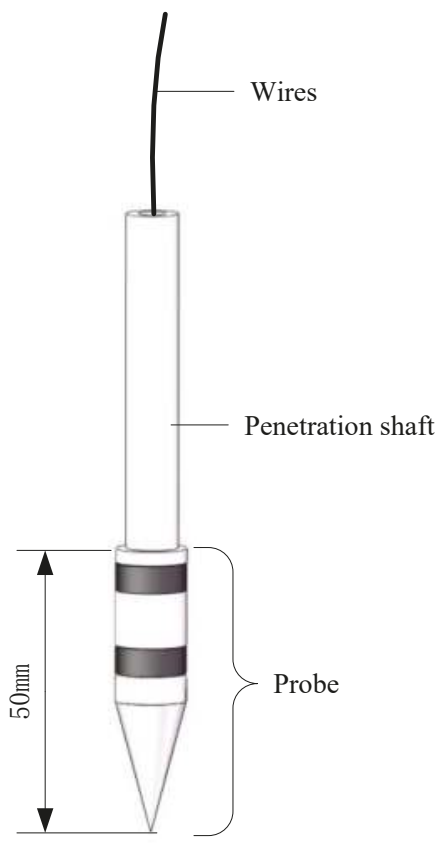

Figure 1. Diagram of the compound sensor.

A photo of the probe of the compound sensor is shown in Figure 2a. The measurement principle of PR is based on the fact that the resistance of a metal conductor varies with the magnitude of its mechanical deformation, that is, the resistance-strain effect. In order to eliminate the influence of the friction on the shaft, resistance-strain gauges are embedded into the probe and Figure $2 b$ illustrates the structure. The hollow metal rod was regarded as an elastic body and four platinum resistance-strain gauges $(350 \Omega$ ) are pasted on the outer surface of it. Wires connect the platinum resistance-strain gauges to form a Wheatstone bridge circuit, as shown in Figure 3. 


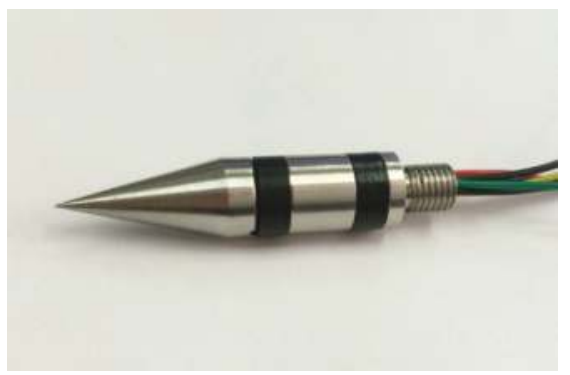

(a)

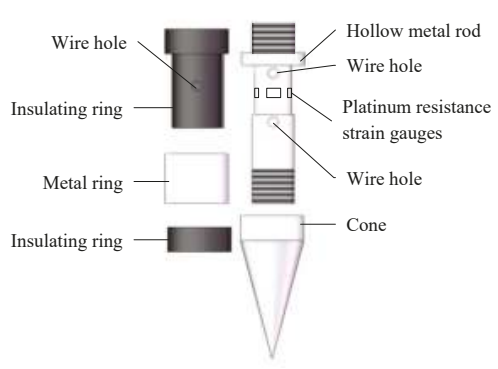

(b)

Figure 2. The probe of the compound sensor. (a) A photo of the probe; (b) Detailed structure of the probe.

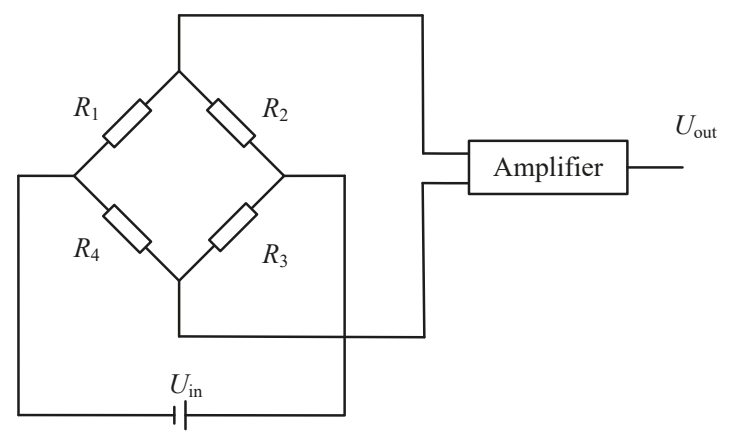

Figure 3. Diagram of the Wheatstone bridge circuit.

In the Wheatstone bridge circuit, each arm of the bridge contains a platinum resistance strain gauge. When the cone is compressed, the hollow metal rod will deform. Based on the resistance-strain effect of metal, the resistance of the strain gauge would change according to the mechanical deformation. The formula for calculating the voltage output $U_{\text {out }}$ of the bridge is

$$
U_{\text {out }}=A \frac{R_{1} R_{3}-R_{2} R_{4}}{\left(R_{1}+R_{2}\right)\left(R_{3}+R_{4}\right)} U_{\text {in }}
$$

where $U_{\text {out }}$ represents the output voltage of the bridge and $U_{\text {in }}$ represents the input voltage of the bridge. $R_{1}, R_{2}, R_{3}$ and $R_{4}$ refer to the resistance values of the bridge arm respectively and $A$ is the amplification factor. The measurement force range of the compound sensor is $0-1 \mathrm{kN}$ and the output signal of the bridge corresponds to $0-2.5 \mathrm{~V}$.

The measurement principle of $\mathrm{MC}$ is based on the dielectric theory, according to which the relative dielectric permittivity of water is 81 , far greater than that of the drying biological materials $\left(\varepsilon_{\mathrm{r} \text {-wood }} \approx 3\right)$ and that of air $\left(\varepsilon_{\mathrm{r} \text {-air }}=1\right)$ [1]. The water content of biological materials directly affects the relative dielectric permittivity. Therefore, the MC of biomaterials can be indirectly obtained by measuring the relative dielectric permittivity. Thus, the moisture content of silage is measured by the frequency domain method [20-23] in this study and the working principle is shown in Figure 4. 


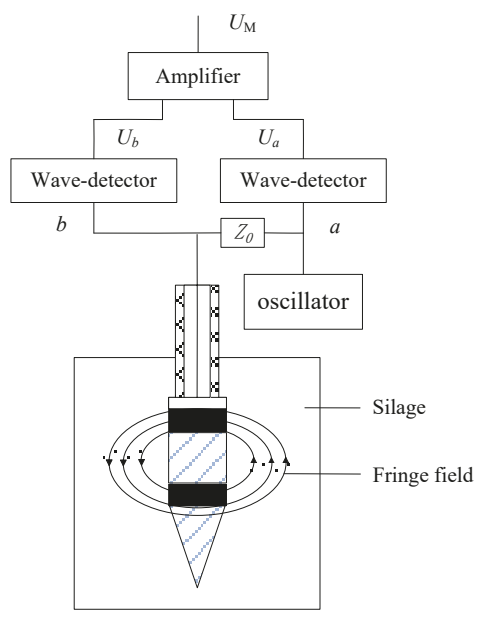

Figure 4. Schematic diagram of the compound sensor for moisture content measurement.

The metal ring and cone, separated by two insulating rings, acted as the moisture electrodes of the compound sensor. A segment of coaxial line cable through the center of the penetration shaft connected both electrodes to an oscillator $(100 \mathrm{MHz})$. Based on the measurement principle of the moisture content, the characteristic impedance of the probe depended on the fringe-effect field across the two electrodes and the relative dielectric permittivity of the silage, which varied with the ratio of water. Therefore, there is a mapping relation between the characteristic impedance of the probe and silage moisture content. $Z_{\mathrm{P}}(\Omega)$, standing for the electric impedance of the probe, can be determined from Equation (2)

$$
Z_{\mathrm{P}}=\frac{Z_{0}}{U_{\mathrm{a}}-U_{\mathrm{b}}} U_{\mathrm{b}}
$$

where $Z_{0}(\Omega)$ is called balance impedance and $U_{\mathrm{a}}$ and $U_{\mathrm{b}}$ are the output signals of each wave detector.

When the length of the transmission line is $\lambda / 4$ ( $\lambda$ is the test frequency wavelength), the MC signal, $U_{\mathrm{M}}$, can be calculated by

$$
U_{\mathrm{M}}=U_{\mathrm{a}}-U_{\mathrm{b}}=2 B \frac{Z_{\mathrm{P}}-Z_{0}}{Z_{\mathrm{P}}+Z_{0}}
$$

where $B$ represents the amplitude of the excitation signal.

\section{Results and Discussion}

\subsection{Force Calibration}

The intention of the force calibration is to establish the relationship between the output signal of the force measuring circuit and the force applied to the compound sensor. To create a reference calibration, a force-testing machine (DWM-10, 0 10 kN, 0.01\%, Suzhou Longsheng Testing Equipment Co. Ltd., Suzhou, China) was utilized to apply force to the compound sensor, as shown in Figure 5. The compound sensor was bolted to the support bracket. As the crossbeam, which was driven by a ball screw, moved down or up, the weights were loaded or unloaded steadily. The calibration includes two processes: power stroke and return stroke. The calibration result (Figure 6) shows that, in each process, there is a linear equation with a determination coefficient $\left(R^{2}\right)$ of 0.99 relating the output of the compound sensor to force values within a range of $0-1 \mathrm{kN}$. 


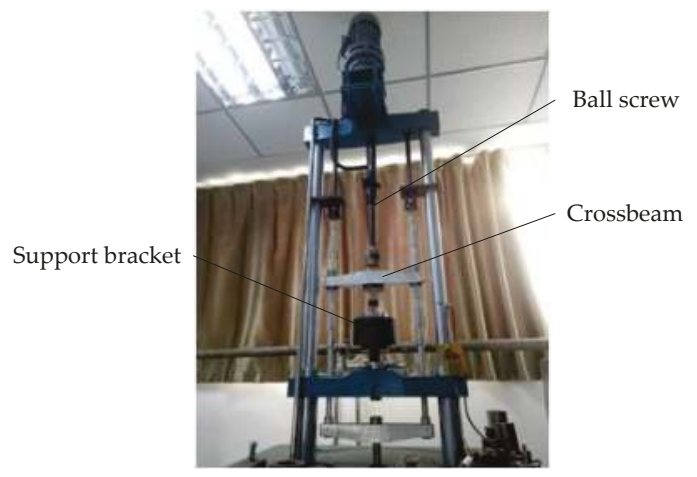

Figure 5. A photo of the force-testing machine.

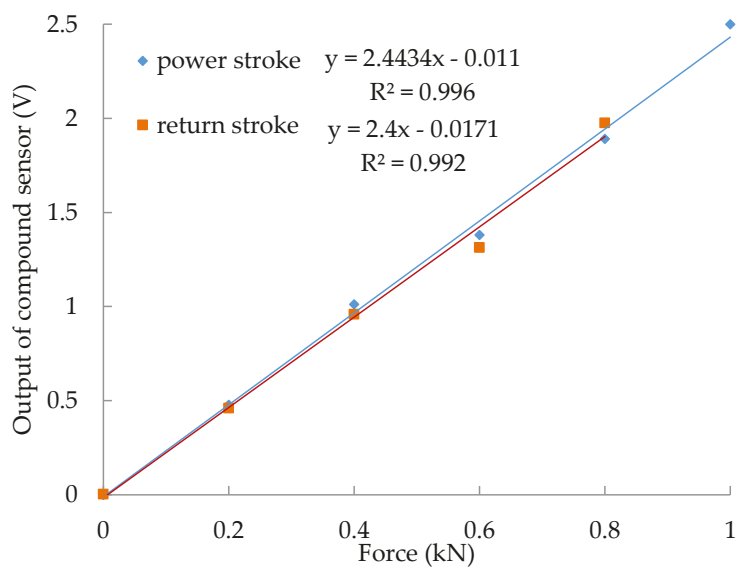

Figure 6. The force calibration results.

\subsection{Calibration of Moisture Content}

The silage material for calibration was chopped maize with moisture content of $63.9 \%$. Before the calibration, eight silage samples were dried at $105^{\circ} \mathrm{C}$ for different degrees and filled into cylinders (inner radius: $200 \mathrm{~mm}$, height: $500 \mathrm{~mm}$ ) with a density of $0.8 \mathrm{~g} / \mathrm{cm}^{3}$ respectively. To ensure that the density was distributed homogeneously, the chopped maize samples were pressured into this cylinder by a material-testing machine (Zwick 1445, Zwick GmbH \& Co. KG, Ulm, Germany) layer by layer. Each sample was sampled three times and the arithmetic average value of the output voltage was recorded. After the measurements, the silage samples were weighed and oven-dried for $24 \mathrm{~h}$ at $105^{\circ} \mathrm{C}$, then weighed again and their moisture content was calculated based on the mass of wet and dry samples. The calibration result (Figure 7) shows a linear relationship between the measured MC values and the output signals of the compound sensor within a range of $34.8 \sim 63.9 \%$ and the correlation is approximated by a linear calibration equation with the determination coefficients $\left(\mathrm{R}^{2}\right)$ of 0.934 . 


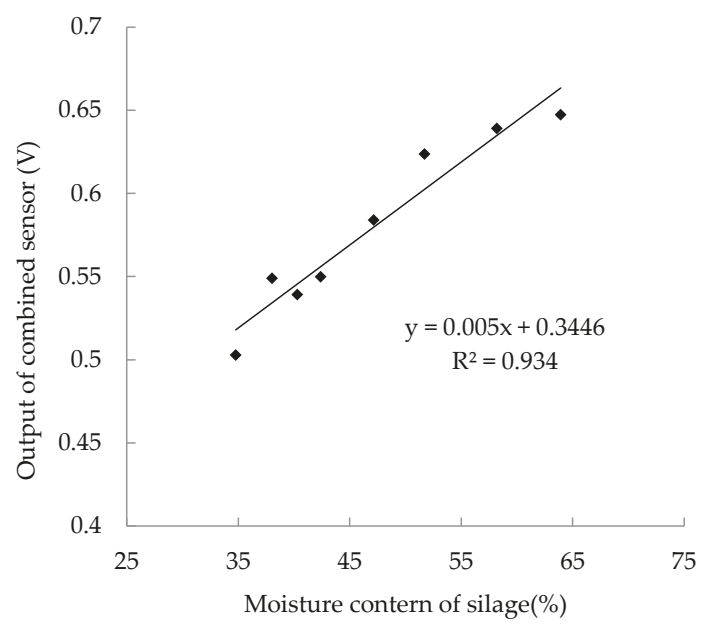

Figure 7. The calibration of moisture content results.

\subsection{Validation Test}

A validation system, which includes a motor-operated penetrometer, a frame and a cylinder, is shown in Figure 8. According to the ASABE Standard, to ensure the penetration speed of the compound sensor is stable at $30 \mathrm{~mm} / \mathrm{s}$ and to improve the operation and control accuracy during the penetration process, the ball screw was selected as the transmission part. The ball screw is driven by a motor $(100 \mathrm{~W}, 1800 \mathrm{r} / \mathrm{min}$, permanent magnet direct current motor, Shanghai Weiting Electric Co. Ltd., Shanghai, China) through a reduction gearbox (reduction ratio: 5:1). A slider is motivated by the ball screw to slide along the axis, thereby converting the rotation of the motor to vertical motion. The compound sensor is installed below the slider and a force sensor (BK-4D, 0 1000 N, 0.5\%, CAAA, Beijing, China) is installed at the upper end of the penetration shaft. When the slider moves downward and the cone penetrates the testing silage, the validation system can synchronously obtain the pressure measurement data of these two sensors in real time. The vertical travel of the penetrometer cone could be adjusted from $100 \mathrm{~mm}$ to $700 \mathrm{~mm}$ and an encoder (500 p/r), acting as a transducer, was set up to output the depth signal of the compound sensor. When the cone reached the predetermined penetration depth or when the PR value exceeded $1000 \mathrm{~N}$, the DC motor would automatically reverse. A micro-controller (MSP430F149, TI, Dallas, TX, USA) was used to log data and coordinate the entire measurement in sequence.

According to the methods described in Section 3.2, 12 cylinders of silage were prepared and the sample information is presented in Table 1. The measurement results of four samples (Cylinder 1 to Cylinder 4) are shown in Figure 9. Within the first insertion where depths are less than $50 \mathrm{~mm}$, the MC signal of the compound sensor (MCC) and the PR signal of the compound sensor (PRC) show great increase. This is due to the fact that, in the initial insertion, the two electrodes of the probe are not fully embedded in the silage and the action and reaction between the cone and the silage do not reach steady state. When insertion depths are greater than $50 \mathrm{~mm}$, the curves of both signals of the compound sensor tend to be horizontal. This shows that MCC keeps close track of PRC, indicating that the compound sensor can determine the packing density and moisture content of the silage simultaneously. 


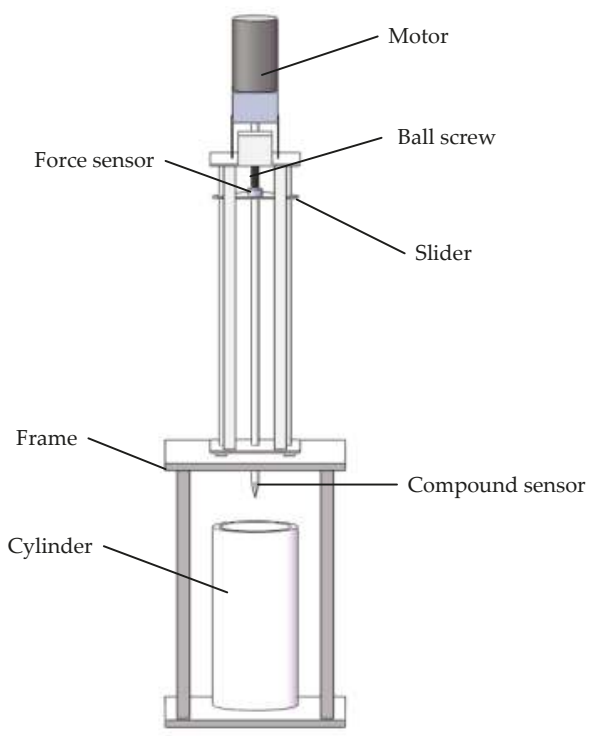

Figure 8. Diagram of the validation system.

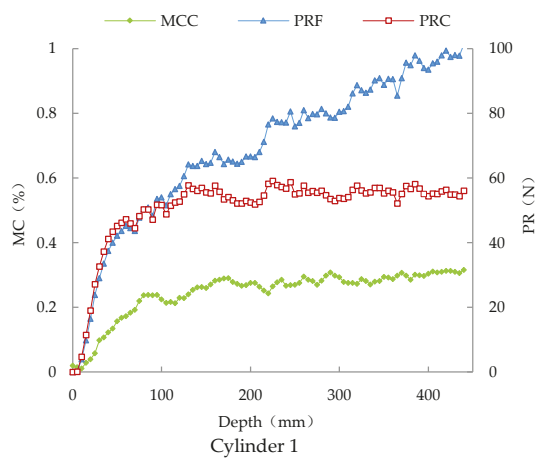

(a)

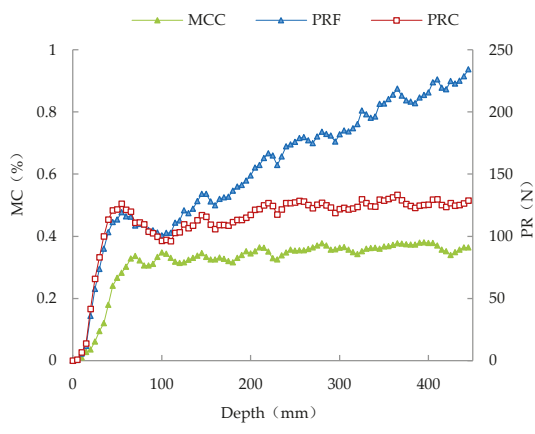

Cylinder 3

(c)

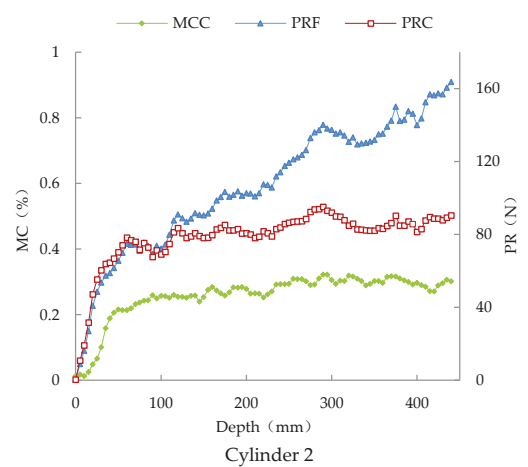

(b)

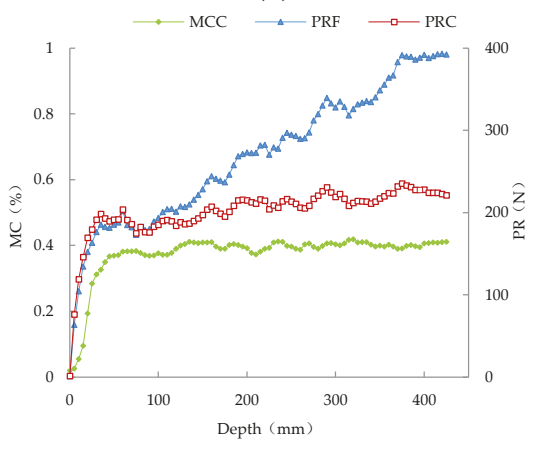

Cylinder 4

(d)

Figure 9. Dynamic measurement results of the compound sensor of Cylinder 1 to Cylinder 4. 
As can be seen from Figure 9, in the initial insertion, the penetration resistance signals of the force sensor (PRF) are basically the same as PRC. Then after the probe was completely embedded in the silage, PRF profiles showed a linear relation to the penetration depth or the contacted area between the shaft and the silage and PRC profiles reached a plateau. For the homogeneous distribution of density in all the cylinders, packing density would change little by depth and this agrees with PR profiles measured with the compound sensor. This shows that the compound sensor designed in this study can effectively eliminate the influence of the friction between the penetration shaft and silage, improving the measurement accuracy.

Table 1. Sample Information.

\begin{tabular}{lcccc}
\hline & Gravimetric Moisture Content $\mathbf{( \% )}$ & Mass $\mathbf{( g )}$ & Volume $\left.\mathbf{( c m}^{3}\right)$ & Dry Matter Density $\left(\mathrm{g} / \mathrm{cm}^{3}\right)$ \\
\hline Cylinder 1 & & 9150 & & 0.284 \\
Cylinder 2 & 49.8 & 10,150 & 0.324 \\
Cylinder 3 & & 11,151 & 0.362 \\
Cylinder 4 & & 12,150 & 0.394 \\
Cylinder 5 & 63.9 & 12,650 & & 0.283 \\
Cylinder 6 & & 14,150 & 15,700 & 0.319 \\
Cylinder 7 & & 15,650 & 0.358 \\
Cylinder 8 & 60.9 & 17,150 & 0.414 \\
Cylinder 9 & 62.4 & 12,022 & \\
Cylinder 10 & 63.2 & 12,487 & \\
Cylinder 11 & 64.0 & 12,756 & \\
Cylinder 12 & & 13,033 & 0.299 \\
\hline
\end{tabular}

Since the 12 samples were evenly packed into cylinders, the average value of the PR at different depths is taken as the PR values of the sample. For samples from Cylinder1 to Cylinder8, the correlation between the dry matter density and the PR value is shown in Figure 10. For the silage samples with the same MC, the PR values increase linearly with the dry matter density and correlations are approximated by tow linear calibration equations with the determination coefficients $\left(\mathrm{R}^{2}\right)$ of 0.999 and 0.926 respectively. On the other hand, for samples with the same dry matter density, the PR of samples with high MC is greater than those with low MC. As expected in Figure 11, there is a linear equation with $\mathrm{R}^{2}$ of 0.988 relating the PR and the MC of four samples (Cylinder 9 to Cylinder 12). It demonstrates that, under the same dry matter density condition, the PR of the silage increases linearly with the MC of the sample.

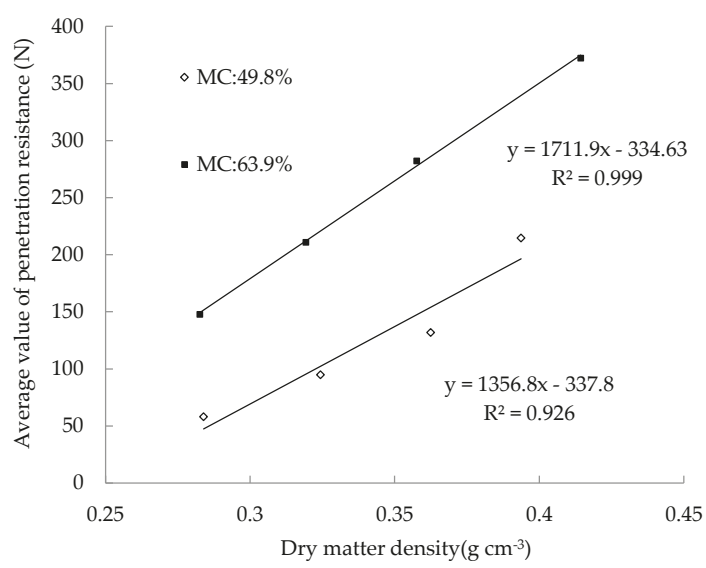

Figure 10. Average value of penetration resistance of silages with two different moisture contents. 


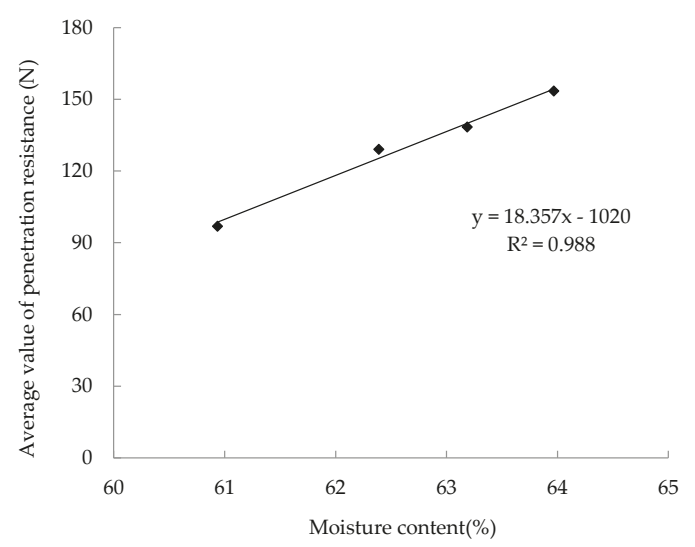

Figure 11. Average value of penetration resistance and volumetric moisture content of silages with the same dry matter density.

\section{Conclusions}

A compound sensor, designed based on ASABE standards, was developed as a novel technique for evaluating the quality of silage. In this compound sensor, the moisture electrode and strain gauges were embedded in an ASABE Standard small cone, which made the compound sensor capable of measuring the packing density and moisture content of silage simultaneously. From the results of relevant calibrations and validation, the following conclusions can be drawn: (1) The packing density and moisture content of the silage are linearly related to the outputs of the compound sensor and both the determination coefficients are greater than 0.93 . This indicates that the designed sensor could obtain the packing density and moisture content of the silage accurately. (2) The compound sensor could determine the packing density and moisture content of the silage simultaneously and eliminate the influence of the friction force between the penetration shaft and the surrounding silage. (3) For the samples with the same moisture content, the packing density increases with the increase of dry matter density. The packing density of the samples with the same dry matter density increases in accordance with the moisture content of these samples.

Acknowledgments: This work was supported by the National Natural Science Foundation of China under Project No. 31401294 and by the Specialized Research Fund for the Doctoral Program of Higher Education under Project No. 20130008120013.

Author Contributions: Fanjia Meng and Delun Meng conceived and designed the experiments; Fanjia Meng and Delun Meng performed the experiments; Delun Meng and Fanjia Meng analyzed the data; Delun Meng, Fanjia Meng and Wei Sun contributed reagents/materials/analysis tools; Delun Meng, Wei Sun and Shuang Deng wrote the paper.

Conflicts of Interest: The authors declare no conflict of interest.

\section{References}

1. Sun, Y.; Cheng, Q.; Meng, F.; Buescher, W.; Maack, C.; Ross, F.; Lin, J. Image-based comparison between a $\gamma$-ray scanner and a dual-sensor penetrometer technique for visual assessment of bale density distribution. Comput. Electron. Agric. 2012, 82, 1-7. [CrossRef]

2. Li, M.; Jungbluth, K.; Sun, Y.; Cheng, Q.; Maack, C.; Buescher, W.; Lin, J.; Zhou, H.; Wang, Z. Developing a Penetrometer-Based Mapping System for Visualizing Silage Bale Density from the Bunker Silo Face. Sensors 2016, 16, 1038. [CrossRef] [PubMed]

3. Meng, F.; Sun, Y.; Buescher, W.; Maack, C.; Cheng, Q.; Berg, A.; Lin, J.; Ross, F.; Zhang, H. An Extended Penetrometer System for the Map-based Assessment of Bale Density Distribution. Appl. Eng. Agric. 2012, 28, 449-455. [CrossRef] 
4. Sun, Y.; Buescher, W.; Lin, J.; Schulze Lammers, P.S.; Ross, F.; Maack, C.; Cheng, Q.; Sun, W. An improved penetrometer technique for determining bale density. Biosyst. Eng. 2010, 105, 273-277. [CrossRef]

5. Li, M.; Sun, Y.; Cheng, Q.; Jungbluth, K.H.; Buescher, W.; Maack, C.; Cheng, H.; Wang, Z. Mapping oxygen-induced temperature patterns of round bale silage based on 3D stepwise-profiling measurement. Measurement 2016, 82, 115-122. [CrossRef]

6. Sun, Y.; Li, M.; Zhou, H.; Shan, G.; Cheng, Q.; Jungbluth, K.H.; Buescher, W.; Maack, C.; Lipski, A.; Wang, Z.; et al. In situ measurements and simulation of oxygen diffusion and heat transfer in maize silage relative to the silo surface. Comput. Electron. Agric. 2017, 137, 1-8. [CrossRef]

7. Toruk, F.; Gonulol, E.; Ulger, P.; Kocabiyik, H. Density, porosity and permeability rates of sunflower silage under different compaction conditions. J. Anim. Vet. Adv. 2009, 8, 1873-1877.

8. Wambacq, E.; Vanhoutte, I.; Audenaert, K.; de Gelder, L.; Haesaert, G. Occurrence, prevention and remediation of toxigenic fungi andcmycotoxins in silage: A review. J. Sci. Food Agric. 2016, 96, 2284-2302. [CrossRef] [PubMed]

9. Pitt, R.E.; Muck, R.E. A diffusion model of aerobic deterioration at the exposed face of bunker silo. J. Agric. Eng. Res. 1993, 55, 11-26. [CrossRef]

10. Zheng, Y.; Yates, M.; Aung, H.; Cheng, Y.; Yu, C.; Guo, H.; Zhang, R.; VanderGheynst, J.; Jenkins, B.M. Influence of moisture content on microbial activity and silage quality during ensilage of food processing residues. Bioprocess Biosyst. Eng. 2011, 34, 987-995. [CrossRef] [PubMed]

11. Fuerll, C.; Schemel, H.; Koeppen, D. Principles for measuring density in silages. Landtechnik 2008, 63, 94-95.

12. Mumme, M.; Katzameryer, J. Mobile test station for the radiometric measurement of density distribution in bales. Landtechnik 2008, 63, 341-343.

13. Perumpral, J.V. Cone penetrometer application: A review. Trans. ASAE 1987, 30, 939-944. [CrossRef]

14. Sun, Y.; Meng, F.; Buescher, W.; Schulze Lammers, P.; Lin, J.; Ross, F.; Maack, C.; Cheng, Q. A study to identify and correct friction-induced error of penetration measurement for agricultural materials. Measurement 2012, 45, 829-835. [CrossRef]

15. Cai, X.; Sun, Y.; Schulze-Lammers, P.; Buescher, W.; Maack, C.; Meng, F.; Lin, J.; Cheng, Q.; Zhang, H. Shaft friction of an ASABE standard soil cone penetrometer. Trans. ASABE 2013, 56, 839-846.

16. ISO 6496:1999. Animal Feeding Stuffs_Determination of Moisture and Other Volatile Matter Content; ISO: Geneva, Switzerland, 1999.

17. Wang, C.; Yang, Z.; Zhang, W. Rapid Determination of Moisture in Feedstuffs with an Infrared Moisture Meter. Cereal Feed Ind. 2003, 2, 47-48.

18. Lin, J.; Sun, Y.; Lammers, P. Evaluating model-based relationship of cone index, soil water content and bale density using dual-sensor penetrometer data. Soil Tillage Res. 2014, 138, 9-16. [CrossRef]

19. ASABE Standards. Procedures for Using and Reporting Data Obtained with Soil Cone Penetromenter; ASAE Standard EP542; ASABE: St. Joseph, MI, USA, 1999.

20. Sun, Y.; Schulze-Lammersb, P.; Ma, D.; Lin, J.; Zeng, Q. Determining soil physical properties by multi-sensor technique. Sens. Actuators A Phys. 2008, 147, 352-357.

21. Naderi-Boldaji, M.; Alimardani, R.; Hemmat, A.; Sharifi, A.; Keyhani, A.; Dolatsha, N.; Keller, T. Improvement and field testing of a combined horizontal penetrometer for on-the-go measurement of soil water content and mechanical resistance. Soil Tillage Res. 2012, 123, 1-10. [CrossRef]

22. Naderi-Boldaji, M.; Sharifi, A.; Jamshidi, B.; Younesi-Alamouti, M.; Minaee, S. A dielectric-based combined horizontal sensor for on-the-go measurement of soil water content and mechanical resistance. Sens. Actuators A Phys. 2011, 171, 131-137. [CrossRef]

23. Shan, G.; Sun, Y.; Cheng, Q.; Wang, Z.; Zhou, H.; Wang, L.; Xue, X.; Chen, B.; Jones, S.B.; Schulze Lammersd, P.; et al. Monitoring tomato root zone water content variation and partitioning evapotranspiration with a novel horizontally-oriented mobile dielectric sensor. Agric. For. Meteorol. 2016, 228-229, 85-94. [CrossRef]

(c) 2017 by the authors. Licensee MDPI, Basel, Switzerland. This article is an open access article distributed under the terms and conditions of the Creative Commons Attribution (CC BY) license (http:/ / creativecommons.org/licenses/by/4.0/). 


\title{
Article \\ Combination of Multi-Agent Systems and Wireless Sensor Networks for the Monitoring of Cattle
}

\author{
Alberto L. Barriuso ${ }^{1, *}$, Gabriel Villarrubia González ${ }^{1}$, Juan F. De Paz ${ }^{1}$, Álvaro Lozano ${ }^{1}$ and \\ Javier Bajo ${ }^{2}$ \\ 1 Faculty of Science, University of Salamanca, Plaza de la Merced s/n, 37002 Salamanca, Spain; \\ gvg@usal.es (G.V.G.); fcofds@usal.es (J.F.D.P.); loza@usal.es (Á.L.) \\ 2 Department of Artificial Intelligence, Polytechnic University of Madrid, Campus Montegancedo s/n, \\ Boadilla del Monte, 28660 Madrid, Spain; jbajo@fi.upm.es \\ * Correspondence: albarriuso@usal.es; Tel.: +34-923-29-44-00
}

Received: 7 November 2017; Accepted: 28 December 2017; Published: 2 January 2018

\begin{abstract}
Precision breeding techniques have been widely used to optimize expenses and increase livestock yields. Notwithstanding, the joint use of heterogeneous sensors and artificial intelligence techniques for the simultaneous analysis or detection of different problems that cattle may present has not been addressed. This study arises from the necessity to obtain a technological tool that faces this state of the art limitation. As novelty, this work presents a multi-agent architecture based on virtual organizations which allows to deploy a new embedded agent model in computationally limited autonomous sensors, making use of the Platform for Automatic coNstruction of orGanizations of intElligent Agents (PANGEA). To validate the proposed platform, different studies have been performed, where parameters specific to each animal are studied, such as physical activity, temperature, estrus cycle state and the moment in which the animal goes into labor. In addition, a set of applications that allow farmers to remotely monitor the livestock have been developed.
\end{abstract}

Keywords: birth sensor; bovine embedded hardware; ambient intelligence; virtual organizations of agents

\section{Introduction}

The last decade saw a breakthrough in the field of Ambient Intelligence (AmI), resulting in an improvement in the quality of people's lives. The main objective of AmI is to adapt technology to the needs of the people in a way that allows users to interact in a natural and effortless manner with the different systems that make up the environment. Technology must act in a transparent way by adapting to the individuals and their context; simplifying the accomplishment of daily tasks and the communication between them and the environment. To achieve this goal, it is necessary to develop new technological models that allow users to interact with multiple devices simultaneously. The devices must collaborate in the accomplishment of daily tasks without the individuals being aware of it. Due to the miniaturization of sensors and the reduction of costs in manufacturing processes, AmI can now provide new solutions to daily problems that were unthinkable just a few years ago. One field to which AmI can be applied to is that of livestock and natural resources management.

Currently in Spain, there are more than 23 million head of swine and more than 18 million of sheep, representing $15 \%$ of the total of the European Union [1]. The continuous growth that the farms are experiencing and the increasing demand for farm produce, make technology an essential instrument of continuous improvement in the development of farms. Requirements are also becoming increasingly strict for both the breeders and the livestock, so the study of factors such as food, physical activity or the animal's health is necessary. On large farms, it is difficult to devote time to observing the behavior of each animal. Technology must provide solutions that simplify farmers' work, especially 
in repetitive and difficult tasks, such as those that entail the study of the factors mentioned above. The research and development of systems that detect anomalies in animals at early stages, is vitally important. Numerous research groups are working on the use of electronic systems in livestock with the aim of improving productivity and reducing operating costs. AmI allows farmers to remotely access up to date information on the animals and obtain their complete traceability. The joint use of information technology and electronic devices, allows to monitor and study parameters as well as the consumption of energy on the farm, the level of food in the feeders, the lighting, climatology, the state of animals' health, their physical activity, etc. Smart-farming or precision farming consists in applying information and communication technologies to livestock and agriculture. Its main objective is to increase the efficiency and quality of production through rapid decision making in cases where some type of problem arises on the farm.

This work presents a low cost wireless network sensor (WSN) interconnected through a multi-agent architecture based on virtual organizations. This system offers remote farm monitoring and traceability service. The aim pursued in this work is to save the farmer from unnecessary movement from place to place and to increase the productivity of the farm, reduce costs and risky situations. A WSN allows to obtain information on the environment and act upon it. The infrastructure of WSNs is capable of distributing communications in dynamic environments, increasing mobility and efficiency, regardless of the location. It is important to inquire into mechanisms that allow constructing computing agent platforms and communication agents that are portable to light devices.

The presence of heterogeneous protocols makes the integration of sensors difficult, since it makes it necessary to incorporate new communication systems between the different elements. When necessary, the proposed system will permit the sending of alerts to the mobiles of users, who will be able to visualize problems of each animal in real time.

By using sensors, it will be possible to detect situations such as the beginning of labor or the estrus cycle, rumination problems, excess body temperature, the distance travelled, events and information of interest to the farmer. The novelty of the system is the implementation of a dashboard which allows people with little technological knowledge to visualize the state of the farm, using a TV.

The article is structured as follows: Section 2 reviews the state of the art and related projects; Section 3 describes the proposed architecture; and outlines the case study that has been conducted to evaluate the system; and finally the results obtained and the conclusions drawn from the work are presented in Section 4. In addition, Appendix A has been included with the hardware design schemes used in this work.

\section{Background}

Due to the miniaturization of hardware, devices are gradually reaching out to all the levels of society [2]. This fact, combined with the lowering production costs of consumer electronics, has favored the rise of Ambient Intelligence (AmI) in different areas such as robotics [3], health [4], education [5] or the transport industry [6]. In short, we can see how the application of AmI techniques has spread to practically every field.

The field of agriculture and livestock is no stranger to the development of AmI projects. In recent years, the implementation of different technological solutions has been promoted in order to move towards intelligent agriculture. In the case of the European Union, such initiatives have been supported by the European Parliament, as evidenced by two reports, which are of great importance to The Alliance for Internet of Things Innovation (AIOTI) [7]: “Technological solutions for sustainable agriculture in the EU" [8] and "Enhancing innovation and economic development in future European farm management" [9].

Smart Farming is based on the use of information and communication technologies, combined with tools that support decision-making and improve the efficiency, productivity and profitability of companies in this sector [10]. The use of intelligent devices allows improving traditionally used tools, by providing them with context awareness and intelligence, as well as automating the accomplishment 
of different tasks or allowing them to be performed remotely [11]. To create a Smart Farm, a network infrastructure is required which allows to connect different low-cost sensors and wireless actuators, used in the monitoring and remote control of facilities and livestock. Data collected by sensors (such as odometry of agricultural machinery, soil moisture, temperature, soil conductivity, $\mathrm{pH}$ level, animal position, body temperature, etc.) are transmitted to a data storage station, where they can be observed and analyzed using artificial intelligence techniques. Thus, farmers can increase their agricultural production through the statistics, patterns or predictions obtained from these data. Moreover, the use of actuators favors the automation and remote operation of certain tasks, such as cattle feeding, the use of irrigation systems, or temperature control in closed facilities where the livestock is located [12].

Santana-Mancilla et al. outline in their work [13] a set of practical considerations that should be taken into account when using and designing Wireless Sensor Networks (WSNs) for livestock farms. Some of the challenges tackled that work are related to: (i) mobility: the sensors used in the monitoring of animal parameters are subject to continuous changes of position, so the topology of networks and the routing of paths must be adapted and respond to the movement of animals; (ii) interferences produced by the animals: in many cases, animals are in herds and their own bodies, especially in cattle, can cause a significant reduction in the radio signal; (iii) limitations in data storage: due to the nature of low cost sensors, their capacity for data storage is limited, so it must be compensated with the use of robust protocols that allow to transmit the information collected by the sensors to central nodes; and (iv) battery life: since replacing batteries in livestock is an expensive process, the optimization of battery life in sensors is crucial; thus, we must work on reducing the energy consumed by data transmission.

Within the scope of livestock, AmI techniques have been most popularly implemented in the beef cattle industry. Given the complexity of breeding beef, the use of AmI allows farmers to perform certain tasks or detect possible problems that their animals may present. In the state of the art work, some of the most relevant studies apply AmI to the following fields:

- Monitoring the position of the animal and analysis of the behavior of the herd: By monitoring the position of livestock, it is possible to study the behavior of both individual animals and the herd as a whole. Since it may be argued that wearable technology annoys animals and alters their habitual behavior, studies that deny this theory have been carried out [14]. Wearable technology designed for animals usually takes the form of necklaces that make use of GPS, although there are also devices that make use of other technologies such as ZigBee [15]. Studies in which the position of cattle is monitored, vary in their objectives, some for example focus on assessing the animals' periods of rest, ingestion and activity $[16,17]$ and others on controlling grazing time in certain areas of the field [15]. In addition, the use of technology allows quickly locating cattle on extensive farms, which is normally a difficult task.

- Intake control/feeding monitoring: Ingestion, rumination and rest are the main activities of ruminant cattle. Thanks to their monitoring, breeders are able to take better decisions in relation to pasture management, as well as the diet supplements that the livestock may require [18]. There are several state of the art works that follow this line of research. For example, in [19], a system is proposed for the monitoring of feed intake in cattle; it is based on the combined use of scales located in self-locking feeders and proximity sensors worn by each animal. With this system, it is possible to identify the amount of food ingested by each animal. Although this is an effective system that accurately measures the ingested quantities, it is only suitable for intensive livestock farming. Other types of systems are required to measure feed intake on extensive farms. In the current state of the art works, the most common trend is the use of microphones to detect acoustic signals and classify them into different events, usually chewing, chewing-biting and biting. The works of Chelotti et al. [20], Clapham et al. [21] and Tani [22] are some of the works that follow this line of research.

- Estrus detection: One of the most influential factors in the productivity of livestock farms is the reproduction of livestock. In recent years, artificial insemination has become more frequent, since it has a higher reproductive efficiency [23]. Therefore, it is crucial to detect the moments in which 
the animals' fertility levels are high. It is estimated that the duration of estrus is between 11 and $21 \mathrm{~h}$; at the end of this period, ovulation occurs, which normally lasts $12 \mathrm{~h}$. It is generally recommended to carry out artificial insemination for 6 to $24 \mathrm{~h}$ before ovulation [24]. Studies that apply AmI to detect estrus are mainly focused on: (i) detection of heat events-period of time before ovulation in which cows are sexually receptive; for example, in the work of Shahriar et al. [25], heat events were identified through the use of accelerometers and unsupervised learning; or (ii) the monitoring of body temperature-there are several studies which determine a relationship between the body temperature of cattle and estrus, in particular, with a mean increase of $0.9^{\circ} \mathrm{C}$ in the vaginal temperature during estrus [26]. Numerous studies have been carried out in this regard, all of them employ sensors to measure the cows' vaginal temperature; in some cases intravaginal sensors are used [27], while in others less intrusive sensors are used, such as in the work of Miura et al. [28], where vaginal temperature is measured by a sensor placed in the outer zone, at the base of the tail.

- Calving detection: Cows frequently experience calving difficulties; to prevent calf loss, human intervention is necessary in many cases. Thanks to calving prediction, cattle breeders are able to assist the parturient, this increases the chances of healthy calves being born and assures cows recover rapidly after calving (of vital importance in animals dedicated to milk production) [29]. In this regard, it is not only important to determine the time of delivery, but also to know the location of the parturient-especially on extensive farms. In some cases, sutured sensors are placed in the parturient vulva [30] to detect labor and a variety of other sensors is used to measure a number of factors; such as body temperature or parturient activity level [31]. The use of collars with integrated GPS systems [32] is the most widespread technique in the detection of animal position.

- $\quad$ Syndromic monitoring systems: Syndromic monitoring systems measure non-specific data that may be related to animal health [33]. The data collected by different sensors help to identify specific pathologies that are related to the appearance of diseases in cattle. In many lines of research, this type of systems is used. There are several studies in the literature, such as the work developed by Park et al. [34], where a complete set of sensors is used to create an animal health monitoring system, or in the work of Frese et al. [35], where electrodes are used to detect symptoms of gastrointestinal diseases related to arrhythmias.

Table 1 shows a collection of different wearables for cattle that can be found in the market today.

Table 1. Cattle Wearables.

\begin{tabular}{ccccc}
\hline Name & Type of Device & Utility & Technologies & Reference \\
\hline $\begin{array}{c}\text { Collar Silent } \\
\text { Herdsman }\end{array}$ & Collar & $\begin{array}{c}\text { Rumination, intake, fertility, } \\
\text { temperature }\end{array}$ & - & {$[36]$} \\
\hline AfiMilk MPC & Milk meter & $\begin{array}{c}\text { Amount of production, milking } \\
\text { trends, conductivity measurement }\end{array}$ & - & {$[37]$} \\
\hline AfiTag Pedometer & Pedometer & $\begin{array}{c}\text { Identification of animals, activity } \\
\text { measurement, estrus detection }\end{array}$ & - & {$[38]$} \\
\hline KV GPS system & Collar & $\begin{array}{c}\text { Location of animals, (battery charge } \\
\text { with photovoltaic system) }\end{array}$ & GPS & {$[39]$} \\
\hline Allflex Tags & Ear tag & Identification of animals & HDX & {$[40]$} \\
\hline $\begin{array}{c}\text { Moocall Calving } \\
\text { Sensor }\end{array}$ & Tail sensor & Prediction of births & GSM & {$[41]$} \\
\hline Telenax Implant & Rumen implant & Theft detection & RF & {$[42]$} \\
\hline
\end{tabular}

As stated above, different ambient and artificial intelligence techniques have been applied in this field of study. Thanks to the great advance of technology, a new point of view arises in the investigation of artificial intelligence [43]: Distributed Artificial Intelligence. It is dedicated to the 
study and definition of semi-autonomous computer systems that attempt to solve problems in which a collective behavior is more efficient than an individual one [44]. Within this area, we can talk about multi-agent systems (MAS), which deal with the intelligent coordination of a set of autonomous agents, including their knowledge, goals, properties and plans for solving problems or making decisions [43]. MAS have been successfully used in different domains thanks to their capabilities of solving computationally difficult (or spatially distributed) problems and their aptitude to develop social behaviors [45]. Currently, the idea of modeling systems through the iterations that occur between their agents is opening the way to a new trend: modeling the behavior of the system from an organizational point of view. There are different platforms that facilitate the design and implementation of MAS (e.g., Java Agent Development Framework (JADE) [46] and Mason [47]), but few that support virtual organizations. PANGEA [48], created by the BISITE research group, is an alternative which does support virtual organizations and offers inter-compatibility with multi-agent platforms using the FIPA-Agent Communication Language (FIPA-ACL). PANGEA employs methods that allow to reorganize and balance the load according to the computational needs of the system, using organizational concepts. It allows for case study modeling to be done using debugging tools, with a set of libraries for all types of IoT controllers and devices. Moreover, it offers a series of tools that facilitate platform creation and management in general. PANGEA provides the necessary elements to allow communication between agents in an easy and safe way. In this way, it favors the distribution of resources and facilitates the control of the nodes where the agents are deployed.

After a detailed review of the state of the art, we found that there was a research gap in a multi-agent architecture that would combine a variety of final services with the aim of improving livestock farm management. This is because all the works published so far have focused on detecting or resolving specific problems. The main objective of this work is to design a platform which makes use of technology that allows for the intelligent and remote management of a livestock farm. It was necessary to implement a MAS in the designed architecture due to the use of sensors with computationally limited hardware as well as the need for together with the need for the efficient use of the battery. These needs can be satisfied by a MAS which has lightweight and fast connection protocols. One of the benefits of multi-agent systems is their scalability, since they are inherently modular. This feature makes it possible to incorporate new features and capabilities much more easily than in a traditional system [49]. The use of a multi-agent architecture allows for the integration of any sensor, independently of its manufacturer; this makes it much simpler to conduct case studies. The multi-agent system PANGEA was chosen for this case study in particular due to its communication protocols, which can be adapted to the design and deployment of agents embedded in ATMega microcontrollers. The main novelties of this work are: (a) the deployment of computationally limited sensors using PANGEA; (b) the ability of the platform to predict estrus, calving, as well as the monitoring of cows' feed intake; and (c) the design of a platform that allows monitoring and controlling a livestock farm remotely using low cost hardware as well as software that can be used easily by people who have limited knowledge of IT.

\section{Proposal}

This section details the architecture designed for the remote monitoring of a beef cattle farm. The following monitoring system detects heat and calving time and measures the levels of feed in the feeders. In addition, several applications are developed which allow to monitor and visualize the farm remotely, from a TV, computer or mobile phone.

The proposal is organized into several subsections to reduce its complexity. Section 3.1 addresses the topic of the MAS platform design and outlines the different virtual organizations that constitute the proposal. Section 3.2 deals with the deployed data connectivity. Section 3.3 describes the sensors used. Section 3.4 describes the employed sensing techniques. Finally, Section 3.5 outlines the applications developed for end users. 


\subsection{Multi-Agent Architecture}

To conduct the case study, we decided to develop a MAS based on virtual organizations. The use of a MAS that allows to develop virtual organizations of agents (VO) provides the system with flexibility and dynamism; it equips the platform with new adaptability, reorganization and learning capabilities. The agents that orchestrate the system belong to different organizations, depending on the nature of the functionality that they perform. VO group agents with similar roles in one organization, with the aim of retrieving, merging and processing the information in an automatic way. This makes the proposed system extensible, favoring a high adaptability to the real demands of the environment. Since the proposal is oriented towards the modeling of MAS as a virtual organization of agents, we must be able to define structure, roles and hierarchies, as well as a series of rules that regulate interaction and communication between agents. PANGEA is perfectly suited to the needs of the environment in which we work, where it will use agents embedded in different wireless sensors. PANGEA also allows for the quick and simple deployment of agents embedded in computationally limited devices.

As can be seen in Figure 1, the general architecture of the system is made up of two large blocks: the lower part shows the agents corresponding to the PANGEA platform, while the upper part shows the virtual organizations of the agents in the proposed system, which are: WSN Organization, Prediction Organization and Application Interface Organization. The main tasks of each of the organizations within the system are listed below:

- WSN Organization: It is responsible for adapting the information of the different hardware sensors that are used in the case study, to a protocol or common language that can be read by all the elements of the platform. The main function is to adapt or translate the raw data into a JSON-type format. This organization is made up of Accelerometer, GPS, Thermometer and Food agents. Each of these agents has the functionality that it needs to be able to obtain the information of each of the sensors. Each of the agents mentioned above, communicate only with a central agent who is responsible for the organization. The main capability of this agent is to establish a bidirectional communication with entities that do not belong to the same virtual organization. These entities may consist of agents that access databases, or other virtual organizations of agents that interpret or analyze the data.

- Prediction Organization: It is responsible for retrieving the data obtained by the WSN organization and processing them. The objective of each agent will be to detect or anticipate specific events that are crucial for the farm's productive processes of the cattle yield and to send an alarm. The agents within this organization analyze events associated with animal feeding, calving or estrus (AlarmFood, predictHeat, and predictLabor agents). When an event is detected by any of these agents, it is communicated to the central agent who is in charge of notifying this event to the rest of the agents in other organizations.

- Application Interface Organization: For the end user to be able to view the information that has been collected and analyzed, it has to be represented by a software application. The central agent of this organization is responsible for obtaining the heterogeneous information from other organizations to adapt it to the standards used in the different applications, where the agents responsible for collecting this information are placed for later viewing: Mobile App, Web App, and TV App are the agents responsible for the visualization layer of the applications for mobile, web and Smart TV respectively. 


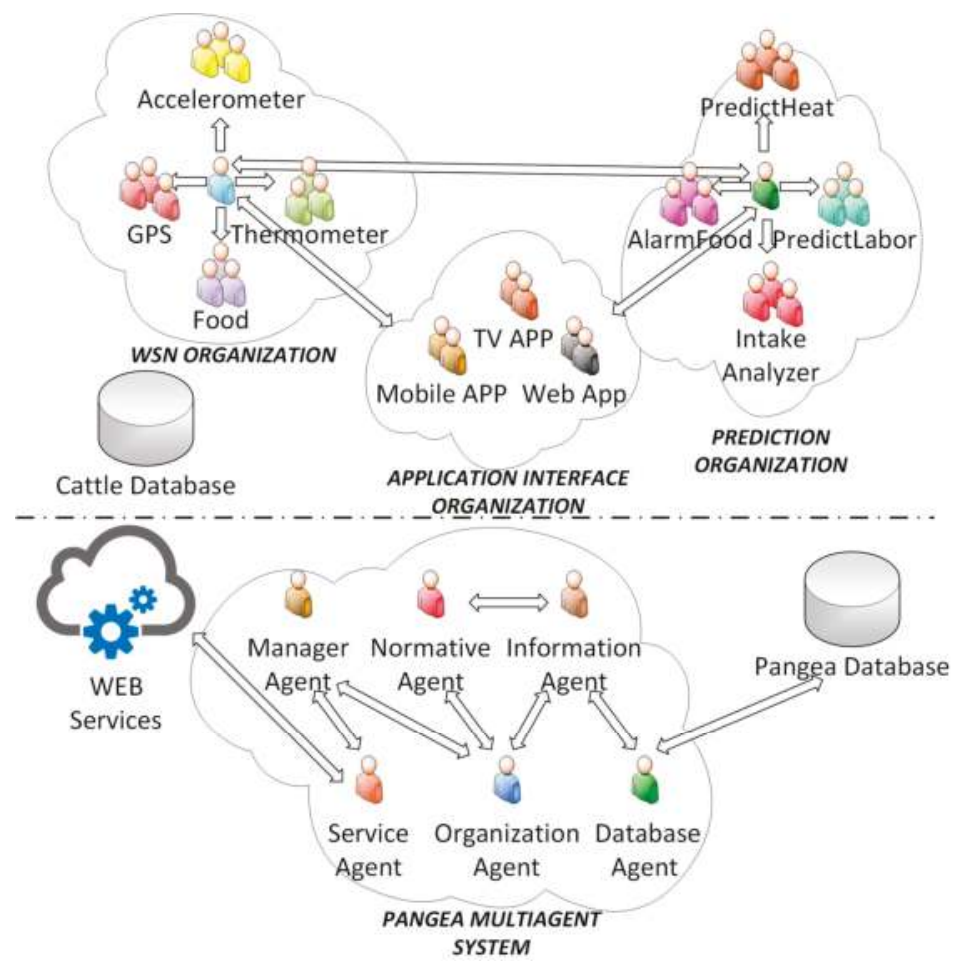

Figure 1. Virtual organization of agents overview.

Furthermore, the proposed system makes use of a set of agents that are part of the PANGEA MAS [48], which has been used as a base line for this work. These agents are described below.

- Manager agent: It is responsible for supervising tasks, ensuring that the system works correctly, checking that there are no overloads in the different functionalities of the system or that errors of communication between agents do not occur.

- Information agent: When a new agent becomes part of the organization, the agent notifies the information agent of the services it offers. In this way, the information agent is aware of all the services available in the organization, thus being able to serve as a directory which the other agents can consult to know of the services that they can access.

- Service agent: This agent is responsible for recording and controlling the operation of the services offered by agents. In addition, it works as a gateway, as it is in charge of the distribution of the services that offered by the agents through web services, so that they are accessible from outside the organization.

- Normative agent: This agent ensures compliance with all rules defined in the organization, always ensuring that agents use those services for which they have permission, warranting the security of communications made within the system.

- Database agent: This agent in charge of accessing the PANGEA database to obtain the pertinent information. This database agent is responsible for representing and storing the contextual information of the system: what organizations and sub-organizations of agents it contains, what type of topology they form, what roles the different agents fulfill, what services they offer, etc. 
- Organization agent: This is the agent in charge of managing organizations and sub-organizations. It is responsible for verifying the entry and exit of the agents to the system, as well as assigning a role to each of the agents in the organization.

One of the greatest advantages of the use of PANGEA is that it allows for the use of different communication protocols, such as HTTP or Message Queue Telemetry Transport (MQTT) among others. MQTT is a messaging protocol specially designed for communication between embedded devices with few computational resources, with low bandwidth and in high latency networks. Due to these characteristics, this protocol has been widely used in the field of Internet of Things (IoT). To verify that the use of this type of protocols provides greater advantages when used in machine-to-machine communication, than the advantages it offers when used with traditional systems based on HTTP protocols with service-oriented architectures, a comparison has been made between both paradigms.

This comparison is shown in Figure 2, the illustrated results have been obtained after sending identical messages from an agent embedded in the feeder devices under the following conditions: MQTT over 3G, MQTT over WiFi, HTTP over 3G, and HTTP over Wi-Fi. Figure 2A shows the percentage of battery consumed by the device during one hour and Figure $2 \mathrm{~B}$ shows the number of messages sent in one hour for the four conditions indicated above. In either case, it can be seen that the choice of MQTT vs. HTTP is properly justified, not only for efficiency in sending messages (which is outstanding in the case of 3G networks) but mainly for the battery saving rates it offers. This aspect is very important in a system like the one proposed in this work, since the replacement of batteries is an expensive process for the cattle ranchers, so the battery saving in the different sensors, also supposes a greater efficiency in productive processes. The flexibility offered by PANGEA enables the use of the most suitable protocol for each use case where, in the case of sensors communication, MQTT has been proven being appropriate.

The general operation of the processes occurring in the platform can be seen in Figure 3. In the first place, the agents embedded in the sensors carry out a data acquisition process. These agents send the collected raw information to the WSN Central agent. This agent is in charge of storing the received information in a standardized way, as well as sharing this information with the other organizations, restricting their access only to the services that require it. To do a data analysis or prediction (such as labor or heat detection), the WSN Central Agent will be responsible for providing information from the different sources to the Prediction Central Agent, which will in turn send it to the corresponding Analyzer Agent. These agents present reactive behavior defined by rules, and are responsible for performing the data analysis. After establishing this information, the analyzer agent will send it to the Prediction Central Agent, who is responsible for storing it so that, if an agent needs it, it is sent on demand (or in other cases, information can be spread from this agent to others automatically, as in the case of estrus detection). When a user makes use of a certain application (mobile application, web application or Smart TV application) and wants to view some kind of information, the software agent which is part of the application will make a request for the desired information to the Prediction Central Agent, which will be returned if available. The communications are controlled (although it is not represented in the figure in order to make it simpler to understand) through the PANGEA layer, which already includes rules that define communications between agents. The Normative Agent ensures that agents in the platform only use those services for which they have permission. 


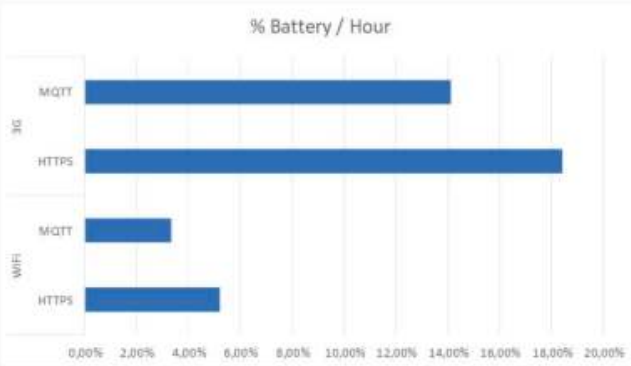

A)

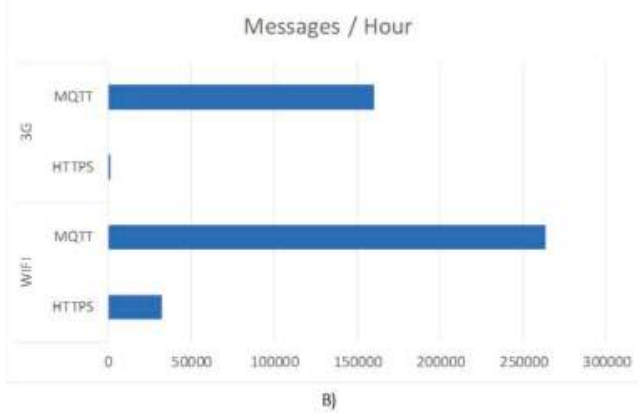

Figure 2. (A) Battery percentage consumed per hour and (B) messages sent per hour (results grouped by protocols and networking technologies).

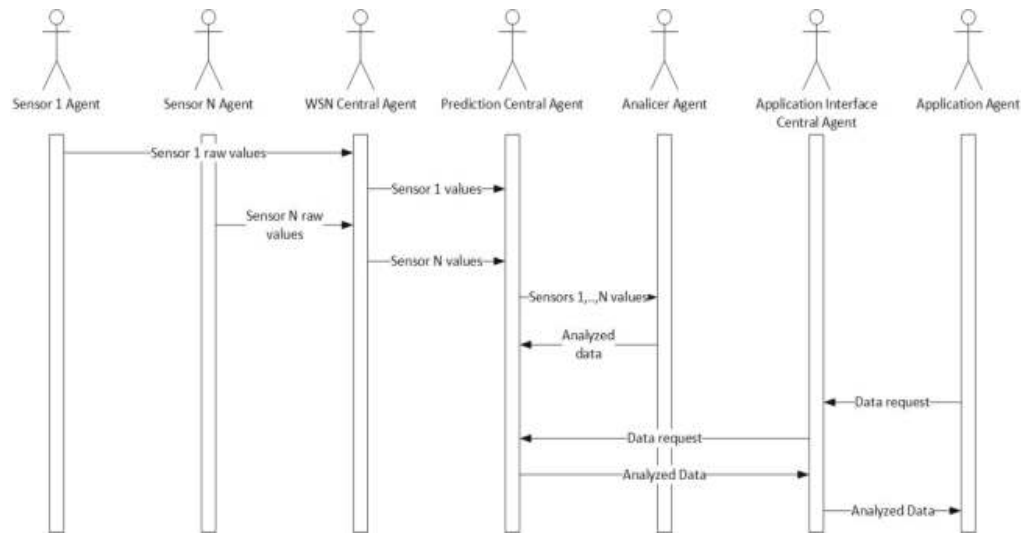

Figure 3. Interaction performed between agents.

\subsection{Farm Connectivity}

To provide a tele-monitoring service on the farm, data connectivity is required. To deploy a data network and offer an internet connection on the farm, several possibilities were studied. One of the alternatives was the hiring of a satellite line based on WiMAX technology. The necessary equipment had a high cost and the monthly quota of the data line was exorbitant, reason why this option had to be discarded. An alternative idea was to hire a conventional ADSL line. However, no service provider had coverage in the area where the farm is situated $(40.796575,-6.241120)$. Finally, we thought of 
making a radio link. The farm where the connection was to be established is located near a town called La Fuente de San Esteban, Salamanca $(40.8013959,-6.2624964)$. This core had a pre-installed broadband connection in the City Hall. To be able to share a data connection in a legal and free way with the inhabitants and farmers of the area, a collaboration agreement was signed with the Regional Government of Salamanca, Junta of Castile and León. This agreement required that, to share the Town Hall connection with the rest of the inhabitants and workers in the area, each connection should be limited to a $512 \mathrm{Kbit} / \mathrm{s}$ downstream speed. The farm did not have direct view with the Town Hall that it needed to connect, since it is located in the center of the village and is surrounded by houses, thus an intermediate point was chosen to act as a gateway. The highest point of the municipality was the Silo $(40.795884,-6.251096)$ with a height of $50 \mathrm{~m}$ above ground level, and had electrical connection. In the upper part of the roof of the Silo and in each geographical orientation $\mathrm{N}, \mathrm{S}, \mathrm{E}, \mathrm{W}$ an Ubiquiti brand antenna of type Ti AM-V5 G-Ti $(5 \mathrm{GHz})$, was installed with a sectorial opening configuration of 120 degrees and a resulting gain of $15 \mathrm{dBi}$. Figure 4 shows the behavior of the signal in the antennas used with an aperture of $60^{\circ}, 90^{\circ}$ and $120^{\circ}$ [50].

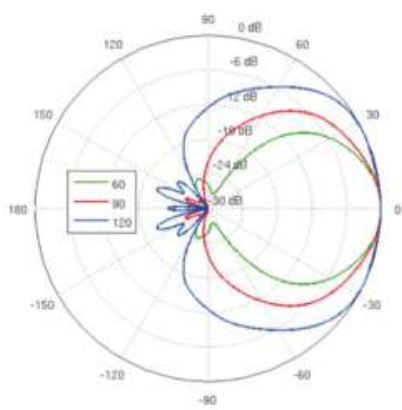

(a)

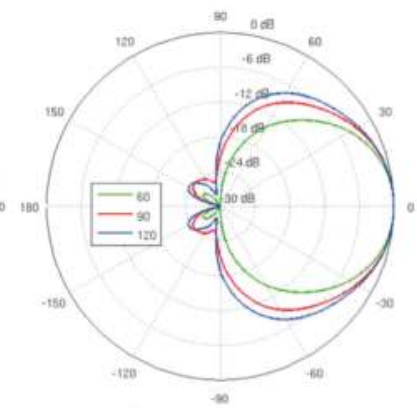

(b)

Figure 4. Behavior of the used antennas signal: Ti AM-V5 G-Ti (5 GHz): (a) horizontal Azimuth and (b) vertical Azimuth.

Once the place where the connection could be made was determined, different point-to-point connections were made with nearby urban nucleus that did not have any kind of broadband connection. The radio link between the silo and the Town Hall was made using a $5 \mathrm{GHz}$ equipment (PowerStation 5 antennas [51]). Figure 5 shows the altimetry profile of the ground between the two points, where it can be verified that there is direct vision and that the quality of the link is correct, with a capacity of $175.51 \mathrm{Mbps}$.

The modulation of the antennas in the link is 64 QAM using a polling policy based on Time Division Multiple Access (AIRMAX) that prioritizes the most active systems through a technique of intelligent surveys, achieving a greater use of the medium. Figure 6 illustrates the network architecture, the distances between each place and actual photos of the antennas.

From the image, we can see that the data connection that already existed in the municipality is distributed and is redirected to the highest point in the town to be able to share that connection with another urban nucleus that did not have a basic Internet connection. Nodes that want to connect to the Internet, such as laptops or smartphones that are approximately $300 \mathrm{~m}$ away from the town hall or silo do not require additional hardware to use the connection. Since the case study contemplates the possibility of monitoring the field by video streaming, we decided to use a NanoStation LOCO M5 $5 \mathrm{GHz}$ from the Ubiquiti brand, configured in client mode with a throughput of $150 \mathrm{Mbps}$.

Thanks to the network infrastructure described above, it was possible to deploy the proposed platform on the farm and to conduct the case study that proves the system's feasibility. 

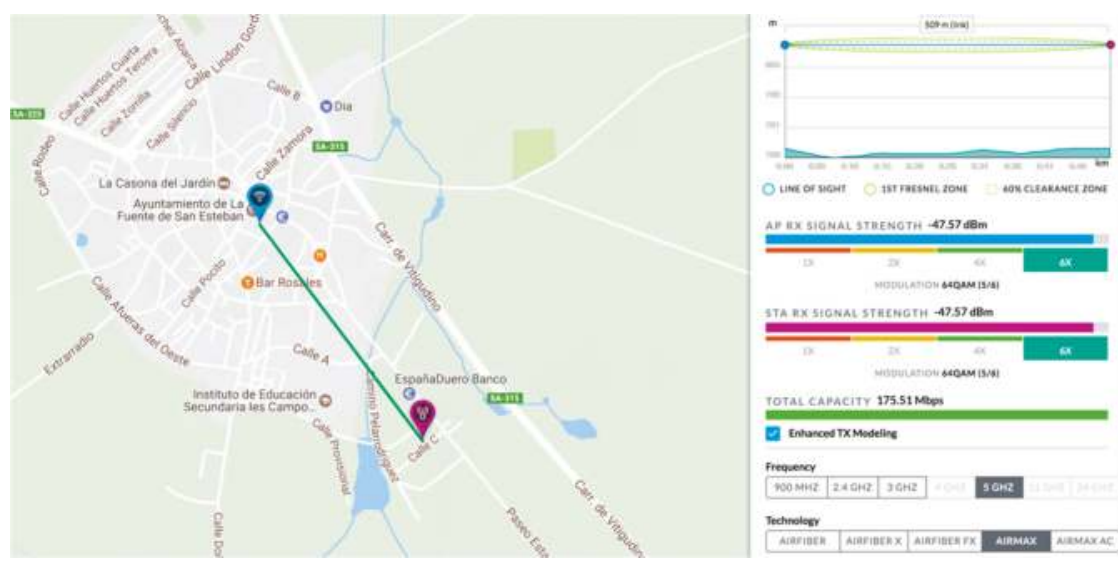

Figure 5. Altimetry and quality of the radio link.

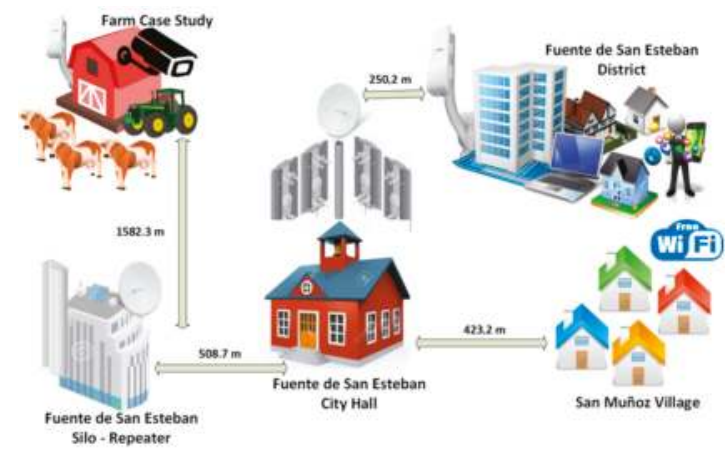

Figure 6. Network architecture diagram.

\subsection{Sensors in Livestock Farming}

Figure 7 shows the general structure of the proposed architecture in the case study. The central part of this system is a WSN, whose main objective is the collection of different data from sensors placed on the cattle that are part of the farm. Depending on the type of sensor, the collected data will be sent in different ways: radio frequency, GPRS or Wi-Fi. To support these types of communication, it was necessary to incorporate additional communication elements, such as radio frequency stations or the previously mentioned NanoStation LOCO antennas. In either case, the final destination of the data is the same: a central server in which these data are stored for later analysis and interpretation. With artificial intelligence techniques, it is possible to automatically detect different events, whose identification can be of great help to the farmer. Thanks to the use of different applications, the farmer can receive alerts which notify when these events occurred. Additionally, he is also able to check the data collected by the sensors in real time. The following sections detail the operation of each of the sensors and the analysis performed on the collected data. 


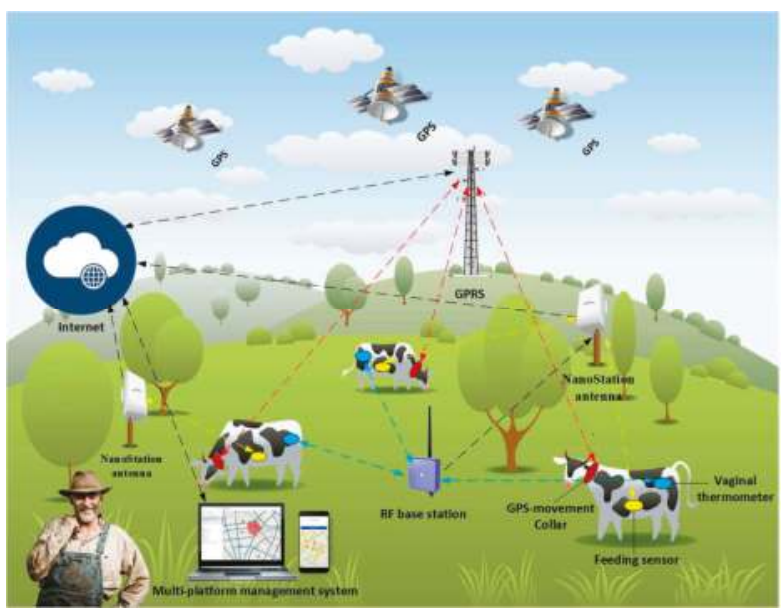

Figure 7. Infrastructure of the proposed system.

In this organization we have GPS, movement, temperature and food sensors. The sensors that were used can be seen in Figure 8A-E.

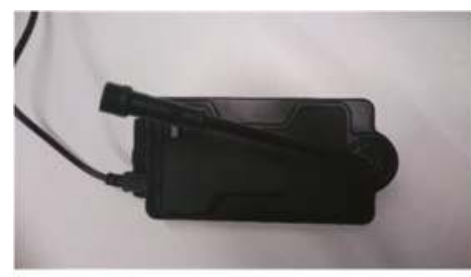

(A)

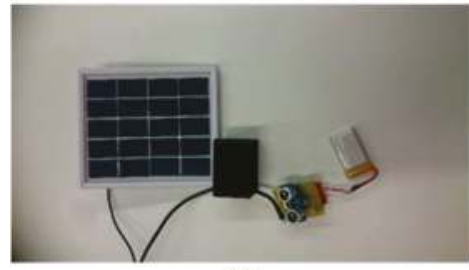

(C)

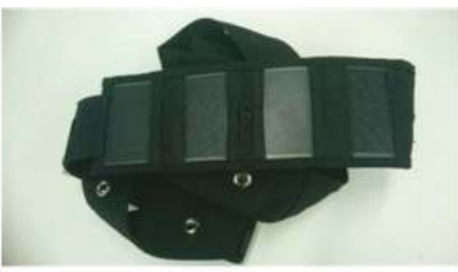

(B)

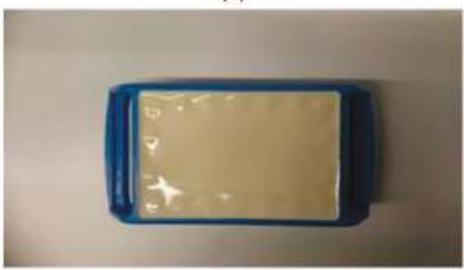

(D)

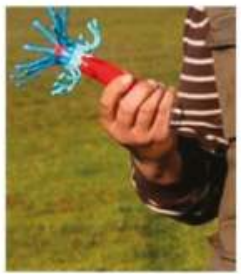

(E)

Figure 8. (A) GPS sensor; (B) solar collar for GPS sensor; (C) food sensor (ultrasonic sensor and solar battery); (D) motion sensor; and (E) vaginal thermometer. 
The GPS sensor retrieves the GPS locations and the time stamps associated with each of them. This sensor has a SIM module that sends the location data to a server via GPRS. With the purpose of making this device energetically self-sufficient, a solar collar that charges its battery has been included. Additionally, a motion sensor is included, so the GPS is only activated when activity is recorded. This motion sensor is also used to detect the activity of the animal, since, as mentioned in the state of the art, a change in the activity of the animal is related to estrus.

As to the thermometer used, it is a vaginal thermometer that sends the data by radiofrequency to a base that subsequently sends them to a server, from which the information can later be extracted. The frequency of sending can be parameterizable, in this case study every $45 \mathrm{~s}$. In the case the sensor cannot transmit the value to the RF base for some reason or because the distance between it and the sensor is too great, the sensor has an internal memory that temporarily stores the values, until the connection with the base RF is successfully reestablished.

The food sensor incorporates ultrasound sensors to measure the volume of feed. The sensor incorporates an Arduino-based controller to retrieve sensor information from the sensor and then send it remotely via WiFi technology to the remote server. In addition, the sensor includes a battery and a photovoltaic panel that makes the device autonomous; there is no need to connect it to electricity. To be more efficient energetically and thus have low consumption rates, sensor data are sent every $60 \mathrm{~min}$. A low cost solar plate is used, with an operating voltage of $5 \mathrm{~V}$, an output power of $1 \mathrm{~W}$ and a current of 0-200 MA.

As a summary, Table 2 shows which kind of data each sensor collects, and in which systems of analysis or prediction the data collected by the sensor are used. The detailed description of each of the analysis and prediction systems is shown in the next section.

Table 2. Relationship between the sensors and the analysis systems.

\begin{tabular}{ccc}
\hline Sensor & Data Collected & $\begin{array}{c}\text { Analysis/Prediction System } \\
\text { Where It Is Used }\end{array}$ \\
\hline GPS Sensor & Cattle Geolocation & $\begin{array}{c}\text { System for the monitoring of ingestion and } \\
\text { system for calving detection }\end{array}$ \\
\hline Motion Sensor & Physical acceleration & $\begin{array}{c}\text { System for the monitoring of ingestion and } \\
\text { system for estrus detection }\end{array}$ \\
\hline Vaginal thermometer & Corporal temperature & System for calving detection \\
\hline Food sensor & Distance & System for the detection of food levels in \\
the feeders
\end{tabular}

\subsection{Analysis and Prediction Systems}

The agents are capable of predicting each of the problems posed in this case study. Some of the sensors require a system that is adaptive for each of the animals and that evolves with time. However, other sensors only require the definition of predefined rules, whose behavior as a result is reactive. In the next subsection, the functioning of sensors is described.

\subsubsection{System for the Detection of Feed Levels in the Feeders}

To implement this system, ultrasonic HC-SR04 sensors have been used. These sensors allow measuring distances in a range of $2-450 \mathrm{~cm}$, with a $0.3 \mathrm{~cm}$ accuracy. To verify the correct functioning of the system, sensors have been installed on 4 feeders. Figure 9A shows one of the feeders where the sensors are installed. The sensors are placed at the base of the feeder's roof. Since there are no electricity sockets close to the feeders, batteries have been chosen to supply power to the sensors. Moreover, photovoltaic plates have been used to charge the batteries and provide the system with complete autonomy. Figure 9B shows the sensor, battery and photovoltaic panel that were used in the case study. 


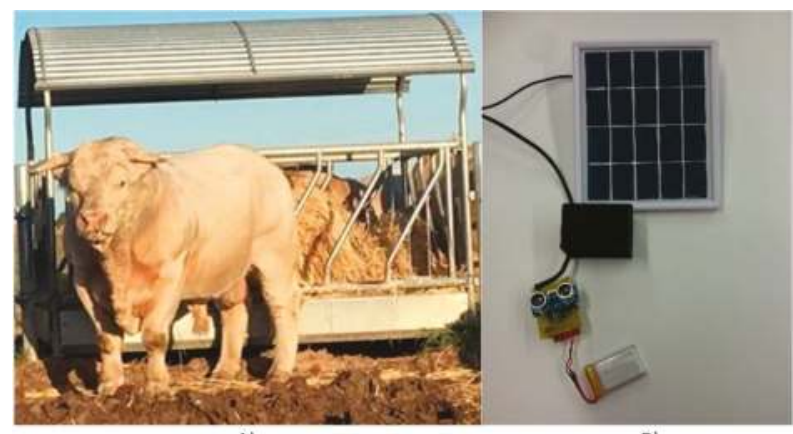

A)

B)

Figure 9. (A) Feeder; and (B) measuring system.

To measure the level of feed, the sensor is installed at the base of the roof of the feeder and measures the distance to the base on which the food is placed. The distance from the roof to the base is $210 \mathrm{~cm}$. On this farm, cattle are fed with bales of straw which measure $90 \mathrm{~cm} \times 100 \mathrm{~cm} \times 210 \mathrm{~cm}$ on average. A single bale is placed per feeder when feeding the cattle. It can therefore be extrapolated that a measurement of $210 \mathrm{~cm}$ recorded by the ultrasonic sensor corresponds to a level of feed of $0 \%$ (the height of the ceiling relative to the base), and that a measurement of $110 \mathrm{~cm}$ in the height between the ceiling and the bale), corresponds to a $100 \%$ feed level. In view of maximum and minimum values of feed in the feeders, the levels of feed can be calculated with the data collected by the sensor. There may be straw residues when new bales are being placed in the feeders, which will cause the feed height to exceed the upper limit that determines 100\% of the feeder filling. In these cases, the level of feed will also be considered to be $100 \%$.

\subsubsection{System for the Monitoring of Ingestion}

A fundamental aspect of livestock farms is the correct feeding of livestock, since it has a direct impact on production rates, whether meat or milk. Although cattle feed on forages, the incorporation of concentrated foods in their diet helps to improve production to a great extent. For this improvement to be effective, it will be necessary to elaborate optimal feed portions for different groups of animals (steers, heifers, bulls, gestating cows, dry cows or production cows). This, in addition to boosting farm production, will in many cases help reduce costs, since the unnecessary use of feed will be largely avoided. To be able to correctly plan animal feeding, a system that allows for the monitoring of the animals' feed intake, has been incorporated in the platform.

To control the animals' feed intake, we developed a system which establishes what kind of food an animal has been ingesting (and for how long). Two sensors placed of the animals' collars have been used to collect the necessary data. These sensors consist of an accelerometer and a GPS module, in which the agents are embedded: the Accelerometer agent and the GPS Agent, both belong to the WSN Organization. The main function of these agents is to collect the information from the sensors and transmit it to the central agent of the organization, which is responsible for providing the information from the sensors' different heterogeneous sources to other organizations, such as the Prediction Organization. In this organization, the Intake Analyzer agent is in charge of merging information with the data recorded by the Accelerometer agent and the GPS agent. On the one hand, thanks to the data variation analysis done by the accelerometer, we can determine when the animal was eating or performing another activity. Furthermore, when geofencing techniques (to delimit the geographical areas where the feeders are located) are applied to GPS data, it is possible to determine if the animal is in an enclosed or open area. Thus, with the combined use of both sources of information, it can be specified whether an animal was eating or not, whether it was in one zone or another and 
thus, we can infer what kind of food it ingested; pasture in the intensive area or concentrated food in an enclosed area.

To develop a system that differentiates the animal's intake periods from the data recorded by the accelerometer, tests have been carried out with data obtained from 6 Charolais breed cattle during a three-week period. During this period, these animals wore a collar that incorporates a LIS3DH accelerometer, which allows acceleration measurement in three axes, with a dynamically configurable detection range of $\pm 2 \mathrm{~g} / \pm 4 \mathrm{~g} / \pm 8 \mathrm{~g} / \pm 16$, and which offers a base consumption rate of $6 \mu \mathrm{A}$ in low-power mode. The procedure to establish if grazing activity is occurring, is the one described by Rayas-Amor et al. in their work [52]. This procedure establishes a relationship between the acceleration and tilt that can be used to estimate the grazing time. On the one hand, the median acceleration readings in the $x$ axis in a range between 0.175 and $0.95 \mathrm{~m} / \mathrm{s}^{2}$ establish grazing activity. On the other hand, the degree of vertical tilt in the $y$ axis can be used to establish grazing position in a range of readings between $0^{\circ}$ and $61^{\circ}$.

The second component involved in the control of intake, aims to determine if the animal has been ingesting pasture or concentrated feed at a given time. There are two well differentiated areas within the farm where the study was carried out: the area where the feeders are located, and the extensive pasture area. As explained previously, it will be possible to determine whether an animal has been ingesting either grass or concentrated feed, based on the animal's geographical location (by differentiating if it is in the zone of feeders or on extensive pasture). To develop this system, GPS sensors Neo- $6 \mathrm{~m}$, placed in the collars of the animals, have been used to continuously monitor the position of the animals. Thanks to the position provided by the GPS, and using geofencing techniques which allow to encircle a certain geographical area, it will be possible to determine if an animal is within one of these zones at any given time by means of a simple system of rules. Figure 10 shows the aerial view of the farm where the study was carried out. Two enclosures can be seen on the photograph, a yellow one and a red one, which correspond to the virtual fences. The yellow fence delimits the geographical space where the feeders are located and the red one delimits the geographical space of the whole farm. Thanks to the delimitation of these zones, we can establish if: (i) the animal is in the zone of the feeders; (ii) the animal is in the area of extensive pasture; or (iii) the animal is outside the farm area.

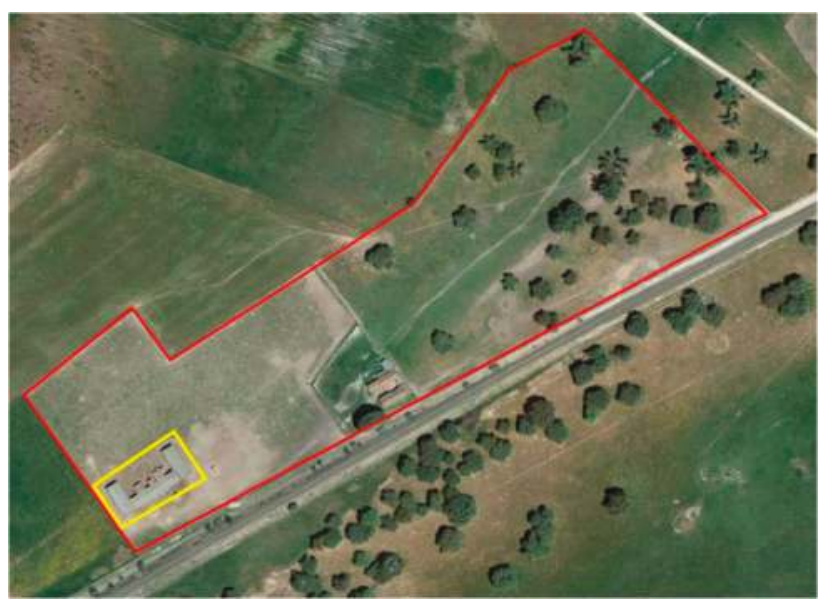

Figure 10. Aerial view of the farm, virtual enclosures. 


\subsubsection{System for Estrus Detection}

The motion sensor is used to predict estrus. Every hour, the motion sensor sends the information from the accelerometers to the central server, where the activity of the animal is estimated. The procedure that is followed to calculate activity is the one described by Yin et al. [53]. Basically, the algorithm for a time interval of $5 \mathrm{~s}$ takes the mean acceleration values for the $\mathrm{x}$-axis, $y$-axis, and $z$-axis and then calculates the value difference for the $x, y, z$ axis between two contiguous intervals. These three values are used together with the sum of their absolute values in order to calculate the activity of the animals (Equation (1)).

$$
d_{i}=\{x, y, z,|x|+|y|+|z|\}
$$

From the values, three clusters are created by applying PAM, so that groups g1, g2, and g3 have one for high, medium and low activity (Equation (2)). In the original algorithm, k-means was used, but to avoid problems with atypical values, PAM is used.

$$
G=\left\{\left\{g_{1}, g_{2}, g_{3}\right\} / g_{1} \cup g_{2} \cup g_{3}=\cup d_{i}, g_{1} \cap g_{2} \cap g_{3}=\varnothing\right\}
$$

To subsequently determine whether the cow is in heat or not, it is necessary to compare the number of elements in each group, with the elements from the groups obtained in previous days at the same hour. We used the indications provided in the work of Yin et al [53] to calculate the activity index of a cow for a particular time t.

$$
a_{t}=\sum_{i=1}^{3} \# g_{i} * w_{i}
$$

where $\# g_{i}$ is the number of elements in group $i$, and $w_{i}$ is the weight of group $i$, by default in this order: $\{0,0.1,0.9\}$.

To calculate the activity index, compare the number of the value of Equation (3) according to the three days prior to the same hour as well as with the previous hour.

$$
v_{i}=\frac{3 a_{t}-\left(a_{t-24}-a_{t-48}-a_{t-72}\right)}{\left(a_{t-24}-a_{t-48}-a_{t-72}\right)}+\frac{\left(a_{t}-a_{t-1}\right)}{a_{t-1}}
$$

To consider aspects associated with the movement of livestock or situations in which cattle may be more active e.g., food, strangers on the premises, animals present, etc. The above formulas are modified to consider changes in the general activity of the control group of cows. These situations are established using Equation (5).

$$
I_{t}^{c}=v_{t}^{c}-\left|\bar{v}_{t}\right|
$$

where $v_{t}^{c}$ is the activity index, calculated from Equation (4) for the cow $c$ in the particular time $t,\left|\bar{v}_{t}\right|$ is the average activity index for all the cows in $t$. By applying Equation (5) it is possible to determine a variation in the activity index of the animals, in comparison to the cows that were previously used as a reference for this study. The selected animals for this study correspond to cows that are already pregnant and can be observed visually, or with cows which are already pregnant that are not observed visually, but the estrus has not been detected during three periods.

The estrus detection thresholds obtained by Equation (5) based on data are established manually at the beginning.

\subsubsection{System for Calving Detection}

In cattle farming, human assistance is often required during labor to ensure a birth without any complications, safeguarding the health of both the parturient and the calf. If the farm is large or if the calving takes place at night, the danger is even greater; the farmer must look for the cow in the whole field and loses critical time on which the health of livestock depends. Thus, it is crucial for the farmer to know exactly the time and the geolocation of the place where the birth will start. 
To solve this problem, a sensor has been designed consisting of an accelerometer and a temperature sensor, which is introduced into the cow's matrix. This sensor should be placed when the following symptoms are detected in the animal: presence of colostrum in the mammary glands, decrease in body temperature or repetitive tail lifting.

To detect labor, continuous monitoring of the animal's temperature is performed. It is possible to detect calving in an early and approximate way when it is going to occur through the analysis of the cow's body temperature. According to the studies conducted by Lammoglia et al. [54], the body temperature of the animal is significantly reduced in the 48 to $8 \mathrm{~h}$ prior to the labor. On the other hand, when the cow's water breaks, the sensor is ejected out of the matrix, producing a variation in temperature, thus the exact moment at which labor begins, can be detected. The geolocation of the cow is obtained by using the GPS module that the cow carries on a collar and is described in detail in Section 3.4.2. The changes in temperature are determined empirically using the values collected by the temperature sensor. To implement this functionality, it is necessary to establish a temperature threshold that determines the beginning of labor. When the threshold is crossed, the farmer is alarmed that the cow is in labor. Figure 11 shows the steps that follow the procedure described above.

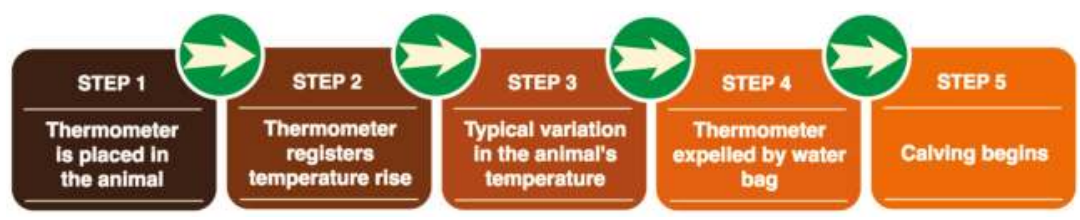

Figure 11. Steps in the detection of labor.

\subsection{Developed Applications}

It is very well known that the world population is getting older. The large number of elderly people in our society can be explained with improved health care and earlier retirement. Despite the internet boom, the older generation is still quite unfamiliar with the use of new technologies. Some are unaware of the benefits offered by technological devices while others, who are interested in using electronic applications and devices, find it too complex to manage them. The distance between technology and older people gets even greater when we look at its high cost. We must take into account that the majority of farm owners are elderly, and therefore the technological solutions offered for farms, should be economic and easy to use. The province of Salamanca has the largest number of cattle in Spain, a total of 6012 , thus this work intends to provide a solution that will allow elderly farmers to correctly control and supervise their farms.

A monitoring prototype has been developed where farmers can view the data on their farm and their animals. They will be able to view these data from their own homes. We chose a low-cost platform design that allows to remotely interact with the farm. A human-machine interface (HMI) has been implemented so that people who have very little knowledge of technology can easily view data and receive notifications. An application for the television has been developed because elderly people are familiar with its use (unlike other electronic devices; computers, mobiles). Users manage the application with a remote control, whose colored buttons are associated with different actions or give access to the submenus of the application.

An alternative is offered to users who are familiar with new technologies; they can display all the data on a website. This website shows real-time information collected by sensors, including the location of animals, cameras, food sensors, water level sensors and alerts on heat, calving or feed levels.

Additionally, an Android application for mobile devices has been developed, where users can also view information and alerts on heat, calving and feeding. This application can be very useful to breeders, because when the system detects an important event, a notification is sent to the user's 
mobile. This provides users with greater comfort and security since they do not have to be constantly checking the application in order to stay informed of the current state of the farm.

\section{Results and Conclusions}

The experimentation and validation phase has been carried out on the Ángel Santiago García Martín cattle farm, located in the town of Fuente de San Esteban town, in the province of Salamanca. The use of PANGEA as the MAS architecture permitted us to design a complex case study where computationally limited device participate in a reduced period. By using the designed architecture, the cattleman ubiquitously tests the algorithms implemented in the platform, allowing for their continuous improvement. The farm where the platform was implanted, has 450 hectares in total, out of which, 300 hectares are dedicated to bovine cattle. The number of livestock on the holding is 200. For the verification tests, a sample of 24 specimens belonging to the Charolais breed have been used.

For the development and validation of the intake monitoring system, tests were carried out with six Charolais breed cattle during a three-week period. Regarding the system that allows establishing what the periods of grazing have been, the use of tri-axial accelerometers has been previously probed to be accurate to estimate grazing time per cow and per day [52], results that could be empirically tested by observing the animals. Regarding the second component involved in the control of the intake, which aims to determine if the animal has been ingesting pasture at a given time, the accuracy of this system is subject to the accuracy of the location obtained by the GPS sensor. During the validation of the system, empirical tests were performed to check the possible fluctuations of the collected values, reaching $3 \mathrm{~m}$ accuracy in the measurements. However, the area around the location of the feeders in this place is so frequented by animals, the grass does not for 3-4 m around it. Thus, the error derived from the measurements in this case is minimal, since there is no food other than the one in the feeders in this space. However, the accuracy of this system in a farm where these conditions are not met, will be subject to the accuracy of the GPS in that location. To deal with this possible problem, a viable alternative may be the use proximity sensors, whose application has been validated in other works [19].

To study the relationship between an increase of physical activity of cows with the onset of heat, a motion sensor was placed in a sample of 8 adult cows. As discussed in Section 3.4.3, an algorithm is applied to calculate the cows' activity index. Figure 12 shows the calculated activity index for one of the cows that participated in the study. During the study, a pronounced increase in the activity index was discovered, as can be seen in the Figure 12, it coincides with the beginning of the animal's zeal. This system has been tested in previous works reaching a 100\% success rates [53]. In this case study, the relationship between the increase in the activity index and the beginning of zeal has been validated by a human expert: the head farmer. The number of the detected heat events has matched with the observed heat events in eight out of the eight studied cows.

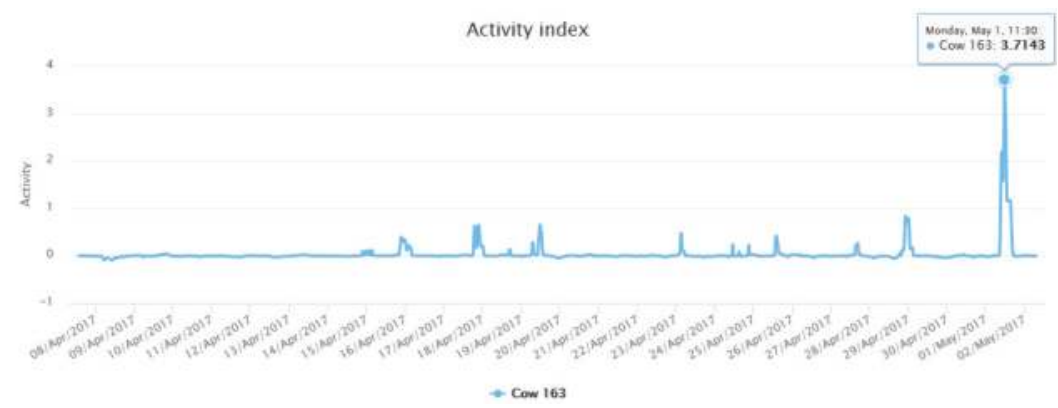

Figure 12. Activity index of a cow. 
To study the temperature variability prior to calving, we opted to monitor six cows on the farm. The objective was to study the temperature pattern to be able to adjust the sensitivity of the designed sensor and to study the variation of the cows' temperature over these days. Table 3 shows the data obtained for six days prior to delivery and the evolution of the temperature variable at the time of delivery. Three of the cows studied were pregnant with a male offspring, while the other three were females.

Table 3. Cows body temperature prior to delivery.

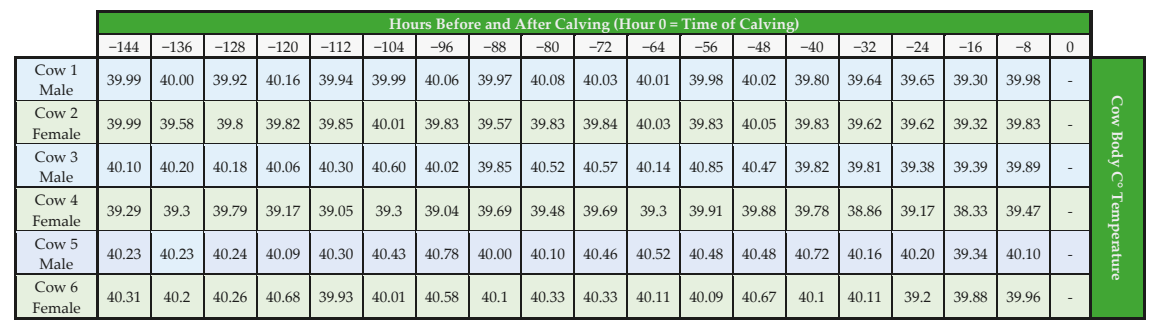

Figure 13 shows the evolution of the average temperature of the offspring according to their gender. As it can be seen in the image, the temperature of the cows lowers $48 \mathrm{~h}$ before the calves were born and when the cow gives birth, the thermometer is expelled. Once it is expelled it measure the temperature of the environment, in this way it is easy to predict labor. In view of the obtained results, the system performance has been verified in all the cows of the control group. The accuracy of this method is subject to two factors: the correct functioning of the temperature sensor and the outside temperature. Assuming that the sensor works properly, the detection of delivery would only fail when the ambient temperature is exactly the same as the body temperature of the cow. Considering that the body temperature of these animals is around $40^{\circ} \mathrm{C}$, and that in these cattle farm the deliveries are scheduled for the months of November, January and February, there is no match possibility between both values.

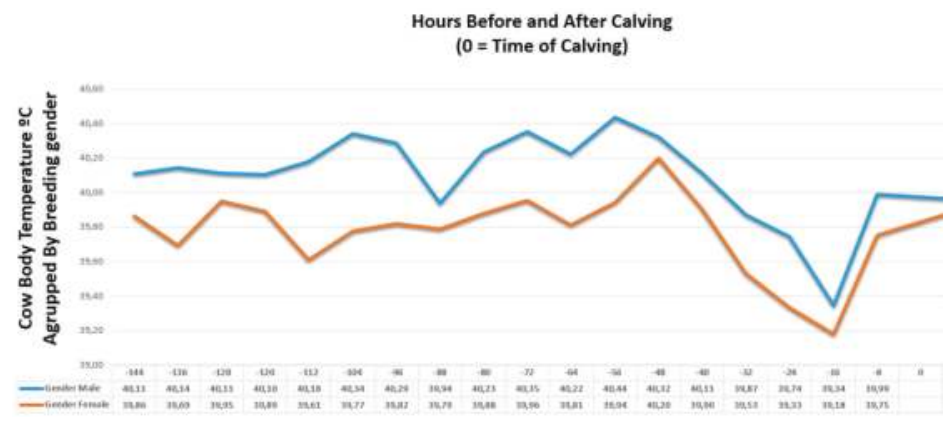

Figure 13. Cow average temperature, grouped by breeding gender.

One of the main results of this work is a monitoring platform that allows to check the state of the exploitation in real time, as well as to receive different alarms of the events detected by the system. As explained in previous sections, in this work, we opted to implement three different applications: one for TVs, one websites and one for smartphones.

The purpose of the farm remote monitoring is to allow the final users who participate in the case study to remotely view the data in an application. Most of the owners of agricultural holdings in the geographical area where the case study has been performed (La Fuente de San Esteban, Salamanca 
$(40.8013959,-6.2624964))$ are elderly people. Adults who live in rural zones are normally reluctant to the use of technology (and more specifically computers) as a central element to access data remotely. For this reason, it was proposed that the monitoring could be carried out from a television. Televisions are present in almost every household and can be operated in a simple way. This idea was provided by the local group Asociación de Mayores San Esteban. In this case of study, six individuals with ages between 40 and 64 years were offered for the development and validation of the monitoring prototype making use of TVs. In the development of the TV application, a Raspberry Pi with an IR sensor (model TSOP38238) has been used as hardware. The connection between the Raspberry Pi and the IR sensor is made using the GPIO pins, as shown in Figure 14.

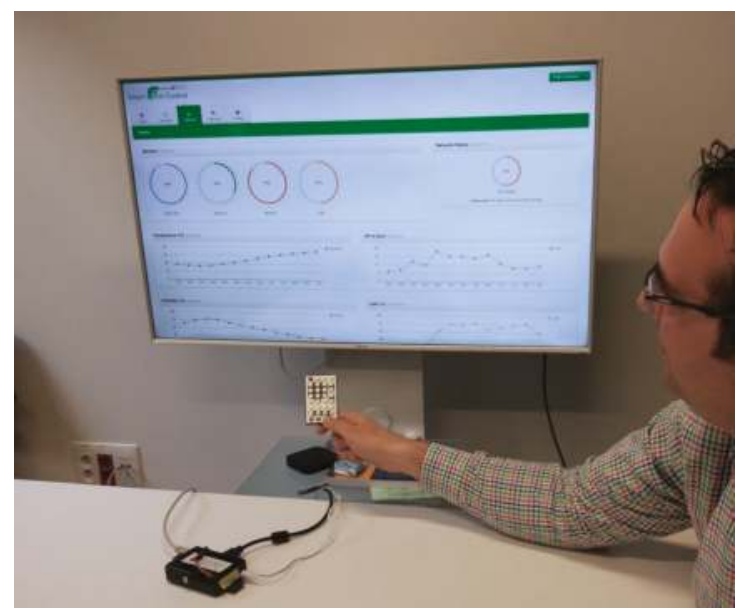

Figure 14. Cow average temperature, grouped by breeding gender.

Pulsations made in the control are used to operate the user's television. This control interface allows users to access through the different options of the designed application. Users highlight the ease of use when accessing the data, since it is not necessary to install or operate a computer, simply plug in the TV and use the conventional control by pressing buttons. The software component designed to identify the buttons that act as an interface between the software and the hardware is based on LIRC (Linux Infrared Remote Control). It allows to associate the pressing of each button with a software event. The different buttons allow to view the different displays provided by the application as well as to move cameras that provide real-time images of the animals. Figure 15 shows the graphical user interface of the TV application. Users who tested and validated the TV prototype highlighted the simplicity of using it, as well as not having to do an initial investment in a computer.

The state of the livestock is also monitored through the web application. Figure 16A shows the information about the alerts that arrive to the system. In this image, you can see three alerts: the first of them indicates that the labor sensor has been activated (has begun to collect measures), the second indicates that there will be a labor in approximately $48 \mathrm{~h}$ and the third warns of the moment the labor begins. Figure 16B shows information about the food sensors, which includes the feed levels and the battery level of the sensor (although the devices are self-sufficient thanks to the use of solar panels that charge the batteries). In addition to the monitoring information, the website includes other information on livestock such as prizes, breeders, sales or cattle raising. 


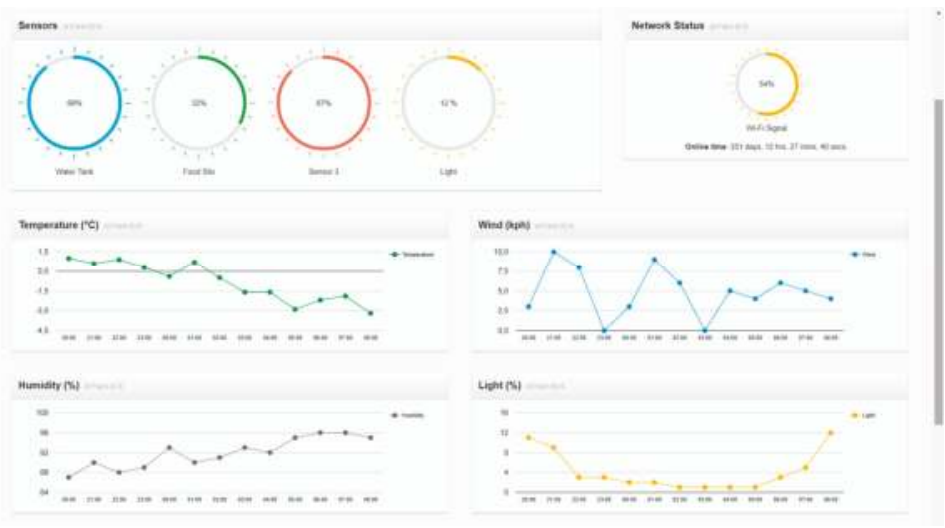

Figure 15. Television application interface.

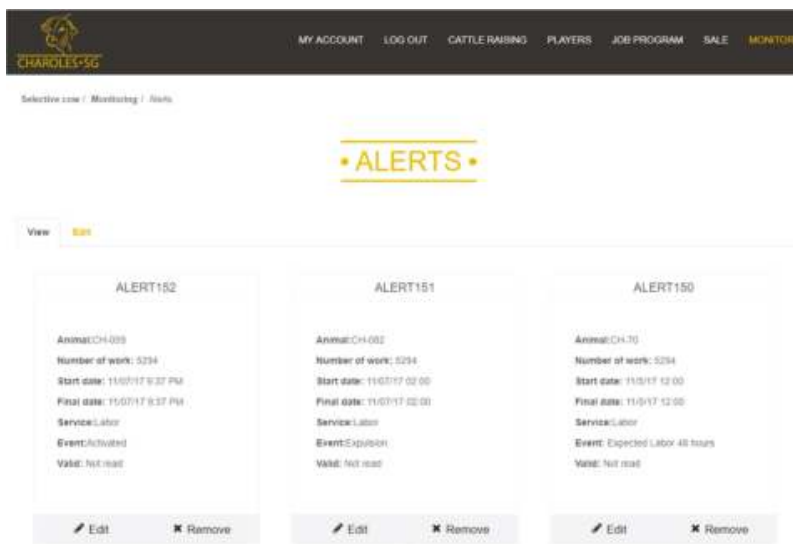

(A)

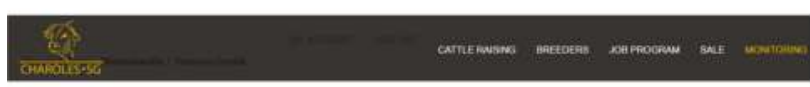

.FOOD SENSORS.

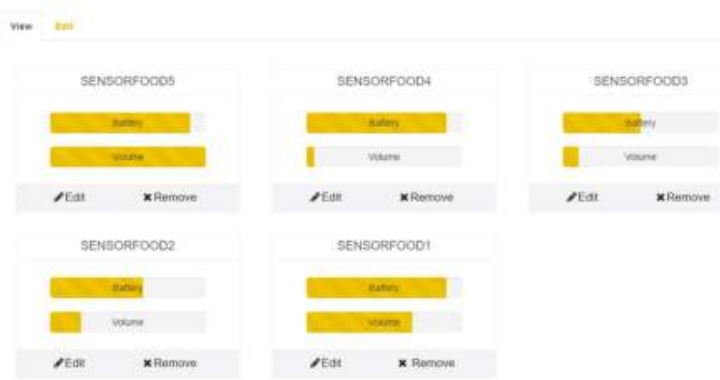

(B)

Figure 16. Web application interface. 
The mobile application provides access to the most important functionality such as alerts on estrus, labor and feeding. The mobile application also allows getting notifications every time a new alert arrives to the system, thus it is not necessary to be continuously looking at the state of livestock. Notifications arrive on the mobile device regardless of whether the application is open or not. They are sent by the agents from the prediction organization. Through the mobile application, you can see alert information in real time, the location of the animals and the cameras. Figure 17A shows the application menu, Figure 17B shows a heat alert for a particular animal; the animal's name, id, date and description can be read in the alert. Figure 17C shows the location of the animals. This location is loaded on a map with the satellite view of google maps, the location of the animals is redrawn synchronously by using websockets, reducing the traffic and updating the location only when there are data. In addition, the different chambers located on the farm can also be viewed from the application.

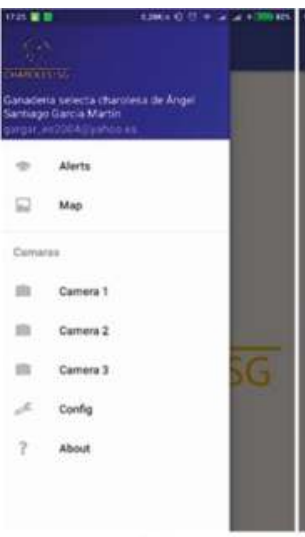

(A)

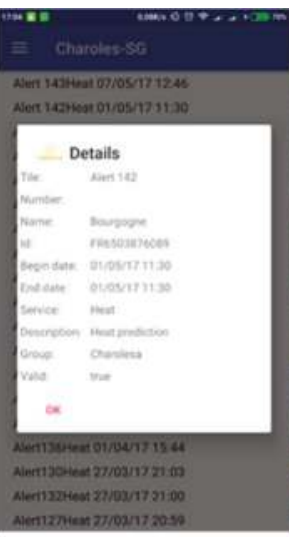

(B)

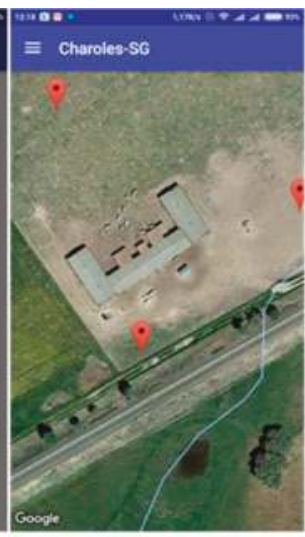

(C)

Figure 17. (A) The application menu; (B) a heat alert for a particular animal, in which you can see the animal's name, id, the date and a description; and $(\mathbf{C})$ the location of the animals.

Cattle farmers have used the system for 12 months, emphasizing the simplicity and the enormous utility of the platform. They were happy with the possibility of monitoring the farm from their television, since the commercial systems that they knew, were based on the sending of SMS to their mobiles, which charge money. From the different utilities offered by the system, the farmers highlighted the benefit of the labor detection system since it allowed them to go to the field and assist the cows whenever it was necessary. The farmer felt overwhelmed by the number of data viewed on the TV application and it had to be reduced to make its use easier. The system for the detection of feeding anomalies, provided greater comfort, since the farmer did not have to spend that much time observing the cattle. The possibility of knowing the state of the feeders and the position of the cows avoids unnecessary displacements. The GPS position of the animal allows to find the animal quickly, which is very important in extensive farming where the farmer must search the animal with a vehicle and would normally spend a lot of time at the task.

Acknowledgments: This work has been supported by project Máquina social para la gestión sostenible de ciudades inteligentes: movilidad urbana, datos abiertos, sensores móviles SA70U16. The project is co-financed with Junta Castilla y León funds. The research of Alberto L. Barriuso has been co-financed by the European Social Fund and Junta de Castilla y León (Operational Programme 2014-2020 for Castilla y León, EDU /128/2015 BOCYL). Álvaro Lozano is supported by the pre-doctoral fellowship from the University of Salamanca and Banco Santander. 
Author Contributions: Alberto L. Barriuso, Gabriel Villarrubia and Juan F. De Paz have developed the system; they made the test and elaborate the review of the state of the art. Javier Bajo and Álvaro Lozano formalized the problem, the algorithms and reviewed the work. All the authors contributed in the redaction of the paper.

Conflicts of Interest: The authors declare no conflicts of interest.

\section{Appendix A}

Since other researchers may decide to use the hardware for their own experimentation, we decided to add electrical diagrams and the interconnection of the components designed for this case study, to this document.

\section{Appendix A.1 Design of the Vaginal Thermometer}

Figure A1 shows the $3 \mathrm{D}$ design of the vaginal thermometer used in the system for calving detection.

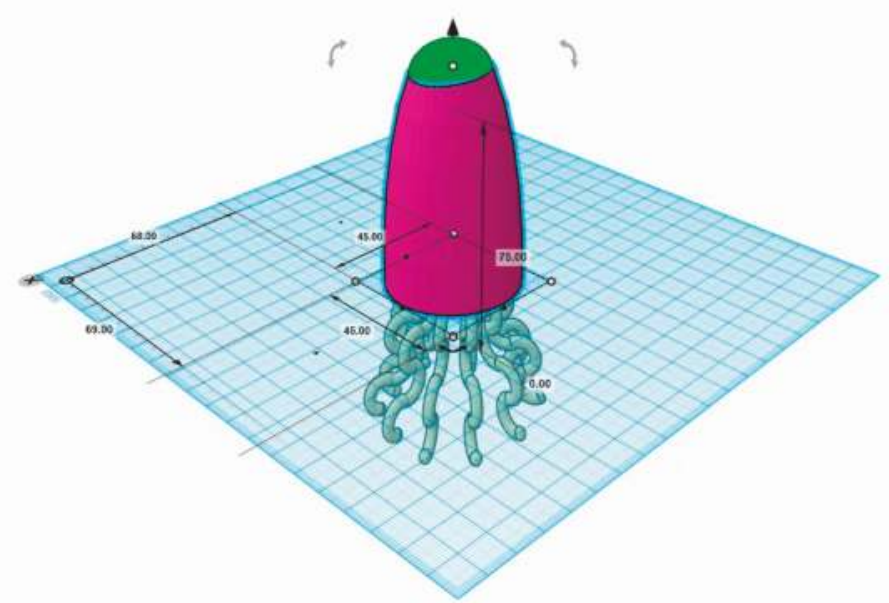

Figure A1. Design of the Vaginal Thermometer.

Appendix A.2 Electric Collar Scheme (GPS + Accelerometer)

Figure A2 shows the electric scheme of the collar that includes both GPS and motion sensors, used in the monitoring of ingestion, estrus detection and calving detection systems. 


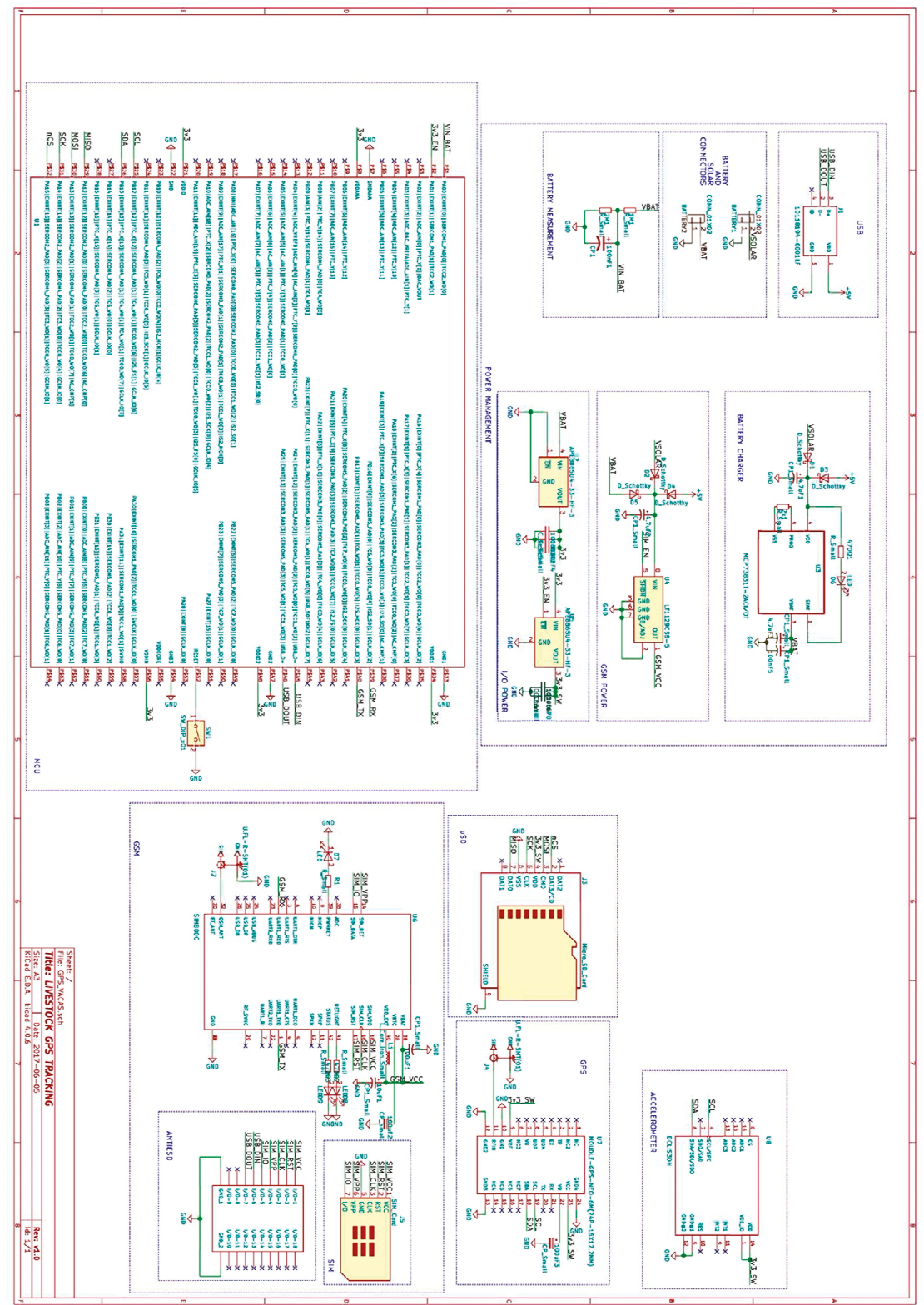

Figure A2. Electric Collar Scheme.

\section{References}

1. Instituto Nacional de Estadística. Censo Agrario, 2009. Available online: http://www.ine.es/CA/Inicio.do (accessed on 28 June 2017).

2. Cook, D.J.; Augusto, J.C.; Jakkula, V.R. Ambient intelligence: Technologies, applications, and opportunities. Pervasive Mob. Comput. 2009, 5, 277-298. [CrossRef] 
3. Adjali, O.; Hina, M.D.; Dourlens, S.; Ramdane-Cherif, A. Multimodal Fusion, Fission and Virtual Reality Simulation for an Ambient Robotic Intelligence. Proc. Comput. Sci. 2015, 52, 218-225. [CrossRef]

4. Roda, C.; Rodríguez, A.C.; López-Jaquero, V.; Navarro, E.; González, P. A Multi-Agent System for Acquired Brain Injury rehabilitation in Ambient Intelligence environments. Neurocomputing 2017, 231, 11-18. [CrossRef]

5. Santana-Mancilla, P.C.; Echeverría, M.A.M.; Santos, J.C.R.; Castellanos, J.A.N.; Díaz, A.P.S. Towards Smart Education: Ambient Intelligence in the Mexican Classrooms. Proc. Soc. Behav. Sci. 2013, 106, 3141-3148. [CrossRef]

6. Lambrou, M.A.; Fjørtoft, K.E.; Sykas, E.D.; Nikitakos, N. Ambient intelligence technologies in support of shipping markets' operations. Telemat. Inform. 2008, 25, 72-83. [CrossRef]

7. Smart Farming Receives the Backing of the European Parliament. Available online: https://ec.europa. eu/digital-single-market/en/news/smart-farming-receives-backing-european-parliament (accessed on 28 June 2017).

8. European Parliament. Report on Technological Solutions for Sustainable Agriculture in the EU-A8-0174/2016. Available online: http:/ / www.europarl.europa.eu/sides/getDoc.do?pubRef=- / EP / /TEXT+REPORT+A8--2016--0174+0+DOC+XML+V0/ / EN\&language=en (accessed on 28 June 2017).

9. European Parliament. Report on Enhancing Innovation and Economic Development in Future European Farm Management-A8-0163/2016. Available online: http://www.europarl.europa.eu/sides/getDoc.do? pubRef=- / /EP / /TEXT+REPORT+A8--2016--0163+0+DOC+XML+V0//EN\&language=en (accessed on 28 June 2017).

10. O'Grady, M.J.; O'Hare, G.M.P. Modelling the smart farm. Inf. Process. Agric. 2017, 4, 321-362. [CrossRef]

11. Wolfert, S.; Ge, L.; Verdouw, C.; Bogaardt, M.J. Big Data in Smart Farming-A review. Agric. Syst. 2017, 153, 69-80. [CrossRef]

12. Kanjilal, D.; Singh, D.; Reddy, R.; Mathew, J. Smart Farm: Extending Automation To The Farm Level. Int. J. Sci. Technol. Res. 2014, 3, 109-113.

13. Kwong, K.H.; Wu, T.-T.; Goh, H.G.; Sasloglou, K.; Stephen, B.; Glover, I.; Shen, C.; Du, W.; Michie, C.; Andonovic, I. Practical considerations for wireless sensor networks in cattle monitoring applications. Comput. Electron. Agric. 2012, 81, 33-44. [CrossRef]

14. Manning, J.K.; Cronin, G.M.; González, L.A.; Hall, E.J.S.; Merchant, A.; Ingram, L.J. The effects of global navigation satellite system (GNSS) collars on cattle (Bos taurus) behaviour. Appl. Anim. Behav. Sci. 2016, 187, 54-59. [CrossRef]

15. Nadimi, E.S.; Søgaard, H.T.; Bak, T.; Oudshoorn, F.W. ZigBee-based wireless sensor networks for monitoring animal presence and pasture time in a strip of new grass. Comput. Electron. Agric. 2008, 61, 79-87. [CrossRef]

16. Williams, M.L.; Parthaláin, N.M.; Brewer, P.; James, W.P.J.; Rose, M.T. A novel behavioral model of the pasture-based dairy cow from GPS data using data mining and machine learning techniques. J. Dairy Sci. 2016, 99, 2063-2075. [CrossRef] [PubMed]

17. Mora-Delgado, J.; Nelson, N.; Fauchille, A. Application of GPS and GIS to study foraging behavior of dairy cattle. Agron. Costarric. 2016, 40, 81-88. [CrossRef]

18. Deniz, N.N.; Chelotti, J.O.; Galli, J.R.; Planisich, A.M.; Larripa, M.J.; Rufiner, H.L.; Giovanini, L.L. Embedded system for real-time monitoring of foraging behavior of grazing cattle using acoustic signals. Comput. Electron. Agric. 2017, 138, 167-174. [CrossRef]

19. Bach, A.; Iglesias, C.; Busto, I. Technical Note: A Computerized System for Monitoring Feeding Behavior and Individual Feed Intake of Dairy Cattle. J. Dairy Sci. 2004, 87, 4207-4209. [CrossRef]

20. Chelotti, J.O.; Vanrell, S.R.; Milone, D.H.; Utsumi, S.A.; Galli, J.R.; Rufiner, H.L.; Giovanini, L.L. A real-time algorithm for acoustic monitoring of ingestive behavior of grazing cattle. Comput. Electron. Agric. 2016, 127, 64-75. [CrossRef]

21. Clapham, W.M.; Fedders, J.M.; Beeman, K.; Neel, J.P.S. Acoustic monitoring system to quantify ingestive behavior of free-grazing cattle. Comput. Electron. Agric. 2011, 76, 96-104. [CrossRef]

22. Tani, Y.; Yokota, Y.; Yayota, M.; Ohtani, S. Automatic recognition and classification of cattle chewing activity by an acoustic monitoring method with a single-axis acceleration sensor. Comput. Electron. Agric. 2013, 92 , 54-65. [CrossRef]

23. López-Gatius, F. Factors of a noninfectious nature affecting fertility after artificial insemination in lactating dairy cows. A review. Theriogenology 2012, 77, 1029-1041. [CrossRef] [PubMed] 
24. Nelson, S.T.; Haadem, C.S.; Nødtvedt, A.; Hessle, A.; Martin, A.D. Automated activity monitoring and visual observation of estrus in a herd of loose housed Hereford cattle: Diagnostic accuracy and time to ovulation. Theriogenology 2017, 87, 205-211. [CrossRef] [PubMed]

25. Shahriar, M.S.; Smith, D.; Rahman, A.; Freeman, M.; Hills, J.; Rawnsley, R.; Henry, D.; Bishop-Hurley, G. Detecting heat events in dairy cows using accelerometers and unsupervised learning. Comput. Electron. Agric. 2016, 128, 20-26. [CrossRef]

26. Kyle, B.; Kennedy, A.; Small, J. Measurement of vaginal temperature by radiotelemetry for the prediction of estrus in beef cows. Theriogenology 1998, 49, 1437-1449. [CrossRef]

27. Hillman, P.E.; Gebremedhin, K.G.; Willard, S.T.; Lee, C.N.; Kennedy, A.D. Continuous Measurements of Vaginal Temperature of Female Cattle Using A Data Logger Encased in a Plastic Anchor. Appl. Eng. Agric. 2009, 25, 291-296. [CrossRef]

28. Miura, R.; Yoshioka, K.; Miyamoto, T.; Nogami, H.; Okada, H.; Itoh, T. Estrous detection by monitoring ventral tail base surface temperature using a wearable wireless sensor in cattle. Anim. Reprod. Sci. 2017, 180, 50-57. [CrossRef] [PubMed]

29. Mee, J.F. Managing the dairy cow at calving time. Vet. Clin. N. Am. Food Anim. Pract. 2004, 20, 521-546. [CrossRef] [PubMed]

30. Calcante, A.; Tangorra, F.M.; Marchesi, G.; Lazzari, M. A GPS/GSM based birth alarm system for grazing cows. Comput. Electron. Agric. 2014, 100, 123-130. [CrossRef]

31. Rutten, C.J.; Kamphuis, C.; Hogeveen, H.; Huijps, K.; Nielen, M.; Steeneveld, W. Sensor data on cow activity, rumination, and ear temperature improve prediction of the start of calving in dairy cows. Comput. Electron. Agric. 2017, 132, 108-118. [CrossRef]

32. Schoenbaum, I.; Kigel, J.; Ungar, E.D.; Dolev, A.; Henkin, Z. Spatial and temporal activity of cattle grazing in Mediterranean oak woodland. Appl. Anim. Behav. Sci. 2017, 187, 45-53. [CrossRef]

33. Santman-Berends, I.M.G.A.; Brouwer-Middelesch, H.; van Wuijckhuise, L.; de Bont-Smolenaars, A.J.G.; van Schaik, G. Surveillance of cattle health in The Netherlands: Monitoring trends and developments using routinely collected cattle census data. Prev. Vet. Med. 2016, 134, 103-112. [CrossRef] [PubMed]

34. Park, M.; Jung, H.; Kim, T.; Ha, O. Design of cattle health monitoring system using wireless bio-sensor networks. In Proceedings of the 4th International Conference on Electronics, Communications and Networks (CECNET IV), Shanghai, China, 12-15 December 2015; p. 325.

35. Frese, D.A.; Thomason, J.D.; Reinhardt, C.; Bartle, S.; Rethorst, D.; Loneragan, G.H.; Thomson, D. Twenty-four hour Holter monitoring in finishing cattle housed outdoors. J. Vet. Cardiol. 2017, 19, 153-159. [CrossRef] [PubMed]

36. Afimilk. Silent Herdsman Collar. Available online: http://www.afimilk.com/es/products/cows/sensors / afimilk-silent-herdsman-neck-collar (accessed on 28 June 2017).

37. Afimilk. MPC Milk Meters. Available online: http://www.afimilk.com/es/products/cows/sensors/milkmeters (accessed on 28 June 2017).

38. Afimilk. AfiTag Pedometers. Available online: http://www.afimilk.com/es/products/cows/sensors/ afitag-pedometers (accessed on 28 June 2017).

39. KV. Solar GPS locator collar. Available online: http://www.espiamos.com/localizador-gps-solar-paraanimales-con-autonomia-ilimitada.html (accessed on 28 June 2017).

40. Cattle Tags. Matched Pair Tags. Available online: http://www.cattletags.com/matched-pair-tags?sort= priceasc (accessed on 28 June 2017).

41. Moocall. Calving Sensors. Available online: https://moocall.com (accessed on 28 June 2017).

42. TELENAX. Rumen Implant. Available online: http:/ / telenax.com/es/ ?option=com_k2\&view=item\&id=35: radio-transmisores-para-ganado\&Itemid=144 (accessed on 28 June 2017).

43. Bond, A.H.; Gasser, L. Readings in Distributed Artificial Intelligence; Morgan Kaufmann: Burlington, MA, USA, 1988.

44. Durfee, E.H.; Lesser, V.R.; Corkill, D.D. Trends in cooperative distributed problem solving. IEEE Trans. Knowl. Data Eng. 1989, 1, 63-83. [CrossRef]

45. Di Bitonto, P.; Laterza, M.; Roselli, T.; Rossano, V. An evaluation method for multi-agent systems. In Agent and Multi-Agent Systems: Technologies and Applications: 5th KES; Springer: Berlin, Germany, 2010; pp. 32-41. 
46. Bellifemine, F.; Poggi, A.; Rimassa, G. JADE: A FIPA2000 compliant agent development environment. In Proceedings of the 5th International Conference on Autonomous Agents (AGENTS '01), Montreal, QC, Canada, 28 May-1 June 2001; pp. 216-217.

47. Luke, S.; Cioffi-Revilla, C.; Panait, L.; Sullivan, K. Mason: A new multi-agent simulation toolkit. In Proceedings of the 2004 Swarmfest Workshop; George Mason University: Fairfax, VA, USA, 2004; Volume 8, pp. 316-327.

48. Zato, C.; Villarrubia, G.; Sánchez, A.; Barri, I.; Rubión, E.; Fernández, A. PANGEA-Platform for Automatic coNstruction of orGanizations of intElligent Agents. Distrib. Comput. Artif. Intell. 2012, 151, 229-239.

49. Stone, P.; Veloso, M. Multiagent Systems: A Survey from a Machine Learning Perspective. Auton. Robot. 2000, 8, 345-383. [CrossRef]

50. AirMAXTM Titanium Sector Antennas Datasheet. Available online: https://dl.ubnt.com/datasheets/ titaniumsector/airMAX_Ti_Sector_DS.pdf (accessed on 20 December 2017).

51. Power Station 5 Datasheet. Available online: https://dl.ubnt.com/ps5_datasheet.pdf (accessed on 20 December 2017).

52. Rayas-Amor, A.A.; Morales-Almaráz, E.; Licona-Velázquez, G.; Vieyra-Alberto, R.; García-Martínez, A.; Martínez-García, C.G.; Cruz-Monterrosa, R.G.; Miranda-de la Lama, G.C. Triaxial accelerometers for recording grazing and ruminating time in dairy cows: An alternative to visual observations. J. Vet. Behav. Clin. Appl. Res. 2017, 20, 102-108. [CrossRef]

53. Yin, L.; Hong, T.; Liu, C. Estrus Detection in Dairy Cows from Acceleration Data using Self-learning Classification Models. JCP 2013, 8, 2590-2597. [CrossRef]

54. Lammoglia, M.A.; Bellows, R.A.; Short, R.E.; Bellows, S.E.; Bighorn, E.G.; Stevenson, J.S.; Randel, R.D. Body temperature and endocrine interactions before and after calving in beef cows. J. Anim. Sci. 1997, 75, 2526-2534. [CrossRef] [PubMed]

(C) 2018 by the authors. Licensee MDPI, Basel, Switzerland. This article is an open access article distributed under the terms and conditions of the Creative Commons Attribution (CC BY) license (http://creativecommons.org/licenses/by/4.0/). 
Article

\title{
Application of Near Infrared Reflectance Spectroscopy for Rapid and Non-Destructive Discrimination of Hulled Barley, Naked Barley, and Wheat Contaminated with Fusarium
}

\author{
Jongguk Lim ${ }^{1}$, Giyoung Kim ${ }^{1}$, Changyeun Mo ${ }^{1}$, Kyoungmin $\mathrm{Oh}^{1}$, Geonseob Kim ${ }^{1}$, \\ Hyeonheui Ham ${ }^{2}$, Seongmin $\mathrm{Kim}^{3, *}$ and Moon S. Kim ${ }^{4}$ \\ 1 Department of Agricultural Engineering, National Institute of Agricultural Sciences, Rural Development \\ Administration, 310 Nongsaengmyeng-ro, Wansan-gu, Jeonju 54875, Korea; limjg@korea.kr (J.L.); \\ giyoung@korea.kr (Gi.K.); cymoh100@korea.kr (C.M.); yoonmine@korea.kr (K.O.); \\ kgs1733@naver.com (Ge.K.) \\ 2 Microbial Safety Team, National Institute of Agricultural Sciences, Rural Development Administration, \\ 166 Nongsaengmyeong-ro, Iseo-myeon, Wanju-gun 55365, Korea; hhham@korea.kr \\ 3 Department of Bioindustrial Machinery Engineering, College of Agriculture \& Life Sciences, \\ Chonbuk National University, 567 Baekje-daero, deokjin-gu, Jeonju 54896, Korea \\ 4 Environmental Microbial and Food Safety Laboratory, Agricultural Research Service, US Department of \\ Agriculture, 10300 Baltimore Avenue, Beltsville 20705, MD, USA; Moon.Kim@ars.usda.gov \\ * Correspondence: smkim@jbnu.ac.kr; Tel.: +82-63-270-2617; Fax: +82-63-270-2620
}

Received: 14 October 2017; Accepted: 28 December 2017; Published: 2 January 2018

\begin{abstract}
Fusarium is a common fungal disease in grains that reduces the yield of barley and wheat. In this study, a near infrared reflectance spectroscopic technique was used with a statistical prediction model to rapidly and non-destructively discriminate grain samples contaminated with Fusarium. Reflectance spectra were acquired from hulled barley, naked barley, and wheat samples contaminated with Fusarium using near infrared reflectance (NIR) spectroscopy with a wavelength range of 1175-2170 nm. After measurement, the samples were cultured in a medium to discriminate contaminated samples. A partial least square discrimination analysis (PLS-DA) prediction model was developed using the acquired reflectance spectra and the culture results. The correct classification rate (CCR) of Fusarium for the hulled barley, naked barley, and wheat samples developed using raw spectra was $98 \%$ or higher. The accuracy of discrimination prediction improved when second and third-order derivative pretreatments were applied. The grains contaminated with Fusarium could be rapidly discriminated using spectroscopy technology and a PLS-DA discrimination model, and the potential of the non-destructive discrimination method could be verified.
\end{abstract}

Keywords: Fusarium; near infrared; discrimination; hulled barely; naked barley; wheat

\section{Introduction}

Barley (Hordeum vulgare L.) is one of the four major grains, and is the next most important crop after rice in South Korea. It is now mainly used for mixed rice, barley tea for children, and various organic food materials [1]. Barley has many functional nutrients such as $\beta$-glucan, arabinoxylan, and hydrocyanic acid, which other general grains do not have, and has effects that aid in the prevention of adult diseases, such as heart disease (by lowering the blood cholesterols) and obesity (by inhibiting body fat accumulation) [2,3]. Barleys can be generally classified into hulled barley, which is not easily peeled and is used as a feed, and naked barley, which is easily peeled and can be used for food [4]. Recently, as consumers' interest in health has increased, hulled barley has been actively developed 
into processed products such as barley tea, malt, and barley sprouts [5,6]. Wheat (Triticum aestivum L.) is second only to corn in world grain production, and is rich in nutrients such as saccharides, proteins, and vitamins. However, most of the wheats consumed in Korea depend on imports. The wheats produced domestically are used for producing bread, noodles, and pasta, and we are trying to increase the self-sufficiency of Korea's wheat production $[7,8]$.

Fusarium (particularly F. graminearum) occurs worldwide in Asia, Canada, Europe, and South America [9]. In Korea, Fusarium occurred on a large scale in the southern part of the country in 1963 , and the production of barleys decreased by $40-80 \%$, which became a big social issue [10]. Fusarium is one of the most destructive plant pathogens; it reduces crop yields through causing red rot fungus in barley and wheat, ear and stem-rotting disease in corn, and root-rotting disease in many other plants [11]. The grains contaminated with Fusarium turn into empty grains and decrease in weight, thus reducing yields and causing enormous economic losses [12]. In particular, the secondary metabolite mycotoxin, which is produced by such molds, is generated during the growing, storage, and distribution of contaminated agricultural products, since it is stable to heat and does not disappear, even when heated. Thus, when contaminated grains are ingested with normal grains by people and livestock, mycotoxins can accumulate in the body and cause serious acute and chronic disorders. Above all, mycotoxin is classified as a carcinogen in nature [13].

Quantification methods using high performance liquid chromatography are often used for the inspection of grain contamination by Fusarium and mycotoxin in research and official analysis methods. For the contamination of Fusarium, the main method involves cultivating the spores that appear as fungi growth and checking them with the naked eye. For the discrimination of mycotoxin, liquid chromatography [14], gas chromatography (GC) [15,16], GC with mass spectrometry [17], and enzyme-linked immunosorbent assay (ELISA) $[18,19]$ are used. These culture and chemical analysis methods are highly sensitive and accurate. However, they require the destruction of the samples, experts who can handle expensive analysis equipment, and are limited in rapid discrimination because it takes a long time [20]. Meanwhile, near infrared reflectance spectroscopy is being used as a technique for quickly and non-destructively discriminate grains contaminated with Fusarium.

Near infrared reflectance spectroscopy (NIRS) is an analytical method that uses a 700-2500 nm wavelength band based on the absorption of near infrared rays by organic compounds and water. More than 60 years have passed since the first practical application of this technique. Karl Norris, the pioneer of NIRS, first attempted this technique in the 1960s to measure the water content of grains and seeds [21].

Since then, instrumentation, statistical methods, and software have improved, and application techniques have increased exponentially in many different fields. Now, the American Association of Cereal Chemists (AACC 39-00) and the American Oil Chemist Association (AOCS am 1-9) have approved NIRS as an analysis method for grains and seeds. The NIR techniques have several important advantages, such as short analysis time, minimal sampling process, and non-destructiveness with a performance comparable to chemistry analytical methods. There are many calibration algorithms for spectra acquired from NIRS, which share the same principles with and are similar to the methods that are widely used in quantitative analysis, such as multiple linear regression (MLR), principal component regression (PCR), and partial least squares (PLS) [22].

PLS and PCR show very similar results, and MLR is more advantageous when handling areas with short wavelengths or data points that have little correlations. In particular, PLS is used as a statistical modeling tool for obtaining correlations between multivariate input variables (independent variables) and output variables (dependent variables) using data acquired from experiments [23]. It is actively used to develop linear multivariate model in diverse industrial fields, including chemical process [24-26]. This method offers the advantage of a model with a higher prediction reliability than general multiple regression analysis, even if there is multicollinearity or a lot of noise in the data, because the input variables have high correlations with one another. The partial least square 
discrimination analysis (PLS-DA) is a supervised classification method used in NIRS, because it allows near infrared reflectance (NIR) variables (wavelengths) with high correlations.

This study was conducted to develop a technology to discriminate hulled barley, naked barley, and wheat contaminated with Fusarium using a near infrared spectroscopy measurement system. For this purpose, a PLS-DA model was developed by applying various mathematical pretreatments to a reflectance spectrum acquired from a spectrometer with a wavelength range of $1175-2170 \mathrm{~nm}$, and its prediction performance was verified through correct classification rate (CCR).

\section{Materials and Methods}

\subsection{Hulled Barley, Naked Barley, and Wheat Samples}

The hulled barley, naked barley, and wheat samples in this study used grains harvested from various regions in 2015, as shown in Figure 1. Hulled barley samples were collected from seven locations, including three locations in Gyeongsangbuk-do, one location in Gyeongsangnam-do, two locations in Jeollabuk-do, one location in Jeollanam-do, and two locations in Chungcheongnam-do. Naked barley samples were collected from one location each in Gyeonggi-do, Jeollanam-do, and Gyeongsangnam-do. Wheat samples were collected from one location each in Gangwon-do, Jeollabuk-do, and Gyeongsangnam-do. Each sample was kept separately in an individual container at a low temperature of $4{ }^{\circ} \mathrm{C}$ to prevent the multiplication of Fusarium. The hulled barley, naked barley, and wheat samples in which no Fusarium was found in the first culture experiment were used as the control group.

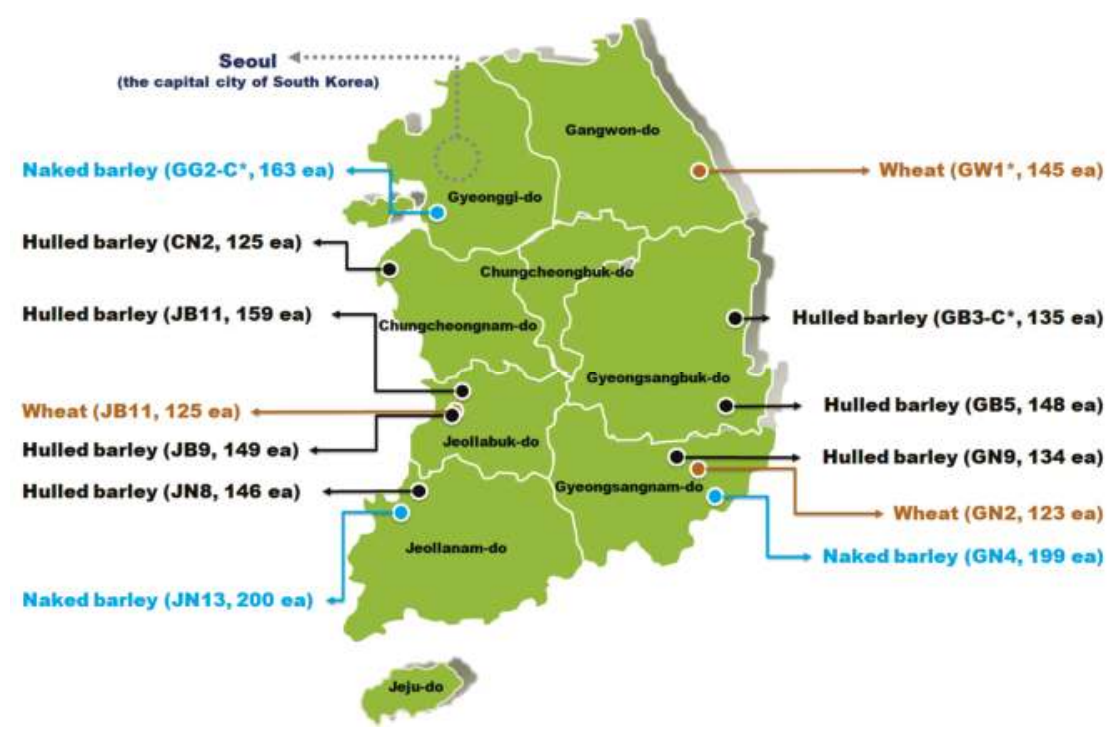

Figure 1. Geographic locations and numbers of hulled barley, naked barley, and wheat used in the experiments from each location. Sample groups marked with an asterisk $\left(^{*}\right)$ are control groups.

As shown in Table 1, the hulled barley samples of the GB3-C group from the Gyeongsangbuk-do region were used as the control group, because no samples contaminated with Fusarium were found in the first culture result. The hulled barley samples collected from the other six locations were used as the experimental group. For the naked barley samples, the GG2-C group from the Gyeonggi-do region were used as the control group, and the samples collected from the other two locations were used as the experimental group. For wheat samples, the GW1-C group from the Gangwon-do region 
were used as the control group, because no wheat contaminated with Fusarium was found, and the samples collected from the other two locations were used as the experimental group. The hulled barley, naked barley, and wheat samples used in this experiment were assigned individual sample numbers. As shown in Table 1, the grain samples were placed separately in a tray with $10 \times 10$ indented cells in the shape of grains.

Table 1. Pictures and quantity of hulled barley, naked barley, and wheat samples.

\begin{tabular}{|c|c|c|c|c|}
\hline Grains & Geographic Locations & Group & Quantity & Pictures \\
\hline \multirow{7}{*}{ Hulled barley } & $\begin{array}{c}\text { Gyeongsangbuk-do } \\
\text { (Yeongdeok-gun) }\end{array}$ & GB3-C $*$ & 135 & \\
\hline & Gyeongsangbuk-do (Gyeongju-si) & GB5 & 148 & \\
\hline & $\begin{array}{l}\text { Gyeongsangnam-do } \\
\text { (Changnyeong-gun) }\end{array}$ & GN9 & 134 & \\
\hline & Jeollabuk-do (Iksan-si) & JB11 & 159 & \\
\hline & Jeollabuk-do (Gimje-si) & JB9 & 149 & \\
\hline & Jeollanam-do (Yeonggwang-gun) & JN8 & 146 & \\
\hline & $\begin{array}{l}\text { Chungcheongnam-do } \\
\text { (Taean-gun) }\end{array}$ & $\mathrm{CN} 2$ & 125 & \\
\hline \multirow{3}{*}{ Naked barley } & Gyeonggi-do (Hwaseong-si) & GG2-C * & 163 & \\
\hline & Jeollanam-do (Hampyeong-gun) & JN13 & 200 & \\
\hline & Gyeongsangnam-do (Gimhae-si) & GN4 & 199 & \\
\hline \multirow{3}{*}{ Wheat } & Gangwon-do (Donghae-si) & GW1-C * & 145 & \\
\hline & Jeollabuk-do (Gimje-si) & JB11 & 125 & \\
\hline & Gyeongsangnam-do (Milyang-si) & GN2 & 123 & \\
\hline
\end{tabular}

* Control group. 


\subsection{NIRS Measurement System}

As shown in Figure 2, the NIRS measurement system is comprised of a NIR spectroscopic sensor, a tungsten-halogen lamp, a fiber optic probe, a sample table, a fiber holder, and a measurement computer.

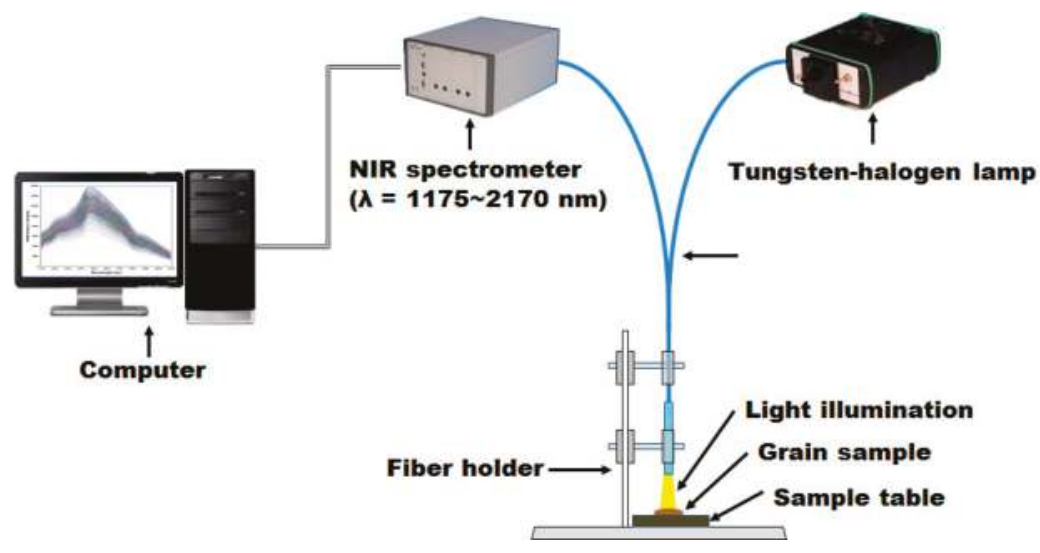

Figure 2. A schematic diagram of the near infrared reflectance spectroscopy (NIRS) measurement system for the discrimination of Fusarium contamination of cereals.

The NIR spectroscopic sensor (Avaspec-NIR256-2.2TC, Avantes BV Inc., Apeldoorn, The Netherlands) has a wavelength range of $1175-2170 \mathrm{~nm}$, a pixel pitch of $3.4 \mathrm{~nm}, 256$ pixels in total with a pixel size of $50 \times 500 \mu \mathrm{m}$, and an indium gallium arsenide (InGaAs) linear array sensor. For the tungsten-halogen lamp used to supply light in the NIR wavelength range, a light source with $10 \mathrm{~W}$ power (Avalight-HAL, Avantes BV Inc., Apeldoorn, The Netherlands) and a wavelength range of 360-2500 nm was used. The bifurcated fiber-optic probe (FCR-7IR400-2-ME, Avantes BV Inc., Apeldoorn, The Netherlands), which transmits light from the light source and receives the light reflected from the grain sample with the NIR spectrometer, consists of seven optic fibers with a diameter of $400 \mu \mathrm{m}$. Furthermore, the AVA soft program (Avasoft-basic, Avantes BV Inc., Apeldoorn, The Netherlands) was used to acquire and store the reflectance spectrum data from the grain samples.

\subsection{NIR Reflectance Spectrum Acquisition}

To acquire the NIR reflectance spectra of hulled barley, naked barley, and wheat, the grains were moved to the dedicated template for measurement, as shown in Figure 3. A total of six measurements were made for each grain sample to acquire six NIR reflectance spectra. The distance between the grain sample and the end of the fiber-optic probe was maintained at 10-15 mm, and the light from the light source was irradiated to the surface of the grain samples as much as possible. A total of 5976 reflectance spectra were acquired from 996 hulled barley samples, a total of 3372 reflectance spectra were acquired from 562 naked barley samples, and a total of 2358 spectra were acquired from 393 wheat samples. The measurement condition was $200 \mathrm{~ms}$ of light integration time, and the cumulative average was obtained from three measurements. In addition, smoothing pretreatment was applied by default for the acquisition of reflectance spectrum. 


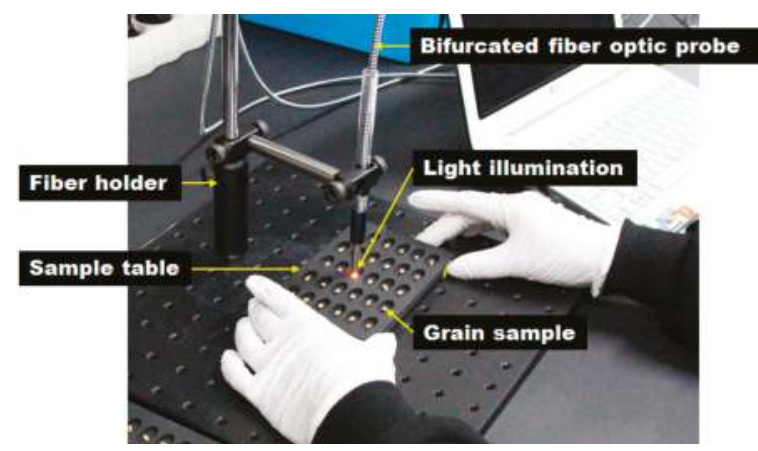

Figure 3. Bifurcated fiber-optic probe and light illumination in near infrared reflectance (NIR) spectrum acquisition for hulled barley, naked barley, and wheat.

\subsection{Culturing Fusarium}

After hulled barley, naked barley, and wheat were measured for the acquisition of the NIR reflectance spectrum, a culture experiment was performed to determine Fusarium contamination for each grain of the samples. First, the medium was prepared to promote the cultivation of Fusarium latent in the grains. For the medium, $23.4 \mathrm{~g}$ and $5.04 \mathrm{~g}$ of Difco ${ }^{\mathrm{TM}}$ potato dextrose agar and Bacto ${ }^{\mathrm{TM}}$ agar, respectively, in powder state were weighed and mixed. Then, $900 \mathrm{~mL}$ of distilled water was added to two types of mixed medium powders, and $225 \mathrm{~mL}$ each of the mixture was poured into each of four bottles. The four medium solutions in the bottles were sufficiently stirred with hotplate with magnetic stirrers (MSH-30A, DAIHAN Scientific Co., Ltd., Wonju, Korea) until they were fully dissolved. The prepared medium solutions were sterilized for 15 minutes at $120^{\circ} \mathrm{C}$ with a high-pressure sterilizer (AC-1 60 L, Universal Scientific Co., Ltd., Gimpo, Korea) and left on a clean bench for about one hour to cool down to $40{ }^{\circ} \mathrm{C}$. Two types of antibiotics were injected to the medium solutions to prevent the growth of germs other than Fusarium. For the first antibiotic, $0.5 \mathrm{~g}$ Neomycin was melted in $50 \mathrm{~mL}$ distilled water and filtered through a $0.2 \mu \mathrm{m}$ filter; then, $10.8 \mathrm{~mL}$ of the filtered antibiotic was injected into the medium solution. For the second antibiotic, $2.5 \mathrm{~g}$ Streptomycin was melted in $50 \mathrm{~mL}$ distilled water and filtered through a $0.2 \mu \mathrm{m}$ filter; then, $18 \mathrm{~mL}$ filtered antibiotic was injected to the $900 \mathrm{~mL}$ medium solution. The injected two antibiotics were sufficiently mixed by stirring for $30 \mathrm{~s}$ using the hotplate with magnetic stirrers. Half of the medium solution with antibiotics added was poured into a $125 \times 125 \mathrm{~mm}$ square dish (SP11125, SPL Life Sciences Co., Ltd., Pocheon, Korea), which could hold 36 grains and had been gas-sterilized. The dish was left for $2-3 \mathrm{~h}$ to harden the medium. Then, 36 grains were placed on the medium in each prepared square dish using sterilized tweezers. The used tweezers were sterilized with an alcohol lamp and cooled off before using again. The square dish with 36 grains was covered and sealed with tape so that no germs would enter it. The sealed square dishes were cultured for $2-4$ days in an incubator at $25^{\circ} \mathrm{C}$, and the final Fusarium spores were verified by visual inspection.

\subsection{Development of Fusarium Discrimination Prediction Model}

The prediction model used to discriminate the grains contaminated with Fusarium was PLS-DA. For the developed model, the CCRs (\%) for normal and contaminated samples were calculated, respectively. Unlike the partial least square regression model-in which a linear regression model is developed with dependent variables—and spectra data — which is an independent variable-PLS-DA, through using the actually measured concentration data $[27,28]$, develops a regression model by specifying the groups to be discriminated as dummy variables instead of using the concentration values as the dependent variable [29]. The independent variables used to develop the PLS-DA 
model were the NIR reflectance spectra acquired from the hulled barley, naked barley, and wheat samples. For the dependent variables, the spectra acquired from the normal hulled barley, naked barley, and wheat samples that were not contaminated with Fusarium was specified as ' 0 ' as a random virtual variable, and the spectra acquired from the contaminated grain samples was specified as ' 1 '.

For the raw spectrum data, spectra pretreatment was applied by various mathematical methods to correct light scattering by irregular surface appearance and develop a good discrimination prediction model [30]. To correct the movement of the spectra baseline due to surrounding environmental conditions such as the changed lighting and temperature when measuring spectra, first derivative, second derivative, and third derivative pretreatments were applied. To remove the light scattering effect during spectrum measurement, mean scattering correction (MSC) and standard normal variate (SNV) were used. Cross-validation statistics were applied to estimate the predictive ability of the PLS-DA calibration models developed during this research. The accuracy of the developed models was determined by its coefficient of determination for calibration (RC2), the standard errors of calibration (SEC), the coefficient of determination for validation (RV2), the standard error of prediction (SEP), and the principal component (PC). Furthermore, the baseline method was applied to correct the minimum values of spectra identically. A multivariate data analysis software application (Unscrambler v9.2, Camo Co., Oslo, Norway) was used for the pretreatment of the acquired reflectance spectra, the development of the PLS-DA model with the converted spectra, and the correction and validation of the developed model.

\section{Results and Discussion}

\subsection{Culture Results of Hulled Barley, Naked Barley and Wheat}

Table 2 shows the culture results for hulled barley, naked barley, and wheat. The first culture results in column (a) show the results of preliminary culture experiment for samples, which were used to determine whether the samples collected from each region were a contaminated group or a normal group. In this study, the control group and experimental group of the hulled barley, naked barley, and wheat samples were classified by the first culture experiment results. The number of grain samples was identical to the number of grains used in the preliminary culture experiment. The second culture experiment was carried out after the near infrared reflectance spectrum was acquired, and the results are shown in column (b).

Table 2. Culture results for control group and experimental group of hulled barley, naked barley, and wheat samples contaminated with Fusarium.

\begin{tabular}{|c|c|c|c|}
\hline Types of Grain & & $\begin{array}{l}\text { (a) Primary Culture Results } \\
\left({ }^{1)} \mathrm{NCF}+{ }^{2)} \mathrm{CF}=\text { Sum) }\right.\end{array}$ & $\begin{array}{c}\text { (b) Secondary Culture } \\
\text { Results (NCF + CF = Sum) }\end{array}$ \\
\hline \multirow{7}{*}{ Hulled barley } & GB3-C * & $135+0=135$ & $135+0=135$ \\
\hline & GB5 & $147+1=148$ & $147+1=148$ \\
\hline & GN9 & $39+95=134$ & $28+106=134$ \\
\hline & JB11 & $158+1=159$ & $159+0=159$ \\
\hline & JB9 & $107+42=149$ & $123+26=149$ \\
\hline & JN8 & $117+29=146$ & $131+15=146$ \\
\hline & CN2 & $114+11=125$ & $123+2=125$ \\
\hline \multirow{3}{*}{ Naked barley } & GG2-C * & $163+0=163$ & $149+14=163$ \\
\hline & JN13 & $166+34=200$ & $188+12=200$ \\
\hline & GN4 & $172+27=199$ & $178+21=199$ \\
\hline \multirow{3}{*}{ Wheat } & GW1-C * & $145+0=145$ & $145+0=145$ \\
\hline & JB11 & $25+100=125$ & $92+33=125$ \\
\hline & GN2 & $73+50=123$ & $60+63=123$ \\
\hline
\end{tabular}

1) NCF: Not contaminated with Fusarium; ${ }^{2)} \mathrm{CF}$ : Contaminated with Fusarium; ${ }^{*}$ Control group. 
For the hulled barley samples, a total of 996 grains including the GB3-C samples, which were the control group, and the samples collected from six regions, which were used as the experimental group, were placed in the square dishes- 36 grains per dish-and were cultured. After four days, the cultured samples were discriminated by experts with the naked eye for contamination with Fusarium. In the control group (GB3-C), no spore contaminated with Fusarium was observed in the preliminary and second culture experiments. In the case of JB11, which was used as an experimental group, one grain was found to be contaminated with Fusarium in the preliminary culture experiment, but in the main experiment, no contaminated hulled barley grain was found. In the other experimental groups, Fusarium spores were observed, although the quantities were different. Therefore, 135 GB3 grains were used as the control group samples for discriminating hulled barley grains contaminated with Fusarium. For the experimental groups samples, a total of 150 grains were used, including GB5 (1 grain), GN9 (106 grains), JB9 (26 grains), JN8 (15 grains), and CN2 (2 grains) for hulled barley samples contaminated with Fusarium. Figure 4 shows the status of the GB3 samples after culture, which were the hulled barley samples that were used as the control group. Normal hulled barley germinated with no generation of spores.
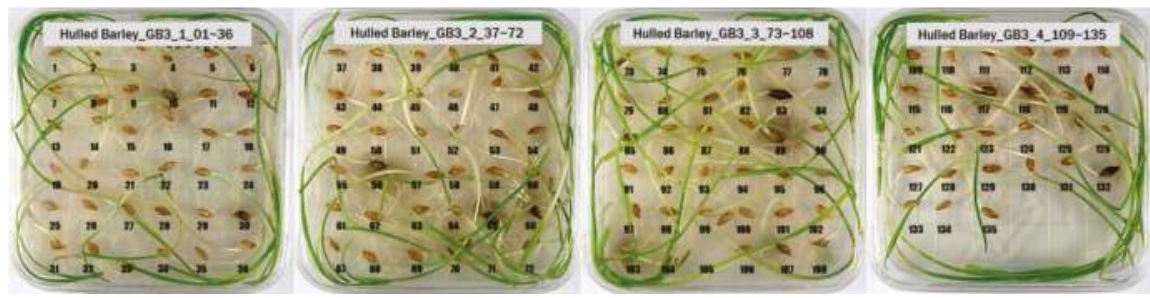

Figure 4. Culture results for hulled barley (GB3-C) in the control group, showing that the Fusarium spore was not observed.

Figure 5 shows the state after culture of the GN9 samples, in which 106 grains generated Fusarium spores: the largest number among the hulled barley sample groups that were used as experimental groups. The samples that generated spores were marked by red dotted lines.
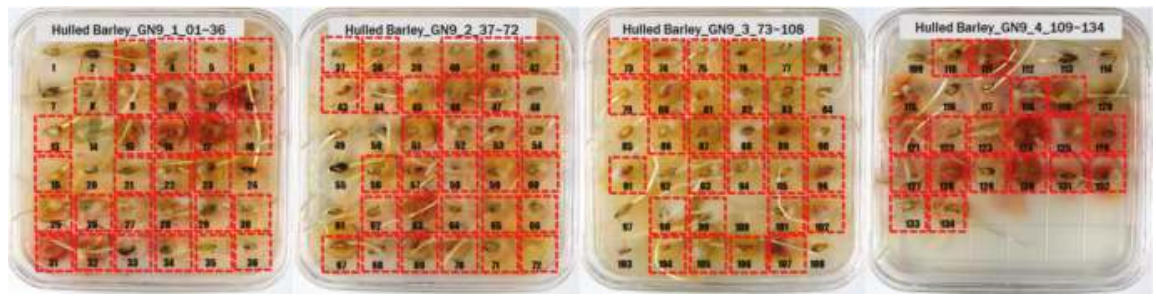

Figure 5. Culture results for hulled barley (GN9) in the experimental group, showing that the Fusarium spore was observed. The hulled barley samples that generated spores were marked by red squares.

For the naked barley samples, a total of 562 grains, including GG2-C samples, which were the control group, and the samples collected from the other two regions, which were used as the experimental groups, were placed and cultured in the square dishes. No Fusarium spores were found in the GG2-C sample group, which was the control group, in the preliminary culture experiment results. In the second culture experiment, however, 14 grains were contaminated with Fusarium among the 163 grains in total, as shown in Figure 6. 

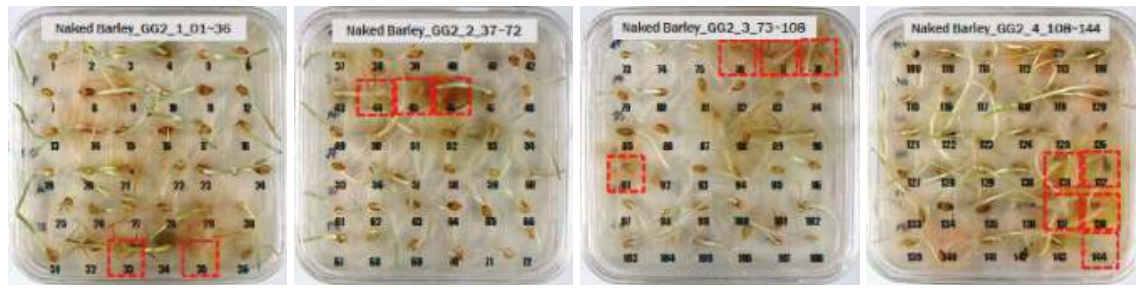

Figure 6. Culture results for naked barley (GG2-C) in the control group, showing that the Fusarium spore was observed. The naked barley samples that generated spores were marked by red squares.

Among the experimental groups, 12 out of the 200 grains in the JN13 sample group and 21 out of the 199 grains in the GN4 group were contaminated. Figure 7 shows the samples of the GN4 group, which were the naked barley samples that were used as the experimental group, in which Fusarium spores were generated. For the control group for discriminating naked barley samples contaminated with Fusarium, 149 grains that were not contaminated with Fusarium in the GG2-C group were used. For the experimental group, a total of 33 grains, including JN13 (12 grains) and GN4 (21 grains), were used.
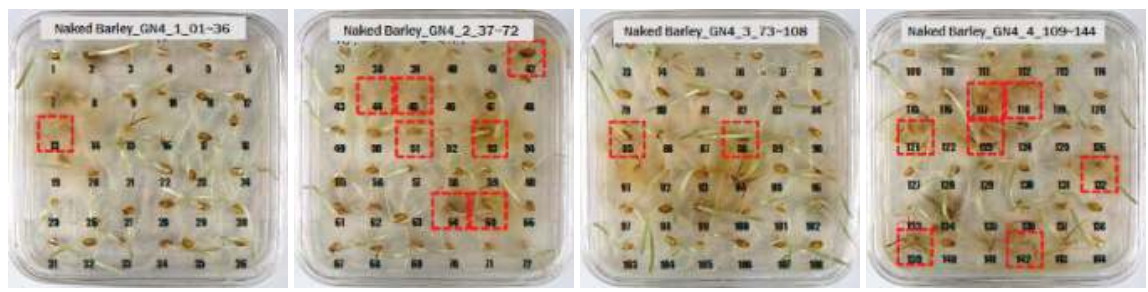

Figure 7. Culture results for naked barley (GN4) in the experimental group, showing that the Fusarium spore was observed. The naked barley samples that generated spores were marked by red squares.

For wheat samples, a total of 393 grains were placed and cultured in square dishes, including GW1-C samples of the control group, and the samples collected from the other two regions in the experimental groups. In the GW1-C wheat samples of the control group, no spores contaminated with Fusarium were found, and normal wheats were germinated, as the second culture experiment results show in Figure 8.
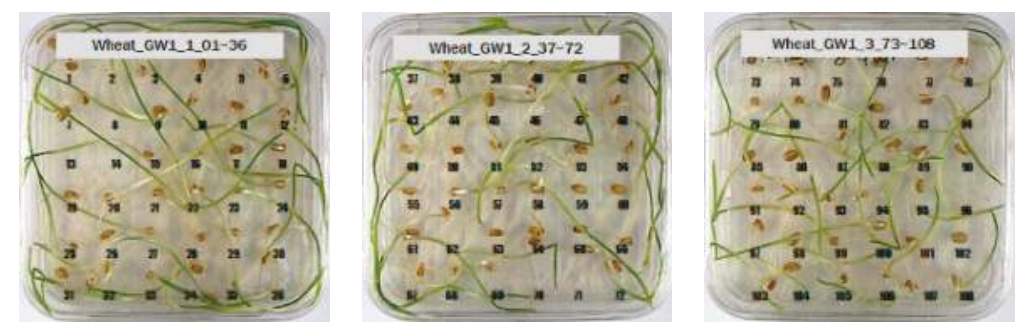

Figure 8. Culture results for wheat (GW1) in the control group, showing that the Fusarium spore was not observed. 
Among the wheat samples in the experimental groups, a total of 96 grains were found to be contaminated with Fusarium, including 33 grains in the JB11 group, and 63 grains in the GN2 group. Figure 9 shows wheat samples of the GN2 group in which Fusarium spores were observed.
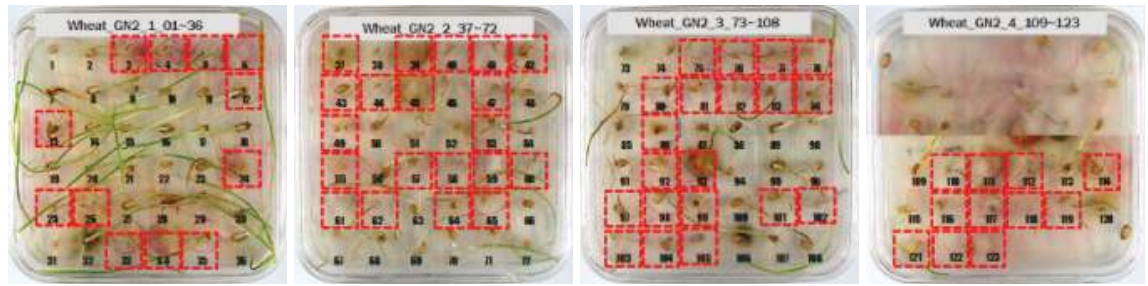

Figure 9. Culture results for wheat (GN2) in the control group, showing that the Fusarium spore was observed. The wheat samples that generated spores were marked by red squares.

\subsection{NIR Reflectance Spectra of Hulled Barley, Naked Barley, and Wheat}

Figure 10 shows the reflectance spectra of 1710 (Not contaminated with Fusarium (NCF): 810, contained with Fusarium (CF): 900) obtained from 285 hulled barley samples (NCF: 135, CF: 150). Figure 10a shows the raw reflectance spectra acquired using NIR256 spectroscopic sensor for 135 grains in the hulled barley samples of the control group that were not contaminated with Fusarium. First, 810 reflectance spectra were collected by measuring 135 samples six times. The highest rising peak occurred at $1575 \mathrm{~nm}$, and the strength of reflectance at this time was 15,500 counts at the maximum. A rising peak also occurred in the $1335 \mathrm{~nm}$ wavelength, and a falling peak occurred at the $1922 \mathrm{~nm}$ wavelength. Figure 10b shows the 900 NIR reflectance spectra acquired from 150 grains of hulled barley samples that were contaminated with Fusarium, which show a similar trend as the reflectance spectra of hulled barley samples. However, the second rising peak occurred at the $1327 \mathrm{~nm}$ wavelength, resulting in a shift.

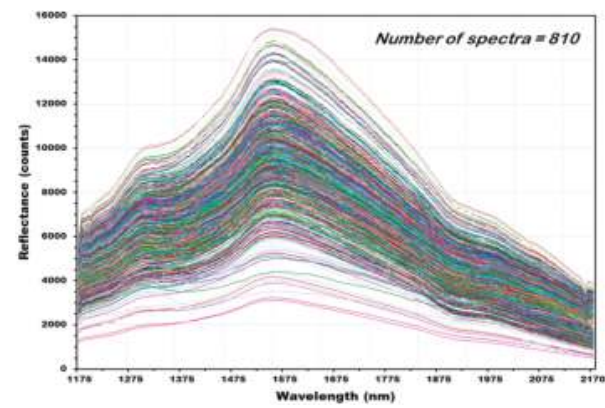

(a)

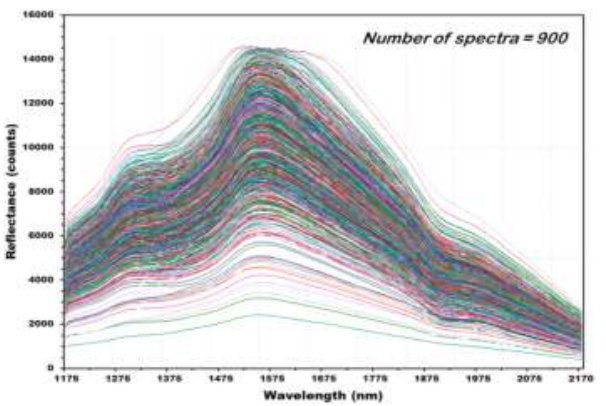

(b)

Figure 10. All of the NIR reflectance spectra of hulled barley samples not contaminated with Fusarium (NCF) (a) and contaminated Fusarium (CF) (b).

Figure 11 shows the reflectance spectra of 1092 (NCF: 894,CF: 198) obtained from 182 naked barley samples (NCF: 149, CF: 33). Figure 11a shows 894 raw reflectance spectra acquired from 149 grains in total of the group naked barley samples in the control group that were not contaminated with Fusarium. Similar to the hulled barley, the maximum rising peak of the naked barley samples occurred at $1579 \mathrm{~nm}$. However, the strength of the reflected light decreased, because the surface of naked barleys with no skin absorbed more light, and the maximum strength of reflectance was around 7500 counts, which is low. The second rising peak occurred at the $1309 \mathrm{~nm}$ wavelength, and the maximum strength 
of reflectance was 6500 counts. The falling peak occurred at $1933 \mathrm{~nm}$. Figure $11 \mathrm{~b}$ shows the $198 \mathrm{raw}$ NIR reflectance spectra acquired from 33 naked barley grains contaminated with Fusarium.

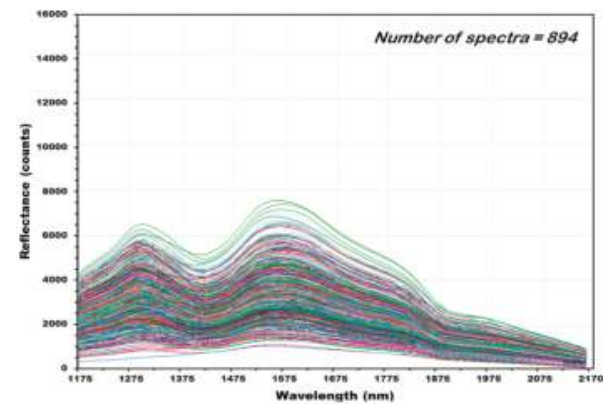

(a)

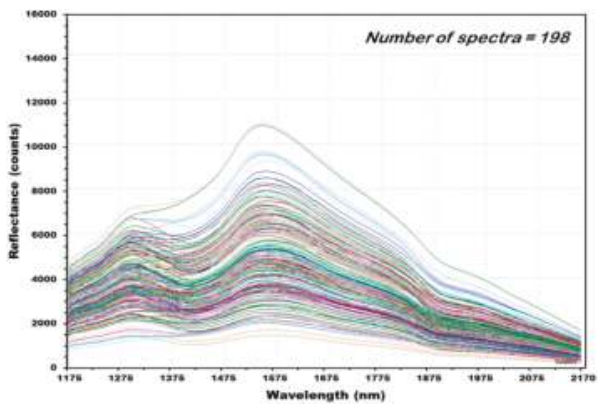

(b)

Figure 11. All NIR reflectance spectra of naked barley samples NCF (a) and CF (b).

Figure 12 shows the reflectance spectra of 1446 (NCF: 870, CF: 576) obtained from 241 wheat samples (NCF: 145, CF: 96). Figure 12a shows 870 raw reflectance spectra acquired from 145 grains of wheat samples that were not contaminated with Fusarium. The maximum rising peak occurred at $1579 \mathrm{~nm}$ wavelength, where those of hulled barley and naked barley grains occurred. The second rising peak occurred at $1314 \mathrm{~nm}$. Figure $12 \mathrm{~b}$ shows 576 raw reflectance spectra acquired from 96 grains of wheat samples contaminated with Fusarium. The first and second rising peaks occurred at wavelengths similar to those of normal wheat.

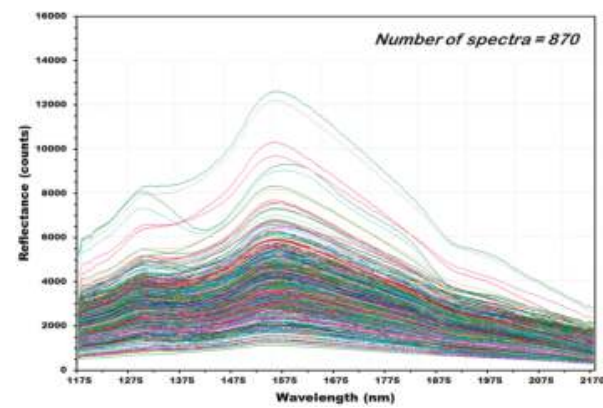

(a)

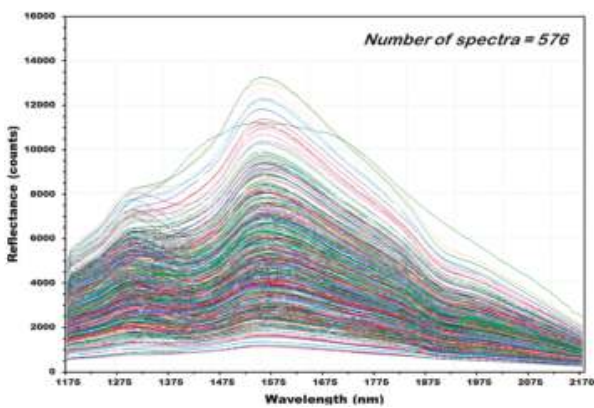

(b)

Figure 12. All NIR reflectance spectra of wheat samples NCF (a) and CF (b).

\subsection{Results of the PLS-DA Prediction Model for Fusarium Discrimination}

A PLS-DA prediction model was developed to discriminate Fusarium contamination using the result of culturing Fusarium after acquiring near infrared reflectance spectrum from hulled barley, naked barley, and wheat samples. Table 3 shows the CCR result for the calibration and validation performance of the PLS-DA models developed by applying various mathematical pretreatments to the NIR reflectance spectra acquired from hulled barley, naked barley, and wheat samples. The CCR of the PLS-DA discrimination prediction model for hulled barley, naked barley, and wheat that has been developed with acquired raw reflectance spectra is $98 \%$ or higher, indicating good results. The CCR for the validation performance of the raw reflectance spectra to which no pretreatment was applied was $100 \%(810 / 810)$ for the NCF of hulled barley samples, and $99.44 \%(895 / 900)$ for the CF, with 
99.72\% on average. The CCR of the naked barley samples was 99.89\% (893/894) for NCF and 99.48\% (195/198) for CF, with $99.18 \%$ on average. The CCR of wheat samples was $99.66 \%(867 / 870)$ for NCF and $99.83 \%(576 / 576)$ for $\mathrm{CF}$, with $99.74 \%$ on average, showing the best discrimination performance. For the mathematical pretreatments for the three types of grain samples, the derivative pretreatment showed the most appropriate and improved performance. In the validation performance of hulled barley samples, the discrimination prediction performance of the second derivative improved to $R_{V}{ }^{2}=$ 0.946 and SEP $= \pm 0.116$. The CCRs of NCF and CF were $100 \%$ and $99.78 \%(898 / 900)$, respectively. For the validation performance of naked barley samples, all of the CCRs of NCF and CF in the first, second, and third derivatives improved to $100 \%$. Thus, the spectra pretreatment that applied derivatives effectively improved the discrimination performance. In particular, the validation performance applying the third derivative pretreatment was $\mathrm{R}_{\mathrm{V}}^{2}=0.948$ and SEP $= \pm 0.088$, showing the best performance among all of the samples. In the wheat samples, too, the CCR of the spectra pretreatment applying derivative improved. The prediction model of the PLS-DA that developed by applying second derivative pretreatment showed the best results at $\mathrm{R}_{\mathrm{V}}^{2}=0.933$ and SEP $= \pm 0.127$.

Table 3. Performance of the partial least square discrimination analysis (PLS-DA) calibration and validation models for Fusarium-contaminated hulled barley samples, as well as the classification accuracy for normal and contaminated hulled barley, naked barley, and wheat.

\begin{tabular}{|c|c|c|c|c|c|c|c|c|c|}
\hline \multirow{3}{*}{ Pretreatment } & \multirow{3}{*}{ 1) $\mathrm{PC}$} & \multicolumn{4}{|c|}{ Performances of Calibration } & \multicolumn{4}{|c|}{ Performances of Validation } \\
\hline & & \multirow{2}{*}{ 2) $R_{c}^{2}$} & \multirow{2}{*}{ 3) SEC } & \multicolumn{2}{|c|}{ 4) CCR (\%) } & \multirow{2}{*}{ 5) $R_{v}^{2}$} & \multirow{2}{*}{ 6) SEP } & \multicolumn{2}{|c|}{$\operatorname{CCR}(\%)$} \\
\hline & & & & NCF & $\mathrm{CF}$ & & & NCF & $\mathrm{CF}$ \\
\hline \multicolumn{10}{|l|}{ Hulled barley } \\
\hline Non-pretreatment & 16 & 0.888 & 0.167 & 100 & 99.56 & 0.881 & 0.172 & 100 & 99.44 \\
\hline 1st order Derivative & 11 & 0.918 & 0.143 & 100 & 100 & 0.911 & 0.149 & 100 & 99.56 \\
\hline 2nd order Derivative & 10 & 0.950 & 0.112 & 100 & 99.78 & 0.946 & 0.116 & 100 & 99.78 \\
\hline 3rd order Derivative & 9 & 0.934 & 0.128 & 100 & 99.89 & 0.928 & 0.134 & 100 & 99.89 \\
\hline Mean ${ }^{7)}$ Nor & 13 & 0.863 & 0.185 & 98.52 & 99.00 & 0.855 & 0.190 & 98.52 & 98.89 \\
\hline Maximum Nor & 13 & 0.855 & 0.190 & 98.52 & 99.00 & 0.846 & 0.196 & 98.15 & 98.78 \\
\hline Range Nor & 14 & 0.869 & 0.181 & 98.89 & 99.11 & 0.860 & 0.187 & 98.77 & 99.00 \\
\hline 8) MSC & 12 & 0.835 & 0.203 & 98.02 & 99.11 & 0.826 & 0.208 & 97.90 & 98.78 \\
\hline Baseline & 15 & 0.875 & 0.177 & 100 & 99.44 & 0.868 & 0.182 & 99.88 & 99.22 \\
\hline 9) $\mathrm{SNV}$ & 13 & 0.836 & 0.202 & 97.90 & 99.11 & 0.827 & 0.208 & 97.90 & 98.78 \\
\hline \multicolumn{10}{|l|}{ Naked barley } \\
\hline Non-pretreatment & 13 & 0.868 & 0.140 & 99.89 & 98.99 & 0.859 & 0.145 & 99.89 & 98.48 \\
\hline 1st order Derivative & 10 & 0.939 & 0.095 & 100 & 100 & 0.932 & 0.101 & 100 & 100 \\
\hline 2nd order Derivative & 7 & 0.944 & 0.091 & 100 & 100 & 0.941 & 0.093 & 100 & 100 \\
\hline 3rd order Derivative & 7 & 0.950 & 0.086 & 100 & 100 & 0.948 & 0.088 & 100 & 100 \\
\hline Mean Nor & 12 & 0.810 & 0.168 & 99.22 & 98.99 & 0.800 & 0.174 & 99.22 & 98.99 \\
\hline Maximum Nor & 13 & 0.810 & 0.170 & 99.22 & 98.99 & 0.792 & 0.176 & 99.22 & 98.99 \\
\hline Range Nor & 13 & 0.807 & 0.169 & 99.22 & 98.99 & 0.792 & 0.176 & 99.22 & 98.99 \\
\hline MSC & 11 & 0.777 & 0.182 & 99.33 & 98.99 & 0.758 & 0.190 & 98.99 & 98.48 \\
\hline Baseline & 13 & 0.865 & 0.142 & 99.89 & 98.99 & 0.855 & 0.147 & 99.89 & 98.48 \\
\hline SNV & 12 & 0.781 & 0.181 & 99.33 & 98.48 & 0.763 & 0.188 & 99.11 & 98.48 \\
\hline \multicolumn{10}{|l|}{ Wheat } \\
\hline Non-pretreatment & 12 & 0.908 & 0.149 & 99.66 & 100 & 0.900 & 0.155 & 99.66 & 99.83 \\
\hline First-order Derivative & 8 & 0.923 & 0.136 & 100 & 100 & 0.909 & 0.147 & 100 & 99.83 \\
\hline $\begin{array}{l}\text { Second-order } \\
\text { Derivative }\end{array}$ & 7 & 0.941 & 0.119 & 100 & 100 & 0.933 & 0.127 & 100 & 99.83 \\
\hline Third`order Derivative & 5 & 0.916 & 0.141 & 100 & 99.83 & 0.911 & 0.146 & 100 & 99.83 \\
\hline Mean Nor & 9 & 0.939 & 0.121 & 99.89 & 99.83 & 0.934 & 0.126 & 99.89 & 99.83 \\
\hline Maximum Nor & 10 & 0.933 & 0.127 & 99.89 & 99.83 & 0.928 & 0.132 & 99.89 & 99.83 \\
\hline Range Nor & 10 & 0.928 & 0.131 & 99.54 & 100 & 0.921 & 0.138 & 99.54 & 99.83 \\
\hline MSC & 9 & 0.912 & 0.145 & 99.54 & 100 & 0.906 & 0.150 & 99.54 & 99.65 \\
\hline Baseline & 13 & 0.909 & 0.147 & 99.89 & 100 & 0.901 & 0.154 & 99.89 & 99.65 \\
\hline SNV & 10 & 0.913 & 0.144 & 99.54 & 100 & 0.908 & 0.149 & 99.54 & 99.65 \\
\hline
\end{tabular}

1) PC: Principle component number; $\left.{ }^{2}\right) \mathrm{R}_{\mathrm{c}}{ }^{2}$ : Coefficient of determination for calibration; ${ }^{3)} \mathrm{SEC}$ : Standard error of calibration; ${ }^{4)}$ CCR: Correct classification rate; ${ }^{5)} \mathrm{R}_{\mathrm{V}}{ }^{2}$ : Coefficient of determination for validation; ${ }^{6)}$ SEP: Standard error of prediction; ${ }^{7)}$ Nor: Normalization; ${ }^{8)}$ MSC: Mean scattering correction; ${ }^{9)}$ SNV: Standard normal variate. 
3.3.1. Prediction Results of the PLS-DA Model for Discrimination of Contaminated with Fusarium (CF)-Hulled Barley

Figure 13a shows the validation result of the PLS-DA model developed with no application of pretreatment to the raw reflectance spectra acquired from the hulled barley samples. In the raw data, five out of 900 spectra acquired from 150 grains of CF hulled barley were predicted as false positive at 0.5 or lower at the reference value of \pm 1 , resulting in $99.44 \%(895 / 900)$ CCR. The 810 spectra from the hulled barley samples that were not contaminated showed $100 \%$ CCR. In this study, various mathematical spectra pretreatments were applied to improve the accuracy of the prediction model. As shown in Figure 13b, the third-order derivative result predicted only one of the 900 spectra acquired from CF as higher than 1.5, which is the reference value, resulting in a CCR of 99.89\% (899/900). The CCR of the third-order derivative pretreatment was $100 \%(810 / 810)$ for NCF. The validation model applying the third-order derivative pretreatment showed $\mathrm{R}_{\mathrm{V}}^{2}=0.928$, which improved from 0.881 , and is the result of the raw spectra in Figure 13a, and SEP decreased to \pm 0.134 , proving the effect of spectra pretreatment.

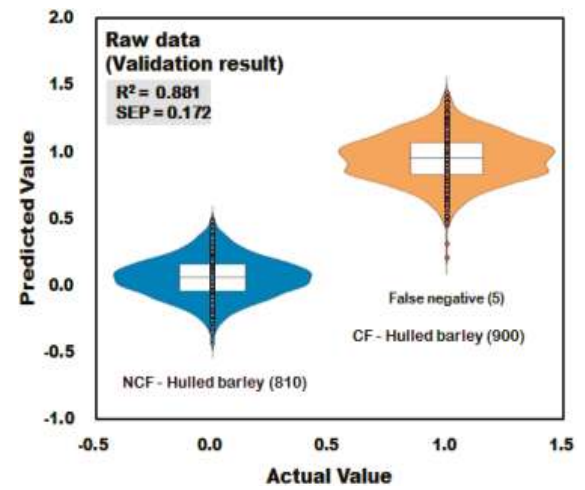

(a)

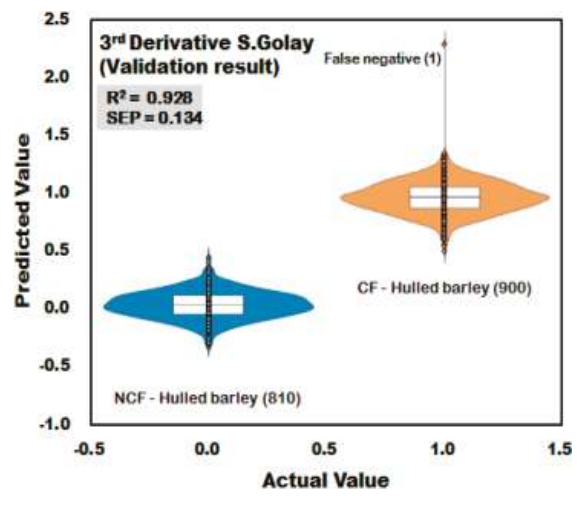

(b)

Figure 13. Validation results for Fusarium discrimination of the PLS-DA model developed using raw reflectance spectra (a) and the third-order derivative pretreatment (b) obtained from hulled barley.

\subsubsection{Prediction Result of PLS-DA Model for Discrimination of CF-Naked Barley}

Figure 14a shows the result of the PLS-DA model developed using the raw spectra acquired from naked barley samples. In the raw data in Figure 14a, one of the 894 spectra acquired from NCF-naked barley samples was predicted as higher than 0.5 , which is the reference value, resulting in a CCR of 99.89\% (893/894). In the CF-naked barley samples, three of the 198 spectra was predicted as lower than 0.5 , which is the reference value, resulting in a CCR of $98.48 \%(195 / 198)$. Figure $14 b$ shows the PLS-DA model developed by applying third-order derivative pretreatment to the raw spectra acquired from naked barley samples. Validation performance of NCF-naked barley and CF-naked barley showed $100 \%$ CCR. The validation model improved to $\mathrm{R}_{\mathrm{V}}{ }^{2}=0.948$ and decreased to SEP $= \pm 0.088$. 


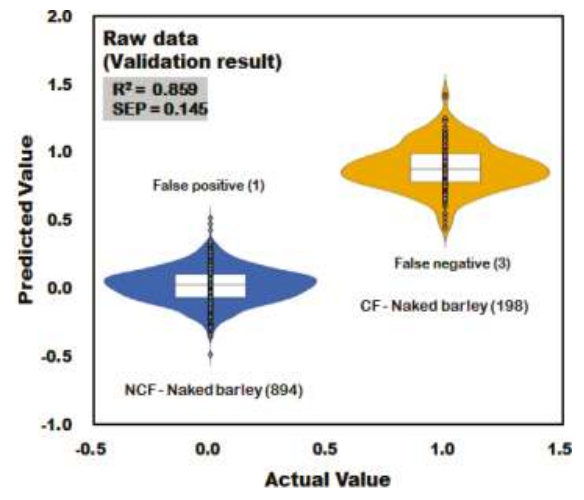

(a)

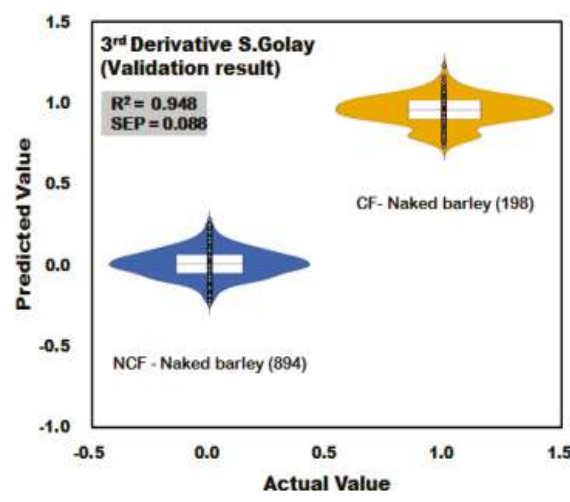

(b)

Figure 14. Validation results for Fusarium discrimination of the PLS-DA model developed using raw reflectance spectra (a) and the third-order derivative pretreatment (b) obtained from naked barley.

\subsubsection{Prediction Result of PLS-DA Model for Discrimination of CF-Wheat}

Figure 15a shows the result of validation model of PLS-DA developed using raw spectra acquired from wheat. In the developed validation model, one of the 576 spectra acquired from CF-wheat samples was predicted as higher than 1.5 , which is the reference value, resulting in a CCR of $99.83 \%$ $(575 / 576)$, and one of the 870 spectra acquired from the NCF wheat samples was predicted as lower than -0.5 , which is the reference value, resulting in a CCR of $99.66 \%(867 / 870)$. Figure $15 \mathrm{~b}$ shows the validation result of the PLS-DA model developed by applying second derivative pretreatment to raw spectra acquired from wheat samples. One of the 576 spectra acquired from the wheat samples contaminated with Fusarium was predicted as higher than 1.5, which is the reference value, resulting in a CCR of $99.83 \%(575 / 576)$. The $\mathrm{R}_{\mathrm{V}}^{2}$ of the validation model applying the second-order derivative pretreatment was 0.933 , which is higher than the result of the raw spectra, and the SEP decreased to 0.127 , proving the effect of the spectra pretreatment.

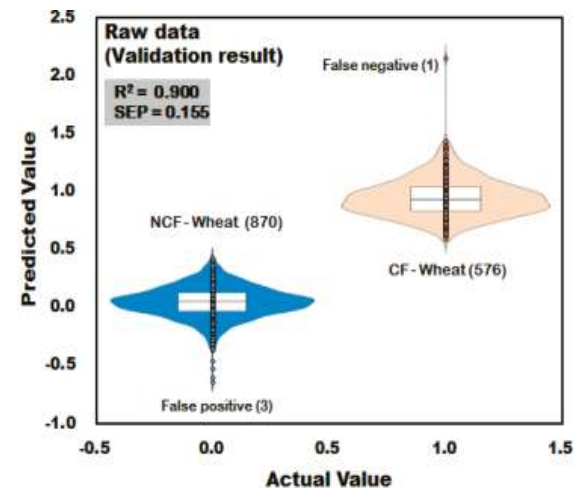

(a)

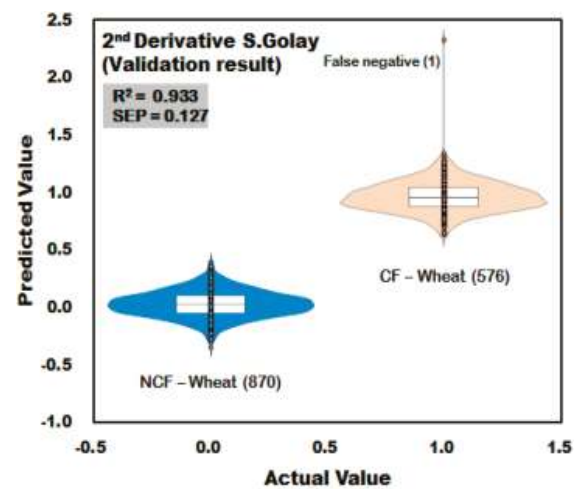

(b)

Figure 15. Validation results for the Fusarium discrimination of the PLS-DA model developed using raw reflectance spectra (a) and the second-order derivative pretreatment (b) obtained from wheat. 


\section{Conclusions and Outlook}

A technology to discriminate hulled barley, naked barley, and wheat contaminated with Fusarium rapidly and non-destructively was developed using near infrared spectroscopy technology. The PLS-DA models that included a first-order derivative, second-order derivative, or third-order pretreatment yielded an improved predictive discrimination accuracy for the infected grain samples $(>99.5 \%)$, compared with the method without a pretreatment. In particular, the naked barley discrimination accuracy was the best when compared with other grains. The PLS-DA model that was developed by applying derivative pretreatment to the acquired reflectance spectra was advantageous in the improvement of the correct classification rate. In our experiment, the use of reflectance spectra based on the NIRS technique has been proposed as an innovative and specific instrument for the discrimination of grains contaminated with fungus. The obtained results suggest the possible applications for discrimination of grains contaminated with other fungi similar to Fusarium. In the future, for the practical validation of the developed PLS-DA model, experiments to discriminate grains contaminated with Fusarium will be performed by adding grain samples produced from other regions or harvested in different years.

Acknowledgments: This study was carried out with the support of "Research Program for Agricultural Science \& Technology Development (Project No. PJ01096901)", National Institute of Agricultural Sciences, Rural Development Administration, Republic of Korea.

Author Contributions: Jongguk Lim and Seongmin Kim contributed to the conception of the study; Giyoung Kim and Changyeun Mo contributed significantly to theoretical analysis and manuscript preparation; Kyoungmin Oh and Hyeonheui Ham performed the test; Geonseob Kim and Moon S. Kim analyzed the data; All authors approved the final version.

Conflicts of Interest: The authors declare no conflict of interest.

\section{References}

1. Lee, K.H.; Lee, S.K.; Park, H.Y.; Sim, E.Y.; Woo, K.S.; Oh, S.K.; Lee, B.K.; Kim, H.J. Quality characteristics of barley Makgeolli prepared with different barley cultivars and milling recovery. Korean J. Food Preser. 2016, 23, 530-553.

2. Kalra, S.; Jood, S. Effect of dietary barley $\beta$-glucan on cholesterol and lipoprotein fractions in rat. J. Cereal Sci. 2000, 31, 141-145. [CrossRef]

3. Song, E.S.; Park, S.J.; Woo, N.R.A.; Won, M.H.; Cho, J.S.; Kim, J.G.; Kang, M.H. Antioxidant capacity of colored barley extracts by varieties. Korean J. Food Nutr. 2005, 34, 1491-1497.

4. Kim, Y.S.; Lee, Y.T.; Seong, H.M. Physicochemical properties of starches from waxy and non-waxy hull-less barleys. J. Korean Soc. Agric. Chem. Biotechnol. 1999, 42, 240-245.

5. Hyun, J.N.; Kweon, S.J.; Park, D.S.; Ko, J.M.; Han, S.I.; Lim, S.G.; Suh, S.J. A new high quality and yielding barley variety "Geungangbori" with lodging resistance. Korean J. Breed. Sci. 2008, 40, 474-478.

6. Seo, K.H.; Kim, Y.H.; Lee, Y.M.; Ghosh, M.; Park, K.M.; Park, D.H.; Kim, J.S.; Lim, B.O. Evaluation of Anti-Oxidant and Anti-Inflammatory Activities of Ganoderma lucidum Cultured on Hulled Barley. Korean J. Med. Crop Sci. 2017, 25, 29-36. [CrossRef]

7. Kim, J.Y.; Yi, Y.H. pH, acidity, color, amino acids, reducing sugars, total sugars, and alcohol in puffed millet powder containing millet Takju during fermentation. Korean J. Food Sci. Technol. 2010, 42, 727-732.

8. Shin, D.S.; Choi, Y.J.; Jeong, S.T.; Sim, E.Y.; Lee, S.K.; Kim, H.J.; Woo, K.S.; Kim, S.J.; Oh, S.K.; Park, H.Y. Quality Characteristics of Mixed Makgeolli with Barley and Wheat. Korean J. Food Nutr. 2016, 29, 565-572. [CrossRef]

9. McMullen, M.; Jones, R.; Gellenberg, D. Scab of wheat and barley: A re-emerging disease of devastating impact. Plant Dis. 1997, 81, 1340-1348. [CrossRef]

10. Chung, H.S. Cereal scab causing mycotoxicosis in Korea and present status of mycotoxin researches. Korean J. Mycol. 1975, 3, 31-36.

11. Marasas, W.F.O.; Kriek, N.P.J.; Fincham, J.E.; van Rensburg, S.J. Primary liver cancer and oesophageal basal cell hyperplasia in rats caused by Fusarium moniliforme. Int. J. Cancer 1984, 34, 383-387. [CrossRef] [PubMed] 
12. Delwiche, S.R. Classification of scab- and other mold-damaged wheat kernels by near-infrared reflectance spectroscopy. Trans. ASAE. 2003, 46, 731-738. [CrossRef]

13. Ren, Y.; Zhang, Y.; Shao, S.; Cai, Z.; Feng, L.; Pan, H.; Wang, Z. Simultaneous determination of multi-component mycotoxin contaminants in foods and feeds by ultra-performance liquid chromatography tandem mass spectrometry. J. Chromatogr. A 2007, 1143, 48-64. [CrossRef] [PubMed]

14. Trenholm, H.L.; Warner, R.M.; Prelusky, D.B. Assessment of extraction procedures in the analysis of naturally contaminated grain products for deoxynivalenol (vomitoxin). J. AOAC 1985, 68, 645-649.

15. Scott, P.M.; Kanhere, S.R.; Tarter, E.J. Determination of nivalenol and deoxynivalenol in cereals by electron-capture gas chromatography. J. AOAC 1986, 69, 889-893.

16. Tacke, B.K.; Casper, H.H. Determination of deoxynivalenol in wheat, barley, and malt by column cleanup and gas chromatography with electron capture detection. J. AOAC Int. 1996, 79, 472-475. [PubMed]

17. Scott, P.M.; Lau, P.Y.; Kanhere, S.R. Gas chromatography with electron capture and mass spectrometric detection of deoxynivalenol in wheat and other grains. J. AOAC 1981, 64, 1364-1371.

18. Usleber, E.; Schneider, E.; Märtlbauer, E.; Terplan, G. Two formats of enzyme immunoassay for 15-acetyldeoxynivalenol applied to wheat. J. Agric. Food Chem. 1993, 41, 2019-2023. [CrossRef]

19. Abramson, D.; Clear, R.M.; Usleber, E.; Gessler, R.; Nowicki, T.W.; Märtlbauer, E. Fusarium species and 8-keto-trichothecene mycotoxins in Manitoba barley. Cereal Chem. 1998, 75, 137-141. [CrossRef]

20. De Girolamo, A.; Cervellieri, S.; Visconti, A.; Pascale, M. Rapid Analysis of Deoxynivalenol in Durum Wheat by FT-NIR Spectroscopy. Toxins 2014, 6, 3129-3143. [CrossRef] [PubMed]

21. Hart, J.R.; Norris, K.H.; Golumbic, C. Determination of the moisture content of seeds by near-infrared spectrophotometry of their methanol extracts. Cereal Chem. 1962, 39, 94-99.

22. Wold, H. Path Models with Latent Variables: The NIPALS Approach. In Quantitative Sociology: International Perspectives on Mathematical and Statistical Modeling; Blalock, H.M., Aganbegian, A., Borodkin, F.M., Boudon, R., Capecchi, V., Eds.; Academic Press: New York, NY, USA, 1975; pp. 307-357.

23. Wold, S.; Sjostrom, M.; Eriksson, L. PLS-Regression: a Basic Tool of Chemometrics. Chemometr. Intell. Lab. 2001, 58, 109-130. [CrossRef]

24. Han, I.S.; Kim, M.; Lee, C.H.; Cha, W.; Ham, B.K.; Jeong, J.H.; Lee, H.; Chung, C.B.; Han, C. Application of Partial Least Squares Methods to a Terephthalic Acid Manufacturing Process for Product Quality Control. Korean J. Chem. Eng. 2003, 20, 977-984. [CrossRef]

25. Han, I.S.; Han, C. Modeling of Multistage Air-Compression Systems in Chemical Processes. Ind. Eng. Chem. Res. 2003, 42, 2209-2218. [CrossRef]

26. Han, I.S.; Han, C.; Chung, C.B. Melt Index Modeling with Support Vector Machines, Partial Least Squares, and Artificial Neural Networks. J. Appl. Polym. Sci. 2004, 95, 967-974. [CrossRef]

27. Lee, K.J.; Hruschka, W.R.; Abbott, J.A.; Noh, S.H.; Park, B.S. Predicting the soluble solids of apples by near infrared spectroscopy(II)-PLS and ANN moddels. J. Korean Soc. Agric. Mach. 1998, 23, 571-582.

28. Son, J.R.; Lee, K.J.; Kang, S.W.; Yang, G.M.; Seo, Y.W. Development of prediction model for sugar content of strawberry using NIR spectroscopy. Food Eng. Prog. 2009, 13, 297-301.

29. Alexandrakis, D.; Downey, G.; Scannell, A.G.M. Detection and identification of bacteria in an isolated system with near-infrared spectroscopy and multivariate analysis. J. Agric. Food Chem. 2008, 56, 3431-3437. [CrossRef] [PubMed]

30. Hwang, I.G. Development of On-Line Apple (Fuji) Sorting System by the Soluble Solid and Acid Contents Using VIS/NIR Spectroscopy. Ph.D. Thesis, Seoul National University, Seoul, Korea, 2000.

(C) 2018 by the authors. Licensee MDPI, Basel, Switzerland. This article is an open access article distributed under the terms and conditions of the Creative Commons Attribution (CC BY) license (http:/ / creativecommons.org/licenses/by/4.0/). 
Article

\title{
Evaluation of Apple Maturity with Two Types of Dielectric Probes
}

\author{
Marcin Kafarski ${ }^{1, *}$, Andrzej Wilczek ${ }^{1}$, Agnieszka Szypłowska ${ }^{1}$, Arkadiusz Lewandowski ${ }^{2}$, \\ Piotr Pieczywek ${ }^{1}$, Grzegorz Janik ${ }^{3}$ and Wojciech Skierucha ${ }^{1}$ \\ 1 Institute of Agrophysics, Polish Academy of Sciences, Doświadczalna 4, 20-290 Lublin, Poland; \\ a.wilczek@ipan.lublin.pl (A.W.); a.szyplowska@ipan.lublin.pl (A.S.); p.pieczywek@ipan.lublin.pl (P.P.); \\ w.skierucha@ipan.lublin.pl (W.S.) \\ 2 Institute of Electronic Systems, Warsaw University of Technology, Nowowiejska 15/19, 00-665 Warsaw, \\ Poland; a.lewandowski@elka.pw.edu.pl \\ 3 Institute of Enviromental Protection and Development, Wroclaw University of Environmental and Life \\ Sciences, Pl. Grunwaldzki 24, 50-363 Wrocław, Poland; grzegorz.janik@upwr.edu.pl \\ * Correspondence: m.kafarski@ipan.lublin.pl; Tel.: +48-81-7445061
}

Received: 26 September 2017; Accepted: 29 December 2017; Published: 4 January 2018

\begin{abstract}
The observed dielectric spectrum of ripe apples in the last period of shelf-life was analyzed using a multipole dielectric relaxation model, which assumes three active relaxation processes: primary $\alpha$-process (water relaxation) and two secondary processes caused by solid-water-ion interactions $\alpha^{\prime}$ (bound water relaxations), as well as $\beta^{\prime}$ (Maxwell-Wagner effect). The performance of two designs of the dielectric probe was compared: a classical coaxial open-ended probe (OE probe) and an open-ended probe with a prolonged central conductor in a form of an antenna (OE-A-probe). The OE-A probe increases the measurement volume and consequently extends the range of applications to other materials, like granulated agricultural products, soils, or liquid suspensions. However, its measurement frequency range is limited as compared to the OE probe because, above $1.5 \mathrm{GHz}$, the probe with the antenna generates higher propagation modes and the applied calibrations and calculations are not sufficient. It was shown that data from measurements using the OE-A probe gave slightly stronger correlations with apples' quality parameters than using the typical OE probe. Additionally, we have compared twelve multipole fitting models with different combinations of poles (eight three-pole and four two-pole models). It was shown that the best fit is obtained using a two-pole model for data collected for the OE-A probe and a three-pole model for the OE probe, using only Cole-Cole poles in both cases.
\end{abstract}

Keywords: dielectric probe; apple shelf-life; dielectric dispersion

\section{Introduction}

The interest in dielectric properties of agricultural products is driven by a practical need for the development of fast and non-destructive techniques for the measurement of vital quality properties such as moisture, chemical composition, structure, texture, etc. [1]. Direct determination of these properties of agricultural products is either difficult, laborious, expensive, or destructive to the samples [2]. Dielectric measurements that can be performed using a variety of time- and frequency-domain techniques proved to be fast, relatively inexpensive in comparison to chemical analyses, easily adaptable to automatic monitoring applications, and non-destructive or minimally invasive to the tested material [3-10]. Especially, water content is an example of a vital property that is easily measured using dielectric sensors $[4,6,11]$.

In the case of apples, there is a need to determine the optimal harvesting time and to monitor their quality during storage using non-destructive means. Microwave dielectric spectroscopy techniques 
could be potentially applied for this purpose, either alone or in combination with other non-destructive methods, such as biospeckle activity monitoring [12]. However, the development of such techniques requires the detailed knowledge of broadband dielectric properties of apples, the distribution of dielectric permittivity in the volume of an apple, and the determination of specific factors influencing the dielectric permittivity in given frequency ranges.

Broadband dielectric response of apples was examined by many researchers. Bhosale et al. [13] predicted the firmness of apples during shelf life using of a parallel plate sensor at low frequencies: $132,640,880 \mathrm{kHz}$. Guo et al. [14] measured 10-18,000 MHz dielectric spectrum of the external surface and the interior tissue of three apple cultivars at $24^{\circ} \mathrm{C}$ over 10 weeks of storage at $4{ }^{\circ} \mathrm{C}$ and correlated the results with quality factors of apples: soluble solids content (SSC), firmness, moisture content, and $\mathrm{pH}$. Although a high correlation was observed in a linear relationship between the dielectric constant divided by SSC and the dielectric loss factor divided by SSC in the complex plane, the SSC was not predicted well from that relationship, and no high correlations were found between the dielectric properties and SSC, moisture content, firmness, or $\mathrm{pH}$. The dielectric constant and loss factor remained essentially constant during the 10-week storage period. Castro-Giráldez et al. [15] developed a dielectric spectroscopy technique from $500 \mathrm{MHz}$ to $20 \mathrm{GHz}$ for the determination of apple (Granny Smith cultivar) maturity in the form of a newly-defined dielectric maturity index (MI) as the difference between the loss factors at the dipolar relaxation frequency and at $0.5 \mathrm{GHz}$. The choice of selected frequencies was used due the fact that apple maturation produces an increase in the sugar content (decreasing loss factor at relaxation frequency) and an increase in the malate content (increasing loss factor at $0.5 \mathrm{GHz}$ ). The authors of [16] tested dielectric properties of the external surface, internal tissue and the juice of Fuji apples during the last two months of tree-ripening. The apples were measured with an open-ended coaxial-line probe and a network analyzer at $24{ }^{\circ} \mathrm{C}$ from 0.01 to $4.5 \mathrm{GHz}$. No obvious correlations were found between permittivity and firmness, moisture content or $\mathrm{pH}$. They concluded that further studies were needed to assess the potential usefulness of dielectric properties for sensing the apple maturity or internal quality. A report on dielectric constants and loss factors of apples with skin and without skin, and flesh juice of the same Fuji apples cultivar, during 21-week storage at $5{ }^{\circ} \mathrm{C}$ and measured at a three-week interval and $24{ }^{\circ} \mathrm{C}$ from 0.01 to $4.5 \mathrm{GHz}$ was presented in [17]. The $\mathrm{pH}$ increased and firmness decreased with storage time. There was no obvious change or trend in the moisture content, SSC, conductivity, and permittivity during storage. The depths of penetration in skin-on and skin-off apples, and apple juice decreased with increased frequency. Weak correlation between the apple dielectric permittivity and the internal qualities and limited penetration depth shows that sensing apple internal qualities from permittivity of a skin-on apple, a skin-off apple and the apple juice is difficult. In conclusions the authors stressed weak correlations between permittivity and quality indices, which suggest that sensing the apple quality from their dielectric properties might not be practical under experimental conditions.

It seems that broadband dielectric measurement of apples is not sufficiently effective in the form of producing a reliable quality index, especially before and at early stage of maturity. The results presented in [15] are quite promising but the destructive measurements presented in [15] were performed in the senescence period of apples, in which they are losing their commercial value.

The objectives of research presented in this paper were to: (1) test the performance of two types of open-ended dielectric probes, with (OE-A) and without (OE) an antenna in the frequency range $10 \mathrm{MHz}-20 \mathrm{GHz}$ using finite element method (FEM) digital simulations, vector network analyzer (VNA) measurements of liquid reference materials (distilled water, ethanol, and methanol), and apples; and (2) test the change of dielectric properties of ripe apples at the time when they are starting to lose their commercial value and possibly determine the dielectric indicators of apples at the time of reaching the final stage of their commercial value by correlating the measured physicochemical parameters with the parameters of the multipole dielectric model.

The frequency range of $10 \mathrm{MHz}-20 \mathrm{GHz}$ was chosen to cover all frequency ranges presented in available literature and the $20 \mathrm{GHz}$ frequency limit was important for observing free water dielectric 
effect in apples. In other similar studies apples have never been measured using OE-A (only with a typical OE, coaxial-line probe).

\section{Materials and Methods}

\subsection{Apples and Reference Liquids}

The measurements were done in laboratory conditions with a controlled temperature of $20 \pm 1^{\circ} \mathrm{C}$. The air humidity was 50\% $\pm 10 \%$. The tested apples were of the Janagold Decosta variety. They were collected from Stryjno Sad cold store near Lublin, Poland, were they were kept in $2-4{ }^{\circ} \mathrm{C}$ before distribution to local grocery shops. The harvesting time of this variety was the beginning of October and the maximum storage time is four months in cold store conditions. Thus, the measurement time (end of January) was intentionally chosen at the end of their commercial value when they quickly become ripe in room conditions.

After dielectric measurements every apple from each series was tested to determine its firmness and crispness as well as the soluble solid content. Apple firmness (as the maximum force inserted to the apple flesh by the puncture probe) and crispness (as the number of acoustic events when puncturing an apple) were measured using two methods: standard puncture test and acoustic emission described in detail in [18]. Soluble solids content as the measure of sugars, organic and amino acids, and soluble pectins in fruit juice was measured for each punctured apple using a pocket digital refractometer (PAL-BX/RI by Atago CO., LTD., Tokyo, Japan). The refractometer provided measurements in the Brix scale with a high measurement accuracy of $\pm 0.1 \%$. (the refractive index measurement resolution -0.0001). Measuring procedure consisted of extraction of juice from apples parenchyma tissue followed by filtering through a paper filter. Small, $90 \mu \mathrm{L}$ doses of the filtered apple juice were dropped on the refractometer by use of an automatic pipette.

The reference values for the relative dielectric permittivity of water, ethanol and methanol used for the calibration of the sensors were calculated from the Cole-Cole formula [19]:

$$
\varepsilon_{r}^{*}=\varepsilon_{\infty}+\frac{\varepsilon_{S}-\varepsilon_{\infty}}{1+(j \omega \tau)^{1-\alpha}},
$$

where the purities and dielectric permittivity values of methanol, ethanol (Stanlab Sp. J., Lublin, Poland), distilled water at $20 \pm 1^{\circ} \mathrm{C}$ and the value of the $\alpha$-parameter are presented in Table 1.

Table 1. Purities and dielectric permittivity values of methanol, ethanol (Stanlab Sp. J., Poland) and distilled water at $20 \pm 1{ }^{\circ} \mathrm{C}$ used in calibration of the $\mathrm{OE}$ and $\mathrm{OE}-\mathrm{A}$ probes [19].

\begin{tabular}{cccccc}
\hline & Purity & $\mathcal{E}_{s}(-)$ & $\varepsilon_{\infty}(-)$ & $\tau(\mathrm{ps})$ & $\alpha(-)$ \\
\hline Water & Electrical conductivity $<2 \mu \mathrm{S} / \mathrm{cm}$ & 80.18 & 5.54 & 9.57 & 0.00 \\
Ethanol & Min. 99.7\% & 25.16 & 4.54 & 193.98 & 0.14 \\
Methanol & Min $99.8 \%$ & 33.65 & 5.65 & 56.36 & 0.00 \\
\hline
\end{tabular}

\subsection{Simulations and Measurement Method}

Two kinds of probes were used in this work: a coax OE probe and a coax OE-A (Figure 1a left and right, respectively). They were machined from the stainless steel. The dimensions (Figure $1 \mathrm{~b}$ ) were adjusted to have a $50 \Omega$ impedance line between the SMA connector Pasternack PE4403 (Pasternack Enterprises, Inc., Irvine, CA, USA) and the ending fixed in a bead made of epoxy-resin. The dielectric constant of the epoxy resin was $3.8 \pm 0.1$ and was measured by the Agilent impedance analyzer E4980A with the $16451 \mathrm{~B}$ dielectric test fixture.

The measurement setup consisted of a VNA (Anritsu VectorStar $10 \mathrm{MHz}-20 \mathrm{GHz}$ ), a set of calibration and verification materials (calibration-air, distilled water and methanol, verification-ethanol) and a measurement fixture with a balance that assured $0.1 \mathrm{~kg}$ accuracy, which 
gives in conversion $\pm 1 \mathrm{~N}$ force accuracy. The force put on the apple by the probe was equal to $10 \pm 1 \mathrm{~N}$. The whole setup was placed in a stable support frame as presented in Figure 1c.

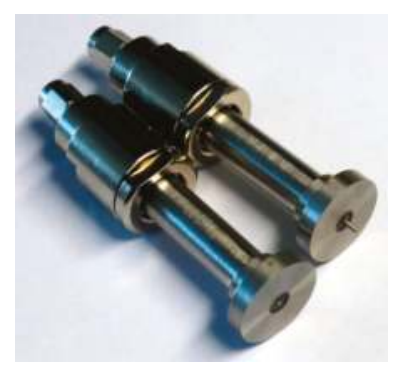

(a)

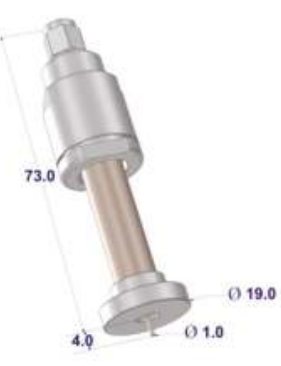

(b)

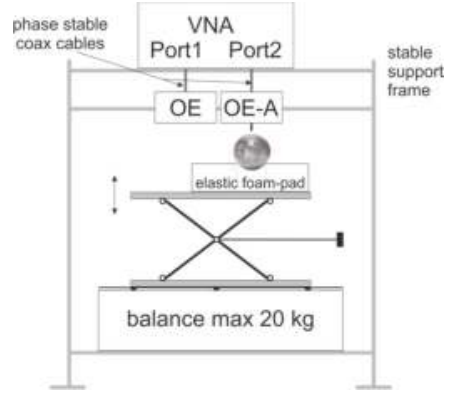

(c)

Figure 1. Tested coaxial probes and the elements of the measurement setup; (a) a coax OE probe (left) and a coax OE-A (right); (b) respective dimensions of the probes (in mm); and (c) experimental setup.

Before measurements, numerical simulations for distilled water were performed using EMPro digital simulation software pack from Keysight (formely Agilent) (Figure 2). The mesh is made automatically by EMPro which chooses the most optimal mesh density, therefore, the Nyquist criterion was fulfilled. Absorbing boundary conditions were applied in simulations. Simulation results proved that the electrical field penetration depth generated by the applied OE-A probe is bigger than for OE probe (Figure 2). Imaginary part of distilled water electrical permittivity and electrical conductivity are smaller as compared to apples. Therefore, similar simulations were also performed for materials with higher conductivity, up to $0.8 \mathrm{~S} / \mathrm{m}$. Obtained results showed that electric field penetration depth was practically the same as presented in Figure 2 for distilled water.

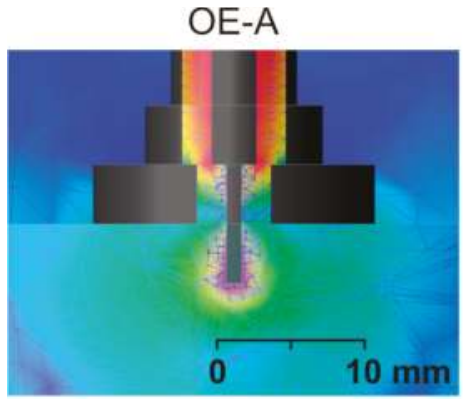

$0 \mathrm{~V} / \mathrm{m}$

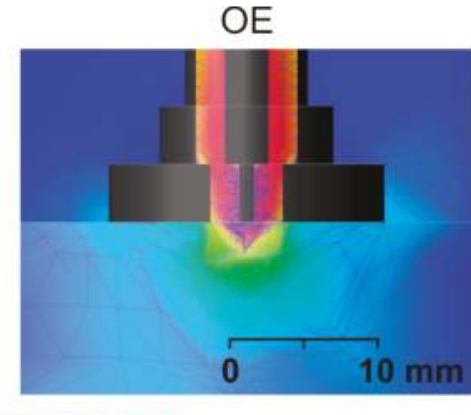

$200 \mathrm{~V} / \mathrm{m}$

Figure 2. EMPro FEM simulations of electrical field penetration depth in distilled water for the frequency of $1.5 \mathrm{GHz}$.

During the measurements the probes were connected to the VNA through phase-stable cables (Anritsu 3671KFKF50-60). Each apple was measured six times with each probe, paying special attention to have no air gap between the probe flat ending and the apple. This was sometimes not possible because of the apple shape. Apple is a solid material with a curved surface and that prevents a good contact of the OE probe [20]. Therefore, two from six measurements were eliminated automatically as 
outliners by a dedicated MATLAB procedure. The outliner elimination procedure tested only the real part of the dielectric permittivity at the arbitrary chosen frequency of $100 \mathrm{MHz}$, where a flat shape of the spectrum begins. Having $n$ curves, the outliner $i$ curve was selected by calculating $n$ standard deviations for the combination of $n$ sets, each without one elementary curve. The minimal standard deviation from the calculated $n$ values, indicated the curve that should be eliminated, leaving $n-1$ curves. The procedure was applied again to eliminate another outliner curve. The same procedure of outliers elimination was repeated to reduce the number of apples from 10 to 7 in each series to make the measurement series representative. Each frequency scan included 1998 points in a logarithmic scale from $10 \mathrm{MHz}-20 \mathrm{GHz}, 600$ points per decade. If the frequency was $300 \mathrm{~Hz}$, a single measurement was performed.

Totally there were 11 measurement series S00-S10, each with 10 apples. After the series S00-S04 performed on five successive days, the time distance between measurements was increased because no significant differences in dielectric spectra were found (see Figures 3-5).

The apples were measured with skin not removed so as to assure nondestructive measurements. This condition was not met in the case of the OE-A probe. The use of an OE-A probe involves the damage of the tested apples, but these tests give a good indication of the general quality of all the other apples. The measurement points were chosen in the middle height of an apple, between the stem and the blossom end.

\subsection{Calibration of the Dielectric Probes}

Before starting each measurement series, the coaxial probes were calibrated on air, water, and methanol according to the procedure described by Bao et al. [21]. This reference shows the proof that the S11 element of the scattering matrix measured at the input of VNA is related to the dielectric permittivity of the sample material at the tip of the OE probe in the form of the following bilinear equation:

$$
S_{11}=\frac{c_{2}+c_{3} \varepsilon_{r}^{*}}{c_{1}+\varepsilon_{r}^{*}}
$$

where the calibration constants $c_{1}, c_{2}$, and $c_{3}$ are complex numbers determined in the open-water-liquid (OWL) calibration [19] using air, distilled water, and methanol as the calibration media. The OWL calibration was chosen because reliable shorting the OE-A probe is rather difficult. Typical calibration of OE probe includes open-water-short (OWS) procedure, where shorting is done with the help of an indium foil [22].

Rewriting the Equation (2) for air, water and methanol gives:

$$
\left[\begin{array}{ccc}
S_{11}^{O} & -1 & -1 \\
S_{11}^{W} & -1 & -\varepsilon_{r, W}^{*} \\
S_{11}^{M} & -1 & -\varepsilon_{r, M}^{*}
\end{array}\right]\left[\begin{array}{c}
c_{1} \\
c_{2} \\
c_{2}
\end{array}\right]=\left[\begin{array}{c}
-S_{11}^{O} \\
-\varepsilon_{r, W}^{*} S_{11}^{W} \\
-\varepsilon_{r, M}^{*} S_{11}^{M}
\end{array}\right]
$$

where the respective superscripts $O, W, M$ stand for open (the measured material is air), distilled water, and methanol, the scattering parameters $S_{11}^{O}, S_{11}^{W}, S_{11}^{M}$ are measured by VNA when the OE or OE-A probe measures air, distilled water, or methanol, $\varepsilon_{r, W}^{*}$ and $\varepsilon_{r, M}^{*}$ represent complex dielectric permittivity of distilled water and methanol. The value of dielectric permittivity of air $\varepsilon_{r, O}^{*}=1$ and therefore the element in the first row of the rightmost vector of the Equation (3) is $-S_{11}^{O}$. The Equation (3) must be solved for each frequency in the scanned range $10 \mathrm{MHz}-20 \mathrm{GHz}$. The validation of the performed calibration was done with the dielectric measurements of ethanol. The calculated spectra of real and imaginary parts of ethanol dielectric permittivity were almost the same for the OE and OE-A probes in the frequency range $10 \mathrm{MHz}-1.5 \mathrm{GHz}$. The relative difference for the Debye parameters were smaller than $2 \%$. 
Having determined the parameters $c_{1}, c_{2}$, and $c_{3}$ from OWL calibration, the dielectric permittivity of the measured material $\varepsilon_{r}^{*}$ was calculated from the apple measured scattering parameter $S_{11}^{\text {apple }}$ using the rearranged form of the Equation (2):

$$
\varepsilon_{r}^{*}=\frac{c_{1} S_{11}^{\text {apple }}-c_{2}}{c_{3}-S_{11}^{a p p l e}}
$$

\subsection{Fitting Data to Multi-Pole Relaxation Model}

The spectrum of $\varepsilon_{r}^{*}$ was determined by fitting real and imaginary data calculated from Equation (4) to the three-pole relaxation model that includes the material conductivity $(\sigma)$ element:

$$
\begin{gathered}
\varepsilon_{r}^{*}=\varepsilon_{\infty}+\sum_{i=1}^{n} \varepsilon_{i}+\frac{\sigma}{j 2 \pi f \varepsilon_{0}} \\
\varepsilon_{i}=\frac{\Delta \varepsilon_{f i}}{1+\frac{j f}{f_{f i}}} \text { Debye type } \\
\varepsilon_{i}=\frac{\Delta \varepsilon_{f i}}{1+\left(\frac{i f}{f_{f i}}\right)^{\alpha}} \text { Cole }- \text { Cole type }
\end{gathered}
$$

where $\varepsilon_{\infty}$ is infinite relative permittivity; $\varepsilon_{0}\left(8.85 \times 10^{-12} \mathrm{~F} / \mathrm{m}\right), \sigma(\mathrm{S})$ stand for air absolute permittivity and low frequency electrical conductivity; $\Delta \varepsilon_{f i}$ and $f_{f i}$ are at each next pole $\Delta \varepsilon_{f w}, \Delta \varepsilon_{b w}$ and $\Delta \varepsilon_{M W}$ stand for the real part dielectric permittivity decrease resulting from the associated relaxation process at $f_{f w}$ (for free water), $f_{b w}$ (for bound water), or $f_{M W}$ (for Maxwell-Wagner interphase effects) frequency $(\mathrm{Hz}) ; f(\mathrm{~Hz})$ is the frequency of the applied electrical field. These values were determined by a nonlinear fitting procedure developed in MATLAB, firstly using the vector fitting approach [23] to determine the preliminary values of the Debye model parameters. Then, the preliminary values are refined using a full nonlinear least-squares fitting implemented with the MATLAB function lsqnonlin. The part: $\sum_{i=1}^{n} \varepsilon_{i}$ of Equation (5) consists of two or three poles. Each of them can be expressed by Debye (D) or Cole-Cole (CC) model, so there are eight different combinations for three-pole and 4 combinations for two-pole model. Therefore, $\varepsilon_{r}^{*}$ can be fitted by 12 different models.

The measurements were performed for the $\mathrm{OE}$ and OE-A probes in the range of $10 \mathrm{MHz}-20 \mathrm{GHz}$ and $10 \mathrm{MHz}-1.5 \mathrm{GHz}$, respectively. Two- and three-pole models were fitted to the measurements data obtained for both, OE and OE-A probes (Table 2).

Apple tissue is a heterogeneous material containing water, dissolved organic molecules, macromolecules, ions, and insoluble matter. The constituents are highly organized in cellular and subcellular structures forming macroscopic elements and soft and hard tissues. Their dielectric properties will, thus, reflect contributions to the polarization from both structure and composition [24].

\section{Results and Discussion}

\subsection{Dielectric Permittivity Spectra of Apples}

Figure $3 \mathrm{a}, \mathrm{b}$ presents example frequency spectra of $\varepsilon_{r}^{*}$ calculated from the Equation (4), for the OE and OE-A probes in the range of $10 \mathrm{MHz}-20 \mathrm{GHz}$ and $10 \mathrm{MHz}-1.5 \mathrm{GHz}$, respectively, each for six measurement points in the apple No. 7, series 10, of the real (upper part) and imaginary (lower part) of $\varepsilon_{r}^{*}$, for the OE and OE-A probes, respectively. Above $1.5 \mathrm{GHz}$ the OE-A probe generates higher propagation modes and the applied calibrations and calculations presented by the Equations (3) and (4) are not sufficient. 
S10, apple No. 7 - all 6 measured points

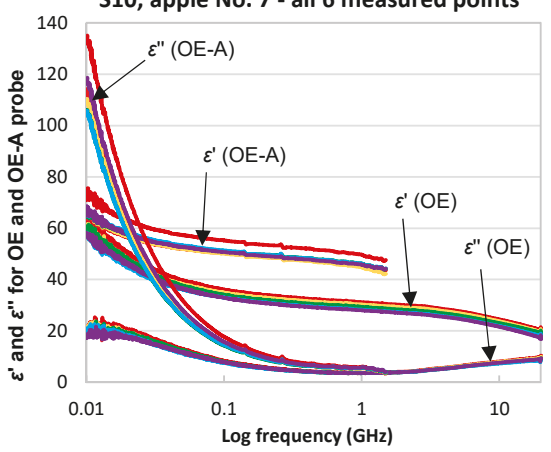

(a)

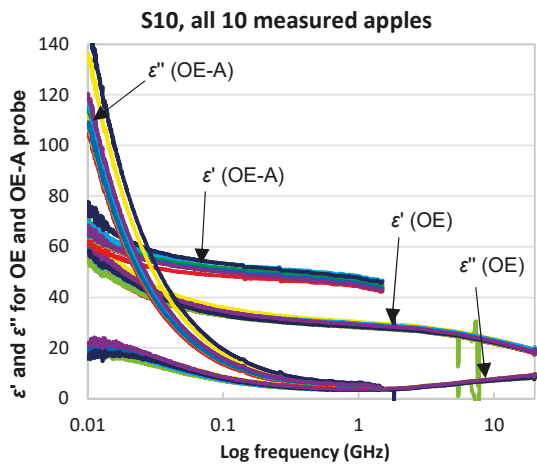

(c)

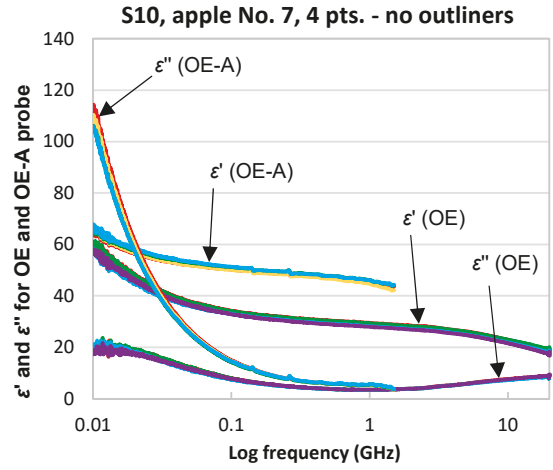

(b)

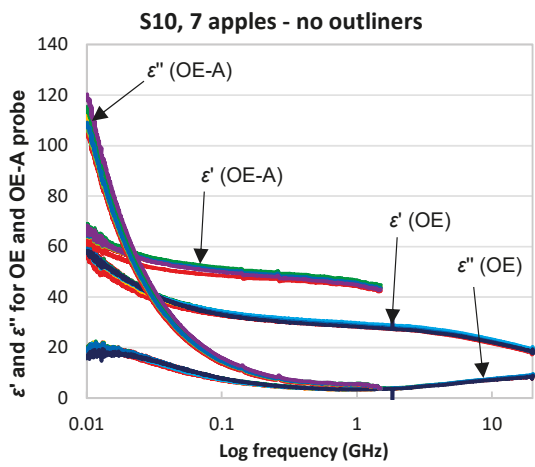

(d)

Figure 3. The scatter of data of recorded $\varepsilon_{r}^{*}$ spectra for the measurement series $\mathrm{S} 10$ collected with the $\mathrm{OE}$ and the OE-A probes, and the results of the applied elimination of outliers: $(\mathbf{a}, \mathbf{b})$ for the apple 7 in series S10; (c,d) for all apples in series S10.

Generally, it was found that, for each measurement point of an individual apple, the spectra of the dielectric permittivity differed, although the OE and OE-A probes were pressed against the apple with the same force and great care was taken to keep the flat ending of the OE and OE-A probes in parallel to the apple surface, which was not flat. The discrepancies were probably due to individual differences between apples, and an imperfect flat interface between the flat sensor and the spherical shape the apple. Therefore, two of six measurement points at each measured apple were eliminated as outlier points to minimize the measurement error.

Having four selected frequency spectra of $\varepsilon_{r}^{*}$ for each apple, the mean value of them was calculated taking ten mean frequency spectra for each measurement series S00-S10, each one for an individual apple in a series. A similar outlier elimination procedure was applied for ten apples in each series so as to finally have seven apples. The outlier elimination procedure decreased the standard error of measurement at least by a factor of 2 .

The differences between real (and imaginary) parts of the spectra measured with the OE and OE-A probes, presented in Figure 3, are caused by an apples' structure. The skin and flesh of apples have different electric parameters and the apple is not a homogenous material (in contrast to, e.g., ethanol). The apples were measured with skin not removed. The OE-A probe has a larger measurement volume than the OE probe. Therefore, the electric field in the case of both probes penetrate the outer layer of an apple (the skin) and flesh of the apple, but the OE-A probe has an antenna which is inserted into the flesh and the volume penetration ratio flesh/skin is larger for the OE-A probe than the OE probe. 
This explains such differences in the measured permittivity spectra. However, the OE-A probe had the upper frequency limit of $1.5 \mathrm{GHz}$ as compared to $20 \mathrm{GHz}$ for the OE probe.

\subsection{Multipole Model Fitting Comparison}

The multipole model fitting was performed for apples in series S00-S10. Data collected using the OE and OE-A probes were fitted by two- and three-pole models. Table 2 presents the root-mean-square-errors (RMSE) of multipole data fitting procedure for S10 apple No. 7.

Table 2. RMSE for two- and three-pole fit procedure for data collected using the OE and OE-A probes.

\begin{tabular}{cccccc}
\hline \multirow{2}{*}{ 3-Pole Model } & \multicolumn{3}{c}{ RMSE } & \multirow{2}{*}{ 2-Pole Model } & \multicolumn{2}{c}{ RMSE } \\
\cline { 2 - 3 } \cline { 5 - 6 } & OE & OE-A & & OE & OE-A \\
\hline D-D-D & 0.3489 & 0.1654 & D-D & 0.8876 & 0.2984 \\
CC-D-D & 0.1143 & 0.3241 & CC-D & 0.4047 & 0.2071 \\
D-CC-D & 0.1810 & 0.8363 & D-CC & 0.3733 & 0.1808 \\
D-D-CC & 0.1835 & 0.6360 & CC-CC & 0.1281 & 0.1796 \\
CC-CC-D & 0.0967 & 0.1942 & & & \\
CC-D-CC & 0.0955 & 0.2271 & & & \\
D-CC-CC & 0.1295 & 0.2642 & & & \\
CC-CC-CC & 0.0951 & 0.1894 & & & \\
\hline
\end{tabular}

In any case the best fit was obtained for models with Cole-Cole poles and the worst with Debye poles (Table 2). Only for OE-A data fitted by the three-pole model the worst fit was obtained using D-CC-D and the best for D-D-D model, but CC-CC-CC also gives very good results. The sequence of poles showed in Table 2 corresponds to $f_{f w}, f_{b w}, f_{M W}$, from left to right, for the three-pole model and $f_{b w}, f_{M W}$ for the two-pole model.

It occurred that the three-pole models gave better fit for OE probe that the two-pole models, while, for the OE-A probe, the two-pole models were generally better. This corresponds to the fact that, in the case of the OE-A probe, the limited frequency range did not enable an accurate fitting of the high-frequency free-water relaxation pole.

Figures $4 \mathrm{a}, \mathrm{b}$ and $5 \mathrm{a}, \mathrm{b}$ present the best and the worst two- and three-pole model data fitting for data collected using the $\mathrm{OE}$ and $\mathrm{OE}-\mathrm{A}$ probes, respectively.

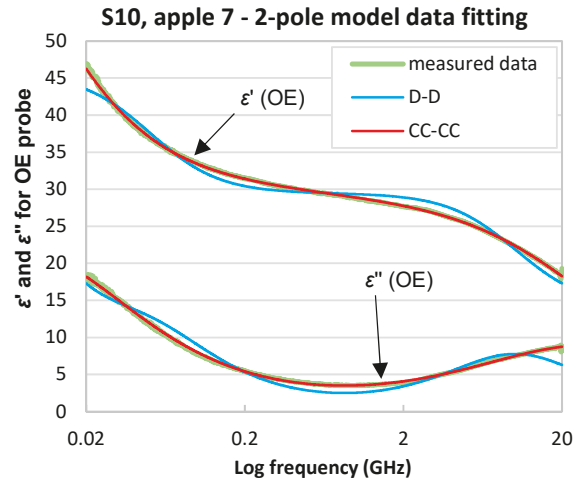

(a)

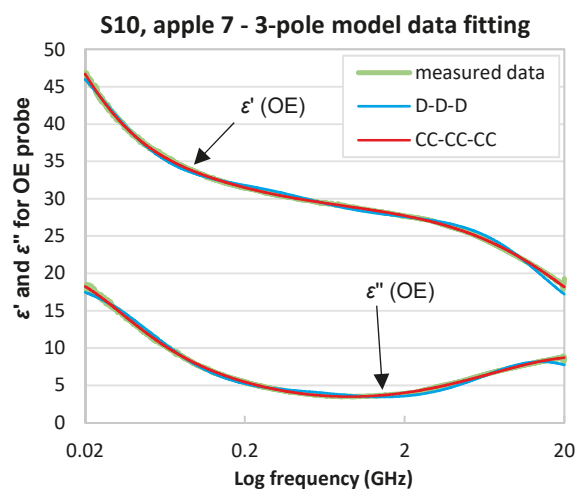

(b)

Figure 4. Results of the best and the worst data fitting of dielectric spectra of $\varepsilon_{r}^{*}$ collected with the OE probe for series S10, apple No. 7 for; (a) two-pole and (b) three-pole models. 
S10, apple 7 - 2-pole model data fitting

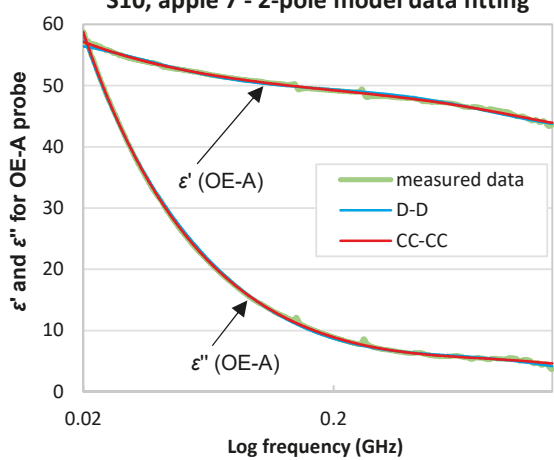

(a)

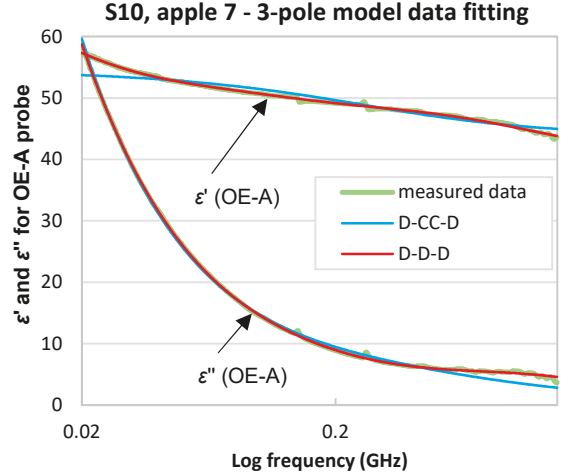

(b)

Figure 5. Results of the best and the worst data fitting of dielectric spectra of $\varepsilon_{r}^{*}$ collected with the OE-A probe for series S10, apple No. 7 for; (a) two-pole and (b) three-pole models.

\subsection{Relations between Quality and Multipole Relaxation Model Fitting Parameters}

There was no correlation found for the OE probe between the apple shelf-life and the parameters of the multipole model described by Equation (5) with three dispersion effects described by the Cole-Cole model (Figure 6). This is probably due to the presence of apple skin, which masks the apple-flesh properties that are most important for the shelf-life period. This is confirmed by slightly better correlations received for the OE-A probe, in the case of electrical conductivity $\sigma$, bound water relaxation frequency $f_{b w}$, and high-frequency dielectric parameter $\varepsilon_{\infty}$ (Figure 7). The respective multipole relaxation model included only two dispersion effects (as the result of $1.5 \mathrm{GHz}$ frequency limit for the OE-A probe) caused by the bound water and the interphase polarization (Maxwell-Wagner effects).

The temporal variability of apple physiochemical parameters in each series is presented in Figure 8. No correlation was found for soluble solids content and the shelf-life, but the apple firmness measured by the puncture and acoustic emission methods showed good correlations $\left(R^{2}>0.8\right)$.

Correlations between dielectric spectrum parameters measured with OE-A probe and shelf-life is better than for the OE probe (Figures 6 and 7). The exceptions are the $\Delta \varepsilon_{\mathrm{bw}}$ and $\Delta \varepsilon_{\mathrm{MW}}$ parameters, which show better correlations for measurements performed using the $\mathrm{OE}$ probe. Figure 8 presents quite good correlations of firmness and acoustic events with shelf-life, with $R^{2}=0.96$ and $R^{2}=0.8$, respectively. Correlation between soluble solid content and shelf-life is much worse $\left(R^{2}<0.1\right)$. Taking into account poor correlations between parameters of the multipole dielectric dispersion model, apple quality parameters, and shelf-life (small $R^{2}$ ), finding a good correlation between dielectric properties and quality parameters is difficult. Figure 9 presents correlations of $\sigma, f_{f w}$, and $f_{M W}$ with firmness. As can be seen, the most reliable correlation can be found for low-frequency electrical conductivity $\sigma$ and firmness, where the second-degree polynomial is fitted with $R^{2} \cong 0.7$. 

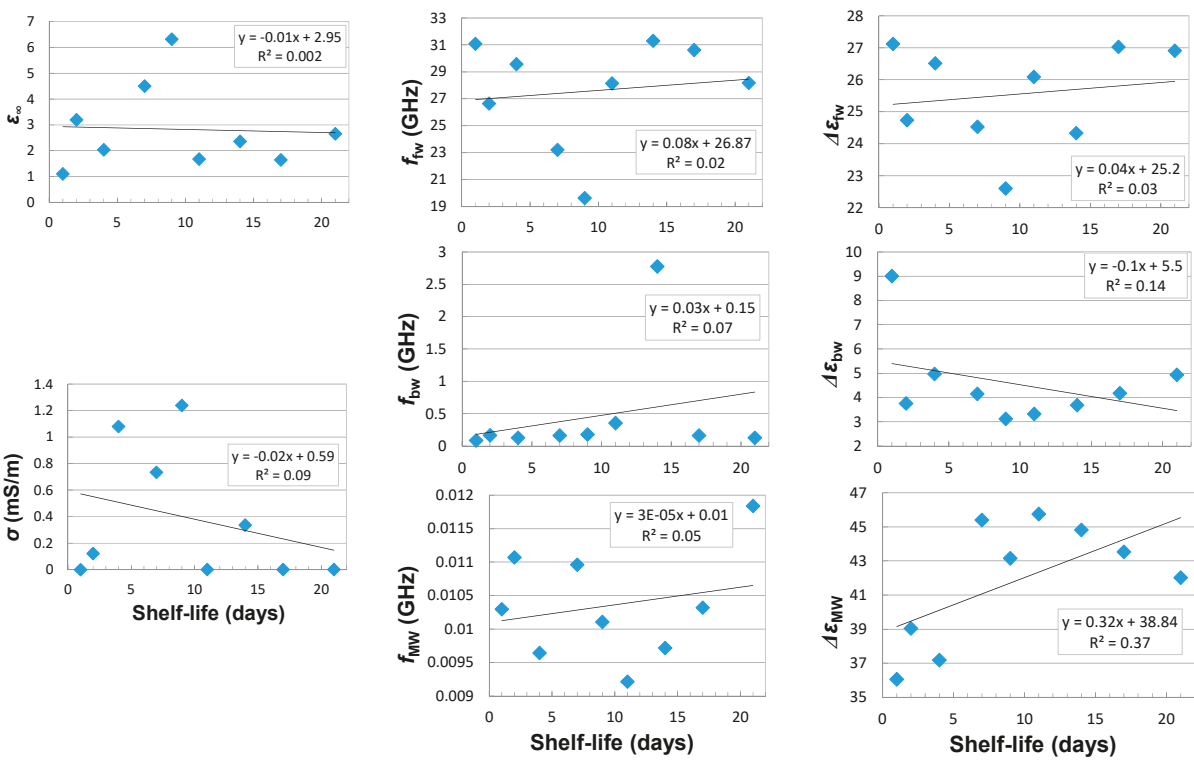

Figure 6. Correlation between the parameters of CC-CC-CC multipole relaxation model and the shelf-life of the tested apples determined by the OE probe (assuming the influence of electrical conductivity $\sigma(\mathrm{mS} / \mathrm{m})$ and three dielectric dispersion effects from Maxwel-Wagner- $M W$, bound water- $b w$ and free water- $f w$ ).
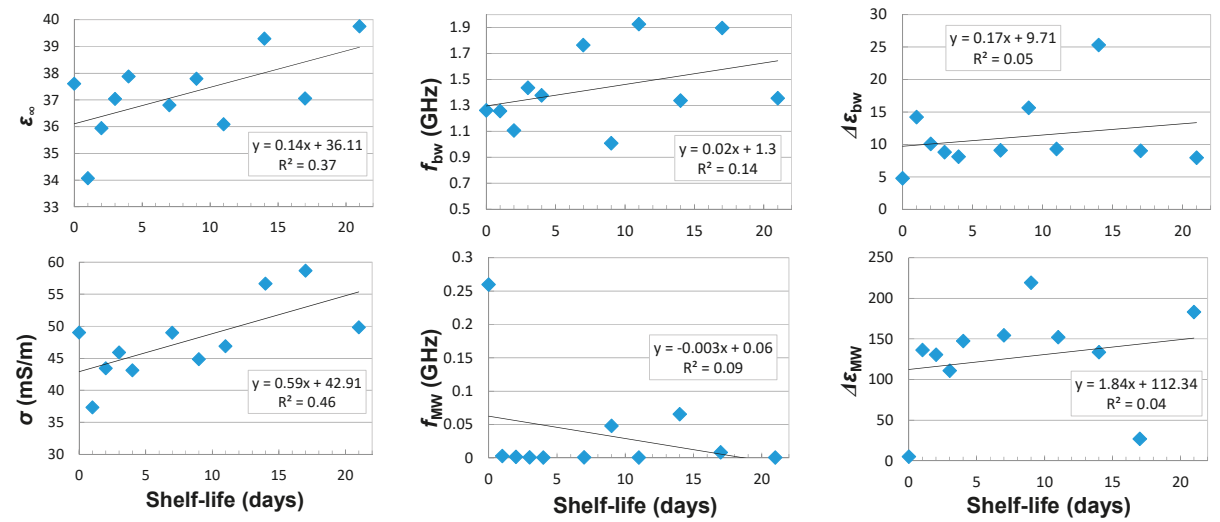

Figure 7. Correlation between the parameters of the multipole model and the shelf-life of the tested apples determined by the OE-A probe (assuming the influence of electrical conductivity $\sigma(\mathrm{mS} / \mathrm{m})$ and two dielectric dispersion effects from Maxwel-Wagner- $M W$ and bound water- $b w$ ). 

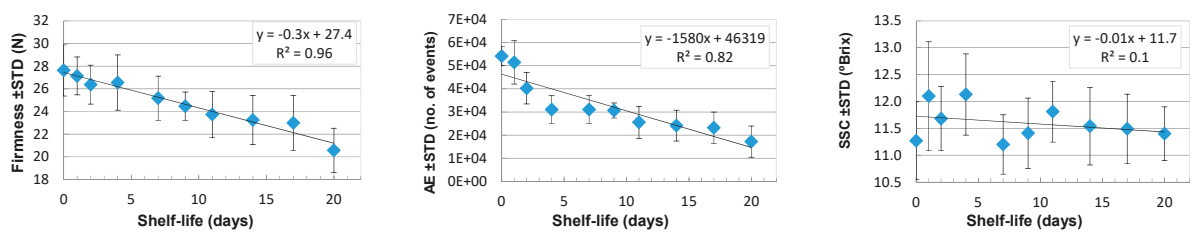

Figure 8. Mean values of firmness - left, acoustic events (AE) - middle, and soluble solids content (SSC) — right variability in apples in each test during the 21-day long shelf-life.
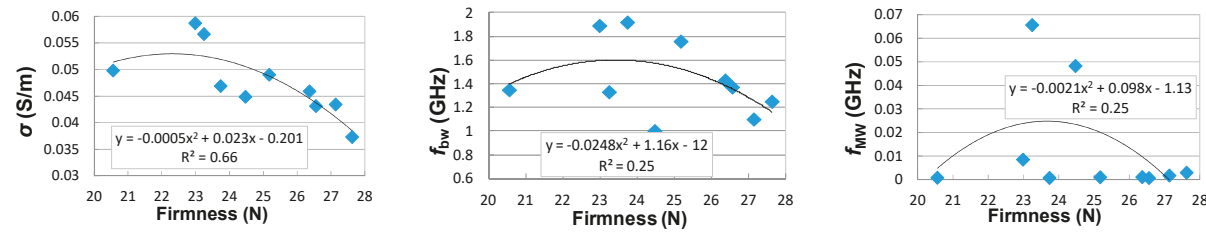

Figure 9. Relation between firmness of the tested apples and selected multipole model parameters (low frequency electrical conductivity $\sigma(\mathrm{mS} / \mathrm{m})$-left, relaxation frequency of bound water effect $b w$ — middle and relaxation frequency of Maxwell-Wagner effect $M W$-right) for $\varepsilon_{r}^{*}$ measured with the OE-A dielectric probe.

\section{Conclusions}

Due to the diversity of apples in a harvested bunch it was necessary to eliminate outliers, i.e., eliminate measurement points in individual apples with outlying results and also eliminate apples with outlying mean-measurements in each measurements series. Outlier elimination decreased the standard deviation of measurement by about two times in each case.

Two- and three-pole data fitting models with different combinations of poles were tested. The best fitting results were obtained using two- and three-pole Cole-Cole models for OE-A and OE probes, respectively.

The dielectric spectrum parameters measured with the probe with an antenna showed quite good correlation of the multipole model parameters with the apples' shelf-life quality parameters, for example, firmness, unlike the spectrum parameters measured with the OE probe. This may have been caused by the greater penetration depth of the electric field in the apples. Especially, the low-frequency conductivity was strongly negatively correlated with the firmness of the apples. However, the $\mathrm{OE}$ probe measures apples with the skin undisturbed and the lack of clear correlations with shelf-life parameters proves that the skin has the greatest influence on the apple dielectric response and has practically the same dielectric properties during apples' shelf-life.

The performed dielectric measurements of the apples confirmed earlier reports that the tests should be conducted on fruits without skin. It seemed that the temporal variability of 21 days was too short to report distinct variability of apples' dielectric permittivity parameters, using nondestructive methods, during the process of their maturity.

Acknowledgments: The presented work was supported by the National Centre for Research and Development, Poland, in the scope of the research project no. PBS1/A9/12/2012.

Author Contributions: M.K. and W.S. conceived and designed the experiments; A.S., M.K. and P.P. performed the experiments; A.W., G.J. and A.L. prepared calculation procedures in MATLAB and analyzed the data; and M.K. and A.S. wrote the paper.

Conflicts of Interest: The authors declare no conflict of interest. 


$\begin{array}{ll}\text { Abbreviations } \\ \text { OE } & \text { Open-ended } \\ \text { OE-A } & \text { Open-ended with antenna } \\ \text { D } & \text { Debye } \\ \text { CC } & \text { Cole-Cole } \\ \text { SSC } & \text { Soluble solid content } \\ \text { AE } & \text { Acoustic events } \\ \text { STD } & \text { Standard deviation } \\ \text { FEM } & \text { Finite Element Method } \\ \text { RMSE } & \text { Root-Mean-Square Error } \\ \text { VNA } & \text { Vector Network Analyzer } \\ \text { OWL } & \text { Open-Water-Liquid calibration } \\ \text { OWS } & \text { Open-Water-Short calibration } \\ \text { fw } & \text { Free water } \\ \text { bw } & \text { Bound water } \\ \text { MW } & \text { Maxwell-Wagner }\end{array}$

\section{References}

1. Khaled, D.E.; Castellano, N.N.; Gazquez, J.A.; Salvador, R.G.; Manzano-Agugliaro, F. Cleaner quality control system using bioimpedance methods: A review for fruits and vegetables. J. Clean. Prod. 2017, 140, 1749-1762. [CrossRef]

2. Khaled, D.E.; Novas, N.; Gazquez, J.A.; Garcia, R.M.; Manzano-Agugliaro, F. Fruit and vegetable quality assessment via dielectric sensing. Sensors 2015, 15, 15363-15397. [CrossRef] [PubMed]

3. Sosa-Morales, M.E.; Valerio-Junco, L.; López-Malo, A.; García, H.S. Dielectric properties of foods: Reported data in the 21st Century and their potential applications. LWT Food Sci. Technol. 2010, 43, 1169-1179. [CrossRef]

4. Skierucha, W.; Wilczek, A.; Szypłowska, A. Dielectric spectroscopy in agrophysics. Int. Agrophys. 2012, 26, 187-197. [CrossRef]

5. Trabelsi, S.; Nelson, S.O. Microwave Sensing of Quality Attributes of Agricultural and Food Products. IEEE Instrum. Meas. Mag. 2016, 19, 36-41. [CrossRef]

6. Skierucha, W.; Wilczek, A.; Szypłowska, A.; Kafarski, M.; Lewandowski, A.; Pieczywek, P. Apple Broadband Dielectric Response. In Proceedings of the 11th International Conference on Electromagnetic Wave Interaction with Water and Moist Substances, ISEMA 2016, Florence, Italy, 23-27 May 2016; pp. 351-358.

7. Nelson, S.O. Dielectric spectroscopy in agriculture. J. Non-Cryst. Solids 2005, 351, 2940-2944. [CrossRef]

8. El Khaled, D.; Castellano, N.; Gázquez, J.; Perea-Moreno, A.-J.; Manzano-Agugliaro, F. Dielectric Spectroscopy in Biomaterials: Agrophysics. Materials 2016, 9, 310. [CrossRef] [PubMed]

9. Szypłowska, A.; Nakonieczna, A.; Wilczek, A.; Paszkowski, B.; Solecki, G.; Skierucha, W. Application of a Coaxial-Like Sensor for Impedance Spectroscopy Measurements of Selected Low-Conductivity Liquids. Sensors 2013, 13, 13301-13317. [CrossRef] [PubMed]

10. Paszkowski, B.; Wilczek, A.; Szypłowska, A.; Nakonieczna, A.; Skierucha, W. A low-frequency sensor for determination of honey electrical properties in varying temperature conditions. J. Food Eng. 2014, 138, 17-22. [CrossRef]

11. Kraszewski, A. Microwave Aquametry: An Effective Tool for Nondestructive Moisture Sensing. Subsurf. Sens. Technol. Appl. 2001, 2, 347-362. [CrossRef]

12. Skic, A.; Szymańska-Chargot, M.; Kruk, B.; Chylińska, M.; Pieczywek, P.M.; Kurenda, A.; Zdunek, A.; Rutkowski, K.P. Determination of the Optimum Harvest Window for Apples Using the Non-Destructive Biospeckle Method. Sensors 2016, 16, 661. [CrossRef] [PubMed]

13. Bhosale, A.A.; Sundaram, K.K. Firmness prediction of the apple using capacitance measurement. Procedia Technol. 2014, 12, 163-167. [CrossRef]

14. Guo, W.; Nelson, S.O.; Trabelsi, S.; Kays, S.J. 10-1800-MHz dielectric properties of fresh apples during storage. J. Food Eng. 2007, 83, 562-569. [CrossRef] 
15. Castro-Giráldez, M.; Fito, P.J.; Chenoll, C. Development of a dielectric spectroscopy technique for the determination of apple (Granny Smith) maturity. Innov. Food Sci. Emerg. Technol. 2010, 11, 749-754. [CrossRef]

16. Guo, W.; Zhu, X.; Nelson, S.O.; Yue, R.; Liu, H.; Liu, Y. Maturity effects on dielectric properties of apples from 10 to $4500 \mathrm{MHz}$. LWT Food Sci. Technol. 2011, 44, 224-230. [CrossRef]

17. Guo, W.; Zhu, X.; Yue, R.; Liu, H.; Liu, Y. Dielectric properties of Fuji apples from 10 to $4500 \mathrm{MHz}$ during storage. J. Food Process. Preserv. 2011, 35, 884-890. [CrossRef]

18. Zdunek, A.; Konopacka, D.; Jesionkowska, K. Crispness and crunchiness judgment of apples based on contact acoustic emission. J. Texture Stud. 2010, 41, 75-91. [CrossRef]

19. Wagner, N.; Schwing, M.; Scheuermann, A. Numerical 3-D FEM and Experimental Analysis of the Open-Ended Coaxial Line Technique for Microwave Dielectric Spectroscopy on Soil. IEEE Trans. Geosci. Remote Sens. 2014, 52, 880-893. [CrossRef]

20. Olmi, R.; Bini, M.; Ignesti, A.; Riminesi, C. Non-destructive permittivity measurement of solid materials. Meas. Sci. Technol. 2000, 11, 1623-1629. [CrossRef]

21. Bao, J.Z.; Swicord, M.L.; Davis, C.C. Microwave dielectric characterization of binary mixtures of water, methanol, and ethanol. J. Chem. Phys. 1996, 104, 4441-4450. [CrossRef]

22. Bobowski, J.S.; Johnson, T. Permittivity measurements of biological samples by an open-ended coaxial line. Prog. Electromagn. Res. 2012, 40, 159-183. [CrossRef]

23. Gustavsen, B.; Semlyen, A. Rational approximation of frequency domain responses by vector fitting. IEEE Trans. Power Deliv. 1994, 14, 1052-1061. [CrossRef]

24. Gabriel, C. Dielectric properties of biological materials. In Bioengineering and Biological Aspects of Electromagnetic Fields, 3rd ed.; Barnes, F.S., Greenebaum, B., Eds.; CRC Press: Boca Raton, FL, USA, 2006; pp. 51-100, ISBN 0849395399.

(C) 2018 by the authors. Licensee MDPI, Basel, Switzerland. This article is an open access article distributed under the terms and conditions of the Creative Commons Attribution (CC BY) license (http:/ / creativecommons.org/licenses/by/4.0/). 


\title{
Plant Pest Detection Using an Artificial Nose System: A Review
}

\author{
Shaoqing Cui ${ }^{1}$, Peter Ling ${ }^{1, *}$, Heping Zhu ${ }^{2}$ and Harold M. Keener ${ }^{1}$ \\ 1 Department of Food, Agricultural and Biological Engineering, The Ohio State University/Ohio Agricultural \\ Research and Development Center, 1680 Madison Ave, Wooster, OH 44691-4096, USA; \\ cui.411@osu.edu (S.C.); keener.3@osu.edu (H.M.K.) \\ 2 United States Department of Agriculture-Agricultural Research Service (USDA-ARS) Application \\ Technology Research Unit, 1680 Madison Ave, Wooster, OH 44691-4096, USA; heping.zhu@ars.usda.gov \\ * Correspondence: ling.23@osu.edu; Tel.: +330-263-3857
}

Received: 10 November 2017; Accepted: 24 January 2018; Published: 28 January 2018

\begin{abstract}
This paper reviews artificial intelligent noses (or electronic noses) as a fast and noninvasive approach for the diagnosis of insects and diseases that attack vegetables and fruit trees. The particular focus is on bacterial, fungal, and viral infections, and insect damage. Volatile organic compounds (VOCs) emitted from plants, which provide functional information about the plant's growth, defense, and health status, allow for the possibility of using noninvasive detection to monitor plants status. Electronic noses are comprised of a sensor array, signal conditioning circuit, and pattern recognition algorithms. Compared with traditional gas chromatography-mass spectrometry (GC-MS) techniques, electronic noses are noninvasive and can be a rapid, cost-effective option for several applications. However, using electronic noses for plant pest diagnosis is still in its early stages, and there are challenges regarding sensor performance, sampling and detection in open areas, and scaling up measurements. This review paper introduces each element of electronic nose systems, especially commonly used sensors and pattern recognition methods, along with their advantages and limitations. It includes a comprehensive comparison and summary of applications, possible challenges, and potential improvements of electronic nose systems for different plant pest diagnoses.
\end{abstract}

Keywords: electronic nose; pest scouting; pest management; gas sensor; noninvasive detection

\section{Introduction}

Reliable disease and pest diagnosis in the early stages of vegetable and fruit production is highly desirable to reduce major production and economic losses. The main purpose of plant pest diagnosis is to assess whether a plant is healthy and to determine the causes of a disorder, if any. However, one major challenge is the difficulty in determining the physical, chemical, and biological changes in plants during the asymptomatic stages of an infection. Another challenge lies in the difficulty of performing the task timely and economically.

To address these challenges, diverse methods or technologies have been developed, which can be divided into two methods: direct and indirect. Direct detection methods include molecular technologies, including polymerase chain reaction (PCR), fluorescence in-situ hybridization (FISH) and serological technologies such as enzyme-linked immunosorbent assay (ELISA) [1-3]. Meanwhile, typical indirect methods detect morphological changes, transpiration rate changes and volatile organic compounds (VOCs) profiles, which correspond to the technologies of fluorescence imaging, hyperspectral techniques and gas chromatography-mass spectrometry (GC-MS) [4-6]. Besides, specific biosensors, such as antibody-based biosensors, DNA/RNA-based affinity biosensors and enzymatic electrochemical biosensors have been developed based on bio-recognition. DNA-based and serological methods are the most available and essential direct detection tools for accurate plant disease diagnosis, 
providing "standard methods" for fungal detection [7]. However, as discussed in other review papers, they are not very reliable at early (asymptomatic) stages and require at least 1-2 days for sample harvest, processing and analysis [8,9]. Thus, there is a need for rapid, reliable diagnostic methods that can be used in the field for crop disease detection at asymptomatic stages. Indirect methods that rely on imaging techniques and VOCs profiles released from infested crops have the potential to address this need. For example, hyperspectral image techniques utilized in both field and greenhouse grown plants for early detection of stress have shown satisfactory classification accuracy. However, some modifications and improvements, such as instantaneous results, are still needed [7]. Biosensors using phage display and bio-photonics have been reported to instantaneously detect infections but still require modifications, improvements and proper validation before being used in the field. In the past three years, the principles behind these technologies, as well as their advantages and disadvantages, have been thoroughly discussed in several review papers and numerous original research papers; thus, this review emphasizes novel sensors and techniques based on VOCs profiles, which are promising technologies for reliable, non-destructive and real-time plant disease monitoring and management [10-12]. The advantages and disadvantages of the aforementioned technologies are discussed in Table 1 [13-26].

Table 1. Comparison of the typical technologies for plant disease detection.

\begin{tabular}{|c|c|c|c|}
\hline Techniques & Advantages & Disadvantages & Refs. \\
\hline PCR & $\begin{array}{l}\text { Mature technology, easy } \\
\text { to operate and portable }\end{array}$ & $\begin{array}{l}\text { Subjected to DNA } \\
\text { extraction, and inhibitors } \\
\text { and polymerase activity }\end{array}$ & {$[13,14]$} \\
\hline FISH & Highly sensitive & Auto-fluorescence & {$[15-17]$} \\
\hline ELISH & $\begin{array}{l}\text { Low-cost, rapid and } \\
\text { visible results }\end{array}$ & $\begin{array}{l}\text { Low-sensitivity to } \\
\text { bacteria }\end{array}$ & [18] \\
\hline Fluorescence imaging & $\begin{array}{l}\text { Sensitive to } \\
\text { abnormalities in } \\
\text { photosynthesis }\end{array}$ & Limited in field setting & {$[19,20]$} \\
\hline $\begin{array}{l}\text { Hyperspectral } \\
\text { Techniques }\end{array}$ & Rapid and highly robust & $\begin{array}{l}\text { Affected by external } \\
\text { factors, such as light, } \\
\text { view angle; relatively } \\
\text { expensive }\end{array}$ & [21] \\
\hline GC-MS & $\begin{array}{l}\text { Providing individual } \\
\text { VOCs information }\end{array}$ & $\begin{array}{l}\text { Expensive, not real-time, } \\
\text { expertise skills needed }\end{array}$ & {$[22,23]$} \\
\hline Enzymatic biosensor & $\begin{array}{l}\text { Real-time and high } \\
\text { specificity }\end{array}$ & $\begin{array}{l}\text { Unstable, easily affected } \\
\text { by } \mathrm{pH} \text {, environment }\end{array}$ & [24] \\
\hline DNA-based biosensor & $\begin{array}{l}\text { Low cost, low limit of } \\
\text { detection }\end{array}$ & $\begin{array}{l}\text { Easily affected by DAN } \\
\text { extraction, not real-time }\end{array}$ & [25] \\
\hline $\begin{array}{l}\text { Antibody-based } \\
\text { biosensor }\end{array}$ & Low cost & Not real-time & [26] \\
\hline
\end{tabular}

Detection of plant infections prior to the onset of visual symptoms is valuable for executing appropriate management strategies and pest control to prevent the spread of diseases [27,28]. Plants emit a large amount of VOCs, which deliver functional information related to their growth, health, and disease [29].

Plants have a broad range of defense mechanisms for combatting infections, attacks by herbivorous insects, and mechanical damage [30]. One of these protection strategies involves emitting specific VOCs to fight potential attackers. These defenses are often divided into direct defenses and indirect defenses. In direct defenses, plants emit repellent VOCs to reduce insect attacks, while, in indirect defenses, VOCs can attract predators to battle pests. It is clear that VOCs play 
significant roles in plant communication and present promising functionality for improving crop protection [31]. VOCs emitted from plants indicate their real-time physiological health status and could provide bio-information that could be used for rapid, non-invasive disease diagnosis. Moreover, the composition of VOCs varies according to the type of damage, such as pathogen infection and herbivore feeding [32]. Some VOCs are present as a strong aromatic gas, but most of them occur at extremely low concentrations that are below the human olfactory threshold. Therefore, novel sensors, sensing technologies, and data analysis methods are required to detect plant VOCs and to interpret the information. The development of such technologies is critical for utilizing VOCs profiling concepts in the field for improved crop production.

GC-MS is a conventional technique used for separating and identifying individual VOCs $[33,34]$. However, GC-MS is expensive and time-consuming; it does not function in real time; and it requires specific expertise for compound determination. Thus, electronic noses (E-noses) are being evaluated to detect plant VOCs $[35,36]$. This paper provides a comprehensive review of this novel technology, including sensor arrays, sampling set design, pattern recognition, and challenges. Applications and potential improvements for using E-noses to diagnose pest-infected plants are also discussed.

\section{Electronic Nose Detecting Technology}

E-noses, also known as artificial olfaction devices, have been widely developed over the past two decades. They have been extensively employed in diverse applications ranging from medical diagnosis to the food industry, environmental protection, and agriculture [37-40]. These systems are designed to mimic the mammalian olfactory system. They are coupled with different types of sensor arrays, which transform the VOCs information into an electronic signal. When gas samples are spread across the sensor array, the odor molecules induce reversible physicochemical changes to the sensing materials. This causes changes in electrical properties such as the resistance and electrical potential. Conditioning circuits are used to modulate the signals, and pattern recognition is used to classify the aromas. Finally, the data can be read, displayed, and saved for pattern recognition analysis, which could provide basic diagnosis results. The most important parts of E-nose system, gas sensors and pattern recognitions, are briefly discussed below.

\subsection{Gas Sensors}

Several commercial gas sensors are available for E-nose systems. They can be grouped according to working mechanisms into three different categories: conductivity sensors, gravimetric sensors, and optical sensors.

\subsubsection{Conductivity Sensors}

Conductivity sensors are based on a conducting polymer (CP) and/or metal oxide semiconductor (MOS), both of which work on the principle of variations in conductivity or resistance upon exposure to particular gases. Although the response mechanisms are different, the physical structures, such as sensing materials, electrodes, and substrates of the conductive sensors, are basically the same. MOS-based sensors need an extra heater.

Conducting polymers have many advantages over other materials when used as gas sensors. Sensors prepared from conducting polymers can operate at room temperature. This is a critical advantage for portable battery-powered E-nose systems since a heater significantly increases power consumption, reducing battery life. More importantly, high discrimination in array sensors can be achieved by using different conducting materials due to the various categories of conducting polymers that are available. However, a main drawback of conducting polymer composites is aging, which can cause sensor drift and poor performance. Furthermore, these materials are not sensitive to certain gases. For example, a sensor based on a graphene oxide-based composite is not sensitive to trimethylamine (TMA), which is a typical VOC released from decaying fish. 
In recent years, due to their simple fabrication and variety, conductive polymer-based sensors have been used in detecting wood decay. The AromaScan $32 \mathrm{~S}$ is a commercial E-nose with 32 organic conductive polymer-based sensors that have been used to determine incipient wood decay caused by fungi. After pre-training on the aroma of pure fungus cultures as well as healthy and decayed wood samples, unknown samples of wood decay fungus were correctly identified based on their VOCs, with up to $93.2 \%$ accuracy [41]. Fast sensor response and good repeatability were also demonstrated in the detection process. Conducting polymers-based sensors, however, have a short life-time, and humidity can affect sensor performance.

MOS sensors are some of the most commonly used gas sensors for constructing sensor arrays due to their cost-effectiveness, reliability, and availability. They have been widely applied in agriculture and forestry industries for diagnosis of plant infection caused by fungus, bacteria, and viruses; insect damage; or mechanical damage [42-47]. The main advantages of MOS sensors are the fast response and recovery times, which mainly depend on the temperatures and the level of interaction between the sensors and gases [48]. MOS sensors are small and can be constructed as integrated circuits. However, the applications of MOS sensors are limited to "moderate" gases such as $\mathrm{CO}_{2}$ and $\mathrm{H}_{2}$, and they are not suitable for sulfur containing gases which can bind with the sensing materials [48-51]. The operation of MOS sensors requires high temperatures of around $200-500{ }^{\circ} \mathrm{C}$, which is beyond the temperature range that a common battery can achieve and, thus, limits practical field applications.

\subsubsection{Gravimetric Sensors}

Two types of gravimetric sensors are employed in E-nose systems: surface acoustic wave (SAW) sensors and quartz crystal microbalance (QCM) sensors. SAW sensors produce a surface wave that travels along the surface of the sensor, while QCM sensors produce a wave that travels through the bulk of the sensor. The working principle of both sensors involves a change in the mass of the piezoelectric sensor coating due to gas absorption, which results in a change in the resonant frequency upon exposure to VOCs [52].

A SAW sensor consists of a piezoelectric substrate with an interdigital input receiving electrode and output transmitting electrode located on the top surface of the substrate. A sensitive thin film is located between the interdigital electrodes. Odor molecules interact with the sensing film and change the mass of the entire sensor unit which leads to a change in the frequency.

QCM-based sensors have a similar operating principle to SAW sensors but a different device structure. The sensor is composed of a quartz chip coated with an absorbing sensing membrane, and a set of gold electrodes attached to the bottom of the chip, with one on each side. Cui et al. investigated the feasibility of an E-nose based on QCM sensors for predicting the shelf life of fruits and meats. The sensor showed promising performance for evaluating food quality $[53,54]$. The sensitivity and selectivity of these sensors strongly depend on the type of sensing material and the interaction between the odor and film compounds. Improving the sensitivity of such sensors relies on developing specific sensing materials for specific VOCs biomarkers. The author's research group developed an ultra-sensitive E-nose system, consisting of a QCM sensor array with four conducting polymers (Figure 1), specifically for infested plants. The primary results indicated that this system has the potential to provide an accurate diagnosis in real-time. The advantages of using SAW and QCM sensors include low cost, small size and high sensitivity. However, they have some disadvantages, such as a complex fabrication process and circuitry and a short life span [55].

\subsubsection{Optical Sensors}

In contrast to the aforementioned sensors, the mechanism of optical sensors is based on changes in chemical properties, such as the reactivity, redox potential, and acid-base interactions [56]. Optical sensors use a wavelength-selectable light source, a light detector, and sensor materials that interact with gases. Colorimetry and fluorometry are the two typical techniques used for analyzing the signal obtained from optical sensors. Suslick and Rakow developed the first colorimetric sensor array in a 
cartridge package for use in odorant detection [57]. A difference map can be obtained from a digital image by digital subtraction of the image of the array before and after exposure. The advantages of colorimetric sensors are their disposability, fast response, and strong robustness for hazardous gas detection. However, a major drawback for many optical sensors is unexpected sensitivity to humidity in the environment, especially for real-time detection, since the humidity varies from day to day and from indoors to outdoors. Research showed that these optical sensors respond to humidity with relative humidity concentrations ranging 10-95\% [58-60]. A significant change in signal caused by a change in humidity can cover up the signals of the target odorants. This issue can be addressed by using hydrophobic materials as substrates for colorimetric or fluorometric sensor arrays. However, a drawback of colorimetric optical sensors is their short lifetime, since molecular dyes used as sensing materials have a limited shelf life [61]. Due to their high sensitivity (sub-ppb), optical sensors and metric arrays have been widely used in the detection of toxic industrial chemicals, explosives, foods and beverages, bacteria, and cancer [62-64]. The advantages and disadvantages of the aforementioned sensors are summarized in Table 2.

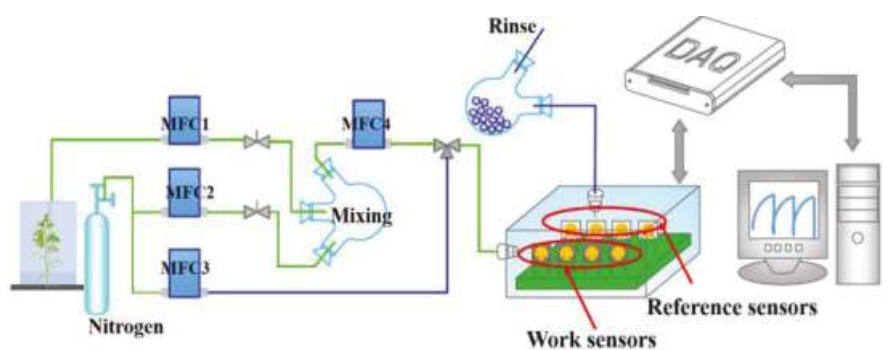

Figure 1. An E-nose system based on QCM sensor array. MFC, mass flow control; DAQ, data acquisition.

Table 2. Summary of advantages and disadvantages of gas sensors applied on E-noses [65-68].

\begin{tabular}{|c|c|c|}
\hline Name & Advantage & Disadvantage \\
\hline $\mathrm{CP}^{1}$ & $\begin{array}{l}\text { Wide range of available conducting } \\
\text { polymers; room temperature operation; fast } \\
\text { response; sensitive to polar compounds }\end{array}$ & $\begin{array}{l}\text { High sensitivity to humidity and } \\
\text { temperature; sensor response drift } \\
\text { with time; short-life time }\end{array}$ \\
\hline $\operatorname{MOS}^{2}$ & $\begin{array}{l}\text { Small size; easy to integrate into } \\
\text { measurement circuitry; fast response and } \\
\text { recovery time; high sensitivity }\end{array}$ & $\begin{array}{l}\text { High-power-consumption; } \\
\text { limited application on portable } \\
\text { systems; blind with sulfur gas; } \\
\text { limited coating materials; sensitive } \\
\text { to humidity }\end{array}$ \\
\hline $\mathrm{SAW}^{3}$ & $\begin{array}{l}\text { Broad applications; high sensitivity; fast } \\
\text { response; diverse sensing materials; small } \\
\text { size; }\end{array}$ & $\begin{array}{l}\text { Relatively poor signal to noise } \\
\text { performance; complex circuitry; } \\
\text { unsatisfactory reproducibility }\end{array}$ \\
\hline $\mathrm{QCM}^{4}$ & $\begin{array}{l}\text { Fast response time; easier fabrication } \\
\text { compared to SAW; high sensitivity; diverse } \\
\text { sensing materials; small }\end{array}$ & $\begin{array}{l}\text { Unsatisfactory reproducibility; } \\
\text { complex circuitry }\end{array}$ \\
\hline $\mathrm{CM}^{5}$ & $\begin{array}{l}\text { High sensitivity; fast response; robustness } \\
\text { in hazardous environment; disposable after } \\
\text { use }\end{array}$ & $\begin{array}{l}\text { Sensitive to humidity; complex } \\
\text { supporting software and } \\
\text { instrument; short life time; only } \\
\text { sensitive to oxygen and VOCs }\end{array}$ \\
\hline
\end{tabular}




\subsection{Sampling Methods}

\subsubsection{Laboratory Sampling}

Odor compounds are drawn into an E-nose via different collection methods, such as headspace sampling, diffusion methods, bubblers, and pre-concentrators. Profiling plant VOCs are conventionally carried out in a sealed chamber or box with controlled temperature and humidity, which simulate the environment of a greenhouse and field. A typical sampling setup is shown in Figure 2. In one study, rice plants with different pest damage were placed in the container, and VOCs were collected after $20 \mathrm{~min}$ to allow for static headspace build up before sampling [46]. In another study, an E-nose was employed to sample the VOCs emitted by powdery mildew and spider mite infected tomato plants, which were housed in clear glass boxes. The humidity and temperature were logged at all times. During the cultivation, clean air was pumped in to create positive pressure to maintain constant environmental parameters and decrease the risk of cross contamination $[69,70]$.

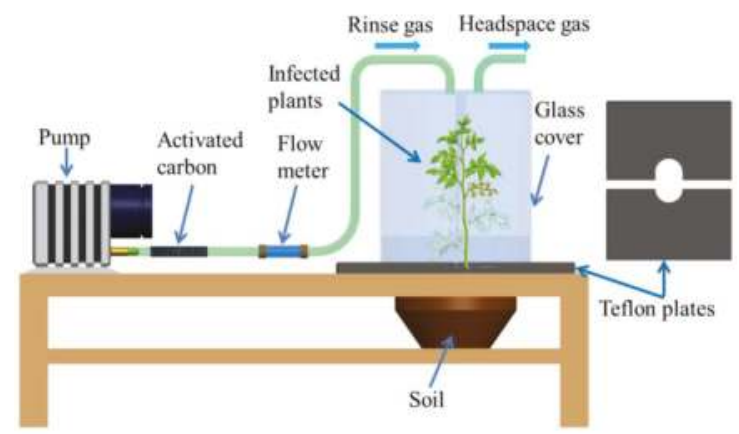

Figure 2. Illustration of VOC collection system for infected plants.

\subsubsection{Field Sampling}

The continuous changes in VOCs, temperatures, and humidity in open space conditions have impeded large scale field applications of E-nose. Recent studies have attempted to address these challenges. One potential solution is cultivating plants in a field environmental control chamber under natural light. In one reported attempt, two-year-old potted apple and pear plants were enclosed in plastic bags or Teflon chambers and cultured under field conditions in a shelter for E-nose detection. The temperature and $\mathrm{CO}_{2}$ assimilation were controlled to maintain consistent levels [71]. Another detection experiment was performed directly at the site of basal stem rot in infected oil palm plants and the surrounding soils using a portable commercial E-nose (Cyranose 320). The results showed $\sim 99 \%$ accuracy in identifying infected trunks and soils from healthy ones [72]. Biondi et al. explored the feasibility of using E-noses for detecting brown rot and ring rot in potatoes in laboratory and field conditions. Results indicated that the E-nose was able to distinguish between healthy potato samples and infected ones under the designed conditions, which included storage in polypropylene bags in a refrigerated chamber [73]. Most of these attempts required an enclosed space and climate control capability to keep the environment relatively stable. A feasible, low cost and easy to use method for field sampling could be gas collection directly from plants or plant branches covered with polyethylene terephthalate bags to form a relatively stable environment. However, the concentrations of VOCs were found to be relatively low for this method, but could be pre-concentrated by extending the sampling time from $30 \mathrm{~min}$ to $3-6 \mathrm{~h}$. The detection of VOCs from plants in open fields or greenhouses is still a big challenge. 


\subsection{Data Analysis Methods}

Data analyses using algorithms are used to perform qualitative classifications and quantitative predictions. There have been significant improvements in pattern recognition technologies, and many advanced algorithms have been introduced for E-nose systems. Two classes of statistical methods are generally used, as shown in Figure 3 The first, supervised methods, include artificial neutral networks (ANN), and are used to classify unknown features of a class that have the most common properties based on prior knowledge or probability distributions from training samples [74]. The other group is unsupervised methods, such as cluster analysis (CA), which separate the input data into different clusters based on feature similarity [75]. To provide a general overview for applications in plant health determination, the sections below review the four most common approaches: cluster analysis (CA), $\mathrm{ANN}$ and random forest (RF).

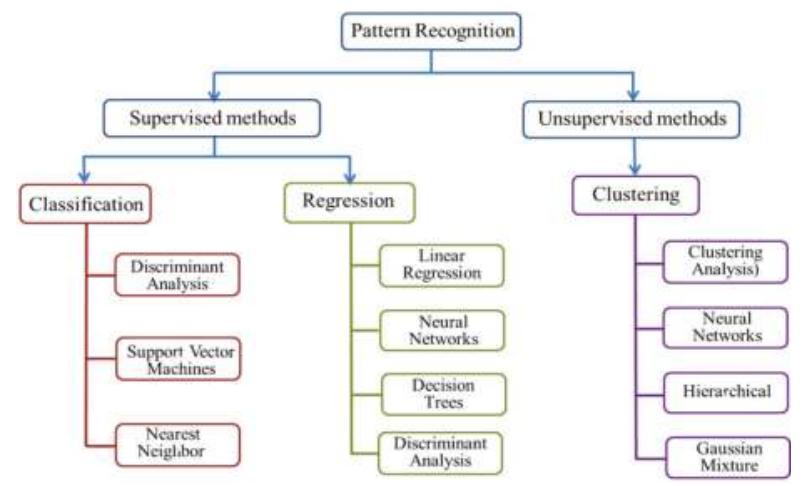

Figure 3. Typical pattern recognition methods applied on E-nose system.

\subsubsection{Unsupervised Statistical Methods}

CA is a widely applied unsupervised classification technique in which clusters are determined based on the distance between each data point [76]. The most common clustering algorithm is Ward's minimum variance method, which minimizes the total data within the cluster variance. The resultant dendrogram shows the connectivity and distance between each of the clusters, in which the shorter the distance, the more similar the samples. A CA dendrogram provides a straightforward way of displaying cluster similarity with semi-quantitative results. Laothaworbkitkul et al. employed an E-nose and cluster analysis to distinguish VOCs emitted from control, artificially damaged, herbivore-damaged, and diseased plants (cucumber, pepper, and tomato plants) [72]. The results of CA clearly showed clusters between damaged and undamaged plants. As expected, CA can also successfully differentiate cucumbers infested with spider mites from wounded cucumbers and healthy cucumbers [77]. The advantages of CA include revealing associations and structures in data which were not previously evident and presenting results in an easy to understand dengrogram. However, some methods are still not clearly established and there is no complete satisfactory method for determining the appropriate number of clusters.

\subsubsection{Supervised Statistical Methods}

ANNs are supervised learning algorithms and are best known for their good adaptability properties in learning, generalization, and noise tolerance, making them suitable for processing nonlinear data. ANNs are capable of learning from input data and optimizing neuron weights in real-time through iterative training and self-adjustment. ANNs consist of multiple layers of neurons, which depend on the complexity of the system. The outputs of ANNs depend on the design of the 
experiment. Due to their robustness and self-adaptability, ANNs have been introduced to E-nose systems to play the role of a "brain" and provide accurate quantitative analysis. Shakaff et al. employed an E-nose combined with an ANN as the main pattern recognition method to detect oil palm trees infested with basal stem rot disease. After training with 240 samples, a typical three-layer network, with one input layer, one hidden layer, and one output layer, was established to analyze another 160 samples. The classification was $100 \%$ successful when using the multilayer perceptron and probabilistic neutral network algorithms, while a $97.5 \%$ success rate was achieved when using the radial basis functions (RBF) algorithm. All three of these methods are ANN methods but use different types of supervision [78]. A back-propagation feed-forward artificial neutral network (BP-ANN) has also been employed to differentiate different apple cultivars, and showed a satisfactory accuracy of $87 \%$ [79]. Compared with unsupervised methods, supervised methods such as ANN require a large amount of training samples, but they provide more robust algorithms and higher accuracy. Besides, ANNs require less formal statistical restrictions on the input variables and they are able to learn and model complex nonlinear relationships between dependent and independent variables. However, the trained "black box" tends to be over-fitted due to the empirical nature of model development.

As the most popular supervised learning algorithm, RF is an ensemble learning method for both classification and regression, which has been widely used as a classifier and predictor in analyzing E-nose data. Briefly, RF is a combination of tree (decision) predictors. The value of a random vector decides a single tree predictor individually and for all the others trees [80]. Its proceeds are generally operated by constructing a multitude of decision trees at training time and providing the class that is the mode of the classification or averaged prediction of the individual trees. Specifically, bootstrap sampling is firstly established and subsets are generated based on bootstrap sampling distributions and randomly original dataset with replacement. For each data subset, a corresponding decision tree model is built. The above three steps are repeated until all the tree models are grown. Finally, the class membership of new samples will be predicted by a maximum vote of the predictions [81]. Due to its good performance in both classification and regression, RF has been intensively adopted in E-nose data analysis. A RF classifier was established to discriminate the difference between healthy maize plants and those at an early stage of Phaeosphaeria leaf spot infestation. Results showed an overall accuracy of $88 \%$ and a kappa value of 0.75 , indicating that RF has potential as a classifier in detecting maize disease infestation [82]. A model to predict powdery mildew infection levels of chardonnay grape brunches was reported to achieve an accuracy of 0.87 in classification of healthy, infected and severely diseased bunches [83]. It is obvious that RF is efficient for a large database and could give an estimate of the important variable in the classification, but it also tends to be over-fitted for some datasets with noisy classification and regression tasks. The advantages and disadvantages of the aforementioned pattern recognition methods were summarized in Table 3.

Table 3. Summary of advantages and disadvantages of CA, ANN and RF.

\begin{tabular}{clll}
\hline Name & \multicolumn{1}{c}{ Functions } & \multicolumn{1}{c}{ Advantages } & \multicolumn{1}{c}{ Disadvantages } \\
\hline CA & Classification & $\begin{array}{l}\text { Reveal associations and structures in } \\
\text { data which are not evident; results are } \\
\text { easy to understand }\end{array}$ & $\begin{array}{l}\text { Some methods are not clearly } \\
\text { established; no satisfactory method for } \\
\text { determining the appropriate number of } \\
\text { clusters }\end{array}$ \\
\hline ANN & $\begin{array}{l}\text { Classification, regression } \\
\text { and prediction }\end{array}$ & $\begin{array}{l}\text { Require less formal statistical } \\
\text { restrictions; able to model complex } \\
\text { nonlinear relationships; able to train } \\
\text { multiple algorithms }\end{array}$ & Big computation burden; tend to overfit \\
\hline RF & $\begin{array}{l}\text { Classification, regression } \\
\text { and prediction }\end{array}$ & $\begin{array}{l}\text { Efficient for large database; estimate the } \\
\text { important variable in the classification; } \\
\text { generate forests for further use }\end{array}$ & $\begin{array}{l}\text { Overfitting for some datasets with noisy } \\
\text { classification and regression tasks }\end{array}$ \\
\hline
\end{tabular}




\section{Applications in Plant Diagnosis}

Plant VOCs play significant roles in responding to pest attacks and are promising targets for pest detection. The composition of VOCs emitted by plants depends on the mode of damage. Biologically, VOCs are produced by a wide range of physiological processes in many different parts of plant tissues. The defense mechanisms to pathogens or viruses are still unclear, but the VOCs have been found to change after plants are infected. Plants require a broad range of defense mechanisms to effectively combat attacks by herbivorous insects or mechanical damage [30]. One of the strategies is to emit specific VOCs to battle potential attacks. While some protective VOCs are emitted at all times, others are induced only in response to herbivore feeding [84]. Accordingly, theses variations of VOCs provide reliable principles of pest detection via E-nose, as shown in Figure 4. Discussed below are advances of using E-noses in detecting fungal and bacterial infections and insect infestations in plants. The discrimination of damaged plants caused by mechanical damage is also discussed, as it is considered as background noise for VOCs detection of insect damage.

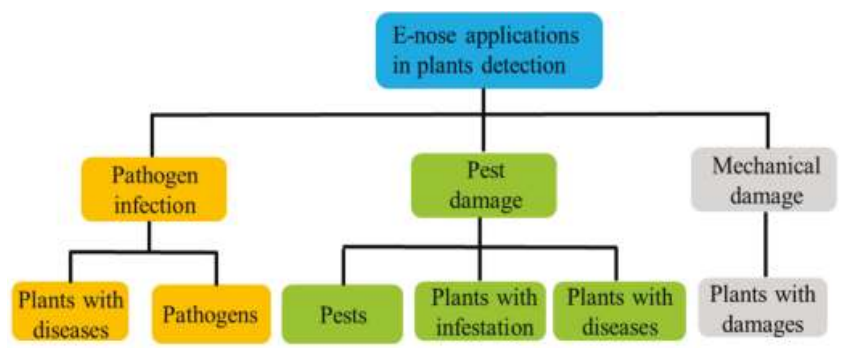

Figure 4. The applications of E-nose in plants disease detection.

\subsection{Fungal and Bacterial Disease Infections}

E-noses have considerable potential in detecting plants with fungal or bacterial infections. The direct approach involves detecting VOCs released from isolated microbes using an E-nose, while the indirect method involves determining changes in VOCs emitted from infected plants that have been inoculated with a target fungus or bacteria.

There has been success using E-noses for early diagnosis to discriminate fire blight (Erwinia amylovor) and blossom blight (Pseudmonas syringgae PV. Syringae) on apple trees under controlled laboratory conditions [85] and field conditions [71]. Researchers have also demonstrated that fire-blight-infected pear trees can be successfully detected in the early stages of infection using an E-nose based on MOS sensors. Grapevines inoculated with tumorigenic strains of Agrobacterium vitis have been correctly differentiated from healthy groups with $83.3 \%$ accuracy using a portable E-nose system [71]. Tomato plants, one of the most valuable greenhouse crops in the world, have attracted the most interest among researchers for investigating VOC fingerprints to monitor the plant's health status. Zhang et al. explored the effects of powdery mildew on the VOCs of infected tomato plants under greenhouse conditions. The results indicated that the disease had a major effect on the VOC profile, and the E-nose was able to discriminate between infected plants and healthy ones with classification accuracy of over $94 \%[69,70]$. Levels of infection by Ceratocystis fagacearum (oak wilt) were predicted with $78.65 \%$ accuracy using E-nose detection [86].

Infection microbes (fungi, bacteria, and viruses) might also contribute to the VOC profile of infected plants. Therefore, it is also essential to detect volatile compounds released from microbial metabolites or during microbial culture. The fire blight pathogen (E. amylovora) was found to have unique volatile characteristics and has been differentiated from other plant-associated bacteria using a MOS-based E-nose, achieving $87.5 \%$ accuracy in discrimination from a reference species [43]. A satisfactory distinction between E. coli and Listeria from individual colonies of suspension was 
accomplished with $92.4 \%$ classification accuracy [87]. VOCs of bacterial strains directly isolated from both chilies and papaya plants were measured and analyzed using an E-nose system, and further confirmed the feasibility of E-nose diagnosis of pathogenic bacteria in plants.

There is also good potential for detecting asymptomatic diseased plants infected with various plant pathogens using E-noses. However, most experiments and validations have been carried out in lab conditions. In field conditions, limitations should be considered and improvements are still needed. For example, plants are usually infected with several diseases during growth and concentrations of volatiles are under the threshold of many commercially available sensors. The unique VOCs associated with a target disease may also be covered or diluted by background VOCs, which are inconsistent in open areas. Therefore, the technology could be greatly improved by developing sensitive and selective sensors; determining specific VOC biomarkers for distinct plant diseases; and validating E-noses or sensor arrays in nurseries, greenhouses, and field conditions.

\subsection{Insect Damage}

E-noses have been successfully demonstrated for detecting insect infested plants and insect population dynamics. Spider mites (Tetranychus urticae Koch) are herbivorous arachnids that can feed on several hundred host plants, including economically important tomato plants cultured in greenhouses. E-noses have been used for extensive investigations of VOCs profiles emitted by tomato plants infested with spider mites, under different growth conditions [69,70]. Tomato plants can be correctly classified, without a priori knowledge, as healthy or infected using this technique.

The reliability of E-nose technology has been confirmed in rice plant diagnosis. Infected rice plants attacked by the striped rice stem borer (Chilo suppressalis) and the brown planthopper (Nilaparvata lugens) can be easily discriminated from healthy plants. Furthermore, the extent of damage to the rice plant as well as the amount of pests can also be successfully estimated based on the sensor response of an E-nose [88]. Volatiles released from insects such as brown planthoppers and stink bugs might be another avenue for revealing different aspects of insect-infested plants. Xu et al. demonstrated the possibility of estimating the age and number of brown planthoppers using an E-nose with classification accuracies of $100 \%$ and $48.93 \%$, respectively [89]. Mating disruption, which involves attracting or confusing males to impede mating and reduce the number of insects, has been widely adopted. Therefore, early recognition of insect gender in a rapid and practical way is critical for applying mating disruption. The gender and species of stink bugs have been precisely predicted using a portable E-nose $[45,46]$. Although the preliminary studies and results discussed thus far show that E-noses with an appropriate detection methodology are promising, the development of intelligent E-noses for specific insect detection is urgently required.

\subsection{Mechanical Damage}

Besides pathogenic and insect damage, mechanical damage also arouses defense responses and causes changes to VOCs fingerprints. The composition of VOCs emitted by damaged plants may depend on the mode of attack [69]. Zhou and Wang investigated differences in the VOCs patterns of rice plants under pest attack and mechanical damage using an MOS-based E-nose. The results indicated that VOCs caused by the mechanical damage were different from those caused by pest attacks, with a classification accuracy of $91.9 \%$ using principle component analysis [46]. Although mechanical damage does not account for a major loss of crop yield, there are plenty of crops that could be damaged by mechanical equipment used in the field. More importantly, the VOCs information from pest damage can be easily confounded by that from mechanical damage. Therefore, detection of VOCs from mechanical damage is recommended during the determination of pest damage. 


\section{Challenges and Improvements}

Extensive studies have demonstrated in laboratory environments that E-noses are promising non-destructive tools for quick and early plant pest damage detection. For field applications, however, several areas of improvement are necessary.

\subsection{Dynamic Nature of VOCs}

VOCs emissions of plants are specific to different tissues, locations, mass, and physiological stages. $\alpha$-thujene, a pheromone in aphids (Homoptera) and allomone in termites (Isoptera), has been reported to accumulate in peduncles of pistachio trees (Pistacia vera), with rarely any amount in the leaves and fruits [90]. The VOCs profile is also dynamic throughout the plant's life cycle. For example, the composition of a flower's odor profiles and the total odor production reach maximum levels when the flower is ready for pollination. After pollination, the amount of VOCs starts to decrease until the end of the flower's life cycle [90-93]. Seasonal variations and location are other factors that significantly affect the VOCs profile. Son et al. investigated the effect of seasonal change on the emission fluxes of monoterpene released from coniferous trees. They found that the amounts of monoterpenes emitted from pitch pine and Korean pine were generally highest in spring, followed by summer and fall, while they were lowest in winter [94]. The VOCs fingerprinting of the same type of American ginseng cultivated in two different locations showed significant differences, indicating that origin strongly influences the VOCs fingerprints $[95,96]$. Therefore, the dynamic nature of VOCs profiles due to differences in region, age, season, and tissues increases complexity in characterizing VOCs biomarkers for the task of pest detection. This is a major challenge for disease diagnosis, even for the same species of plants.

\subsection{Environmental Effects on Sensing}

The influence of humidity and temperature is another challenge in the detection of plant pests, as sensors in E-nose systems are sensitive to these factors. The instability of these two factors causes obvious drift in the sensor response, which reduces the signal to noise ratio $(\mathrm{S} / \mathrm{N})$ for detection of the targeted VOCs. Furthermore, humidity reduces the lifetime of gas sensors, thus limiting their application in long-term and high frequency monitoring of a plants' status. Preconditioning the sample gases and housing the sensors in a dry environment can mitigate the aforementioned limitations; however, the power requirements of these units may constrain their field applications.

\subsection{Detection in Field Conditions}

The performance of E-noses in actual production environments needs to be studied using larger field trials. However, the environmental parameters of open fields, such as temperature, humidity, and background gas compositions [97,98], are uncontrollable and keep changing. Furthermore, the concentrations of many VOCs released from plants are very low and below the detection threshold of currently available E-noses. Background noise generated from the atmosphere can also hide the plants' true VOCs variance caused by pest attacks, fungal infection, or other causes. Therefore, a controlled environment that can maintain temperature, humidity, and even gas compositions, to certain degree, is more suitable for E-nose applications. Further development of sensor arrays with high sensitivity and selectivity are desirable.

\subsection{Plant Pest Specific Detection Technique Optimization}

As previously discussed, the artifact of natural variations of plant released VOCs profiles might overshadow the true changes caused by the presence of targeted pests. Moreover, the characteristic VOCs that reflect a specific pest infection of plants remain unknown. Thus, specific VOCs closely related to a plant pest might be ignored or mistakenly identified; therefore, the identification of distinct volatile biomarkers specific to a particular pest or plant is required, and sensitive and selective gas 
sensors that are specific to these biomarkers need to be optimized. Attempts to address this challenge should firstly determine the characteristic volatile biomarkers through a combination of conventional precision chromatography technologies, such as GC-MS. The next step is to fabricate the corresponding sensing materials that exhibit high sensitivity and selectivity to the target volatile indexes, and finally to develop a special gas sensor array based on these sensing materials.

\subsection{Combinations with Other Advanced Technologies}

It is well known that E-noses are designed to identify the entire fingerprint of VOCs, but not individual volatile components. However, information about specific components is essential when detecting hazardous or toxic gases. Therefore, the advantages of conventional chromatography technologies are obvious. The combination of an E-nose system with mass spectrometry or gas chromatography could address these challenges and extend the application of these technologies. Inspired by comprehensive sensory evaluation of biological senses, an electronic tongue that mimics the human sense of taste could be combined with an E-nose to establish a more robust and more widely applicable platform, especially for applications requiring liquid detection or in areas with high humidity $[87,88]$.

\subsection{Micro E-Noses}

An inexpensive portable E-nose would be preferable for plant pest detection, especially in open field detection. However, the bulky size and high price of most commercially available E-noses limit their potential agricultural applications. One improvement would be to develop a small (one-chip) or micro-level sensor array by using integrated circuit (IC) technologies and micro-electro mechanical systems (MEMS) to reduce the size [99]. Moreover, the price of ICs can be relatively low with mass production. Therefore, a new generation of portable E-noses with extremely small size and low cost could be realized.

With the rapid development of smart phones, a one-chip sensory array could be integrated with smart phones and a user-friendly interface in an app, which could help to realize intelligent, small, multi-functional, and low-cost E-noses. In this scenario, a smart phone with an E-nose chip could diagnose plant pests by placing it near infected plants, and the results could be displayed immediately. Identifying and quantifying VOCs emitted from plants can enhance E-nose sensors to maximize their effectiveness. Further refinements, such as the design and optimization of sensor arrays for specific VOCs markers, are likely to lead to improvements in sensitivity, as well as increase the robustness of the technology in the face of inconsistent environments in production agriculture.

A miniaturization of hardware units, such as signal conditioning and data acquisition components, is another way to reduce the size of E-nose system. The potential advantages of such a system include low cost, small size and wide application due to portability. Extensive research has been done to design portable E-nose systems by adopting a micro-controller equipped with a compact flash memory that assures data acquisition, analysis in real-time, and light-emitting diode (LED) screen $[100,101]$. Those designed portable E-nose systems have been successfully used for food inspection and indoor air quality monitoring [102].

\section{Conclusions}

Plant pests threaten commercial crops, causing reduction of food production and leading to significant economic losses worldwide. To control and manage the damage caused by pests, various technologies and different strategies have been developed and used. Although conventional methods, such as nucleic acid and serology-based technologies, have been commercially available and widely used, their applications for field detection is limited due to the need of a laboratory setting, time consumption for analysis, and cost limitations. The current and newly developed technologies, such as imaging methods or biosensors, have attracted extensive attention but still need improvement. Therefore, developing advanced, real-time, cost efficient and portable devices or technologies for early 
stage detection are needed. A portable E-nose system equipped with sensitive gas sensor arrays and pattern recognition is an innovative method that may meet these requirements, as well as provide some advantages over traditional technologies and address some challenges such as field-application.

An overview of innovative E-nose technologies with an emphasis on applications in plant pest detection were reviewed in this paper. The major advantages of E-noses include being extremely sensitive, providing real-time analysis, and being easy to operate and portable; thus, E-nose technology can provide a new platform for plant pest diagnosis. It has been demonstrated, in both laboratory and field environments, that plant infection symptoms can be successfully diagnosed using E-noses with accurate prediction and satisfactory sensor performance. However, challenges remain in regard to sensor selectivity, interference from the surrounding atmosphere, and the difficulty of detection in open fields, which require further investigation and improvement.

Acknowledgments: This project is supported by funding from USDA NIFA SCRI (Grant No. 2015-51181-24253). The authors would like to thank Mary Wicks for reading the manuscript and providing useful suggestions.

Author Contributions: Shaoqing Cui prepared and wrote this manuscript. Heping Zhu and Peter Ling read the manuscript and provided many useful suggestions. Harold M. Keener gave many suggestions on the manuscript structures and polished the language.

Conflicts of Interest: The authors declare no conflict of interest.

\section{References}

1. Nishi, K.; Isobe, S.-I.; Zhu, Y.; Kiyama, R. Fluorescence-Based Bioassays for the Detection and Evaluation of Food Materials. Sensors 2015, 15, 25831-25867. [CrossRef] [PubMed]

2. Nassuth, A.; Pollari, E.; Helmeczy, K.; Stewart, S.; Kofalvi, S.A. Improved RNA extraction and one-tube RT-PCR assay for simultaneous detection of control plant RNA plus several viruses in plant extracts. J. Virol. Methods 2000, 90, 37-49. [CrossRef]

3. Clark, M.F.; Adams, A.N. Characteristics of the microplate method of enzyme-linked immunosorbent assay for the detection of plant viruses. J. Gen. Virol. 1977, 34, 475-483. [CrossRef] [PubMed]

4. Mahlein, A.K.; Oerke, E.C.; Steiner, U.; Dehne, H.W. Recent advances in sensing plant diseases for precision crop protection. Eur. J. Plant Pathol. 2012, 133, 197-209. [CrossRef]

5. Bürling, K.; Hunsche, M.; Noga, G. Use of blue-green and chlorophyll fluorescence measurements for differentiation between nitrogen deficiency and pathogen infection in winter wheat. J. Plant Physiol. 2011, 168, 1641-1648. [CrossRef] [PubMed]

6. Fang, Y.; Umasankar, Y.; Ramasamy, R.P. Electrochemical detection of p-ethylguaiacol, a fungi infected fruit volatile using metal oxide nanoparticles. Analyst 2014, 139, 3804-3810. [CrossRef] [PubMed]

7. Ray, M.; Ray, A.; Dash, S.; Mishra, A.; Achary, K.G.; Nayak, S.; Singh, S. Fungal disease detection in plants: Traditional assays, novel diagnostic techniques and biosensors. Biosens. Bioelectron. 2017, 87, 708-723. [CrossRef] [PubMed]

8. Martinelli, F.; Scalenghe, R.; Davino, S.; Panno, S.; Scuderi, G.; Ruisi, P.; Villa, P.; Stroppiana, D.; Boschetti, M.; Goulart, L.R.; et al. Advanced methods of plant disease detection. A review. Agron. Sustain. Dev. 2015, 35, 1-25. [CrossRef]

9. Kuzdralinski, A.; Kot, A.; Szczerba, H.; Nowak, M.; Muszyriska, M. A review of conventional PCR Assays for the detection of selected phytopathogens of wheat. J. Mol. Microbiol. Biotechnol. 2017, 27, 175-189. [CrossRef] [PubMed]

10. Rajendran, D.K.; Park, E.; Nagendran, R.; Hung, N.B.; Cho, B.-K.; Kim, K.-H.; Lee, Y.H. Visual Analysis for Detection and Quantification of Pseudomonas cichorii Disease Severity in Tomato Plants. Plant Pathol. J. 2016, 32, 300-310. [CrossRef] [PubMed]

11. Cellini, A.; Biondi, E.; Blasioli, S.; Rocchi, L.; Farneti, B.; Braschi, I.; Savioli, S.; Rodriguez-Estrada, M.T.; Biasioli, F.; Spinelli, F. Early detection of bacterial diseases in apple plants by analysis of volatile organic compounds profiles and use of electronic nose. Ann. Appl. Biol. 2016, 168, 409-420. [CrossRef]

12. Fang, Y.; Ramasamy, R.P. Current and prospective methods for plant disease detection. Biosensors 2015, 5, 537-561. [CrossRef] [PubMed] 
13. Eun, A.J.C.; Huang, L.; Chew, F.T.; Li, S.F.Y.; Wong, S.M. Detection of two orchid viruses using quartz crystal microbalance (QCM) immunosensors. J. Virol. Methods 2002, 99, 71-79. [CrossRef]

14. Eun, A.J.-C.; Huang, L.; Chew, F.-T.; Fong-Yau Li, S.; Wong, S.-M. Detection of two orchid viruses using quartz crystal microbalance-based DNA biosensors. Phytopathology 2002, 92, 654-658. [CrossRef] [PubMed]

15. Fang, Y.; Umasankar, Y.; Ramasamy, R.P. A novel bi-enzyme electrochemical biosensor for selective and sensitive determination of methyl salicylate. Biosens. Bioelectron. 2016, 81, 39-45. [CrossRef] [PubMed]

16. Perera, R.M.M.; Marriott, P.J.; Galbally, I.E. Headspace solid-phase microextraction-Comprehensive two-dimensional gas chromatography of wound induced plant volatile organic compound emissions. Analyst 2002, 127, 1601-1607. [CrossRef] [PubMed]

17. Kesselmeier, J.; Staudt, M. Biogenic Volatile Organic Compound (VOC): An Overview on Emissions, Physiology and Ecology. J. Atmos. Chem. 1999, 33, 23-88. [CrossRef]

18. Lowe, A.; Harrison, N.; French, A.P. Hyperspectral image analysis techniques for the detection and classification of the early onset of plant disease and stress. Plant Methods 2017, 13, 1-12. [CrossRef] [PubMed]

19. Csefalvay, L.; Gaspero, G.D.; Matous, K.; Bellin, D.; Ruperti, B.; Olejnickova, J. Pre-symptomatic detection of Plasmopara viticola infection in grapevine leaves using chlorophyll fluorescence imaging. Eur. J. Physical. Med. Rehab. 2009, 125, 291-302. [CrossRef]

20. Perez, E.; Saavedra, J.; Crawford, P.; Arias, S. Recovery and creative practices in people with severe mental illness: Evaluating well-being and social inclusion. Disabil. Rehabilit. 2018, 40, 905-911. [CrossRef]

21. López, M.M.; Bertolini, E.; Olmos, A.; Caruso, P.; Gorris, M.T.; Llop, P.; Penyalver, R.; Cambra, M. Innovative tools for detection of plant pathogenic viruses and bacteria. Int. Microbiol. 2003, 6, 233-243. [CrossRef] [PubMed]

22. Wallner, G.; Amann, R.; Beisker, W. Optimizing fluorescent in situ hybridization with rRNA-targeted oligonucleotide probes for flow cytometric identification of microorganisms. Cytometry 1993, 14, 136-143. [CrossRef] [PubMed]

23. DeLong, E.; Wickham, G.; Pace, N. Phylogenetic stains: Ribosomal RNA-based probes for the identification of single cells. Science 1989, 243, 1360-1363. [CrossRef] [PubMed]

24. Schaad, N.W.; Frederick, R.D. Real-time PCR and its application for rapid plant disease diagnostics. Can. J. Plant Pathol. 2002, 24, 250-258. [CrossRef]

25. Lau, H.Y.; Botella, J.R. Advanced DNA-Based Point-of-Care Diagnostic Methods for Plant Diseases Detection. Front. Plant Sci. 2017, 8, 1-14. [CrossRef]

26. Moter, A.; Göbel, U.B. Fluorescence in situ hybridization (FISH) for direct visualization of microorganisms. J. Microbiol. Methods 2000, 41, 85-112. [CrossRef]

27. Sankaran, S.; Mishra, A.; Ehsani, R.; Davis, C. A review of advanced techniques for detecting plant diseases. Comput. Electron. Agric. 2010, 72, 1-13. [CrossRef]

28. Shaikh, P.M.M.; Butala, N.; Sharma, S.; Sukumaran, S. Review on Diagnosis the Plant Disease at Early Stage. Int. J. Adv. Res. Comput. Commun. Eng. 2017, 6, 321-324. [CrossRef]

29. Baldwin, I.T.; Halitschke, R.; Paschold, A.; von Dahl, C.C.; Preston, C.A. Volatile Signaling in Plant-Plant Interactions: "Talking Trees" in the Genomics Era. Science 2006, 311, 812-815. [CrossRef] [PubMed]

30. Choudhary, D.K.; Johri, B.N.; Prakash, A. Volatiles as priming agents that initiate plant growth and defence responses. Curr. Sci. 2008, 94, 595-604.

31. Pare, P.W.; Tumlinson, J.H. Update on plant-insect interactions plant volatiles as a defense against insect herbivores. Plant Physiol. 1999, 121, 325-331. [CrossRef] [PubMed]

32. Maffei, M.E. Sites of synthesis, biochemistry and functional role of plant volatiles. S. Afr. J. Bot. 2010, 76, 612-631. [CrossRef]

33. Nusaibah, S.A.; Siti Nor Akmar, A.; Idris, A.S.; Sariah, M.; Mohamad Pauzi, Z. Involvement of metabolites in early defense mechanism of oil palm (Elaeis guineensis Jacq.) against Ganoderma disease. Plant Physiol. Biochem. 2016, 109, 156-165. [CrossRef] [PubMed]

34. Phillips, M.; Flematti, G.R.; Davis, T.M.; Suzuki, M.; Homig, H.; Benz-Bohm, G.; Kreuder, J.; Younossi-Hartenstein, A.; Sauvanet, A.; Couvelard, A.; et al. Detection of carbon disulfide in breath and air: A possible new risk factor for coronary artery disease. Int. Arch. Occup. Environ. Health 1992, 64, 119-123. [CrossRef] [PubMed] 
35. Ghaffari, R.; Laothawornkitkul, J.; Iliescu, D.; Hines, E.; Leeson, M.; Napier, R.; Moore, J.P.; Paul, N.D.; Hewitt, C.N.; Taylor, J.E. Plant pest and disease diagnosis using electronic nose and support vector machine approach. J. Plant Dis. Prot. 2012, 119, 200-207. [CrossRef]

36. Wilson, A.D. Diverse applications of electronic-nose technologies in agriculture and forestry. Sensors 2013, 13, 2295-2348. [CrossRef] [PubMed]

37. Xu, X.; Tian, F.; Yang, S.X.; Li, Q.; Yan, J.; Ma, J. A solid trap and thermal desorption system with application to a medical electronic nose. Sensors 2008, 8, 6885-6898. [CrossRef] [PubMed]

38. Estefania Nunez Carmona, E.; Sberveglieri, V.; Ponzoni, A.; Galstyan, V.; Zappa, D.; Pulvirenti, A.; Comini, E. Detection of food and skin pathogen microbiota by means of an electronic nose based on metal oxide chemiresistors. Sens. Actuators B Chem. 2017, 238, 1224-1230. [CrossRef]

39. Xu, S.; Lü, E.; Lu, H.; Zhou, Z.; Wang, Y.; Yang, J.; Wang, Y. Quality Detection of Litchi Stored in Different Environments Using an Electronic Nose. Sensors 2016, 16, 852. [CrossRef] [PubMed]

40. Messina, V.; Domínguez, P.G.; Sancho, A.M.; Walsöe de Reca, N.; Carrari, F.; Grigioni, G. Tomato Quality during Short-Term Storage Assessed by Colour and Electronic Nose. Int. J. Electrochem. 2012, 2012, 1-7. [CrossRef]

41. Baietto, M.; Wilson, A.D.; Bassi, D.; Ferrini, F. Evaluation of Three Electronic Noses for Detecting Incipient Wood Decay. Sensors. 2010, 10, 1062-1092. [CrossRef] [PubMed]

42. Zou, H.Q.; Li, S.; Huang, Y.H.; Liu, Y.; Bauer, R.; Peng, L.; Tao, O.; Yan, S.R.; Yan, Y.H. Rapid identification of Asteraceae plants with improved RBF-ANN classification models based on MOS sensor E-nose. Evid. Based Complement. Altern. Med. 2014, 2014. [CrossRef] [PubMed]

43. Spinelli, F.; Cellini, A.; Vanneste, J.L.; Rodriguez-Estrada, M.T.; Costa, G.; Savioli, S.; Harren, F.J.M.; Cristescu, S.M. Emission of volatile compounds by Erwinia amylovora: Biological activity in vitro and possible exploitation for bacterial identification. Trees Struct. Funct. 2012, 26, 141-152. [CrossRef]

44. Chang, K.P.P.; Zakaria, A.; Nasir, A.S.A.; Yusuf, N.; Thriumani, R.; Shakaff, A.Y.M.; Adom, A.H. Analysis and Feasibility Study of Plant Disease using E-Nose. In Proceedings of the 2014 IEEE International Conference on Control System, Computing and Engineering (ICCSCE 2014), Batu Ferringhi, Malaysia, 28-30 November 2014

45. Lan, Y.-B.; Zheng, X.-Z.; Westbrook, J.K.; Lopez, J.; Lacey, R.; Hoffmann, W.C. Identification of Stink Bugs Using an Electronic Nose. J. Bionic Eng. 2008, 5, 172-180. [CrossRef]

46. Zhou, B.; Wang, J. Discrimination of different types damage of rice plants by electronic nose. Biosyst. Eng. 2011, 109, 250-257. [CrossRef]

47. Costa, A.; Tangorra, F.M.; Zaninelli, M.; Oberti, R.; Cavalchini, A.G.; Savoini, G.; Lazzari, M. Evaluating an e-nose ability todetect biogas plant efficiency: a case study. Ital. J. Anim. Sci. 2016, 15, 116-123. [CrossRef]

48. Penza, M.; Cassano, G.; Tortorella, F. Gas recognition by activated $\mathrm{WO}_{3}$ thin-film sensors array. Sens. Actuators B Chem. 2001, 81, 115-121. [CrossRef]

49. Gebicki, J. Application of electrochemical sensors and sensor matrixes for measurement of odorous chemical compounds. TrAC Trends Anal. Chem. 2016, 77, 1-13. [CrossRef]

50. Wang, C.; Yin, L.; Zhang, L.; Xiang, D.; Gao, R. Metal oxide gas sensors: Sensitivity and influencing factors. Sensors 2010, 10, 2088-2106. [CrossRef] [PubMed]

51. Kim, Y.; Pham, C.; Chang, J.P. Potentials and challenges of integration for complex metal oxides in CMOS devices and beyond. J. Phys. D Appl. Phys. 2015, 48, 63001. [CrossRef]

52. Albert, K.J.; Lewis, N.S.; Schauer, C.L.; Sotzing, G.A.; Stitzel, S.E.; Vaid, T.P.; Walt, D.R. Cross-Reactive Chemical Sensor Arrays. Chem. Rev. 2000, 100, 2595-2626. [CrossRef] [PubMed]

53. Cui, S.; Yang, L.; Wang, J.; Wang, X. Fabrication of a sensitive gas sensor based on $\mathrm{PPy} / \mathrm{TiO}_{2}$ nanocomposites films by layer-by-layer self-assembly and its application in food storage. Sensors Actuators B Chem. 2016, 233, 337-346. [CrossRef]

54. Cui, S.; Wang, J.; Wang, X. Fabrication and design of a toxic gas sensor based on polyaniline/titanium dioxide nanocomposite film by layer-by-layer self-assembly. RSC Adv. 2015, 5, 58211-58219. [CrossRef]

55. Arshak, K.; Moore, E.; Lyons, G.M.; Harris, J.; Clifford, S. A Review of Gas Sensors Employed in Electronic Nose Applications. Sens. Rev. 2004, 24, 181-198. [CrossRef]

56. Askim, J.R.; Mahmoudi, M.; Suslick, K.S. Optical sensor arrays for chemical sensing: The optoelectronic nose. Chem. Soc. Rev. 2013, 42, 8649-8682. [CrossRef] [PubMed] 
57. Rakow, N.A.; Suslick, K.S. A colorimetric sensor array for odour visualization. Nature 2000, 406, 710-713. [CrossRef] [PubMed]

58. Suslick, K.S. An optoelectronic nose: "Seeing" smells by means of colorimetric sensor arrays. MRS Bull. 2004, 29, 720-725. [CrossRef] [PubMed]

59. Janzen, M.C.; Ponder, J.B.; Bailey, D.P.; Ingison, C.K.; Suslick, K.S. Colorimetric sensor Arrays for volatile organic compounds. Anal. Chem. 2006, 78, 3591-3600. [CrossRef] [PubMed]

60. Feng, L.; Musto, C.J.; Kemling, J.W.; Lim, S.H.; Zhong, W.; Suslick, K.S. Colorimetric sensor array for determination and identification of toxic industrial chemicals. Anal. Chem. 2010, 82, 9433-9440. [CrossRef] [PubMed]

61. Kemling, J.W.; Suslick, K.S. Nanoscale porosity in pigments for chemical sensing. Nanoscale 2011, 3, $1971-1973$. [CrossRef] [PubMed]

62. Martin, G.S.; Mannino, D.M.; Eaton, S.; Moss, M. The epidemiology of sepsis in the United States from 1979 through 2000. N. Engl. J. Med. 2003, 348, 1546-1554. [CrossRef] [PubMed]

63. Mazzone, P.J.; Wang, X.F.; Xu, Y.; Mekhail, T.; Beukemann, M.C.; Na, J.; Kemling, J.W.; Suslick, K.S.; Sasidhar, M. Exhaled breath analysis with a colorimetric sensor array for the identification and characterization of lung cancer. J. Thorac. Oncol. 2012, 7, 137-142. [CrossRef] [PubMed]

64. Kiani, S.; Minaei, S.; Ghasemi-Varnamkhasti, M. Application of electronic nose systems for assessing quality of medicinal and aromatic plant products: A review. J. Appl. Res. Med. Aromat. Plants 2016, 3, 1-9. [CrossRef]

65. Wilson, A.D.; Baietto, M. Advances in electronic-nose technologies developed for biomedical applications. Sensors 2011, 11, 1105-1176. [CrossRef] [PubMed]

66. Filenko, D.D. Chemical Gas Sensors based on Functionalized Self-Actuated Piezo-Resistive Cantilevers. Dissertation zur Erlangung des akademischen Grades eines Doktors. Ph.D. Thesis, Universität Kassel, Kassel, Germany, 2008.

67. Chiu, S.W.; Tang, K.T. Towards a chemiresistive sensor-integrated electronic nose: A review. Sensors 2013, 13, 14214-14247. [CrossRef] [PubMed]

68. Tewari, J.C.; Irudayaraj, J.M.K. Floral classification of honey using mid-infrared spectroscopy and surface acoustic wave based z-Nose sensor. J. Agric. Food Chem. 2005, 53, 6955-6966. [CrossRef] [PubMed]

69. Ghaffari, R.; Zhang, F.; Iliescu, D.D.; Hines, E.L.; Leeson, M.S.; Napier, R. Detection of Diseases and Volatile Discrimination of Plants: An Electronic Nose and Self-Organizing Maps Approach. In Intelligent Systems for Machine Olfaction: Tools and Methodologies; IGI Global: Hershey, PA, USA, 2011; pp. 214-230.

70. Zhang, F.; Iliescu, D.D.; Hines, E.L.; Leeson, M.S. Tomato Plant Health Monitoring: An Electronic Nose Approach. In Intelligent Systems for Machine Olfaction: Tools and Methodologies; IGI Global: Hershey, PA, USA, 2011; pp. 231-248.

71. Spinelli, F.; Noferini, M.; Vanneste, J.L.; Costa, G. Potential of the electronic-nose for the diagnosis of bacterial and fungal diseases in fruit trees. EPPO Bull. 2010, 40, 59-67. [CrossRef]

72. Markom, M.A.; Shakaff, A.Y.M.; Adom, A.H.; Ahmad, M.N.; Hidayat, W.; Abdullah, A.H.; Fikri, N.A. Intelligent electronic nose system for basal stem rot disease detection. Comput. Electron. Agric. 2009, 66, 140-146. [CrossRef]

73. Biondi, E.; Blasioli, S.; Galeone, A.; Spinelli, F.; Cellini, A.; Lucchese, C.; Braschi, I. Detection of potato brown rot and ring rot by electronic nose: From laboratory to real scale. Talanta 2014, 129, 422-430. [CrossRef] [PubMed]

74. Yangali-Quintanilla, V.; Verliefde, A.; Kim, T.U.; Sadmani, A.; Kennedy, M.; Amy, G. Artificial neural network models based on QSAR for predicting rejection of neutral organic compounds by polyamide nanofiltration and reverse osmosis membranes. J. Membr. Sci. 2009, 342, 251-262. [CrossRef]

75. Wu, B.; Quilot, B.; Kervella, J.; Génard, M.; Li, S. Analysis of genotypic variation of sugar and acid contents in peaches and nectarines through the Principle Component Analysis. Euphytica 2003, 132, 375-384. [CrossRef]

76. Belous, O.; Malyarovskaya, V.; Klemeshova, K. Diagnostics of subtropical plants functional state by cluster analysis. Potravinarstvo 2016, 10, 237-242. [CrossRef]

77. Laothawornkitkul, J.; Moore, J.P.; Taylor, J.E.; Possell, M.; Gibson, T.D.; Hewitt, C.N.; Paul, N.D. Discrimination of plant volatile signatures by an electronic nose: A potential technology for plant pest and disease monitoring. Environ. Sci. Technol. 2008, 42, 8433-8439. [CrossRef] [PubMed] 
78. Abdullah, A.H.; Shakaff, A.Y.M.; Zakaria, A.; Saad, F.S.A.; Shukor, S.A.A.; Mat, A. Application Specific Electronic Nose (ASEN) for Ganoderma boninense detection using artificial neural network. In Proceedings of the 2014 2nd International Conference on Electronic Design (ICED), Penang, Malaysia, 19-21 August 2014; pp. 148-152. [CrossRef]

79. Xiaobo, Z.; Jiewen, Z. Comparative analyses of apple aroma by a tin-oxide gas sensor array device and GC/MS. Food Chem. 2008, 107, 120-128. [CrossRef]

80. Breiman, L. Random forests. Mach. Learn. 2001, 45, 5-32. [CrossRef]

81. Qiu, S.; Wang, J. The prediction of food additives in the fruit juice based on electronic nose with chemometrics. Food Chem. 2017, 230, 208-214. [CrossRef] [PubMed]

82. Adam, E.; Deng, H.; Odindi, J.; Abdel-Rahman, E.M.; Mutanga, O. Detecting the Early Stage of Phaeosphaeria Leaf Spot Infestations in Maize Crop Using in situ Hyperspectral Data and Guided Regularized Random Forest Algorithm. J. Spectrosc. 2017, 2017, 1-8. [CrossRef]

83. Knauer, U.; Matros, A.; Petrovic, T.; Zanker, T.; Scott, E.S.; Seiffert, U. Improved classification accuracy of powdery mildew infection levels of wine grapes by spatial-spectral analysis of hyperspectral images. Plant Methods 2017, 13,1-15. [CrossRef] [PubMed]

84. Frost, C.J.; Mescher, M.C.; Carlson, J.E.; De Moraes, C.M. Plant defense priming against herbivores: Getting ready for a different battle. Plant Physiol. 2008, 146, 818-824. [CrossRef] [PubMed]

85. Fuentes, M.T.; Lenardis, A.; Fuente de la, E.B. Insect assemblies related to volatile signals emitted by different soybean - weeds - herbivory combinations. Agric. Ecosyst. Environ. 2018, 255, 20-26. [CrossRef]

86. Wilson, A.D.; Lester, D.G.; Oberle, C.S. Development of conductive polymer analysis for the rapid detection and identification of phytopathogenic microbes. Phytopathology 2004, 94, 419-431. [CrossRef] [PubMed]

87. Green, G.C.; Chan, A.D.C.; Dan, H.; Lin, M. Using a metal oxide sensor (MOS)-based electronic nose for discrimination of bacteria based on individual colonies in suspension. Sens. Actuators B Chem. 2011, 152, 21-28. [CrossRef]

88. Cheng, S.-M.; Wang, J.; Wang, Y.-W.; Wei, Z.-B. Discrimination of Different Types Damage of Tomato Seedling by Electronic Nose. ITM Web Conf. 2017, 11, 1-8. [CrossRef]

89. Xu, S.; Zhou, Z.; Lu, H.; Luo, X.; Lan, Y.; Zhang, Y.; Li, Y. Estimation of the age and amount of brown rice plant hoppers based on bionic electronic nose use. Sensors 2014, 14, 18114-18130. [CrossRef] [PubMed]

90. Agostini, F.; dos Santos, A.C.; Rossato, M.; Pansera, M.R.; dos Santos, P.L.; Serafini, L.A.; Molon, R.; Moyna, P. Essential oil yield and composition of Lamiaceae species growing in Southern Brazil. Braz. Arch. Biol. Technol. 2009, 52, 473-478. [CrossRef]

91. Cerreta, M.M.; Furton, K.G. An assessment of detection canine alerts using flowers that release methyl benzoate, the cocaine odorant, and an evaluation of their behavior in terms of the VOCs produced. Forensic Sci. Int. 2015, 251, 107-114. [CrossRef] [PubMed]

92. Dudareva, N.; Murfitt, L.M.; Mann, C.J.; Gorenstein, N.; Kolosova, N.; Kish, C.M.; Bonham, C.; Wood, K. Developmental regulation of methyl benzoate biosynthesis and emission in snapdragon flowers. Plant Cell 2000, 12, 949-961. [CrossRef] [PubMed]

93. Jones, K.N.; Reithel, J.S.; Irwin, R.E. A trade-off between the frequency and duration of bumblebee visits to flowers. Oecologia 1998, 117, 161-168. [CrossRef] [PubMed]

94. Son, Y.S.; Kim, K.J.; Jung, I.H.; Lee, S.J.; Kim, J.C. Seasonal variations and emission fluxes of monoterpene emitted from coniferous trees in East Asia: Focused on Pinus rigida and Pinus koraiensis. J. Atmos. Chem. 2015, 72, 27-41. [CrossRef]

95. Cui, S.; Wu, J.; Wang, J.; Wang, X. Discrimination of American ginseng and Asian ginseng using electronic nose and gas chromatography-mass spectrometry coupled with chemometrics. J. Ginseng Res. 2017, 41, 85-95. [CrossRef] [PubMed]

96. Cui, S.; Wang, J.; Yang, L.; Wu, J.; Wang, X. Qualitative and quantitative analysis on aroma characteristics of ginseng at different ages using E-nose and GC-MS combined with chemometrics. J. Pharm. Biomed. Anal. 2015, 102, 64-77. [CrossRef] [PubMed]

97. Al Makky, A.; Alaswad, A.; Gibson, D.; Olabi, A.G. Renewable energy scenario and environmental aspects of soil emission measurements. Renew. Sustain. Energy Rev. 2017, 68, 1157-1173. [CrossRef]

98. Marć, M.; Zabiegała, B.; Namieśnik, J. Testing and sampling devices for monitoring volatile and semi-volatile organic compounds in indoor air. TrAC Trends Anal. Chem. 2012, 32, 76-86. [CrossRef] 
99. Vasiliev, A.A.; Pisliakov, A.V.; Sokolov, A.V.; Samotaev, N.N.; Soloviev, S.A.; Oblov, K.; Guarnieri, V.; Lorenzelli, L.; Brunelli, J.; Maglione, A.; et al. Non-silicon MEMS platform for gas sensor. Sens. Actuactor B Chem. 2016, 224, 700-713. [CrossRef]

100. Zhang, L.; Tian, F.; Liu, S.; Dang, L.; Peng, X.; Yin, X. Chaotic time series prediction of E-nose sensor drift in embedded phase space. Sens. Actuators, B Chem. 2013, 182, 71-79. [CrossRef]

101. Sadeghifard, S.; Esmaeilani, L. A new embedded e-nose system to identify smell of smoke. In Proceedings of the 2012 7th International Conference on System of Systems Engineering (SoSE), Genova, Italy, 16-19 July 2012; pp. 253-257. [CrossRef]

102. Kumbhar, A.; Gharpure, D.C.; Botre, B.A.; Sadistap, S.S. Embedded e-nose for food inspection. In Proceedings of the 2012 1st International Symposium on Physics and Technology of Sensors (ISPTS-1), Pune, India, 7-10 March 2012; pp. 311-314. [CrossRef]

(C) 2018 by the authors. Licensee MDPI, Basel, Switzerland. This article is an open access article distributed under the terms and conditions of the Creative Commons Attribution (CC BY) license (http:/ / creativecommons.org/licenses/by/4.0/). 
Article

\title{
Research on the Effects of Drying Temperature on Nitrogen Detection of Different Soil Types by Near Infrared Sensors
}

\author{
Pengcheng $\mathrm{Nie}^{1,2,3}$, Tao Dong ${ }^{1,2}$, Yong $\mathrm{He}^{1,2, *}$ and Shupei Xiao ${ }^{1,2}$ \\ 1 College of Biosystems Engineering and Food Science, Zhejiang University, Hangzhou 310058, China; \\ npc2012@zju.edu.cn (P.N.); 21613052@zju.edu.cn (T.D.); xsp941230@163.com or 180312@zju.edu.cn (S.X). \\ 2 Key Laboratory of Sensors Sensing, Ministry of Agriculture, Zhejiang University, Hangzhou 310058, China \\ 3 State Key Laboratory of Modern Optical Instrumentation, Zhejiang University, Hangzhou 310058, China \\ * Correspondence: yhe@zju.edu.cn; Tel.: +86-0571-8898-2143
}

Received: 7 December 2017; Accepted: 22 January 2018; Published: 29 January 2018

\begin{abstract}
Soil is a complicated system whose components and mechanisms are complex and difficult to be fully excavated and comprehended. Nitrogen is the key parameter supporting plant growth and development, and is the material basis of plant growth as well. An accurate grasp of soil nitrogen information is the premise of scientific fertilization in precision agriculture, where near infrared sensors are widely used for rapid detection of nutrients in soil. However, soil texture, soil moisture content and drying temperature all affect soil nitrogen detection using near infrared sensors. In order to investigate the effects of drying temperature on the nitrogen detection in black soil, loess and calcium soil, three kinds of soils were detected by near infrared sensors after $25^{\circ} \mathrm{C}$ placement (ambient temperature), $50^{\circ} \mathrm{C}$ drying (medium temperature), $80^{\circ} \mathrm{C}$ drying (medium-high temperature) and $95{ }^{\circ} \mathrm{C}$ drying (high temperature). The successive projections algorithm based on multiple linear regression (SPA-MLR), partial least squares (PLS) and competitive adaptive reweighted squares (CARS) were used to model and analyze the spectral information of different soil types. The predictive abilities were assessed using the prediction correlation coefficients $\left(R_{P}\right)$, the root mean squared error of prediction (RMSEP), and the residual predictive deviation (RPD). The results showed that the loess $\left(R_{P}=0.9721\right.$, RMSEP $\left.=0.067 \mathrm{~g} / \mathrm{kg}, \mathrm{RPD}=4.34\right)$ and calcium soil $\left(R_{P}=0.9588\right.$, RMSEP $\left.=0.094 \mathrm{~g} / \mathrm{kg}, \mathrm{RPD}=3.89\right)$ obtained the best prediction accuracy after $95^{\circ} \mathrm{C}$ drying. The detection results of black soil $\left(R_{P}=0.9486, R M S E P=0.22 \mathrm{~g} / \mathrm{kg}, \mathrm{RPD}=2.82\right)$ after $80^{\circ} \mathrm{C}$ drying were the optimum. In conclusion, drying temperature does have an obvious influence on the detection of soil nitrogen by near infrared sensors, and the suitable drying temperature for different soil types was of great significance in enhancing the detection accuracy.
\end{abstract}

Keywords: nitrogen; near infrared sensors; drying temperature; SPA-MLR; PLS; CARS

\section{Introduction}

Soil, which provides nutrients in the process of plant growth, is the foundation and plays an important role in agriculture. Thus, it is of great importance to obtain soil nutrient elements such as soil nitrogen quickly and accurately for precision fertilization and agricultural production [1,2]. Many conventional soil analytical techniques such as Dumas combustion are often complex, with multi-component interactions [3]. At present, near infrared sensors (NIR) have been successfully applied to the fields of agriculture, food, medicine, petroleum and chemistry, and are one of the most important analytical methods, indispensable in qualitative and quantitative analysis [4,5]. In recent years, many scholars have used near infrared sensors to detect soil nitrogen and improved the detection accuracy in the aspects of soil pretreatments, spectral data processing, characteristic band selection and algorithm optimization. 
Dalal predicted soil nitrogen with the multiple linear regression method at the spectral bands of $1702 \mathrm{~nm}, 1870 \mathrm{~nm}$ and $2052 \mathrm{~nm}$ [6]. Lee et al. found that the sensitive bands of soil total nitrogen were not only affected by soil type, but also determined by sampling depth [7]. He et al. detected N, P, K, organic matter $(\mathrm{OM})$ and $\mathrm{pH}$ content in a loamy mixed soil by NIR. The results showed that the correlation coefficient between measured and predicted values of N, OM and pH were $0.93,0.93$ and 0.91 , respectively, but were not suitable for predicting $\mathrm{P}$ and $\mathrm{K}[8,9]$. Stoner et al. described the spectral reflectance curves and their relationships with five soil types in detail according to the characteristics of the spectral reflectance of surface soil samples from the United States and Brazil [10]. Ramirez et al. analyzed the soil organic matter, total nitrogen, organic carbon, clay and calcium. The results suggested that the forecast equation spectrum of soil organic matter and total nitrogen performed better [11]. Brunet's research pointed out that the grinding treatment of soil reduced the prediction accuracy of organic carbon, while grinding had no obvious effects on total nitrogen detection [12]. Barthès et al. studied how the NIR prediction of carbon and nitrogen content were affected by sample grinding, drying and replication. The results showed that the prediction accuracy was the highest with oven-dried, $0.2 \mathrm{~mm}$ ground soil samples [13]. Hernandez et al. found that the soil particle size and high water content would affect the accuracy of soil organic nitrogen prediction [14]. Nie's research indicated that the soil with the strictest pretreatment (dried, ground, sieved and pressed) had the highest accuracy in predicting the soil nitrogen content [15]. Cozzolino et al. found that the prediction correlation coefficients among coarse sand $(2-0.25 \mathrm{~mm})$, fine sand $(0.25-0.05 \mathrm{~mm})$ and clay sand $(<0.05 \mathrm{~mm})$ were $0.90,0.92$ and 0.96 respectively based on NIR [16]. Nocita et al. used visible near infrared sensors to detect soil organic carbon content under different soil moisture gradients. The soil samples were collected and dried, and then 0.005 grams of water was added to each soil sample. It was found that water content could affect the detection accuracy, and the $\mathrm{R}^{2}$ of the optimum model was 0.74 [17-19].

However, there is little research about the influence of soil drying temperature on soil nitrogen detection by near infrared sensors. Soil texture, soil moisture content and external temperature all affect soil nitrogen detection $[20,21]$. Particularly, the temperature has an influence on the water removal and activity of urease [22]. Coarse samples were dried at $20^{\circ} \mathrm{C}$ for $48 \mathrm{~h}$ for the prediction of $\mathrm{C}$ and $\mathrm{N}$ content using visible near infrared spectroscopy and the comparative method was used to detect their potential mineralization in heterogeneous soil samples [23]. The flat-dry samples were dried at $35^{\circ} \mathrm{C}$ for $12 \mathrm{~h}$ to explore the effects of soil sample pretreatments and standardized rewetting [24]. Soil was subjected to oven drying at $60^{\circ} \mathrm{C}$ for $24 \mathrm{~h}$, after which they were ground and sieved with a $0.002 \mathrm{~m}$ sieve [25] or soil samples were dried at $95^{\circ} \mathrm{C}$ for $24 \mathrm{~h}$ [26] and then sieved for estimating total nitrogen using NIR. Besides this, soil samples were dried at $80^{\circ} \mathrm{C}$ for $8 \mathrm{~h}$ to detect soil nitrogen with different pretreatments using NIR on He's research [27]. Moreover, soil samples were dried naturally, rolled and broken into pieces, then sieved with a 2-mm screen to estimate the organic matter or soil properties using NIR $[28,29]$. However, the impact of drying temperature on soil nitrogen detection has been studied little and the mechanism was not clear yet.

The objective of this study was to investigate the influence of temperature on soil nitrogen detection of loess, calcium soil and black soil as well as find the suitable drying temperature for different soil types to enhance the nitrogen detection accuracy by near infrared sensors. Besides this, this study compared the differences of the near infrared spectra of different soils and different temperatures, and the prediction models established by SPA-MLR, PLS and CARS were analyzed from the perspective of temperatures, soil properties and algorithms.

\section{Materials and Methods}

\subsection{Experimental Materials and Sample Preparation}

The experimental soils included black soil, loess and calcium soil, which were collected from different regions with different physical and chemical properties in China. Among them, black soil comes from the Greater Khingan region, whose $\mathrm{pH}$ value is neutral to slightly alkaline. Loess comes from $\mathrm{Xi}^{\prime}$ an, 
Shanxi province, whose soil properties are loose and porous. Calcium soil with the features of loose and poor structure is from Jinan, Shandong province. The soil sample preparation process was as follows. First, the soil samples were sieved with a 40 mesh sieve $(0.425 \mathrm{~mm})$ and grinded; in addition, the urea solutions with different concentrations were prepared. Second, different nitrogen concentration gradients for three kinds of soils were prepared, that were, loess $(0.09-0.93 \mathrm{~g} / \mathrm{kg}, 0.1 \mathrm{~g} / \mathrm{kg}$ per gradient), calcium soil (0.32-1.17 g/ kg, $0.1 \mathrm{~g} / \mathrm{kg}$ per gradient), black soil (0.46-2.15 g/ kg, $0.2 \mathrm{~g} / \mathrm{kg}$ per gradient). Meanwhile, the three kinds of soils without urea added were set as references. There were 16 samples for each concentration, and each soil type contained 11 nitrogen gradients. Third, the experiments were carried out in four groups, each group containing three soil types. Black soil, loess and calcium soil were dried after $50{ }^{\circ} \mathrm{C}$ for $24 \mathrm{~h}$ (group I), $80^{\circ} \mathrm{C}$ for $18 \mathrm{~h}$ (group II) and $95^{\circ} \mathrm{C}$ for $12 \mathrm{~h}$ (group III) respectively. Other soil samples were dried and then placed at $25^{\circ} \mathrm{C}$ for 12 days (group IV).

\subsection{Spectrometric Determination}

The portable near infrared optical instrument is from Isuzu Optics Corp (Shanghai, China). It is an interferometer instrument which is reflective with two integrated tungsten halogen lamps. The instrument collects spectral information in the range of $900-1700 \mathrm{~nm}$, whose optical resolution is $10 \mathrm{~nm}$ and the signal-noise ratio is 5000:1 in a $1 \mathrm{~s}$ scan; the size is $120 \times 85 \times 54 \mathrm{~mm}$ and the weight is $900 \mathrm{~g}$. The soil detection platform is shown in Figure 1.

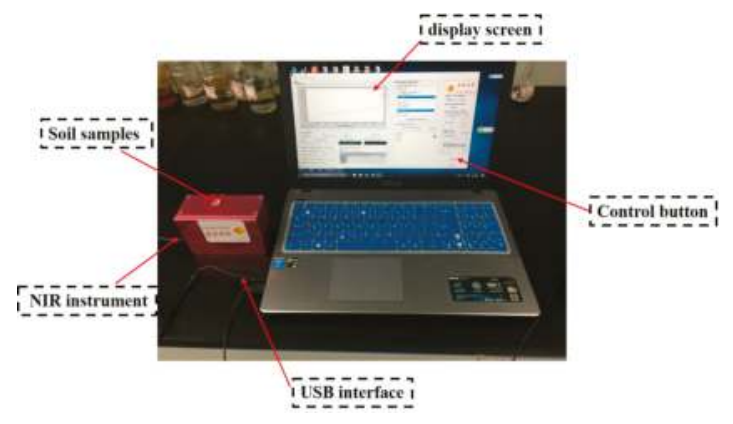

Figure 1. Near infrared (NIR) spectrum soil detection platform.

When the spectrum of soil were measured, the samples were placed on the light source window, which avoided the phenomenon of light leakage since the size of the soil sample is larger than that of the light source window. Before performing the spectroscopic measurement, the instrument should be preheated for $15 \mathrm{~min}$ and be prepared with blackboard and whiteboard correction operation. In order to maintain the integrity of the original soil spectra and the rapidity of the detection process, the spectral acquisition parameter is set up as 400 points, and the spectrum is obtained by averaging three scans.

\subsection{Data Analysis}

Near infrared light is an electromagnetic wave between the infrared and visible light whose wavelength range is from $780 \mathrm{~nm}$ to $2526 \mathrm{~nm}$ [30]. The spectral information originates from the vibration of the $\mathrm{O}-\mathrm{H}, \mathrm{C}-\mathrm{H}$ and $\mathrm{N}-\mathrm{H}$ groups, which can reflect the variety of organic matter in the characteristic signal of the spectral region [31]. According to Lambert absorption law [32], the spectral characteristics would change as material composition or structure changes. However, at the same time, it can also be affected by the soil surface texture, density and uneven distribution of internal components, which is very difficult for all the redundant information of the spectral data to be eliminated, such as the overlap. Therefore, in order to achieve the purpose of qualitative or quantitative analysis of complex mixtures, it is necessary to extract and analyze the weak chemical information by chemometrics method in the spectral analysis. 
In this paper, the original spectra were preprocessed by Savitzky-Golay (S-G) smoothing. Then three modeling methods were used to model and analyze the spectral information. The SPXY method [33] was used to divide the three soil samples into two groups according to the proportion of 2:1, among which 118 soil samples (N1) were calibrated and 58 soil samples (N2) were validated at different temperatures and different soils. All data analysis was based on MATAB R2014a (The Math-Works, Natick, MA, USA).

\subsection{Spectral Preprocessing Method}

Savitzky-Golay (S-G) smoothing [34], also known as polynomial smoothing, uses the weighted average method to quantize the data in the moving window by polynomial least squares fitting as well as emphasizing the central role of the center point. The formula of average wavelengths after S-G smoothing is

$$
x_{k, \text { smooth }}=\overline{x_{i}}=\frac{1}{H} \sum_{i=-w}^{+w} x_{k+1} h i
$$

where $H$ is the normalization factor, $h i$ is the smoothing coefficient and $H=\sum_{I=-W}^{+W} h_{i}$. The measured value multiplied by the smoothing coefficient minimizes the smoothing influence on the useful information. In the experiment, the S-G was used to remove the background noise of the instrument and the noise of the spectrum.

\subsection{SPXY Method}

SPXY, the method of choosing the calibration sample, was put forward on the basis of KS methods by Galvao et al. [35]. The basic principle is that spectrum and concentration variables are considered at the same time to calculate the distance of the samples, the distance formula is as follows:

$$
d_{x y}=\frac{d_{x}(i, j)}{\max _{i, j \in(1, z)}\left[d_{x}(i, j)\right]}+\frac{d_{y}(i, j)}{\max _{i, j \in(1, z)}\left[d_{y}(i, j)\right]}, i, j \in[1, z]
$$

In the formula, $d_{x}(i, j)$ is based on spectral characteristic parameters for the calculation of the distance between the samples, while $d_{y}(i, j)$ is based on concentration characteristic parameters for the calculation of the distance between the samples-which makes the sample in spectrum space and concentration space have the same weightiness-divided by their corresponding maximum standardizing, respectively. $z$ is spectral space.

\subsection{Modeling Method}

\subsubsection{Partial Least Squares Method}

Partial least squares regression (PLSR) is one of the most widely used methods for quantitative correction in chemometrics. In the PLS model, the principal components of the matrix $X$ and the matrix $Y$ are decomposed in order to extract the most comprehensive variables with respect to the dependent variables and maximize the correlation between the principal component and the concentration, which overcomes the negative effects of the multiple correlation of variables and further improves the reliability of the model [36]. In this paper, the whole band spectral data are used as independent variable $X$, and the nitrogen content are considered as the dependent variable $Y$. The minimum cross validation is used to verify the root mean square error cross validation (RMSECV) to determine the optimum number of principal factors.

\subsubsection{Successive Projections Algorithm-Multiple Linear Regression (SPA-MLR)}

Araujo et al. [37] first proposed the selection of spectral variables by means of the successive projections algorithm (SPA). Soares [38] used SPA for cross-classification analysis. The SPA, a forward variable selection method, uses vector projection analysis to find the variable group with minimal 
redundancy information to effectively eliminate the collinear, singular and instable variables in the spectra. Since it reduces the number of variables used in the model and lowers the complexity of the model, the collinear between the vectors is minimized. The multiple liner regression (MLR) adopts the least squares method to estimate the coefficient matrix, resulting in the samples whose numbers are more than the number of spectral variables. Extracting feature wavelength modeling based on SPA-MLR has significance in actual detection because of the useful information for mining spectral data with latent variables [39].

\subsubsection{Competitive Adaptive Weighting Method (CARS)}

The competitive adaptive weighted algorithm method, imitating the evolution of "survival of the fittest" principle, phases out of the invariable wavelength [40]. It uses Monte Carlo sampling or random sampling method to select a part of the sample from the calibration set samples for PLS modeling and repeats this process for hundreds of iterations. In the process of wavelength variable selection, the adaptive weighted sampling method is used to preserve the wavelength variable with the absolute value of PLS regression coefficient, and the wavelength invariable with small absolute value of regression coefficient is removed. In order to obtain a series of wavelength variable subsets, each subset of wavelength variables is modeled by cross validation, and the optimal wavelength variable subset is selected according to the RMSECV value [41].

\subsection{Model Evaluation Index}

In this experiment, the modeling effect is evaluated by the correlation coefficient $R$, the root mean square error (RMSE) and the residual predictive deviation (RPD). The correlation coefficient $\mathrm{R}$ reflects the level of intimacy between variables, root mean square error (RMSE) reflects the accuracy of the model, and RPD reflects the prediction ability of the model. The higher the R and RPD and the lower the RMSE, the better the performance of the prediction model. In this paper, $R_{c}$ and $R_{p}$ represent the correlation coefficient of calibration set and prediction set, respectively, and RMSEC and RMSEP represent the root mean square error of the calibration set and prediction set respectively. Besides this, RPD was suggested to be at least 3 for agriculture applications; $2<\mathrm{RPD}<3$ indicates a model with a good prediction ability; $1.4<\mathrm{RPD}<2$ is an intermediate model needing some improvement; and the $\mathrm{RPD}<1.4$ indicates a poor prediction ability of the model [42].

\section{Results and Discussion}

\subsection{Temperature and Soil Reflectance}

In this experiment, the spectral information of three kinds of soil samples at four temperatures were collected. According to Figure 2, the abscissa of the curve is the wavelength and the ordinate of the curve is the average spectral reflectance.

Figure $2 \mathrm{~A}-\mathrm{D}$ shows the near infrared reflectance curves of the four soils after $50^{\circ} \mathrm{C}, 80^{\circ} \mathrm{C}, 95^{\circ} \mathrm{C}$ drying and $25^{\circ} \mathrm{C}$ placement respectively. First, the near infrared spectra of different soils vary from each other, but the overall trends are similar. The physical properties, chemical properties and soil colors would have certain influence on the absorption of near infrared spectra [43], which results in the differences of spectral curves.

Second, the temperature does affect reflectance strength. The reflectance of the black soil spectral curve at $25^{\circ} \mathrm{C}$ placement is significantly lower than other temperatures. The reason is that the water in the soil cannot be completely dried when soil was placed at $25^{\circ} \mathrm{C}$ and the water absorption of near-infrared spectroscopy is very sensitive. The loess spectral curves are less affected by temperature because the loess are relatively loose, and porous, thus the water content in loess are easy to evaporate while drying. 

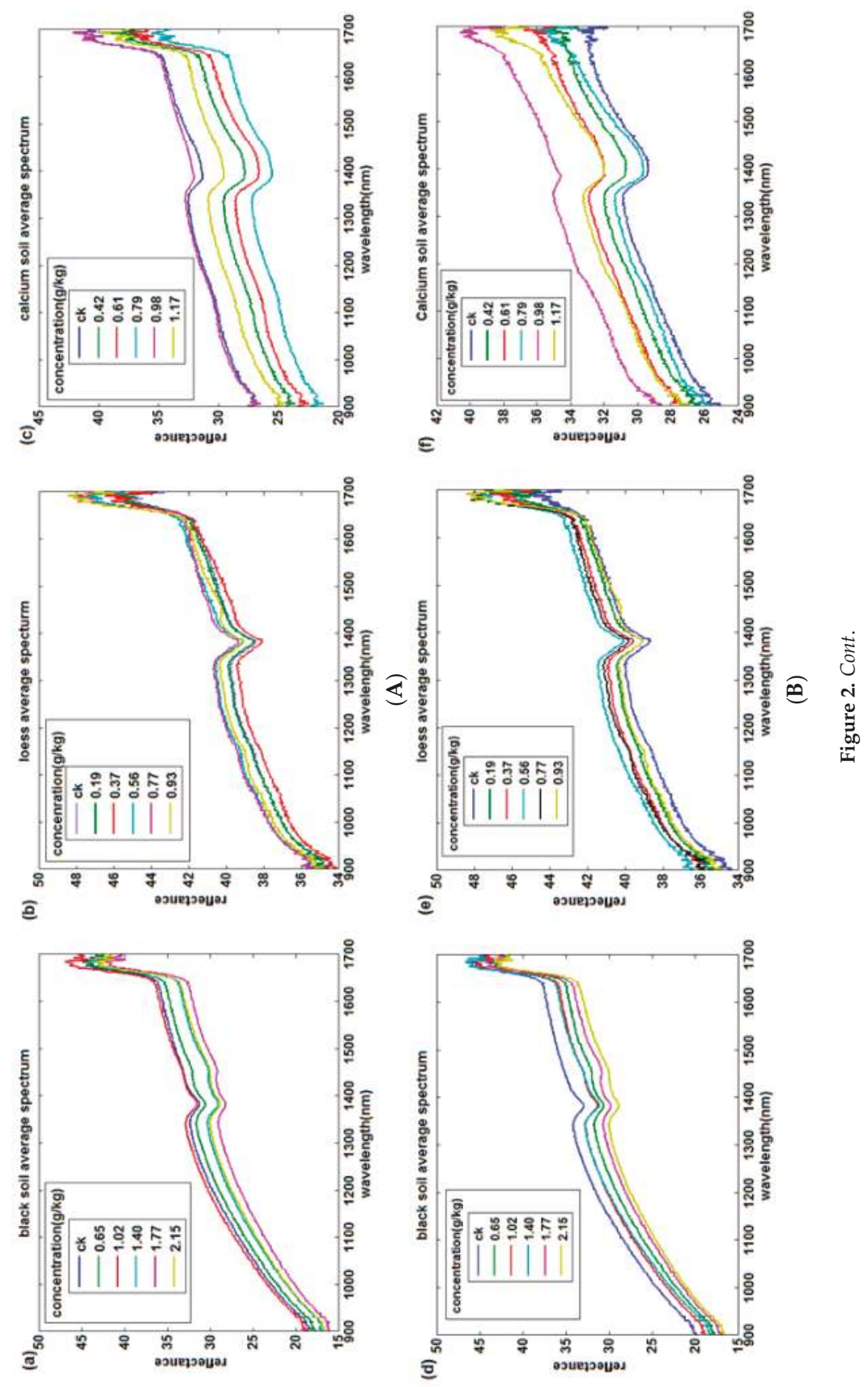

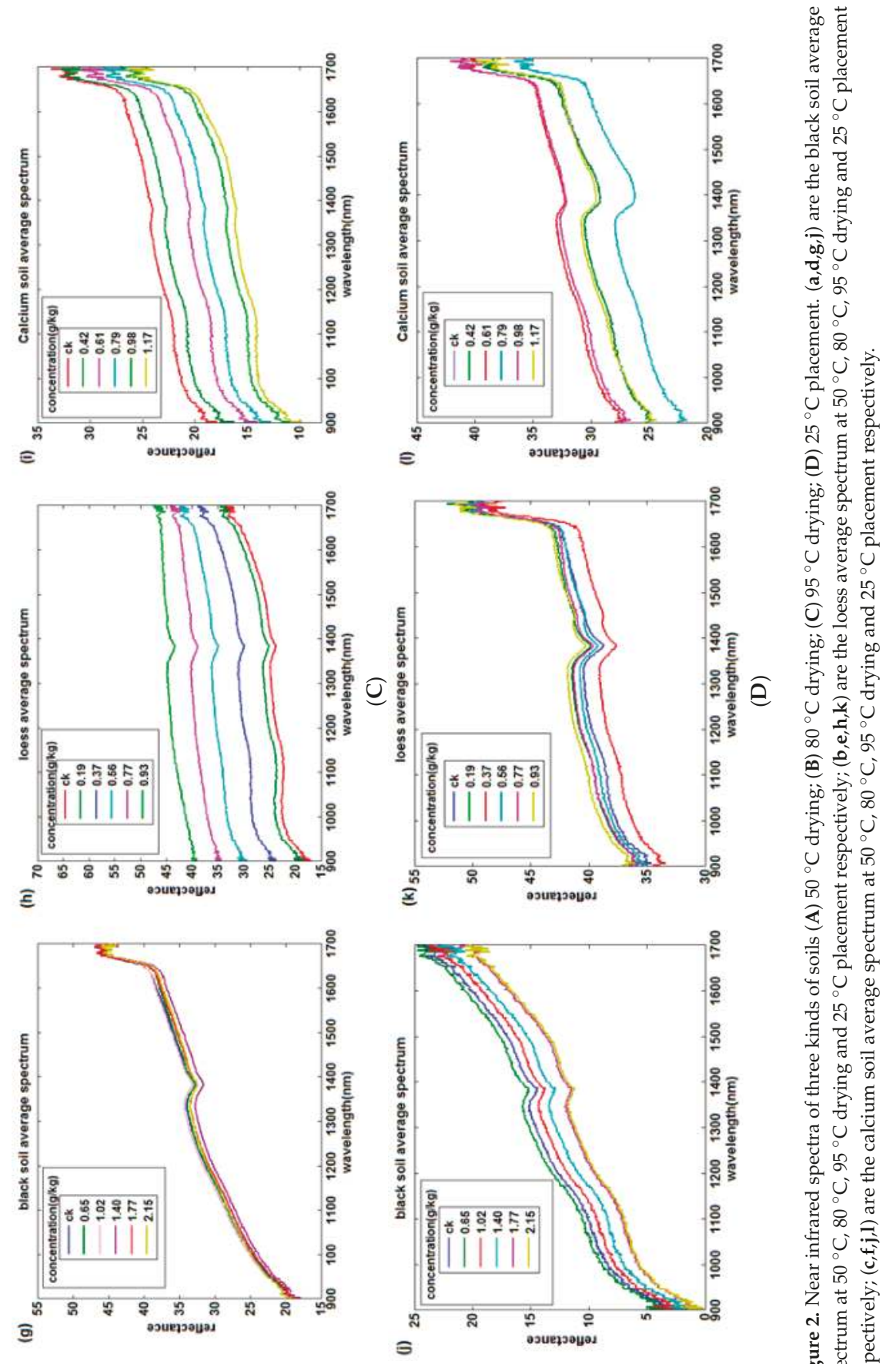

bò 斿 ๑ 눙 ๑ิ

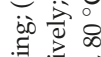

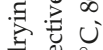
บ เก 苞䒕 « क्ष $\frac{0}{0}$ है पै 0 节害 ¿ై 艺昰节 ฮี के ईू छ

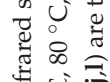
苛帠 䒕

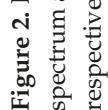


Third, the spectral absorption characteristics of those three soils are different. There is an obvious decrease trend in the band $1385 \mathrm{~nm}$ among the black soil, loess and calcium soil, which is caused by the vibration of $\mathrm{O}-\mathrm{H}$ [44]. However, different soils have different characteristic bands. It is suggested in Figure $2 \mathrm{a}$ that the spectral reflectance of black soil decreases gradually at $1470 \mathrm{~nm}$ with the increase of nitrogen concentration of soil. Figure $2 \mathrm{~h}$ shows that the spectral reflectance of loess decreases weakly near the band $1160 \mathrm{~nm}$ and Figure 2i displays that calcium soil has a spectral absorption at band $1145 \mathrm{~nm}$ when the drying temperature is $95^{\circ} \mathrm{C}$. Those mentioned above might be the characteristic bands of soil total nitrogen in different kinds of soils.

\subsection{Data Modeling Prediction and Analysis}

\subsubsection{SPA-MLR Model}

The maximum number of selected variables was set up to 30 , and the wavelength variables were selected from the 400 spectral variables based on the minimum error, which are shown in Table 1. Figure 3 presents the SPA wavelength number of loess, calcium soil and black soil, where Figure 3A-D represents the variable number of SPA after soil $50{ }^{\circ} \mathrm{C}, 80^{\circ} \mathrm{C}, 95{ }^{\circ} \mathrm{C}$ drying and $25{ }^{\circ} \mathrm{C}$ placement respectively. It is indicated that although the variable numbers and bands differed in the same soil at different temperatures after the variable selection, the characteristic bands are similar when the temperatures varied small. The variable numbers and bands for different soils on the same drying temperature are not the same, suggesting that both soil type and drying temperature have a great influence on wavelength variables selected by SPA-MLR.

Table 1. Successive projections algorithm based on multiple linear regression (SPA-MLR) algorithm variable number and wavelength.

\begin{tabular}{|c|c|c|c|}
\hline Soil Type & Temperature & Variable Number & Wavelength (nm) \\
\hline \multirow{4}{*}{ Loess } & $50{ }^{\circ} \mathrm{C}$ & 15 & $\begin{array}{c}915,1428,1695,1694,1693,1692,1487,1550,1683 \\
1676,1673,1675,1686,1582,1650\end{array}$ \\
\hline & $80^{\circ} \mathrm{C}$ & 7 & $1160,1660,1582,1682,1675,1489,1428$ \\
\hline & $95^{\circ} \mathrm{C}$ & 4 & $1160,1428,1675,1486$ \\
\hline & $25^{\circ} \mathrm{C}$ & 4 & $1424,1488,1694,1428$ \\
\hline \multirow{4}{*}{ Calcium soil } & $50^{\circ} \mathrm{C}$ & 10 & $1651,1154,1438,910,1301,979,1450,1675,1246,1677$ \\
\hline & $80^{\circ} \mathrm{C}$ & 7 & $1651,1675,1678,979,1677,1058,1244$ \\
\hline & $95^{\circ} \mathrm{C}$ & 7 & $1552,1675,1487,1491,1673,921,1650$ \\
\hline & $25^{\circ} \mathrm{C}$ & 5 & $1651,1675,1146,979,1167$ \\
\hline \multirow{4}{*}{ Black soil } & $50{ }^{\circ} \mathrm{C}$ & 5 & $1423,928,1654,1496,1694$ \\
\hline & $80^{\circ} \mathrm{C}$ & 10 & $928,1654,1681,1682,1694,1496,1423,915,1684,1662$ \\
\hline & $95^{\circ} \mathrm{C}$ & 18 & $\begin{array}{c}1650,1680,1682,1694,915,1684,1050,1429,1491 \\
1662,928,925,910,916,918,1662,1675,1690\end{array}$ \\
\hline & $25^{\circ} \mathrm{C}$ & 5 & $1423,925,1681,1496,1694$ \\
\hline
\end{tabular}

The prediction results of SPA-MLR are shown in Table 2 and Figure 4. Both loess $\left(R_{P}=0.9758\right.$, $\mathrm{RMSEP}=0.07 \mathrm{~g} / \mathrm{kg}, \mathrm{RPD}=4.35)$ and calcium soil $\left(\mathrm{R}_{\mathrm{P}}=0.9517, \mathrm{RMSEP}=0.103 \mathrm{~g} / \mathrm{kg}, \mathrm{RPD}=3.24\right)$ obtain the best detection effect after $95^{\circ} \mathrm{C}$ drying, while black soil has a better detection effect after $50{ }^{\circ} \mathrm{C}\left(\mathrm{R}_{\mathrm{P}}=0.9486, \mathrm{RMSEP}=0.22 \mathrm{~g} / \mathrm{kg}, \mathrm{RPD}=2.82\right)$ and $80^{\circ} \mathrm{C}\left(\mathrm{R}_{\mathrm{P}}=0.9373, \mathrm{RMSEP}=0.234 \mathrm{~g} / \mathrm{kg}\right.$, $\mathrm{RPD}=2.55)$ drying, and the three kinds of soils have the worst effect when soils were placed in the $25^{\circ} \mathrm{C}$ environment. 
Table 2. The modeling results of different soils and temperatures by SPA-MLR. RMSEC: root mean square error (RMSE) of the calibration set; RMSEP: RMSE of the prediction set; RPD: residual predictive deviation.

\begin{tabular}{ccccccccc}
\hline \multirow{2}{*}{ Group } & \multirow{2}{*}{ Soil Type } & \multicolumn{3}{c}{ Calibration Set } & \multicolumn{3}{c}{ Prediction Set } \\
\cline { 3 - 8 } & & $\mathbf{N 1}$ & $\mathbf{R}_{\mathbf{c}}$ & $\mathbf{R M S E C}(\mathbf{g} / \mathbf{k g})$ & $\mathbf{N 2}$ & $\mathbf{R}_{\mathbf{p}}$ & RMSEP (g/kg) & RPD \\
\hline \multirow{3}{*}{$1\left(50{ }^{\circ} \mathrm{C}\right)$} & Black soil & 118 & 0.9725 & 0.11 & 58 & 0.9486 & 0.22 & 2.82 \\
& Loess & 118 & 0.9649 & 0.072 & 58 & 0.9265 & 0.13 & 2.34 \\
& Calcium soil & 118 & 0.9681 & 0.039 & 58 & 0.9290 & 0.120 & 2.63 \\
\hline \multirow{2}{*}{$2\left(80^{\circ} \mathrm{C}\right)$} & Black soil & 118 & 0.9203 & 0.251 & 58 & 0.9373 & 0.234 & 2.55 \\
& Loess & 118 & 0.9727 & 0.067 & 58 & 0.9541 & 0.090 & 3.20 \\
& Calcium soil & 118 & 0.9492 & 0.108 & 58 & 0.9320 & 0.162 & 2.12 \\
\hline \multirow{3}{*}{$3\left(95^{\circ} \mathrm{C}\right)$} & Black soil & 118 & 0.9692 & 0.156 & 58 & 0.9132 & 0.282 & 2.16 \\
& Loess & 118 & 0.9660 & 0.075 & 58 & 0.9758 & 0.070 & 4.35 \\
& Calcium soil & 118 & 0.9670 & 0.087 & 58 & 0.9517 & 0.103 & 3.24 \\
\hline \multirow{2}{*}{$4\left(25^{\circ} \mathrm{C}\right)$} & Black soil & 118 & 0.6061 & 0.486 & 58 & 0.7129 & 0.418 & 1.25 \\
& Loess & 118 & 0.5473 & 0.247 & 58 & 0.6217 & 0.246 & 1.20 \\
& Calcium soil & 118 & 0.4391 & 0.302 & 58 & 0.5824 & 0.365 & 0.94 \\
\hline
\end{tabular}



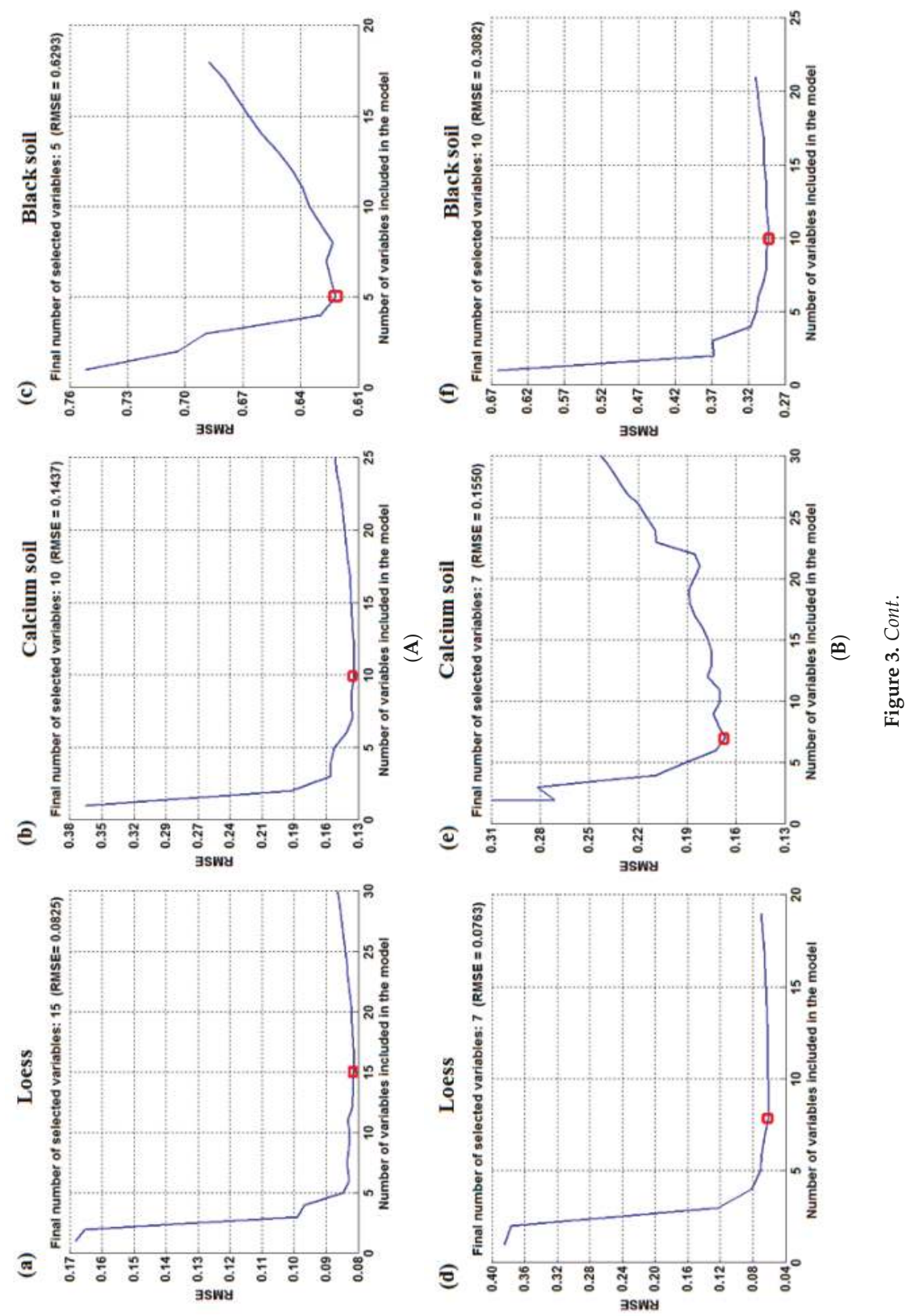

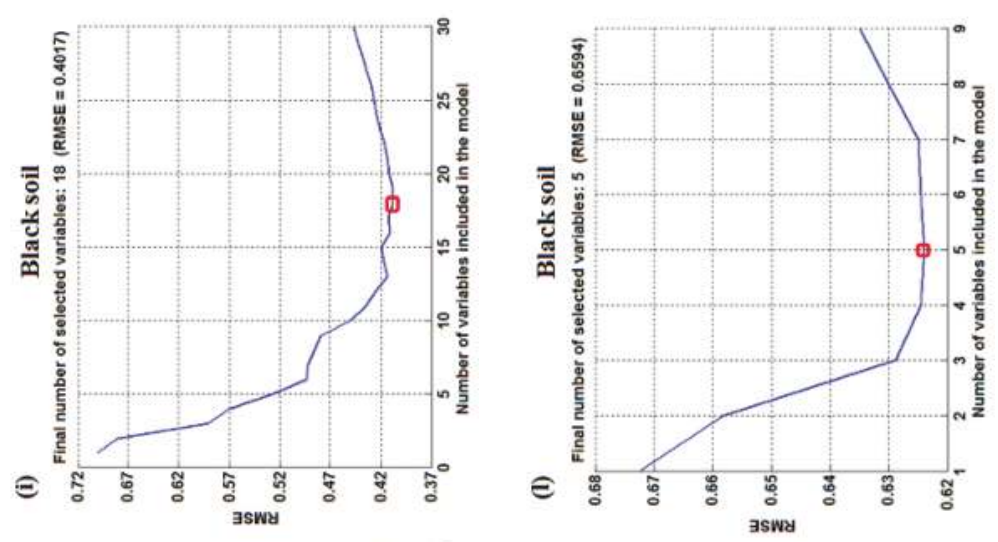

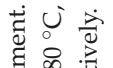

80

范

U.

늘 छัญ

อิ है छ

के̀ चै

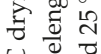

บ ए

늘 छ

प्ञ

के चु ป

究器

U

$\infty$ 这文
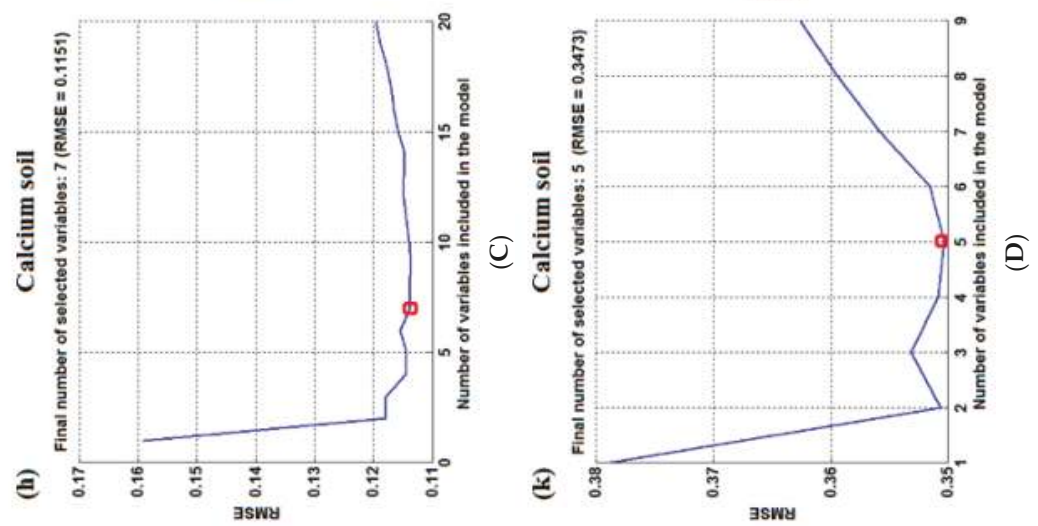

ê.

क.

60

त्र

บ

เి

¿

हू

का $\frac{0}{2}$

ล ن

놀

造 壳

की

कृ ⿷

붕

늠 조
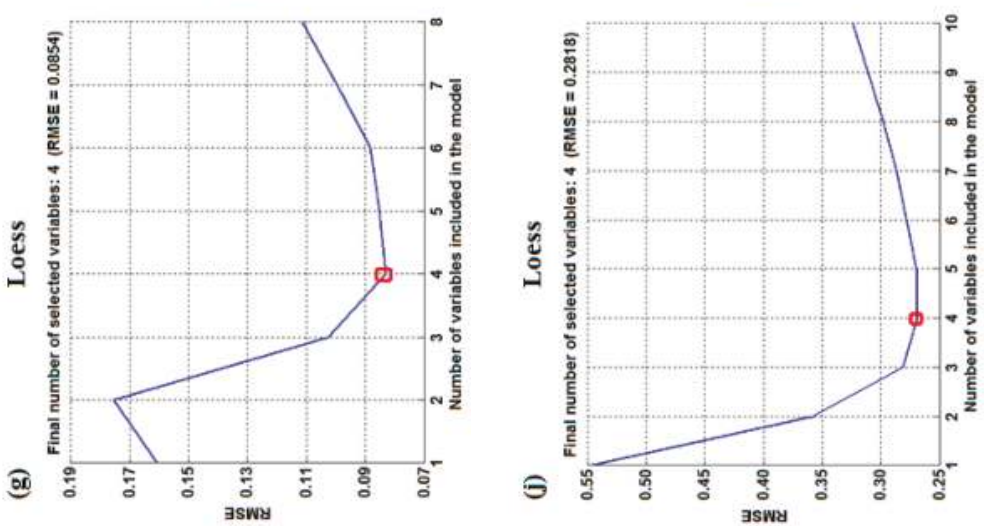

ฮิ

⿷匚

엉

ช

के

उั हैं

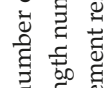

墕

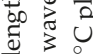

范

उ.

气ิ సี

ญ

整突它 
On the one hand, the reason might be that medium and high temperature could stimulate the activity of soil urease and remove fully water in soil [45]. Meanwhile, soil water content was preserved little when soils were placed at $25^{\circ} \mathrm{C}$ during the long time. Compared with $\mathrm{O}-\mathrm{H}$ bond, the $\mathrm{N}-\mathrm{H}$ bond exists mostly in multiple frequency or combination frequency, which was relatively weak in soil spectra and affects the extraction of soil nitrogen information [46].

On the other hand, the information of physical and chemical properties, including iron oxides, particle size distribution and surface roughness vary dramatically in different soils, which reduce or even obscure the spectral effect of nitrogen in soil [43]. Among them, loess mainly contains $\mathrm{SiO}_{2}$, $\mathrm{Al}_{2} \mathrm{O}_{3}$ and $\mathrm{CaO}$ with the properties of being loose and porous, and calcium soil mainly consisted of $\mathrm{CaCO}_{3}$. Both loess and calcium soil have few $\mathrm{O}-\mathrm{H}$ bonds when they were dried, which interferes with the NIR spectrum to a small extent [47].

Hence, the prediction accuracy of loess and calcium soil was the optimum among three kinds of soils. However, the black soil obtains relatively low prediction accuracy because the abundant organic matter and humus in black soil have a strong absorption in NIR, resulting in adverse interference for nitrogen detection [48].

Figure 4 indicates that black soil nitrogen detection ranges from $0.93 \mathrm{~g} / \mathrm{kg}$ to $1.87 \mathrm{~g} / \mathrm{kg}$, and the detection of nitrogen in loess is concentrated in the vicinity of $0.47 \mathrm{~g} / \mathrm{kg}$, while the soil nitrogen calcium concentrates from $0.47 \mathrm{~g} / \mathrm{kg}$ to $0.93 \mathrm{~g} / \mathrm{kg}$ when soils were placed at $25^{\circ} \mathrm{C}$, which largely deviates from the true values of the nitrogen in soil. The reason might be that the water content in soil was relatively higher when it was placed at $25{ }^{\circ} \mathrm{C}$ than when drying at other medium and high temperatures, which affects the extraction of soil nitrogen information [49].

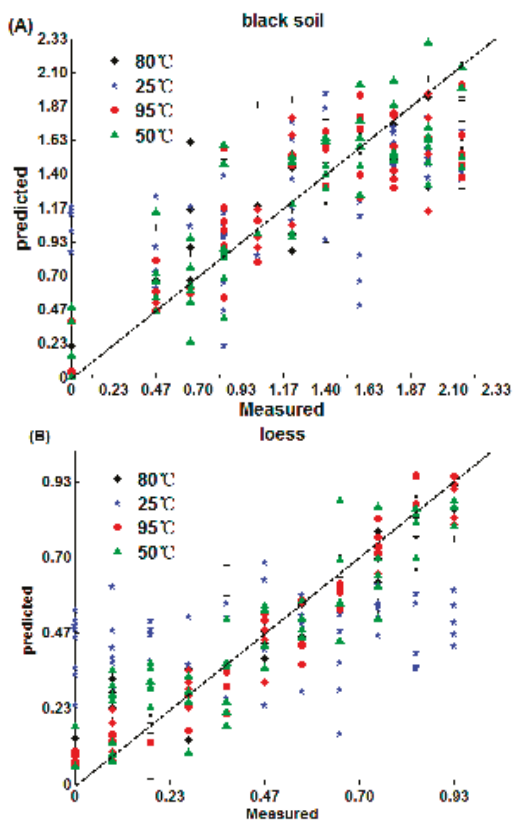

Figure 4. Cont. 


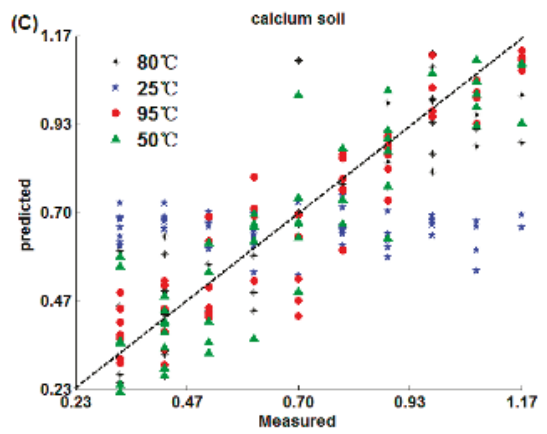

Figure 4. SPA-MLR algorithm prediction results: (A) black soil; (B) loess; (C) calcium soil.

\subsubsection{PLS Method Model}

The prediction results of PLS are shown in Table 3 and Figure 5. The detection accuracy from high to low is loess, calcium soil and black soil, in that order. Moreover, both loess $\left(R_{P}=0.9721\right.$, RMSEP $=0.067 \mathrm{~g} / \mathrm{kg}$, RPD $=4.34)$ and calcium soil $\left(\mathrm{R}_{\mathrm{P}}=0.9588, \mathrm{RMSEP}=0.094 \mathrm{~g} / \mathrm{kg}, \mathrm{RPD}=3.89\right)$ have the best detection accuracy after $95^{\circ} \mathrm{C}$ drying. However, the black soil $\left(R_{P}=0.9216, R M S E P=0.228 \mathrm{~g} / \mathrm{kg}\right.$, $\mathrm{RPD}=2.72$ ) after $50{ }^{\circ} \mathrm{C}$ drying achieves the best detection accuracy of black soil. Moreover, the results of PLS and SPA-MLR are similar, which indicates that medium and high temperatures are helpful for the soil nitrogen detection and the reasons have been discussed in Section 3.2.1.

Table 3. The modeling results of different soil types and temperatures by partial least squares (PLS).

\begin{tabular}{|c|c|c|c|c|c|c|c|c|}
\hline \multirow{2}{*}{ Group } & \multirow{2}{*}{ Soil Type } & \multicolumn{3}{|c|}{ Calibration Set } & \multicolumn{4}{|c|}{ Prediction Set } \\
\hline & & N1 & $\mathbf{R}_{\mathrm{c}}$ & RMSEC (g/kg) & N2 & $\mathbf{R}_{\mathrm{p}}$ & RMSEP (g/kg) & RPD \\
\hline \multirow{3}{*}{$1\left(50^{\circ} \mathrm{C}\right)$} & Black soil & 118 & 0.9525 & 0.198 & 58 & 0.9216 & 0.228 & 2.72 \\
\hline & Loess & 118 & 0.9609 & 0.077 & 58 & 0.9466 & 0.112 & 2.71 \\
\hline & Calcium soil & 118 & 0.9881 & 0.057 & 58 & 0.9258 & 0.128 & 2.69 \\
\hline \multirow{3}{*}{$2\left(80^{\circ} \mathrm{C}\right)$} & Black soil & 118 & 0.9417 & 0.216 & 58 & 0.9368 & 0.217 & 2.82 \\
\hline & Loess & 118 & 0.9935 & 0.033 & 58 & 0.9568 & 0.090 & 3.31 \\
\hline & Calcium soil & 118 & 0.9173 & 0.132 & 58 & 0.9316 & 0.119 & 2.75 \\
\hline \multirow{3}{*}{$3\left(95^{\circ} \mathrm{C}\right)$} & Black soil & 118 & 0.9906 & 0.086 & 58 & 0.9065 & 0.273 & 2.22 \\
\hline & Loess & 118 & 0.9739 & 0.066 & 58 & 0.9721 & 0.067 & 4.34 \\
\hline & Calcium soil & 118 & 0.9269 & 0.129 & 58 & 0.9588 & 0.094 & 3.89 \\
\hline \multirow{3}{*}{$4\left(25^{\circ} \mathrm{C}\right)$} & Black soil & 118 & 0.7773 & 0.391 & 58 & 0.6849 & 0.480 & 1.26 \\
\hline & Loess & 118 & 0.3507 & 0.267 & 58 & 0.4529 & 0.287 & 1.09 \\
\hline & Calcium soil & 118 & 0.5332 & 0.286 & 58 & 0.5568 & 0.258 & 1.34 \\
\hline
\end{tabular}

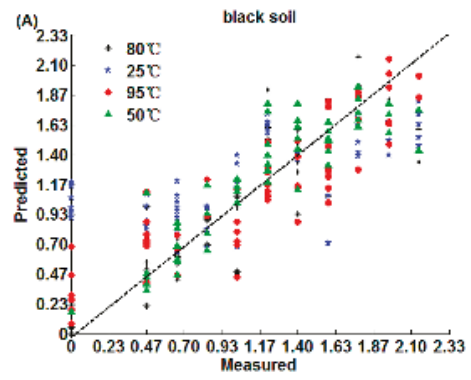

Figure 5. Cont. 


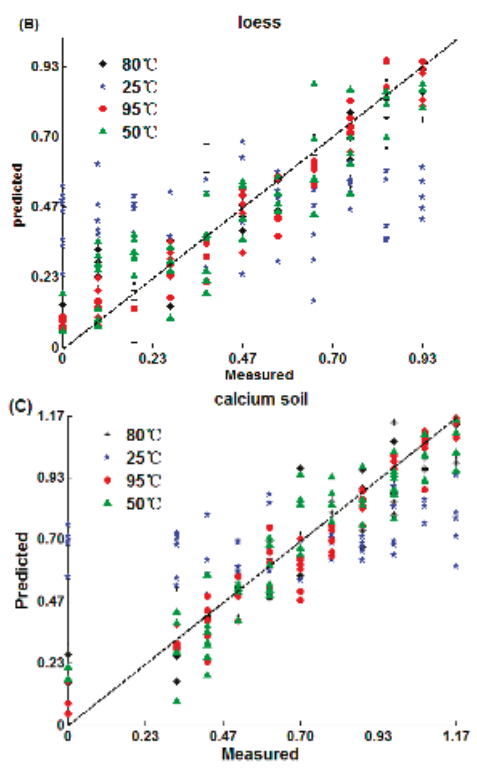

Figure 5. The prediction effect by PLS: (A) black soil; (B) loess; (C) calcium soil.

\subsubsection{CARS Model Methods}

The setting times of the CARS variable selection was 500, and the variable selection process are shown in Figure 6. Figure $6 \mathrm{a}-\mathrm{d}$ show the variable selection process of loess after $50{ }^{\circ} \mathrm{C}, 80^{\circ} \mathrm{C}$ and $95^{\circ} \mathrm{C}$ drying and $25^{\circ} \mathrm{C}$ placement, respectively. The number of final variable numbers and principal components are shown in Table 4. After the selection, the number of variables and bands differ in the same soil at different drying temperatures and the number of variables and bands in different soil types on the same drying temperature vary from each other as well, indicating that both soil type and soil drying temperature have a great influence on wavelength variables selected by CARS.

Table 4. The selected variables and principal component number.

\begin{tabular}{cccc}
\hline Soil Type & Temperature & Selected Variables Number & Principal Component Number \\
\hline \multirow{4}{*}{ Loess } & $50^{\circ} \mathrm{C}$ & 20 & 5 \\
& $80^{\circ} \mathrm{C}$ & 40 & 6 \\
& $95^{\circ} \mathrm{C}$ & 19 & 3 \\
\hline \multirow{2}{*}{ Calcium } & $25^{\circ} \mathrm{C}$ & 29 & 3 \\
soil & $50^{\circ} \mathrm{C}$ & 18 & 3 \\
& $80^{\circ} \mathrm{C}$ & 26 & 5 \\
\hline \multirow{3}{*}{ Black soil } & $95^{\circ} \mathrm{C}$ & 20 & 5 \\
\hline & $25^{\circ} \mathrm{C}$ & 14 & 3 \\
\hline & $50^{\circ} \mathrm{C}$ & 21 & 5 \\
& $80^{\circ} \mathrm{C}$ & 11 & 5 \\
\hline
\end{tabular}




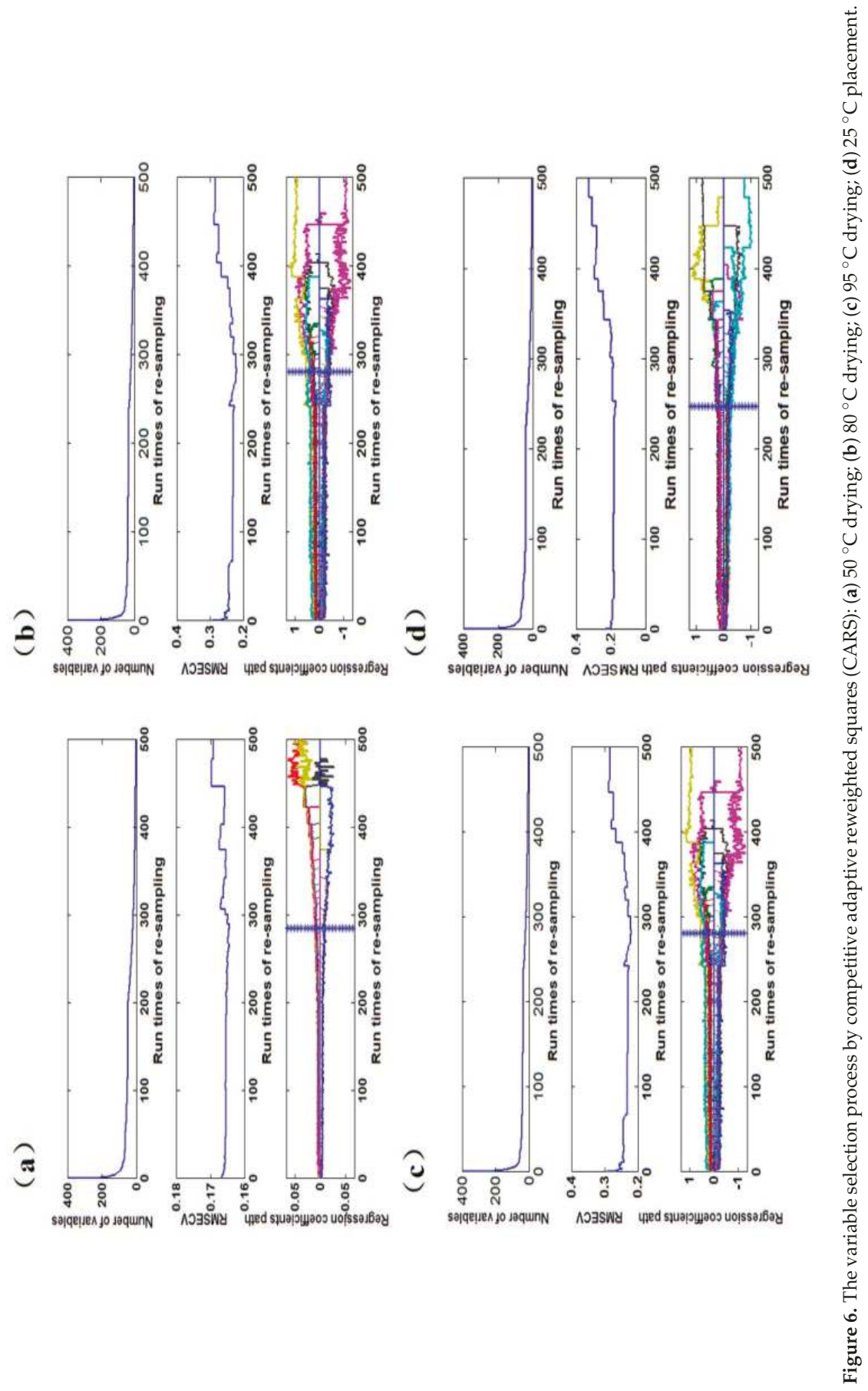


The prediction results of the CARS are shown in Figure 7 and Table 5. The results of nitrogen prediction of loess $\left(R_{P}=0.9612, R M S E P=0.079 \mathrm{~g} / \mathrm{kg}, \mathrm{RPD}=3.92\right)$ and calcium soil $\left(\mathrm{R}_{\mathrm{P}}=0.9472, \mathrm{RMSEP}=0.112 \mathrm{~g} / \mathrm{kg}\right.$, $\mathrm{RPD}=3.07$ ) are the best after $95{ }^{\circ} \mathrm{C}$ drying. While the black nitrogen prediction was best after $80{ }^{\circ} \mathrm{C}$ drying. Also, the three kinds of soils have the worst effects when soils were placed at $25^{\circ} \mathrm{C}$. The results are similar to the SPA-MLR and PLS, which indicates that medium and high temperatures are beneficial to soil nitrogen detection and the reasons have been discussed in Section 3.2.1.

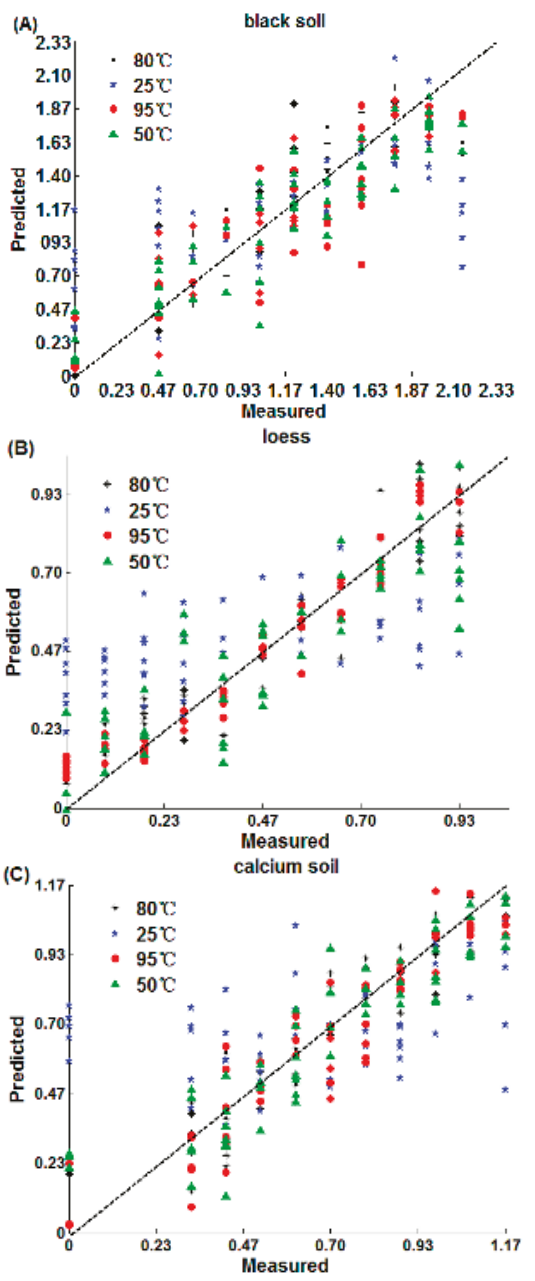

Figure 7. CARS prediction results: (A) black soil; (B) loess; (C) calcium soil.

Table 5. The modeling results of different soils and temperatures by CARS.

\begin{tabular}{lcccccccc}
\hline \multirow{2}{*}{ Group } & \multirow{2}{*}{ Soil Type } & \multicolumn{4}{c}{ Calibration Set } & \multicolumn{3}{c}{ Prediction Set } \\
\cline { 3 - 9 } & & $\mathbf{N} 1$ & $\mathbf{R}_{\mathbf{c}}$ & RMSEC $(\mathbf{g} / \mathbf{k g})$ & $\mathbf{N} 2$ & $\mathbf{R}_{\mathbf{p}}$ & RMSEP $(\mathbf{g} / \mathbf{k g})$ & $\mathbf{R P D}$ \\
\hline \multirow{2}{*}{$1\left(50^{\circ} \mathrm{C}\right)$} & Black soil & 118 & 0.9625 & 0.163 & 58 & 0.9416 & 0.185 & 2.95 \\
& Loess & 118 & 0.9009 & 0.1875 & 58 & 0.8966 & 0.1885 & 1.61 \\
& Calcium soil & 118 & 0.9281 & 0.1549 & 58 & 0.8977 & 0.1414 & 2.43 \\
\hline
\end{tabular}


Table 5. Cont.

\begin{tabular}{ccccccccc}
\hline \multirow{2}{*}{$2\left(80^{\circ} \mathrm{C}\right)$} & Black soil & 118 & 0.9205 & 0.25 & 58 & 0.9288 & 0.237 & 2.68 \\
& Loess & 118 & 0.93 & 0.106 & 58 & 0.9412 & 0.105 & 2.90 \\
& Calcium soil & 118 & 0.9117 & 0.136 & 58 & 0.9258 & 0.119 & 2.79 \\
\hline \multirow{3}{*}{$3\left(95^{\circ} \mathrm{C}\right)$} & Black soil & 118 & 0.9731 & 0.146 & 58 & 0.9021 & 0.277 & 2.24 \\
& Loess & 118 & 0.9609 & 0.077 & 58 & 0.9612 & 0.079 & 3.92 \\
& Calcium soil & 118 & 0.9381 & 0.118 & 58 & 0.9472 & 0.112 & 3.07 \\
\hline \multirow{3}{*}{$4\left(25^{\circ} \mathrm{C}\right)$} & Black soil & 118 & 0.5458 & 0.551 & 58 & 0.5763 & 0.476 & 1.31 \\
& Loess & 118 & 0.5615 & 0.247 & 58 & 0.3862 & 0.265 & 1.15 \\
& Calcium soil & 118 & 0.3698 & 0.322 & 58 & 0.4241 & 0.304 & 1.13 \\
\hline
\end{tabular}

\subsection{Analysis and Comparison of Results}

Figure 8 shows the results of different models of three kinds of soils at different temperatures. First, the soil nitrogen detection effect ranking of loess and calcium soil was $95^{\circ} \mathrm{C}, 80^{\circ} \mathrm{C}, 50^{\circ} \mathrm{C}$ and $25^{\circ} \mathrm{C}$, while in black soil, the effect of soil nitrogen detection was $50^{\circ} \mathrm{C}, 80^{\circ} \mathrm{C}, 95^{\circ} \mathrm{C}$ and $25^{\circ} \mathrm{C}$, in that order.

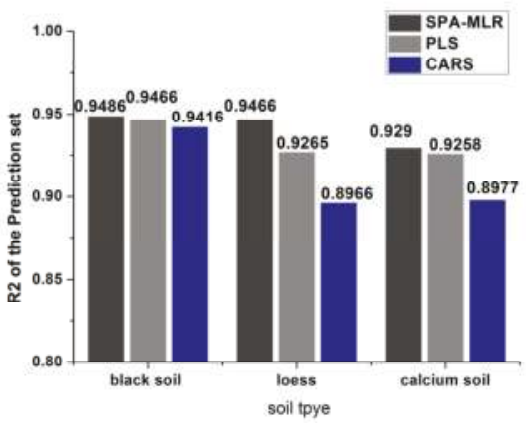

(A)

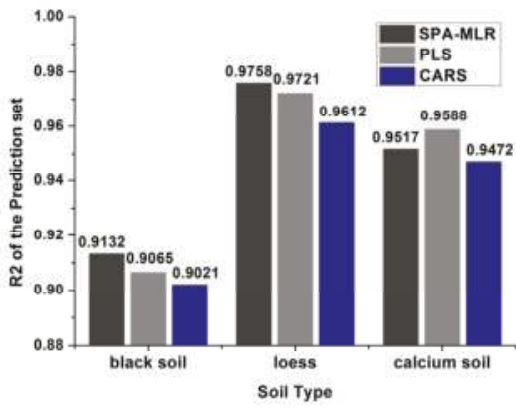

(C)

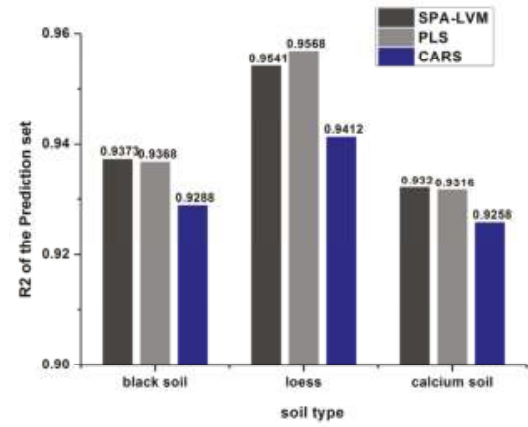

(B)

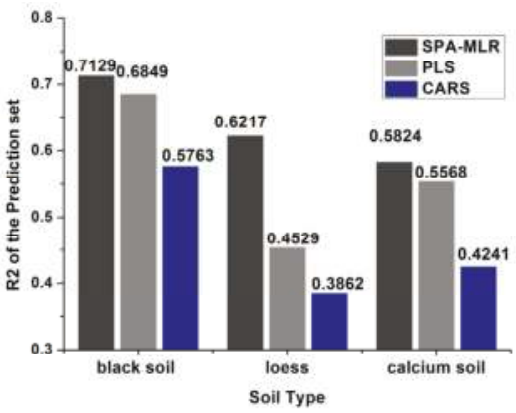

(D)

Figure 8. The prediction results of three kinds of soils at different drying temperatures based on three algorithms: (A) $50{ }^{\circ} \mathrm{C}$ drying; (B) $80^{\circ} \mathrm{C}$ drying; (C) $95^{\circ} \mathrm{C}$ drying; (D) $25^{\circ} \mathrm{C}$ placement.

This indicated that urease in soil was activated and the water content was easier to fully evaporate under medium and high drying temperatures, thus the detection effects were obviously better. However, the information of physical and chemical properties including iron oxides and particle size distribution were different in soil [43], which caused the different detection results when different soils were dried at the same temperature. Moreover, the prediction effects of black soil in $50^{\circ} \mathrm{C}, 85^{\circ} \mathrm{C}$ and $90^{\circ} \mathrm{C}$ were worse than that of the loess and the calcium soil, but the results were better than those of other soils when soils were placed at $25^{\circ} \mathrm{C}$, which not only indicated that the physicochemical properties of the black soil caused 
the difference of the results, but also suggested that the little water preserved in the drying process had the least influence on the nitrogen detection in black soil than other soils.

Second, different algorithms had different effects on soil nitrogen detection based on the same temperature. The overall detection effect ranking from better to worse was SPA-MLR, PLS and CARS, and the prediction accuracy of SPA-MLR and PLS was similar. The reason might be that SPA-MLR could efficiently eliminate redundant variables, which made the results more accurate and the detection precision higher [39]. The comprehensive variables extracted by PLS performed well in summarizing the information of independent variables, explaining dependent variables and eliminating noise interference in the system, which effectively handled the variables multiple correlation problem [42]. In the CARS, it was difficult to find the best or optimal value of the noise threshold and select the randomness of the characteristic variables, which leaded to the poor prediction results [41].

Figure 9 shows the prediction effects of soils under different drying temperatures using different algorithms.

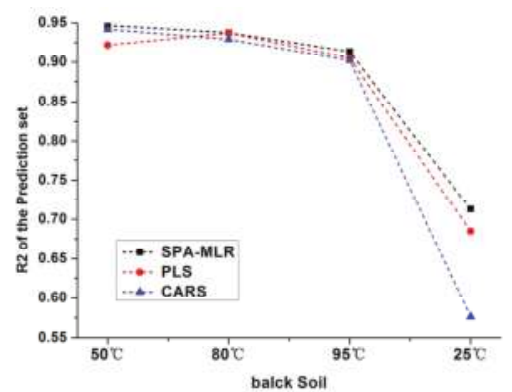

(A)

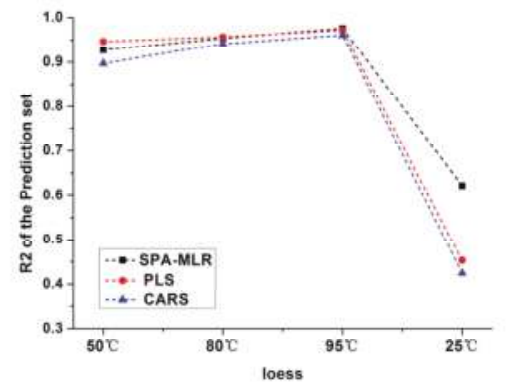

(B)

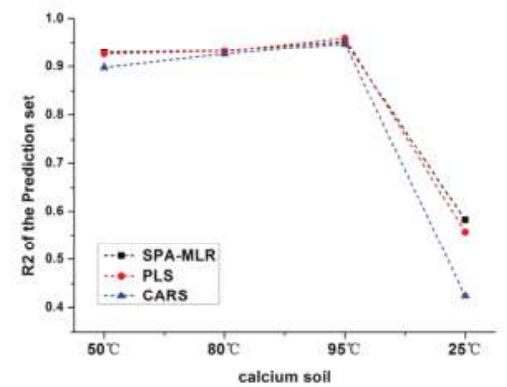

(C)

Figure 9. Prediction results of three kinds of soil based on different temperatures algorithms: (A) black soil; (B) loess; (C) calcium soil. 
As can be seen from the Figure 9, no matter which algorithm was used, the prediction accuracy of black soil was better when the drying temperatures ranged from $50{ }^{\circ} \mathrm{C}$ to $80^{\circ} \mathrm{C}$. While the detection accuracy of loess and calcium soil nitrogen obtained better results when the temperatures were in the range of $80^{\circ} \mathrm{C}$ to $90^{\circ} \mathrm{C}$.

\section{Conclusions}

In this paper, three kinds of soils were used to investigate the effects of drying temperature on soil nitrogen detection using near infrared sensors. The NIR spectra of different soils varied greatly and the spectra of the same soil type changed greatly under different drying temperatures.

The main conclusions are as follows: (1) The drying temperature does have an influence on soil nitrogen detection by near infrared sensors and the suitable drying temperatures for different soils were different, which indicated that it was necessary to find the suitable drying temperature for different soil types to enhance the detection accuracy; (2) the drying temperatures ranged from $50{ }^{\circ} \mathrm{C}$ to $80{ }^{\circ} \mathrm{C}$ for black soil nitrogen detection accuracy were better than other temperatures, while the loess and calcium soil nitrogen detection had better results when the drying temperature was $95{ }^{\circ} \mathrm{C}$. The $\mathrm{O}-\mathrm{H}$ bonds of water in soil might be the main factor influencing the prediction accuracy when soils were placed at $25^{\circ} \mathrm{C}$. Besides this, the suitable drying temperatures for more soil types should be further researched; (3) the SPA-MLR and PLS models obtained a better prediction effect for soils, while CARS performed worse. In conclusion, drying temperature had an obvious influence on the detection of soil nitrogen by near infrared sensors, and the suitable drying temperature for different soil types was of great significance in enhancing the detection accuracy.

Acknowledgments: This work is supported by National keypoint research and invention program of the thirteenth (2016YFD0700304) and Natural Science Foundations of China (Grant No. 61405175).

Author Contributions: This work presented here was carried out collaborations among all authors. Pengcheng Nie and Tao Dong conceived the idea. Pengcheng Nie, Tao Dong, Shupei Xiao worked together on associated data and carried out the experimental work. Pengcheng Nie and Tao Dong drafted the manuscript. Shupei Xiao and Yong He provided their experience and co-wrote the paper with Pengcheng Nie. All authors contributed, reviewed and improved the manuscript.

Conflicts of Interest: The authors declare no conflict of interest.

\section{References}

1. Todorova, M.; Atanassova, S.; Lange, H.; Pavlov, D. Estimation of total N, total P, pH and electrical conductivity in soil by near-infrared reflectance sensors. Agric. Sci. Technol. 2011, 3, 50-54.

2. Muñozhuerta, R.F.; Guevaragonzalez, R.G.; Contrerasmedina, L.M.; Torrespacheco, I.; Pradoolivarez, J.; Ocampovelazquez, R.V. A Review of Methods for Sensing the Nitrogen Status in Plants: Advantages, Disadvantages and Recent Advances. Sensors 2013, 13, 10823-10843. [CrossRef] [PubMed]

3. Liu, Y.D.; Xiong, S.S.; Liu, D.L. Applications of near infrared reflectance sensors technique (NIRS) to soil attributes research. Sens. Spectr. Anal. 2014, 34, 2639-2644.

4. Bevilacqua, M.; Bucci, R.; Materazzi, S.; Marini, F. Application of near infrared (NIR) sensors coupled to chemometrics for dried egg-pasta characterization and egg content quantification. Food Chem. 2013, 140, 726-734. [CrossRef] [PubMed]

5. Filippi, J.J.; Belhassen, E.; Baldovini, N.; Brevard, H.; Meierhenrich, U.J. Qualitative and quantitative analysis of vetiver essential oils by comprehensive two-dimensional gas chromatography and comprehensive two-dimensional gas chromatography/mass spectrometry. J. Chromatogr. A 2013, 1288, 127-148. [CrossRef] [PubMed]

6. Dalal, R.C. Simultaneous determination of moisture, organic carbon, and total nitrogen by near infrared reflectance spectrophotometry. Soil Sci. Soc. Am. J. 1986, 50, 120-123. [CrossRef]

7. Lee, K.S.; Lee, D.H.; Sudduth, K.A.; Chung, S.O.; Kitchen, N.R.; Drummond, S.T. Wavelength identification and diffuse reflectance estimation for surface and profile soil properties. Trans. ASABE 2009, 52, 683-695. [CrossRef] 
8. Cen, Y.L.; Song, T.; He, Y.; Bao, Y.D. Rapid detection method of soil organic matter contents using visible/near infrared diffuse reflectance spectral data. J. Zhejiang Univ. 2011, 37, 300-306.

9. He, Y.; Huang, M.; García, A.; Hernández, A.; Song, H. Prediction of soil macronutrients content using near-infrared sensors. Comput. Electron. Agric. 2007, 58, 144-153. [CrossRef]

10. Stoner, E.R.; Baumgardner, M.F. Characteristic variations in reflectance of surface soils. Soil Sci. Soc. Am. J. 1981, 45, 1161-1165. [CrossRef]

11. Ramirez-Lopez, L.; Behrens, T.; Schmidt, K.; Stevens, A.; Demattê, J.A.M.; Scholten, T. The spectrum-based learner: A new local approach for modeling soil vis-NIR spectra of complex datasets. Geoderma 2013, 195-196, 268-279. [CrossRef]

12. Brunet, D.; Barthès, B.G.; Chotte, J.L.; Feller, C. Determination of carbon and nitrogen contents in alfisols, oxisols and ultisols from africa and brazil using NIRS analysis: Effects of sample grinding and set heterogeneity. Geoderma 2007, 139, 106-117. [CrossRef]

13. Barthès, B.; Brunet, D.; Ferrer, H.; Chotte, J.L.; Feller, C. Determination of total carbon and nitrogen content in a range of tropical soils using near infrared sensors: Influence of replication and sample grinding and drying. J. Near Infrared Sens. 2006, 14, 341-348. [CrossRef]

14. Hermamez, A.H.; He, Y.; Pereira, A.G. Non-destructive measurement of acidity, soluble solids and firmness of satsuma mandarin using Vis/NIR-sensors techniques. J. Food Eng. 2006, 77, 313-319.

15. Nie, P.C.; Dong, T.; He, Y.; Qu, F.F. Detection of soil nitrogen using near infrared sensors based on soil pretreatment and algorithms. Sensors 2017, 17, 1102. [CrossRef] [PubMed]

16. Cozzolino, D.; Morón, A. Potential of near-infrared reflectance sensors and chemometrics to predict soil organic carbon fractions. Soil Tillage Res. 2006, 85, 78-85. [CrossRef]

17. Nocita, M.; Kooistra, L.; Bachmann, M.; Müller, A.; Powell, M.; Weel, S. Predictions of soil surface and topsoil organic carbon content through the use of laboratory and field sensors in the Albany Thicket Biome of Eastern Cape Province of South Africa. Geoderma 2011, 167, 295-302. [CrossRef]

18. Nocita, M.; Stevens, A.; Noon, C.; Wesemael, B.V. Prediction of soil organic carbon for different levels of soil moisture using Vis-NIR sensors. Geoderma 2013, 199, 37-42. [CrossRef]

19. Nocita, M.; Stevens, A.; Toth, G.; Panagos, P.; Wesemael, B.V.; Montanarella, L. Prediction of soil organic carbon content by diffuse reflectance sensors using a local partial least square regression approach. Soil Biol. Biochem. 2014, 68, 337-347. [CrossRef]

20. Chen, S.K.; Subler, S.; Edwards, C.A. Effects of agricultural biostimulants on soil microbial activity and nitrogen dynamics. Appl. Soil Ecol. 2002, 19, 249-259. [CrossRef]

21. Chen, S.K.; Edwards, C.A.; Subler, S. The influence of two agricultural biostimulants on nitrogen transformations, microbial activity, and plant growth in soil microcosms. Soil Biol. Biochem. 2003, 35, 9-19. [CrossRef]

22. Cartes, P.; Jara, A.A.; Demanet, R.; de la Mora, M. Urease activity and nitrogen mineralization kinetics as affected by temperature and urea input rate in Southern Chilean Andisols. J. Soil Sci. Plant Nutr. 2009, 9, 69-82. [CrossRef]

23. Fystro, $\mathrm{G}$. The prediction of $\mathrm{C}$ and $\mathrm{N}$ content and their potential mineralisation in heterogeneous soil samples using Vis-NIR spectroscopy and comparative methods. Plant Soil 2002, 246, 139-149. [CrossRef]

24. Bo, S. Effects of soil sample pretreatments and standardised rewetting as interacted with sand classes on Vis-NIR predictions of clay and soil organic carbon. Geoderma 2010, 158, 15-22.

25. Mouazen, A.M.; Baerdemaeker, J.D.; Ramon, H. Towards development of on-line soil moisture content sensor using a fibre-type NIR spectrophotometer. Soil Tillage Res. 2005, 80, 171-183. [CrossRef]

26. Kuang, B.; Mouazen, A.M. Non-biased prediction of soil organic carbon and inorganic nitrogen with Vis-NIR spectroscopy, as affected by soil moisture content and texture. Biosyst. Eng. 2013, 114, 249-258. [CrossRef]

27. He, Y.; Xiao, S.; Nie, P.; Dong, T.; Qu, F.; Lin, L. Research on the optimum water content of detecting soil nitrogen using near infrared sensor. Sensors 2017, 17, 2045. [CrossRef] [PubMed]

28. Tian, Y.; Zhang, J.; Yao, X.; Cao, W.; Zhu, Y. Laboratory assessment of three quantitative methods for estimating the organic matter content of soils in china based on visible/near-infrared reflectance spectra. Geoderma 2013, 202, 161-170. [CrossRef]

29. Nduwamungu, C.; Ziadi, N.; Tremblay, G.F.; Parent, L.É. Near-infrared reflectance spectroscopy prediction of soil properties: Effects of sample cups and preparation. Soil Sci. Soc. Am. J. 2009, 73, 1896-1903. [CrossRef] 
30. Ntziachristos, V.; Bremer, C.; Weissleder, R. Fluorescence imaging with near-infrared light: New technological advances that enable in vivo molecular imaging. Eur. Radiol. 2003, 13, 195-208. [PubMed]

31. Sarkhel, S.; Desiraju, G.R. N-H . . O, O-H . . O, and C-H . . O hydrogen bonds in protein-ligand complexes: Strong and weak interactions in molecular recognition. Proteins 2004, 54, 247-259. [CrossRef] [PubMed]

32. Fuwa, K.; Valle, B.L. The physical basis of analytical atomic absorption spectrometry. The pertinence of the beer-lambert law. Anal. Chem. 1963, 35, 942-946. [CrossRef]

33. Zou, X.B.; Zhao, J.W.; Povey, M.J.; Holmes, M.; Mao, H.P. Variables selection methods in near-infrared spectroscopy. Anal. Chim. Acta 2010, 667, 14-32.

34. Chen, J.; Jönsson, P.; Tamura, M.; Gu, Z.; Matsushita, B.; Eklundh, L. A simple method for reconstructing a high-quality NDVI time-series data set based on the Savitzky-Golay filter. Remote Sens. Environ. 2004, 91, 332-344. [CrossRef]

35. Lindberg, W.; Persson, J.A.; Wold, S. Partial least-squares method for spectrofluorimetric analysis of mixtures of humic acid and lignin sulfonate. Anal. Chem. 1983, 55, 643-648. [CrossRef]

36. Zhan, X.R.; Zhu, X.R.; Shi, X.Y.; Zhang, Z.Y.; Qiao, Y.J. Determination of hesperidin in tangerine leaf by near-infrared spectroscopy with SPXY algorithm for sample subset partitioning and Monte Carlo cross validation. Spectrosc. Spectr. Anal. 2009, 29, 964-968.

37. Araújo, M.C.U.; Saldanha, T.C.B.; Galvão, R.K.H.; Yoneyama, T.; Chame, H.C.; Visani, V. The successive projections algorithm for variable selection in spectroscopic multicomponent analysis. Chemom. Intell. Lab. Syst. 2001, 57, 65-73. [CrossRef]

38. Soares, S.F.C.; Galvão, R.K.H.; Araújo, M.C.U.; Silva, E.C.D.; Pereira, C.F.; Leite, F.C. A modification of the successive projections algorithm for spectral variable selection in the presence of unknown interferents. Anal. Chim. Acta 2011, 689, 22-28. [CrossRef] [PubMed]

39. Diniz, P.H.G.D.; Gomes, A.A.; Pistonesi, M.F.; Band, B.S.F.; Araújo, M.C.U.D. Simultaneous classification of teas according to their varieties and geographical origins by using NIR sensors and SPA-LDA. Food Anal. Methods 2014, 7, 1712-1718.

40. Yun, Y.H.; Wang, W.T.; Deng, B.C.; Lai, G.B.; Liu, X.B.; Ren, D.B.; Xu, Q.S. Using variable combination population analysis for variable selection in multivariate calibration. Anal. Chim. Acta 2015, 862, 14-23. [CrossRef] [PubMed]

41. Krakowska, B.; Custers, D.; Deconinck, E.; Daszykowski, M. The Monte Carlo validation framework for the discriminant partial least squares model extended with variable selection methods applied to authenticity studies of Viagra ${ }^{\circledR}$ based on chromatographic impurity profiles. Analyst 2016, 141, 1060-1070. [CrossRef] [PubMed]

42. D'Acqui, L.P.; Pucci, A.; Janik, L.J. Soil properties prediction of western mediterranean islands with similar climatic environments by means of mid-infrared diffuse reflectance spectroscopy. Eur. J. Soil Sci. 2010, 61, 865-876. [CrossRef]

43. Sudduth, K.A.; Hummel, J.W. Soil Organic Matter, CEC, and Moisture Sensing with a Portable NIR Spectrophotometer. Trans. ASAE 1993, 36, 1571-1582.

44. Libowitzky, E. Correlation of $\mathrm{O}-\mathrm{H}$ stretching frequencies and $\mathrm{O}-\mathrm{H} \ldots$. O hydrogen bond lengths in minerals. Korrelation von O-H-Streckfrequenzen und O-R ... O-Wasserstoffbrückenlängen in Mineralen. Monatshefte Für Chemie 1999, 130, 1047-1059.

45. Dalton, D.A.; Evans, H.J.; Hanus, F.J. Stimulation by nickel of soil microbial urease activity and urease and hydrogenase activities in soybeans grown in a low-nickel soil. Plant Soil 1985, 88, 245-258. [CrossRef]

46. Alahmadi, Y.J.; Gholami, A.; Fridgen, T.D. The protonated and sodiated dimers of proline studied by IRMPD spectroscopy in the N-H and O-H stretching region and computational methods. Phys. Chem. Chem. Phys. 2014, 16, 26855-26863. [CrossRef] [PubMed]

47. Ketterings, Q.M.; Bigham, J.M. Soil color as an indicator of slash-and-burn fire severity and soil fertility in Sumatra, Indonesia. Soil Sci. Soc. Am. J. 2000, 64, 1826-1833. [CrossRef] 
48. Liu, D.; Wang, Z.; Zhang, B.; Song, K.; Li, X.; Li, J.; Li, F.; Duan, H. Spatial distribution of soil organic carbon and analysis of related factors in croplands of the black soil region, Northeast China. Agric. Ecosyst. Environ. 2006, 113, 73-81. [CrossRef]

49. Knadel, M.; Deng, F.; Alinejadian, A.; Jonge, L.W.D.; Moldrup, P.; Greve, M.H. The effects of moisture conditions-from wet to hyper dry-on visible near-infrared spectra of Danish reference soils. Soil Sci. Soc. Am. J. 2014, 78, 422-433. [CrossRef]

(C) 2018 by the authors. Licensee MDPI, Basel, Switzerland. This article is an open access article distributed under the terms and conditions of the Creative Commons Attribution (CC BY) license (http:/ / creativecommons.org/licenses/by/4.0/). 
Article

\title{
Specim IQ: Evaluation of a New, Miniaturized Handheld Hyperspectral Camera and Its Application for Plant Phenotyping and Disease Detection
}

\author{
Jan Behmann ${ }^{1, *}$, Kelvin Acebron ${ }^{2}$, Dzhaner Emin ${ }^{2}$, Simon Bennertz ${ }^{2}$, Shizue Matsubara ${ }^{2}$, \\ Stefan Thomas ${ }^{1}$, David Bohnenkamp ${ }^{1}$, Matheus T. Kuska ${ }^{1}$, Jouni Jussila ${ }^{4}$, Harri Salo ${ }^{4}$, \\ Anne-Katrin Mahlein ${ }^{1,3}$ and Uwe Rascher ${ }^{2}$ \\ 1 INRES-Plant Diseases and Plant Protection, University of Bonn, 53115 Bonn, Germany; \\ jbehmann@uni-bonn.de (J.B.); stefan.thomas@uni-bonn.de (S.T.); davidb@uni-bonn.de (D.B.); \\ mkuska@uni-bonn.de (M.T.K.); amahlein@uni-bonn.de (A.-K.M.) \\ 2 IBG-2, Forschungszentrum Jülich (FZJ), Jülich, 52428 Germany; k.acebron@fz-juelich.de (K.A.); \\ d.emin@fz-juelich.de (D.E.); s.bennertz@gmail.com (S.B.); s.matsubara@fz-juelich.de (S.M.); \\ u.rascher@fz-juelich.de (U.R.) \\ 3 Institute of Sugar Beet Research (IFZ), 37079 Göttingen, Germany \\ 4 Specim Ltd., FI-90571 Oulu, Finland; jouni.jussila@specim.fi (J.J.); harri.salo@specim.fi (H.S.) \\ * Correspondence: jbehmann@uni-bonn.de; Tel.: +49-228-73-4998
}

Received: 30 October 2017; Accepted: 24 January 2018; Published: 2 February 2018

\begin{abstract}
Hyperspectral imaging sensors are promising tools for monitoring crop plants or vegetation in different environments. Information on physiology, architecture or biochemistry of plants can be assessed non-invasively and on different scales. For instance, hyperspectral sensors are implemented for stress detection in plant phenotyping processes or in precision agriculture. Up to date, a variety of non-imaging and imaging hyperspectral sensors is available. The measuring process and the handling of most of these sensors is rather complex. Thus, during the last years the demand for sensors with easy user operability arose. The present study introduces the novel hyperspectral camera Specim IQ from Specim (Oulu, Finland). The Specim IQ is a handheld push broom system with integrated operating system and controls. Basic data handling and data analysis processes, such as pre-processing and classification routines are implemented within the camera software. This study provides an introduction into the measurement pipeline of the Specim IQ as well as a radiometric performance comparison with a well-established hyperspectral imager. Case studies for the detection of powdery mildew on barley at the canopy scale and the spectral characterization of Arabidopsis thaliana mutants grown under stressed and non-stressed conditions are presented.
\end{abstract}

Keywords: hyperspectral camera; handheld; sensor evaluation; case studies

\section{Introduction}

This study introduces the novel, handheld hyperspectral camera Specim IQ and evaluates it with respect to applications in plant physiology and plant pathology. The sensor is designed as a mobile, hand-held and stand-alone camera (cf., Figure 1) with ground-based application areas. For monitoring the status of plants, field, greenhouse and laboratory-based measurements can be performed. By measuring spectral reflectance from 400 to $1000 \mathrm{~nm}$, several important plant traits can be covered by the sensor. The sensor relies on the established push broom principle but integrates a scanner system and allows focusing in the image plane. All investigations presented in this publication were performed on a prototype of the Specim IQ sensor and by using the corresponding software Specim IQ Studio during the pilot phase. 

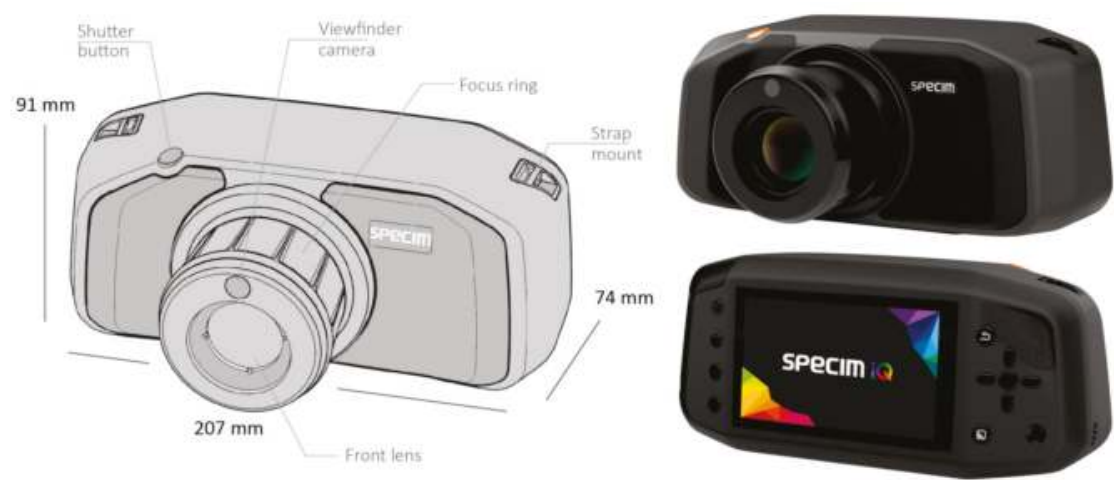

Figure 1. Vector visualization of the Specim IQ (Specim Ltd., Oulu, Finland) with annotations and dimensions on the left side and RGB renderings on the right side.

Hyperspectral imaging (HSI) is a non-invasive method, which can provide detailed and highly resolved reflectance characteristics of target materials on different scales. The reflected light of the target is recorded with a high spectral and spatial resolution of a two-dimensional image [1]. In hyperspectral images, every pixel has more than hundred consecutive bands, containing the reflectance values of the target for specific wavelengths called the spectral signature [1]. Many sensors address the visible part of the electromagnetic spectrum (400-700 nm, VIS) and are also able to measure the near-infrared wavelength (700-1000 nm, NIR) specifying them as VISNIR sensors. The shortwave-infrared (1000-2500 nm, SWIR) part of the electromagnetic spectrum is also important for specific applications but typically not all spectral regions can be covered by one sensor.

In recent times, an increasing number of successful studies prove the high versatility of applications of hyperspectral imaging. Differences in the material composition of the measured target-e.g., living plants, fruits, geological samples-are reflected in the corresponding spectral profiles. This allows the detection of important parameters in food production, soil science and precision agriculture [2-6]. Hyperspectral imaging is applied for an improved quality and safety monitoring in food production [7-9]. Successful applications have been shown e.g., for lesion detection on apple skin using a hyperspectral NIR camera [10], prediction of moisture in soybeans by VISNIR imaging [11] and the acidity levels of grapevines from seven varieties by NIR hyperspectral imaging [12]. Pigment concentration and other quality parameters of bell pepper were determined using VISNIR imaging and Partial Least Squares Regression [13]. In soil science, hyperspectral imaging is used for the investigation of soil composition and evaluation of soil quality. The spatial distribution of three classes of organic matter within $10 \times 30 \mathrm{~cm}$ soil samples was determined using k-means clustering [3]. In a similar approach, a snapshot camera with the potential for outdoor application was applied for visualizing the spatial heterogeneity and estimating soil properties like nitrogen or clay content by Partial Least Squares Regression [2]. Using the SWIR range, a spectral library has been developed that allows the detection of crude oil contamination in different soil types [14].

In precision agriculture, HSI can be applied for site-specific fertilization and precise plant protection applications [15-17]. In recent years, HSI was introduced in greenhouse and field phenotyping for non-invasive quantification of structural and functional plant traits [18]. Integrated multi sensor platforms allow the application in the field, e.g., for grapevine phenotyping [19]. Studies in plant pathology showed the advantage of a high spatial resolution in close range imaging to detect and characterize plant diseases and even subtle resistance mechanisms of plants to diseases [20,21]. On the other side of the scale, hyperspectral sensors are increasingly used for ecosystem monitoring and remote sensing of vegetation [22,23]. In this context, Vegetation Indices (VIs) are commonly used to remotely evaluate vegetation covers both quantitatively and qualitatively $[24,25]$. These spectral 
ratios were shown to be sensitive to changes in plant functional status which helps in estimating gross [26,27] and net photosynthesis [28] down to its efficiency [29].

With the increase of different application scenarios, the demand for tailored hyperspectral cameras rises. Every hyperspectral sensor type has specific strengths and limitations, which need to match the requirements of the measured object and aim of investigation. Push broom and whisk broom scanners capture the spectral information of a line or point on the measured object, respectively. To compose the hyperspectral image, the object is scanned through movement or rotation [21]. However, the required scanner process limits the application when the object is in motion.

Airborne applications mostly aim at plant status sensing or variety mapping and rely on whisk or push broom systems. The spatial referencing is performed with suitable accuracy by using additional correction parameters from an inertial measurement unit and global navigation satellite systems [30]. Light-weight platforms, like UAVs, have the advantage of lower flight altitudes but do not provide such high-quality correction signals.

Triggered by the trend towards UAVs, full frame and snapshot hyperspectral cameras arouse intense attention. Usually, the image data is spatially referenced by established structure-from-motion software in post processing [31]. The underlying measurement principles are well-known but now significant technical development allows to produce light-weight, reliable sensors with sufficient radiometric accuracy for many applications. The main approaches for multi- and hyperspectral frame cameras are spectral scanners [32], multi-point spectrometer [2], mosaic sensors and multi-sensor systems. The Rikola hyperspectral camera (Senop, Kangasala, Finland) as a Fabry-Pérot-filter camera specifies the wavelength of the light illuminating the panchromatic sensor array [32]. By fast changing of the passing wavelength, hyperspectral images of up to $1010 \times 1010 \mathrm{px}$ and 380 bands can be captured in the current version of the camera. A particular feature of the camera is to define the needed bands and to reduce measurement time by reducing the number of bands. Multi-point spectrometers, such as the Cubert S 185 (Cubert GmbH, Ulm, Germany), are capturing the whole spectral characteristics in one shot by projecting the spectra of different pixel locations onto one 2D sensor [32,33]. The low spatial resolution of the hyperspectral image, $50 \times 50 \mathrm{px}$ and 125 bands for the Cubert S 185, is partially compensated by an additional panchromatic image with higher spatial resolution allowing pan-sharpening. Mosaic cameras in contrast are basically multispectral cameras that expand the principle of common RGB cameras by adding more and narrow band filters in front of individual pixels [34]. In one image frame, up to 25 bands can be captured. A variant uses a line layout capturing up to 70 bands in one shot but only one band for each pixel location [35]. To restore a hyperspectral image, proper referencing and 3D reconstruction with multiple images at different positions are necessary. Other multispectral designs integrate up to six sensor arrays, each sensing up to three bands. They can be classified as multi camera systems with several optics and sensor arrays (e.g., the popular TetraCam [36]). The Specim IQ shares some characteristics with these snapshot cameras especially with the spectral scanner, e.g., capturing a full hyperspectral image without external movement and the slight temporal delay of capturing different parts (spatial or spectral) of the image. Latter will cause distortions if a moving object is observed. The Specim IQ may allow UAV applications as well, but due to its focus on direct manual control it is not possible in the current version.

Ground-based hyperspectral sensing includes applications in the field, the greenhouse and the laboratory with moving or static platforms. Moving field platforms and field robots rely mainly on line scanner systems as the camera weight is a minor factor and the movement is already provided by the platform $[17,37,38]$. Distortions as a result of vibrations do not affect the spectral signal, but only affect the spatial image and are typically not corrected. For chlorophyll estimation in sugar beet for example, a moving platform was combined with a linear stage [39]. A similar set-up was also recently adopted to use high performance and thus heavy imaging spectrometers to retrieve subtle differences between different breeding lines in the field $[40,41]$. In the greenhouse, more compact measurement stations are required. For water stress detection, a line scanner camera was combined with a rotating mirror to obtain hyperspectral images [42]. In Reddy et al. [43], a line scanner was used with a linear stage in 
the greenhouse and furthermore, the same line scanner was attached to a tractor for field observations in order to detect glyphosate-resistant weeds.

In the laboratory and in high-throughput systems, the use of line scanners is common as under optimal measurement conditions the spatial and spectral measurement quality is superior $[21,39,44]$. Ge et al. [44] derived a variety of plant-physiological parameters using a conveyor belt-based high-throughput facility with a hyperspectral sensor cabin. A mirror-based push broom sensor was used to capture images of single sugar beet leaves and to derive disease specific vegetation indices [21]. Jay et al. [39] used a fixed camera with attached illumination while the plant probe was moved by a translation stage. They obtained multi-angle data and derived radiative transfer models especially suited for the close range. Microscopic observations for the investigation of resistance reactions of a plant against a fungal pathogen were also observed by a line scanner attached to a microscope optic [20]. The image was gathered by moving the probe during the measurement.

High amounts of spectral data are collected with each hyperspectral image, making it difficult to extract relevant information from the images. This leads to the requirement of advanced data analysis methods in order to work efficiently with hyperspectral sensors [45]. For a successful application, a suitable sensor needs to be accompanied by an appropriate data analysis pipeline, which needs to be concerted to the sensor characteristics.

In the present study, the characteristics of the novel hyperspectral camera Specim IQ are presented, and a direct qualitative comparison on radiometric accuracy with the well-established sensor Specim HS-V10E-CL-30 $[21,40,41,46,47]$ (denoted in the manuscript as Specim V10E (Specim Ltd., Oulu, Finland)) was conducted. Further details on this sensor are provided in Section 3. The IQ sensor is accompanied by the software Specim IQ Studio (Specim Ltd., Oulu, Finland) which relies on the Spectral Angle Mapper (SAM) algorithm to analyze the images and to develop SAM applications which can be executed on the device itself. The performance of the sensor and the accompanying data analysis software were investigated in two lifelike case studies. Performance for the detection of powdery mildew on barley and classical differentiation of Arabidopsis thaliana mutants are demonstrated.

\section{Technical Description of the Specim IQ Camera System}

This section provides the technical background of the sensor and gives an overview of the different normalization possibilities and measurement modes. Specim IQ is a handheld hyperspectral camera, which performs hyperspectral data capturing, data processing and visualization of a classification result in one single integrated unit. The camera is supported by Specim IQ Studio software . Using the software, the user is enabled to develop and download its own applications to the camera as it possesses embedded processing capability. Detailed technical specifications are given in Table 1.

Table 1. Specim IQ technical specification.

\begin{tabular}{lc}
\hline Parameter & Value \\
\hline Spectral camera & VNIR 400-1000 nm (CMOS) \\
Viewfinder camera & 5 Mpix \\
Focus camera & 1.3 Mpix \\
User interface & SW Specim \\
Processor & NVIDIA Tegra K1 \\
Storage SD card & max. 32 GB \\
Data format & Specim data set with ENVI compatible data files \\
Battery & 5200 mAh Li-Ion (Type 26650) \\
Operational time & 100 measurements with one SD card and battery \\
Display \& keyboard & $4.3^{\prime \prime}$ touch screen \& 13 physical buttons \\
\hline
\end{tabular}


Table 1. Cont.

\begin{tabular}{lc}
\hline Parameter & Value \\
\hline Camera interface & USB Type-C \\
Connectivity & GPS \\
Size & $207 \times 91 \times 74 \mathrm{~mm}$ (depth with lens $125.5 \mathrm{~mm})$ \\
Weight & $1.3 \mathrm{~kg}$ \\
F/number & 1.7 \\
Wavelength range & $400-1000 \mathrm{~nm}$ \\
Spectral resolution FWHM & $7 \mathrm{~nm}$ \\
Slit length & $42 \mu \mathrm{m}$ \\
Slit height & $11.7 \mathrm{~mm}$ \\
Spatial sampling & $512 \mathrm{px}$ \\
Spectral bands & 204 \\
Image resolution & $512 \times 512 \mathrm{px}$ \\
Data output & $12 \mathrm{bit}$ \\
QE peak & $>45 \%$ \\
Full well capacity & $>32,000 \mathrm{e}-$ \\
Peak SNR & $>400: 1$ \\
Working distance & $150 \mathrm{~mm}-\infty$ \\
FOV & $31^{\circ} \times 31^{\circ}$ \\
FOV at 1 m distance & $0.55 \mathrm{~m} \times 0.55 \mathrm{~m}$ \\
Temperature, operational & $0{ }^{\circ} \mathrm{C}$ to $+40^{\circ} \mathrm{C}$ \\
\hline
\end{tabular}

The measurements are performed based on the line scanner, i.e., push broom principle and comprise the wavelength range $400-1000 \mathrm{~nm}$. Its spatial sampling, i.e., the number of pixel per line is 512, and the spectral resolution is $7 \mathrm{~nm}$ with 204 spectral bands across the wavelength range. By adjusting the binning in spectral dimension, the amount of spectral bands can be adjusted. The number of imaged lines is static with 512 lines and thus the camera captures always a square image with a resolution of $512 \times 512$ px. Specim IQ camera is equipped with a touch display, physical buttons, chargeable battery, replaceable memory card and USB connector. The camera employs a processor (NVIDIA Tegra K1 (NVIDIA Corporation, Santa Clara, USA)), spectral camera (CMOS technology), viewfinder camera ( $5 \mathrm{Mpix}$ ), focus camera and a scanner with the motor for optics movement. The fore optic of the camera provides a $31^{\circ} \times 31^{\circ}$ field of view and focusing is set manually. The focus range of the camera is from $150 \mathrm{~mm}$ to infinity. As an example, with $1 \mathrm{~m}$ distance to the target, the viewable area is $0.55 \times 0.55 \mathrm{~m}$, resulting in a spatial resolution of $1.07 \mathrm{~mm}$ on the target.

Specim IQ is controlled via a touch screen and relies on a graphical user interface that guides the user through the entire imaging pipeline (Figure 2). The usability has been designed to have similarities with a standard system camera and to require only minimal user input. One main character of the camera is its ability to convert the recorded hyperspectral data into instant classification results displayed on the screen. For this purpose, the Specim IQ uses a workflow which is shown in Figure 2.

The measurement process is divided into five steps. During the establishment of the imaging setup, the hyperspectral camera is directed to the target using the viewfinder camera, i.e., a RGB camera with identical viewing direction and small vertical offset. As common for any hyperspectral measurement, a suitable illumination providing a continuous spectrum over the wavelength range of interest is required. This could be sunlight outdoors or halogen lamps indoors. Typically, the characteristics of the illumination are captured by the measurement of a white reference panel, which has to be located next to the sample.

After this, the integration time and camera focus needs to be adjusted. The viewfinder camera image gives a preset for the integration time of the spectral camera. The preset value and a preferable range are proposed to the user, but a manual adjustment of the integration time in the range 1-500 ms is possible. The focusing of the system is done manually, but supported by the focus camera image. In contrast to a traditional line scanner camera, this approach uses a normal camera image and highlights sharp edges. The parallax between the viewfinder camera and actual spectral camera 
is compensated by an automatic calibration done by edge detection between viewfinder and focus cameras. The vertical position of the viewfinder camera image is virtually adjusted accordingly or alternatively, based on a manual parallax correction. The adjustment is needed to overlay the spectral and viewfinder camera images with suitable accuracy.

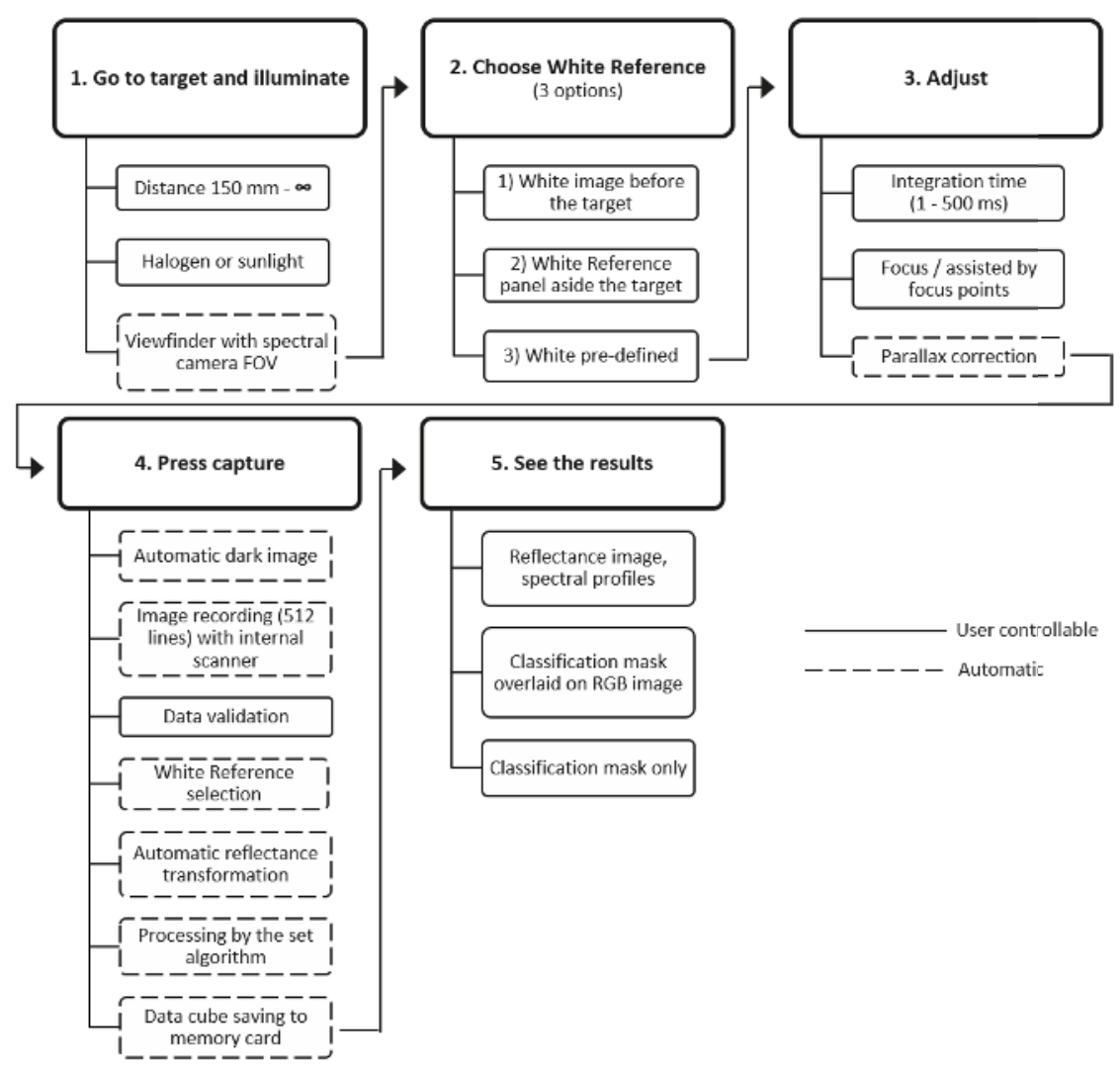

Figure 2. The standard workflow of the Specim IQ hyperspectral camera.

After these initial adjustment steps, image recording is started by pressing the capture button. At first, the dark reference, representing the sensor noise without incoming light is recorded automatically. This is done on the spectral camera home position that is blocked from incoming light. After the dark reference acquisition, the spectral camera is moved to the measurement starting position and the actual data acquisition is started.

After the full $512 \times 512$ px image is scanned, the user can check focus and integration time in the data validation view. There, a RGB image derived from the hyperspectral image is shown together with an intensity histogram. Based on the validation view, the image can be rejected, kept for the further workflow or it can be selected to save only raw, uncorrected data. For the white reference measurement, Specim IQ offers three possibilities. The white reference can be recorded prior to the actual measurement (custom white reference). Alternatively, the white reference can be recorded simultaneously with the actual sample (simultaneous white reference). In cases where white reference recording is not possible, device memory provides a general halogen spectrum from 400 to $1000 \mathrm{~nm}$ (pre-defined white reference). In the first two possibilities, an area scan over the white reference is 
performed, in which 100 random spectra are averaged and used for data reflectance transformation. The latter approach uses the pre-defined spectrum directly to convert the measured data to reflectance. In all cases, the reflectance transformation is performed automatically for the data.

For data processing, Specim IQ provides three different modes: Default Recording Mode (without any processing), Automatic Screening Mode (ASM, one-class classifier) and Application Mode (deployed processing model developed by Specim IQ Studio).

In Default Recording Mode, all the raw data and reflectance data are saved into separate data cubes on the data set. The data set is a folder structure, where the data is stored together with meta information. The ASM can be used to build a single class classifier, at the site, and directly from the user interface of the Specim IQ. The ASM mode is based on a Spectral Angle Mapper [48]. The user has a possibility to select the reference spectra, as well as threshold for class creation. Prior to the classification, the reflectance transformation is performed as described above, as well as automatic wavelength range selection to avoid using noisy parts of the spectra. By using the Specim IQ Studio software, it is possible to build applications that can be uploaded to the Specim IQ camera. In the Application Mode it is possible for the user to define data recording, visualization and saving. Furthermore, the data classification can be defined by creating several classes, class groups, data preprocessing (Savitzky-Golay algorithm for data smoothing [49]), and classification for the targeted application. The Specim IQ Studio software allows to create applications without any programming directly from a graphical user interface.

If Specim IQ is used in the Default Recording Mode, the RGB image derived from reflectance data is shown to the user after measurement. There are possibilities for the user to check the spectra, add tags and other information to the dataset. If the data processing capabilities of Specim IQ are used, either with Automatic Screening or Application Mode, the classification mask is visualized as an overlay on a RGB image or without RGB information. Using this visual feedback, a direct assessment of imaging quality and flexible real-time analysis of samples using hyperspectral images is possible. Importing own spectral algorithms beyond the SAM to Specim IQ is not possible and models in Specim IQ Studio are limited to SAM only. However, importing reference spectra for the basis of SAM classification model is supported and processing time can be optimized with wavelength range selection and averaging spectral bands.

\section{Qualitative Comparison of Specim IQ with Specim V10E}

Comparative measurements of the Specim IQ system and Specim V10E sensor were conducted to assess the measurement quality of the Specim IQ camera and to provide an impression for which applications the new sensor is suited.

The Specim V10E sensor is an established and representative system of the class of hyperspectral push broom cameras in the same spectral range ( $400-1000 \mathrm{~nm}$ ) as the Specim IQ. In this study, four-fold spectral binning is applied to provide a similar sampling distance using 211 bands. Capturing 1600 pixel per line, it has in general a higher spatial resolution than the Specim IQ, but it is dependent on an external movement which is provided by a linear stage in the present setup [21,47]. The sensors were compared in an indoor setting with halogen illumination and an outdoor setting with natural light to capture the measuring performance for multiple relevant scenarios. Both sensors were positioned in nadir orientation above different test materials, such as paper and polyethylene of different color (in the following called color cards). In the laboratory, the Specim V10E-including the light sources-was moved by a linear drive across the object of interest whereas the Specim IQ used the internal scanning properties and a spatially fixed illumination.

During outdoor measurements, sunlight from full-midday sun ( 90.000 lx) was used as the only light source. In both scenarios, the cameras were placed as close to each other as possible with a maximum distance of $20 \mathrm{~cm}$ during the movement of the line scanner. The test materials were placed at a distance of $1 \mathrm{~m}$. All images were normalized using a 99\% barium sulfate white reference (Specim), which was positioned within the field of view during image acquisition. Due to the different 
measurement principles (push broom vs. static), the comparability of measurement conditions is limited by the spatially variable light conditions in the laboratory. As a result of the moving light sources of the Specim V10E, both cameras observed the white reference with slightly different light intensity. To remove this effect and obtain comparable reflectance values, the spectral signatures of the Specim IQ were scaled to attain the same mean reflectance value as the Specim V10E spectra.

The mean reflectance, its standard deviation and the mean and maximum distance between the Specim IQ reflectance spectrum and the Specim V10E reflectance spectrum for all color cards were calculated from more than $400 \mathrm{px}$. A subset of representative spectra comparing the sensor performance is given in Figure 3 for indoor measurements and in Figure 4 for outdoor measurements. All parameters illustrating the accuracy and the concordance of both sensors using all color cards for the laboratory and for the outdoor scenario are given in the appendix.

The results allow to group the materials in paper and polyethylene which show a low in-group variability of the accuracy parameters (Table A1). In the indoor experiment, a mean absolute distance between the spectra of Specim V10E and Specim IQ of 0.007 and 0.009 and maximum absolute distance of 0.025 and 0.030 was observed for the paper and polyethylene materials, respectively. The standard deviation of 0.017 for the Specim V10E and 0.021 for the Specim IQ represents the combined effect of measurement noise and material heterogeneity. In the outdoor experiment, a mean absolute distance of 0.054 and 0.032 and maximum absolute distance of 0.0207 and 0.164 was observed for the paper and polyethylene materials, respectively (Table A2). In contrast to the indoor experiment, the standard deviation of 0.038 and 0.020 for the Specim V10E and 0.042 and 0.017 for the Specim IQ reveals significant differences between the two material groups. The increased standard deviation of the paper color cards measured outdoors is presumably caused by angular effects as the paper was not completely flat due to fixation. As the same parts of the paper cards are averaged in the images of both sensors, the majority of this effect is excluded from the further analysis.
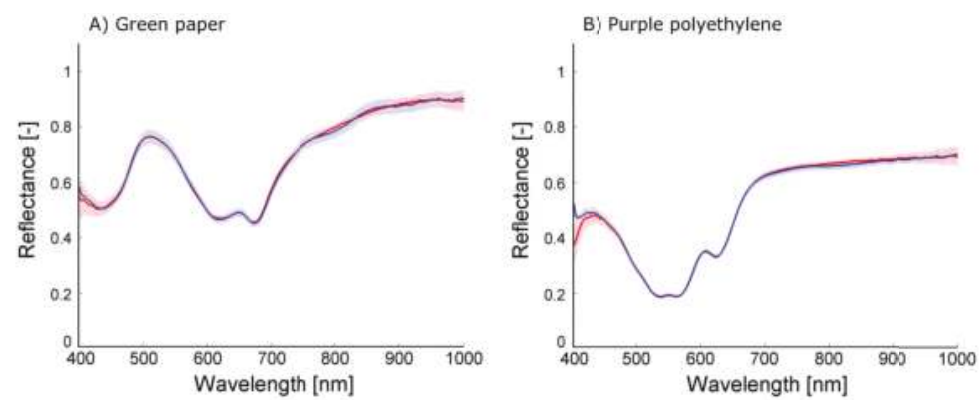

C) Used color cards
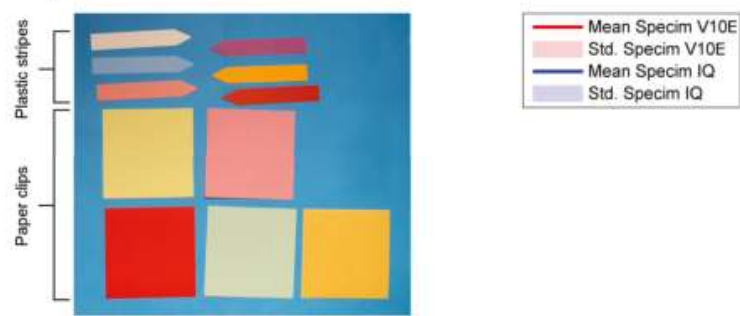

Figure 3. The mean spectra including the standard deviation of green paper (A) and purple polyethylene (B) as representatives of the observed reference objects of different color in the indoor setting (C). 

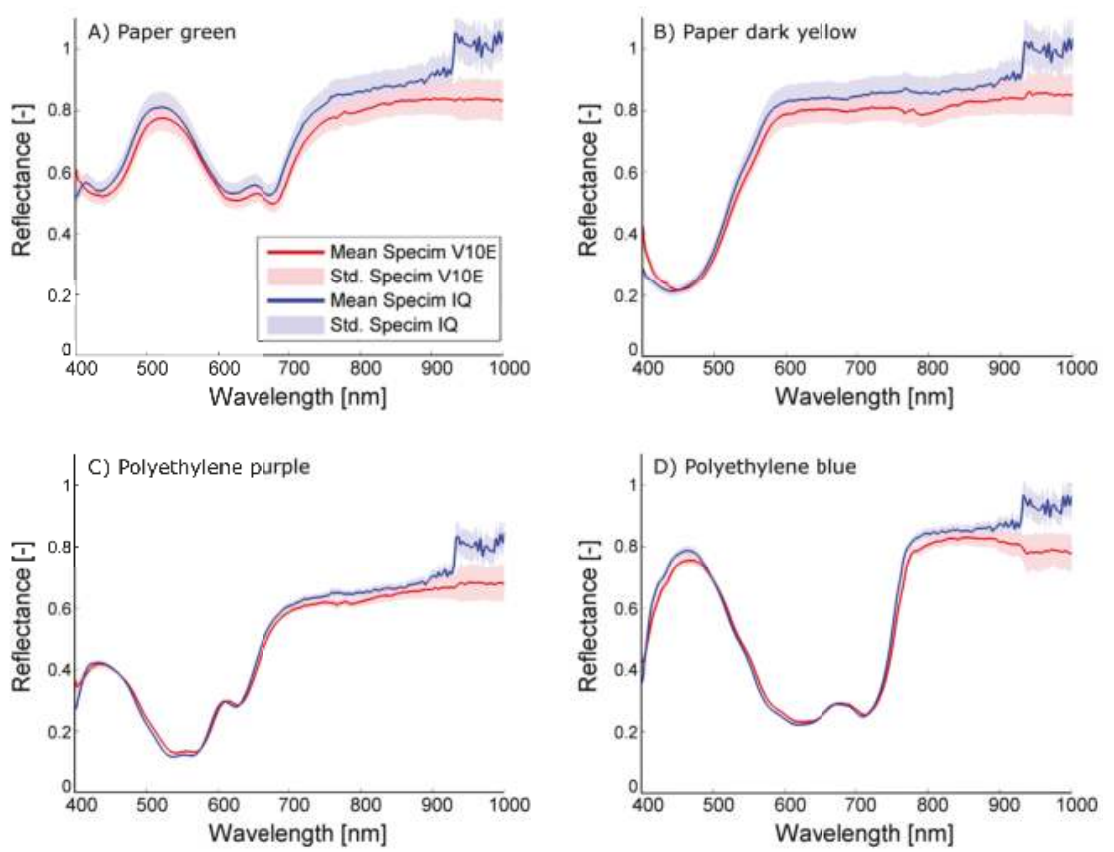

Figure 4. Comparison of spectral reflectance spectra from the outdoor experiment (A-D): paper green, paper dark yellow, Polyethylene purple, Polyethylene blue. Beside the increased reflectance observed by the Specim IQ in the NIR, all spectra reveal a high level of congruence.

Overall, it turns out that the sensors give comparable results. The shape of the observed spectra corresponds to a high degree, resulting in mean differences of 0.009 in the laboratory and 0.043 outdoors. The mean standard deviation of Specim IQ and Specim V10E are on the same scale indoors $(0.017$ and 0.021 ) and identical outdoors (0.029 and 0.029), but for the Specim IQ it is more homogeneous whereas the Specim V10E has a higher noise level in the spectral border regions (400-450 nm and 900-1000 nm, cf. Figure 3A). As negative characteristics, outdoor observations with the Specim IQ showed an increased reflectance between 925 and $1000 \mathrm{~nm}$. The remaining part of the spectrum was not affected. This results in comparably high maximum deviations (on average 0.186 ) and may be related to the low integration time of $1 \mathrm{~ms}$. According to the manufacturer, this effect was caused by the low raw signal level in addition to the atmospheric $\left(\mathrm{H}_{2} \mathrm{O}\right)$ absorption band in the range between 925 and $970 \mathrm{~nm}$. This results in the prompt increase in the reflectance signal. The remedy for this would be to use spectral flattening filter in front of the fore optics and increase the integration time. Furthermore, a line pattern was visible in all images (Figure 5). According to the manufacturer, it is induced by the changing optical geometry at the slit during the scanning process. As a consequence, the slit width is not completely identical for single lines of the image. Nevertheless, due to the averaging of multiple lines, the mean spectra were not affected. 

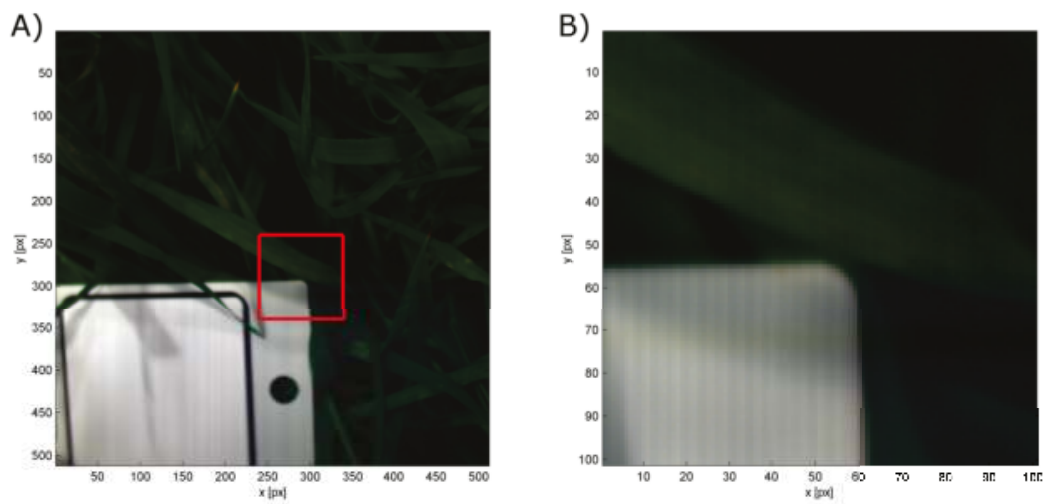

Figure 5. RGB visualization of a reflectance test image to show the line pattern. The highlighted image part in (A) is visualized in zoom view in (B). On the white reference the line pattern is visible, whereas on the plants it is mainly covered by natural variability.

\section{Case Studies}

In addition to the qualitative evaluation in comparison to an established sensor, the performance of the Specim IQ was investigated in two case studies. In the first case study, the reflectance characteristics of three different genotypes of Arabidopsis thaliana were recorded to test the capacity of the camera to resolve subtle differences that are based on slightly different leaf pigment composition. The second case study aimed to quantify the level of infestation of two barley cultivars inoculated with powdery mildew, using the machine learning method Support Vector Machine (SVM [50]). By this selection, two relevant applications in plant and crop science, dealing with different plant functional traits were investigated.

\subsection{Use of Specim IQ Camera System as a Tool for Understanding Physiological Response of} Arabidopsis thaliana Mutants Adapted in Stressed and Non-Stressed Condition

Vegetation indices (VIs) are used by plant scientists to evaluate structural and functional vegetation traits quantitatively and qualitatively across different scales $[24,25,51,52]$. They are used for example to assess the green biomass [53], canopy structure [54], leaf area index [55], chlorophyll content [56-58], fraction of absorbed photosynthetically active radiation (fAPAR) [55,59], and light-use efficiency [29] of plants and canopies [60]. With the development of portable hyperspectral imaging sensors, reflectance from visible $(400-700 \mathrm{~nm})$ and near infrared $(700-1000 \mathrm{~nm})$ spectrum can be easily derived to study and screen mutant plants to identify genes and understand its physiological function in a high-throughput way in phenotyping environments.

In this case study, two mutant lines of Arabidopsis thaliana acclimated in non-stressful (NSA) and stressful (SA) condition were used as the main subject. The selected mutants are deficient in either PsbS protein (npq4) [61] or violaxanthin de-epoxidase (npq1) [62], both resulting in a limited ability to thermally dissipate excessive light energy via a process called non-photochemical quenching (NPQ). In addition, the lack of violaxanthin de-epoxidase in npq1 mutant inhibits light-dependent enzymatic conversion of carotenoids. This conversion, which is a part of NPQ regulation, causes a very subtle spectral change in leaf reflectance that is invisible to human eyes. Both mutations are not fatal and the plants develop normally under greenhouse conditions. Under high light conditions, however, the two mutants are unable to adjust light energy harvesting by NPQ to protect their photosystems against photo-damage. Along with these mutants, the "normal" plants without mutations (Col-0) were used as a control group. Thus, by using these mutants we tested whether the Specim IQ can detect subtle differences in leaf pigments and physiological traits. 
Plants were sown in $7 \times 7 \mathrm{~cm}$ pots (one plant per pot) filled with soil. Three plants from each genotype were grown and acclimated to non-stressful conditions in the greenhouse while another three plants were acclimated for at least two days to natural light and temperature conditions in the field that are more stressful for plants. All plants were randomly distributed within the imaging frame.

The Specim IQ camera system was used to take reflectance images of Arabidopsis plants inside the greenhouse with white panel ( $90 \%$ reflectance) as a reference target. As the Specim IQ software does not support the calculation of vegetation indices, the hyperspectral imagery data was imported into ENVI Classic 5.3 (Harris Geospatial Solutions, Broomfield, USA) resolving the regions of interest (ROI) using Normalized Difference Vegetation Index (NDVI [63], Equation (1)) and manual tracing of individual plants. For the case study, two VIs were calculated that are correlated to leaf chlorophyll content, namely the NDVI and the Red Edge Inflection Point (REIP [64,65], Equation (2) by Matlab 2013a and Signal Processing Toolbox 6.19 (The MathWorks, Inc., Natick, USA)). To derive REIP, plant spectra was smoothed using Savitzky-Golay filtering [49] before calculating the first derivative. REIP was identified as the maximum value of the first derivative between 690 and $720 \mathrm{~nm}$ after spline interpolation with $0.1 \mathrm{~nm}$ resolution. As a third VI, the Photochemical Reflectance Index (PRI [66], Equation (3)) was calculated, which is described to be sensitive to the activation of the NPQ pathway and carotenoid conversion (for more details on these indices and their functional meaning see [25]. Using the RStudio software V1.0.143 (RStudio, Inc., Boston, USA), analysis of variance (ANOVA) was carried out using agricolae package for all the calculated average VIs in a single plant. Likewise, pairwise mean comparison using least square difference (LSD, $\alpha=0.05$ ) was performed as a post hoc analyses.

$$
\begin{aligned}
\mathrm{NDVI} & =\frac{R_{800}-R_{680}}{R_{800}+R_{680}} \\
\mathrm{REIP} & =\underset{690 \leq x \leq 720}{\arg \max R_{x}^{\prime}} \\
\mathrm{PRI} & =\frac{R_{531}-R_{570}}{R_{531}+R_{570}}
\end{aligned}
$$

Vegetation indices computed from the ROIs of the images of the SA and NSA plants revealed distinct differences in NDVI, REIP and PRI. Plants' spectral reflectance (Figure 6B) varied in green $(\sim 530 \mathrm{~nm})$, red edge $(\sim 700 \mathrm{~nm})$ and near-infrared $(>700 \mathrm{~nm})$ regions of the spectrum where light-use efficiency, narrowband and broadband greenness are estimated, respectively. For both NDVI (Figure 6C,D) and REIP (Figure 6E,F), the two npq mutants showed significant changes between SA and NSA while no significant difference was observed in Col-0 plants. Although the shift in red edge inflection point is small $(\sim 2 \mathrm{~nm})$, LSD test showed significant differences between SA and NSA plants of $n p q 1$ and $n p q 4$. Previous studies $[67,68]$ reported that the red edge shift towards the shorter wavelength was related to a reduced chlorophyll content in leaves. This demonstrates the sensitivity of these indices to subtle changes in pigment ratios and leaf structures which arose in leaves of the NPQ-deficient mutants during acclimation to the stressful condition. The third index, PRI, revealed stress response (Figure 6G) in all plants. The PRI values decreased in all SA plants on average by 1.4-fold and the three genotypes significantly differed $(\alpha=0.01)$ in the extent of the decrease (Figure $6 \mathrm{H})$. Moreover, fair resolution of pixel patterns within a single plant was captured by the calculated VIs (Figure 6C,E,G) which can further provide spatial information on the relative distribution of different pigments ratios reflecting plant status.

This case study demonstrates the usability of a portable hyperspectral camera to simultaneously detect relative changes in both chlorophyll content (NDVI and REIP) and the xanthophyll cycle (PRI) in Arabidopsis plants. While this case study only showed representative vegetation indices, it offers more opportunity to compute other established VIs (including those which are based on derivatives of spectra) to gain more physiological information in plants. The magnitude of change between the SA and NSA plants can provide quantitative information indicative of stress levels, as shown here in simple rosette of Arabidopsis thaliana. 

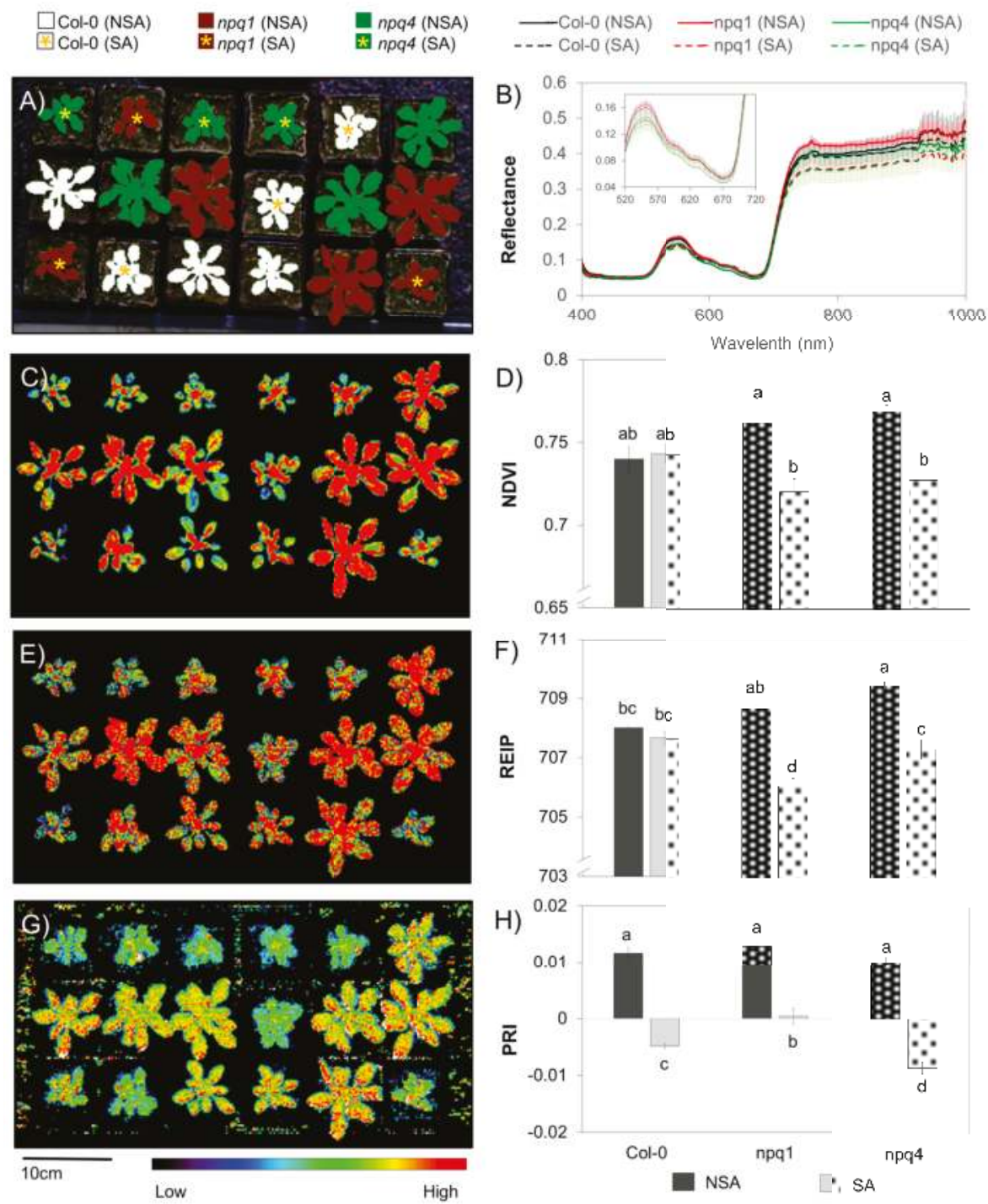

Figure 6. Differences observed between non-stress acclimated (NSA) and stress acclimated (SA) Arabidopsis wildtype (Col-0) and NPQ-deficient mutants (npq1 and npq4) as shown by computed spectral ratios. Left panel shows the false-colour images of selected ROIs (A); NDVI (C); REIP (E); and PRI (G) computed from spectral information captured by the Specim IQ camera. Right panel shows the computed means \pm standard errors of reflectance values (B); NDVI (D); REIP (F); and PRI (H) from three individual plants randomly distributed in the imaging frame. Different letters indicate significant differences based on LSD $(\alpha=0.05)$.

\subsection{Quantification of Powdery Mildew Infection on Barley}

Information on disease severity of crop plants is relevant for the evaluation of the susceptibility of host plants against specific plant pathogens. It is a crucial information source for breeders and essential for plant protection measures in precision agriculture. Hyperspectral imaging, as a 
non-invasive and objective method, has shown great promise for phenotyping applications in previous studies $[5,21,69]$. Furthermore, the possibility to create automated measurement series directly in phenotyping greenhouses and fields could lead to an increased throughput for phenotyping applications, reducing the time required for the development of new pathogen resistant cultivars [70]. In the field of precision farming, the early and precise detection of plant diseases is also highly relevant as it determines the efficiency of countermeasures [5].

Therefore, the Specim IQ was tested in a scenario for the assessment of powdery mildew disease severity of different barley cultivars on the canopy scale. The cultivars Milford and Tocada, which have different susceptibility ratings against powdery mildew with 4 and 7 according to the German cultivar list (Descriptive Variety List; Bundessortenamt, Hanover, Germany), were investigated with both camera systems for comparison and evaluated according to the results of subsequent image analysis. The goal of the case study was to accurately detect powdery mildew symptoms on both cultivars. Furthermore, it was of interest to achieve a quantification of the disease symptoms over the image in order to assess the different disease severities of the cultivars through hyperspectral imaging in combination with data analysis methods.

For the collection of reflectance characteristics, measurements were conducted using the Specim IQ in a Mini-Plot phenotyping facility in a greenhouse (Thomas et al., in preparation). The "Mini-Plots" are containers which allow the observation of small canopies of up to 360 barley plants with sufficient soil, to enable a root system development similar to barley plants under field conditions. The plants can grow under natural light conditions, while a curtain system at the movable measurement platform allows the application of stable artificial light conditions with diffuse light. The diffuse light is provided by a setup of six $120 \mathrm{~W}$ halogen lamps, which are spread over the measurement platform for stable lighting conditions. The properties of the diffuse light lowers the impact of the canopy architecture on the measurement results, due to the reduction of shadowing and different reflection angles in the canopy. Observations were performed on 11/23/2016 at 26 days after inoculation (dai) under controlled light conditions with a measurement distance of around $60 \mathrm{~cm}$ and oblique view. The images were normalized using a white reference within the image (cf. Figure 7) and the built-in function of the Specim IQ. A representative result of the SAM analysis of the Specim IQ Studio is given in Figure 7B. Compared to the SVM classification in Figure 7C, the SAM detects similar regions with symptoms in the upper leaves, but misses some of the older, brownish symptoms which are instead not assigned to a class. This may be related to the limited number of samples used for the SAM. Therefore, an increased number of samples may enable competitive results. A quantitative evaluation of the SAM result was not supported by the used version of Specim IQ Studio.

To assess the suitability of the recorded images for the quantification of susceptibility, a SVM classification model for disease symptoms was derived based on manual annotation. "Background", "healthy leaf tissue" and "leaf tissue with powdery mildew symptoms" were used as target classes. The linear SVM model was learned using 15 annotated samples for each of the three classes. Using the more complex non-linear SVM with a radial function kernel did not improve the result accuracy. The classified background was removed from the further analysis and the ratio of symptomatic pixels to all plant pixels was calculated. For the inoculated (inoc.) cv. Milford and cv. Tocada, disease symptoms were detected at $25.8 \%$ and $4.4 \%$ of all plant pixels, whereas for the healthy plots (cont.) only $2.0 \%$ and $2.2 \%$ were determined, respectively. Existing misclassifications were mainly the effect of mixed pixels at the border of the white reference as they appear like the white mycelium on the leaf surface. To remove this systematic bias, the disease severity was determined by subtracting the amount of pixels classified falsely as symptomatic (inoculated-control). Strong difference of $2.2 \%$ to $23.7 \%$ for the analyzed cultivars was predicted. This values represent the visual impression of the RGB pixels but do not represent the rated susceptibility given by the German cultivar list. The main reason for this is the specific selection of the measured region (approximately $30 \times 30 \mathrm{~cm}$ ), which does not represent the disease severity within the whole plot. 
Using the Specim IQ, it was possible to assess the disease severity of complex canopies by measurements in the greenhouse. Controlled illumination conditions support the high signal quality. The spatial resolution was sufficient to identify single symptoms on the barley leaves.

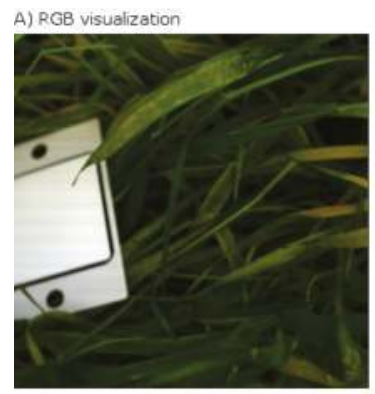

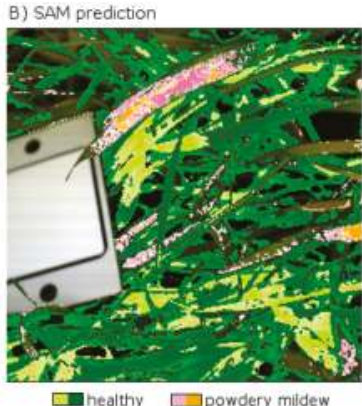

$\square$ nealthy $\square$ powdery mildew

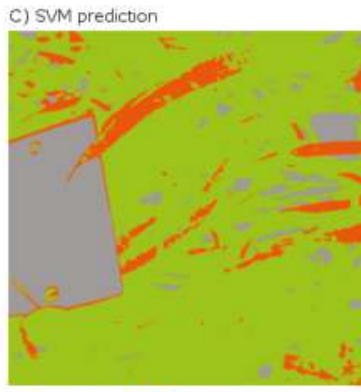

$\square$ healthy $\square$ powdery mildew

Figure 7. Classification of powdery mildew using the Spectral Angle Mapper (SAM) and Support Vector Machine (SVM). Powdery mildew detection with the SAM is based on two reference spectra for "symptoms" and two reference spectra for "healthy tissue". The SVM prediction is based on 15 training samples for each class. The image contains the white reference panel on the left side.

\section{Discussion}

The investigations performed in this study evaluate the Specim IQ camera with regard to spectral imaging quality and usability in typical applications in current plant phenotying research. The comparison of the new Specim IQ camera with the established Specim V10E revealed the competitiveness of the handheld device, but also its limits. In laboratory experiment, high level of conformity was testified on the observed test materials. The visualized spectra in Figure 3 and the mean distances between the spectra of averaged 0.009 (min: 0.004 and max: 0.016) show the overall good measurement quality. At some materials the phenomenon was observed that going from $400 \mathrm{~nm}$ to $450 \mathrm{~nm}$ the reflectance is decreasing even if the Specim V10E detects a increasing reflectance (cf. Figure 3B). This effect leads to increased distances between the observed spectra if the material has a decreasing reflectance in this region but has nearly no effect if the reflectance is increasing. One reason could be the influence of stray light that adds a systematic effect to first few bands. The option to compensate this effect by calibration or hardware adaption needs further investigations. In the daylight observations, both cameras still show a good coherency, but to a lower degree. Natural variation in the light intensity and composition causes a higher standard deviation of 0.029 compared to 0.019 and an increased mean distance of 0.043 (Figure 4, Table A2). A systematic deviation of the Specim IQ with an absolute value of up to 0.10 was visible in the near infrared from $925 \mathrm{~nm}$ to $1000 \mathrm{~nm}$. A line pattern, caused by slight instabilities of the slit width of the Specim IQ sensor resulting in a variability of up to $5 \%$ in reflectance, was visible in all images of the Specim IQ (Figure 5). Its effect on the reference measurements was minimized as a region of the image was averaged. However, the Specim IQ showed suitable imaging characteristics in both environments that allow a reliable sensing of reflectance characteristics. The performance under different environmental conditions can be mostly deduced as they will be a mixture of the presented extreme conditions.

The application of the Specim IQ for the investigation of Arabidopsis thaliana mutants with different rates of NPQ reveals the suitability of selected VIs and the potential of the Specim IQ to record these subtle parameters (Figure 6). For instance, chlorophyll content was estimated with the use of REIP, which requires a continuous hyperspectral signal as it is based on the first derivative of the vegetation spectra. Using the three indices shown, it was possible to detect a deviation from the wildtype (Col-0) while significant difference was observed between the two mutants under stress condition. In general, hyperspectral imagers are able to provide such comprehensive reflectance information that allow a 
flexible sensing of various target parameters applicable for high-throughput phenotyping in the lab and in the field. The Specim IQ provides sufficient spectral resolution and radiometric accuracy to record reliably VI values. Thus, a characterization of subtle differences in plant characteristics can be performed by the Specim IQ given a suitable selection of VIs. Hereinafter, it is recommended to investigate the possibility to detect steady-state chlorophyll a fluorescence based on proper derivatives of plant spectra taken under controlled conditions [71].

The application of the Specim IQ for the detection and quantification of powdery mildew on barley plants in the Mini-Plot facility showed promising results. The SVM analysis determines reasonable predictions with a good concordance to the visual perception (Figure 8). Applied in larger experiments, the Specim IQ can be used to rank the resistance level of multiple varieties providing significant input in resistance breeding. The quantification of powdery mildew infections based on hyperspectral images is a challenging task due to the likelihood of confusion with specular reflections and senescent leaf parts. Consequently, the SVM classifier was applied, which has been proven to provide convincing results in hyperspectral data sets (Figure 7). A detection of powdery mildew using the SAM, which is integrated in Specim IQ Studio, may be possible by combining multiple reference spectra. Using only few reference spectra, it produces incomplete detection results as not all appearances of the disease were covered. Moreover, a qualitative assessment of the detection accuracy was not possible as an export of classification results was not possible with the software version available during the experiments. The definition and optimization of tailored data interpretation models is decisive for the detection accuracy, whereas the data quality of Specim IQ camera is not a limiting factor.
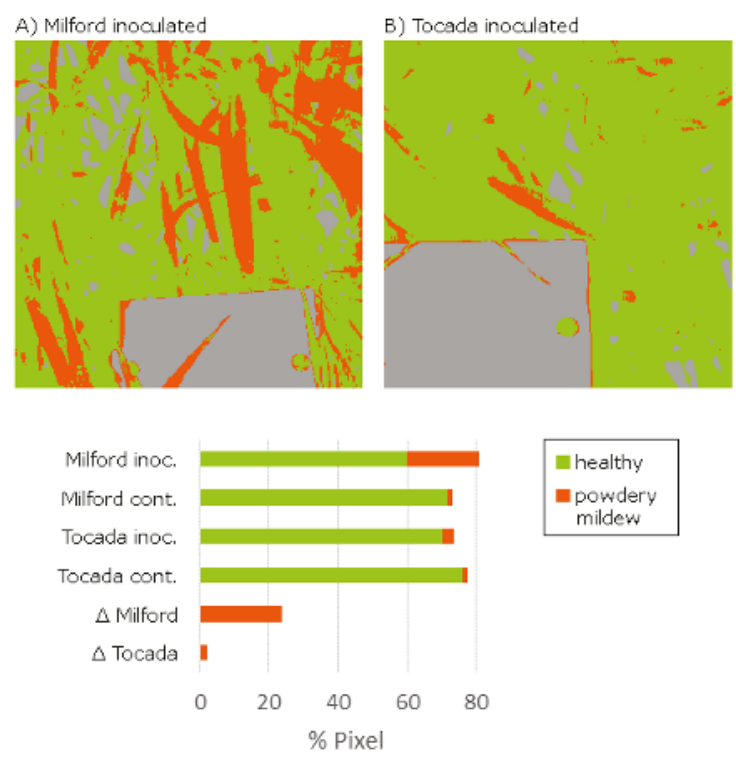

Figure 8. Evaluation for the images of inoculated barley plants by a Support Vector Machine classification model (green: healthy, orange: symptom, gray: background): Cultivar (cv.) Milford shows significantly more affected pixels whereas the $\mathrm{cv}$. Tocada shows only a few symptoms in the measured part of the canopy. Percentage of affected pixels is given for inoculated (inoc.) and healthy control (cont.) plants.

The handling of the Specim IQ is determined by the predefined imaging pipeline (Figure 2). Within this pipeline, all relevant parameters are requested before the image is taken and operating errors like a missing white reference capture are, as far as possible, prevented. The interface is 
designed to lower the initial hurdle for the unexperienced operator. The integrated touch screen supports the user by clear instructions compared to the alternative multi-purpose buttons. If suitable analysis programs are deployed on the device, on the fly imaging and analysis can be performed by an inexperienced person after a short introduction. On the other side, such dialogue approach prevents a high level of automation. Furthermore, a remote control from a computer was not supported in the prototype version. Summarizing, in setups with a high level of repetitions, such as static measurement or high-throughput platforms, other sensors are preferable. The Specim IQ sensor is developed for mobile and highly flexible applications in the laboratory, the greenhouse and in the field.

\section{Conclusions}

The new Specim IQ camera was evaluated with regard to the measurement quality, the handling and the performance in real-world scenarios of plant-physiological and agricultural experiments. The radiometric evaluation of the provided Specim IQ prototype revealed a high level of concordance to the established Specim V10E in indoor and outdoor settings. Limitations are revealed for the bands $400-415 \mathrm{~nm}$ at some materials and for the bands $925-1000 \mathrm{~nm}$ in direct sunlight. Applications are possible indoors and outdoors. The results obtained from the VI analysis suggest potential applications in the context of plant research and phenotyping strategies. For the assessment of powdery mildew, the Specim IQ showed sufficient measurement capabilities and in combination with SVM a high level of consistency to visual assessment in quantification.

The Specim IQ may become a promising hyperspectral camera device with multiple opportunities in plant science. The high measurement quality in combination with compactness, mobility and integrated processing capabilities creates the conditions to acquire new application fields. Trade-off situations between data quality, throughput and stability of environmental conditions are common in plant phenotyping. The Specim IQ allows now to transfer sensor technique at the quality level of laboratory equipment to the greenhouse and the field without any carrier platform or control and storage devices. Thus, the flexible and robust Specim IQ supports the technology transfer to the field and has the potential to increase the overall impact of hyperspectral sensing technologies.

Acknowledgments: This study was funded by the German Federal Ministry of Education and Research (BMBF) within the scope of the competitive grants program "Networks of excellence in agricultural and nutrition research-CROP.SENSe.net" (Funding code: 0315529), Junior Research Group "Hyperspectral phenotyping of resistance reactions of barley" and due to financial support of the Daimler and Benz foundation and Bayer CropScience. We thank the reviewers for the constructive and valuable remarks.

Author Contributions: J.B., K.A., D.E., A.-K.M. and U.R. conceived and designed the experiments; J.B., K.A., S.B., S.M., S.T., D.B. and M.T.K. performed the experiments; J.B., K.A., and S.T. analyzed the data; J.B., K.A., S.T, J.J., H.S., A.-K.M. and U.R. wrote the paper. The manuscript was revised and approved by all authors.

Conflicts of Interest: SPECIM, Spectral Imaging Oy Ltd., provided two prototypes of the Specim IQ, a training and prepared Section 2. Technical description of the Specim IQ camera system. Additionally, Specim provided financial support to perform the evaluation of the sensor and payed the APC. Scientists from the Forschungszentrum Jülich and Bonn University designed the scientific test cases, analysed the results, produced the graphs and wrote the text. Representatives of the company were not involved in the design of the comparison with the Specim V10E, the case studies and the writing of the text of the case studies. 


\section{Appendix A}

Table A1. Comparative performance parameters of the Specim IQ and Specim V10E observed in the laboratory under artificial light conditions. Mean standard deviation of the homogeneous color cards, the mean absolute distance between the mean spectra, the maximum absolute distance and the mean relative distance in percent.

\begin{tabular}{llllll}
\hline Indoor & $\begin{array}{l}\text { Std. } \\
\text { Specim V10E [-] }\end{array}$ & $\begin{array}{l}\text { Std. } \\
\text { Specim IQ [-] }\end{array}$ & $\begin{array}{l}\text { Mean } \\
\text { Abs. Distance [-] }\end{array}$ & $\begin{array}{l}\text { Maximum } \\
\text { Abs. Distance [-] }\end{array}$ & $\begin{array}{l}\text { Mean } \\
\text { Rel. Distance [\%] }\end{array}$ \\
\hline Paper, Yellow & 0.017 & 0.022 & 0.008 & 0.022 & 0.019 \\
Paper, Green & 0.017 & 0.023 & 0.004 & 0.015 & 0.006 \\
Paper, Red & 0.015 & 0.016 & 0.016 & 0.033 & 0.098 \\
Paper, bright Yellow & 0.018 & 0.022 & 0.008 & 0.031 & 0.013 \\
Paper, Pink & 0.017 & 0.018 & 0.006 & 0.023 & 0.011 \\
Polyethylene, Pink & 0.017 & 0.025 & 0.008 & 0.038 & 0.013 \\
Polyethylene, Blue & 0.017 & 0.024 & 0.010 & 0.030 & 0.020 \\
Polyethylene, White & 0.020 & 0.032 & 0.007 & 0.032 & 0.033 \\
Polyethylene, Red & 0.015 & 0.017 & 0.006 & 0.022 & 0.013 \\
Polyethylene, Orange & 0.016 & 0.016 & 0.006 & 0.037 & 0.008 \\
Polyethylene, Purple & 0.015 & 0.013 & 0.004 & 0.021 & 0.022 \\
\hline Average & 0.016 & 0.021 & 0.008 & 0.028 & \\
\hline
\end{tabular}

Table A2. Comparative performance parameters of the Specim IQ and Specim V10E observed outdoors under natural light conditions. Mean standard deviation of the homogeneous color cards, the mean absolute distance between the mean spectra, the maximum absolute distance and the mean relative distance in percent.

\begin{tabular}{llllll}
\hline Outdoor & $\begin{array}{l}\text { Std. } \\
\text { Specim V10E [-] }\end{array}$ & $\begin{array}{l}\text { Std. } \\
\text { Specim IQ [-] }\end{array}$ & $\begin{array}{l}\text { Mean } \\
\text { Abs. Distance [-] }\end{array}$ & $\begin{array}{l}\text { Maximum } \\
\text { Abs. Distance [-] }\end{array}$ & $\begin{array}{l}\text { Mean } \\
\text { Rel. Distance [\%] }\end{array}$ \\
\hline Paper, Yellow & 0.040 & 0.046 & 0.054 & 0.183 & 0.072 \\
Paper, Green & 0.041 & 0.047 & 0.060 & 0.222 & 0.077 \\
Paper, Red & 0.041 & 0.035 & 0.037 & 0.156 & 0.090 \\
Paper, bright Yellow & 0.031 & 0.035 & 0.050 & 0.193 & 0.065 \\
Paper, Pink & 0.036 & 0.045 & 0.051 & 0.237 & 0.065 \\
Paper, White & 0.040 & 0.043 & 0.071 & 0.254 & 0.081 \\
Polyethylene, Pink & 0.020 & 0.017 & 0.024 & 0.138 & 0.043 \\
Polyethylene, Blue & 0.020 & 0.016 & 0.039 & 0.185 & 0.057 \\
Polyethylene, White & 0.023 & 0.022 & 0.023 & 0.145 & 0.025 \\
Polyethylene, Red & 0.018 & 0.014 & 0.041 & 0.171 & 0.130 \\
Polyethylene, Orange & 0.021 & 0.018 & 0.035 & 0.183 & 0.084 \\
Polyethylene, Purple & 0.018 & 0.015 & 0.031 & 0.160 & 0.062 \\
\hline Average & 0.029 & 0.029 & 0.043 & & 0.071 \\
\hline
\end{tabular}

\section{References}

1. Jensen, J.R. Remote Sensing of Environment: An Earth Ressource Perspective; Pearson: London, UK, 2006.

2. Jung, A.; Vohland, M.; Thiele-Bruhn, S. Use of a portable camera for proximal soil sensing with hyperspectral image data. Remote Sens. 2015, 7, 11434-11448.

3. Steffens, M.; Kohlpaintner, M.; Buddenbaum, H. Fine spatial resolution mapping of soil organic matter quality in a Histosol profile. Eur. J. Soil Sci. 2014, 65, 827-839.

4. Cheng, W.; Sun, D.W.; Pu, H.; Wei, Q. Chemical spoilage extent traceability of two kinds of processed pork meats using one multispectral system developed by hyperspectral imaging combined with effective variable selection methods. Food Chem. 2017, 221, 1989-1996.

5. Mahlein, A.K. Plant disease detection by imaging sensors-Parallels and specific demands for precision agriculture and plant phenotyping. Plant Dis. 2016, 100, 241-251.

6. Sendin, K.; Williams, P.J.; Manley, M. Near infrared hyperspectral imaging in quality and safety evaluation of cereals. Crit. Rev. Food Sci. Nutr. 2016, 13,1-16.

7. Gowen, A.; O'Donnell, C.; Cullen, P.; Downey, G.; Frias, J. Hyperspectral imaging-An emerging process analytical tool for food quality and safety control. Trends Food Sci. Technol. 2007, 18, 590-598.

8. Wu, D.; Sun, D.W. Advanced applications of hyperspectral imaging technology for food quality and safety analysis and assessment: A review-Part I: Fundamentals. Innov. Food Sci. Emerg. Technol. 2013, 19, 1-14. 
9. Pu, Y.Y.; Feng, Y.Z.; Sun, D.W. Recent progress of hyperspectral imaging on quality and safety inspection of fruits and vegetables: A review. Compr. Rev. Food Sci. Food Saf. 2015, 14, 176-188.

10. Nicolaï, B.M.; Lötze, E.; Peirs, A.; Scheerlinck, N.; Theron, K.I. Non-destructive measurement of bitter pit in apple fruit using NIR hyperspectral imaging. Postharvest Biol. Technol. 2006, 40, 1-6.

11. Huang, M.; Wang, Q.; Zhang, M.; Zhu, Q. Prediction of color and moisture content for vegetable soybean during drying using hyperspectral imaging technology. J. Food Eng. 2014, 128, 24-30.

12. Baiano, A.; Terracone, C.; Peri, G.; Romaniello, R. Application of hyperspectral imaging for prediction of physico-chemical and sensory characteristics of table grapes. Comput. Electron. Agric. 2012, 87, 142-151.

13. Schmilovitch, Z.; Ignat, T.; Alchanatis, V.; Gatker, J.; Ostrovsky, V.; Felföldi, J. Hyperspectral imaging of intact bell peppers. Biosyst. Eng. 2014, 117, 83-93.

14. Scafutto, R.D.M.; de Souza Filho, C.R.; Rivard, B. Characterization of mineral substrates impregnated with crude oils using proximal infrared hyperspectral imaging. Remote Sens. Environ. 2016, 179, 116-130.

15. Mahlein, A.K.; Oerke, E.C.; Steiner, U.; Dehne, H.W. Recent advances in sensing plant diseases for precision crop protection. Eur. J. Plant Pathol. 2012, 133, 197-209.

16. Simko, I.; Jimenez-Berni, J.A.; Sirault, X.R. Phenomic approaches and tools for phytopathologists. Phytopathology 2017, 107, 6-17.

17. West, J.S.; Bravo, C.; Oberti, R.; Lemaire, D.; Moshou, D.; McCartney, H.A. The potential of optical canopy measurement for targeted control of field crop diseases. Annu. Rev. Phytopathol. 2003, 41, 593-614.

18. Fiorani, F.; Rascher, U.; Jahnke, S.; Schurr, U. Imaging plants dynamics in heterogenic environments. Curr. Opin. Biotechnol. 2012, 23, 227-235.

19. Kicherer, A.; Herzog, K.; Bendel, N.; Klück, H.C.; Backhaus, A.; Wieland, M.; Rose, J.C.; Klingbeil, L.; Läbe, T.; Hohl, C.; et al. Phenoliner: A New Field Phenotyping Platform for Grapevine Research. Sensors 2017, 17, 1625, doi:10.3390/s17071625.

20. Kuska, M.; Wahabzada, M.; Leucker, M.; Dehne, H.W.; Kersting, K.; Oerke, E.C.; Steiner, U.; Mahlein, A.K. Hyperspectral phenotyping on the microscopic scale: Towards automated characterization of plant-pathogen interactions. Plant Methods 2015, 11, 28, doi:10.1186/s13007-015-0073-7

21. Mahlein, A.K.; Steiner, U.; Hillnhütter, C.; Dehne, H.W.; Oerke, E.C. Hyperspectral imaging for small-scale analysis of symptoms caused by different sugar beet diseases. Plant Methods 2012, 8, 3, doi:10.1186/1746-4811-8-3.

22. Malenovskỳ, Z.; Mishra, K.B.; Zemek, F.; Rascher, U.; Nedbal, L. Scientific and technical challenges in remote sensing of plant canopy reflectance and fluorescence. J. Exp. Bot. 2009, 60, 2987-3004.

23. Drusch, M.; Moreno, J.; Del Bello, U.; Franco, R.; Goulas, Y.; Huth, A.; Kraft, S.; Middleton, E.M.; Miglietta, F.; Mohammed, G.; et al. The FLuorescence EXplorer Mission Concept-ESA's Earth Explorer 8. IEEE Trans. Geosci. Remote Sens. 2017, 55, 1273-1284.

24. Fang, S.; Tang, W.; Peng, Y.; Gong, Y.; Dai, C.; Chai, R.; Liu, K. Remote Estimation of Vegetation Fraction and Flower Fraction in Oilseed Rape with Unmanned Aerial Vehicle Data. Remote Sens. 2016, 8, 416, doi: $10.3390 /$ rs8050416.

25. Rascher, U.; Nichol, C.J.; Small, C.; Hendricks, L. Monitoring spatio-temporal dynamics of photosynthesis with a portable hyperspectral imaging system. Photogramm. Eng. Remote Sens. 2007, 73, 45-56.

26. Running, S.W.; Nemani, R.R. Relating seasonal patterns of the AVHRR vegetation index to simulated photosynthesis and transpiration of forests in different climates. Remote Sens. Environ. 1988, 24, 347-367.

27. Sellers, P.J. Canopy reflectance, photosynthesis and transpiration. Int. J. Remote Sens. 1985, 6, 1335-1372.

28. Choudhury, B.J. Relationships between vegetation indices, radiation absorption, and net photosynthesis evaluated by a sensitivity analysis. Remote Sens. Environ. 1987, 22, 209-233.

29. Inoue, Y.; Peñuelas, J. Relationship between light use efficiency and photochemical reflectance index in soybean leaves as affected by soil water content. Int. J. Remote Sens. 2006, 27, 5109-5114.

30. Rossini, M.; Nedbal, L.; Guanter, L.; Ač, A.; Alonso, L.; Burkart, A.; Cogliati, S.; Colombo, R.; Damm, A.; Drusch, M.; et al. Red and far red Sun-induced chlorophyll fluorescence as a measure of plant photosynthesis. Geophys. Res. Lett. 2015, 42, 1632-1639.

31. Aasen, H.; Burkart, A.; Bolten, A.; Bareth, G. Generating 3D hyperspectral information with lightweight UAV snapshot cameras for vegetation monitoring: From camera calibration to quality assurance. ISPRS J. Photogramm. Remote Sens. 2015, 108, 245-259. 
32. Näsi, R.; Honkavaara, E.; Tuominen, S.; Saari, H.; Pölönen, I.; Hakala, T.; Viljanen, N.; Soukkamäki, J.; Näkki, I.; Ojanen, H.; et al. UAS based tree species identification using the novel FPI based hyperspectral cameras in visible, NIR and SWIR spectral ranges. Int. Arch. Photogramm. Remote Sens. Spat. Inf. Sci. 2016, XLI-B1, 1143-1148.

33. Bareth, G.; Aasen, H.; Bendig, J.; Gnyp, M.L.; Bolten, A.; Jung, A.; Michels, R.; Soukkamäki, J. Low-weight and UAV-based hyperspectral full-frame cameras for monitoring crops: Spectral comparison with portable spectroradiometer measurements. Photogramm. Fernerkund. Geoinf. 2015, 2015, 69-79.

34. Geelen, B.; Tack, N.; Lambrechts, A. A compact snapshot multispectral imager with a monolithically integrated per-pixel filter mosaic. Proc. SPIE, 2014, 8974, 89740L, doi:10.1117/12.2037607.

35. Delalieux, S.; Delauré, B.; Tits, L.; Boonen, M.; Sima, A.; Baeck, P. High resolution strawberry field monitoring using the compact hyperspectral imaging solution COSI. Adv. Anim. Biosci. 2017, 8, 156-161.

36. Vega, F.A.; Ramírez, F.C.; Saiz, M.P.; Rosúa, F.O. Multi-temporal imaging using an unmanned aerial vehicle for monitoring a sunflower crop. Biosyst. Eng. 2015, 132, 19-27.

37. Wendel, A.; Underwood, J. Self-supervised weed detection in vegetable crops using ground based hyperspectral imaging. In Proceedings of the 2016 IEEE International Conference on Robotics and Automation (ICRA), Stockholm, Sweden, 16-21 May 2016; pp. 5128-5135.

38. Busemeyer, L.; Mentrup, D.; Möller, K.; Wunder, E.; Alheit, K.; Hahn, V.; Maurer, H.P.; Reif, J.C.; Würschum, T.; Müller, J.; et al. Breedvision-A multi-sensor platform for non-destructive field-based phenotyping in plant breeding. Sensors 2013, 13, 2830-2847.

39. Jay, S.; Bendoula, R.; Hadoux, X.; Féret, J.B.; Gorretta, N. A physically-based model for retrieving foliar biochemistry and leaf orientation using close-range imaging spectroscopy. Remote Sens. Environ. 2016, 177, 220-236.

40. Pinto, F.; Müller-Linow, M.; Schickling, A.; Cendrero-Mateo, M.P.; Ballvora, A.; Rascher, U. Multiangular Observation of Canopy Sun-Induced Chlorophyll Fluorescence by Combining Imaging Spectroscopy and Stereoscopy. Remote Sens. 2017, 9, 415, doi:10.3390/rs9050415.

41. Pinto, F.; Damm, A.; Schickling, A.; Panigada, C.; Cogliati, S.; Müller-Linow, M.; Balvora, A.; Rascher, U. Sun-induced chlorophyll fluorescence from high-resolution imaging spectroscopy data to quantify spatio-temporal patterns of photosynthetic function in crop canopies. Plant Cell Environ. 2016, 39, 1500-1512.

42. Katsoulas, N.; Elvanidi, A.; Ferentinos, K.; Bartzanas, T.; Kittas, C. Calibration methodology of a hyperspectral imaging system for greenhouse plant water stress estimation. Acta Hortic. 2016, 1142, 119-126.

43. Reddy, K.N.; Huang, Y.; Lee, M.A.; Nandula, V.K.; Fletcher, R.S.; Thomson, S.J.; Zhao, F. Glyphosate-resistant and glyphosate-susceptible Palmer amaranth (Amaranthus palmeri S. Wats.): Hyperspectral reflectance properties of plants and potential for classification. Pest Manag. Sci. 2014, 70, 1910-1917.

44. Ge, Y.; Bai, G.; Stoerger, V.; Schnable, J.C. Temporal dynamics of maize plant growth, water use, and leaf water content using automated high throughput RGB and hyperspectral imaging. Comput. Electron. Agric. 2016, 127, 625-632.

45. Behmann, J.; Mahlein, A.K.; Rumpf, T.; Römer, C.; Plümer, L. A review of advanced machine learning methods for the detection of biotic stress in precision crop protection. Precis. Agric. 2015, 16, 239-260.

46. Zhou, K.; Deng, X.; Yao, X.; Tian, Y.; Cao, W.; Zhu, Y.; Ustin, S.L.; Cheng, T. Assessing the Spectral Properties of Sunlit and Shaded Components in Rice Canopies with Near-Ground Imaging Spectroscopy Data. Sensors 2017, 17, 578, doi:10.3390/s17030578.

47. Behmann, J.; Mahlein, A.K.; Paulus, S.; Kuhlmann, H.; Oerke, E.C.; Plümer, L. Calibration of hyperspectral close-range pushbroom cameras for plant phenotyping. ISPRS J. Photogramm. Remote Sens. 2015, $106,172-182$.

48. Kruse, F.A.; Lefkoff, A.; Boardman, J.; Heidebrecht, K.; Shapiro, A.; Barloon, P.; Goetz, A. The spectral image processing system (SIPS)-interactive visualization and analysis of imaging spectrometer data. AIP Conf. Proc. 1993, 283, 192-201.

49. Savitzky, A.; Golay, M.J. Smoothing and differentiation of data by simplified least squares procedures. Anal. Chem. 1964, 36, 1627-1639.

50. Cortes, C.; Vapnik, V. Support Vector Networks. Machine Learning 1995, 20, 273-297.

51. Bannari, A.; Morin, D.; Bonn, F.; Huete, A.R. A review of vegetation indices. Remote Sens. Rev. 1995, 13, 95-120. 
52. Purevdorj, T.S.; Tateishi, R.; Ishiyama, T.; Honda, Y. Relationships between percent vegetation cover and vegetation indices. Int. J. Remote Sens. 1998, 19, 3519-3535.

53. Tucker, C.J. Red and photographic infrared linear combinations for monitoring vegetation. Remote Sens. Environ. 1979, 150, 127-150.

54. Gamon, J.A.; Field, C.B.; Goulden, M.L.; Griffin, K.L.; Hartley, A.E.; Joel, G.; Peñuela, J.; Valentini, R. Relationships between NDVI, canopy structure, and photosynthesis in three Californian vegetation types. Ecol. Appl. 1995, 5, 28-41.

55. Asrar, G.; Fuchs, M.; Kanemasu, E.T.; Hatfield, J.L. Estimating absorbed photosynthetic radiation and leaf area index from spectral reflectance in wheat. Agron. J. 1984, 76, 300-306.

56. Daughtry, C.S.T.; Walthall, C.L.; Kim, M.S.; Colstoun, E.B.D. Estimating Corn Leaf Chlorophyll Concentration from Leaf and Canopy Reflectance. Remote Sens. Environ. 2000, 74, 229-239.

57. Wu, C.; Niu, Z.; Tang, Q.; Huang, W. Estimating chlorophyll content from hyperspectral vegetation indices: Modeling and validation. Agric. For. Meteorol. 2008, 148, 1230-1241.

58. Gitelson, A.A.; Merzlyak, M.N. Remote estimation of chlorophyll content in higher plant leaves. Int. J. Remote Sens. 1997, 18, 2691-2697.

59. Iwata, H.; Ueyama, M.; Iwama, C.; Harazono, Y. A variation in the fraction of absorbed photosynthetically active radiation and a comparison with MODIS data in burned black spruce forests of interior Alaska. Polar Sci. 2013, 7, 113-124.

60. Nichol, C.J.; Rascher, U.; Matsubara, S.; Osmond, B. Assessing photosynthetic efficiency in an experimental mangrove canopy using remote sensing and chlorophyll fluorescence. Trees 2006, 20, 9 , doi:10.1007/s00468-005-0005-7.

61. Li, X.P.; Björkman, O.; Shih, C.; Grossman, A.; Rosenquist, M.; Jansson, S.; Niyogi, K. A pigment-binding protein essential for regulation of photosynthetic light harvesting. Nature 2000, 403, 391-395.

62. Niyogi, K.K.; Grossman, A.R.; Björkman, O. Arabidopsis mutants define a central role for the xanthophyll cycle in the regulation of photosynthetic energy conversion. Plant Cell 1998, 10, 1121-1134.

63. Jackson, R.; Slater, P.; Pinter, P. Discrimination of growth and water stress in wheat by various vegetation indices through clear and turbid atmospheres. Remote Sens. Environ. 1983, 13, 187-208.

64. Herrmann, I.; Pimstein, A.; Karnieli, A.; Cohen, Y.; Alchanatis, V.; Bonfil, D. LAI assessment of wheat and potato crops by VEN $\mu$ S and Sentinel-2 bands. Remote Sens. Environ. 2011, 115, 2141-2151.

65. Horler, D.; Dockray, M.; Barber, J. The red edge of plant leaf reflectance. Int. J. Remote Sens. 1983, 4, $273-288$.

66. Gamon, J.A.; Serrano, L.; Surfus, J.S. The photochemical reflectance index: An optical indicator of photosynthetic radiation use efficiency across species, functional types, and nutrient levels. Oecologia 1997, 112, 492-501.

67. Vogelmann, J.; Rock, B.; Moss, D. Red edge spectral measurements from sugar maple leaves. Remote Sens. 1993, 14, 1563-1575.

68. Le Maire, G.; Francois, C.; Dufrene, E. Towards universal broad leaf chlorophyll indices using PROSPECT simulated database and hyperspectral reflectance measurements. Remote Sens. Environ. 2004, 89, 1-28.

69. Chin, J.A.; Wang, E.C.; Kibbe, M.R. Evaluation of hyperspectral technology for assessing the presence and severity of peripheral artery disease. J. Vasc. Surg. 2011, 54, 1679-1688.

70. Virlet, N.; Sabermanesh, K.; Sadeghi-Tehran, P.; Hawkesford, M.J. Field Scanalyzer: An automated robotic field phenotyping platform for detailed crop monitoring. Funct. Plant Biol. 2017, 44, 143-153.

71. Zarco-Tejada, P.; Pushnik, J.; Dobrowski, S.; Ustin, S. Steady-state chlorophyll a fluorescence detection from canopy derivative reflectance and double-peak red-edge effects. Remote Sens. Environ. 2003, 84, 283-294.

(C) 2018 by the authors. Licensee MDPI, Basel, Switzerland. This article is an open access article distributed under the terms and conditions of the Creative Commons Attribution (CC BY) license (http:/ / creativecommons.org/licenses/by/4.0/). 


\title{
Article \\ Two Solutions of Soil Moisture Sensing with RFID for Landslide Monitoring
}

\author{
Sérgio Francisco Pichorim ${ }^{1, *}$, Nathan J. Gomes ${ }^{2}$ and John C. Batchelor ${ }^{2}$ \\ 1 Graduate School of Electrical Engineering and Computer Science (CPGEI-DAELN), \\ Federal University of Technology, Paraná (UTFPR), 80230-901 Curitiba, Brazil \\ 2 School of Engineering and Digital Arts, University of Kent, CT2 7NT Canterbury, UK; \\ N.J.Gomes@kent.ac.uk (N.J.G.); J.C.Batchelor@kent.ac.uk (J.C.B.) \\ * Correspondence: pichorim@utfpr.edu.br; Tel.: +55-41-3310-4684
}

Received: 13 November 2017; Accepted: 29 January 2018; Published: 3 February 2018

\begin{abstract}
Two solutions for UHF RFID tags for soil moisture sensing were designed and are described in this paper. In the first, two conventional tags (standard transponders) are employed: one, placed close to the soil surface, is the sensor tag, while the other, separated from the soil, is the reference for system calibration. By transmission power ramps, the tag's turn-on power levels are measured and correlated with soil condition (dry or wet). In the second solution, the SL900A chip, which supports up to two external sensors and an internal temperature sensor, is used. An interdigital capacitive sensor was connected to the transponder chip and used for soil moisture measurement. In a novel design for an UHF RFID tag the sensor is placed below the soil surface, while the transponder and antenna are above the soil to improve communication. Both solutions are evaluated practically and results show the presence of water in soil can be remotely detected allowing for their application in landslide monitoring.
\end{abstract}

Keywords: soil; moisture; sensor; landslide

\section{Introduction}

In Brazil, due to serious social problems, human occupation occurs in hillsides usually evaluated as risk areas. This exposes people to many hazards, especially landslide risks. In summer, tropical rains soak the soil of hillsides, causing tragedies. Unfortunately, landslides have repeatedly occurred in the last decade. Landslide disasters are related to several factors and natural events, such as earthquakes, volcanic activity, and, in particular, the degree of rain and soil water saturation. The ability to monitor the soil moisture, at many points of an urban slope where there are risk areas, could be a very important way of evaluating the degree of danger and, therefore, predicting and/or preventing accidents and deaths. Some studies have shown a clear and direct relationship between rainfall, soil moisture and landslide events [1,2]. Sensing networks and telemetric instrumentation have been applied to monitor soil moisture levels for landslide forecasting [1-3].

Ultra-high-frequency radio-frequency identification (UHF RFID) devices may be an interesting solution for soil moisture measurement because the sensors are low-cost, can be passive (no battery) and can provide communication distances of some meters [4,5]. A set of RFID-based soil moisture sensors properly installed on a hillside could provide real-time landslide monitoring.

Moisture level or liquid identification have been frequent applications of UHF RFID tags in the literature [6-15], for example, monitoring the beverage volume in cup or bottle [11,12], water in concrete [5], moisture in wall [13] or in soil $[9,10,14,15]$. The electrical properties of a tag can be modified by the presence of water in its proximity, causing, for example, changes or shifts in resonance frequency, the backscattered signal, or the activation energy (tag's turn-on power) [11,12,14-16]. Different approaches can be found using cheap and conventional tags (i.e., standard non-sensing 
transponders) [8,11-13] or proposing new designs, where special probes connect the tag to the medium under measurement $[5,14,15]$. For system calibration, an interesting solution is the use of two tags, one affected by moisture and the other (as a reference) protected against moisture [6,13]. By frequency sweeping [6] or reader power ramping [17], the responses of tags can be measured and differences between them can be correlated to humidity level.

This paper presents two solutions for the use of UHF passive RFID tags in soil moisture measurement, targeting particularly landslide monitoring.

\section{Two Methods of Measurement}

For the use of UHF RFID tag as a soil moisture monitor, two solutions were proposed and designed. For the first approach, two conventional tags (adhesive labels) are placed in the same spot, where one works as an analog sensor and other as a reference. The sensitive tag (sensor) has its antenna characteristics modulated by soil moisture. This will change the threshold of its activation. The other tag (reference), which is made moisture insensitive, is used as a distance or power reference. The reader works at a constant frequency but with power sweeping (ramp). The difference between the responses of the tags can be used to determine soil moisture. Many UHF chip tags can be applied in this solution. For example, the G2XL is a chip for passive, intelligent tags and labels. Although it does not support any external sensor, it can be used as a tag sensor by changing the antenna tuning, impedance matching, or signal level $[4,11,12,17]$. In this case, the sensing is achieved through capacitive coupling arising from the antenna proximity to the soil surface.

For the second approach, a dedicated tag chip with external sensor input is used. There are few UHF chip tags available with sensor input, however some solutions are possible. For example, the chip G2iL+ is a 4-pin chip, allowing the tamper alarm application [18]; this 1-bit input (on or off) could be used as a digital sensor for soil moisture. For this purpose, a threshold between dry and wet soil must be triggered by a specific circuit.

A better solution for the second approach is the chip SL900A, which is an EPC global Class 3 sensor tag chip with automatic data logging [19]. It works with an internal temperature sensor and can read two external analog sensors [20-23], including resistive, capacitive and optic sensors. This solution will be developed and described further in this paper.

\section{First Approach: Two Conventional Tags}

Initially, a test using a conventional UHF RFID tag (FT-G1210 RFID Inlays, NXP, Eindhoven, Netherlands) on dry and wet soil was conducted to understand the influence of soil moisture on the tag's power on threshold. The influence was evaluated at different separation distances $(H)$ between the tag and soil surface.

The soil moisture sensor tag is placed close to the ground (Figure 1) at height $H$. The highest sensitivity of the tag was found to be when $H$ was a few millimeters, as will be described below. A second tag, which is used as a reference by the reader (distance $R$ ), is separated from the soil with height $A$; it must be placed far from the soil (in air), some centimeters high, in order to be insensitive to soil moisture. Both tags are parallel to the soil but perpendicular to each other, as seen in Figure 1. 


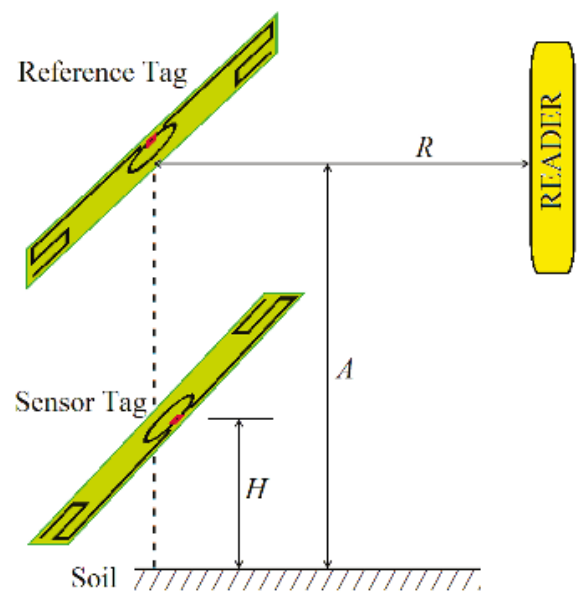

Figure 1. System using two adhesive UHF RFID tags for soil moisture monitoring. Sensor and reference tags, with distance $H$ and $A$, respectively, from soil. The reading distance is $R$.

The behavior of the tags was measured by a Tagformance Lite Reader (Voyantic, Espoo, Finland) in order to determine the best height $H$. This equipment applies ramps of power and analyses the response of a tag population. Thus, the transmission power necessary to turn on the tags $\left(P_{\operatorname{Tag} \mathrm{On}}\right)$ can be evaluated. Frequencies between $800 \mathrm{MHz}$ and $1 \mathrm{GHz}$ were tested.

The setup shown in Figure 1 was placed on dry and wet soils and the Tagformance Lite Reader's antenna was oriented to avoid signal reflections from the soil. Initially, the distance between reader antenna and tag $(R)$ was set to $7 \mathrm{~cm}$.

The test was conducted using $2200 \mathrm{~cm}^{3}$ of topsoil (black earth collected in coordinates 51.297254 North and 1.063208 East), which was dried before the tests. After the first tests (for dry soil situation), a volume of $500 \mathrm{~cm}^{3}$ of tap water was added to the soil. Then, the wet soil tests were started some minutes later, after the water had completely percolated. This volume of water represents a humidity of $22.7 \%$ (volume ratio), which can be considered a very wet soil (soaked). For black earth with bulk density of $1.1 \mathrm{~g} / \mathrm{cm}^{3}$, this volume ratio represents a weight ratio of $20.7 \%$ of water in soil. It is a level that can be assumed within a risk range for a landslide event [2].

In Figure 2 the behavior of a sensor tag over dry and wet soil (dashed and solid lines, respectively) was observed for a tag height $(H)$ of $26 \mathrm{~mm}$ as a function of the reader's frequency. The presence of water in the soil modifies the power necessary to turn on the tag, especially around $870 \mathrm{MHz}$. For the European UHF RFID frequency ( $868 \mathrm{MHz}$, marked with a cursor in Figure 2), the turn-on power $\left(P_{\text {TagOn }}\right)$ changes from about 1.5 to $5 \mathrm{dBm}$ as a function of soil humidity. This significant variation can be exploited by a soil moisture monitoring system. Analyzing the results of Figure 2, it is possible to see that, at $868 \mathrm{MHz}$, the tag is more sensitive to soil moisture (dry and wet) than at $915 \mathrm{MHz}$. Of course, other tag models, or other antenna designs, may exhibit different behavior. 


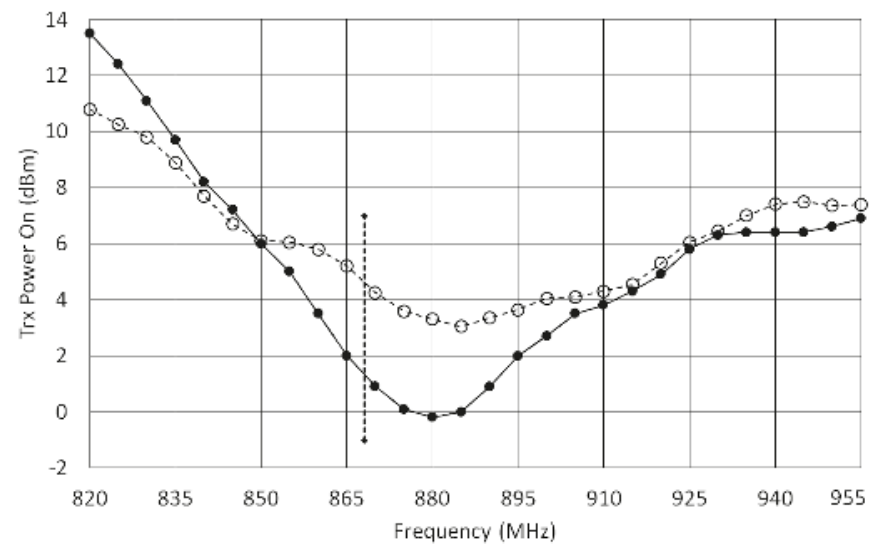

Figure 2. Transmission power to turn on the tag (for $H=26 \mathrm{~mm}$ ) as a function of frequency. Solid line is for wet soil and dashed line is for dry soil. The cursor marks the frequency of $868 \mathrm{MHz}$.

Adopting the frequency of $868 \mathrm{MHz}$, a second test was made to evaluate the tag's behavior as a function of the height $H$, with measurements obtained for $H$ from 1 to $60 \mathrm{~mm}$ over both dry and wet soil, as shown in Figure 3. When the tag is very close to the soil ( $H$ lower than $5 \mathrm{~mm}$ ) the transmission power $P_{\operatorname{Tag} O n}$ is greater than $12 \mathrm{dBm}$ for both soil situation (dry and wet), thus the tag becomes insensitive. Of course, for greater heights the sensor tag will be also insensitive. A maximum sensitivity is obtained when the tag is placed 10 to $30 \mathrm{~mm}$ above the soil, as can be seen in Figure 3 .

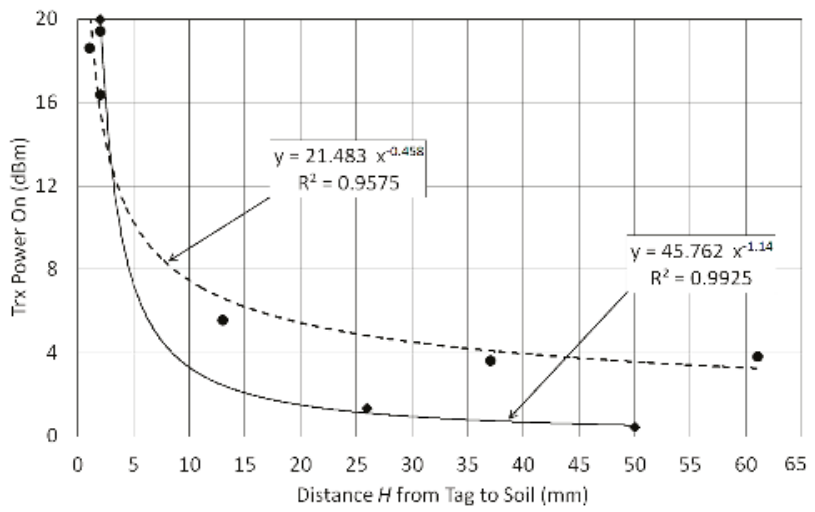

Figure 3. Transmission power to turn on the tag (for $868 \mathrm{MHz}$ ) as a function of the distance between tag and soil $(H)$. Diamonds (solid line) are for wet soil and dots (dashed line) are for dry soil.

Using the results of Figure 3, a system with two conventional UHF RFID tags (adhesive labels) was designed. The sensor tag was fixed with $H=12 \mathrm{~mm}$ and a second tag (the reference one) was placed with $A=100 \mathrm{~mm}$, where the tag was assumed to be insensitive to soil moisture. A photo in Figure 4 shows how these tags are attached. This prototype, only for indoor evaluation, was constructed with a structure of expanded polystyrene blocks, which is assumed to be an UHF neutral material. The reading distances $(R)$ of 30,60 and $90 \mathrm{~cm}$ were used to measure the transmission power $P_{\text {TagOn }}$ for sensor and reference tags over dry and wet soils, as seen in Figure 5. 


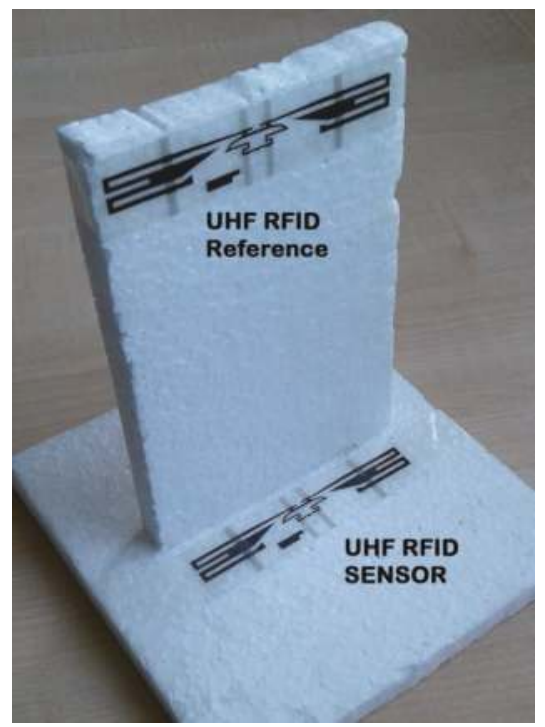

Figure 4. Prototype of Soil Moisture Sensor using two adhesive UHF RFID tags. The Sensor tag is place $12 \mathrm{~mm}$ over the soil. A Reference tag is $100 \mathrm{~mm}$ apart from the soil.

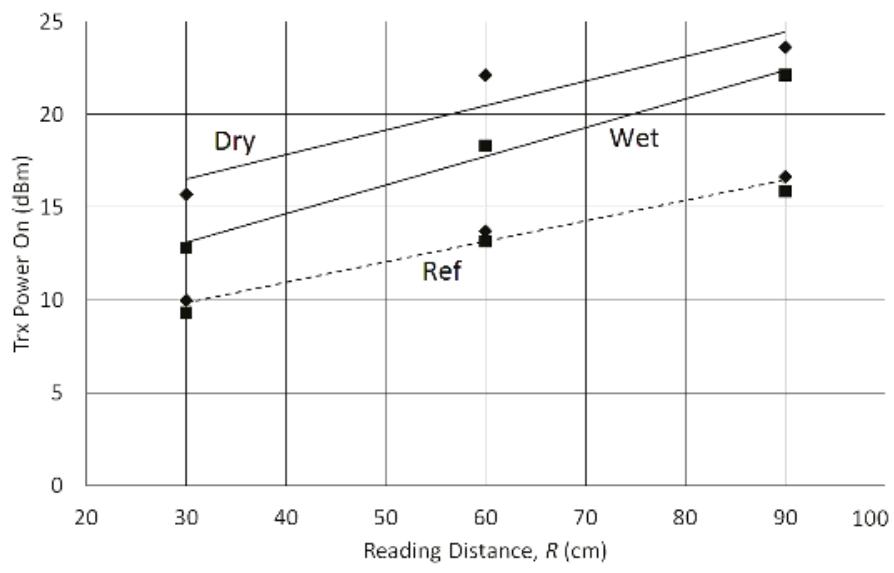

Figure 5. Transmission power to turn on the tags (for $868 \mathrm{MHz}$ ) as a function of the reading distance between tags and reader $(R)$. Solid lines are for Sensor Tag and dashed line for Reference Tag. Diamonds are for dry soil and squares are for wet soil.

According to test results with the Tagformance Lite Reader, a theoretical read range $R$ up to $4 \mathrm{~m}$ can be expected. There is a difference of about 7 to $8 \mathrm{~dB}$ between sensor and reference tags when the soil surface is dry. However, this difference decreases to about 4 to $5 \mathrm{~dB}$ when the soil is wet. These differences are relatively constant for variations of distance $R$.

It can be noticed that the reference sensor (dashed lines) is almost completely insensitive to soil moisture. On the other hand, a significant variation is observed for sensor tag response. Therefore, this behavior can be exploited as a system to monitor soil moisture using two conventional tags. 


\section{Second Approach: Using SL900A Chip}

The SL900A (AMS AG, Premstätten, Austria) is an UHF RFID chip with an internal temperature sensor and two other inputs for external analog sensors. These inputs can support many types of sensor, such as, resistive, conductive, capacitive, current, voltage, resistive bridge and photodiode [19]. The Sensor Front End (SFE) of the SL900A is composed of an integrated 10-bit Analog to Digital Converter (ADC) with selectable voltage references (Vo1 and Vo2). In addition, a current source is used for sensor excitation (data field "Seti"). An operational amplifier feedback resistance (data field "Rang") and the signal frequency (data field "Df") can be configured in the SFE (see Figure 6). The frequency of the RTC oscillator can be calibrated from 800 to $1165 \mathrm{~Hz}$. The references of the ADC are selected for Vo1 (160 to $510 \mathrm{mV}$ ) and Vo2 (260 to $610 \mathrm{mV}$ ). These voltage references are individually selectable in $50 \mathrm{mV}$ steps [19]. For a capacitive sensor, the circuit is connected as shown in Figure 6.

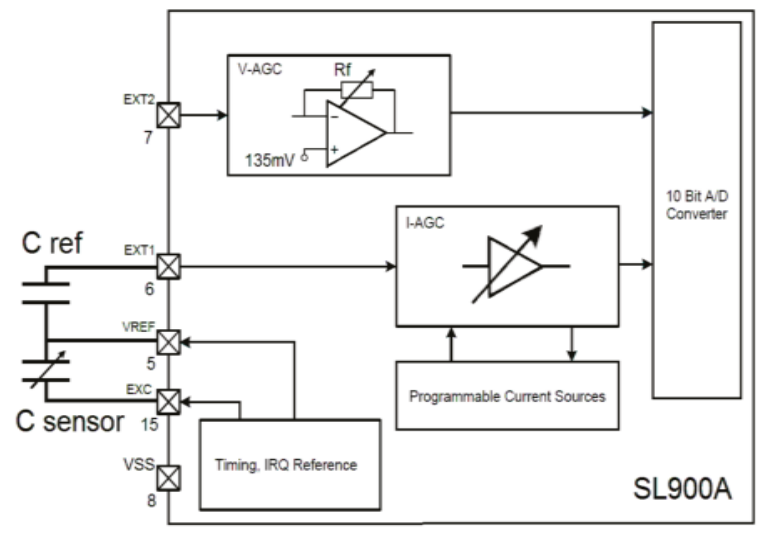

Figure 6. Block diagram of External Sensor Interface of SL900A. For a capacitive sensor (C sensor) a reference capacitor ( $\mathrm{C}$ ref) must be inserted in Ext1 pin.

The SL900A chip uses commands EPC (Gen2) and cool-Log ${ }^{\circledR}$ for its configuration, communication and setting. These commands are transmitted by the tag's reader using a Graphic User Interface (GUI) program. A reader kit DK-R902 LP2 (IDS Microchip, Wollerau, Switzerland) and its GUI, R90xG Demonstrator Control (version 2.12), was used to configure and read the developed tag sensor. The basic commands, used for configuring the chip's SFE interface and reading the chip's ADC output, are:

- Get measurement setup (bytes 0xE0 and 0xA3). Tag replies 128 bits with setup information;

- Get calibration data (bytes 0xE0 and 0xA9). Tag replies 72 bits with current calibration;

- Set SFE parameters (bytes 0xE0 and 0xA4). Reader sends 16 bits for sensor front end setup;

- Set calibration data (bytes 0xE0 and 0xA5). Reader sends 56 bits for tag's calibration; and

- Get sensor value (0xE0 and 0xAD). Tag answers 6 bits of status and 10 bits of ADC value.

Using the above commands from the GUI software, the sensor front end of SL900A was appropriately configured.

The complete circuit for this second approach is shown in Figure 6, where the SL900A is configured for a capacitive sensor in Ext1. Initially, a simulation of capacitive sensor input was performed, in order to test the communication, configuration and response of the circuit and chip. Some capacitors were connected to yield a range from 15 to $88 \mathrm{pF}$. Five readouts, obtained by the GUI and reader, were collected for each input capacitance. The reference capacitor ( $\mathrm{C}$ ref) of $27 \mathrm{pF}$ was determined in function of ADC voltage limits and the range of a real moisture sensor's capacitance. A correlation of 
0.9969 between input capacitances and the GUI readouts was calculated, with an RMS error of 5.15\%. When the input capacitance is low, the readouts are more unstable, probably, due to stray capacitances of wires, or even noise.

To avoid this instability, an interdigital sensor was designed to work at higher capacitances (ranging to about hundreds of picofarad). The capacitive sensor for the soil moisture measurement is a set of 12 interdigital tracks of copper constructed directly on the printed circuit board (PCB) of the UHF RFID tag, as shown in Figure 7. The sensor area $\left(70 \times 30 \mathrm{~mm}^{2}\right)$ is covered with PCB ink for protection against corrosion. The tracks are designed with $2 \mathrm{~mm}$ of width and gaps of $0.5 \mathrm{~mm}$ between them.

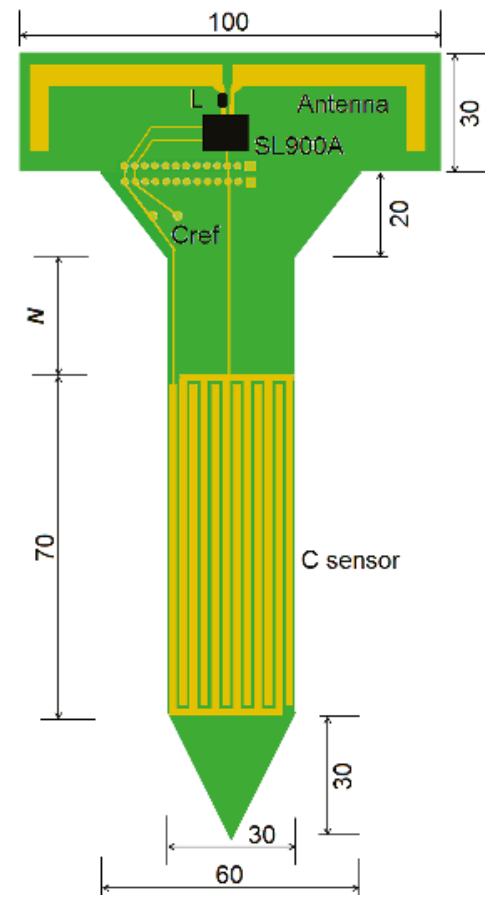

Figure 7. Design of the Soil Stick Sensor using UHF RFID chip (SL900A) and an interdigital capacitive humidity sensor ( $C$ sensor). A neck of $N=30 \mathrm{~mm}$ was defined. The arrow shape allows the tag to be stuck into soil. The whole sensor area must be buried. All dimensions are in millimeter $(\mathrm{mm})$.

The sensor's range of capacitance variation depends on the PCB substrate and ink permittivity, as well soil characteristics and moisture level. In practice, the total sensor capacitance varied between about 70 to $700 \mathrm{pF}$, for dry and wet soil, respectively. Thus, a reference capacitor (C ref) of $220 \mathrm{pF}$ was chosen and inserted between the pins 5 and 6 of chip SL900A.

In this work, the RFID tag sensor is a passive device (no battery), although the SL900A can also work actively with a 3-V coin battery. The tag's antenna is a dipole (L shape) with an SMD inductor (39 $\mathrm{nH})$ for impedance matching [20,24]. The total height of the tag can be increased. The tag's neck (length $N$ in Figure 7) can be defined or changed as a function of how deep the sensor area ( $C$ sensor) must be inserted into the soil while the tag's antenna is elevated from the soil, aiming towards communication range improvement.

A new test was performed with the complete tag to analyze the system performance in soil with different moisture levels. The UHF RFID tag (Figure 7) was stuck into a soil sample in the laboratory (as can be seen in Figure 8). The soil moisture was estimated by the weight ratio of moist and dried soil, 
using a digital scale (resolution of $0.1 \mathrm{~g}$ ). For this test, the topsoil (volume of $650 \mathrm{~cm}^{3}$ ) was collected from coordinates 25.533168 South and 49.200655 West.

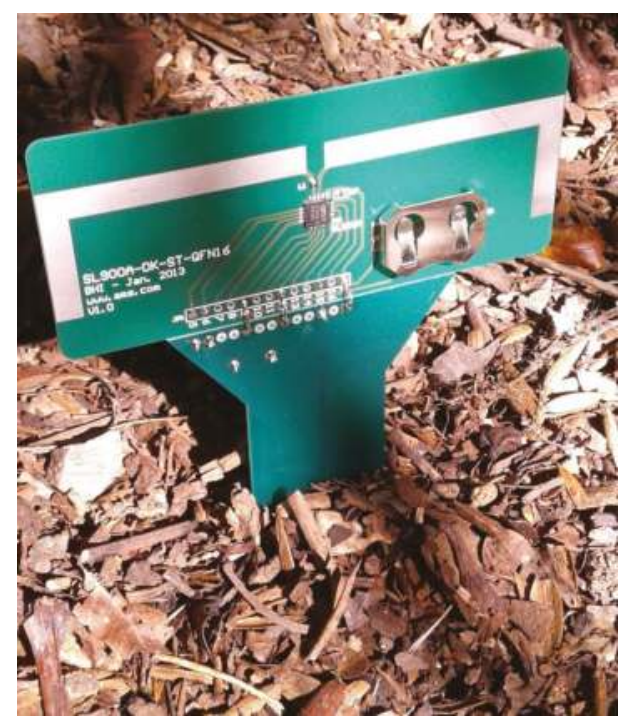

Figure 8. Example of the Soil Stick Sensor for Humidity in a real situation.

The soil was wetted $\left(140 \mathrm{~cm}^{3}\right.$ of tap water) and the moisture level was monitored during consecutive days, while it was drying naturally. The sensor capacitance was measured by an impedance analyzer (model 4294A, Agilent, Santa Clara, CA, USA). Figure 9 shows the relationship between soil moisture (weight ratio, $h$ ) and the sensor capacitance, where a nonlinear behavior is observed. A simple approximation, using parabolic curve fitting, was applied to describe its tendency. The plotted curve presented a correlation of 0.9992 .

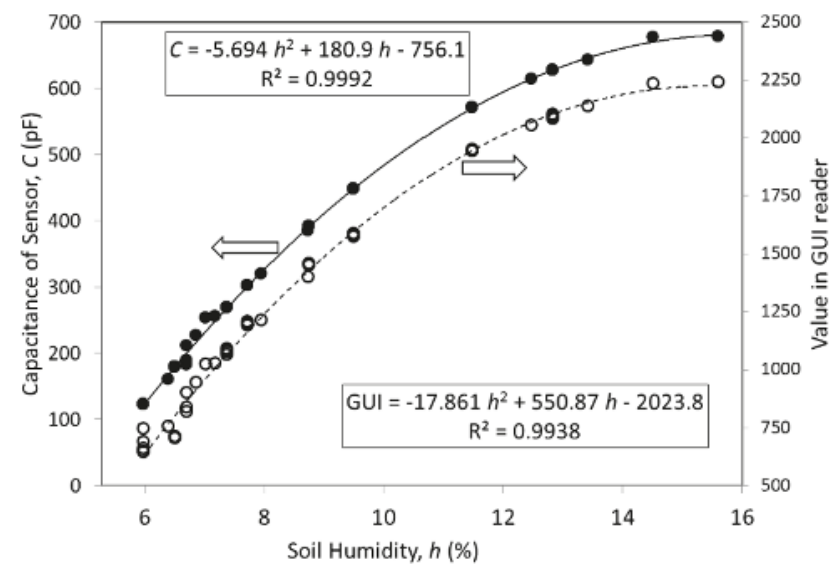

Figure 9. Sensor capacitance (solid dots and line) as a function of soil moisture $h$ (weight ratio). Dashed line and hollow dots are values obtained by GUI of UHF RFID reader. Curves are parabolic approximations. 
The sensor capacitance (C) can be read by the tag's IC (SL900A) and by the UHF reader's GUI software, as shown on the right axis of Figure 9.

For a very dry soil ( $h$ smaller than $6 \%$ ), the sensor capacitance tends to a saturation value of about $100 \mathrm{pF}$. When the soil is soaked ( $h$ greater than $16 \%$ ), the capacitance becomes saturated at about $680 \mathrm{pF}$.

\section{Discussion}

The prevention of disasters involving landslides, especially those caused by excess water or moisture in the soil is the major motivation of this research. In this paper two solutions were presented to monitor soil moisture through RFID technology, especially using UHF tags. The novelty presented in this paper is the use of two passive tag options (with and without built-in sensor) to solve the problem of landslide on slopes with human occupation. The main advantage of using RFID tags is the absence of batteries in the remote modules (RFID sensors) and the power supply is only in the reader modules, which may be installed together with public lighting posts, for example.

Two conventional UHF RFID tags can be utilized to monitor soil moisture. Significant differences in turn-on power levels of $7 \mathrm{~dB}$ and $5 \mathrm{~dB}$ between sensor tag and reference tag for dry and wet soils, respectively, were observed in practice. Although this first solution uses ultra-low-cost tags, the determination of soil moisture needs an UHF RFID reader with power sweep functionality, i.e., an ability to produce power ramps to determine $P_{T a g O n}$. A reader with this facility is a high-cost piece of equipment; therefore, the first solution presents some problems when used in an urban area.

For the second option, the use of a dedicated chip (SL900A), its reader kit and the software (GUI) delivered by the manufacturer, simplified technically the project. Because of the dedicated sensor interface and 10-bit analog-to-digital conversion, the best results were obtained. Moreover, the interdigital capacitive sensor allows the determination of soil moisture level with higher accuracy and resolution. Although the SL900A tag is more expensive than conventional tags, its reader is simpler and relatively low-cost, allowing an outdoor implementation. Finally, this last solution is interesting, from the research and scientific point of view, if a second external sensor is inserted in the UHF RFID tag, since this input (Ext2) is available with the SL900A. In this case, beyond temperature and soil moisture measurements, other landslide parameters, such as rain precipitation level, soil movement or displacement, etc., could be monitored.

Briefly, the main advantage of the first solution is the simplicity of the sensor, which uses just ordinary tags, however its disadvantage is the high cost of the reader module. On the other hand, the second solution has the advantage of low cost, although it needs the design of a dedicated tag with sensor for the function. Another advantage of the latter solution is the possibility of including other type of sensor, or even two sensors. These characteristics can guide the definition of which solution is better to be used.

The main advantage of both solutions presented in this paper is that the RFID sensors are for long-term applications, due to the sensors are passive, i.e., they work without internal battery. The use of internal battery, in a sensor network, for example, requires periodic interventions in the system for batteries' replacement.

Although there is a direct relation between soil moisture level and landslide risk, other geological and climatic parameters, such as, precipitation or rainfall [2], should be considered for landslide forecasting. However, soil moisture is one of the most important and frequently used as a first indication of landslide alert. The volume ratios of water in soil, used in these experiments, represent high levels that can be assumed within a risk range for a landslide event [1-3].

It is important to point out that the presented experiments have been tested in a sufficient number of times to demonstrate that both techniques are functional in practice. For the implementation in a real environment (urban slope), the tests must be remade and repeated in a greater number of times (including statistical analysis) to obtain the behavior curves of the tags and the sensor more accurate with the local soil. Thus, the final performance must be evaluated thoroughly. This is because, for both solutions, the system calibration has a strong dependence on the soil characteristics (composition, 
density, porosity, etc.) of the hillsides, which will be monitored. Of course, this procedure is beyond the scope of this paper.

For the passive RFID approach, the distances over which the sensors can be interrogated is limited to below few meters, assuming good, directive antenna design and matching. Thus, the location of the sensors must be known and the reader antennas should be placed on street furniture such as lamp-posts on the outskirts of the populated area to be protected against landslide. The availability of electrical power at the lamp-posts will enable relatively high power interrogating signals, too. Multiple antenna heads can be placed on one lamp-post, aimed at different sensors but connected to a single reader. In this scenario, the RFID readers would be interconnected by any telecommunications infrastructure, with data eventually hopped back via a gateway node, collecting the information from multiple readers, through the internet to a monitoring station.

Acknowledgments: The authors thank Mohamed Ali Ziai, Luis Carlos Vieira, Diego Dias Reis and Adriano Gamba for helping in sensor manufacturing, practical tests and technical support. This work was supported by the Newton Research Collaboration Programme-Royal Academy of Engineering (NRCP1516/1/139) and the Brazilian Council for Scientific and Technological Development (CNPq 307509/2015-0). The costs to publish in open access were covered by Federal University of Technology_Paraná and University of Kent.

Author Contributions: Nathan J. Gomes was the project's leader; all authors conceived and designed the experiments; Sérgio Francisco Pichorim performed the experiments and the data analysis; John C. Batchelor contributed with materials and instrumentation; all authors wrote the paper.

Conflicts of Interest: The authors declare no conflict of interest.

\section{References}

1. Brocca, L.; Ponziani, F.; Moramarco, T.; Melone, F.; Berni, N.; Wagner, W. Improving landslide forecasting using ASCAT-derived soil moisture data: A case study of the Torgiovannetto landslide in central Italy. Remote Sens. 2012, 4, 1232-1244. [CrossRef]

2. Ray, R.L.; Jacobs, J.M. Relationships among remotely sensed soil moisture, precipitation and landslide events. Nat. Hazards 2007, 43, 211-222. [CrossRef]

3. Takayama, S.; Akiyama, J.; Fujiki, T.; Mokhtar, N.A.B. Wireless sensing node network management for monitoring landslide disaster. J. Phys. Conf. Ser. 2013, 459, 1-6. [CrossRef]

4. Ziai, M.A.; Batchelor, J.C. Temporary on-skin passive UHF RFID transfer tag. IEEE Trans. Antennas Propag. 2011, 59, 3565-3571. [CrossRef]

5. Manzari, S.; Musa, T.; Randazzo, M.; Rinaldi, Z.; Meda, A.; Marrocco, G. A passive temperature radio-sensor for concrete maturation monitoring. In Proceedings of the IEEE RFID Technology and Applications Conference (RFID-TA), Tampere, Finland, 8-9 September 2014; pp. 121-126. [CrossRef]

6. Virtanen, J.; Ukkonen, L.; Björninen, T.; Elsherbeni, A.Z.; Sydänheimo, L. Inkjet-printed humidity sensor for passive UHF RFID systems. IEEE Trans. Instrum. Meas. 2011, 60, 2768-2777. [CrossRef]

7. Manzari, S.; Occhiuzzi, C.; Nawale, S.; Catini, A.; Natale, C.D.; Marrocco, G. Humidity Sensing by Polymer-Loaded UHF RFID Antennas. IEEE Sens. J. 2012, 12, 2851-2858. [CrossRef]

8. Gao, J.; Siden, J.; Nilsson, H.E. Printed Electromagnetic Coupler with an Embedded Moisture Sensor for Ordinary Passive RFID Tags. IEEE Electron Device Lett. 2011, 32, 1767-1769. [CrossRef]

9. Kim, S.; Le, T.; Tentzeris, M.M.; Harrabi, A.; Collado, A.; Georgiadis, A. An RFID-enabled inkjet-printed soil moisture sensor on paper for 'smart' agricultural applications. In Proceedings of the 2014 IEEE SENSORS, Valencia, Spain, 2-5 November 2014; pp. 1507-1510. [CrossRef]

10. Bauer-Reich, C.; Tan, K.C.; Haring, F.; Schneck, N.; Wick, A.; Berge, L.; Hoey, J.; Sailer, R.; Ulven, C. An investigation of the viability of UHF RFID for subsurface soil sensors. In Proceedings of the IEEE International Conference on Electro/Information Technology, Milwaukee, WI, USA, 5-7 June 2014; pp. 577-580. [CrossRef]

11. Bhattacharyya, R.; Florkemeier, C.; Sarma, S. RFID tag antenna based sensing: Does your beverage glass need a refill? In Proceedings of the IEEE International Conference on RFID (IEEE RFID 2010), Orlando, FL, USA, 14-16 April 2010; pp. 126-133. [CrossRef] 
12. Li, W.; Zhang, W.; Tam, K.W.; Chiang, C.P.; Chiang, K.H. On the measurements of liquid sensing based on UHF RFID. In Proceedings of the IEEE International Conference on RFID Technology and Applications (RFID-TA), Foshan, China, 21-23 September 2016; pp. 107-110. [CrossRef]

13. Sidén, J.; Zeng, X.; Unanber, T.; Koptyug, A.; Nilsson, H.E. Remote moisture sensing utilizing ordinary RFID tags. In Proceedings of the 2007 IEEE SENSORS, Atlanta, GA, USA, 28-31 October 2007; pp. 308-311. [CrossRef]

14. Hasan, A.; Bhattacharyya, R.; Sarma, S. Towards pervasive soil misture sensing using RFID tag antenna-based sensors. In Proceedings of the IEEE International Conference on RFID Technology and Applications (RFID-TA), Tokyo, Japan, 16-18 September 2015; pp. 165-170. [CrossRef]

15. Hasan, A.; Bhattacharyya, R.; Sarma, S. A monopole-coupled RFID sensor for pervasive soil moisture monitoring. In Proceedings of the IEEE Antennas and Propagation Society International Symposium (APSURSI), Orlando, FL, USA, 7-13 July 2013; pp. 2309-2310. [CrossRef]

16. Pichorim, S.F.; Marcis, V.A.; Laskoski, G.T. Humidity in sandy soil measured by passive, wireless and resonant sensor with bifilar coil. J. Phys. Conf. Ser. 2012, 407, 1-9. [CrossRef]

17. Ziai, M.A.; Batchelor, J.C. Multi-state logging freeze detection passive RFID tags. IEEE Trans. Antennas Propag. 2014, 62, 6406-6411. [CrossRef]

18. Ćika, D.; Draganić, M.; Šipuš, Z. Active wireless sensor with radio frequency identification chip. In Proceedings of the 35th International Convention MIPRO, Opatija, Croatia, 21-25 May 2012; pp. 727-732.

19. AMS. Datasheet of SL900A Chip. Available online: https://ams.com/eng/content/download/755603/ 1912935/file/SL900A_DS000294_3-00.pdf (accessed on 9 October 2017).

20. Salmerón, J.F.; Rivadeneyra, A.; Martí, F.M.; Vallvey, L.F.C.; Palma, A.J.; Carvajal, M.A. Passive UHF RFID tag with multiple sensing capabilities. Sensors 2015, 15, 26769-26782. [CrossRef] [PubMed]

21. Rennane, A.; Abdelnour, A.; Kaddour, D.; Touhami, R.; Tedjini, S. Development of Passive UHF RFID Tag on Flexible Foil for Sport Balls Pressure Monitoring. In Proceedings of the 32nd General Assembly and Scientific Symposium of the International Union of Radio Science (URSI GASS), Montreal, QC, Canada, 19-26 August 2017

22. Escobedo, P.; Carvajal, M.A.; Capitán-Vallvey, L.F.; Fernández-Salmerón, J.; Martínez-Olmos, A.; Palma, A.J. Passive UHF RFID Tag for Multispectral Assessment. Sensors 2016, 16, 1085. [CrossRef] [PubMed]

23. Falco, A.; Salmerón, J.F.; Loghin, F.C.; Lugli, P.; Rivadeneyra, A. Fully Printed Flexible Single-Chip RFID Tag with Light Detection Capabilities. Sensors 2017, 17, 534. [CrossRef] [PubMed]

24. AMS. Datasheet of SL900A-DK-STQFN16 Kit. Available online: http:/ /ams.com/eng/Support/Demoboards/ Wireless-Connectivity/Sensor-Tags-Interfaces/SL900A-Demo-Kit (accessed on 9 October 2017).

(C) 2018 by the authors. Licensee MDPI, Basel, Switzerland. This article is an open access article distributed under the terms and conditions of the Creative Commons Attribution (CC BY) license (http:/ / creativecommons.org/licenses/by/4.0/). 


\title{
Comparative Study of the Detection of Chromium Content in Rice Leaves by $532 \mathrm{~nm}$ and $1064 \mathrm{~nm}$ Laser-Induced Breakdown Spectroscopy
}

\author{
Jiyu Peng ${ }^{1,2,+}$, Fei Liu ${ }^{1,2,+}$, Tingting Shen ${ }^{1}$, Lanhan $\mathrm{Ye}^{1}{ }^{1}$, Wenwen Kong ${ }^{3}$, Wei Wang ${ }^{1}$, \\ Xiaodan Liu $^{1}$ and Yong $\mathrm{He}^{1,2, *}$ \\ 1 College of Biosystems Engineering and Food science, Zhejiang University, Hangzhou 310058, China; \\ jypeng@zju.edu.cn (J.P.); fliu@zju.edu.cn (F.L.); shentingtingstt@163.com (T.S.); lena_ye15@163.com (L.Y.); \\ 15236193955@163.com (W.W.); m15307266704@163.com (X.L.) \\ 2 Key Laboratory of Spectroscopy Sensing, Ministry of Agriculture, Hangzhou 310058, China \\ 3 School of Information Engineering, Zhejiang A \& F University, Linan 311300, China; zjukww@163.com \\ * Correspondence: yhe@zju.edu.cn; Tel.: +86-571-88982143 \\ + These authors contribute equally to this work.
}

Received: 13 December 2017; Accepted: 13 February 2018; Published: 18 February 2018

\begin{abstract}
Fast detection of toxic metals in crops is important for monitoring pollution and ensuring food safety. In this study, laser-induced breakdown spectroscopy (LIBS) was used to detect the chromium content in rice leaves. We investigated the influence of laser wavelength (532 $\mathrm{nm}$ and $1064 \mathrm{~nm}$ excitation), along with the variations of delay time, pulse energy, and lens-to-sample distance (LTSD), on the signal (sensitivity and stability) and plasma features (temperature and electron density). With the optimized experimental parameters, univariate analysis was used for quantifying the chromium content, and several preprocessing methods (including background normalization, area normalization, multiplicative scatter correction (MSC) transformation and standardized normal variate (SNV) transformation were used to further improve the analytical performance. The results indicated that $532 \mathrm{~nm}$ excitation showed better sensitivity than $1064 \mathrm{~nm}$ excitation, with a detection limit around two times lower. However, the prediction accuracy for both excitation wavelengths was similar. The best result, with a correlation coefficient of 0.9849 , root-mean-square error of $3.89 \mathrm{mg} / \mathrm{kg}$ and detection limit of $2.72 \mathrm{mg} / \mathrm{kg}$, was obtained using the SNV transformed signal (Cr I $425.43 \mathrm{~nm}$ ) induced by $532 \mathrm{~nm}$ excitation. The results indicate the inspiring capability of LIBS for toxic metals detection in plant materials.
\end{abstract}

Keywords: rice leaves; chromium content; laser-induced breakdown spectroscopy; laser wavelength; preprocessing methods

\section{Introduction}

Toxic metal detection is important for monitoring environmental pollution and ensuring food safety, which is directly related to human's health. As the second most common metal contaminant in soil, water and sediments, chromium $(\mathrm{Cr})$ can cause severe morphological, physiological and biochemical process damage in plants [1]. Because of the advantages of fast analytical speed, non-contact detection and little sample preparation, the laser-induced breakdown spectroscopy (LIBS) technique has been proven efficient in the determination of elements in biological samples, such as plant materials [2,3], algal biomass [4], and biomedical samples [5].

Several studies concerning the detection of chromium [6,7], copper [6,8], lead [9] and silver [10] contents in plant materials have been published recently. However, due to the moderate detection limits of LIBS, the samples used for analysis usually had a high toxic metal contents [9], and the 
sample number was limited [6], making it difficult to evaluate the quantification capability of the LIBS technique. On the other hand, the application of LIBS in toxic metals detection is also limited by the instability of signal, which is mainly dependent of the sampling approach (laser ablation), sampling mass (usually around $\mu \mathrm{g} / \mathrm{pulse}$ ) and complexity of the laser-sample interaction [11]. The very characteristics of LIBS might be considered as some of the most important issues for quantification of toxic metals in plant materials.

In general, the analytical performance of LIBS could be improved by choosing suitable experimental parameters (e.g., laser properties, beam guiding strategies and detector parameters). The laser wavelength is one of important parameters that is related to the laser-sample and laser-plasma interactions, which might further affect the LIBS signal. The influence of laser wavelength on the absorption properties of substance and plasma has been discussed by Noll et al. in his book [12]. It has been also reported that shorter wavelengths had higher photon energies that might lead to shorter optical penetration depth, higher ablation rate and less fractionation [13]. In contrast, longer wavelengths might have stronger plasma shielding and inverse bremsstrahlung [14]. Stronger emissions and weaker signal stability were observed in IR irradiation [15]. Zhang et al. compared the signal intensity and stability under $266 \mathrm{~nm}$ and $1064 \mathrm{~nm}$ excitations when analyzing trace elements in holly leaves [16]. Better repeatability was found under $266 \mathrm{~nm}$ excitation. The Nd:YAG laser operated at its fundamental wavelength $(1064 \mathrm{~nm})$ is mostly preferred by researchers because of its economic and powerful features. In this case, some efforts are carried out to evaluate the influence of laser wavelength.

In addition to the laser wavelength, the analytical performance of LIBS is also greatly affected by laser energy, lens-to-sample distance (LTSD) and delay time [12]. All these factors along with laser wavelength determine the overall performance of LIBS. De Carvalho et al. investigated the effects of fluence on the detection of nutrient elements in plant materials using LIBS. They found that the sensitivity and measurement precision increased with laser fluence [17]. In addition, the gate width, delay time, amplification gain and number of pulses for detection of nutrient elements in plants materials were optimized based on a neuro-genetic approach [18]. However, few studies have considered the influence of wavelength as well as the correlation with other factors, especially for plant materials. Different from other samples (e.g., metals), the constituents of plant materials are complex, inhomogeneous, and it may be sensitive to the experimental parameters.

From the point of view of modeling, data preprocessing is necessary for LIBS data management. Because of the laser ablation process in LIBS, the accuracy and precision of analytical results might be affected by random or systematic changes of the abovementioned parameters [19]. It has been proven that normalization and outliers filtering could be used to reduce the interference matrix and to improve the calibration models [20-22]. For the normalization strategies, it mainly consists of single-variate and multivariate corrections, which has been reviewed by Zorov et al. in detail [23]. A single reference signal (e.g., individual line, background, spectral area) is used in single-variate corrections, while more than one reference signal is used in multivariate corrections. In this study, a self-developed routine was used to detect outliers and four different normalization methods were used to reduce shot-to-shot fluctuation.

In this work, we aimed to detect chromium content in rice leave using fundamental wavelength $\left(1064 \mathrm{~nm}, E_{p}=1.17 \mathrm{eV}\right)$ and second harmonic $\left(532 \mathrm{~nm}, E_{p}=2.34 \mathrm{eV}\right)$ of Nd:YAG Q-switched pulse laser. The specific objectives of this study were (1) to compare the influence of wavelength on signal (sensitivity and repeatability), plasma features (electron density and temperature) and quantification capability; (2) to optimize the experimental parameters for both $532 \mathrm{~nm}$ and $1064 \mathrm{~nm}$ laser wavelengths; (3) to establish univariate models for the fast detection of chromium content in rice leaves, and further improve the performance with preprocessing methods (background normalization, area normalization, standardized normal variate (SNV) and multiplicative scatter correction (MSC)). 


\section{Materials and Methods}

\subsection{Sample Preparation}

Rice (Oryza sativa L.) leaves with different chromium stress were used for analysis. Rice seeds with genotype Chunyou 84 were obtained from the China National Rice Research Institute (Hangzhou, China). The cultivation of rice plants was carried out according to Zeng et al. [24], and the nutrient solution was prepared according to Yoshida et al. [25]. After growing in complete nutrient solution for 7 days, five treatments including control group and experiment groups of $25 \mu \mathrm{M}, 50 \mu \mathrm{M}$, $75 \mu \mathrm{M}$ and $100 \mu \mathrm{M}$ chromium stress (prepared using $\mathrm{K}_{2} \mathrm{Cr}_{2} \mathrm{O}_{7}$ ) were adopted. The chromium stress of $50 \mu \mathrm{M}$ and $100 \mu \mathrm{M}$ could be considered as moderate pollution and severe pollution, respectively [26]. In addition, an extra group with a chromium stress of $60 \mu \mathrm{M}$ was prepared to investigate the influence of wavelength on the variations of delay time, pulse energy and LTSD. After exposure to chromium stress for 8 weeks, 43 samples with the five treatments (see Table 1) were collected, and all rice leaves of the extra group were used to constitute one sample. In order to reduce shot-to-shot fluctuation, all the samples were dried, ground and pressed into pellets. The leaves were ground with a tissue milling machine. The samples were placed in a $5 \mathrm{~mL}$ centrifuge tube, and ground at $60 \mathrm{~Hz}$ for $1 \mathrm{~min}$. The pellets were prepared by placing $100 \mathrm{mg}$ of leaf powder into a squared die set and then pressed with $600 \mathrm{MPa}$ of pressure for $1 \mathrm{~min}$. The pellets were square with $10 \mathrm{~mm}$ length on each side.

\subsection{Experimental Setup}

The experiment was carried out with a self-assembled LIBS setup, which mainly consists of a Nd:YAG Q-switched pulse laser for ablation, optics for beam guiding, spectrograph for light dispersion and intensified charge coupled device (ICCD) camera for detection. A detailed description of our LIBS device has been given elsewhere [27].

In this work, $532 \mathrm{~nm}$ and $1064 \mathrm{~nm}$ excitations were produced when laser operated at the fundamental and second harmonics, respectively. The beam profile of the laser was nearly a flat top distribution. The beam diameter and pulse duration were $7 \mathrm{~mm}$ and $8 \mathrm{~ns}$, respectively. In addition, the RSD of pulse-to-pulse energy was less than $2 \%$. With the help of a plano-convex lens ( $\mathrm{f}=100 \mathrm{~mm}$, $532 / 1064 \mathrm{~nm} \mathrm{~V}$ coat), the laser beams were focused onto samples. In order to avoid ablating the same spot continuously, the samples were moved by an X-Y-Z motorized stage every five accumulation shots, and a total of 16 positions were ablated for each sample. The relative gain of ICCD was set at 26 . Before the experiment, wavelength of spectrograph and signal intensity of ICCD were calibrated using a mercury argon lamp (HG-1, Ocean Optics, Minneola, FL, USA) and a deuterium halogen source (DH-2000-BAL-CAL, Ocean Optics, Minneola, FL, USA).

\subsection{Reference Method for Chromium Content Determination}

The reference value of chromium content of rice leaves was determined with inductively coupled plasma mass spectrometry (ICP-MS), using a modification of method described by Aziz et al. [28]. In addition, the trueness of the reference method was evaluated with citrus leaves (GBW10020), and the result was consistent with the certified value. Before the measurement, $100 \mathrm{mg}$ of ground leaves was weighted into the Teflon vessels and microwave digested with $4 \mathrm{~mL}$ of $65 \% \mathrm{HNO}_{3}$ and $1 \mathrm{~mL}$ of $30 \%$ $\mathrm{H}_{2} \mathrm{O}_{2}$. After digestion, they were translated into $25 \mathrm{~mL}$ volumetric flasks and diluted to the mark with distilled water. Finally, the solutions were analyzed with ICP-MS (ELAN DRC-e, PerkinElmer, Waltham, MA, USA). The duration of sample preparation (regardless of drying and grinding) for ICP-MS was about $3 \mathrm{~h}$, and it was 2 min for LIBS. The results for chromium content determination were shown in Table 1. 
Table 1. ICP-MS measured chromium content in rice leaves with five different treatments (range: the range of chromium content, mean $\pm \mathrm{SD}$ : the mean and standard deviation of chromium content).

\begin{tabular}{cccc}
\hline Cr Stress Level $(\mu \mathbf{M})$ & Sample Number & Range $(\mathbf{m g} / \mathbf{k g})$ & Mean $\pm \mathbf{S D}(\mathbf{m g} / \mathbf{k g})$ \\
\hline 0 & 9 & $0.58-0.80$ & $0.68 \pm 0.08$ \\
25 & 11 & $2.67-12.37$ & $7.84 \pm 2.65$ \\
50 & 11 & $14.35-32.37$ & $21.52 \pm 4.87$ \\
75 & 7 & $22.49-57.63$ & $39.00 \pm 10.86$ \\
100 & 5 & $56.03-86.60$ & $67.32 \pm 11.62$ \\
\hline
\end{tabular}

\subsection{Data Analysis}

In LIBS analysis, abnormal spectra might result due to the fluctuation of experimental parameters. It has been reported that removing the outliers in data analysis could improve the precision and repeatability of LIBS measurement [29]. After LIBS data acquisition, a self-developed routine was used to remove abnormal spectra based on median absolute deviation (MAD) [30]. In order to meet our particular case, we used the peak intensity of emission CN $388.29 \mathrm{~nm}$ as a variable to identify outliers. Because the emission of molecule band $\mathrm{CN}$ usually appeared in organic samples, and it was relatively stable. First, the median and MAD of peak intensity of CN $388.29 \mathrm{~nm}$ was calculated. Then the spectrum was considered as an outlier when the difference between its intensity of CN $388.29 \mathrm{~nm}$ and median was beyond 2.5 times MAD. Outlier detection was performed until no outlier was identified or the number of remaining spectra was less than $75 \%$ of the number of original spectra. In order to obtain the real response of the system, the process of outliers filtering was not applied in optimization, while it was only performed during the modeling.

In order to compare the signal quality and performance metrics of LIBS with different experimental parameters, we used limit of detection (LOD), signal-to-noise $(\mathrm{S} / \mathrm{N})$ and signal-to-background (S/B) ratios to evaluate sensitivity and relative standard deviation (RSD) to evaluate signal stability. These indicators were calculated with following equations:

$$
\begin{aligned}
\frac{\mathrm{S}}{\mathrm{N}} & =\frac{I_{\text {net signal }}}{\sigma_{\text {background }}} \\
\frac{\mathrm{S}}{\mathrm{B}} & =\frac{I_{\text {net signal }}}{\bar{I}_{\text {background }}} \\
\mathrm{RSD} & =\frac{\sigma_{\text {signal }}}{\bar{I}_{\text {signal }}} \times 100 \%
\end{aligned}
$$

where $I_{\text {net signal }}$ is the net signal of interested element, $\sigma_{\text {background }}$ is the standard deviation of the background intensities, $\bar{I}_{\text {background }}$ is the averaged intensity of background near to analytical line, $\sigma_{\text {signal }}$ is standard deviation of signal of interested element with three replicate measurements, and $\bar{I}_{\text {signal }}$ is averaged intensity of signal of interested element with three replicate measurements.

In addition, electron density and temperature were used to characterize the plasma features. Because the electron density was related to the full width at half maximum (FWHM) of the Stark broadening lines, it could be calculated with following equation:

$$
\Delta \lambda_{\text {stark }}=2 \omega\left[\frac{N_{e}}{10^{16}}\right]
$$

where $N_{e}$ is the electron density $\left(\mathrm{cm}^{-3}\right), \omega$ is the electron impact width parameter [31], and $\Delta \lambda_{\text {stark }}$ is FWHM of Stark broadening lines (i.e., $\mathrm{Mg}$ II $280.27 \mathrm{~nm}$ in this case, and no self-absorption and overlapping were observed). Because the measured line broadening could be dominated by Stark 
broadening and instrumental broadening, we used a Voigt function to fit the experimental data. The term $\Delta \lambda_{\text {stark }}$ could be deduced with following expression [32]:

$$
\Delta \lambda_{\text {exp }}=\frac{\Delta \lambda_{\text {stark }}}{2}+\left[\left(\frac{\Delta \lambda_{\text {stark }}}{2}\right)^{2}+\left(\Delta \lambda_{\text {inst }}\right)^{2}\right]^{1 / 2}
$$

where $\Delta \lambda_{\text {exp }}$ is the FWHM of experimental broadening of Mg II $280.27 \mathrm{~nm}$, and $\Delta \lambda_{\text {inst }}$ is the instrumental broadening of the spectrograph, which was measured using a mercury light source. In this case, $\Delta \lambda_{\text {inst }}$ was determined to be $0.055 \mathrm{~nm}$ (in air).

The temperature of plasma was estimated by spectrum simulation of the $\mathrm{CN}$ emissions in the spectral window around $388 \mathrm{~nm}$ using LIFBASE2.1 software [33].

After investigating the influences and optimizing the values of experimental parameters, univariate analysis was used to compare the quantification performance with 532/1064 nm excitations. The order of samples $(n=43)$ was rearranged according to the chromium content from low to high. Then, three samples with an interval of four were assigned to a calibration set $(n=32)$, and the rest were in the prediction set $(n=11)$.

In addition, normalization was used to eliminate the matrix effect and shot-to-shot fluctuation and further improve the calibration performance [23]. In this case, different prepocessing methods were compared, including background normalization, area normalization, SNV and MSC. MSC is a transformation method that is originally used to compensate for additive and multiplicate scattering in reflectance spectroscopy [34]. It consists of fitting a separate regression line to each spectrum using mean spectrum of a set of samples, and the coefficients are used to correct the variables in original spectrum. SNV is another transformation method that is also used for correcting multiplicate scattering in spectroscopy [35]. Different from MSC, it removes the scatter effect by centering and scaling each spectrum only with data from that spectrum. Background and area normalizations were obtained by normalizing each spectrum to background intensity and the total emission integrated intensity, respectively. SNV and MSC transformations were performed in the Unscrambler X (CAMO AS, Oslo, Norway).

The calibration results were evaluated with correlation coefficient $(R)$ and root of mean squared error (RMSE). RMSE is a measurement of the average difference between reference values and model predicted values. It is obtained by calculating the square root of the average of squared errors. The sensitivity of proposed methods was compared using LOD, which could be deduced with following equation:

$$
\mathrm{LOD}=\frac{3 \sigma_{\text {background }}}{b}
$$

where $\sigma_{\text {background }}$ is the standard deviation of the background intensities, $b$ is the slope of calibration curve.

\section{Results}

\subsection{Spectral Characteristics}

Figure 1 shows the averaged spectra of sample from extra group (60 $\mu \mathrm{M}$ chromium stress) with 532/1064 nm excitations. In order to investigate the influence of laser wavelength, the experimental parameters of pulse energy, LTSD, delay time and gate width were set at $90 \mathrm{~mJ}, 98 \mathrm{~mm}, 4 \mu \mathrm{s}$ and $16 \mu \mathrm{s}$, respectively. As seen in Figure 1, the emission lines from macro-nutrients (such as $\mathrm{Ca}, \mathrm{Mg}, \mathrm{K}, \mathrm{Si}$ ) and micro-nutrients $(\mathrm{Na})$ were observed, as well as the main organic constituent $(\mathrm{C})$. Distinguishing differences in line intensity were observed for 532/1064 nm excitations, and the differences were associated with the wavelength of emission lines. In the spectrum near UV region, the intensity with $1064 \mathrm{~nm}$ excitation was more intense than that from $532 \mathrm{~nm}$ excitation. However, in the visible and near-IR region, the intensity of most emission lines with $532 \mathrm{~nm}$ excitation was higher than that from $1064 \mathrm{~nm}$ 
excitation. This might be related to the transition energy of emission lines. According to the equation $\Delta E=h c / \lambda$, the transition energy was inversely proportional to the wavelength. Hence, the emission line with longer wavelength corresponded to lower transition energy. Because photon energy of $532 \mathrm{~nm}$ excitation $\left(E_{p}=2.34 \mathrm{eV}\right)$ was higher than $1064 \mathrm{~nm}$ excitation $\left(E_{p}=1.17 \mathrm{eV}\right)$, the processes of bonding breaking and ionization were more intense [36]. Therefore, more transitions with $532 \mathrm{~nm}$ excitation might occur for lower energy gap (i.e., longer wavelength region).

For the emission lines of chromium (Cr I 425.43, $428.48 \mathrm{~nm}$ ), the intensities with 532/1064 nm excitations in this experimental condition were similar. This was accordant with above conclusion that the spectral intensity was affected both by transition energy and inverse bremsstrahlung. As seen in Table 2, the transition energies for Cr I $425.43 \mathrm{~nm}$ and Cr I $428.48 \mathrm{~nm}$ were $2.91 \mathrm{eV}$ and $2.90 \mathrm{eV}$, respectively. However, the prominent mechanism of the excitation wavelength differed with different experimental conditions (delay time, laser energy and LTSD). In order to find the best experimental parameters, we further investigated the influence of wavelength under above mentioned experimental parameters.

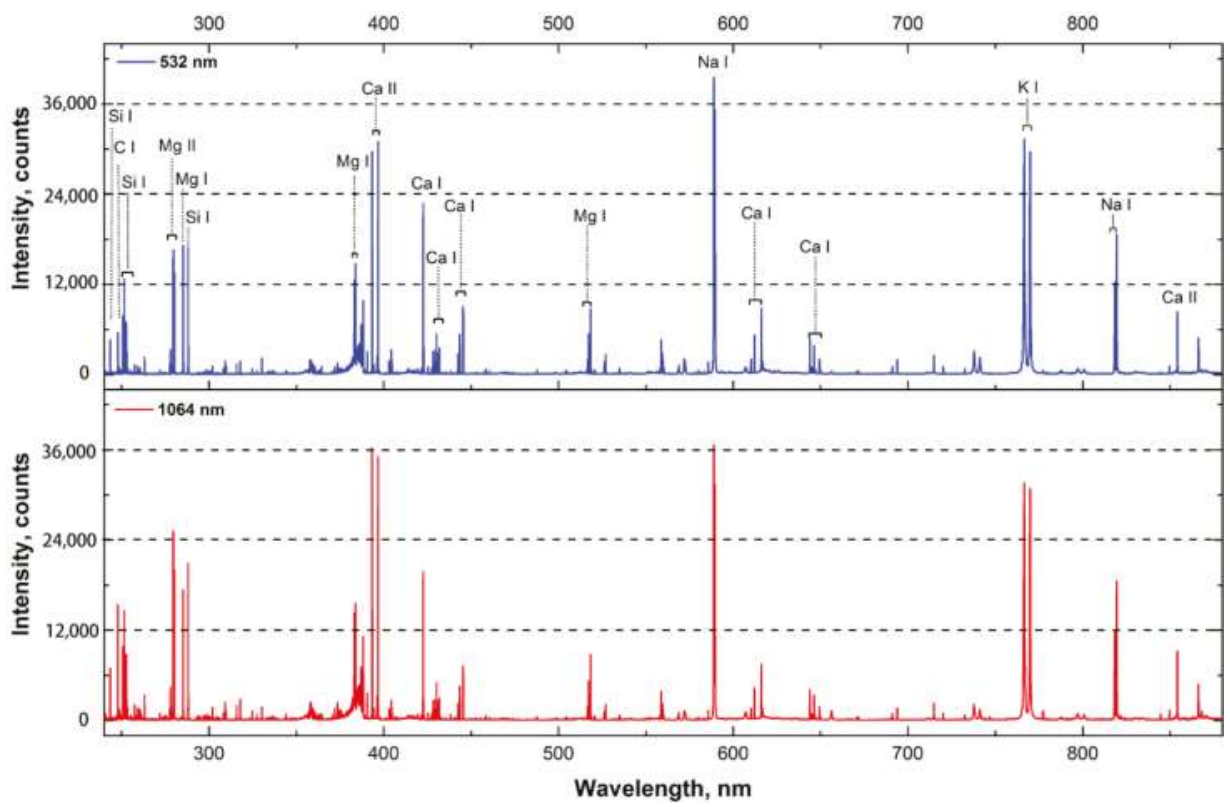

Figure 1. Averaged spectra of sample from extra group $(60 \mu \mathrm{M}$ chromium stress) in the range of 240-880 nm. Experimental parameters of energy, LTSD, delay time, gate width for $532 \mathrm{~nm}$ and $1064 \mathrm{~nm}$ excitations are $90 \mathrm{~mJ}, 98 \mathrm{~mm}, 4 \mu \mathrm{s}$ and $16 \mu \mathrm{s}$, respectively.

Table 2. Spectral properties of two emission lines of chromium $\left(\lambda_{k i}\right.$ : transition wavelength, $A_{k i}$ : transition probability, E: energy, i and k: lower and higher levels).

\begin{tabular}{cccc}
\hline & $\lambda_{k i}(\mathrm{~nm})$ & $A_{\boldsymbol{k} i}\left(\mathrm{~s}^{-\mathbf{1}}\right)$ & $\boldsymbol{E}_{\boldsymbol{i}}-\boldsymbol{E}_{\boldsymbol{k}}(\mathrm{eV})$ \\
\hline $\mathrm{Cr}(\mathrm{I})$ & 425.43 & $3.15 \times 10^{7}$ & $0-2.91$ \\
$\mathrm{Cr}(\mathrm{I})$ & 427.48 & $2.2 \times 10^{7}$ & $0-2.90$ \\
\hline
\end{tabular}




\subsection{Influence of Delay Time}

Delay time is an important parameter in LIBS detection, which describes the delay from initiation of plasma to detection. In the first period of plasma radiation process, the background continuum emission is strong, and it is mainly due to bremsstrahlung process. In this case, time-resolved analysis of plasma light allows for the determination of the suitable delay when the signal from interested emissions predominate. In addition, gate width allows for recording time period of the interested emissions, which is also called as integral time. In the pre-experiment, the life time of emission lines (Cr I 425.43, $427.48 \mathrm{~nm}$ ) was estimated around $20 \mu \mathrm{s}$. In order to capture all the signal during the life time and eliminate the influence of dark current, the sum of delay time and gate width was equal to $20 \mu \mathrm{s}$. In addition, the laser energy and LTSD for both 532/1064 nm excitations were set at $80 \mathrm{~mJ}$ and $98 \mathrm{~mm}$, respectively.

Figure 2a shows the time-resolved spectral intensity in the range of $424-428 \mathrm{~nm}$. In the early delay time, background continuum emissions were strong for both 532/1064 nm excitations, which resulted in low SNR/SBR for interested signal (see Figure 2b). As the increase of delay time, the background continuum emissions decreased quickly, while the intensity of emissions Cr I 425.43, $427.48 \mathrm{~nm}$ decreased slowly during the delay time of $0-4 \mu$ s. However, the RSD of interested signal continually increased as the increase of delay time, which indicated the decay of signal stability. The trend of RSD was contrast with those of temperature and electron density (Figure $2 b, c$ ). At long delay times, the plasma began to cool down, which caused the signal instability.
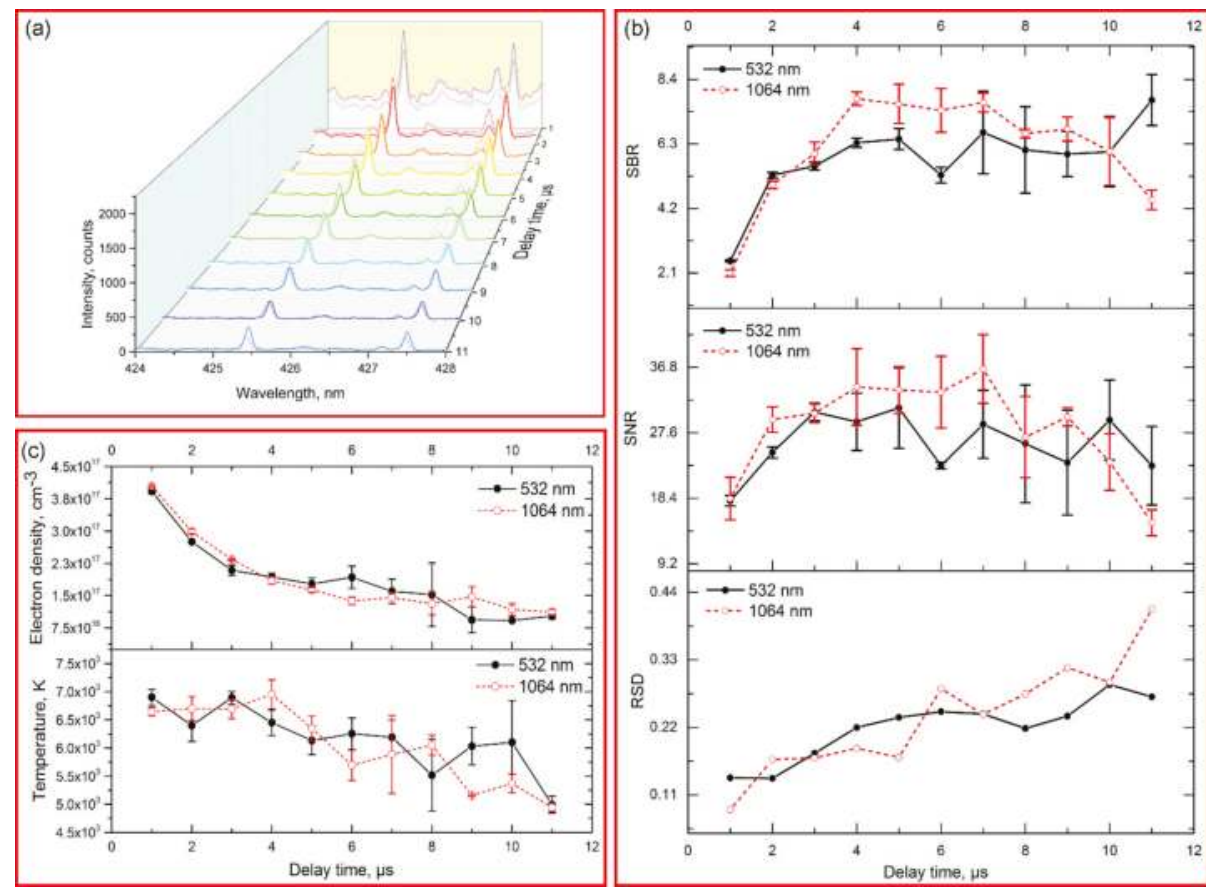

Figure 2. (a) Spectral intensity in the range of $424-428 \mathrm{~nm}$ with different delay times. The solid line indicated the spectra from $532 \mathrm{~nm}$ excitation, and dash line indicated the spectra from $1064 \mathrm{~nm}$ excitation; (b) Comparison of SBR, SNR and RSD of emission Cr I $425.43 \mathrm{~nm}$ with different delay time under $532 \mathrm{~nm}$ and $1064 \mathrm{~nm}$ excitations; (c) Time-resolved analysis of electron density and temperature under $532 \mathrm{~nm}$ and $1064 \mathrm{~nm}$ excitations. 
Some differences were observed for 532/1064 nm excitations. Compared with $532 \mathrm{~nm}$ excitation, both background continuum and analytical emissions for $1064 \mathrm{~nm}$ excitation were strong in the first delay period, while those decayed quickly as the increase of delay time. It was known that the first stage of plasma was predominated by inverse bremsstrahlung (free-free transition), whose cross-section was proportional to the cube of laser excitation wavelength [15]. Hence, higher absorption was observed in $1064 \mathrm{~nm}$ wavelength laser induced plasma. In the long delay time $(\geq 10 \mu \mathrm{s})$, the emissions from $1064 \mathrm{~nm}$ excitation were weaker than those from $532 \mathrm{~nm}$ excitation. It indicated that the emissions from $1064 \mathrm{~nm}$ excitation might decay quickly than that from $532 \mathrm{~nm}$ excitation. Take overall consideration of SBR, SNR and RSD, the optimal delay time for 532/1064 nm excitations were both $4 \mu \mathrm{s}$.

\subsection{Influence of Pulse Energy}

Laser pulse energy is an important laser related parameter, which affects the ablation and plasma formation [37]. More precisely, the pulse energy used for ablation can be transformed to fluence (energy per unit area, $\mathrm{J} / \mathrm{cm}^{2}$ ), which can better describe the energy delivered to the target. In this experiment, the energy was adjustable in the range of 20-130 mJ with an attenuator of half wave plate and polarizer, and the LTSD was maintained at $98 \mathrm{~mm}$ (i.e., the laser beam focused $2 \mathrm{~mm}$ below the target). Hence, the fluence used for ablation was in the range of $5.1-33.8 \mathrm{~J} / \mathrm{cm}^{2}$ with interval of $2.55 \mathrm{~J} / \mathrm{cm}^{2}$. In addition, the delay time and gate width of ICCD were optimized at $4 \mu \mathrm{s}$ and $16 \mu \mathrm{s}$ according to the results obtained from above section.

Figure 3a shows the spectral intensity in the range of $424-428 \mathrm{~nm}$ with 12 different pulse energies of the laser $(532 / 1064 \mathrm{~nm}, 8 \mathrm{~ns}, 1 \mathrm{~Hz})$. The signal intensities for chromium increased with the increase of energy, while they began to flatten in high energy. The variation of background continuum emission was similar to the signal for chromium. The increase of spectral intensity might be related to the greater ablated particle with the increase of fluence (see Figure 4). Figure $3 \mathrm{c}$ shows the electron density and plasma temperature with different pulse energies. The electron density in low pulse energy $(\leq 30 \mathrm{~mJ})$ of $1064 \mathrm{~nm}$ excitation was not available, because the emission line used for calculation was disappeared. The electron density and temperature of plasma increased as the increase of energy (see Figure 3c). It indicated that more electrons were generated, and greater energy of photons were absorbed by electrons, which caused the increase of temperature [38]. In addition, when the fluence was low, the RSD were relatively high (Figure 3b). As the fluence used for the samples was around the ablation threshold, the produced plasma was likely unstable. 

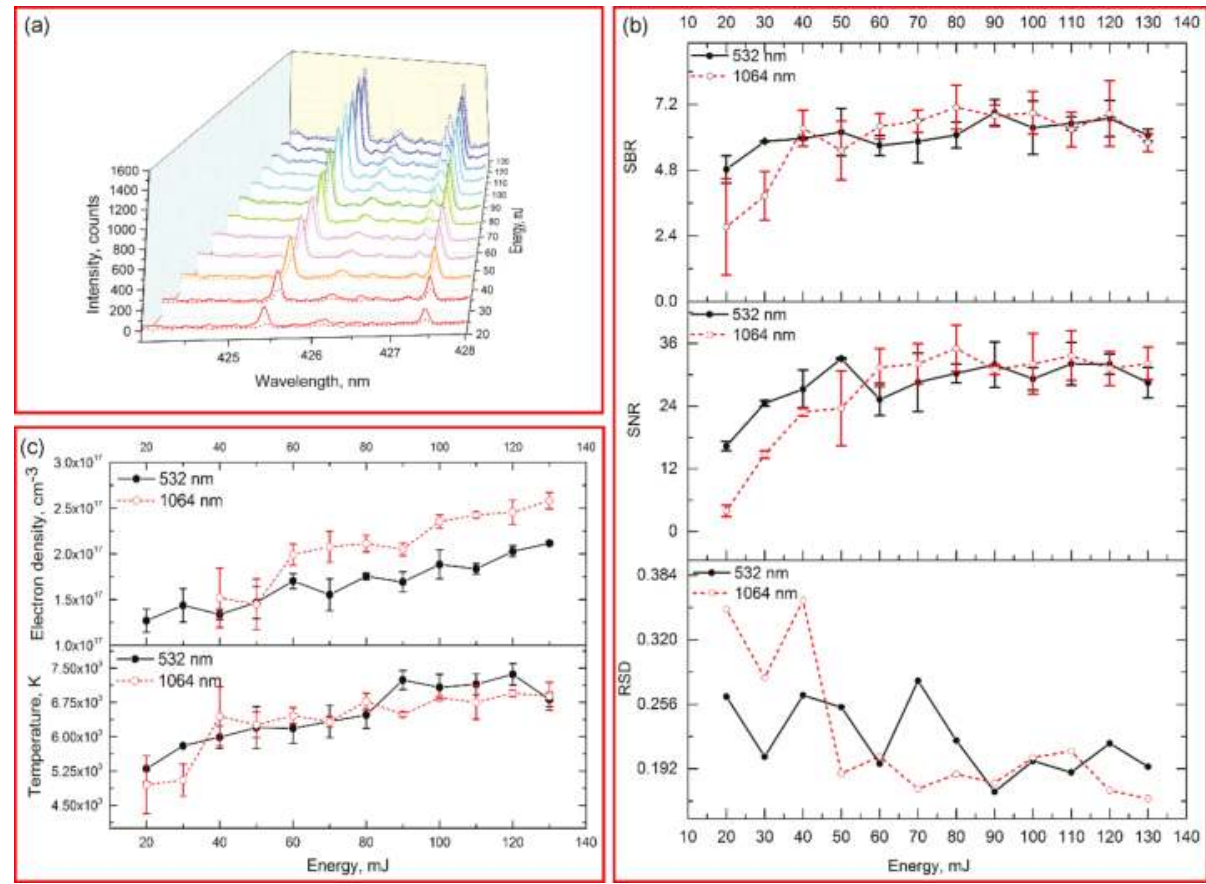

Figure 3. (a) Spectral intensity in the range of $424-428 \mathrm{~nm}$ with different laser energies. The solid line indicated the spectra from $532 \mathrm{~nm}$ excitation, and dash line indicated the spectra from $1064 \mathrm{~nm}$ excitation; (b) Comparison of SBR, SNR and RSD of emission Cr I $425.43 \mathrm{~nm}$ with different laser energies under $532 \mathrm{~nm}$ and $1064 \mathrm{~nm}$ excitations; (c) Electron density and temperature with different laser energy under $532 \mathrm{~nm}$ and $1064 \mathrm{~nm}$ excitations.

The differences of 532/1064 nm excitations with different pulse energies were also compared. In the low pulse energy, especially for the energy lower than $40 \mathrm{~mJ}$, the signal intensity, SBR and SNR from $532 \mathrm{~nm}$ excitation were obviously higher than those from $1064 \mathrm{~nm}$ excitation (see Figure 3a,b). This phenomenon might be related to the actual energy for ablation in low pulse energy. Because of the plasma shielding effect, greater pulse energy of $1064 \mathrm{~nm}$ excitation was absorbed by the plasma, causing less energy for ablation [39]. As the increase of energy, the increase of ablation efficiency of $1064 \mathrm{~nm}$ excitation was obvious. As seen in Figure 4, the depth and ablated volume of crater from $1064 \mathrm{~nm}$ excitation increased greatly compared with those from $532 \mathrm{~nm}$ excitation. Thus, higher emissions were induced by $1064 \mathrm{~nm}$ excitation with high pulse energy. However, the SNR and SBR of $532 / 1064 \mathrm{~nm}$ excitations were similar when the energy higher than $80 \mathrm{~mJ}$. For practical applications, using $532 \mathrm{~nm}$ excitation or $1064 \mathrm{~nm}$ excitation for element detection was similar when high pulse energy was required, while $532 \mathrm{~nm}$ excitation was suitable for low destruction applications, such as biological samples. The optimized energy for $532 \mathrm{~nm}$ and $1064 \mathrm{~nm}$ excitations in this case were $90 \mathrm{~mJ}$ and $80 \mathrm{~mJ}$, of which the SBR/SNR reached the maximal and the RSD was relatively low. 

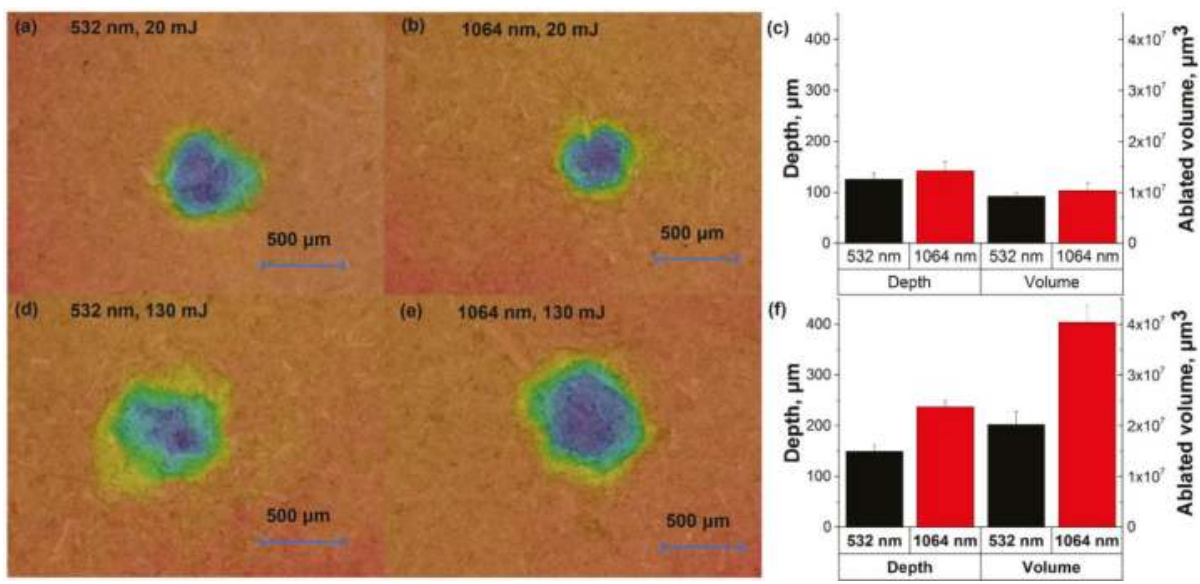

Figure 4. Morphologies of craters with different pulse energies under $532 \mathrm{~nm}$ and $1064 \mathrm{~nm}$ excitations: (a) $532 \mathrm{~nm}$ excitation, pulse energy = $20 \mathrm{~mJ}$; (b) $1064 \mathrm{~nm}$ excitation, pulse energy = $20 \mathrm{~mJ}$; (d) $532 \mathrm{~nm}$ excitation, pulse energy = $130 \mathrm{~mJ}$; (e) $1064 \mathrm{~nm}$ excitation, pulse energy $=130 \mathrm{~mJ}$. The colors in $(\mathbf{a}, \mathbf{b}, \mathbf{d}, \mathbf{e})$ indicated the depth of craters. Plots of statistic parameters (depth and ablated volume) of craters with different pulse energies: (c) pulse energy = $20 \mathrm{~mJ}$; (f) pulse energy $=130 \mathrm{~mJ}$. The morphologies of craters were measured using digital microscopy (VHX-6000, Keyence, Osaka, Japan).

\subsection{Influence of Lens-To-Sample Distance}

We also investigated the influence of lens-to-sample distance on the signal. LTSD not only affects the fluence delivered to the target, but also has an effect on signal stability. In fact, the LTSD is a brief description of the laser spot on the target with fixed experimental parameters, which is dependent of the focal length of lens and the diameter of laser beam. In this experiment, the focal length of lens was $100 \mathrm{~mm}$, and the diameter of laser beam was around $7 \mathrm{~mm}$. In addition, the delay time and gate width of camera were set at $4 \mu$ s and $16 \mu$ s, and the pulse energy used in this case was $80 \mathrm{~mJ}$.

Figure 5a shows the spectral intensity in 424-428 nm spectral window with LTSD in the range of 93-102 mm. When the LTSD was larger than $100 \mathrm{~mm}$, the laser ablated the atmosphere. As the increase of LTSD, the signal achieved its maximal values at $98 \mathrm{~mm}$ and $99 \mathrm{~mm}$ for 532/1064 nm excitations and then decreased. The emissions of chromium might not be directly related with crater's depth and ablated volume. As seen in Figure 6, the ablated volume and depth decrease, while the emissions increase with the LTSD changes from 93 to $98 \mathrm{~mm}$. Hence, the variance of emission intensity might be credited to the ablation efficiency between laser beam and target, which was related to ablation fluence and ablation spot. As the LTSD increased $(<100 \mathrm{~mm})$, the ablation fluence increased while the ablation spot decreased. The maximal values of signal might indicate the optimized values of LTSD with the largest ablation efficiency, which were based on the trade-off between the fluence and the ablation spot diameter. The trends of SNR and SBR were consistent with that of signal (Figure 5b). For the RSD, it deceased with LTSD from 93 to $100 \mathrm{~mm}$, then it greatly increased. As seen in Figure 5c, the temperature and electron density increased with the increase of LTSD (when it was shorter than $100 \mathrm{~mm}$ ). It indicated that the used pulse energy with short LTSD might be insufficient to produce enough electrons and maintain high plasma temperature, which induced instability of the signal. For the LTSD larger than the focal length of lens, the laser beam mainly caused the breakdown in the air rather than the samples. In the practical application, it should be strictly avoided. Above all, the optimal LTSD for $532 \mathrm{~nm}$ and $1064 \mathrm{~nm}$ excitations were $98 \mathrm{~mm}$ and $99 \mathrm{~mm}$, respectively. 

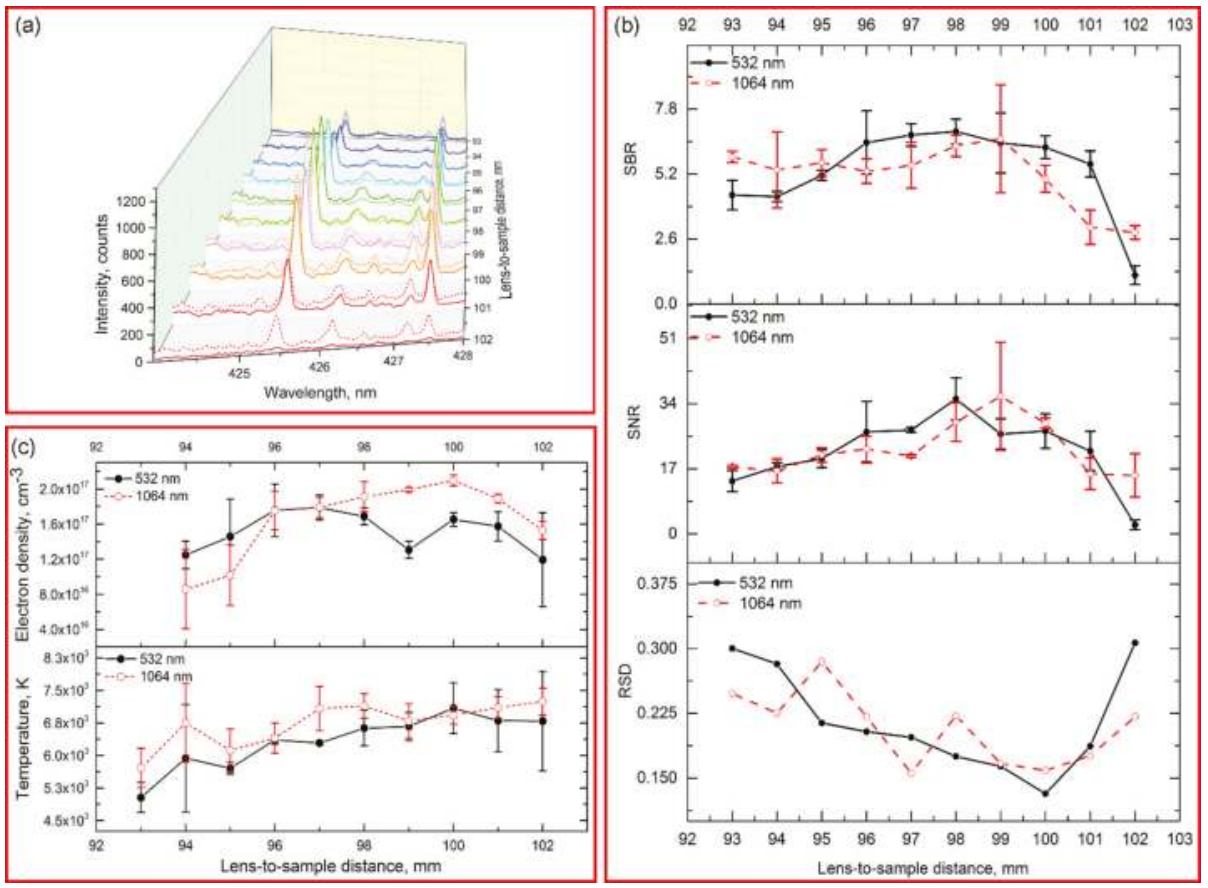

Figure 5. (a) Spectral intensity in the range of $424-428 \mathrm{~nm}$ with different LTSDs. The solid line indicated the spectra from $532 \mathrm{~nm}$ excitation, and dash line indicated the spectra from $1064 \mathrm{~nm}$ excitation; (b) Comparison of SBR, SNR and RSD of emission Cr I $425.43 \mathrm{~nm}$ with different LTSDs under $532 \mathrm{~nm}$ and $1064 \mathrm{~nm}$ excitations; (c) electron density and temperature with different LTSDs under $532 \mathrm{~nm}$ and $1064 \mathrm{~nm}$ excitations.

We also compared the influence of wavelength with the variation of LTSD. When the LTSD was in the range of $95 \mathrm{~mm}$ to $98 \mathrm{~mm}$, the signal intensity and background continuum emission from $532 \mathrm{~nm}$ excitation was higher than those from $1064 \mathrm{~nm}$ excitation. It was contrary for the remaining values of LTSDs. The phenomenon might be credited to the greater ablation efficiency of $532 \mathrm{~nm}$ excitation and plasma shielding effect of $1064 \mathrm{~nm}$ excitation. As seen in Figure 6, the craters caused by $532 \mathrm{~nm}$ laser beam were apparently with smaller depth and ablated volume, and it was more obvious for $98 \mathrm{~mm}$ LTSD. For the LTSD larger than the focal length of lens, although similar depth and ablated volume were found with 532/1064 nm excitations, the emissions from $1064 \mathrm{~nm}$ excitation were apparently more intense than that from $532 \mathrm{~nm}$ excitation. Thanks to the greater optical penetration depth of $1064 \mathrm{~nm}$ excitation, more mass of sample was evaporated and transferred into plasma, which might emit intense chromium radiation. The differences between 532 and $1064 \mathrm{~nm}$ excitations could be credited to the different ablation efficiency and plasma shielding effect, which might be related to the ablation fluence and ablation spot. Hence, further study is necessary to improve the ablation efficiency and reduce the effect of plasma shielding by optimizing the experimental parameters. 

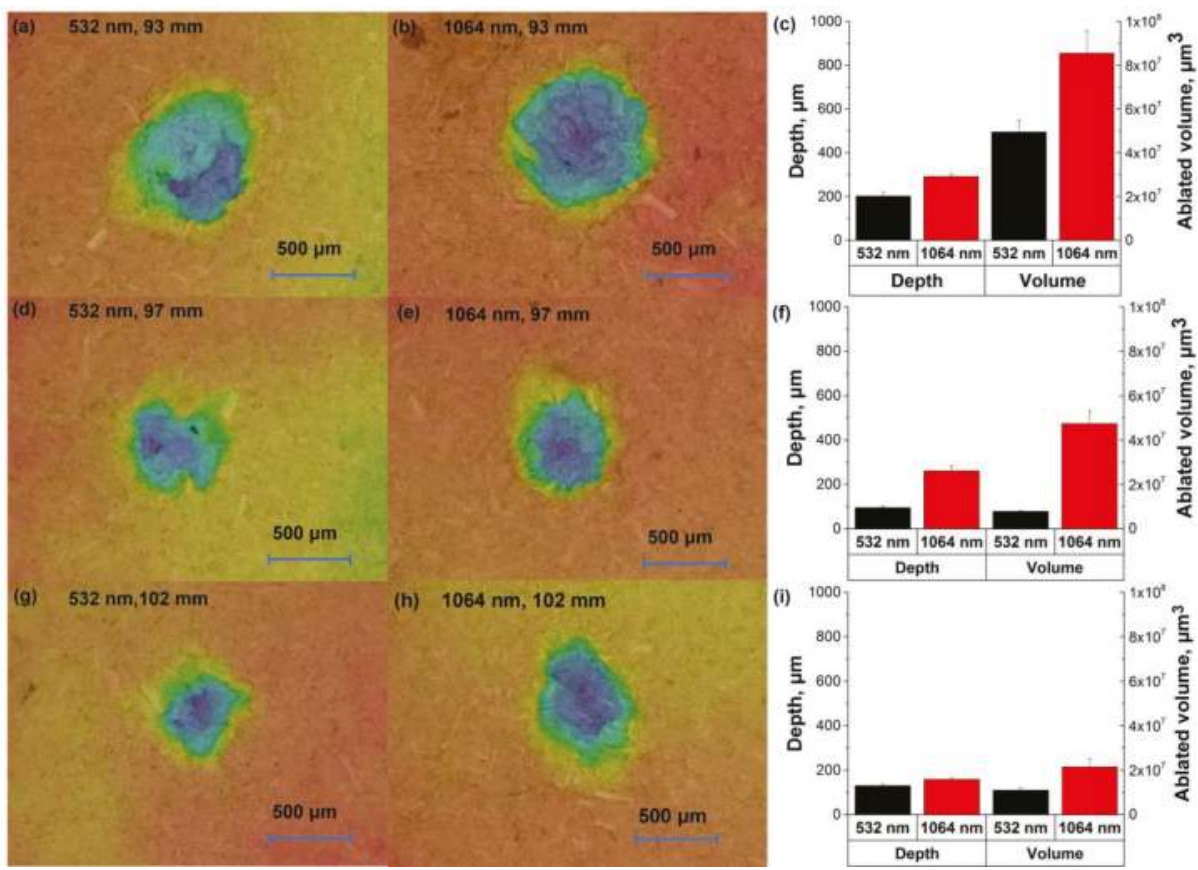

Figure 6. Morphologies of craters with different LTSDs under $532 \mathrm{~nm}$ and $1064 \mathrm{~nm}$ excitations: (a) $532 \mathrm{~nm}$ excitation, LTSD = $93 \mathrm{~mm}$; (b) $1064 \mathrm{~nm}$ excitation, LTSD = $93 \mathrm{~mm}$; (d) $532 \mathrm{~nm}$ excitation, LTSD = $97 \mathrm{~mm}$; (e) $1064 \mathrm{~nm}$ excitation, LTSD = $97 \mathrm{~mm}$; (g) $532 \mathrm{~nm}$ excitation, LTSD = $102 \mathrm{~mm}$; (h) $1064 \mathrm{~nm}$ excitation, LTSD $=102 \mathrm{~mm}$. The colors in $(\mathbf{a}, \mathbf{b}, \mathbf{d}, \mathbf{e}, \mathbf{g}, \mathbf{h})$ indicated the depth of craters. Plots of statistic parameters (depth and ablated volume) of craters with different LTSDs: (c) LTSD = $93 \mathrm{~mm}$; (f) $\operatorname{LTSD}=97 \mathrm{~mm}$; (i) LTSD $=102 \mathrm{~mm}$.

\subsection{Analytical Figures-Of-Merit}

After optimization of the experimental parameters under 532/1064 nm excitations, we further investigated the influence of wavelength on the calibration results. Pulse energy, delay time, gate width, LTSD for 532/1064 nm excitations were set at 90/80 mJ, 4/4 $\mu \mathrm{s}, 16 / 16 \mu \mathrm{s}$ and 98/99 mm, respectively. Figure 7a shows the spectra of two representative samples with chromium content of $6.99 \mathrm{mg} / \mathrm{kg}$ and $56.03 \mathrm{mg} / \mathrm{kg}$, respectively. After parameter optimization, the chromium signal and background continuum emission induced by $1064 \mathrm{~nm}$ excitation were both stronger than those from $532 \mathrm{~nm}$ excitation. However, it was hard to evaluate the influence of wavelength on the detection simply from two samples.

Figure $7 \mathrm{~b}-\mathrm{e}$ show the spectra after the treatments of background normalization, area normalization, MSC transformation and SNV transformation, respectively. As seen in Figure 7, the baselines of the spectra with background normalization, area normalization and SNV transformation were similar, while the baseline of MSC transformed and raw spectra show distinguished difference between 532 and $1064 \mathrm{~nm}$ excitations. This might be credited to the normalization principal of each method. Because MSC standardized each spectrum using the mean spectrum of each set as reference, the corrected spectra might retain basic features of experimental conditions and sample characteristics. Hence, the MSC normalization might be more suitable for similar sample sets, and the experimental conditions should be similar. This very feature might limit the practical application. In addition, the preprocessed spectra of background normalization, 
area normalization and SNV normalization showed similar trend, and the chromium emissions from $532 \mathrm{~nm}$ excitation were greatly stronger than that from $1064 \mathrm{~nm}$ excitation. As seen in Figure 7a, the raw chromium signal of 532/1064 nm excitations was similar. Hence, the decreased chromium signal in preprocessed spectra might be attributed to the variables used for normalization. It also indicated that $1064 \mathrm{~nm}$ excitation might induce greater background, integral area and noise.
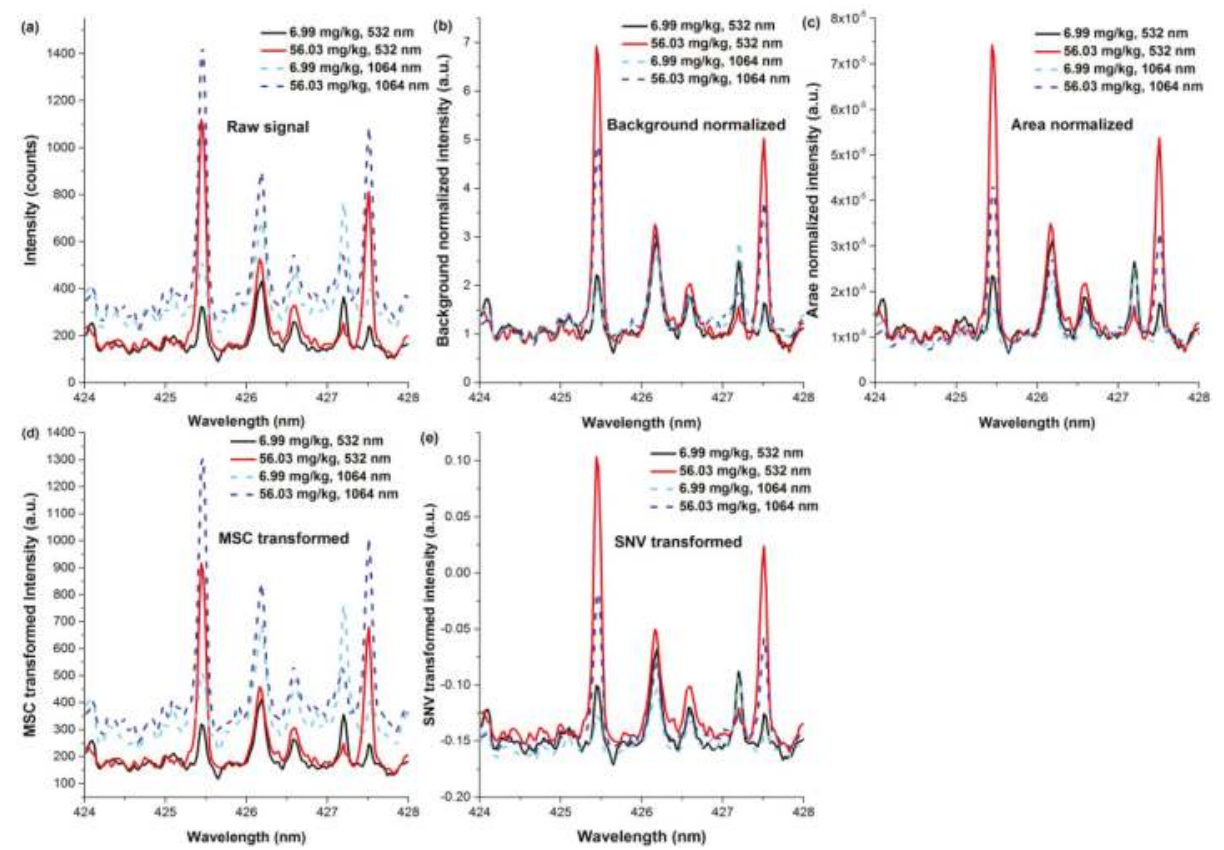

Figure 7. Spectra of two representative samples (chromium content of $6.99 \mathrm{mg} / \mathrm{kg}$ and $56.03 \mathrm{mg} / \mathrm{kg}$ ) in the spectral range of 424-248 nm. The spectra were processed by different pretreatments: (a) raw; (b) background normalization; (c) area normalization; (d) MSC transformation; (e) SNV transformation.

We further compared the calibration results with different preprocessing methods in Table 3. The analytical signal used for calibration was the maximal value within three pixels around Cr I at $425.43 \mathrm{~nm}$ and $427.48 \mathrm{~nm}$. The model performance was evaluated with RMSE and R, and the sensitivity was evaluated with LOD. 
Table 3. Calibration results based on 532/1064 nm excitations with different preprocessing methods.

\begin{tabular}{|c|c|c|c|c|c|c|c|}
\hline \multirow{2}{*}{ Wavelength } & \multirow{2}{*}{ Variables $(\mathrm{nm})$} & \multirow{2}{*}{ Preprocessing Methods } & \multicolumn{3}{|c|}{ Calibration } & \multicolumn{2}{|c|}{ Prediction } \\
\hline & & & $\mathbf{R}$ & $\begin{array}{c}\text { RMSE } \\
\text { (mg/kg) }\end{array}$ & LOD (mg/kg) & $\mathbf{R}$ & $\begin{array}{c}\text { RMSE } \\
\text { (mg/kg) }\end{array}$ \\
\hline \multirow{10}{*}{$532 \mathrm{~nm}$} & \multirow{5}{*}{425.43} & raw & 0.9789 & 4.61 & 4.01 & 0.9872 & 4.62 \\
\hline & & background normalization & 0.9780 & 4.69 & 4.83 & 0.9680 & 6.38 \\
\hline & & area normalization & 0.9857 & 3.76 & 4.53 & 0.9834 & 4.40 \\
\hline & & SNV transformation & 0.9847 & 3.89 & 2.72 & 0.9790 & 4.62 \\
\hline & & MSC transformation & 0.9829 & 4.11 & 2.69 & 0.9805 & 4.49 \\
\hline & \multirow{5}{*}{427.48} & raw & 0.9725 & 5.26 & 5.34 & 0.9828 & 4.75 \\
\hline & & background normalization & 0.9705 & 5.46 & 6.44 & 0.9697 & 5.95 \\
\hline & & area normalization & 0.9845 & 3.91 & 6.07 & 0.9831 & 4.24 \\
\hline & & SNV transformation & 0.9852 & 3.82 & 3.61 & 0.9820 & 4.33 \\
\hline & & MSC transformation & 0.9848 & 3.87 & 3.56 & 0.9836 & 4.30 \\
\hline \multirow{10}{*}{$1064 \mathrm{~nm}$} & \multirow{5}{*}{425.43} & raw & 0.9721 & & 7.56 & 0.9782 & 4.35 \\
\hline & & background normalization & 0.9715 & & 9.02 & 0.9509 & 6.66 \\
\hline & & area normalization & 0.9764 & & 8.40 & 0.9707 & 5.04 \\
\hline & & SNV transformation & 0.9784 & & 4.28 & 0.9703 & 5.15 \\
\hline & & MSC transformation & 0.9743 & & 4.40 & 0.9818 & 4.11 \\
\hline & \multirow{5}{*}{427.48} & raw & 0.9791 & & 11.35 & 0.9779 & 4.38 \\
\hline & & background normalization & 0.9755 & & 13.74 & 0.9722 & 4.87 \\
\hline & & area normalization & 0.9864 & & 12.70 & 0.9842 & 3.67 \\
\hline & & SNV transformation & 0.9823 & & 6.24 & 0.9846 & 3.83 \\
\hline & & MSC transformation & 0.9704 & & 6.61 & 0.9930 & 2.63 \\
\hline
\end{tabular}

In general, $532 \mathrm{~nm}$ laser pulse obtained better calibration results, with lower RMSEs, LODs and higher values of $R$. The sensitivity of $532 \mathrm{~nm}$ excitation was obviously higher than that from $1064 \mathrm{~nm}$ excitation, with a LODs around two times lower. It might be credited to the higher noise induced by $1064 \mathrm{~nm}$ excitation. However, the improvement in the aspect of prediction accuracy was limited when using $532 \mathrm{~nm}$ excitation. In addition, calibration with the emission from Cr I $425.43 \mathrm{~nm}$ showed better overall sensitivity than that from Cr I $427.48 \mathrm{~nm}$. However, the prediction accuracy for both emission lines was similar. Among the preprocessing methods, best calibration results were achieved with the MSC and SNV normalizations. It has been proven that SNV normalization could be used to compensate the laser fluctuation [40]. The improvement of background and area normalizations was limited, and the sensitivity of those was even reduced. Scatter plots that describe the calibration performance of raw signal, MSC normalized signal and SNV normalized signal with 532/1064 nm excitations are shown in Figure 8. 

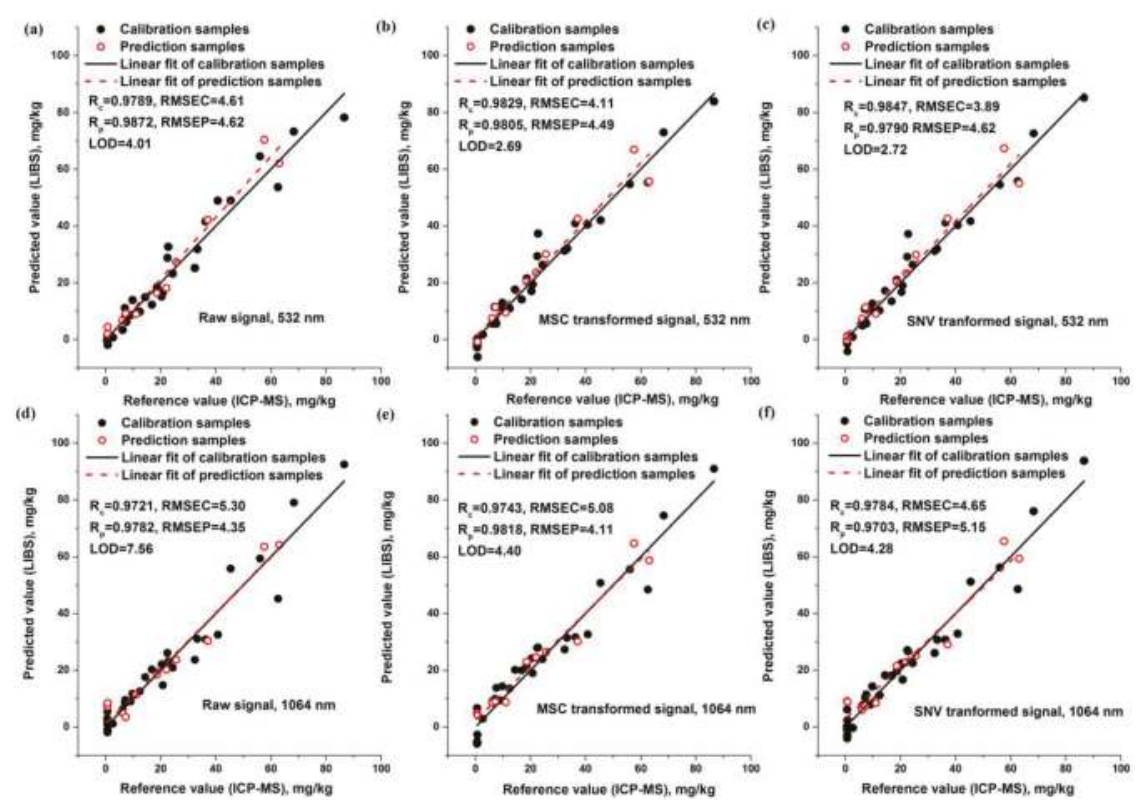

Figure 8. Relationship between reference value and LIBS measured value that calibrated with the variables of (a) raw signal of $532 \mathrm{~nm}$ excitation; (b) MSC transformed signal of $532 \mathrm{~nm}$ excitation; (c) SNV transformed signal of $532 \mathrm{~nm}$ excitation; (d) raw signal of $1064 \mathrm{~nm}$ excitation; (e) MSC transformed signal of $1064 \mathrm{~nm}$ excitation; (f) SNV transformed signal of $1064 \mathrm{~nm}$ excitation. $\left(\mathrm{R}_{\mathrm{c}}: \mathrm{R}\right.$ in calibration set, $\mathrm{R}_{\mathrm{p}}: \mathrm{R}$ in prediction set, RMSEC: RMSE in calibration set, RMSEP: RMSE in prediction set).

Apparently, the samples after normalization dispersed closely to the linear fitting line, and the sensitivity was greatly improved. Since SNV normalization could be applied under different experimental conditions, it might be suited for further application. Hence, using the combination of $532 \mathrm{~nm}$ excitation and SNV normalized signal of Cr I $425.43 \mathrm{~nm}$ was recommended for the detection of chromium content in rice leaves.

\section{Conclusions}

In this study, we investigated the influence of laser wavelength on the signal (sensitivity and stability) and plasma features (electron density and temperature) for the detection of chromium content in rice leaves. We found that the predominant laser wavelength mechanism might differ with the variations of delay time, pulse energy and LTSD. The effect of wavelength on chromium signal might be the result of transition energy, inverse bremsstrahlung and ablation efficiency. With the comparison of the sensitivity and stability, the experimental parameters including delay time, gate width, pulse energy and LTSD were optimized at $4 / 4 \mu \mathrm{s}, 16 / 16 \mu \mathrm{s}, 90 / 80 \mathrm{~mJ}$ and $98 / 99 \mathrm{~mm}$ for 532/1064 nm excitations, respectively. In the aspect of quantification capability, $532 \mathrm{~nm}$ excitation gave better detection sensitivity than $1064 \mathrm{~nm}$ excitation, with a LOD two times lower. However, the improvement of $532 \mathrm{~nm}$ excitation in prediction accuracy was limited. The best calibration result was achieved by relating the SNV normalized signal of Cr I $425.43 \mathrm{~nm}$ with reference chromium content, with R of 0.9849 , RMSE of $3.89 \mathrm{mg} / \mathrm{kg}$, LOD of $2.72 \mathrm{mg} / \mathrm{kg}$ for $532 \mathrm{~nm}$ excitation and R of 0.9784, RMSE of $4.65 \mathrm{mg} / \mathrm{kg}$, LOD of $4.28 \mathrm{mg} / \mathrm{kg}$ for $1064 \mathrm{~nm}$ excitation. The proposed approach might provide the first proof-of-principle data for application of LIBS for quantification of chromium content in rice leaves with improved sensitivity and accuracy. 
Acknowledgments: This study was supported by the National Key Research and Development Program of China (2016YFD0200603), National Natural Science Foundation of China (31671579), Development of Scientific Research Foundation of Zhejiang A \& F University (2015FR025) and Zhejiang Provincial Natural Science Foundation of China (LY15C130003).

Author Contributions: Jiyu Peng, Fei Liu, and Yong He conceived and designed the experiments and made guidance for writing of the manuscript; Tingting Shen, Lanhan Ye, Wei Wang and Xiaodan Liu performed the experiments; Wenwen Kong contributed to data analysis; Jiyu Peng and Fei Liu analyzed the data and wrote the whole paper; All authors reviewed the manuscript.

Conflicts of Interest: The authors declare no conflict of interest.

\section{References}

1. Zeng, F.; Wu, X.; Qiu, B.; Wu, F.; Jiang, L.; Zhang, G. Physiological and proteomic alterations in rice (Oryza sativa L.) seedlings under hexavalent chromium stress. Planta 2014, 240, 291-308. [CrossRef] [PubMed]

2. Peng, J.; Liu, F.; Zhou, F.; Song, K.L.; Zhang, C.; Ye, L.H.; He, Y. Challenging applications for multi-element analysis by laser-induced breakdown spectroscopy in agriculture: A review. Trends Anal. Chem. 2016, 85, 260-272. [CrossRef]

3. Santos, D.; Nunes, L.C.; de Carvalho, G.G.A.; Gomes, M.D.; de Souza, P.F.; Leme, F.D.; dos Santos, L.G.C.; Krug, F.J. Laser-induced breakdown spectroscopy for analysis of plant materials: A review. Spectrochim. Acta B 2012, 71, 3-13. [CrossRef]

4. Porizka, P.; Prochazkova, P.; Prochazka, D.; Sladkova, L.; Novotny, J.; Petrilak, M.; Brada, M.; Samek, O.; Pilat, Z.; Zemanek, P.; et al. Algal biomass analysis by laser-based analytical techniques-A review. Sensors 2014, 14, 17725-17752. [CrossRef] [PubMed]

5. Rehse, S.J.; Salimnia, H.; Miziolek, A.W. Laser-induced breakdown spectroscopy (libs): An overview of recent progress and future potential for biomedical applications. J. Med. Eng. Technol. 2012, 36, 77-89. [CrossRef] [PubMed]

6. Gondal, M.A.; Habibullah, Y.B.; Baig, U.; Oloore, L.E. Direct spectral analysis of tea samples using $266 \mathrm{~nm}$ uv pulsed laser-induced breakdown spectroscopy and cross validation of libs results with icp-ms. Talanta 2016, 152, 341-352. [CrossRef] [PubMed]

7. Kumar, R.; Tripathi, D.K.; Devanathan, A.; Chauhan, D.K.; Rai, A.K. In-situ monitoring of chromium uptake in different parts of the wheat seedling (Triticum aestivum) using laser-induced breakdown spectroscopy. Spectrosc. Lett. 2014, 47, 554-563. [CrossRef]

8. Arantes de Carvalho, G.G.; Moros, J.; Santos, D., Jr.; Krug, F.J.; Laserna, J.J. Direct determination of the nutrient profile in plant materials by femtosecond laser-induced breakdown spectroscopy. Anal. Chim. Acta 2015, 876, 26-38. [CrossRef] [PubMed]

9. Haider, Z.; Ali, J.; Arab, M.; Munajat, Y.B.; Roslan, S.; Kamarulzman, R.; Bidin, N. Plasma diagnostics and determination of lead in soil andphaleria macrocarpaleaves by ungated laser induced breakdown spectroscopy. Anal. Lett. 2015, 49, 808-817. [CrossRef]

10. Krizkova, S.; Ryant, P.; Krystofova, O.; Adam, V.; Galiova, M.; Beklova, M.; Babula, P.; Kaiser, J.; Novotny, K.; Novotny, J.; et al. Multi-instrumental analysis of tissues of sunflower plants treated with silver(i) ions-Plants as bioindicators of environmental pollution. Sensors 2008, 8, 445-463. [CrossRef] [PubMed]

11. Tognoni, E.; Cristoforetti, G. [invited] signal and noise in laser induced breakdown spectroscopy: An introductory review. Opt. Laser Technol. 2016, 79, 164-172. [CrossRef]

12. Noll, R. Laser-Induced Breakdown Spectroscopy: Fundamentals and Applications; Springer: Berlin/Heidelberg, Germany, 2012.

13. Russo, R.E.; Mao, X.L.; Liu, H.C.; Gonzalez, J.; Mao, S.S. Laser ablation in analytical chemistry-A review. Talanta 2002, 57, 425-451. [CrossRef]

14. Galbacs, G. A critical review of recent progress in analytical laser-induced breakdown spectroscopy. Anal. Bioanal. Chem. 2015, 407, 7537-7562. [CrossRef] [PubMed]

15. Fornarini, L.; Spizzichino, V.; Colao, F.; Fantoni, R.; Lazic, V. Influence of laser wavelength on libs diagnostics applied to the analysis of ancient bronzes. Anal. Bioanal. Chem. 2006, 385, 272-280. [CrossRef] [PubMed]

16. Zhang, D.C.; Ma, X.W.; Wen, W.Q.; Zhang, P.J.; Zhu, X.L.; Li, B.; Liu, H.P. Influence of laser wavelength on laser-induced breakdown spectroscopy applied to semi-quantitative analysis of trace-elements in a plant sample. Chin. Phys. Lett. 2010, 27. [CrossRef] 
17. De Carvalho, G.G.A.; Santos, D.; Nunes, L.C.; Gomes, M.D.; Leme, F.D.; Krug, F.J. Effects of laser focusing and fluence on the analysis of pellets of plant materials by laser-induced breakdown spectroscopy. Spectrochim. Acta B 2012, 74, 162-168. [CrossRef]

18. Nunes, L.C.; da Silva, G.A.; Trevizan, L.C.; Santos Júnior, D.; Poppi, R.J.; Krug, F.J. Simultaneous optimization by neuro-genetic approach for analysis of plant materials by laser induced breakdown spectroscopy. Spectrochim. Acta Part B 2009, 64, 565-572. [CrossRef]

19. Hahn, D.W.; Omenetto, N. Laser-induced breakdown spectroscopy (libs), part ii: Review of instrumental and methodological approaches to material analysis and applications to different fields. Appl. Spectrosc. 2012, 66, 347-419. [CrossRef] [PubMed]

20. Castro, J.P.; Pereira-Filho, E.R. Twelve different types of data normalization for the proposition of classification, univariate and multivariate regression models for the direct analyses of alloys by laser-induced breakdown spectroscopy (libs). J. Anal. At. Spectrom. 2016, 31, 2005-2014. [CrossRef]

21. Pořízka, P.; Klus, J.; Prochazka, D.; Képeš, E.; Hrdlička, A.; Novotný, J.; Novotný, K.; Kaiser, J. Laser-induced breakdown spectroscopy coupled with chemometrics for the analysis of steel: The issue of spectral outliers filtering. Spectrochim. Acta Part B 2016, 123, 114-120. [CrossRef]

22. Pořízka, P.; Klus, J.; Hrdlička, A.; Vrábel, J.; Škarková, P.; Prochazka, D.; Novotný, J.; Novotný, K.; Kaiser, J. Impact of laser-induced breakdown spectroscopy data normalization on multivariate classification accuracy. J. Anal. At. Spectrom. 2017, 32, 277-288. [CrossRef]

23. Zorov, N.B.; Gorbatenko, A.A.; Labutin, T.A.; Popov, A.M. A review of normalization techniques in analytical atomic spectrometry with laser sampling: From single to multivariate correction. Spectrochim. Acta B 2010, 65, 642-657. [CrossRef]

24. Zeng, F.; Qiu, B.; Ali, S.; Zhang, G. Genotypic differences in nutrient uptake and accumulation in rice under chromium stress. J. Plant Nutr. 2010, 33, 518-528. [CrossRef]

25. Yoshida, S.; Forno, D.A.; Cock, J.H.; Gomez, K.A. Laboratory Manual for Physiological Studies of Rice; International Rice Research Institute: Los Banos, Philippines, 1976.

26. DalCorso, G.; Farinati, S.; Maistri, S.; Furini, A. How plants cope with cadmium: Staking all on metabolism and gene expression. J. Integr. Plant Biol. 2008, 50, 1268-1280. [CrossRef] [PubMed]

27. Peng, J.; Song, K.; Zhu, H.; Kong, W.; Liu, F.; Shen, T.; He, Y. Fast detection of tobacco mosaic virus infected tobacco using laser-induced breakdown spectroscopy. Sci. Rep. 2017, 7, 44551. [CrossRef] [PubMed]

28. Aziz, R.; Rafiq, M.T.; Li, T.; Liu, D.; He, Z.; Stoffella, P.J.; Sun, K.; Xiaoe, Y. Uptake of cadmium by rice grown on contaminated soils and its bioavailability/toxicity in human cell lines (caco-2/hl-7702). J. Agric. Food Chem. 2015, 63, 3599-3608. [CrossRef] [PubMed]

29. Sobron, P.; Wang, A.; Sobron, F. Extraction of compositional and hydration information of sulfates from laser-induced plasma spectra recorded under mars atmospheric conditions-Implications for chemcam investigations on curiosity rover. Spectrochim. Acta Part B 2012, 68, 1-16. [CrossRef]

30. Leys, C.; Ley, C.; Klein, O.; Bernard, P.; Licata, L. Detecting outliers: Do not use standard deviation around the mean, use absolute deviation around the median. J. Exp. Soc. Psychol. 2013, 49, 764-766. [CrossRef]

31. Griem, H.R. Plasma Spectroscopy; Mcgraw-Hill Book Company: New York, NY, USA; San Francisco, CA, USA; Toronto, ON, Canada; London, UK, 1964.

32. Hahn, D.W.; Omenetto, N. Laser-induced breakdown spectroscopy (libs), part I: Review of basic diagnostics and plasma-particle interactions: Still-challenging issues within the analytical plasma community. Appl. Spectrosc. 2010, 64, 335a-366a. [CrossRef] [PubMed]

33. Luque, J.; Crosley, D.R. Lifbase: Database and spectral simulation program. SRI Int. Rep. MP 1999, $99,009$.

34. Tomas, I.; Tormod, N. The effect of multiplicative scatter correction (msc) and linearity improvement in nir spectroscopy. Appl. Spectrosc. 1988, 42, 1273-1284.

35. Barnes, R.J.; Dhanoa, M.S.; Lister, S.J. Standard normal variate transformation and de-trending of near-infrared diffuse reflectance spectra. Appl. Spectrosc. 1989, 43, 772-777. [CrossRef]

36. Coons, R.W.; Harilal, S.S.; Hassan, S.M.; Hassanein, A. The importance of longer wavelength reheating in dual-pulse laser-induced breakdown spectroscopy. Appl. Phys. B 2012, 107, 873-880. [CrossRef]

37. Fortes, F.J.; Moros, J.; Lucena, P.; Cabalin, L.M.; Laserna, J.J. Laser-induced breakdown spectroscopy. Anal. Chem. 2013, 85, 640-669. [CrossRef] [PubMed] 
38. De Giacomo, A. A novel approach to elemental analysis by laser induced breakdown spectroscopy based on direct correlation between the electron impact excitation cross section and the optical emission intensity. Spectrochim. Acta Part B 2011, 66, 661-670. [CrossRef]

39. Kasem, M.A.; Gonzalez, J.J.; Russo, R.E.; Harith, M.A. Effect of the wavelength on laser induced breakdown spectrometric analysis of archaeological bone. Spectrochim. Acta Part B 2014, 101, 26-31. [CrossRef]

40. Syvilay, D.; Wikie-Chancellier, N.; Trichereau, B.; Texier, A.; Martinez, L.; Serfaty, S.; Detalle, V. Evaluation of the standard normal variate method for laser-induced breakdown spectroscopy data treatment applied to the discrimination of painting laysers. Spectrochim. Acta Part B 2015, 114, 38-45. [CrossRef]

(C) 2018 by the authors. Licensee MDPI, Basel, Switzerland. This article is an open access article distributed under the terms and conditions of the Creative Commons Attribution (CC BY) license (http:/ / creativecommons.org/licenses/by/4.0/). 


\title{
Using a Mobile Device "App" and Proximal Remote Sensing Technologies to Assess Soil Cover Fractions on Agricultural Fields
}

\author{
Ahmed Laamrani ${ }^{1,2, *}$, Renato Pardo Lara ${ }^{1}$, Aaron A. Berg ${ }^{1}$, Dave Branson ${ }^{3}$ and Pamela Joosse ${ }^{2}$ \\ 1 Department of Geography-Hutt Building, University of Guelph, Guelph, ON N1G 2W1, Canada; \\ rpardo@uoguelph.ca (R.P.L.); aberg@uoguelph.ca (A.A.B.) \\ 2 Agriculture and Agri-Food Canada (AAFC)-Science and Technology Branch, 174 Stone Road West, \\ Guelph, ON N1G 4S9, Canada; pamela.joosse@agr.gc.ca \\ 3 FieldTRAKS Solutions Inc., 6367 McCordick Road, North Gower, Ottawa, ON K0A 2T0, Canada; \\ d.branson@fieldtraks.ca \\ * Correspondence: alaamran@uoguelph.ca; Tel.: +1-519-824-4120 (ext. 58950)
}

Received: 15 January 2018; Accepted: 21 February 2018; Published: 27 February 2018

\begin{abstract}
Quantifying the amount of crop residue left in the field after harvest is a key issue for sustainability. Conventional assessment approaches (e.g., line-transect) are labor intensive, time-consuming and costly. Many proximal remote sensing devices and systems have been developed for agricultural applications such as cover crop and residue mapping. For instance, current mobile devices (smartphones \& tablets) are usually equipped with digital cameras and global positioning systems and use applications (apps) for in-field data collection and analysis. In this study, we assess the feasibility and strength of a mobile device app developed to estimate crop residue cover. The performance of this novel technique (from here on referred to as "app" method) was compared against two point counting approaches: an established digital photograph-grid method and a new automated residue counting script developed in MATLAB at the University of Guelph. Both photograph-grid and script methods were used to count residue under 100 grid points. Residue percent cover was estimated using the app, script and photograph-grid methods on 54 vertical digital photographs (images of the ground taken from above at a height of $1.5 \mathrm{~m}$ ) collected from eighteen fields ( 9 corn and 9 soybean, 3 samples each) located in southern Ontario. Results showed that residue estimates from the app method were in good agreement with those obtained from both photograph-grid and script methods ( $R^{2}=0.86$ and 0.84 , respectively). This study has found that the app underestimates the residue coverage by $-6.3 \%$ and $-10.8 \%$ when compared to the photograph-grid and script methods, respectively. With regards to residue type, soybean has a slightly lower bias than corn (i.e., $-5.3 \%$ vs. $-7.4 \%$ ). For photos with residue $<30 \%$, the app derived residue measurements are within $\pm 5 \%$ difference (bias) of both photograph-grid- and script-derived residue measurements. These methods could therefore be used to track the recommended minimum soil residue cover of $30 \%$, implemented to reduce farmland topsoil and nutrient losses that impact water quality. Overall, the app method was found to be a good alternative to the point counting methods, which are more time-consuming.
\end{abstract}

Keywords: agricultural land; field crops; land cover; photograph-grid method; remote sensing; data validation and calibration; mobile app

\section{Introduction}

The amount of crop residue left in the field after harvest is of great importance for water storage [1], soil erosion control [2-4] and assessment and modeling of soil carbon sequestration [5]. Retaining crop 
residue is of interest in agro-ecosystems in North America, such as the mid-West and Great Lakes states where agricultural practices, including tillage practices (preparing land for growing crops), can affect water quality of the Gulf of Mexico and Great Lakes [6]. Such practices are of greater importance in the Canadian agro-ecosystem of southwestern Ontario, an area where, retaining crop residue cover $\geq 30 \%$ on the surface is considered a conservation tillage practice [7-9] and is an important objective of the Great Lakes Agricultural Stewardship Initiative funded by Agriculture and Agri-food Canada (AAFC) and the Ontario Ministry of Agriculture, Food and Rural Affairs (OMAFRA, [10]). Previous studies have found that conservation tillage and crop residue cover are important for reducing time and fuel consumption, improving water and soil quality [11-13], increasing the amount of organic matter [14-16], reducing greenhouse gas emissions [17] and reducing soil erosion by up to $75 \%$ by maintaining a corn crop residue cover of 15\% [18]. Crop residue cover estimation has been used to qualify specific fields for federal or provincial conservation programs (i.e., Land Stewardship I and II Programs offered by OMAFRA from 1987 to 1994). Such quantitative information on the amount of crop residue cover by field, which can then be extrapolated to regions, is essential to understand the state of soil management and the capacity for additional change in an area of interest.

The ability of tillage and planting systems to maintain soil residue cover is currently measured by using one or more of the following methods: Line-transect method (e.g., knotted rope), Meter stick method, Photograph comparison method, Calculation method and Photographic-grid method. Each of these techniques, described more completely by Dodd et al. [19], has various advantages and disadvantages. A common feature of each, however, is that they tend to be laborious and time-consuming to complete properly. These might be reasons why few landowners directly measure their field residue levels. Line-transect and digital photograph-grid methods are the two main approaches that have been widely used to quantify crop residue cover from ground observations. However, the standard ground-based line-transect method requires significant effort to collect an optimal number of samples [20], is time consuming, labor intensive and cannot provide continuous data over large areas, as percent residue cover is estimated at spatially and temporally disconnected fields. Several studies have found that the digital photograph-grid method can be a suitable alternative to the line-transect method (i.e., $[5,20])$. However, one of the main issues in the use of photographs to derive percent residue cover is that multiple manipulations are required (i.e., photograph collection, grid preparation, visual counting by different observers, script or spreadsheet calculation of percentage cover). Remote sensing imagery is a valuable tool to assess and map tillage practices, crop residue cover and cover crops over large areas. However, monitoring and deriving information on crop residue cover from space has been restricted in the past by low spectral and spatial-temporal resolution and availability of ground truth data [21]. Therefore, there is a need to identify low-cost, reliable, quick and easy to implement methods for residue estimation.

Mobile application technologies are becoming more abundant in farm management around the world. For instance, Dehnen-Schmutz et al. [22] found that $84 \%$ of British and French farmers used their smartphone for farm management. In the province of Ontario about 39\% of farmers had used their smartphones/tablets for farm management in 2015; 56\% had used computers/laptops [23]. The fact that many mobile devices (i.e., smartphones, tablets) have technical components such as GPS, camera and core processing power makes them suitable for several proximal remote sensing purposes. They can offer valuable, accurate and rapid information, calculations, measurements, or evaluations of agriculture attributes. Numerous studies have used mobile device applications (apps) to assess green crop cover [24], ground cover [25], fractional green canopy cover [26], turf coverage [27], leaf area index [28,29], or chlorophyll content in corn plants [30], or citizen science and/or participatory sensing [22,31,32]. One advantage of these devices is that they are equipped with digital cameras and processors, which allow users (i.e., farmers and/or crop advisors) to capture images and perform crop residue analysis efficiently. Despite the increased interest in using proximal remote sensing mobile devices in different agricultural applications, reported attempts to estimate percent crop cover residue using these technologies are limited. Here, we tested and validated a free 
Crop Residue Estimator (i.e., recently developed by @FieldTRAKS Solutions [33]) that can be deployed on various platforms including web applications and mobile technologies. This app uses an automated color threshold image classification process method. This method requires the selection of pixels (RGB) that represent the target object (i.e., residue cover or soil surface). The image is classified using the onboard processing capabilities of the device and is based upon a histogram of selected RGB pixel values. The method produces a 2-class thematic image representing either crop residue or soil surface. The pixels of each class are summed to determine the percent cover. One advantage of this method is that the mobile device returns immediate results which may be saved to create a statistical summary. FieldTRAKS Solutions created this application as a proof of concept and this is the first time that it has been tested in a comparison with an accepted method of calculating crop residue to evaluate its performance before it can be broadly recommended.

The objectives of this paper are to test the feasibility and strength of this mobile device app in assessing residue cover and to qualitatively compare this new method's performance against existing ground survey techniques. Specifically, we compared the residue cover estimates derived from the "app" method to those obtained using digital photograph-grid method and an automated residue counting script procedure. In a recent study [20], the photograph-grid method was found strongly correlated to the line-transect method which is considered a standard and robust method for assessing crop residue and was the "ground truth" in the context of that study. In this study, both photograph-grid and script methods were used to count residue under 100 grid points and are considered as reference methods in this evaluation. The three methods (app, photograph-grid and script) were evaluated using the same digital photograph dataset. The ultimate goal is to demonstrate if using a proximal remote sensing mobile device with a color threshold app can be a convenient alternative to methods that use digital photograph intersection counting for crop residue cover assessment.

\section{Materials and Methods}

\subsection{Study Area}

This study is conducted within the Lake Erie basin of southwestern Ontario, Canada (Figure 1). This area is primarily agricultural, with corn (Zea mays), soybean (Glycine max) and winter wheat (Triticum aestivum L.) as the dominant crops grown in rotation in this region. The topography is generally characterized by a combination of flat and rolling terrain, occasionally interspersed with steep ravines. Soils in the sample area range in texture from clay loam to loamy sand. The southwestern region of Ontario has the largest tillable land area in the province with a total of 3,026,576 ha [34] and an estimated biomass of residue (from three common crops, i.e., corn, soybean and winter wheat) that could be removed annually ranging between 6963 and $7223 \mathrm{~kg} / \mathrm{ha}$ [35]. The area has a climate typically characterized by long moderate winters (November-April) and hot and humid summers. The average annual temperature is $8.7^{\circ} \mathrm{C}$ with June, July and August as the warmest months (mean of $20.1^{\circ} \mathrm{C}$ ); and December, January and February as the coldest months (mean of $-3.2^{\circ} \mathrm{C}$ ). Total annual precipitation is $993 \mathrm{~mm}$, of which about one-third falls during the peak vegetative growth period, between early May and August (Environment Canada, 2011; St. Thomas weather station, about $15 \mathrm{~km}$ $\mathrm{W}$ of the study area). The bulk of the precipitation (2/3) falls during the non-growing season as fall or spring rain, or winter snow which then leaves the landscape after melting during winter thaw periods or as spring runoff. This is of significance as it means that the bulk of the precipitation occurs during the non-growing season when the landscape is most vulnerable to soil erosion; this emphasizes why retaining sufficient crop residue cover is an important and effective soil management practice in this region [6]. 


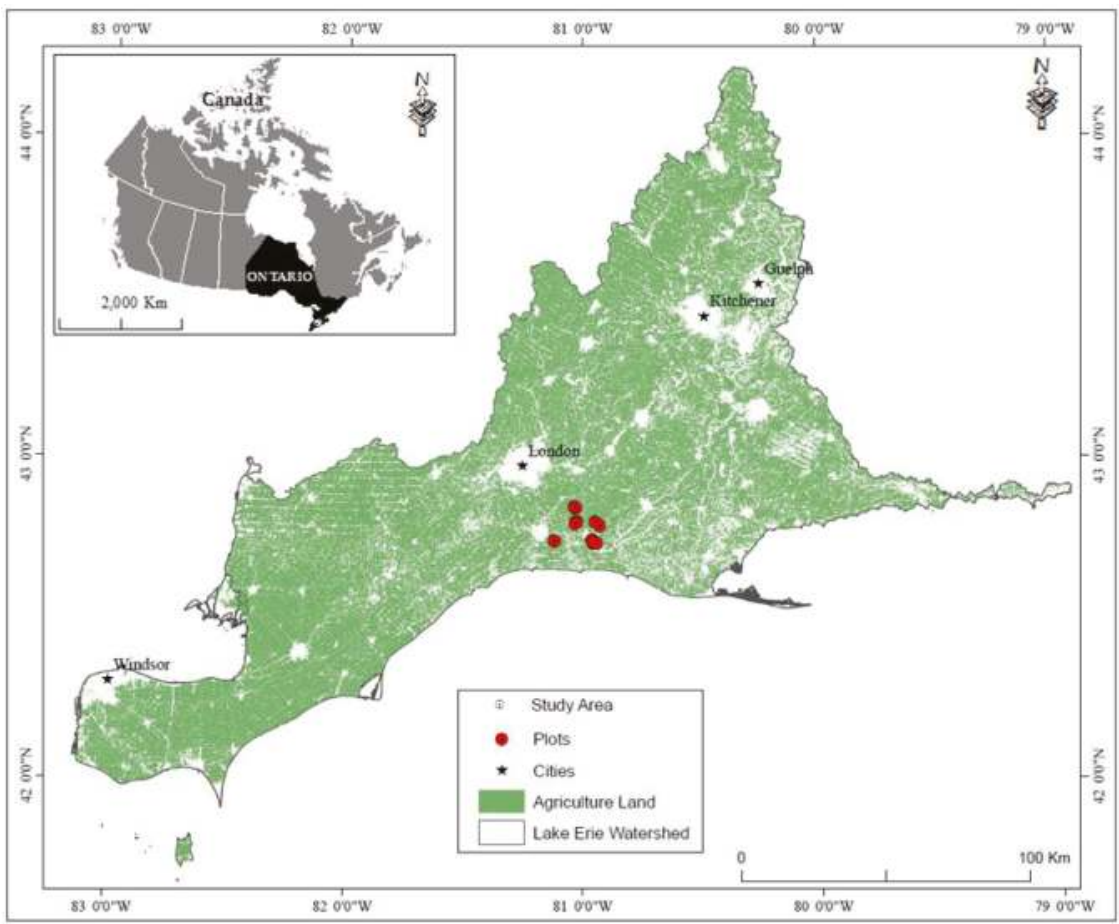

Figure 1. Study area within the Lake Erie watershed, southern Ontario, Canada. The 18 plots used in this study were located south of the city of London.

\subsection{Sampling Design and Field Data Collection Field}

Our study involved fields that had been established as part of a larger project that dealt with the use of multi-temporal remote sensing for mapping and quantification of soil cover on southern Ontario agricultural fields over the Lake Erie basin (see Figure 1 and [20]). Eighteen square plots (one $15 \mathrm{~m} \times 15 \mathrm{~m}$ plot per field) were established throughout the study area and sampled in fall 2014 after harvest. These 18 separate agricultural fields were located on privately-owned farms. The harvested crops on these 18 plots were corn (9 plots) and soybean ( 9 plots); and three samples (i.e., three photographs) per plot were collected. We aimed to sample three residue levels from each crop a priori from a visible assessment (i.e., 3 low residue corn, 3 low soybean, 3 medium corn, 3 medium soybean, 3 high corn and 3 high soybean where: low: $<30 \%$, medium: $30-60 \%$ and high: $>60 \%$ residue cover respectively); fields were assigned to the appropriate category after they were measured, resulting in more samples in the low residue category (22) and fewer in the medium (15) and high (17) residue categories than originally planned (18 samples each).

\subsubsection{Photograph-Grid Method}

In a recent study, we have found that percentage of crop residue estimates derived from either five or three digital photographs per plot were both strongly correlated to those derived from a reference method (i.e., line-transect). Therefore, among the 5 photos used in that study [20], only three photographs per plot were randomly selected and used here, for a total of 54 photographs. These 54 photographs (three per plot), were taken using a digital camera (i.e., Nikon COOLPIX P7800 12.2 MP Digital Camera with 7.1× Optical Zoom, China). At each plot, photos were taken at a 90-degree angle to 
the ground (perpendicular to soil surface) as this orientation is preferable for soil cover assessment [36] and at a fixed height $(1.5 \mathrm{~m})$. To do so, a $75 \times 100 \mathrm{~cm}$ quadrat was placed on the ground with its longest side perpendicular to tillage direction, or planting direction if there was no tillage (Figure 2a). Once photographs of the quadrat were taken, percent residue cover from those photographs was derived using a uniformly spaced 10 by 10 digital grid (i.e., 100 intersections; Figure $2 \mathrm{~b}$ ). To do so, first, the digital grid was super-imposed on each digital photograph, as in $[5,20,21]$, so that the grid spans the majority of the photo. The grid-line thickness was matched to that of a wooden dowel specification ( $\geq 3 / 32$ inches or $\geq 0.24 \mathrm{~cm}$ in diameter) following Shelton et al.'s [37] method using the scale of the quadrat. Second, intersections of residue with the grid were counted visually from a computer screen and percent cover was then calculated as the percent of total intersections that overlay residue.

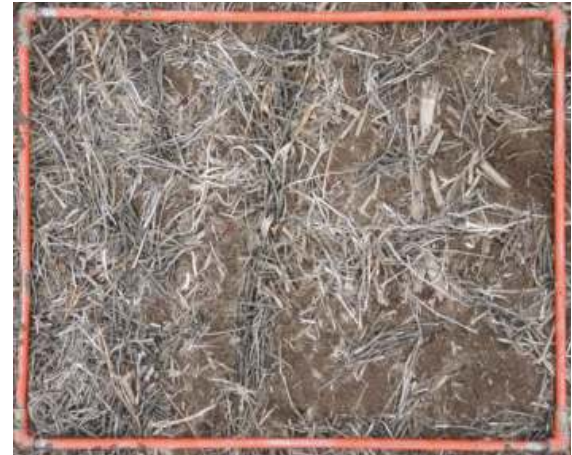

(a)

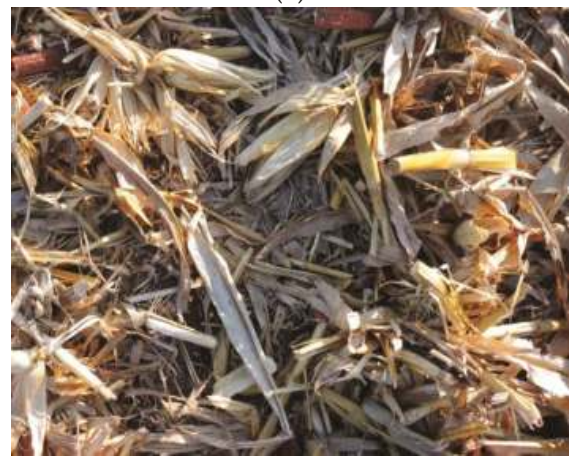

(c)

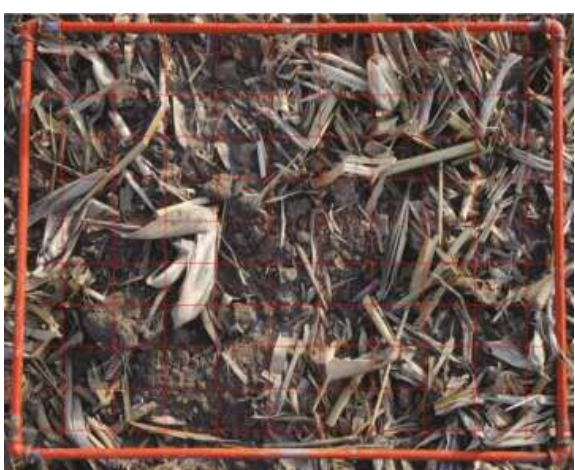

(b)

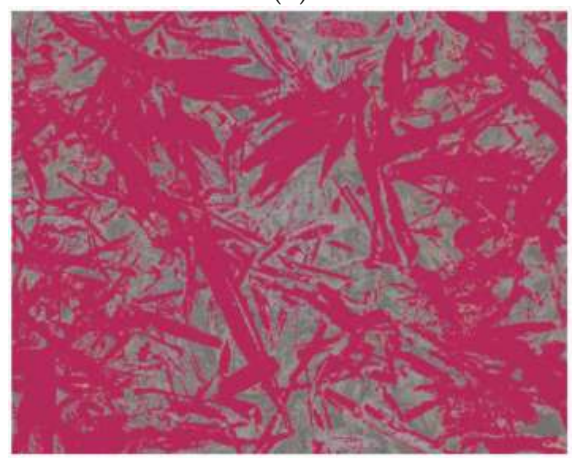

(d)

Figure 2. Examples of vertical photographs taken over soybean (a) and corn (c) fields. The photograph-grid method used superimposed, uniformly spaced 10 by 10 digital grid intersections (b). The app estimated crop residue is displayed in (d) as a thematic classified map using a two-color palette legend (residue class in red vs. no-residue class in gray. (c,d) show an example of the app method input and output. This example shows an evident underestimation of the app-derived crop residue compared to that from the photograph-grid method (i.e., $62 \%$ vs. $88 \%$, respectively).

\subsubsection{App Method}

The same set of 54 photographs was downloaded to an Android tablet and analyzed for crop residue cover using a mobile application technology. The app used in this study was developed by FieldTRAKS Solutions Inc. [33] and was partially funded through the Ontario Water Resource Adaptation and Management Initiative (2013). It was developed as a hybrid application using the Adobe PhoneGap cross-platform framework which aims to combine the convenience of developing 
with HTML5, JavaScript and CSS with the power of the native apps access to device's the APIs and sensors. FieldTRAKS Solutions views the application to be a prototype or proof of concept and has made it freely available in its' present form as an APK install for Android device (available for download at www.fieldtraks.ca). It employs an in-house crop residue image analysis algorithm that can be deployed in various platforms including web application and mobile technologies. This technique creates an automated color threshold image classification based on RGB pixel values only (i.e., without any normalization) through the selection of points (i.e., training dataset) which are representative of the target crop residue of the current processed digital photograph. The processing algorithm creates a histogram of RGB color values and selects values that fall within the color ranges created by selecting representative pixels of the target (e.g., corn residue or soybean residue). The histogram and target values are relative to the current image and no adjustments were made for shadows or any other sun effects. It is assumed that any such effects will be consistent throughout the image and affect target and non-target areas equally as all photos were taken under the same illumination conditions. As points are selected, an internal histogram of RGB values is constructed that represents the colour ranges of the crop residue in the image. Pixels with RGB values outside of the histogram are viewed to be soil or non-crop residue material. For the purposes of this study, sampling points of residue were chosen on every photo and used as training to direct the image processing software for the classification of all other pixels in the digital photo.

No optimal number of sampling points was provided by the app developer but an arbitrary minimum of four training sample points on each photo was suggested. To determine the appropriate number to be used as reference for this study, we tested multiple numbers of sampling points (i.e., 4,8 , 12) and have found that an increase in the number of sampling points (i.e., 4 to 12) is associated with a notable decrease of the correlation coefficient (data not shown) between residue percent derived from photograph-grid and app methods, leading to an overestimation of the residue percent. Nevertheless, four and eight sampling points had lower overestimation of the residue percent. Therefore, four to eight different sample residue pieces (i.e., training points) per photo were used for this study. Generally, a minimum of four sampling points were selected if the residue was homogeneous with respect to contrast and colour and more (i.e., maximum of 8) were selected in the more heterogeneous cases. The following selection procedures/guidelines were used to optimize the residue colour selection and capture variation across the photo for the $4-8$ sample points selected: (i) the sampling points were selected from across all the image and not from one corner; (ii) in each photo, the brightest and the darkest pieces of residue, then a mix of larger and smaller piece of residue had to be chosen; and (iii) if more than one type of crop residue (i.e., soybean and corn) is present in the photo, each type of residue (darkest and brightest) was selected. It is important to mention that photos with $>60 \%$ residue cover required selection of the maximum number of points (i.e., up to 8), whereas photos with less than $30 \%$ residue had a narrower range of residue colours and thus required fewer points selected (i.e., minimum 4).

Once all the sampling points were chosen (Figure 2c), the classification analysis was performed by the app and the estimated crop residue was displayed as a percentage on the tablet screen together with a thematic classified image (Figure 2d) using a two-color palette legend (residue and no-residue classes).

\subsubsection{Residue Cover Obtained from Digital Photograph-Grid Counting and the App Classification}

Similar to a pre-established set of rules used in a recent study [20], the residue cover percent for each digital photograph for both the photograph-grid and app methods was determined by an average of two assessors. To begin, independent assessments by three persons were carried out on a photo using each method. When the estimates of a minimum of two persons for a method were within $\pm 5 \%$ difference of the residue estimate of an individual photograph (i.e., either photograph-grid or the app counting), the two estimates with the smallest difference were averaged and used as the photograph's and app's datum. When assessments by all three persons disagreed by $>5 \%$, the estimate provided by the most accurate person (considered the counter with the most experience) was averaged with the 
estimate of the second counter in closest agreement with the most accurate estimate. Estimates from only two persons, for each photo, were averaged in order to minimize the human error that might affect the counting process and to increase the accuracy of the estimated percent crop residue cover.

\subsubsection{Crop Residue Cover Estimation Using the Script Method}

In addition to the photograph-grid method and the app method, a MATLAB script developed at the University of Guelph in 2016 was tested on the same set of 54 photographs. The script method aims to make the residue counting procedure more accurate, efficient, semi-automatic, rapid and transparent. As in Booth et al. (2006), the script method automates the manual pixel classification (MPC) procedure and the ensuing analysis, while only using the keyboard's arrow keys. On the other hand, manual pixel classification methods are particularly useful when calibrating automatic color threshold methods (Booth et al. 2006; i.e., the app). The script method was conducted by overlaying a 10 by 10 digital grid (for a total of 100 -intersection) on each digital photograph, as in [5,20,21]. It is important to mention that a script with a denser grid (i.e., 400-intersections) was also tested on the same 54 photographs to evaluate whether this would substantially improve the correlation coefficient between the app and script results. This quadrupling in sample size led to a marginal increase in the coefficient of determination ( $0.84 \mathrm{vs.} 0.82)$ while increasing the evaluation time four-fold. The script has the advantages of automating the overlay of grid intersections so there is less photo manipulation of creating a record of the classification decisions.

Before running the script on every photograph, each of the 100 grid intersections was identified with a centered red circular marker; once classified the marker turns either green, indicating residue, or black, indicating no residue. The crosshairs, created by the grid intersection and circular marker, allow for greater viewing of both adjacent pixels and the sample pixel using keyboard symbols. The script automatically locates and zooms in $(2 \times)$ on the grid intersections, where the user records whether the pixel contains residue $(\downarrow)$ or not $(\rightarrow)$, the user also has the option to zoom in $(\uparrow)$ up to $32 \times$ or reclassify to the previous intersection $(\leftarrow)$. The zoom function repeats the original image pixels in two dimensions to yield the visual effect of the single square pixel growing but not changing in color or sharpness. The user is taken from one point to the next until each intersection is classified. The script also automates the calculation of the image percent cover and estimates error statistics (based on a binomial distribution). The number and location of intersections classified as residue is automatically recorded and the script outputs a spreadsheet for each photo, listing the intersection coordinates and classification decision, a low-resolution duplicate of the photo showing the classification decisions at each intersection and a meta-spreadsheet that tabulates the results for each image analyzed. Only one person's assessment was used for the script-method to determine percent residue.

\subsection{Statistical Analysis}

Crop residue cover estimates derived from both digital photograph counting methods and the app were compared using linear regression models. Accuracy measures included the coefficient of determination $\left(R^{2}\right)$, root mean squared error (RMSE; Equation (1)) and Bias (Equation (2)). $R^{2}$ was used to explore the strength of the relationship between crop residue measured by the different methods, while RMSE was used to determine the magnitude of error among the methods. Bias is a quantitative term describing the difference between the average of measurements from reference method (i.e., photograph-grid and script) and the app method made on the same dataset of photos. RMSE and Bias were determined from Equations (1) and (2) cited in Molinier et al. [32].

$$
\begin{aligned}
\text { RMSE } & =\sqrt{\sum_{i} \frac{\left(y_{i}-\hat{y}_{i}\right)^{2}}{n}} \\
B I A S & =\frac{\sum_{i}\left(y_{i}-\hat{y}_{i}\right)}{n}
\end{aligned}
$$


where $y_{i}$ is the observed values from digital photograph reference data, $\hat{y}_{i}$ represents the app measurements and $n$ the total number of measurements. P-values were determined and significance was declared at $\alpha=0.05$. Statistical analyses were conducted in $\mathrm{R}$ [38].

As an additional statistical analysis, we also tested whether alternative distributions to model the data could improve the relationships between the methods (app vs. photograph-grid and app vs. script). In this context, non-linear regressions were performed using ordinary least squares (OLS) methods with logarithmic transformations of the response variable (Equation (3)) and logit transformations of the intersection-normalized response variable (Equation (4)).

$$
\begin{gathered}
\ln y_{i}=\beta_{0}+\beta_{1} x_{i} \Longleftrightarrow y_{i}=e^{\beta_{0}} e^{\beta_{1} x_{i}} \\
\operatorname{logit}\left(\frac{y_{i}}{\mathbf{1 0 0}}\right)=\ln \left(\frac{y_{i} / 100}{1-\left(y_{i} / 100\right)}\right)=\beta_{0}+\beta_{1} x_{i} \Longleftrightarrow \frac{y_{i}}{100}=\frac{e^{\beta_{0}+\beta_{1} x_{i}}}{1+e^{\beta_{0}+\beta_{1} x_{i}}}
\end{gathered}
$$

where $y_{i}$ represent the app measurements, $\beta_{0}$ and $\beta_{1}$ are regression coefficients and $x_{i}$ are the observed values from digital photograph reference data.

Ordinary Least Squares (OLS) regressions are based on the assumption that the response variable is normally distributed; this is not the case for this data set given its discrete and bounded nature. Another assumption of linear regression is homoscedasticity. OLS regression analysis using heteroscedastic data will still provide an unbiased estimate for the relationship between the predictor and response variables but standard errors and therefore inferences obtained from data analysis are suspect. Generalized Poisson [39,40] and Beta Regression [41-43] with a logit link function were also used to model the variance of the relationship between the app and both the photograph and script methods. In essence, the log-transformed OLS and Generalized Poisson models establish the relationship between the predictor and response variables similarly but consider the variance using different distributions; the same applies to the logit transformation and the beta regression with a logit link function.

\section{Results}

\subsection{App-, Photograph-Grid and Script-Derived Residue Cover}

Tables 1 and 2 show the estimated crop residue as evaluated by app, photograph-grid and script methods in the 18 plots (Table 1) and for the different residue type and levels (Table 2). For the photograph-grid method residue cover ranged from $2-97 \%$ for corn $(47 \pm 6.4 \%$; mean \pm Standard Error (SE)) and 6-83\% for soybean (35 \pm 4.9$)$, while for the app method the percentage of residue cover varied from 4 to 89 for corn $(39 \pm 5.3 \%)$ and 5 to 72 for soybean $(29 \pm 4.3)$. For the script method, the corn and soybean range were $3-90 \%(49 \pm 5.9)$ and $10-80 \%(41 \pm 4.7)$, respectively. Table 1 also shows that mean residue cover measured for all the data $(n=54)$ using the app method were on average slightly lower than the photograph-grid- and script-derived measurements (i.e., $34 \%$ vs. $41 \%$ and $34 \%$ vs. $45 \%$, respectively; Table 2 ).

Table 1. Summary statistics for residue cover percent derived from app, photograph-grid and script methods in the studied 18 plots.

\begin{tabular}{ccccccccccc}
\hline \multirow{2}{*}{ Plot ID } & \multirow{2}{*}{ Type } & \multicolumn{3}{c}{ Photograph-Grid-Derived } & \multicolumn{3}{c}{ Script-Derived } & \multicolumn{3}{c}{ App-Derived } \\
\cline { 3 - 10 } & & Mean & Range & SE & Mean & Range & SE & Mean & Range & SE \\
\hline 1 & CR & 4 & $2-5$ & 0.9 & 9 & $3-14$ & 3.3 & 9 & $7-13$ & 2.1 \\
2 & CR & 5 & $4-6$ & 0.7 & 12 & $10-14$ & 1.3 & 14 & $12-16$ & 1.3 \\
3 & CR & 8 & $4-14$ & 3.1 & 11 & $7-19$ & 3.8 & 6 & $4-9$ & 1.3 \\
4 & CR & 47 & $35-62$ & 8.1 & 49 & $39-64$ & 7.6 & 38 & $36-40$ & 1.1 \\
5 & CR & 49 & $39-68$ & 9.5 & 54 & $43-71$ & 8.7 & 27 & $21-40$ & 6.4 \\
6 & CR & 59 & $41-80$ & 11.3 & 61 & $50-73$ & 6.6 & 43 & $25-54$ & 9.3 \\
7 & CR & 74 & $70-80$ & 2.9 & 77 & $72-83$ & 3.2 & 65 & $61-73$ & 3.7 \\
\hline
\end{tabular}


Table 1. Cont.

\begin{tabular}{ccccccccccc}
\hline \multirow{2}{*}{ Plot ID } & \multirow{2}{*}{ Type } & \multicolumn{3}{c}{ Photograph-Grid-Derived } & \multicolumn{3}{c}{ Script-Derived } & \multicolumn{3}{c}{ App-Derived } \\
\cline { 3 - 10 } & & Mean & Range & SE & Mean & Range & SE & Mean & Range & SE \\
\hline 8 & CR & 87 & $82-92$ & 2.9 & 81 & $76-85$ & 2.6 & 75 & $62-88$ & 7.7 \\
9 & CR & 88 & $74-97$ & 7.2 & 85 & $78-90$ & 3.6 & 77 & $70-89$ & 6.3 \\
10 & SB & 7 & $6-8$ & 0.5 & 15 & $12-18$ & 1.8 & 7 & $6-7$ & 0.5 \\
11 & SB & 8 & $6-10$ & 1.0 & 18 & $8-14$ & 1.6 & 11 & $8-14$ & 1.6 \\
12 & SB & 11 & $4-21$ & 5.1 & 21 & $11-34$ & 6.8 & 11 & $6-17$ & 3.5 \\
11 & SB & 12 & $9-16$ & 2.2 & 14 & $10-16$ & 1.9 & 8 & $5-9$ & 1.2 \\
14 & SB & 38 & $29-48$ & 5.6 & 41 & $37-47$ & 3.2 & 39 & $20-54$ & 10.2 \\
15 & SB & 48 & $42-52$ & 3.1 & 55 & $51-58$ & 2.2 & 32 & $28-34$ & 1.7 \\
16 & SB & 58 & $48-73$ & 7.7 & 68 & $62-79$ & 5.4 & 48 & $19-72$ & 15.6 \\
17 & SB & 59 & $47-70$ & 6.6 & 68 & $65-71$ & 1.8 & 50 & $43-55$ & 3.6 \\
18 & SB & 70 & $54-83$ & 8.5 & 71 & $66-80$ & 4.4 & 58 & $42-67$ & 8.1 \\
\hline
\end{tabular}

$\mathrm{CR}=$ Corn; $\mathrm{SB}=$ Soybean; $\mathrm{SE}$ refers to Standard Error. Every plot presents the average residue cover percent of three photographs. Plots are cauterized as: Low residue cover $(<30 \%)$; Medium residue cover $(30-60 \%)$; or High residue cover $(>60 \%)$. Plots from fields with $<30 \%$ and $\geq 30 \%$ residue cover are considered conventional and conservation practices, respectively.

Table 2. Summary statistics for residue cover percent grouped by residue type and levels.

\begin{tabular}{ccccccccccc}
\hline \multirow{2}{*}{ Residue } & \multirow{n}{*}{$\mathbf{n}$} & \multicolumn{3}{c}{ Photograph-Grid-Derived } & \multicolumn{3}{c}{ Script-Derived } & \multicolumn{3}{c}{ App-Derived } \\
\cline { 3 - 10 } & & Mean & Range & SE & Mean & Range & SE & Mean & Range & SE \\
\hline All & 54 & 41 & $2-97$ & 4.1 & 45 & $3-90$ & 3.8 & 34 & $4-89$ & 3.5 \\
Corn & 27 & 47 & $2-97$ & 6.5 & 49 & $3-90$ & 5.9 & 39 & $4-89$ & 5.3 \\
Soybean & 27 & 35 & $4-83$ & 4.9 & 41 & $10-80$ & 4.7 & 29 & $5-72$ & 4.3 \\
Low & 22 & 9 & $2-29$ & 1.3 & 16 & $3-38$ & 1.7 & 11 & $4-42$ & 1.7 \\
Medium & 15 & 46 & $35-57$ & 1.7 & 53 & $37-66$ & 2.5 & 35 & $19-54$ & 3.2 \\
High & 17 & 77 & $62-97$ & 2.6 & 77 & $64-90$ & 1.8 & 64 & $38-89$ & 3.4 \\
$\geq 30 \%$ & 32 & 63 & $35-97$ & 3.2 & 65 & $37-90$ & 2.6 & 50 & $19-89$ & 3.5 \\
\hline
\end{tabular}

SE refers to Standard Error. Residue type level low, medium, and high represent fields with $<30 \%, 30-60 \%$, and $>60 \%$, respectively.

\subsection{Relationship between Residue Cover Obtained by App vs. Photograph and Script Methods}

Estimates of residue cover from each of the 54 photos were obtained from the app and compared to those obtained from photograph-grid and scripts methods. Analyses showed that percentage of residue cover estimates using the app method were strongly correlated to those estimated from photograph-grid $\left(R^{2}=0.86 ; P<0.001 ; n=54 ;\right.$ Table 3 and Figure $\left.3 a\right)$ and script methods $\left(R^{2}=0.84\right.$; $\mathrm{P}<0.001 ; \mathrm{n}=54$; Table 3 and Figure 4a). When the three individual estimates per plot were averaged this correlation was even higher (photograph-grid: $R^{2}=0.95, P<0.001, n=18$; script: $R^{2}=0.92$, $\mathrm{P}<0.001, \mathrm{n}=18$; figures not shown here). With regards to estimation by residue type, corn showed higher $\mathrm{R}^{2}$ than soybean for both photograph-grid ( $\left.0.86 \mathrm{vs.} 0.85\right)$ and script method ( $\left.0.86 \mathrm{vs.} 0.79\right)$ (Table 3; Figures $3 \mathrm{~b}$ and $4 \mathrm{~b}$ ). When the data were stratified by residue cover level (i.e., low, medium and high), the correlation between the app and photograph-grid was significant for low $\left(R^{2}=0.40\right.$; $\mathrm{P}<0.001 ; \mathrm{n}=22)$, medium $\left(\mathrm{R}^{2}=0.40 ; \mathrm{P}<0.05 ; \mathrm{n}=15\right)$ and high $\left(\mathrm{R}^{2}=0.27 ; \mathrm{P}<0.05 ; \mathrm{n}=17\right)$ residue estimates. As for the correlation between the app and script methods, only medium residue level was not significant $\left(\mathrm{R}^{2}=0.13, \mathrm{P}<=0.17, \mathrm{n}=15\right.$; Table 3$)$. Fields with less than $30 \%$ residue cover (low) displayed statistically significant correlation and the lowest error (photograph-grid: $\mathrm{R}^{2}=0.40$, RMSE $=6.5$; script: $R^{2}=0.45$, RMSE $=7.9$, respectively). Fields with more than $30 \%$, residue, considered a conservation tillage practice, showed a higher correlation but also a higher error (photograph-grid: $\mathrm{R}^{2}=0.70, \mathrm{P}<0.001, \mathrm{RMSE}=16.4$, script: $\mathrm{R}^{2}=0.65, \mathrm{P}<0.001, \mathrm{RMSE}=19.0, \mathrm{n}=32$; Table 3). 
Table 3. Summary statistics of relationships between residue cover percent as determined by app compared to photograph-grid and script methods in corn and soybean fields.

\begin{tabular}{|c|c|c|c|c|c|c|c|c|c|c|c|c|c|}
\hline \multirow{2}{*}{ Residue } & \multirow{2}{*}{$\mathbf{n}$} & \multicolumn{6}{|c|}{ App vs. Photograph-Grid } & \multicolumn{6}{|c|}{ App vs. Script } \\
\hline & & $\mathbf{R}^{2}$ & $\mathbf{P}$ & $m$ & $b$ & RMSE & Bias & $\mathbf{R}^{2}$ & $\mathbf{P}$ & $m$ & $b$ & RMSE & Bias \\
\hline All & 54 & 0.86 & 0.00 * & 0.77 & 2.84 & 13.3 & -6.3 & 0.84 & 0.00 * & 0.84 & -3.45 & 15.4 & -10.8 \\
\hline Corn & 27 & 0.86 & 0.00 * & 0.76 & 4.06 & 15.0 & -7.4 & 0.86 & 0.00 * & 0.84 & -1.77 & 14.7 & -9.6 \\
\hline Soybean & 27 & 0.85 & 0.00 * & 0.80 & 1.59 & 11.2 & -5.3 & 0.79 & $0.00 *$ & 0.81 & -4.05 & 16.2 & -12.0 \\
\hline Low & 22 & 0.40 & $0.00 \dagger$ & 0.81 & 3.80 & 6.5 & 2.1 & 0.45 & $0.00 *$ & 0.66 & 0.63 & 7.9 & -4.6 \\
\hline Medium & 15 & 0.40 & $0.00 \ddagger$ & 1.18 & -19.47 & 14.6 & -11.2 & 0.13 & 0.17 & 0.47 & 9.81 & 21.8 & -18.0 \\
\hline High & 17 & 0.27 & $0.03 \ddagger$ & 0.70 & 10.40 & 17.7 & -13.0 & 0.43 & $0.00+$ & 1.25 & -31.67 & 16.2 & -12.3 \\
\hline$\geq 30 \%$ & 32 & 0.70 & 0.00 * & 0.91 & -6.73 & 16.4 & -12.1 & 0.65 & 0.00 * & 1.09 & -20.8 & 19.0 & -15.0 \\
\hline
\end{tabular}

All data $=$ all the sampled residue data were used $(3$ replicates per field; $\mathrm{n}=54) ; \mathrm{LR}=$ Low residue cover $(\mathrm{CR}+\mathrm{SB})$; $\mathrm{MR}=$ Medium residue cover $(\mathrm{CR}+\mathrm{SB}) ; \mathrm{HR}=$ High residue cover $(\mathrm{CR}+\mathrm{SB}) ; \leq 30 \%$ and $>30 \%$ represent plots with less and greater than $30 \%$ residue left on the field. $\mathrm{n}=$ total number of samples (measurements) used in the correlation. P-value $<0.001 *$ and $<0.01+$; the results is significant at $\mathrm{P}<0.05 . m$ is the slope of the line, and $\mathrm{b}$ is the $\mathrm{y}$-intercept of the line. RMSE and Bias are expressed in percent $(\%)$.

The bias between the app measurements and both the photograph-grid and script methods were analyzed in this study (Table 3). For the photograph-grid method, the biases were generally under $10 \%$ for all the data $(-6.3 \%)$ with a notable exception for fields with $\geq 30 \%$ residue covers (bias of $-12.1 \%)$. For the script method, the biases were higher than those for the photograph-grid method $(-10.8 \%)$ and under $15 \%$ overall (except for medium level residue; Table 3$)$. Among all the significantly correlated residue estimates, fields with $<30 \%$ residue cover showed the lowest biases $(2.1 \%$ and $-4.6 \%$ ) and RMSE (6.5 and 7.9\%) for both methods, respectively. This study also showed that soybean and corn residue covers have different trends in regard to the method used. Indeed, the bias of the app residue measurement compared to the photograph-grid method has higher for corn than soybean $(-6.4 \%$ vs. $-5.3 \%)$; whereas, corn residue measurements compared to the script method has lower bias than soybean $(-9.6 \%$ vs. $-12 \%)$. This finding (soybean residue cover has higher bias than corn) is consistent with other residue estimating studies in which errors were higher on soybeans compared to corn [20,21].

Finally, by dividing the field data between those with less than and greater than $30 \%$ residue cover, considered a conservation practice, results displayed the lowest $(-4.1 \%)$ and highest $(-15 \%)$ biases, respectively (data not shown in Table 3).
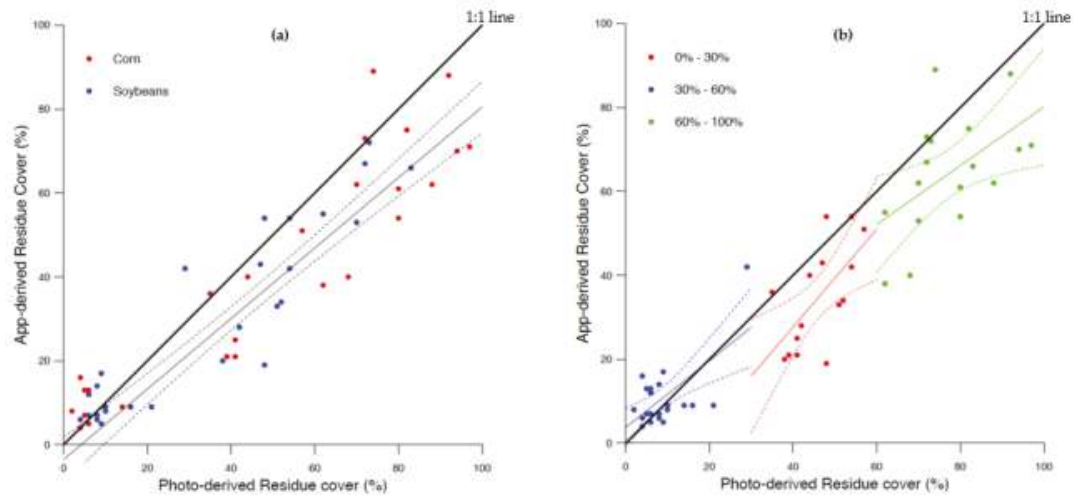

Figure 3. Relationships between residue cover percent as determined by photograph-grid and app: (a) residue cover $($ corn $=$ red dots and soybean $=$ blue dots); and $(b)$ residue levels (Low = blue, medium $=$ red and high $=$ green). Related statistics are shown in Table 2. 

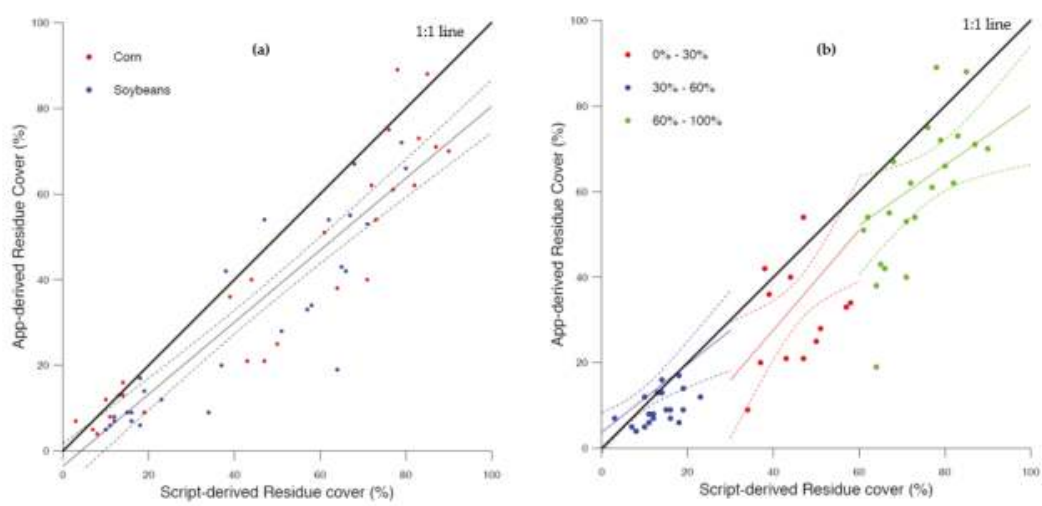

Figure 4. Relationships between residue cover percent as determined by script and app: (a) residue cover $($ corn = red dots and soybean = blue dots); and $(\mathbf{b})$ residue levels $($ Low $<30 \%$, medium $=30-60 \%$ and high $>60 \%$ ). Related statistics (i.e., $\mathrm{R}^{2}$ ) are shown in Table 3.

\subsection{Exploring Non-Linear Relationship Alternative to Modelling the Residue Cover Data}

Results of alternative distributions to model the data (app vs. photograph-grid and app vs. script) are summarized in Figures 5 and 6 and Table 4. Exploratory analysis of the data was indicative of an exponential or logistic relationship between the app and script methods (Figures 5 and 6). The relationship can be approximated using log and logit transformations of the response variable.
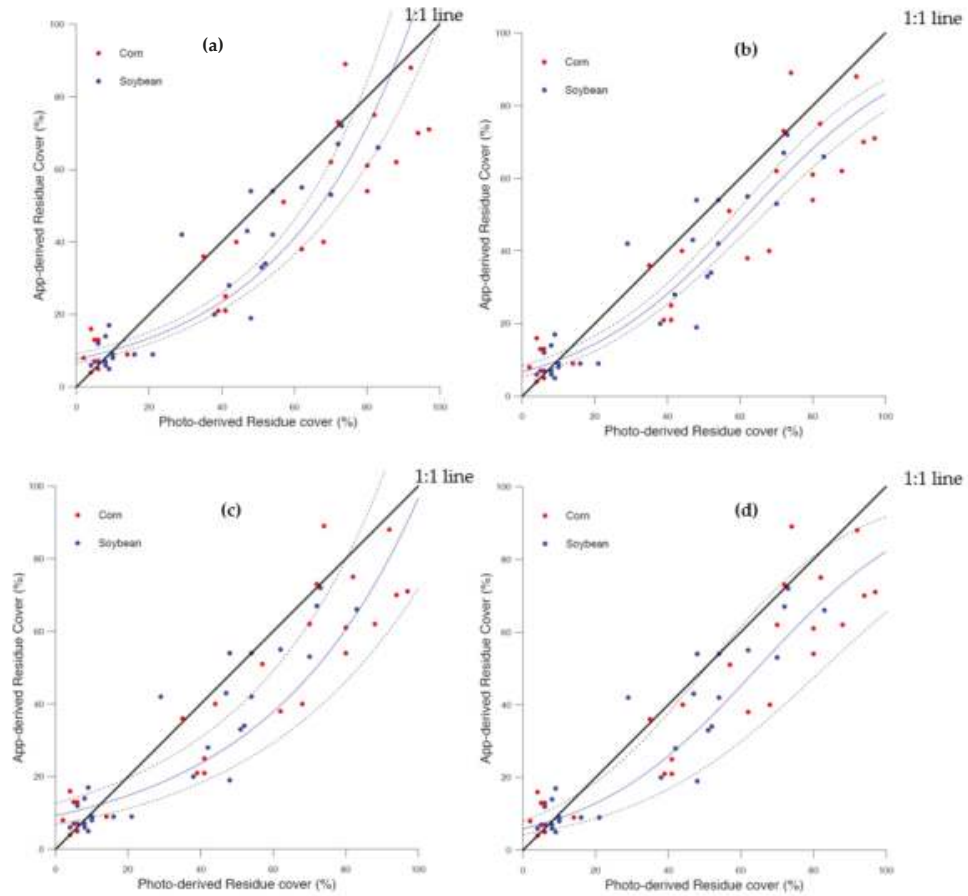

Figure 5. Relationships between residue cover percent as determined by photograph-grid and app with 95\% CI: (a) OLS log-linear regression; (b) OLS logit-linear regression, (c) generalized Poisson regression; (d) Beta regression with logit link. 

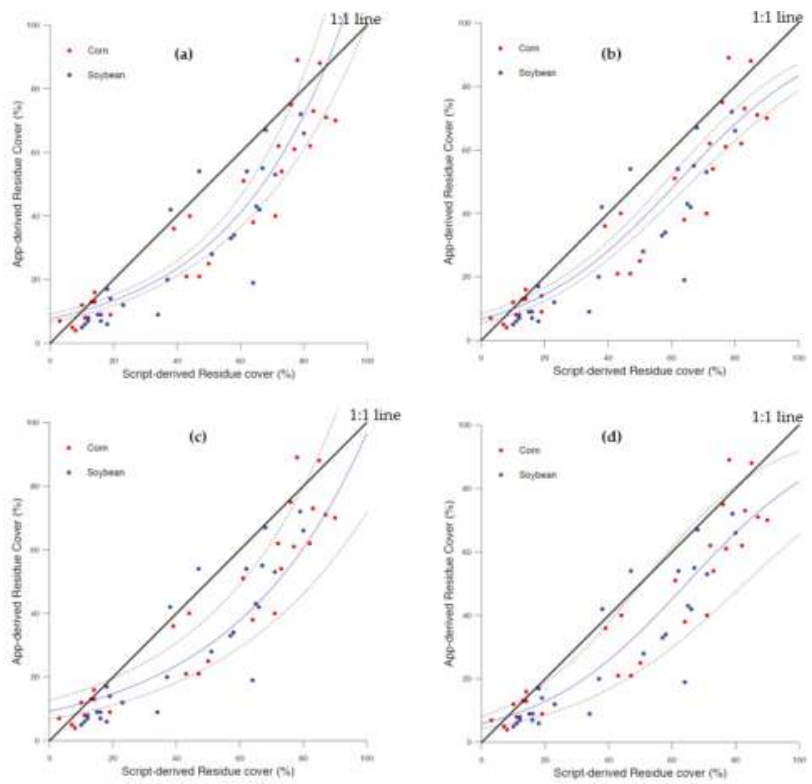

Figure 6. Relationships between residue cover percent as determined by script and app with 95\% CI: (a) OLS log-linear regression; (b) OLS logit-linear regression; (c) generalized Poisson regression; (d) Beta regression with logit link.

Table 4. Summary statistics of non-linear regression models between residue cover percent as determined by photograph-grid and script versus app methods.

\begin{tabular}{lcccccccc}
\hline \multirow{2}{*}{ Regression } & \multicolumn{3}{c}{ App vs. Photograph-Grid } & \multicolumn{4}{c}{ App vs. Script } \\
\cline { 2 - 8 } & $\mathbf{R}^{\mathbf{2}}$ & P-Value & $\boldsymbol{m}\left(\times \mathbf{1 0}^{-\mathbf{2}}\right)$ & $\boldsymbol{b}$ & $\mathbf{R}^{\mathbf{2}}$ & $\mathbf{P}$-Value & $\boldsymbol{m}\left(\times \mathbf{1 0}^{\mathbf{2}}\right)$ & $\boldsymbol{b}$ \\
\hline $\begin{array}{l}\text { Log } \\
\text { Transform }\end{array}$ & 0.84 & $0.00 *$ & 2.79 & 2.04 & 0.86 & $0.00 *$ & 3.11 & 1.78 \\
$\begin{array}{l}\text { Logit } \\
\text { Transform }\end{array}$ & 0.86 & $0.00 *$ & 4.24 & -2.63 & 0.86 & $0.00 *$ & 4.66 & -3.00 \\
$\begin{array}{l}\text { Generalized } \\
\text { Poisson }\end{array}$ & $\mathrm{N} / \mathrm{A}$ & $0.00 *$ & 2.35 & 1.73 & $\mathrm{~N} / \mathrm{A}$ & $0.00 *$ & 2.79 & 1.50 \\
Beta & $0.86^{* *}$ & $0.00 *$ & 3.94 & -2.43 & $0.86 *$ & $0.00 *$ & 4.29 & -2.77 \\
\hline
\end{tabular}

P-value: * $<0.001$. ${ }^{*}$ Pseudo $\mathrm{R}^{2}$ refers to the squared correlation between the linear predictor for the mean and the link-transformed response [44]). N/A: Generalized Poisson models using R do not output a coefficient of determination or correlation coefficient.

\section{Discussion}

Overall, this study has found that residue estimates from the FieldTRAKS app were in good agreement with those obtained with the photograph-grid method. There was a slight underestimation $(-6.3 \%)$ and corn has a higher contribution to the bias than soybean, which could be explained by illumination phenomena. Analysis was done of freshly harvested fields in the fall and we believe that corn fields with high amounts of residue created a higher contrast between residue in shadow and illuminated areas in the same photograph, which leads to confusion for the app classifier. This can be supported by the fact that the high residue level has the highest RMSE and bias compared to low and medium classes (Table 3) and with most likely a higher contribution of corn plots (i.e., plot 9 in Table 1) to the bias. The results of this study suggest that the RMSE and bias are low for fields with $<30 \%$ residue cover. This is particularly encouraging because these fields which are the ones most 
likely to need additional conservation practices, are more likely to be reliably assessed and identified using the app.

Similarly, the results indicate that residue estimates from the FieldTRAKS App were, overall, in good relative agreement (accuracy) with those obtained with the script method. Indeed, the app showed an underestimation of $-10.8 \%$ relative to the script results. Contrary to the photograph-grid comparison, soybean showed a higher contribution to the bias than corn most likely because soybean residue pieces are relatively thinner than most corn pieces, increasing the likelihood to not be identified by the assessor. Second, there was a lower contrast between soil and soybean residue which lead to confusion for the app classifier. This study found that at high contrast or low contrast it is difficult for the app to adequately select training points to represent residue range, compared to grid count where forced to assess set points.

Exploratory analysis of the data was indicative of an exponential or logistic relationship between the app and script methods (Figures 5 and 6). Inferences based on the standard errors reported using these ordinary least squares (OLS) regression estimates may be invalid and indeed seem too restrictive when $95 \% \mathrm{CI}$ are considered. The data appear to be bimodal and/or under dispersed, meaning that the error terms are underestimated using OLS. It is recommended that a generalized Poisson or Beta regression be performed when modeling the variance of these data.

This study also found that residue cover estimated from the script method overestimates that obtained by the photograph-grid method although they are strongly correlated (Bias $=4.2 \%$; RMSE $=7.5 ; R^{2}=0.96$; regression results not shown). Difference in bias between these two methods could be attributed to the fact that grids used for grid point counting methods do not coincide and the script method, unlike the photograph-grid method, does not take into consideration the minimum residue size (i.e., to be counted, a piece of residue had to be $\geq 0.24 \mathrm{~cm}$ in diameter according to Shelton et al. [35]) as a rule of residue discrimination. Future iterations of the script method should require the implementation of this condition to be more comparable to field measurements.

Aside from reducing processing time and aiming to reduce effects from different users visually processing the photos, the script method is being developed to accumulate training and validation data sets for supervised and unsupervised statistical and machine learning approaches to residue estimation. The discrepancies and biases in the app vs. photo-grid and app vs. script methods, sampling error is a concern, as the reference counting method (photograph-grid and script) only sample a minute fraction of the millions of image pixels that the app classifies. The data appear to be bimodal and/or non-normally distributed, meaning that the error terms are underestimated using OLS. It is recommended that a generalized Poisson or Beta regression be performed when modeling the variance of these data. Even so, this study showed that the app can provide reliable and consistent measurements and that there is a potential for using apps (i.e., the FieldTRAKS Crop. Residue Estimator) to estimate residue over agricultural fields faster (i.e., each photo can be captured and classified in less than $3 \mathrm{~min}$ ) provided the underestimation is corrected (i.e., by applying the regression equation).

There are many advantages of using the Crop Residue Estimator app. It could be employed: (i) to provide the farmers and crop advisors who manage the province's cropland (i.e., corn, soybean and other grains) with the means to evaluate their crop residue cover easily; (ii) to provide farmers with convenient means of recording the results of the soil and water conservation practices applied on their farm (i.e., crop residue cover after harvest); (iii) to take advantage of mobile/communication technologies (i.e., tablets, smart phones) that are already purchased and used by landowners for other farm-related activities, such as collecting and organizing their field level operations; and (iv) to provide opportunities for farmers to demonstrate to government agencies and consumers their concern for soil and water conservation and their individual and collective on-farm efforts to improve environmental performance and sustainability of their land. These app-derived residue measurements could also be used for remote sensing data calibration and validation or for keeping track of the minimum recommended $30 \%$ soil cover throughout the year to reduce farmland topsoil and nutrient losses 
that impact water quality. Our future work will focus on exploring the use of app-derived residue estimates to determine whether very high resolution multi-spectral remote sensing datasets derived from Unmanned Aerial Vehicles (UAVs) could be used for future data collection efforts for high quality ground truth datasets of residue cover.

\section{Conclusions}

Mobile device remote sensing technologies and apps have attracted a great deal of interest from agricultural specialists, researchers and farmers in recent years. The interest in the use of these technologies in agriculture for deriving valuable information, measurements, or calculations has expanded rapidly over the last decade in conjunction with changes in technology as well as increase in computing and open-source image analysis software accessibility. There is no doubt that the use of apps among farmers will keep growing in the agricultural field and their use requires field testing and validation for different agriculture attributes. Therefore, studies aimed to evaluate apps' potential usefulness in estimating agricultural residue percentage are of value. In this study, we assessed whether the analysis methods employed in the Crop Residue Estimator app developed by FieldTRAKS Solutions can be suitable for crop residue cover assessment and therefore, can provide a convenient and rapid measurement alternative to conventional methods (i.e., line-transect) which are considered laborious and time consuming. Overall, this study found that crop residue cover estimates obtained by the app showed a high correlation and an underestimation with those achieved by reference methods (photograph-grid and script methods). In comparison to the complexity of the photograph-grid or script method for residue counting, the app can easily obtain residue estimates on fields through a simple supervised classification process using 4-8 representative training points from the image. Finally, this study showed that mobile devices equipped with apps can perform adequate digital crop residue analysis particularly for lower residue amounts $(<30 \%$ residue cover) below the threshold considered a conservation practice. However, for remote sensing studies where more accurate measurements of residue are necessary for providing ground-based corroboration datasets, the photograph-grid or the script based approach are recommended.

Acknowledgments: The authors gratefully acknowledge funding from Agriculture and Agri-Food Canada (AAFC), Ontario Ministry of Agriculture Food and Rural Affairs (OMAFRA) and the Water Resource Adaptation and Management Initiative (WRAMI) program for funding the development of the app, the script-based approach and evaluation of the methods. Special thanks to Kevin McKague of OMAFRA for encouraging development of the FieldTRAKS app and use of a tablet for app evaluation. The authors would like to thank all the farmers for access to their land for this study and Natalie Feisthauer from AAFC-STB Guelph and many students from the Universities of Waterloo and Guelph who assisted with fieldwork and processing the data. We also thank the editor and two anonymous reviewers for their helpful comments which greatly improved earlier versions of the manuscript.

Author Contributions: Ahmed Laamrani and Pamela Joosse conceived and designed the field experiments and photograph-grid analysis and app comparison while Ahmed was working at Agriculture and Agri-Food Canada. Renato Pardo created the MATLAB script and Dave Branson contributed to the app development. Ahmed, Renato and Aaron analyzed and interpreted the data at the University of Guelph which is the current affiliation/address of the three authors. Ahmed wrote the manuscript and has been responsible for handling correspondence at all stages of refereeing and publication. Renato and Dave contributed to the writing of MATLAB script and app sections, respectively. All the authors have contributed to revising the manuscript.

Conflicts of Interest: The authors declare no conflict of interest.

Disclaimer: The use: evaluation and reference to particular products by the authors of this manuscript should not be regarded as an endorsement by Agriculture and Agri-Food Canada or the University of Guelph.

\section{References}

1. Daughtry, C.S.T.; Hunt, E.R., Jr. Mitigating the effects of soil and residue water contents on remotely sensed estimates of crop residue cover. Remote Sens. Environ. 2008, 112, 1647-1657. [CrossRef]

2. Kumar, K.; Goh, K.M. Crop residues and management practices: Effects on soil quality, soil nitrogen dynamics, crop yield and nitrogen recovery. Adv. Agron. 1999, 68, 197-319. 
3. Mailapalli, D.R.; Burger, M.; Horwath, W.R.; Wallender, W.W. Crop residue biomass effects on agricultural runoff. Appl. Environ. Soil Sci. 2013, 2013, 805206. [CrossRef]

4. Enciso, J.; Nelson, S.D.; Perea, H.; Uddameri, V.; Kannan, N.; Gregory, A. Impact of residue management and subsurface drainage on non-point source pollution in the Arroyo Colorado. Sustain. Water Qual. Ecol. 2014, 3-4, 25-32. [CrossRef]

5. Aguilar, J.; Evans, R.; Daughtry, C.S.T. Performance assessment of the cellulose absorption index method for estimating crop residue cover. J. Soil Water Conserv. 2012, 67, 202-210. [CrossRef]

6. Molder, B.; Cockburn, J.; Berg, A.; Lindsay, J.; Woodrow, K. Sediment-assisted nutrient transfer from a small, no-till, tile drained watershed in Southwestern Ontario, Canada. Agric. Water Manag. 2015, 152, 31-40. [CrossRef]

7. Shelton, D.P.; Dickey, E.C.; Jasa, P.J.; Kanable, R.; Smydra Krotz, S. Using the Line-Transect Method to Estimate Percent Residue Cover. Biol. Syst. Eng. 1990, G-20. Available online: https:/ /digitalcommons.unl. edu/biosysengfacpub/289/ (accessed on 21 February 2018).

8. Huggins, D.R.; Reganold, J.P. No-till: The quiet revolution. Sci. Am. 2008, 299, 70-77. [CrossRef] [PubMed]

9. Lal, R. Sequestering carbon and increasing productivity by conservation agriculture. J. Soil Water Conserv. 2015, 70, 55A-62A. [CrossRef]

10. OMAFRA. Great Lakes Agricultural Stewardship: Helping Ontario Farmers Keep the Great Lakes Healthy. 2015. Available online: https://news.ontario.ca/omafra/en/2015/02/great-lakes-agriculturalstewardship. html (accessed on 21 February 2018).

11. Clarke, N.D.; Stone, J.A.; Vyn, T.J. Conservation tillage expert system for southwestern Ontario: Multiple experts and decision techniques. AI Appl. Nat. Resour. Manag. 1990, 4, 78-84.

12. Patni, N.K.; Masse, L.; Jui, P.Y. Groundwater quality under conventional and no-tillage: I. Nitrate, electrical conductivity and pH. J. Environ. Qual. 1998, 27, 869-877. [CrossRef]

13. Yang, W.; Sheng, C.; Voroney, P. Spatial targeting of conservation tillage to improve water quality and carbon retention benefits. Can. J. Agric. Econ. 2005, 53, 477-500. [CrossRef]

14. Kochsiek, A.E.; Knops, J.M.H.; Brassil, C.E.; Arkebauer, T.J. Maize and soybean litter-carbon pool dynamics in three no-till systems. Soil Sci. Soc. Am. J. 2013, 77, 226-236. [CrossRef]

15. Congreves, K.A.; Smith, J.M.; Németh, D.D.; Hooker, D.C.; Van Eerd, L.L. Soil organic carbon and land use: Processes and potential in Ontario's long-term agro-ecosystem research sites. Can. J. Soil Sci. 2014, 94, 317-336. [CrossRef]

16. Van Eerd, L.L.; Congreves, K.A.; Hayes, A.; Verhallen, A.; Hooker, D.C. Long-term tillage and crop rotation effects on soil quality, organic carbon and total nitrogen. Can. J. Soil Sci. 2014, 94, 303-315. [CrossRef]

17. Smith, P.; Martino, D.; Cai, Z.; Gwary, D.; Janzen, H.; Kumar, P.; McCarl, B.; Ogle, S.; O’Mara, F.; Rice, C.; et al. Greenhouse gas mitigation in agriculture. Philos. Trans. R. Soc. 2008, 363, 789-813. [CrossRef] [PubMed]

18. Ketcheson, J.W.; Stonehouse, D.P. Conservation tillage in Ontario. J. Soil Water Conserv. 1983, 38, $253-254$.

19. Dodd, V.A.; Grace, P.M.; Dickey, E.C.; Shelton, D.P.; Meyer, G.E.; Fairbanks, K.T. Determining crop residue cover with electronic image analysis. Biol. Syst. Eng. 1989, 3057-3063. Available online: https:/ / digitalcommons.unl.edu/biosysengfacpub/238/ (accessed on 21 February 2018).

20. Laamrani, A.; Joosse, P.; Feisthauer, N. Determining the number of measurements required to estimate crop residue cover by different methods. Soil Water Conserv. 2017, 72, 471-479. [CrossRef]

21. Pacheco, A.; McNairn, H. Evaluating multispectral remote sensing and spectral unmixing analysis for crop residue mapping. Remote Sens. Environ. 2010, 114, 2219-2228. [CrossRef]

22. Dehnen-Schmutz, K.; Foster, G.L.; Owen, L.; Persello, S. Exploring the role of smartphone technology for citizen science in agriculture. Agron. Sustain. Dev. 2016, 36, 1-9. [CrossRef]

23. Statistics Canada. Table 004-0243-Census of Agriculture, Farms Reporting Technologies in the Year Prior to the Census. 2016. Available online: http://www5.statcan.gc.ca/cansim/a26?lang=eng\&retrLang=eng\&id= 0040243\&tabMode=dataTable\&p1=-1\&p2=11\&srchLan=-1 (accessed on 22 February 2018).

24. Booth, T.D.; Cox, S.E.; Berryman, R.D. Point sampling digital imagery with "SamplePoint". Environ. Monit. Assess. 2006, 123, 97-108. [CrossRef] [PubMed]

25. Lynch, T.M.H.; Barth, S.; Dix, P.J.; Grogan, D.; Grant, J.; Grant, O.M. Ground Cover Assessment of Perennial Ryegrass Using Digital Imaging. Agron. J. 2015, 107, 2347-2352. [CrossRef]

26. Patrignani, A.; Ochsner, T.E. Canopeo: A Powerful New Tool for Measuring Fractional Green Canopy Cover. Agron. J. 2015, 107, 312-2320. [CrossRef] 
27. Karcher, D.E.; Richardson, M.D. Batch Analysis of Digital Images to Evaluate Turfgrass Characteristics. Crop Sci. 2005, 45, 1536-1539. [CrossRef]

28. Confalonieri, R.; Foi, M.; Casa, R.; Aquaro, S.; Tona, E.; Peterle, M.; Boldini, A.; De Carli, G.; Ferrari, A.; Finotto, G.; et al. Development of an app for estimating leaf area index using a smartphone. Trueness and precision determination and comparison with other indirect methods. Field Crops Res. 2013, 96, 67-74. Available online: http:/ /dx.doi.org/10.1016/j.compag.2013.04.019 (accessed on 21 February 2018). [CrossRef]

29. Campos-Taberner, M.; Garcia-Haro, F.; Moreno, A.; Gilabert, M.; Sanchez-Ruiz, S.; Martinez, B.; Camps-Valls, G. Mapping leaf area index with a smartphone and Gaussian processes. IEEE Geosci. Remote Sens. Lett. 2015, 12, 2501-2505. [CrossRef]

30. Vesali, F.; Omid, M.; Mobli, H.; Kaleita, A. Feasibility of using smart phones to estimate chlorophyll content in corn plants. Photosynthetica 2017, 55, 603-610. [CrossRef]

31. Salk, C.F.; Sturn, T.; See, L.; Fritz, S.; Perger, C. Assessing quality of volunteer crowdsourcing contributions: Lessons from the Cropland Capture game. Int. J. Digit. Earth 2016, 9, 410-426. [CrossRef]

32. Molinier, M.; López-Sánchez, C.A.; Toivanen, T.; Korpela, I.; Corral-Rivas, J.J.; Tergujeff, R.; Häme, T. Relasphone-Mobile and Participative In Situ Forest Biomass Measurements Supporting Satellite Image Mapping. Remote Sens. 2016, 8, 869. [CrossRef]

33. FieldTRAKS Solutions. (C) FieldTRAKS Solutions 2004-2016. Available online: http:/ /www.fieldtraks.ca/ (accessed on 15 January 2018).

34. Kludze, H.; Deen, B.; Dutta, A. Report on Literature Review of Agronomic Practices for Energy Crop Production under Ontario Conditions. 2011, pp. 494-504. Available online: https://ofa.on.ca/ uploads/userfiles/files/u\%20of\%20g\%20ofa\%20project-final\%20report\%20july\%2004-2011\%20 (accessed on 15 January 2018).

35. Kludze, H.; Deen, B.; Weersink, A.; van Acker, R.; Janovicek, K.; De Laporte, A.; McDonald, A. Estimating sustainable crop residue removal rates and costs based on soil organic matter dynamics and rotational complexity. Biomass Bioenergy 2013, 56, 607-618. [CrossRef]

36. Rasmussen, J.; Norremark, M.; Bibby, B.M. Assessment of leaf cover and crop soil cover in weed harrowing research using digital images. Weed Res. 2007, 47, 299-310. [CrossRef]

37. Shelton, D.P.; Kanable, R.; Jasa, P.J. Estimating Percent Residue Cover Using the Line-Transect Method. Bol. Syst. Eng. 1993. Available online: digitalcommons.unl.edu/cgi/viewcontent.cgi?article=1255\&context= biosysengfacpub (accessed on 21 February 2018).

38. R Development Core Team. A Language and Environment for Statistical Computing. 2011. Available online: http:/ / www.R-project.org/ (accessed on 15 January 2018).

39. Hilbe, J.M. Modeling Count Data; Cambridge University Press: New York, NY, USA, 2014; Chapter 9.

40. Singh, K.P.; Famoye, F. Analysis of Rates Using a Generalized Poisson Regression Model. Biom J. 1993, 35, 917-923. [CrossRef]

41. Smithson, M.; Verkuilen, J. A Better Lemon Squeezer? Maximum-Likelihood Regression with Beta-Distributed Dependent Variables. Psychol. Methods 2006, 11, 54-71. [CrossRef] [PubMed]

42. Cribari-neto, F.; Zeileis, A. Beta Regression in R. J. Stat. Softw. 2010, 34, 1-24. [CrossRef]

43. Ryu, D.; Famiglietti, J.S. Characterization of footprint-scale surface soil moisture variability using Gaussian and beta distribution functions during the Southern Great Plains 1997 (SGP97) hydrology experiment. Water Resour. Res. 2005, 41,1-13. [CrossRef]

44. Ferrari, S.; Cribari-neto, F.; Ferrari, S.L.P.; Cribari-neto, F. Beta Regression for Modelling Rates and Proportions. J. Appl. Stat. 2004, 31, 799-815. [CrossRef] 
Article

\title{
A Framework to Design the Computational Load Distribution of Wireless Sensor Networks in Power Consumption Constrained Environments
}

\author{
David Sánchez-Álvarez *, Marino Linaje and Francisco-Javier Rodríguez-Pérez \\ School of Technology, University of Extremadura, 10003 Caceres, Spain; mlinaje@unex.es (M.L.); \\ fjrodri@unex.es (F.-J.R.-P.) \\ * Correspondence: davidsa@unex.es; Tel.: +34-927-257-195
}

Received: 26 January 2018; Accepted: 20 March 2018; Published: 23 March 2018

\begin{abstract}
In this paper, we present a work based on the computational load distribution among the homogeneous nodes and the Hub/Sink of Wireless Sensor Networks (WSNs). The main contribution of the paper is an early decision support framework helping WSN designers to take decisions about computational load distribution for those WSNs where power consumption is a key issue (when we refer to "framework" in this work, we are considering it as a support tool to make decisions where the executive judgment can be included along with the set of mathematical tools of the WSN designer; this work shows the need to include the load distribution as an integral component of the WSN system for making early decisions regarding energy consumption). The framework takes advantage of the idea that balancing sensors nodes and Hub/Sink computational load can lead to improved energy consumption for the whole or at least the battery-powered nodes of the WSN. The approach is not trivial and it takes into account related issues such as the required data distribution, nodes, and Hub/Sink connectivity and availability due to their connectivity features and duty-cycle. For a practical demonstration, the proposed framework is applied to an agriculture case study, a sector very relevant in our region. In this kind of rural context, distances, low costs due to vegetable selling prices and the lack of continuous power supplies may lead to viable or inviable sensing solutions for the farmers. The proposed framework systematize and facilitates WSN designers the required complex calculations taking into account the most relevant variables regarding power consumption, avoiding full/partial/prototype implementations, and measurements of different computational load distribution potential solutions for a specific WSN.
\end{abstract}

Keywords: wireless sensor networks (WSN); energy efficiency; distributed systems; processing of sensed data; WSN distribution algorithms; recognition patterns; agriculture

\section{Introduction}

The breakthrough in wireless communications and electronics has enabled the rapid growth of Wireless Sensor Networks (WSNs). Low-cost hardware is enabling the massive deployment of WSNs due to their connection advantages, e.g., avoiding wiring infrastructure or places, allowing sensing of hard-to-reach locations or facilitating the transparency proposed in the ubiquitous computing vision of Weiser, among others. WSNs are used in many application areas (e.g., health, military, home, agriculture, etc).

Even though WSN is a very general term that includes many variations, authors generally agree that WSNs are composed of sensor nodes, which consist of detection/acting hardware (e.g., sensors and actuators), data processing capabilities (processor and memory-related hardware), communication hardware (e.g., transceiver, receiver, antenna and so on) and energy (e.g., power line, battery and related components). In WSNs, nodes can be homogeneous or heterogeneous and a special node 
(usually referred as sink, hub or gateway in the literature, according to its capabilities) is added when the network needs to coordinate or communicate with outside networks [1]. Not all WSNs need an explicit hub/sink, but we have included this special node in the research work because it is part of many WSNs, especially those used in agriculture, where usually a LAN communication infrastructure does not exist and WAN communications are performed by the sink/gateway. Compared to the sensor nodes, this special node may have different hardware and software including different sleep-wake intervals. Therefore, it may also require different power constraints in relation to the sensor nodes.

Currently, each WSN deployment supposes the study of many different alternatives and potential solutions during the analysis and design phases. What microcontrollers, communication modules, and protocols or network topologies are viable for a particular project? The project context is also especially relevant during the design phase. Is the WSN going to be deployed in a city? (where some communication networks may already exist). Do the nodes need to be battery operated due to the lack of electricity in the deployment area? These and other questions establish the requisites and impose constraints to the WSN that the designer must propose for the particular project. Even when designers may use state of the art and/or standard components, the way all these components are mixed and restrictions imposed by the deployment context leads many times to specific/ad-hoc WSN solutions.

The agricultural context has specific constraints where sensor nodes tend to be small, with limited processing resources, trying to cope with the low-cost deployments where many sensor nodes are required [1]. Even when the sensor nodes processing capabilities are not extraordinary, these nodes are able to detect, sampling information from the environment and, based on some local decision process, transmit the detected data to the user. In addition, for many WSN-related projects, including agriculture solutions, the battery is a common power source for a sensor node due to the lack of power-lines. In some cases, energy harvesting hardware, such as solar panels, are added depending on the suitability of the environment in which the sensor will be deployed. Anyway, battery-operated sensor nodes impose energy constraints that play a pivotal role in many scenarios. Designing a WSN capable to cope with these constraints can lead to viable or inviable solutions.

In [2] some of these elements are discussed and communication issues are identified as the most important power consumers. WSN design is complex and it requires many resources, mainly people, time and budget. Therefore, for many resource-constrained projects and according to the designer expertise, only a few solutions are evaluated.

This work focuses on a very specific aspect that, to the best of our knowledge, the literature does not fully cover, namely how computational load distribution can be used to decrease power consumption. According to our experience, this is a quite complex and a major time-consuming stage during the design phase. Thus, supporting designer while taking decisions during early phases of the WSN development will avoid the implementation, deployment, and measurement of different processing load distributions for the different potential WSN implementations.

To support the WSN designer, we have created a framework which energy efficiency goal is to maximize the useful life of the battery-operated nodes while coping with the rest of WSN requirements. The proposed framework helps with complex calculations, avoiding full/partial/ prototype implementations, deployments and measurements of different potential processing load distribution solutions for a specific WSN. Therefore, this framework should provide other theoretical advantages that we have not tested in this research work, such as decreasing cost and time-to-market, while supporting early design decisions during the analysis and design phases of a project involving a WSN. In this work, we also analyze how load distribution affects the energy consumption when the system scales. Our goal is to integrate our experience as well as the knowledge from other authors into the framework in order to facilitate designers work, getting to the most optimal solution regarding computation load distribution for greater battery-operated lifetimes.

The last part of this paper is used to apply the framework to a real scenario in a context quite common in our region, Extremadura, a mainly rural area of Spain where agriculture and cattle raising are very important pieces of the Gross Domestic Product (GDP). Results from the proposed framework 
are coincident with those exposed by other authors as well as our own experience as it is shown in the case study.

The rest of this document is organized as follows: in Section 2, the related works are presented. Next, we describe in Section 3 the materials and methods used and followed for the realization of this work. Section 4 details the design and implementation. In Section 5, the results obtained in the research are shown and discussed. Finally, in Section 6 the main contributions of this paper and some insights are provided.

\section{Related Work}

This Section details related works that represent most closely related research to our works, and focused on processing load distribution in WSNs. Load balancing is a well-known research topic in many computational contexts such as multicore processors, distributed networks and so on. One of the existing general methods for optimizing data processing is the optimal division of the processing load [3]. This leads to processing time saving, which in turn could be transmitted in energy saving at least taking into account only the processing elements of a sensor node. In order to realize the optimal division of the processing load, the first one that obtains the data optimally divide the processing load into $\mathrm{N}$ smaller parts (being $\mathrm{N}$ the number of processors).

The solution proposed by the authors in [3] is that when the $N$-th processor has the solution of its calculation, it begins to send back the solution to its immediate neighbor. The transmission takes a time $T$ and when the solution is received, it is sent with the solutions from previous neighbor and this transmission will have a duration of $2 T$.

For WSNs, another feature that must be taken into account is to differentiate when the data processing and transmission of the results can be done simultaneously, and when to do it sequentially. Depending on the full system requirements, some methods can take advantage over others. For example, in the case of performing the two things simultaneously, the required time for the processor to complete the task is usually lower, but power consumption is higher being the transceiver always on/sending. From the hub/sink point of view, the time to receive the data is higher, but consumption is lower because it only carries out shipment to the final node, so the communication part will only be active during the transmission time.

While the previous works make sense for many different processing contexts, due to the nature of the nodes and the connectivity that WSNs impose, it is not viable to apply directly the processing load balancing approaches from different research areas while maintaining power consumption low. Due to duty cycles of nodes in WSNs to minimize energy consumption, they are not always wake up, not always or never synchronized to other nodes, sleeping as long as possible. These features make load distribution term as used in this work more specific from the more general term "load balancing" which primary goal is to distribute a set of resources that will execute the work.

Many WSNs can be considered event-based distributed systems that differ from traditional communication networks in several ways: sensor networks have severe energy constraints, low-rate data, and many-to-one flows. The end-to-end routing schemes that have been proposed in the literature for mobile ad-hoc networks are not appropriate under these settings.

In [4], the potential performance improvement gained by balancing the traffic throughout the WSN is analyzed. It shows that sending the data generated by each sensor node through multiple paths, instead of a single path, allows significant energy conservation. A new analytical model for load-balanced systems is complemented by simulation to quantitatively evaluate the benefits of the proposed load-balancing technique. Specifically, is derived the set of paths to be used by each sensor node and the associated weights (i.e., the proportion of utilization) that maximize the network lifetime.

In [5], the usefulness of Error Correction Codes (ECCs) is evaluated from an energy perspective, the energy consumed in the coding-decoding and the transmission of additional "redundant" bits in relation to the energy saved. The authors present a framework for evaluating several ECCs based on an integral energy model of a sensor node. The framework supports the exploration of the design 
space of the sensor node with parameters related to the application and the implementation, such as the distance, the bit error rate, the path loss exponent, as well as the modulation scheme and the parameters of ECC. The results show that, compared to the transmission of non-coded data, the optimal energy ECC saves $15-60 \%$ of node energy for the given parameters.

The authors in [6] present a distributed algorithm to find the location of a vehicle. They prove that by distributing the calculation, the energy consumption can be greatly improved. An approach for such a distribution algorithm firstly estimates the processing where the data is produced and then transmitting the result to the sink/concentrator. Secondly, it estimates the load when all sensor nodes transmit their raw data to be processed in the hub/sink. The work shows that the power consumption depends not only on the amount of data of process load but also on the distance between the sensor and the base station.

In [7] a collaborative system for WSNs with limited energy and low processing capabilities is proposed. The WSN acts as a distributed signal processor, taking advantage of each sensor node processor, using a distributed algorithm, and collaborative communication scheme. Authors detect that the computation time decreases but it comes to a point where increasing the number of nodes, and computation time begins to increase, losing load distribution efficiency. Authors detail that regarding energy consumption, the greater the number of computational nodes and the required communications are, the greater power consumption is for each node. In this research work, the study is intentionally limited to the Fourier Transform algorithm and it is not extensible to other algorithms because it does not take into account different degrees of distribution.

In [8] power consumption while distributing computing load in a WSNs is studied. The paper focuses on the problem of scheduling information processing e.g., the sequence of message passing and calculations in WSNs. Processing time, energy consumption, and the rate of calculations are measured. The authors analyzed some specific computational algorithms, obtaining the scaling behavior for the computation time and the energy consumption. As in our study, and in order to be predictable, it is also assumed that the network topology is fixed and known.

The authors in [9] propose a data communication scheme that uses the adaptive Hierarchical Least Mean Square (HLMS) filter. The HLMS prediction techniques that predict the measured values at both the sensor node and the hub are analyzed and then sensor nodes are required only to send those readings that deviate from the prediction by an error threshold. This data reduction strategy achieves significant energy savings by reducing the amount of data sent by each node.

In [10], the authors design an efficient routing scheme in combination with energy saving techniques to improve the useful life of the WSN nodes. This document proposes a methodology that applies a data reduction scheme based on predictions to design an energy efficient routing protocol. From the results of the simulation, they inferred that the proposed protocol shows a better performance compared to the LEACH protocol and it increases the overall service life of WSNs.

In [11] the maximum rate at which computation and communication to the sink node are studied. The work is based on the fact that in many situations, an agent is not interested getting all the data from all the sensor nodes, but collecting from a sink node a relevant function of the sensor node measurements (sensor fusion, data aggregation or similar). The work focuses on symmetric functions, where only the data from a sensor is important, not its identity. Their analysis provides interesting insights about the complexity of the algorithms that could be performed by the sensor nodes or by the hub.

Even when none of the previous studies solve the issues stated in the Introduction, many of them provide valuable insights and knowledge that we will include in the proposed framework to support early decisions during the WSN design. The framework leverages the idea that balancing the nodes of the sensors and computational load hub/sink can lead to the improvement of the energy for all or at least the WSN battery-powered nodes. The approach is not trivial and takes into account issues such as the distribution of data required, nodes and connectivity and hub/sink availability due to its characteristics of connectivity and duty cycle. 


\section{Materials and Methods}

The use of WSNs in the agricultural context imposes a set of typical constraints, which may change from one project to another. More precisely, in extensive agriculture, the most common one in our region, WSNs generally requires a large number of sensors and a combination of medium and long-range communications. Thus, this context faces many problems such as power restrictions and signal propagation in the environment.

Regarding power restrictions, the process of routing data in WSNs can be affected largely by energy considerations, path, and radio link. When a WSN consists of a large number of sensors (on the order of tens of thousands or more) dispersed over a large area and depending on the time interval between samples, it may be more energy efficient to send measurement data from sensors to end nodes using data aggregation. Sensors are expected to have a useful life for a considerable period, e.g., months or years, which adds further energy consumption restrictions.

Regarding propagation in the environment, the sensor nodes deployed in the ground are sensitive to signal attenuation due to vegetal coverage, but also nodes deployed higher if they are between trees. Dense vegetal coverage may include even protected forest inside a farm. In large farms, the probability to have some points with no direct line of sight between the sensor and the hub/sink nodes is high.

Another energy problem comes from the WSNs in which the sensor nodes communicate with another node(s) in order to get their data to the hub/sink node because the distance is too far. Many authors have tried to cope with the problems of keeping the nodes awake for longer periods to be able to receive data and some synchronous solutions have been presented and standardized based on multi-hop and mesh architectures and protocols. While these solutions provide benefits in some situations, at least in our practice using some of them (e.g., ZigBee or RF mesh) they consume a larger amount of energy in comparison to other centralized architectures/protocols, that in opposite requires a central node always awake. This is understandable because they have to transmit more data due to the retransition/multihop as well as transmitting and receiving for longer periods to avoid exact synchronization. Thus, the sleeping periods in the microcontroller and/or the communication hardware are reduced and consequently the power consumption is increased.

In order to calculate the energy consumption of a sensor node, another parameter to take into account is the size of the packet that will be transmitted. Since the power required to transmit package depends on the packet size and the distance to the receiver node, the larger the size of the package is, the bigger the energy consumption of the node will be. Many WSNs, also in agriculture, can take advantage of telemetry protocols, decreasing packet overhead. Two restrictions that can arise are when data is larger than the one that the node can process (e.g., due to memory limitations) and when the operations required by the algorithm are not included in the microcontroller, making it processing last longer. Thus, when these restrictions arise, they could make inviable to process the data locally and it would be required to send it to the hub for processing. Therefore, microprocessors computational capabilities are quite relevant for this work.

Regardless of the schematic type of the sensor networks, WSN designers try to support the power saving operation modes for the nodes. The most obvious way to conserve energy is to turn off the transmitter when it is not needed. Although this energy-saving method apparently provides significant energy gains, an important point that should not be overlooked is that especially in connection-oriented protocols or when the transmission rate must be close to instantaneous, waking up the transmitter and stabling the connection can be greater than maintain the connection alive. As a result, operation in an energy-saving mode is only effective when the energy consumption balance between sleeping mode and wake up mode is positive. Depending mainly on the microcontroller and the transmitter operation modes, there may be a variable number of sensor node states while switching on/off the microcontroller(s), the memory(ies), A/D converter(s), and transmitter(s) and receiver(s) among others. State changes are characterized by their power consumption but also introduce some latency overhead. The threshold time is determined for the transition time from one mode to another and the individual power consumption of the modes in question. 
One method to calculate power consumption is by measuring the processing time for each microprocessor to perform the specified algorithm, which can be calculated by measuring the clock cycles necessary to process the data. Performing the calculations in a systematic way with our framework help designers to take into account all the involved variables. Furthermore, designers benefit from the framework capability to calculate the processing time required for a specific hardware to process a specific algorithm and estimate the time for other potential microprocessors that may be used in the project.

\subsection{Initial Approach}

One of the problems while designing a WSN is the time invested in communication issues such as the bandwidth required by the solution, technologies, speed, impact on the battery and so on. This complex design process is even more complicated due to the variability involved in distributing the data and/or processing between the hub/sink and sensor nodes. This variability also involves choosing whether such data is sent in different states from the sensor nodes to the hub/sink (e.g., raw, pre-processed or processed applying some kind of filtering or sensor fusion). The number of potential combinations is high and due to time or project cost constraints, many projects do not contemplate most of the possibilities and rush by choosing one or a small set of them for further study/ prototyping.

The solution we propose makes available to WSN designers and solution implementers a framework for early decision support, whose central body is a set of formulas. Based on different aspects such as the microprocessors used where and how data processing is performed or the quantity/time required for transmission/reception of the data among others, the result of the formulas would indicate where the main energy consumption is concentrated.

This framework supports decisions such as choosing the best-suited communication technology and processor for the nodes, both for the hub/sink node and for the sensor nodes. That is, the framework systematizes the estimation over a wide range of technologies and it shows the power consumption impact of each design choice almost instantly with little effort. The framework also facilitates the comparison when applying different or the same software centralized or distributed algorithms over the same or different hardware platforms.

The framework has been tested in real scenarios and it has shown its consistency with the knowledge that WSN experts have acquired through the years, e.g., in those solutions with battery operated nodes and a large number of samples per time unit, processing distribution (that may include data transmission) it is usually a good choice processing the data locally and sent it later only the event/resumed data. However, even for experts it may require quite a time to detect limits to this behavior, e.g., until what point will energy consumption decrease? This fundamental question and related ones can be easily answered by the numerical results systematized by our proposal. The framework does not take apart designers expertise. Indeed, this expertise can be also used to limit the variability of the potential solutions, decreasing, even more, the costs and development time. It has been designed to take into account several variations that WSN components may expose, such as using microcontrollers that may include or not communication capabilities inside a single IC or different power consumption modes among others. The proposed framework also simplifies unnecessary issues, e.g., it obviates sensor related issues, the sampling phase, since it is common to any of the potential solutions for a specific project so, sensoring power consumption is canceled when comparing different solutions. Many microcontrollers include many operation modes, allowing switching on/off different part of the controller (e.g., ADC, RTC when available, and so on). Cancelling sampling phase power consumption measures, the operation modes can also be simplified to three operation modes, i.e., processing, active and sleep modes.

The study was performed with the following requirements and restrictions:

(1) R0: The WSN is static. 
(2) R1: The WSN includes a hub/sink node that can receive and transmit data to the rest of the sensor nodes. Thus, communications between two sensor nodes go through the hub/sink in order to increase sleep time in sensor nodes.

(3) R2: Clustering between nodes is not used. Even when we think that the framework is extensible enough to include it, we have not tested in real scenarios. Therefore, we have not included it in this work.

(4) R3: All sensor nodes use the same hardware. Just for the framework to be easier to understand and direct apply it, while is direct to include heterogeneous sensor nodes.

(5) R4: Constrained power supply for sensor nodes, i.e., battery-operated sensor nodes. The uninterrupted power supply in the hub/sink (that can be achieved as in the case study through solar panels or other energy harvesting solutions when required), since the hub/sink must be always in $\mathrm{RX}$ state listening to asynchronous communications from the nodes.

\subsection{Computing Evaluation Performance Techniques}

Being one of the objectives of this work is to achieve the minimum energy consumption for a particular WSN, we must know the power consumption of each component of the WSN. Therefore, in this section, we analyze the power consumption of the microprocessors and the communication components used in the nodes.

Even being the communication phase the most power hungry one, another important part of the energy consumption occurs during the processing phase while the processor is wake up to process the data collected from the sensors. This power consumption depends on different factors, hardly related to two microprocessor capabilities: its supported operation modes (different awake/sleep modes from different processor vendors and families) and its performance (which depends on the cycles required by each instruction that may include different processing operations such as memory access, arithmetic-logic operations or floating point operations to name some of them).

To evaluate the performance of a processor we can use different techniques:

- Analytical (mathematical) models of the machine.

- Simulation (algorithmic) models of the machine.

- The actual machine.

On the one hand, we discarded using only real measures over the actual machine due to the cost to test a concrete algorithm with all the processors that want to be evaluated to be part of the network nodes. On the other hand, the first two alternatives should be used when the processor is not available physically or when the designer wants to save the time to test each algorithm of interest in each processor to compare them.

\subsubsection{Analytical Models}

The analytical models have limited scope of use due to the difficulty of expressing the detailed behavior of the processor and its workload in the form of mathematical equations. It is a model typically used in very early stages in the design of processors to make general performance estimates. When comparing different processors, it is necessary to establish the measurement criterion that allows quantifying the results of the comparison.

Time is a common unit of measure when comparing several processors, although the points of view of the different observers may lead to different conclusions. Thus, the user of a processor can say that processor A is faster than processor B when A executes its program in a shorter time than $B$. Instead, a person in charge of a computing center may think $B$ is faster than $A$ if the processor executes more jobs per unit of time. The first will be interested in the response time of the processor while the second will be in productivity (throughput). However, in both cases, the key is time: the processor that performs the same amount of work in the shortest possible time will be the fastest, the difference is whether we measure a task (response time) or many (productivity). 
To characterize the performance of a microprocessor, we use the execution time of the microprocessor to compute the data received from the sensors or from other nodes. However, the time it takes for a program to be executed by a computer can be difficult to measure due to multitasking operating systems (O.S.) and I/O devices that have response times that are independent of the computer clock frequency. Therefore, it is necessary to differentiate between the times it takes a CPU to execute the code of a program, the time used by the O.S. to perform their tasks, and the time needed to access the I/O devices. Response time is used as a measure of system performance (with the system not loaded), while CPU performance usually refers to the user's CPU time over an unloaded system [12]. Thus, we also discard its use in the proposed framework.

\subsubsection{Simulation Models}

Simulation models can be built more accurately, collecting detailed design specifications. However, these models require a great computational capacity when all the basic components of the processor are incorporated. An alternative is to use a set of programs representative of the actual workload that the machine will have. These programs are called benchmarks [13]. A general non-exclusive classification of benchmarks may be used according to the scope of the application they represent. Following this criteria, we can classify benchmarks as [14]:

- Integer: applications in which arithmetic is mastered, including search procedures, logical operations and so on, such as SPECint2000.

- Floating point: applications involving intensive real type numerical calculations such as SPECfp2000 and LINPACK.

- $\quad$ Transactions: applications strongly dominated by transactions on databases e.g., TPC-C.

And grouped by the nature of the program they implement:

- $\quad$ Real programs: compilers, word processors and other real applications. With them, we can get the most accurate measurements of real usage.

- Kernels: composed of snippets of code from real programs. Suitable for analyzing specific performance characteristics of a particular machine such as LINPACK and Livermore Loops.

- $\quad$ Benchmark suits: composed by a set of programs that measure the different operating modes of a machine such as SPEC, CoreMark, and TPC.

- $\quad$ Reduced/toy benchmarks: Reduced programs (10-100 lines of code) and known results. They are easy to enter and run on any machine (e.g., quicksort...).

- Synthetic benchmarks: An artificial code that does not belong to any user program and it is used to determine execution profiles such as whetstone and dhrystone.

We have chosen to include the CoreMark v1.0 in the framework proposed in this work, being focused on performance for low power microcontrollers. We apply it to estimate the performance of the processors for a particular algorithm based on the public results of the same processors running the simulation model. The procedure that the framework follows consist of measuring initially the performance of the processor without processing load and then performing the same operation while executing the data processing algorithm that would be deployed in the nodes.

CoreMark provides a method to test only the main features of a processor. The software returns data that can be used to calculate the performance and total consumption of the processor. From these results, we can obtain the performance ratio of the different potential processors to be used in the sensor network.

CoreMark software itself allows us to change parameters such as:

- The number of iterations

- Toolchain options and build/load/execute

- The method of acquiring a data memory block 
- The method of acquiring seed values

- The implementation in core_portme.c

- Settings in core_portme.h

Results obtained running this benchmark are CoreMark (instructions), CoreMark $/ \mathrm{MHz}$ (instructions per second) and CoreMark/Core (instructions per core). For the calculation of the Equation (1), we use the results obtained from the CoreMark/MHz value. The higher these values are, the higher the processor performance is. With these measures, we can already obtain a relation of the performance of a processor and obtain an estimate of the consumption when processing the data.

Once we have obtained the results of the processor performance with our data processing algorithm and without it, we can obtain the relationship that is used by our framework. Specifically, the framework uses this relationship as a correction factor of adjustment that is used to characterize the processing time of any processor in the WSN (Equation (1)). As can be seen from Equation (1), this correction factor is calculated as follows:

$$
F_{C}=\frac{T_{A}}{T_{B}}
$$

where $T_{A}$ is the time that the processor takes to execute the algorithm in the node and $T_{B}$ is the time that the same processor needs to execute the CoreMark software. This formula is used later to calculate the total consumption of the processor.

Equation (1) is one of the important parts of the framework since it calculates the estimated data processing time for the different potential microprocessors used in the WSN design. This approach allows the framework to estimate values quite close to the actual processor performance, without compromising the cost of the design phase, since we only need one hardware platform to run the algorithm once and the rest of the processor's performance can be estimated. In addition, with this measure, we can know how efficient the microprocessor is for a particular algorithm in relation to other microprocessors.

\subsubsection{Energy Consumption}

As mentioned previously, most of the energy consumption occurs while communicating nodes. So choosing a particular communication technology is one of the key decisions to take when designing the WSN. Sending and reception power consumption is different.

In Equation (2), the total power consumption for a node as specified in the support framework:

$$
\begin{aligned}
C_{\text {TotalNode }}= & \left(C_{\text {SLEEP }} \times T_{\text {SLEEP }}\right)+\left(C_{R X} \times T_{R X}\right)+\left(C_{T X} \times T_{T X}\right) \\
& +\left(C_{P R O C} \times T_{P R O C}\right)+\left(C_{A C T} \times T_{A C T}\right)
\end{aligned}
$$

The following pseudocode represents the phases of the different calculations necessary to obtain the results of the framework.

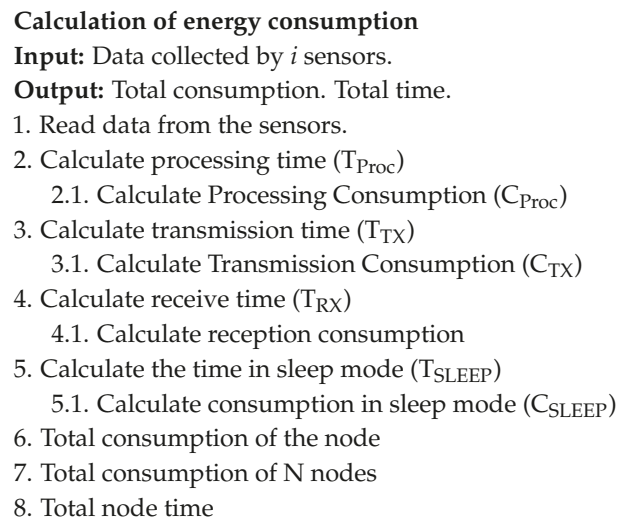


The Total Node Time consumption is the total consumption of the network (node hub/sink and sensor nodes) in the interval of $60 \mathrm{~min}$. In this way, we can know the node power consumption. This is very relevant for the designer, in order to now e.g., when it would be possible to feed the sensor node with a battery (and which type) or when it would require a more complex power source or even a powerline. Through the consumption in active mode $\left(C_{A C T} \times T_{A C T}\right)$ and $\left(C_{P R O C} \times T_{P R O C}\right)$, designers could infer if it is better to send or process data locally. The consumption in sleep mode $\left(C_{\text {SLEEP }} \times T_{\text {SLEEP }}\right)$ will help the designer to decide when it would be better to keep the node in active mode, because of the relation between the sleep mode and the number of times the node has to be activated.

Equation (2) is another important result of the framework since with it calculates the total consumption of the sensors nodes and the hub/sink. With this equation, designers can know an estimate of how the energy consumption will be taking into account the different times and consumptions of the different parts and processing that are performed:

$$
C_{\text {Total }}=\left(C_{\text {SensorNode }} \times D_{\text {NodeSensor }}\right)+\left(C_{\text {Hub/SinkNode }} \times\left(100-D_{\text {SensorNode }}\right)\right)
$$

where $D_{\text {SensorNode }}$ is the volume of data to be processed in the sensor node and $\left(100-D_{\text {SensorNode }}\right)$ is the rest of data that is processed in the hub/sink. $C_{\text {SensorNode }}$ and $C_{\text {Hub/SinkNode }}$ are the energy consumptions in a period of $60 \mathrm{~min}$, of the sensor node and hub/sink.

With Equation (3), the framework calculates an estimation of energy consumption according to the data processing distribution between the sensor node and the Hub/Sink making use of Equation (2).

Depending on the project, there will be cases in which there will not be bidirectional communication because there is the only transmission in a specific node or vice versa when the node only receives data from the WSN. Because sensors nodes can perform scheduled or event-based measurements, the time the microprocessor is in sleep mode is an estimation.

\section{Case Study}

To apply the research work to a real case study, we have selected a project that won a prize from the Telefonica Chair at the University of Extremadura. The project was initially developed without the support framework proposed in this study in order to detect the behavior of the proposed framework over an already developed WSN. The project requires the design of a WSN to detect and finally alert the farmers when their fruit trees are being stolen, providing tranquility to the farmers.

For this particular case study, the processing of the data can be split into two parts. For this specific case, one is the branch movement detection and the other is the theft pattern recognition. The pattern recognition is intended to differentiate the different causes by which the movements in the branches of the trees are produced, e.g., environmental causes (air) or animals. The movement detection is a kind data filtering, while the pattern recognition requires data from all the sensor nodes detecting movement to decide when to alert the farmer or not.

The difference between the two, apart from the precision, is that the tilt module has much less power consumption by far. Furthermore, it allows using simple interrupts keeping the sensor node in sleep mode for longer time periods and avoiding using the microcontroller ADC to decrease even more its power consumption while sampling.

An additional requirement for this project arises in order to camouflage sensor nodes in the branches of the trees, so no solar panel or other highlighters can be used. Thus, sensor nodes are power constrained and they must operate in sleep mode as long as possible. Regarding the hub/sink, when the sensor nodes need to sleep as long as possible, avoiding mesh architecture to eliminate retransmissions and nodes synchronization, it would be necessary to have the hub/sink awake for communication purposes. Without power-line and to protect the hub/sink from thieves, it would be required to install the hub/sink in an unreachable place so, it could use a solar panel. 
Depending on the farm and trees to protect, the number of sensor nodes to be deployed will be different. For the test, we used 15 sensors nodes on a private farm, this is where the importance of using the decision support framework proposed comes into play. It will support the decision of the designer regarding the best solution for the processing distribution while minimizing power consumption. It helps while analyzing different options such as processing the data locally or sending the data to the hub/sink to be processed or choosing a more complex computational load distribution approach, e.g., performing a filtering in each sensor node and process only this filtered information from all the sensor nodes at the hub/sink. We will analyze all of them through the framework that will support these project decisions based on data, not just intuitions and avoiding a tedious job that can lead to mistakes and waste of time.

This saves time and cost due to the hardware and time required to take all the related measures. While prototyping and to decrease costs, for this particular case study regarding the hardware, the use of a Raspberry Pi Zero was evaluated. It includes the Broadcom BCM2835 1GHz processor that we will evaluate to be used as hub/sink node. This node needs two communication modules, a GSM modem to alert the farmer (GSM/GPRS SIM800L with a SIM card for the case study) and another to communicate with the rest of the nodes. For the later, we will analyze ZigBee, Bluetooth, wi-fi and custom RF through a very low power communication module called NRG v1.0 PanStamp. The selected communication module will be also used for the sensor nodes as transceiver while ATmega2560 processor as well as MSP430F5529 are used as microcontrollers for the sensor nodes. These platforms were initially evaluated due to its low power profile. We have also selected them to demonstrate that the decision support framework is also able to deal with hardware that includes inside a single integrated circuit, computation (a Texas Instrument chip from the ultra-low power MSP430 family) and communication (a CC1101RF communication technology operating inside the ISM band at frequencies of 868-915 MHz, achieving an approximate range of 150-200 $\mathrm{m}$ with line of sight).

Based on the results collected by CoreMark and CoreMark for our algorithm, we obtain the correction factor applicable to the Equation (1). This correction factor is equal to 0.97. From this relationship, we can calculate the processing time $\left(T_{P R O C}\right)$ for the processors that we wish to use in the nodes of the network. In Table 1, can see the results of processing time obtained from the Equation (4) for different processors in relation to CoreMark and our algorithm:

$$
T_{A}=0.97 \times T_{B}=T_{P R O C}
$$

Table 1. Results CoreMark.

\begin{tabular}{ccc}
\hline Processor & CoreMark $(\mu \mathrm{s})$ & CoreMark Algorithm $(\mu \mathrm{s})$ \\
\hline Broadcom BCM2835 SoC $1000 \mathrm{MHz}$ & 2.06 & 2.014 \\
MSP430F5529 & 1.11 & 1.085 \\
ATmega2560 & 0.53 & 0.51 \\
\hline
\end{tabular}

As detailed in Table 1, the processor performance is affected when executing the data processing algorithm. This will make consumption and processing time a bit higher and will increase proportionally with the data.

The results of Table 1 are used in the framework in the Equation (4) for estimate the processing time of the data in the processor that you want to use. Therefore, based on the results can find the relationship of the processing time that exists at the time of the processing of data and raw data.

Table 2 shows a summary of different communication technologies in order to detail different parameters of technologies currently in use for WSNs and the typical power consumption. These parameters are those used in the framework for the calculation of consumption in the sending and receiving of data. It is also easy from this table to decrease the number of technologies to be compared due to the huge power consumption of some of them. 
Table 2. Consumption characteristics of different communication technologies.

\begin{tabular}{cccccc}
\hline Technology & $\begin{array}{c}\text { Speed } \\
\text { (Mbps) }\end{array}$ & $\begin{array}{c}\text { Package Size } \\
\text { (Bytes) }\end{array}$ & $\begin{array}{c}\text { Send/Reception } \\
\text { Time Package (ms) }\end{array}$ & $\begin{array}{c}\text { Consumption } \\
\text { Send (W) }\end{array}$ & $\begin{array}{c}\text { Consumption } \\
\text { Reception (W) }\end{array}$ \\
\hline Wi-Fi 802.11g & 54 & 1468 & 0.21 & 1.1 & 0.8 \\
Wi-Fi 802.11b & 11 & 1468 & 1.06 & 1.25 & 0.65 \\
Bluetooth 802.15.1 & 25 & $48^{1}$ & $0.015^{1}$ & $0.1^{1}$ & $0.01^{1}$ \\
ZigBee 802.15.4 & 0.250 & $672^{2}$ & $0.215^{2}$ & $0.001^{2}$ & $0.5^{2}$ \\
RF 868-915 MHz & 0.6 & 1460 & $1.02-6.4$ & 0.112 & 0.105 \\
\hline \multicolumn{7}{c}{$100 \mathrm{~m}$ of distance; ${ }^{2} 10 \mathrm{~m}$ of distance. }
\end{tabular}

The data in Table 2 are used to characterize and calculate the consumption of the sending/receiving of the data between the nodes of the network according to the communication technology that it is desired to use. As can be seen according to the communication technology used, the package size, range, speed and consumption are very different. Therefore, one of the parts to take into account in the design of a WSN is to select the most optimal technology.

In Table 3 a comparison of the power consumption when using different microprocessors/ microcontrollers, using different operation modes is detailed. These values are those provided by the manufacturers of the chips. The excerpt of processors corresponds to well-known vendors such as Texas Instruments, Atmel, and Broadcom. Some vendors do not use in their datasheets exactly the same operation mode names of the table headings. We have homogenized the information from the vendors in order to be able to compare different processors.

Table 3. Comparison of power consumption (in Watts) of different operation modes and processors.

\begin{tabular}{cccc}
\hline Processor & Active Consumption & SleepConsumption & Processing Consumption \\
\hline Broadcom BCM2835 SoC $1000 \mathrm{MHz}$ & 0.5 & $\mathrm{NaN}$ & 1.24 \\
MSP430F5529 & 0.03 & $4.8 \times 10^{-6}$ & 0.057 \\
ATmega2560 & 0.07 & $4.5 \times 10^{-6}$ & 0.413 \\
\hline
\end{tabular}

In Table 3 shows the energy consumption in the different operating modes, which is used in the framework to calculate the energy consumption according to the time in which they are operating in the different modes. For this case study, with the support of the framework, we will perform the calculations for the different parts of the WSN and we will see how the energy consumption behaves for the different processing distributions raised previously.

For the case of the theft detection algorithm, three different scenarios have been proposed:

1. Data is processed in the sensor nodes. On the sensor nodes, the implemented microprocessors have much less computing capacity than on the hub/sink node, which integrates a much more powerful processor. Therefore, as mentioned previously and as can be seen in Table 3, the data processing in the sensor node is much smaller, so to process the data will take more time that translates to a higher consumption. In this case, only $5 \%$ of the data is sent, which has already been processed. Theft detection can be performed on the sensor node because it needs few computational resources. Regarding the power consumption will be higher, since it must be more time in active mode and processing to be able to perform all the processing of the data.

2. Data is processed on the hub/sink node. In this case, there is not the problem of computational capacity, but the energy consumption in the sending of the data by the sensor nodes since they have to send many more data and that leads to greater consumption in the communication part. Consumption is increased because the volume of data is greater, $100 \%$ of the data collected by the sensors need to be sent. So, the number of packages to be sent is higher and it takes more time to perform it. Due to this, many parts of the sensor node hardware are working, especially communications impacting negatively on the node power consumption. 
3. Balanced distribution of the data. Part of the data is processed in the sensor node, and another part of the data is sent to the hub/sink node to be processed: this approach is the most balanced in both power consumption and data processing. In the case of data processing, when performing part of the processing in the sensor node we saved the consumption of having to send all the data. The processing consumption is much lower than the consumption to send the data. In this way, we also solve the problem of computation in the sensor nodes for the recognition of movement patterns. Therefore, some of the data collected by the sensors are sent, the rest of the data is processed at the node. For this third distributed scenario, the power consumption is the most balanced of the three evaluated scenarios, since the sensor nodes send a quarter of the data and have to process a smaller amount of data. The hub/sink node only has to be in charge to analyze the movement patterns of the theft and the few data coming to it from the sensor nodes.

One of the most important constraints is that the WSN will not have an unlimited energy source, only the hub/sink node. Because there can be large signal attenuation due to the forage of the trees, another essential requirement is where the nodes need to be placed. A wrong design decision here would make the communication with the hub/sink node impossible in case of theft, making the alert system fail.

We have initially proposed to place nodes in the different trees for the detection of the movement of the branches. These nodes will communicate with the hub/sink node, which has the communication with the external network to send the alert. The framework would allow us to compare different communication systems between nodes (e.g., Bluetooth, Wi-Fi, or ZigBee, among others) and with the Internet (e.g., GPRS, 3G or 4G, among others).

\section{Results}

This section shows the results obtained from the framework for all the nodes in the case study of fruit trees. Since this final alert is independent of the processing load distribution, the measurements of the hub/sink node have not taken into account the power consumption of sending data outside the network.

\subsection{Processor Performance}

We start looking at the performance of the microprocessors/microcontrollers, as detailed in Equation (1). Once their performance measurements were calculated with CoreMark v1.0, we proceed to perform the same measures, but this time running the thief detection algorithm that would be run by the WSN. In Table 4, the performance of the processors is detailed. It compares how the performance of processors is affected by running our processing algorithm or the CoreMark software.

Table 4. Performance of a selected set of microprocessors.

\begin{tabular}{ccccc}
\hline Processor & CoreMark (itr/s) & $\begin{array}{c}\text { CoreMark/MHz } \\
(\mu \mathbf{s})\end{array}$ & $\begin{array}{c}\text { CoreMark Alg. } \\
\text { Proc. (itr/s) }\end{array}$ & $\begin{array}{c}\text { CoreMark/MHz } \\
\text { Alg. Proc. }(\mu \mathbf{s})\end{array}$ \\
\hline Broadcom BCM2835 SoC 1000 MHz & 2066.91 & 2.06 & 2013.17 & 2.014 \\
MSP430F5529 & 27.70 & 1.11 & 26.9798 & 1.085 \\
ATmega2560 & 4.25 & 0.53 & 4.14 & 0.516 \\
\hline
\end{tabular}

As it is shown in Table 4, when executing the processing algorithm, the microcontrollers are slower and perform fewer instructions per second, i.e., the MSP430 and the ATMega cannot perform as many instructions per second as the Broadcom. At this point, we had a problem because both the MSP430 and the ATMega, cannot perform the same number of instructions as the Broadcom, getting blocked because they have less RAM to store data and results, so the decision was automatically made and the motion recognition patterns are executed on the Hub/Sink node. For the sensor nodes, the ATMega is discarded for the sensor node because its processing time and energy consumption in the different modes, as can be seen in Table 3, is greater than the MSP430. 


\subsection{Analysis of Energy Consumption as a Function of the Processing Load Distribution}

For the scope of the case study presented, we use the proposed framework to help with the energy consumption calculations for the different parts of the WSN for the different processing distributions studied. In addition, it will also show how the distribution of the load between the nodes sensor and the hub/sink node affects power consumption.

In Table 5, the energy consumption estimated in watts for the three load distribution scenarios and how their volume of data affects the energy consumption of the network. As can be seen in the table, the larger the size of packages to send is, the higher the consumption is, because the communication has to perform more transmissions.

Table 5. Estimated total network power consumption calculated by means of Equation (2).

\begin{tabular}{ccccccccc}
\hline Package Size (Bytes) & $\mathbf{2 0}$ & $\mathbf{5 0}$ & $\mathbf{1 0 0}$ & $\mathbf{2 0 0}$ & $\mathbf{5 0 0}$ & $\mathbf{1 0 0 0}$ & $\mathbf{2 0 0 0}$ & $\mathbf{5 0 0 0}$ \\
\hline Scenario 1 & 12 & 12 & 12 & 12 & 12.1 & 12.1 & 12.3 & 12.6 \\
Scenario 2 & 11.9 & 12.1 & 12.1 & 12.3 & 12.6 & 13.3 & 14.6 & 18.5 \\
Scenario 3 & 12 & 12 & 12.1 & 12.1 & 12.3 & 12.6 & 13.2 & 15 \\
\hline
\end{tabular}

In Figure 1 is depicted the total power consumption estimated with Equation (2) for the three scenarios studied.

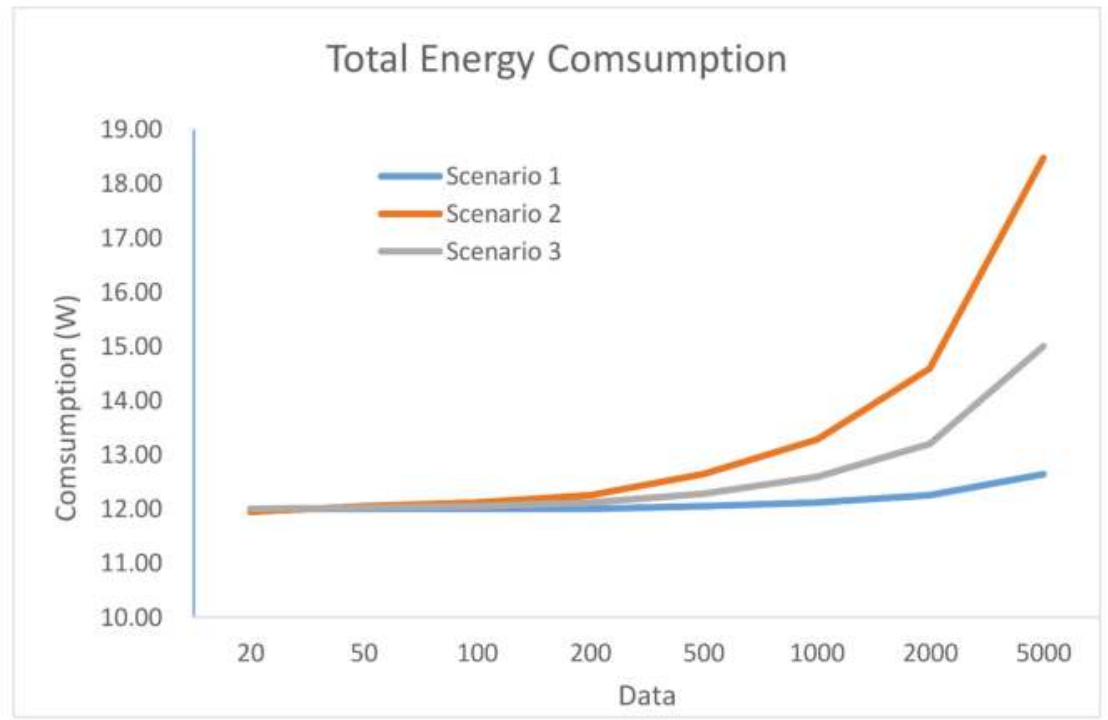

Figure 1. Energy consumption of the network.

As depicted in Figure 2, data transmission power consumption represents the most power hungry part of the total consumption shown in Figure 1. This indicates that it is necessary to look for a balance between processing and sending tasks.

With these results, the designer can take some decisions about how to perform the distribution of the data acquired by the sensor nodes, in order to achieve a balanced load of the data in the network. In this way, the designer also obtains an estimate of how the energetic consumption of the entire WSN will be. As the volume of data increases, the processing time in the sensor node also increases, because the processing is slower. Even when the selected microcontrollers would be able to run the full 
algorithm for the scenario 1 , in which $100 \%$ is processed in the sensor node, it would not be efficient due to its power consumption.

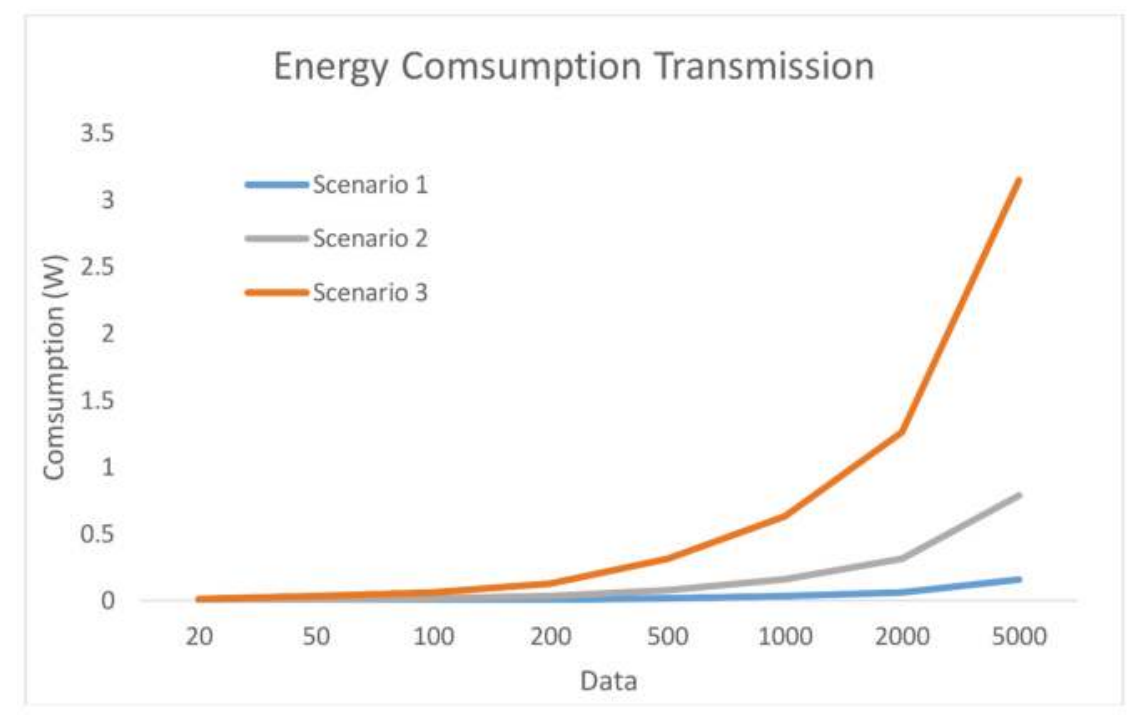

Figure 2. Energy consumption produced by the transmission.

For scenario 2, having to send all the data collected by the sensors nodes to the Hub/Sink, a problem of excessive energy consumption arise. Therefore, this distribution would not be useful to implement it in the network of fruit tree thefts due to the high consumption, especially in the sensor nodes that, according to the project requisites, must be battery-operated.

When considering the energy consumption required by the transmission and the processing time required by the sensor node, the third scenario is the most appropriate for this case study, allowing to achieve a balance between the processing and the consumption of the WSN sensor nodes.

From Equation (2), the energy of the sensor node and the hub/sink node cannot be estimated when the data is processed according to a specific load distribution. This is accomplished by Equation (3). Two load distributions: for the $60 \%$ of data volume processing in the sensor node (labelled Load 1 ) and 100\% (labelled Load 2) have been evaluated. These load distributions have been selected because these two distributions are the energy consumptions of the node and the hub/sink sensor node. For other load distributions, the energy does not converge, i.e., for a load lower than $60 \%$ distributions use the sensor node energy does not exceed the consumption of the hub/sink node.

From Figure 3, we conclude that as the data processing increases, it reaches a point where the energy consumption in the sensor node is greater than in the hub/sink node. It is in the case of Load 1 , so that the intercession of the power consumption of the sensors nodes and the hub/sink serves as a reference to take the right decision regarding load distribution, to achieve a model of energy consumption and more balanced processing time. Therefore, at a level of less than $60 \%$ load, it is more efficient to perform the processing in the sensor node, until the energy consumption of the sensor node begins to be higher than the hub/sink node. For distributions of loads less than $60 \%$, the energy consumption in the sensor node will be smaller, but the data processing time will increase. The load distribution between 60-90\% there is an energy consumption more balanced between the sensor node and hub/sink node. conversely, a load distribution over $90 \%$ power consumption is higher in the $\mathrm{hub} /$ sink node but gets the data processing is carried out at higher speeds. 


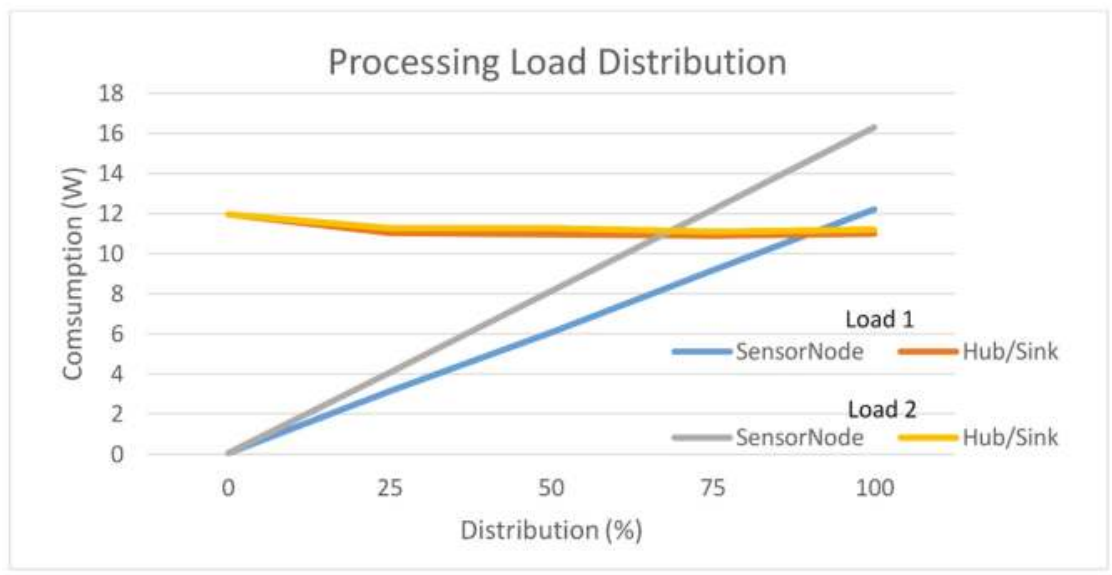

Figure 3. Energy consumption as a function of processing load distribution.

Figure 3 can help to make the decision how to achieve a lower energy consumption in the sensor node at the expense of a longer data processing time or, by the contrary, a greater energy consumption and shorter processing time. Depending on the purpose for which the network is being designed, it will be more interesting for the energy consumption to be lower or for the processing time to be shorter. Another thing to keep in mind is that if designer opts for a lower load distribution, the energy consumption in sensor nodes will be greater, which would shorten the network's useful life. Therefore, with the help of these results obtained with the framework, we can make the necessary decisions regarding load distribution and energy consumption in the design phase, before implementing the WSN.

The sensor nodes will be in sleep mode all the time until an event happens, branch movement, that will cause the node to wake up and send a transmission to the hub/sink node. On the contrary, the hub/sink node will be mainly in active mode and when receiving detection messages from the sensor nodes, the hub/sink node will enter into processing mode to process the pattern recognition.

Therefore, it has been decided that the movement patterns and the larger part of the data processing is carried out on the hub/sink node, apart from that the performance is greater than that of the sensor nodes which do not support a large another of the reasons is that being always in active mode the hub/sink node its consumption will be constant which can save us energy in the sensor nodes.

\subsection{Real Transmission and Coverage Measurement}

The proposed WSN design support framework is primarily based on estimations, no real platforms (only one real platform is required to estimate the relation stated in Equation (9)). In this section, the framework estimations are compared with the actual measurements when implementing the algorithm and deploying the WSN in a real environment. In Figure 4 the real power consumption measured at the sensor node during data transmission is depicted.

As observed in Figure 4, data transmission is programmed to be performed every $5 \mathrm{~s}$ to evaluate how the power consumption in the transmission related to the amount of data sent. As observed in this figure, the greater amount of information sent, the greater the consumption is.

Thinking about extending the theft of fruit to other relatively similar scenarios such as precision farming and thinking that it is not always feasible to visually camouflage the node in the branches effectively, we have included in this final part of the document different scenarios that could be evaluated. The terrain on which the measurements have been made is soil moist, wet due to rainfall the day before the measures, which can be matched to regular irrigation. The farm terrain is planted with 
olive trees and vines, as detailed in the top images of Figure 5. The branches of the trees are a good camouflage for the sensor nodes, but the vines may require the burial of the sensors (see Figure 5 right bottom, where we used a regular plastic Tupperware container just to take the signal measures to check the signal attenuation). Burying the sensors is not only interesting to sense the ground for many projects (e.g., ground $\mathrm{pH}$, humidity or temperature) but also to prevent vandalism and theft of the sensors themselves.

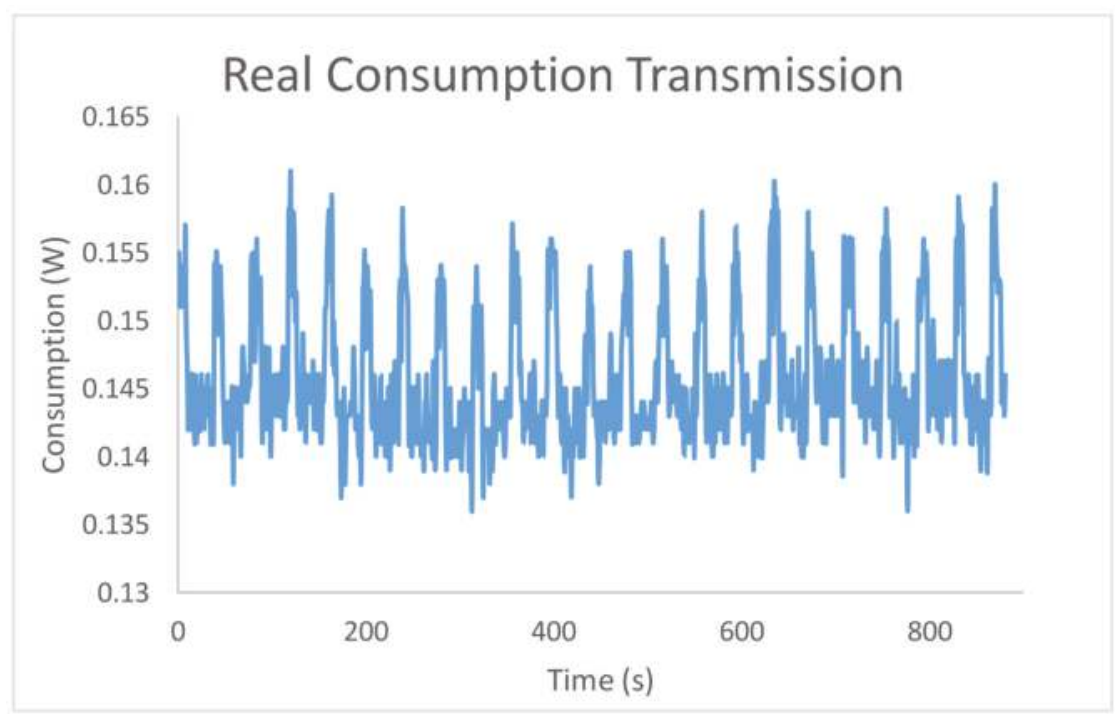

Figure 4. Real consumption of the data transmission.
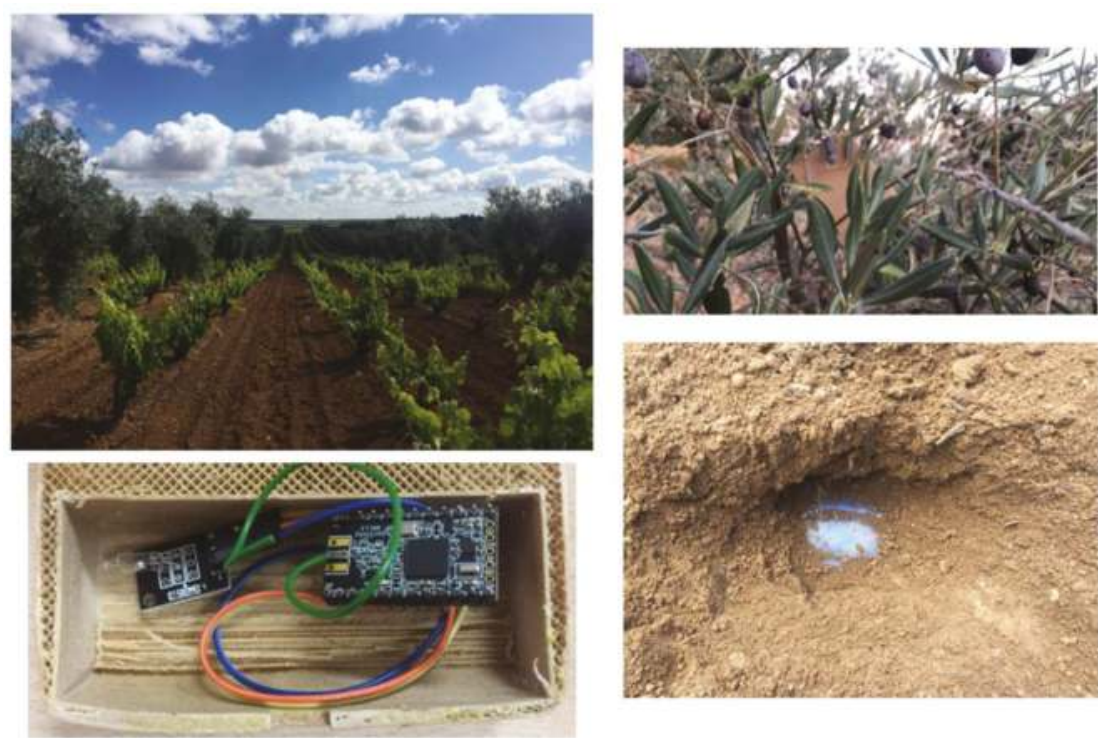

Figure 5. The farm where the measures of coverage and power of the signal were realized. The box includes the sensor node and batteries as well as hardware to check relevant parameters. 
The contemplated cases were as follows:

1. Sensor node at ground level with a line of sight to the hub/sink node.

2. Sensor node at ground level without line of sight to the hub/sink node (due to vegetation).

3. Sensor node below ground level and wet surface.

4. Sensor node above ground level.

Table 6 summarizes the actual distance measured for the different cases that have arisen.

Table 6. Real coverage measure for sensor nodes.

\begin{tabular}{cc}
\hline Scenario & Distance (m) \\
\hline Sensor node at ground level with direct view to the hub/sink node & 34.2 \\
Sensor node at ground level without direct vision to the hub/sink node & 22.5 \\
Sensor node below ground level and wet surface & 16.2 \\
Sensor node above ground level & 217.8 \\
\hline
\end{tabular}

As specified in Table 6, the obstacles (vines) greatly influence the distance at which the signal from the sensor node can reach to communicate with the hub/sink node. In the case where the sensor is above the level of obstacles (vines), the forage of the trees hardly influences the range of the signal. Thus, the distance measured is approximately the same as that indicated theoretically by the vendor. The sensor node is confined inside a hermetic 3D printed case (detailed in Figure 5 bottom left). As consequence, the state of the soil, in this case humid, would be another characteristic to take into account to design the WSN.

After making the measurements to know the power and the distance to which the signal arrives, we have been able to verify that apart from the obstacles that may exist and interfere in the communication making that distance is smaller, the size of the package also influences the distance to which it can be transmitted. The package of more data reaching much less distance than a package with less information.

In Table 7 is summarized the theoretical consumption that the manufacturers of these technologies provide for the different operation modes, using the same hardware that we had used for the case study.

Table 7. Comparison of consumption of the sensor and hub/sink node in the different operation modes.

\begin{tabular}{ccccc}
\hline & Active Mode (W) & Sleep Mode (W) & Processing Mode (W) & Sending Mode (W) \\
\hline PanStamp & 0.03 & $4.8 \times 10^{-6}$ & 0.057 & 0.17 \\
Raspberry Pi Zero & 0.5 & $\mathrm{NaN}$ & 1.24 & 0.67 \\
\hline
\end{tabular}

From the point of view of energy consumption (according to Table 7), there is a large difference in consumption between the hub/sink and sensor nodes. However, it is a good solution when we want to be able to process all the information that comes from the sensor nodes since we also need more computational capacity. The consumption of the hub/sink node with respect to the sensor node over time is also greater since it is always wake up (for that reason this fact is not contemplated in the table). Meanwhile, the sensor nodes most of the time are in sleep mode and they are only activated when one of the sensors activates the microprocessor of the sensor node.

\section{Conclusions}

In this work, we try to find the right computational load distribution between the microprocessors/ microcontrollers to be used in the sensor nodes that typically expose little storage capacity and slow data processing performance. However, the hub/sink may overcome these limitations and even when consuming more energy, it really helps when trying to minimize the power consumption of the whole or relevant parts of the network. Therefore, it is necessary to find a relationship between the processing 
time and the consumption of the transmission, and to be able to achieve a balanced consumption so that in this paper we demonstrate that the implementation of a sensor network in a real case is possible without having to use a continuous source of energy.

This work also proposes a support framework to help WSN designers in the early stages of the development in order to take decisions regarding computational load distribution in the network. Based on different aspects such as the type of microprocessors that are going to be used, where and how the data is going to be processed, or the quantity/time required for the transmission/reception of the data. The proposed framework will indicate to designers the hub/sink power consumption concentration. Thus, WSN designers can calculate effortless how different computational load distributions among sensor nodes and the hub/sink affect power consumption.

This framework will also help designers to take early decisions such as knowing which communication technology and which processor is best suited for the nodes and the hub depending on the complexity of the recognition pattern algorithm to be performed. The framework includes benchmark data, so it is a direct application to compare the performance of software algorithms on the hardware platforms. That is, designers can propose and evaluate in early stages a wide variability of technologies and see the impact of each choice compared to the rest instantly and with little effort.

The framework has been applied to a case study in order to understand better its behavior and usage. For the case study, an experimental network of wireless sensors for the detection of robberies in fruit trees was implemented. Key elements of this case study include detection, classification, and tracking. Three different computational load distributions were proposed and evaluated through the framework, showing the framework its strength helping with complex scenarios calculus and avoiding full/partial implementation that would increase project cost. The real measures were also performed over the same case study to evaluate the differences between the expected measures and the real ones. We really think that this original research based on previous works by other authors, can help WSN designers or support their decisions based on data as well as give advice to more novel ones.

Some papers have been analyzed/evaluated in the related work, but it is quite common in the literature to see hardware or software related works, but it is less common to see mixed ones, maybe due to the research topics imposed by some publications, only focused on hardware or software. Results shown in this research work clearly lead us to the opinion that it is not trivial the time to evaluate different scenarios with different hardware/software combinations. Where data is processed affect where the data must be available and this takes time to transmit/receive the information by the nodes, impacting power consumption of these nodes and in some cases other parts of the network. Due to this complexity, to find viable solutions in cost and power consumption, it is of vital importance to make the right decision in early development stages.

Acknowledgments: Applied Telematics Engineering and Advanced Communications Research Group, Department of Informatics and Telematics Systems Engineering, Department of Computer and Communication Technologies, Project TIN2015-69957-R (MINECO/FEDER, EU), Project International Institute of Investigation and Innovation of Aging (4IE), Ref. 0445_4IE_4_P. Project co-financed by the European Regional Development Fund (ERDF) through the Interreg V-A Spain Portugal Program (POCTEP) 2014-2020.
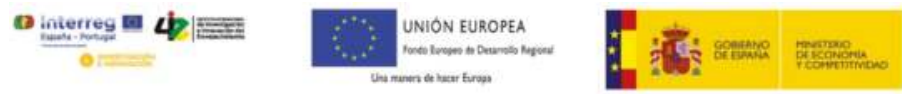

Author Contributions: D.S.-Á., M.L. and F.-J.R.P. conceived and designed the experiments; D.S.-Á. performed the experiments; D.S.-Á. and M.L. analyzed the data; M.L. and F.-J.R.P. contributed reagents/materials/analysis tools; D.S.-Á. wrote the paper.

Conflicts of Interest: The authors declare no conflict of interest. The founding sponsors had no role in the design of the study; in the collection, analyses, or interpretation of data; in the writing of the manuscript, and in the decision to publish the results. 


\section{References}

1. Tubaishat, M.; Madria, S. Sensor networks: An overview. IEEE Potentials 2003, 22, 20-23. [CrossRef]

2. Sohraby, K. Wireless Sensor Networks: Technology, Protocols, and Applications; John Wiley \& Sons: Hoboken, NJ, USA, 2007; p. 328.

3. Yick, J.; Mukherjee, B. Wireless sensor network survey. Comput. Netw. 2008, 52, 2292-2330. [CrossRef]

4. Al-Karaki, J.N.; Ul-Mustafa, R.; Kamal, A.E. Data aggregation in wireless sensor networks-Exact and approximate algorithms. In Proceedings of the 2004 Workshop on High Performance Switching and Routing, Phoenix, AZ, USA, 19-21 April 2004; pp. 241-245.

5. Bouabdallah, F.; Bouabdallah, N.; Boutaba, R. On Balancing Energy Consumption in Wireless Sensor Networks. IEEE Trans. Veh. Technol. 2009, 58, 2909-2924. [CrossRef]

6. Shih, E. Physical layer driven protocol and algorithm design for energy-efficient wireless sensor networks. In Proceedings of the 7th Annual International Conference on Mobile Computing and Networking (MobiCom ‘01), Rome, Italy, 16-21 July 2001; pp. 272-287.

7. Chiasserini, C.F. On the concept of distributed digital signal processing in wireless sensor networks. In Proceedings of the MILCOM, Anaheim, CA, USA, 7-10 October 2002; Volume 1, pp. 260-264. [CrossRef]

8. Khude, N. Time and energy complexity of distributed computation in wireless sensor networks. In Proceedings of the IEEE 24th Annual Joint Conference of the IEEE Computer and Communications Societies, Miami, FL, USA, 13-17 March 2005; Volume 4, pp. 2625-2637.

9. Tan, L.; Wu, M. Data Reduction in Wireless Sensor Networks: A Hierarchical LMS Prediction Approach. IEEE Sens. J. 2016, 16, 1708-1715. [CrossRef]

10. Singh, D.P.; Bhateja, V.; Soni, S.K. Prolonging the lifetime of wireless sensor networks using prediction based data reduction scheme. In Proceedings of the 2014 International Conference on Signal Processing and Integrated Networks (SPIN), Noida, India, 20-21 February 2014; pp. 420-425.

11. Giridhar, A.; Kumar, P.R. Computing and communicating functions over sensor networks. IEEE J. Sel. Areas Commun. 2005, 23, 755-764. [CrossRef]

12. Chouhan, S.; Balakrishnan, M.; Bose, R. A framework for energy consumption based design space exploration for wireless sensor nodes. In Proceedings of the 2008 ACM/IEEE International Symposium on Low Power Electronics and Design (ISLPED), Bangalore, India, 11-13 August 2008; pp. 329-334.

13. Shah-Mansouri, V;; Wong, V.W.S. Distributed maximum lifetime routing in wireless sensor networks based on regularization. In Proceedings of the IEEE GLOBECOM 2007-IEEE Global Telecommunications Conference, Washington, DC, USA, 26-30 November 2007; pp. 598-603.

14. Patterson, D.A.; Hennessy, J.L. Computer Organization and Design; Morgan Kaufmann: Burlington, MA, USA, 2007.

(C) 2018 by the authors. Licensee MDPI, Basel, Switzerland. This article is an open access article distributed under the terms and conditions of the Creative Commons Attribution (CC BY) license (http:/ / creativecommons.org/licenses/by/4.0/). 

MDPI

St. Alban-Anlage 66

4052 Basel

Switzerland

Tel. +41616837734

Fax +41 613028918

www.mdpi.com

Sensors Editorial Office

E-mail: sensors@mdpi.com www.mdpi.com/journal/sensors

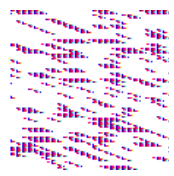



MDPI

St. Alban-Anlage 66

4052 Basel

Switzerland

Tel: +41 616837734

Fax: +41 613028918 